\title{
Bioluminescence Profiling of NanoKAZ/NanoLuc Luciferase Using a Chemical Library of Coelenterazine Analogues
}

Eloi P. Coutant, ${ }^{[\mathrm{a}]}$ Glwadys Gagnot, ${ }^{[\mathrm{a}, \mathrm{b}]}$ Vincent Hervin, ${ }^{[\mathrm{a}]}$ Racha Baatallah, ${ }^{[\mathrm{a}]}$ Sophie Goyard, ${ }^{[\mathrm{c}]}$ Yves Jacob, ${ }^{[\mathrm{d}]}$ Thierry Rose, ${ }^{[c]}$ Yves L. Janin ${ }^{*}[\mathrm{a}]$

[a] Unité de Chimie et Biocatalyse, Institut Pasteur, UMR 3523, CNRS, 28 rue du Dr. Roux, 75724 Paris Cedex 15, France.

[b] Université Paris Descartes, Sorbonne Paris Cité, 12 rue de l'Ecole de Médecine, 75006 Paris, France.

[c] Center for Innovation and Technological Research, Institut Pasteur, 25 rue du Dr. Roux, 75724 Paris Cedex 15, France.

[d] Unité de Génétique Moléculaire des Virus à ARN, Institut Pasteur, UMR 3569, CNRS, 28 rue du Dr. Roux, 75724 Paris Cedex 15, France.

\begin{abstract}
We describe here an extensive structure-bioluminescence relationship study of a chemical library of analogues of coelenterazine, using nanoKAZ/NanoLuc, a mutated luciferase originated from the catalytic subunit of the deepsea shrimp Oplophorus gracilirostris. Out of the $135 \mathrm{O}$-acetylated precursors, prepared using our recently reported synthesis and following their hydrolysis to give solutions of the corresponding luciferins, notable bioluminescence improvements were achieved in comparison with furimazine, currently amongst the best substrates of nanoKAZ/NanoLuc. For instance, the rather more lipophilic analogue 8-(2,3-difluorobenzyl)-2-((5-methylfuran-2yl)methyl)-6-phenylimidazo[1,2-a]pyrazin-3(7H)-one provided a 1.5-fold improvement of the total light output over a two hours period, a close to 3-fold increase of the initial signal intensity and a signal-to-background ratio 5 times greater than furimazine. The kinetic parameters for the enzymatic reaction were obtained for a selection of luciferin analogues and provided unexpected insights in the luciferase activity. Most prominently, along with a general substrate-dependent and irreversible inactivation of this enzyme, in the case of the optimized luciferin mentioned above, the consumption of 2664 molecules was found to be required to produce, by bioluminescence, a single Relative Light Unit (RLU; a luminometer-dependent fraction of a photon).
\end{abstract}

\section{Introduction}

As very well related in Prof. Osamu Shimomura's book, ${ }^{[1]}$ bioluminescence is based on the catalyzed oxidation of a substrate (a luciferin) by enzymes such as luciferases or photoproteins. A remarkably diverse set of marine genera, ${ }^{[2]}$ such as Renilla, Metridia, Gaussia, Periphylla or Oplophorus as well as Aequorea and Obelia, are using coelenterazine (1) as their substrate to produce a blue light, the most visible color undersea, with the help of very different enzymes encoded in their respective genome. ${ }^{[3]}$ The generally accepted mechanism of this photon emission was established using the photoprotein of Aequorea which can, arguably, be considered as a calciumdependent luciferase. ${ }^{[4]}$ As depicted in the scheme 1, this mechanism starts with an oxidative process leading to the hydroperoxide $\mathbf{2}$, which undergoes a cyclisation to give the endoperoxide/dioxetanone $\mathbf{3}$. This is followed by its decarboxylation leading to the occurrence of coelenteramide 4 in an exited state which can relax via the emission of a photon or via non radiative processes.$^{[5]}$ Of note is that, because of its inherent reactivity, coelenterazine can, without the recourse to a luciferin and under a variety of conditions, undergo a similar reaction leading to the production of a photon although in a far less efficient manner. ${ }^{[1]}$ 
<smiles>CC(C)(C)OOC(Cc1ccc(O)cc1)(Cc1ccc(O)cc1)OC1(Nc2ncc(-c3ccc(O)cc3)nc2Cc2ccccc2)OOC1=O</smiles>

Scheme 1. Mechanism for coelenterazine (1) bioluminescence.

Because of the ever-growing usefulness of bioluminescence-based reporting systems in life sciences, ${ }^{[6]}$ research have focused on improving the characteristics of the light signal (intensity, duration, and/or wavelength). In the case of the marine bioluminescent systems using coelenterazine (1), it is from the Oplophorus gracilirostris luciferase that the best optimization was achieved. The investigations on this luciferase first pointed out that few coelenterazine analogues were acceptable substrates ${ }^{[7]}$ and, probably more important, that its rather small $19 \mathrm{kDa}$ catalytic subunit was enough to produce a signal. ${ }^{[8]}$ From these results, an extensive mutation campaign of the catalytic subunit, combined with an array of substrates analogues, provided greatly improved bioluminescent reporting systems. ${ }^{[9]}$ These achievements included a better luciferase expression, a longer protein half-life as well as a far more intense and long-lasting signal. This was especially true when combining the mutant luciferase known as nanoKAZ/NanoLuc with furimazine (5) as its optimized substrate. ${ }^{[9 \mathrm{~d}]}$ However, some other luciferin analogues, such as compounds 6-9 and 10-12 (figure 1), were also reported to provide improved signals. ${ }^{[7,9 a, b, 10]}$ In an attempt to see if even more improvements were possible, we used our recently reported original synthesis of such luciferins ${ }^{[11]}$ and undertook a thorough structure-bioluminescence relationship study using the nanoKAZ/NanoLuc luciferase. 

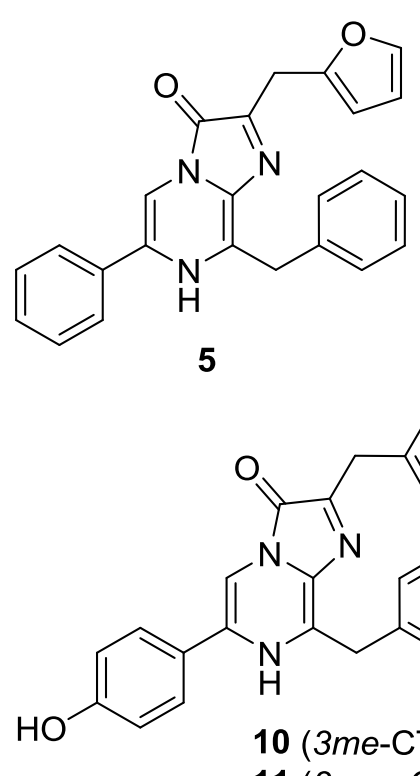

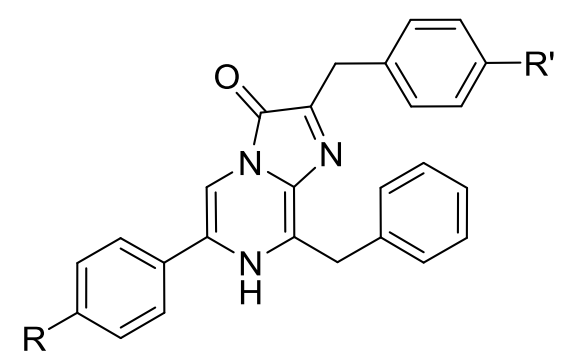

6 ( $h-\mathrm{CTZ}): \mathrm{R}=\mathrm{OH}, \mathrm{R}^{\prime}=\mathrm{H}$

7 (bis-CTZ): $\mathrm{R}=\mathrm{R}^{\prime}=\mathrm{H}$

8 (6h-f-CTZ): $\mathrm{R}=\mathrm{H}, \mathrm{R}^{\prime}=\mathrm{F}$

9 (f-CTZ): $\mathrm{R}=\mathrm{OH}, \mathrm{R}^{\prime}=\mathrm{F}$

Figure 1. Structures of selected artificial imidazo[1,2-a]pyrazin-3(7H)-ones luciferins.

\section{Results and discussion}

As depicted in the general synthetic scheme 2, our original synthetic approach is providing stable $O$-acetylated luciferins $\mathbf{2 5}$ which, for the vast majority, could be stored at room temperature for up to two years (no changes were seen in ${ }^{1} \mathrm{H}$ NMR spectra). The hydrolysis of their acetyl function (step xv, hydrochloric acid in ethanol and DMSO) then provides fresh acidic solutions of the considered luciferins 26. As opposed to the commercially available solution of furimazine (5) which is shipped in dry ice and has to be stored at $-20{ }^{\circ} \mathrm{C}$, these $O$-acetylated luciferins $\mathbf{2 5}$ are thus providing some improvement in the synthesis and handling of this class of reagents. The decision to provide users with the proluciferins 25 (which we have named hikarazines ${ }^{\circledR}$ ) was also based on disastrous purification trials of compounds $\mathbf{2 6}$ using chromatography. Accordingly, LC/MS analysis was the only method used to check the purity of the luciferin solutions resulting from this acidic hydrolysis. When undertaken, the LC/MS monitoring clearly proved that the hydrolysis of compounds $\mathbf{2 5}$ into the luciferins $\mathbf{2 6}$ was clean and complete in two hours at $50{ }^{\circ} \mathrm{C}$ or in 12 hours at $18{ }^{\circ} \mathrm{C}$. Moreover, thanks to the hydrochloric acid present, ${ }^{[1]}$ these solutions appears to be rather stable in closed vial for at least an additional 12 hours at $18^{\circ} \mathrm{C}$ and a relatively modest decay was noted after 14 days (see supplementary info). When stored for up to three months at $-20{ }^{\circ} \mathrm{C}$, such solutions still provided a robust bioluminescence signal. The occurrence of traces amount of, plausibly, a dehydroluciferin was noted in most cases (see supplementary info) but such compounds were absent from the ${ }^{1} \mathrm{H}$ NMR spectra of the starting material. Finally, extensive decomposition (mentioned in the tables below) was observed in few instances but this behavior was also seen for the corresponding $O$-acetylated precursors $\mathbf{2 5}$ (transformation of the isolated solid/wax into a black oil). In this regard, since we could not check the completeness and purity of every solutions made, we assumed that the hydrolysis of clean $O$-acetylated precursors 25, always led to clean solutions of the corresponding luciferins 26. This was of course double-checked to further establish the most important results described in the following (see supplementary info). Concerning the chemistry, to reach even more original luciferin analogues, we also extended the scope of some of the steps in this synthetic pathway. For instance, the hydroxypyrazines 19 were prepared either directly from the piperazin-2-ones 17, by an aromatization using sulfur and heat (step iv), or via an original reaction based on the sodium hydroxide-based dehydration (step vi) of the corresponding nitrone 18. When running reaction on relatively large scale $(20 \mathrm{~g})$ we usually found that undertaking this aromatization step via the nitrone $\mathbf{1 8}$ gave more reproducible results since any excess of sulfur, past two equivalents, tended to over-oxidize compound 19 and thus lower the reaction yield. The vast majority of the halogenated pyrazines were obtained using hot phenylphosphonic dichloride $(\mathbf{2 0}, \mathrm{X}=\mathrm{Cl}$, step vii), but in a few more delicate cases (e.g. $\mathrm{R}_{2}=$ tetrahydrofuran-2-yl) we resorted to a much milder procedure. This started (step viii) with the synthesis of the triflate intermediates $\left(\mathbf{2 1}, \mathrm{X}=\mathrm{OSO}_{2} \mathrm{CF}_{3}\right)$ and the corresponding bromo 
derivatives $(\mathbf{2 2}, \mathrm{X}=\mathrm{Br})$ were then prepared via an aromatic Finkelstein reaction (step ix) using sodium bromide. ${ }^{[12]}$ The $\mathrm{N}$-arylation (step x) of halogenopyrazines $\mathbf{2 0}$ or 22 most often proceeded well in acetonitrile at $60{ }^{\circ} \mathrm{C}$ although, in some problematic cases, the recourse to toluene at $90{ }^{\circ} \mathrm{C}$ greatly improved the yields. From the $N$-arylated compounds 23, a one pot procedure (step xii) proceeding via the sodium salt of acid $\mathbf{2 4}$ and leading directly to the $O$-acetylated luciferins 25 was used. When relevant, the removal of the benzyls protecting the hydroxyl groups to give analogues featuring phenolic functions was then achieved (step xvi). ${ }^{[11 \mathrm{~b}]}$ However, in at least five cases (25, $\mathrm{R}^{2}$ or $\mathrm{R}^{3}=\mathrm{BnOC}_{6} \mathrm{H}_{4}$ ), over-hydrogenation of the heterocyclic system and/or lack of an effective debenzylation became such an issue that we resorted to the preparation of the (deprotected) phenol-bearing acids $\mathbf{2 4}$ (steps xi and xiii). From these, a controlled cyclisation, using two equivalent of acetic anhydride (step xiv), led to the phenolbearing $O$-acetylated luciferins $\mathbf{2 5}$ without too much over-acetylation. 
<smiles>[R]C=C[N+](=O)[O-]</smiles><smiles>[R]CC1NC([R7])CNC1=O</smiles><smiles>[R]Cc1nc([R])cnc1O</smiles>

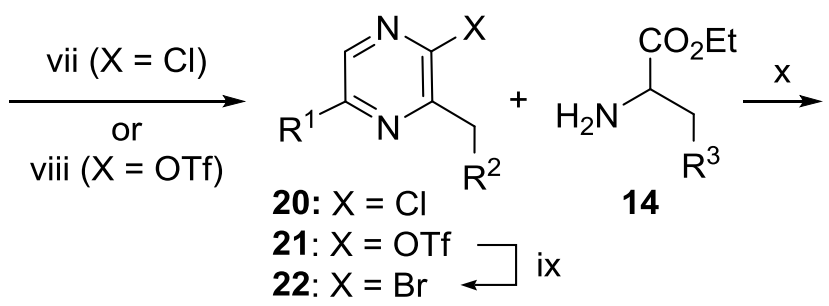<smiles>[R]Cc1nc([R])cnc1NC(C[R])C(=O)O[Na]</smiles>

23

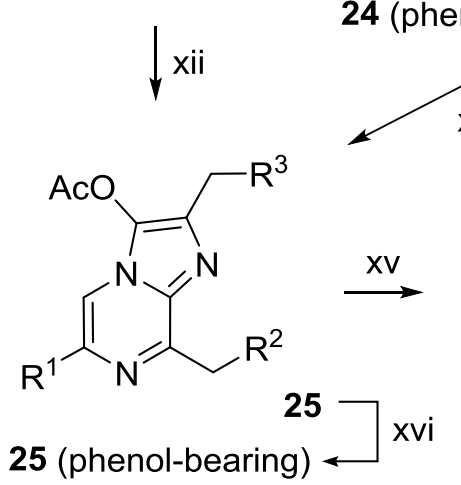<smiles>[R]Cc1[nH]c([R])cn2c(=O)c(C[R])nc1-2</smiles>

Scheme 2. i: neat, $20{ }^{\circ} \mathrm{C}, 10 \mathrm{~min}-12 \mathrm{~h}$. ii: $\mathrm{Zn}, 37 \% \mathrm{H}_{3} \mathrm{O}^{+} \mathrm{Cl}^{-}$, dioxane, $0-20{ }^{\circ} \mathrm{C}, 2 \mathrm{~h}$. iii: neat, $140{ }^{\circ} \mathrm{C}, 3 \mathrm{~h}$. iv: $\mathrm{S}_{8}$, 1,3- $\mathrm{Cl}_{2} \mathrm{C}_{6} \mathrm{H}_{4}$ or decaline, reflux, $10 \mathrm{~h}$. v: $\mathrm{AcOOH}$, AcOEt, $20{ }^{\circ} \mathrm{C}, 12$ h. vi: $\mathrm{NaOH}, \mathrm{EtOH}, 65{ }^{\circ} \mathrm{C}, 1$ h. vii: $\mathrm{PhPOCl}_{2}$, $100{ }^{\circ} \mathrm{C}, 12$ h. viii: $\mathrm{Tf}_{2} \mathrm{O}, \mathrm{NEt}_{3}, \mathrm{CH}_{2} \mathrm{Cl}_{2}, 2{ }^{\circ} \mathrm{C}, 40 \mathrm{~min}$. ix: $\mathrm{NaBr}, \mathrm{TfOH}, \mathrm{DMF}, 120{ }^{\circ} \mathrm{C}, 12 \mathrm{~h} . \mathrm{x}: \mathrm{Cs}_{2} \mathrm{CO}_{3}, \mathrm{Pd}(\mathrm{OAc})_{2}$, BINAP, $\mathrm{MeCN}, 60{ }^{\circ} \mathrm{C}$ or toluene, $90{ }^{\circ} \mathrm{C}, 12 \mathrm{~h}$. xi: a) $\mathrm{NaOH}$, THF, $20{ }^{\circ} \mathrm{C}, 12 \mathrm{~h}$, b) $\mathrm{NH}_{4} \mathrm{Cl}, \mathrm{H}_{2} \mathrm{O}$. xii: a) $\mathrm{NaOH}$, THF, $20{ }^{\circ} \mathrm{C}, 12 \mathrm{~h}$, b) $\mathrm{Ac}_{2} \mathrm{O}, 20{ }^{\circ} \mathrm{C}, 2 \mathrm{~h}$. xiii: $\mathrm{Pd} / \mathrm{C}, \mathrm{NH}_{4}{ }^{+} \mathrm{HCO}_{2}{ }^{-}$, EtOH, reflux, 90 min. xiv: Ac $2 \mathrm{O}$, AcOEt, reflux, $30 \mathrm{~min}$. xv: $37 \% \mathrm{H}_{3} \mathrm{O}^{+} \mathrm{Cl}^{-}$, DMSO, EtOH, $50{ }^{\circ} \mathrm{C}, 2$ h. xvi: $\mathrm{H}_{2}, \mathrm{Pd} / \mathrm{C}, \mathrm{AcOEt}, \mathrm{AcOH}, \mathrm{EtOH}, 20{ }^{\circ} \mathrm{C}, 12 \mathrm{~h}$.

We also explored the alternative preparation of some hydroxypyrazines $\mathbf{1 9}$ based on the regioselective condensation of 1,2-dicarbonyls and $\alpha$-amino amides. ${ }^{[13]}$ As depicted in scheme 3 , the hydroxypyrazine intermediates 19a-c were obtained in rather modest yields from phenylglyoxal (27) and $\alpha$-amino amides 28a-c 
with this attractively simple method. However, we have so far failed to generalize the use of this preparation since, as commented before, ${ }^{[14]}$ a lack of condensation regioselectivity plagued many of our trials, especially when starting from phenylalanine amide $\left(\mathbf{2 8} ; \mathrm{R}^{2}=\mathrm{C}_{6} \mathrm{H}_{5}\right)$. As hinted in the three examples depicted here, the reaction selectivity and yields are regularly better when starting from aliphatic $\alpha$-amino amides than from aromatic ones.

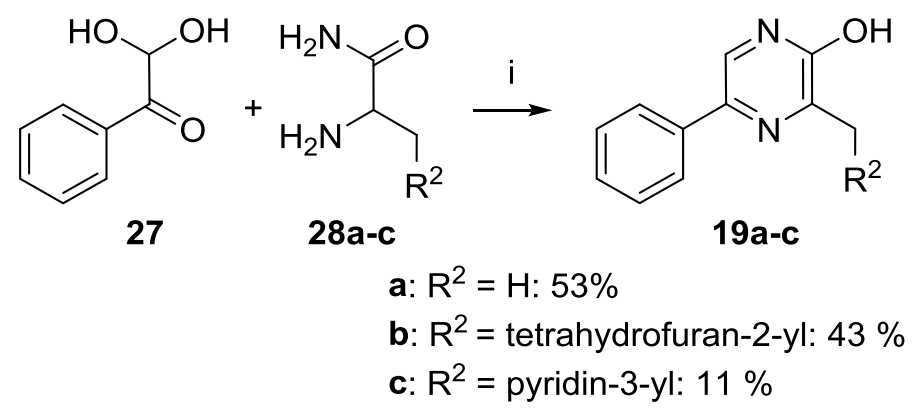

Scheme 3. i: a) $\mathrm{NaOH}, \mathrm{MeOH},-78{ }^{\circ} \mathrm{C}$ b) $\mathrm{H}_{3} \mathrm{O}^{+} \mathrm{Cl}^{-}$

With these approaches, out of selected combinations of 12 different nitrostyrenes $\mathbf{1 3}^{[15]}$ and two distinct $\alpha$-amino esters 14 chosen amongst 60 previously reported, ${ }^{[16]}$ we prepared $135 O$-acetylated imidazo[1,2- $\left.a\right]$ pyrazin-3(7H)ones proluciferins 25, which led after deprotection to the luciferins $\mathbf{2 6}$ listed in tables 1-7. An extensive description of the synthesis of these $O$-acetylated precursors along with a characterization of most of the synthetic intermediates is provided in the supplementary information section. Moreover, as depicted in scheme 4, the chemistry used to prepare the methyl-bearing proluciferins 30, 32 and 34 leading to the corresponding luciferins 35-37, also used the synthetic pathways shown in scheme 2. Compound $\mathbf{3 0}$ was thus obtained in 6 steps from the $\beta$ methyl nitrostyrene 29 and phenylalanine ethyl ester (PheOEt) in an $8 \%$ overall yield. The second proluciferin 32 was easily obtained in two steps from chloropyrazine 20a and the $\beta$-methyl phenylalanine derivative 31. ${ }^{[16 c]}$ One note is that our attempt to prepare a $\beta, \beta$-dimethyl homologue of compound 32 failed when trying to $\mathrm{N}$-arylate (step $\mathrm{x}$ in scheme 2) the corresponding $\beta, \beta$-dimethyl phenylalanine ester. Finally, a two steps preparation of compound 34 was achieved using the methyl-bearing chloropyrazine 33. This chloropyrazine was actually obtained by the more straightforward methylation of the chloropyrazine 20a which avoided a far longer synthetic alternative starting with compound 31. Again, a more detailed description of this chemistry is provided in the supplementary information section. 


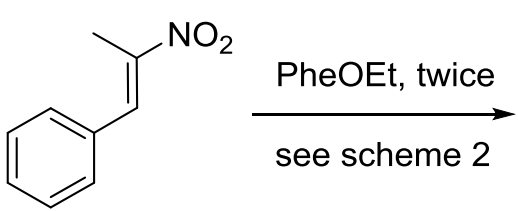

29<smiles>Clc1ncc(-c2ccccc2)nc1Cc1ccccc1</smiles><smiles>CC(c1ccccc1)c1nc(-c2ccccc2)cnc1Cl</smiles>

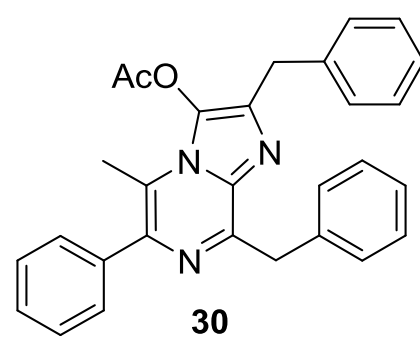

30

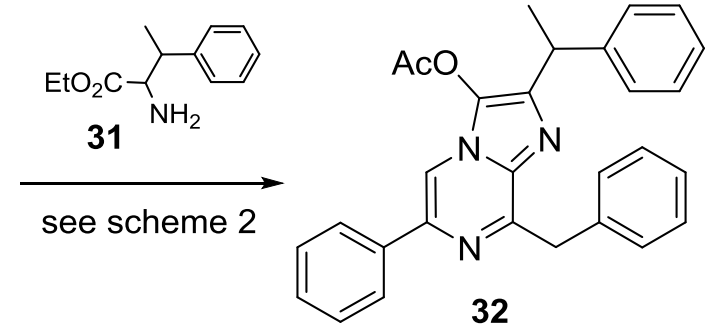

32

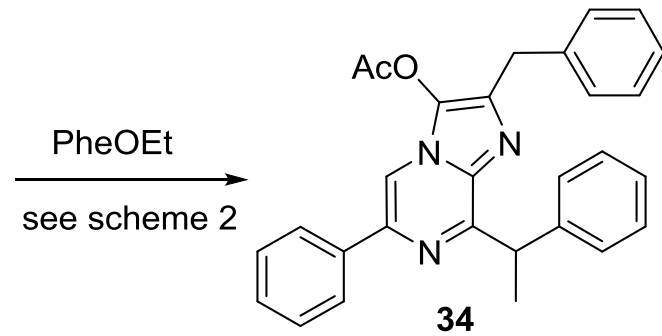

Scheme 4. i: a) LDA, THF, $-78^{\circ} \mathrm{C}$, b) MeI. $-78^{\circ} \mathrm{C}$ to $20{ }^{\circ} \mathrm{C}, 0.5 \mathrm{~h}$.

As listed in tables 1-7, the bioluminescence profiles of the resulting solutions of luciferins 26 were assessed with the nanoKAZ/NanoLuc luciferase using a Berthold Centro $\mathrm{XS}^{3}$ luminometer. These values were obtained in relative light unit (RLU) which only corresponds to a fraction of the photons emitted, this fraction actually depends on the luminometer used as well as its age. In any case, these values are completely appropriate for comparison if the same setting and the same machine are used throughout the experiments. Our assays thus led to bioluminescence profiles over two hours for each compound and we analyzed the results using four values which we normalized to the bioluminescence of furimazine (5) rather than the very weak one obtained with coelenterazine (1). The first value ( $\mathrm{I}_{\max }$ ) corresponds to the maximum intensity. In few cases, this value (as well as the $t_{1 / 2}$ ) can be strongly dependent on the laps of time existing between the addition of the luciferase and the beginning of the bioluminescence monitoring. Indeed, as we previously reported, ${ }^{[1 \mathrm{~b}]}$ initial intensities about three times more intense than furimazine but lasting only a few seconds after the addition of the luciferase in the medium were sometimes observed. In the main experimental setting, a (fixed) delay was unavoidable. For this reason, the $I_{\max }$ reported in table 1-7 may not do justice to the ephemeral brightness of few of the "flashy" luciferins tested. The second value $\left(t_{1 / 2}\right)$ corresponds to a comparison of the time when the signal has reached half of its measured initial intensity (over the two hours monitoring period). It is also dependent on the experimental setting but provides an idea of the signal decay compared to furimazine (5). The third value $\left(\mathrm{S}_{120}\right)$ is the sum of the signal observed over two hours relative to furimazine (5). Finally, measurement of the spontaneous emission of photons in the phosphate buffer in the absence of enzyme (autoluminescence) was systematically performed for all analogues. This provided a signal-to-background value $(\mathrm{S} / \mathrm{B})$, characteristic of the relative stability of these luciferins in this buffer, once again in comparison with furimazine (5).

Table 1 is illustrating some of the avenues we explored in the design of analogues featuring various aryls on position $\mathrm{R}^{3}$. Since variations on this position have been extensively investigated in the past, we only sought a confirmation of some of the results reported when using nanoKAZ. ${ }^{[9 d, 10 a, ~ i, ~ 17] ~}$ As previously reported, ${ }^{[10 a]}$ biscoelenterazine (7) is endowed with signal characteristics in par with furimazine (5) although the $t_{1 / 2}$ and $S_{120}$ values both indicate a faster decay over two hours. The para-fluoro luciferin $6 h$-f-coelenterazine (8) also displayed the reported $^{[10 a, i]}$ strong initial intensity but, as illustrated by its $t_{1 / 2}$ value, it was short-lived in comparison with 
furimazine (5). Interestingly, bioluminescence of the ortho- or meta-fluoro luciferins $26 \mathbf{a}$ and 26b displayed in both cases a loss of this strong initial intensity. An attempt to improve this with the 2,4-fluoroluciferin 26c only led, as reported, ${ }^{[10 \mathrm{i}]}$ to a signal pretty much equal to the 4-fluoroluciferin 8 . Moreover, replacing the 4-fluoro group by a 4-chloro (analog 26d) or a 4-bromo (analog 26e) led to luciferins of lesser interest. We also checked the three possible methyl-bearing luciferins, but only the meta-substituted analogue $\mathbf{2 6 g}$ displayed an improved bioluminescence. Indeed, the corresponding ortho isomer $\mathbf{2 6 f}$ was of no interest and the bioluminescence characteristics of the para isomer $\mathbf{2 6}$ here decreased by a factor of four compared to the meta-methyl isomer $\mathbf{2 6 g}$. A similar investigation with the three possible trifluoromethyl-bearing luciferins (26i-k) or methoxy-bearing luciferins (261-n) pointed out a similar "meta effect" for analogues $\mathbf{2 6 \mathbf { j }}$ and $\mathbf{2 6} \mathbf{m}$ in comparison with their respective isomers. The relatively important initial intensity of the para-methoxy analogue 26n has actually been noted before, ${ }^{[10 i]}$ and the same group claimed ${ }^{[17]}$ the slightly more intense signal for the meta-methoxy analogue 26m which we also observed. The two analogues featuring respectively a 3- and a 4-hydroxyphenyl group on $\mathrm{R}^{3}$, 260 and 26p, turned out to be of little interest in agreement with what has been reported in the past. ${ }^{[10 a, 17]}$ Finally, since the (original) meta-methyl luciferin $\mathbf{2 6 g}$ displayed the strongest initial intensity, we prepared and assayed the five alkyl-bearing analogues listed in at the end of table 1 . However, this met little success aside from the observation that the $\mathrm{t}_{1 / 2}$ of the meta-substituted alkyl derivatives $\mathbf{2 6 q}$ and $\mathbf{2 6} \mathbf{r}$ were rather better than their parasubstituted homologues 26s and $26 \mathbf{u}$.

\begin{tabular}{|c|c|c|c|c|c|}
\hline \multicolumn{6}{|c|}{ Table 1. Variations on $\mathrm{R}^{3}$} \\
\hline \multicolumn{6}{|c|}{$\mathrm{O} \quad-\mathrm{R}^{3}$} \\
\hline \multicolumn{2}{|r|}{$\mathrm{R}^{3}$} & \multirow{2}{*}{$I_{\max }$} & $\mathrm{t}_{1 / 2}$ & $S_{120}$ & S/B \\
\hline 5 & furan-2-yl & & 1 & 1 & 1 \\
\hline 7 & $\mathrm{C}_{6} \mathrm{H}_{5}$ & 1.3 & 0.4 & 0.8 & 1.4 \\
\hline $26 a$ & $2-\mathrm{FC}_{6} \mathrm{H}_{4}$ & 0.4 & $\overline{1.0}$ & $\overline{0.4}$ & 0.6 \\
\hline $26 b$ & $3-\mathrm{FC}_{6} \mathrm{H}_{4}$ & 0.7 & 0.7 & 0.6 & 0.9 \\
\hline 8 & $4-\mathrm{FC}_{6} \mathrm{H}_{4}$ & 1.2 & 0.4 & 0.6 & 1.5 \\
\hline $26 c$ & $2,4-\mathrm{F}_{2} \mathrm{C}_{6} \mathrm{H}_{3}$ & 1.0 & 0.3 & 0.5 & 1.5 \\
\hline 26d & $4-\mathrm{ClC}_{6} \mathrm{H}_{4}$ & 0.4 & 0.4 & 0.2 & 0.5 \\
\hline $26 \mathrm{e}$ & $4-\mathrm{BrC}_{6} \mathrm{H}_{4}$ & 0.3 & 0.2 & 0.1 & 0.5 \\
\hline $26 f$ & $2-\mathrm{MeC}_{6} \mathrm{H}_{4}$ & $<0.1$ & 1.4 & $<0.1$ & $<0.1$ \\
\hline $26 \mathrm{~g}$ & $3-\mathrm{MeC}_{6} \mathrm{H}_{4}$ & 1.3 & 0.6 & 1.0 & 1.6 \\
\hline $26 \mathrm{~h}$ & $4-\mathrm{MeC}_{6} \mathrm{H}_{4}$ & 0.3 & 0.3 & 0.1 & 0.3 \\
\hline $26 \mathbf{i}$ & $2-\mathrm{CF}_{3} \mathrm{C}_{6} \mathrm{H}_{4}$ & 0.1 & 0.9 & 0.1 & 0.2 \\
\hline $26 j$ & $3-\mathrm{CF}_{3} \mathrm{C}_{6} \mathrm{H}_{4}$ & 0.4 & 1.1 & 0.4 & 0.6 \\
\hline $26 \mathbf{k}$ & $4-\mathrm{CF}_{3} \mathrm{C}_{6} \mathrm{H}_{4}$ & 0.2 & 0.4 & 0.1 & 0.2 \\
\hline 261 & 2- $\mathrm{MeOC}_{6} \mathrm{H}_{4}$ & $\overline{0.4}$ & 0.5 & 0.2 & 0.4 \\
\hline $26 \mathrm{~m}$ & $3-\mathrm{MeOC}_{6} \mathrm{H}_{4}$ & 1.2 & 0.7 & 0.9 & 1.3 \\
\hline $26 n$ & $4-\mathrm{MeOC}_{6} \mathrm{H}_{4}$ & 0.7 & 0.3 & 0.3 & 0.9 \\
\hline $260^{[a]}$ & $3-\mathrm{HOC}_{6} \mathrm{H}_{4}$ & 0.2 & 0.1 & $<0.1$ & - \\
\hline $26 \mathbf{p}^{[\mathrm{a}]}$ & 4- $\mathrm{HOC}_{6} \mathrm{H}_{4}$ & 0.1 & 0.1 & $<0.1$ & - \\
\hline $26 q$ & $3-n-\mathrm{PrC}_{6} \mathrm{H}_{4}$ & 0.5 & $\overline{1.0}$ & 0.5 & 0.6 \\
\hline $26 r$ & 3-c- $\mathrm{PrC}_{6} \mathrm{H}_{4}$ & 0.6 & 0.9 & 0.5 & 0.6 \\
\hline $26 s$ & $4-n-\mathrm{PrC}_{6} \mathrm{H}_{4}$ & 0.2 & 0.2 & 0.1 & 0.2 \\
\hline $26 t$ & $4-i-\mathrm{PrC}_{6} \mathrm{H}_{4}$ & 0.3 & 0.4 & 0.1 & 0.3 \\
\hline $26 u$ & $4-c-\mathrm{PrC}_{6} \mathrm{H}_{4}$ & 0.3 & 0.3 & 0.1 & 0.4 \\
\hline ]: Th & . & & & $g$ & \\
\hline
\end{tabular}


Another direction, listed in table 2, was explored with the introduction of whole array of (hetero)cycles on $\mathrm{R}^{3}$. Since many analogues featuring this type of modifications have been previously reported ${ }^{[9 d, 10 i]}$ or patented, ${ }^{[17]}$ we also made and assessed some of these compounds along with original derivatives. In view of the bioluminescence of furimazine (5), the tetrahydrofuranyl and the dioxolane derivatives $\mathbf{2 6 \mathbf { v }}$ and $\mathbf{2 6} \mathbf{w}$ were made. Their very disappointing bioluminescence characteristics hinted for the necessity of an aromatic moiety on position $\mathrm{R}^{3}$. This requirement was further confirmed with the very weak bioluminescence of the cyclohexyl or cyclopentyl-bearing analogues 26x and 26y. Moreover, the benzyl homolog 26z turned out to be of little interest. The 2-pyridyl derivative 26aa was disappointing as the corresponding proluciferin turned out to be very unstable, thus prohibiting a proper assay of this luciferin. In view of all the work already published ${ }^{[10 \mathrm{i}]}$ on many 3-pyridyl derivatives, we did not study further variations on this heterocycle. Interestingly, the isomeric furan-3-yl derivative 26ab was found to provide about half the signal of furimazine (5) and the previously described (without bioluminescence data) ${ }^{[9 \mathrm{~d}]}$ thiophen-2-yl analogue 26ac led to a quite intense signal which decreased twice as fast as furimazine (5). Finally, we assessed the replacement of the furan by an oxazole or an isoxazole ring systems but none of the four analogues 26ad-26ag were of notable interest.

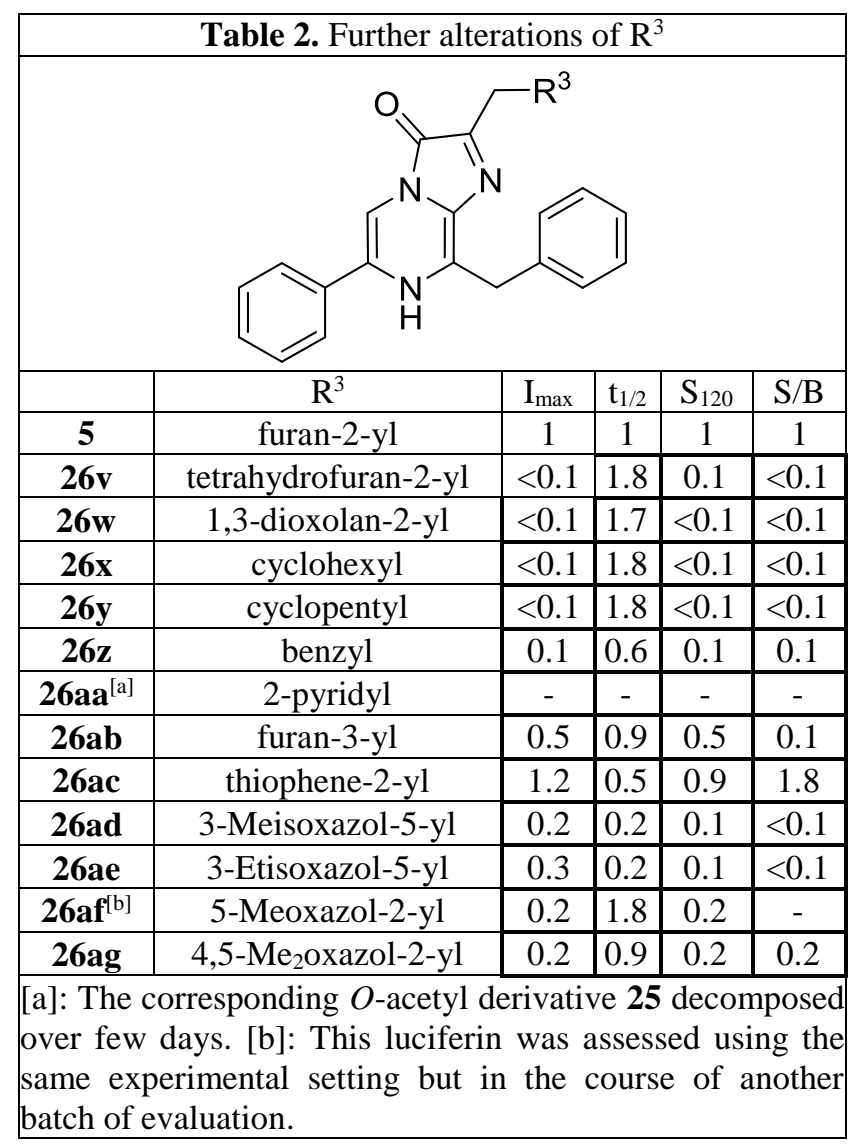

As depicted in table 3, a fairly large number of alkylfurans derivatives were also evaluated. The initial impetus for this part of our work was the half-life improvement seen in table 1 for the meta-tolyl derivative 26g, in comparison with bis-coelenterazine (7), which only differs by one methyl group. This approach turned out to be handsomely rewarding, although not as initially expected. Indeed, the 5-methylfuran-2-yl 26ah turned out to be endowed with a very strong initial intensity and half of the $t_{1 / 2}$ of furimazine (5). A patent published in the course of this study has actually depicted this luciferin analogue but, surprisingly, no mention of its bioluminescence properties was provided. ${ }^{[17]}$ Following these results, 5-alkylfuran-2-yl analogues 26ai-al were made and this pointed out that the methyl of 26ah or the ethyl group of 26ai led to the most intense signals. Moreover, past the $n$-propyl side chain of compound 26ak, the more hindered cyclopropyl side chain of 26aj caused a slight drop in intensity, and the $n$ pentyl-bearing analog 26al displayed a very weak bioluminescence. Of even more interest for these improved luciferins were the signal-to-background ratios which were about three times better than the reference furimazine (5). We also noted that despite a more modest intensity gain, the $n$-propyl derivative 26ak had a signal which 
lasted substantially longer than furimazine (5). A similar phenomenon was observed for the previously claimed ${ }^{[17]}$ 5-trifluoromethyl bearing analogue 26am although this took place with a fivefold loss of initial intensity. Further work led to the 4,5-dimethylfuran-2-yl derivative 26an, which retains some of these improved bioluminescence properties but, as seen for the 4,5,6,7-tetrahydrobenzofuran-2-yl analogue 26ao or the polyalkyl derivatives 26apar, further crowding of these positions was counterproductive. In view of these results, we also tried this approach with the alkylthiophene-bearing derivatives 26at-av. However, in comparison with the unsubstituted thiophenebearing luciferin 26ac, no such drastic improvement was observed. A more hindered 5-iso-butyl-bearing thiophene derivative was previously claimed although it appears to display only a third of furimazine initial intensity. ${ }^{[17]}$ Of note in this study was the much improved signal stability of the 3-methylthiophene derivative 26au.

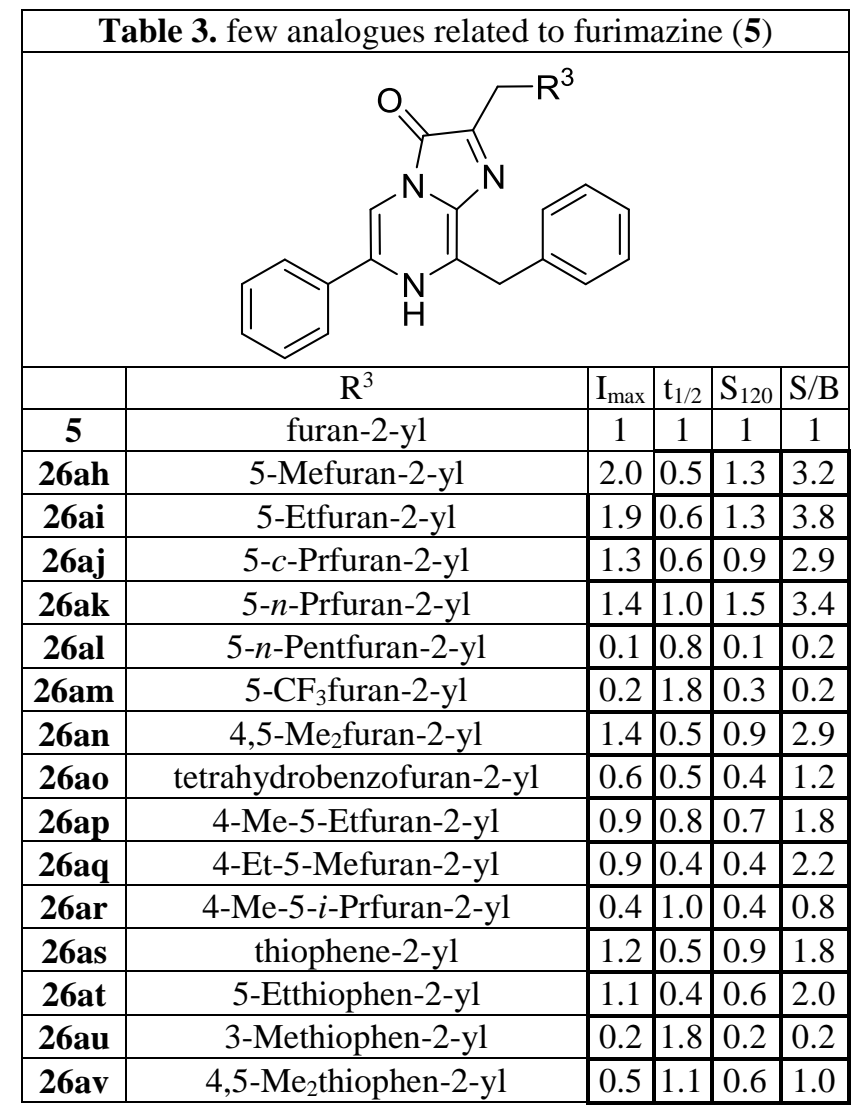

The beginning of table 4 is depicting our efforts to introduce an array of substituents on the aryl $\mathrm{R}^{1}$, while retaining either a 5-methylfuran-2-yl or a furan-2-yl on $\mathrm{R}^{3}$. These pointed out bioluminescence $\mathrm{I}_{\max }$ improvement for the luciferins 26aw, 26ax and 26ay featuring an ortho- or meta-fluorophenyl as $\mathrm{R}^{1}$. However, shifting the fluorine atom on the para position, as seen for compound 26az, drastically lowered this $I_{\max }$. Moreover, if a meta-tolyl as $\mathrm{R}^{1}$ provided luciferin $\mathbf{2 6 b a}$ with a less intense but remarkably stable signal, a similar signal collapse was observed if the methyl group was moved to the para position as seen for compound $\mathbf{2 6 b b}$. No such contrast was seen for the ortho-, meta- or para-methoxy-bearing derivatives $\mathbf{2 6 b c - b e , ~ w h i c h ~ a l l ~ l e d ~ t o ~ a n ~ e v e n ~ w e a k e r ~ b i o l u m i n e s c e n c e ~ t h a n ~}$ the one observed for coelenterazine (1) itself. Concerning hydroxy-bearing derivatives, we have already reported $^{[11 \mathrm{~b}]}$ the bright and short-lived bioluminescence of the furan-bearing coelenterazine analogue 26bf and a very similar observation was made for the 5-methylfuran-2-yl derivative $\mathbf{2 6 b g}$ in contrast with the rather less intense phenyl-bearing $h$-coelenterazine (6). A recent patent is actually confirming this observation for compound 26bf along with the mention that an irreversible inhibition of the luciferase is taking place. ${ }^{[18]}$ Concerning the irreversible aspect mentioned in this document, a two stage oxidation process can be suggested which would lead to reactive quinoid-bearing products. This "flash" behavior was also reported for an array of hydroxy-bearing analogues such as compounds $\mathbf{1 0 - 1 2}$ depicted in figure $1 .{ }^{[10 \mathrm{a}]}$ When moving this hydroxyl group to position 3 of aryl $\mathrm{R}^{1}$, we have already noted ${ }^{[11 \mathrm{~b}]}$ the far more stable signal provided by the furan-bearing analogue 26bh, but the introduction of a methyl on $\mathrm{R}^{3}$ as seen for the 5-methylfuran-2-yl derivative 26bi only slightly increased the initial 
bioluminescence intensity and lasted far less than furimazine (5). Moreover, in all appearance because of its hydroxyl function on aryl $\mathrm{R}^{3}$, compound $\mathbf{2 6 b j}$ - the bis-hydroxylated isomeric analogue of coelenterazine (1) - lost all its signal intensity. Finally, for the last four compounds 26bk-bn listed in this table, we retained a 2fluorophenyl as $\mathrm{R}^{1}$ and varied the nature of the $\mathrm{R}^{3}$ aryl group. For each case, an initial intensity improvement was

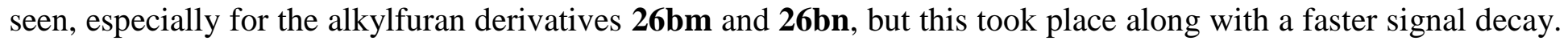
At this stage, we stopped pursuing similar alteration because, as mentioned in the footnote of this table, all too often the corresponding 2-fluoro-bearing proluciferins 25 turned out to be much less stable.

\begin{tabular}{|c|c|c|c|c|c|c|}
\hline \multicolumn{7}{|c|}{ Table 4. More elaborated analogues of furimazine } \\
\hline \multicolumn{7}{|c|}{${ }^{\mathrm{R}^{3}}$} \\
\hline & $\mathrm{R}^{1}$ & $\mathrm{R}^{3}$ & $\mathrm{I}_{\max }$ & $\mathrm{t}_{1 / 2}$ & $\mathrm{~S}_{120}$ & $\mathrm{~S} / \mathrm{B}$ \\
\hline 5 & $\mathrm{H}$ & furan-2-yl & 1 & 1 & 1 & 1 \\
\hline 26ah & $\mathrm{H}$ & 5-Mefuran-2-yl & 2.0 & 0.5 & 1.3 & 3.2 \\
\hline 26aw ${ }^{[a]}$ & $2-\mathrm{F}$ & 5-Mefuran-2-yl & 2.2 & $\overline{0.4}$ & 1.9 & - \\
\hline $26 a x$ & $2-\mathrm{F}$ & furan-2-yl & 1.4 & 0.8 & 1.2 & 1.2 \\
\hline 26ay & $3-\mathrm{F}$ & 5-Mefuran-2-yl & 1.7 & 0.5 & 1.1 & 3.6 \\
\hline $26 a z$ & $4-\mathrm{F}$ & 5-Mefuran-2-yl & 0.1 & 1.8 & 0.1 & 0.1 \\
\hline 26ba & $3-\mathrm{Me}$ & 5-Mefuran-2-yl & 0.4 & 1.2 & 0.4 & 0.7 \\
\hline $26 b b$ & $4-\mathrm{Me}$ & 5-Mefuran-2-yl & $<0.1$ & 1.4 & $<0.1$ & $<0.1$ \\
\hline $26 b c^{[a]}$ & 2-MeO & furan-2-yl & $<0.1$ & 1.8 & $<0.1$ & $<0.1$ \\
\hline 26bd & $3-\mathrm{MeO}$ & 5-Mefuran-2-yl & $<0.1$ & 2.2 & $<0.1$ & - \\
\hline 26be & 4-MeO & 5-Mefuran-2-yl & $<0.1$ & 1.8 & $<0.1$ & $<0.1$ \\
\hline 1 & $4-\mathrm{HO}$ & $4-\mathrm{HOC}_{6} \mathrm{H}_{5}$ & 0.1 & 0.6 & 0.1 & $<0.1$ \\
\hline $26 \mathrm{bf}$ & $4-\mathrm{HO}$ & furan-2-yl & 3.1 & 0.1 & 0.1 & 0.1 \\
\hline $26 b^{[b]}$ & $4-\mathrm{HO}$ & 5-Mefuran-2-yl & 2.6 & $<0.1$ & 0.1 & - \\
\hline 6 & $4-\mathrm{HO}$ & $\mathrm{C}_{6} \mathrm{H}_{5}$ & 0.5 & $<0.1$ & $<0.1$ & 0.1 \\
\hline $26 \mathrm{bh}$ & $3-\mathrm{HO}$ & furan-2-yl & 0.9 & 0.8 & 0.8 & 1.2 \\
\hline 26bi & $3-\mathrm{HO}$ & 5-Mefuran-2-yl & 1.2 & 0.5 & 0.7 & 2.1 \\
\hline $26 \mathrm{bj}$ & $3-\mathrm{HO}$ & $4-\mathrm{HOC}_{6} \mathrm{H}_{5}$ & $<0.1$ & 1.9 & 0.1 & $<0.1$ \\
\hline 26bk & $2-\mathrm{F}$ & $\mathrm{C}_{6} \mathrm{H}_{5}$ & 1.3 & 0.4 & 0.8 & 2.6 \\
\hline 26bl & $2-\mathrm{F}$ & $4-\mathrm{FC}_{6} \mathrm{H}_{4}$ & 1.3 & 0.2 & 0.5 & 2.2 \\
\hline $26 b^{[a]}$ & $2-\mathrm{F}$ & 5-Etfuran-2-yl & 2.6 & 0.4 & 2.3 & - \\
\hline 26bn $^{[\mathrm{a}]}$ & $2-\mathrm{F}$ & 4,5- $\mathrm{Me}_{2}$ furanyl & 2.1 & 0.3 & 2.0 & - \\
\hline \multicolumn{7}{|c|}{$\begin{array}{l}\text { [a]: The corresponding } O \text {-acetylated derivatives } \mathbf{2 5} \text { were } \\
\text { not stable over time, initial bioluminescence results only. } \\
\text { [b]: This luciferin was assessed using the same } \\
\text { experimental setting but in the course of another batch of } \\
\text { evaluation. }\end{array}$} \\
\hline
\end{tabular}

In table 5, we then set out to investigate the influence of substituents on $\mathrm{R}^{2}$, initially while retaining a phenyl on $\mathrm{R}^{3}$.

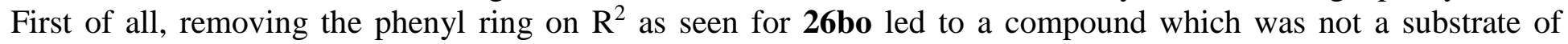
nanoKAZ luciferase. The introduction of the tetrahydrofuran-2-yl group seen in compound 26bp was not so good either and the 3-pyridyl of $\mathbf{2 6} \mathbf{b q}$ led only to a modest increase of initial intensity and overall signal. Moreover, nothing of interest was observed with the three hydroxyl-bearing isomers 26br-bt. On the other hand, when investigating the effect of a fluorine atom on the same $\mathrm{R}^{2}$ ring, the ortho- and meta-fluoro isomers $26 \mathbf{b u}$ and $26 \mathbf{b v}$ turned out to be substrates as good as bis-coelenterazine (1) whereas the para-fluoro analogue 26bw led to a very diminished bioluminescence. The same loss was observed for the para-fluoro derivative $\mathbf{2 6 \mathbf { b x }}$ in which the phenyl on $\mathrm{R}^{3}$ was replaced by a furan-2-yl moiety. Replacing the fluorine atom by a chlorine in position 2 of the $\mathrm{R}^{2}$ aryl led to compounds $26 \mathbf{b y}, \mathbf{2 6 b z}$ and $26 \mathbf{c a}$ which displayed very similar and pretty good bioluminescence profiles 
although of a lesser interest than analogue 26bu, featuring a 2-flurophenyl on $\mathrm{R}^{2}$ and a phenyl on $\mathrm{R}^{3}$, or furimazine (5). Shifting this chlorine atom to the meta position led to compound $\mathbf{2 6 c b}$, which lost a lot of signal intensity in

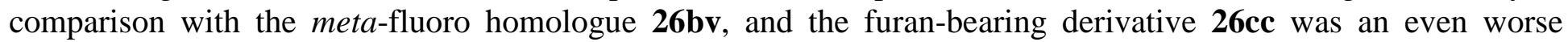

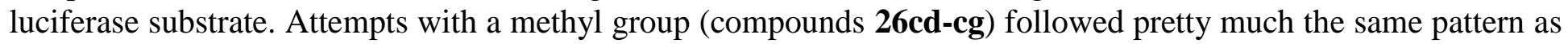
the methoxy-bearing derivative 26ch or the two analogs featuring a trifluoromethyl group 26ci-cj, which all led to an almost complete loss of luminescence properties. We then focused on derivatives featuring a 2-fluorophenyl or a 3-fluorophenyl moiety on $\mathrm{R}^{2}$ and a variety of substituents on $\mathrm{R}^{3}$. For instance, the combination of a 2fluorophenyl on $\mathrm{R}^{2}$ and a 3-methylphenyl for compound 26ck or a 3-methoxyphenyl for compound 26cl both led to improved signals in comparison with the profiles listed in table 1 for compounds $\mathbf{2 6 a}$ and $\mathbf{2 6 g}$. Such synergy on their bioluminescence properties was much less important (or not existing) for the analogues bearing a 3fluorophenyl on $\mathrm{R}^{2}$ when comparing compounds $26 \mathrm{cq}, 26 \mathrm{cr}$ and $26 \mathrm{cs}$ with their phenyl homologues $26 \mathrm{~g}, \mathbf{2 6 \mathrm { m }}$ and 8 listed in table 1 . In any case, this prompted us to combine the alkylfuran groups on $\mathrm{R}^{3}$ with a 2 -fluorophenyl or a 3 -fluorophenyl on $\mathrm{R}^{2}$. For the four analogues featuring a 2-fluorophenyl group (compounds $\mathbf{2 6} \mathbf{c m}$-cp), tangible improvements (often in their initial intensity), were observed in comparison with their phenyl homologue furimazine (5), compounds 26ah, 26ai and 26an listed in table 3. To a lesser extent this was also the case for derivatives with a 3-fluorophenyl group (compounds $26 \mathrm{ct}, 26 \mathrm{cu}, 26 \mathrm{cv}$ and $26 \mathrm{cw}$ ) although the improvements were more tangible on their bioluminescence half-life than on their initial intensity. 


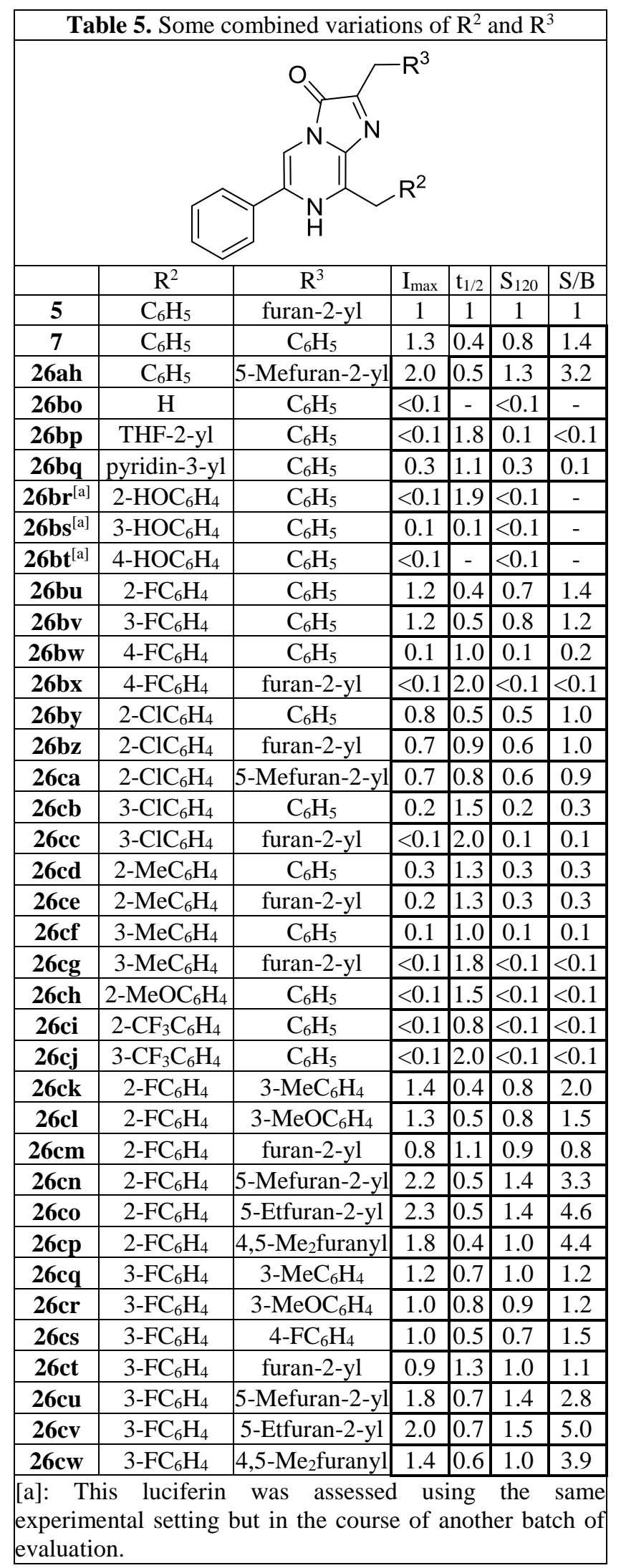

These interesting effects of fluorine drove us to undertake the fairly systematic synthesis and bioluminescence evaluation of luciferins analogues bearing two fluorine atoms on the aryl $\mathrm{R}^{2}$, depicted in table 6 . However, in view

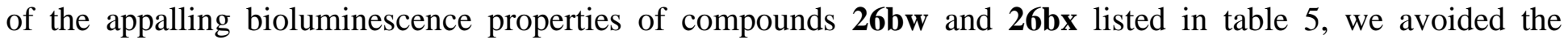
synthesis and evaluation of analogues featuring a fluorine on the para position of $\mathrm{R}^{2}$. The results are pointing out a 
few tendencies. The most prominent one is that, out of the four groups of analogues made, the influence of these two fluorine atoms could be classed. The best luciferins featured a 2,3-difluorophenyl moiety (compounds 26djdr) and this was followed by 2,6-difluorophenyl-bearing derivatives 26cx-da and then the much less bioluminescent analogues 26db-de and 26df-di, respectively harboring a 3,5-difluorophenyl or a 2,5difluorophenyl component. In every case, we studied the effect of small alkyls on the furan ring on $\mathrm{R}^{3}$ and, within these four groups of difluorophenyl derivatives, a contribution pattern was recurrent. Indeed, a methyl and then an ethyl on carbon 5 of the furan provided the most improved initial intensity then, to a lesser degree, a 4,5dimethylfuran had also a positive effect; all this in comparison with the compounds only featuring an unsubstituted furan ring. In view of the really strong bioluminescence improvement of the 2,3-difluorophenyl derivatives 26dl and 26dm, we also prepared further furan-bearing analogues with small alkyl substituents (26do-dr) but no additional improvement was secured and the same pattern observed in table 3 for the phenyl-bearing analogues 26ao-ar was seen. Equally interesting was the profile of compounds 26db or 26df, which displayed only a third or a fifth of the initial intensity of furimazine (5) but had about twice its half-life. Finally, the poor bioluminescence of the last two analogues depicted in this table, the 2,3,5-triflurophenyl-bearing derivatives 26ds and 26dt, answered the question regarding a possible additive effect of a third fluorine atom.

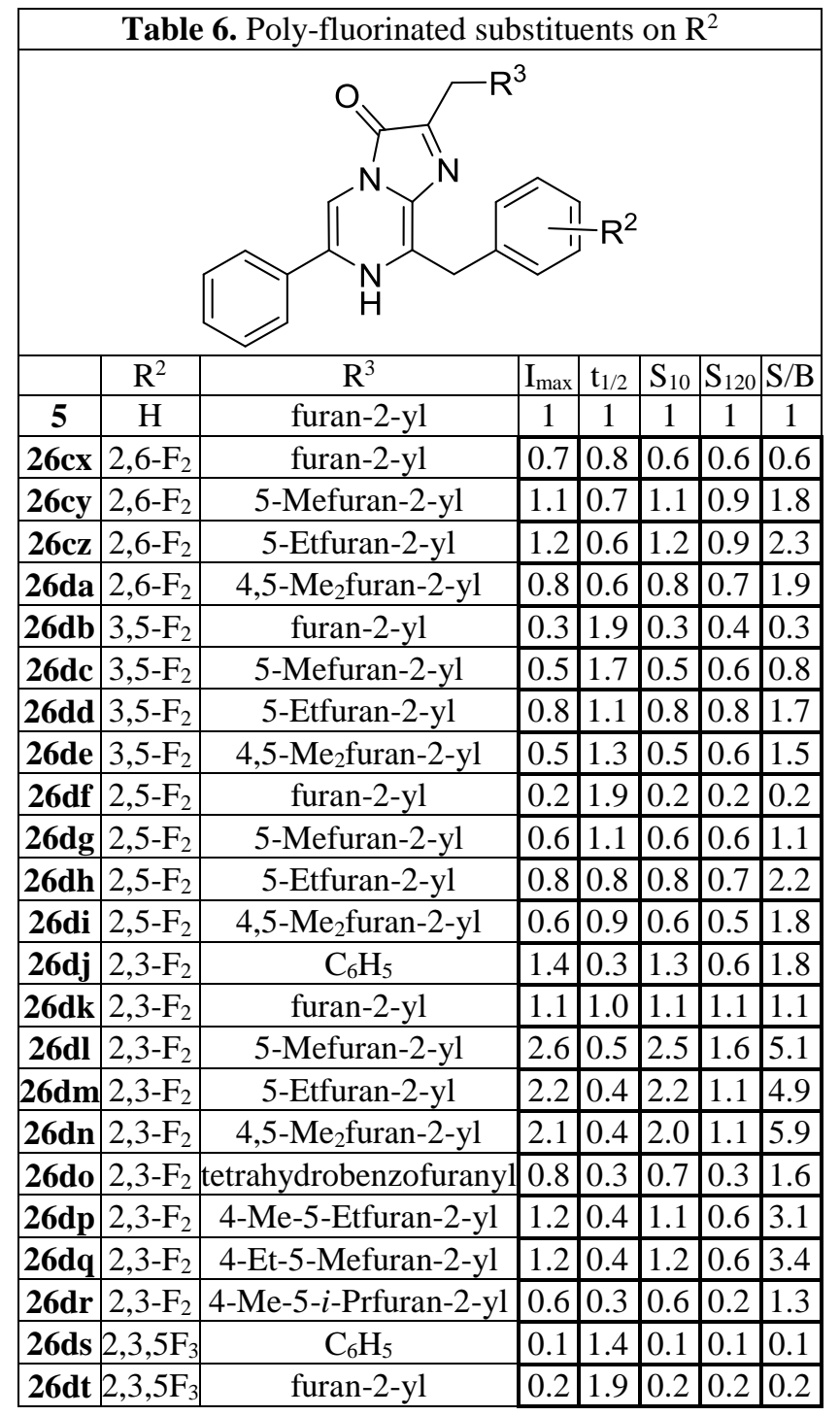

As depicted in table 7, we also investigated the bioluminescence properties of the difluorinated compounds 26du and 26dv, but no additional improvements were observed in comparison with the corresponding monofluorinated luciferins 26ax and 26bk of table 4. Moreover, as for other compounds bearing such a fluorine on $\mathrm{R}^{1}$, the furanbearing $O$-acetyl precursor of luciferin $26 \mathrm{du}$ was not so stable and decomposed over few days. Similarly, the 


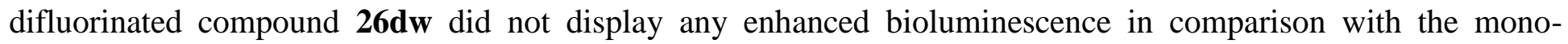
fluorinated analogues $\mathbf{2 6 c u}$ or 26bm. In a different approach, the effects of a methyl group on position 5 of the imidazo[1,2-a]pyrazin-3(7H)-one ring system (compound 35) as well as on $\mathrm{R}^{3}$ (compound 36) or $\mathrm{R}^{2}$ (compound 37) were also studied. Interestingly, in every case this additional methyl had a strongly deleterious effect on the bioluminescence.

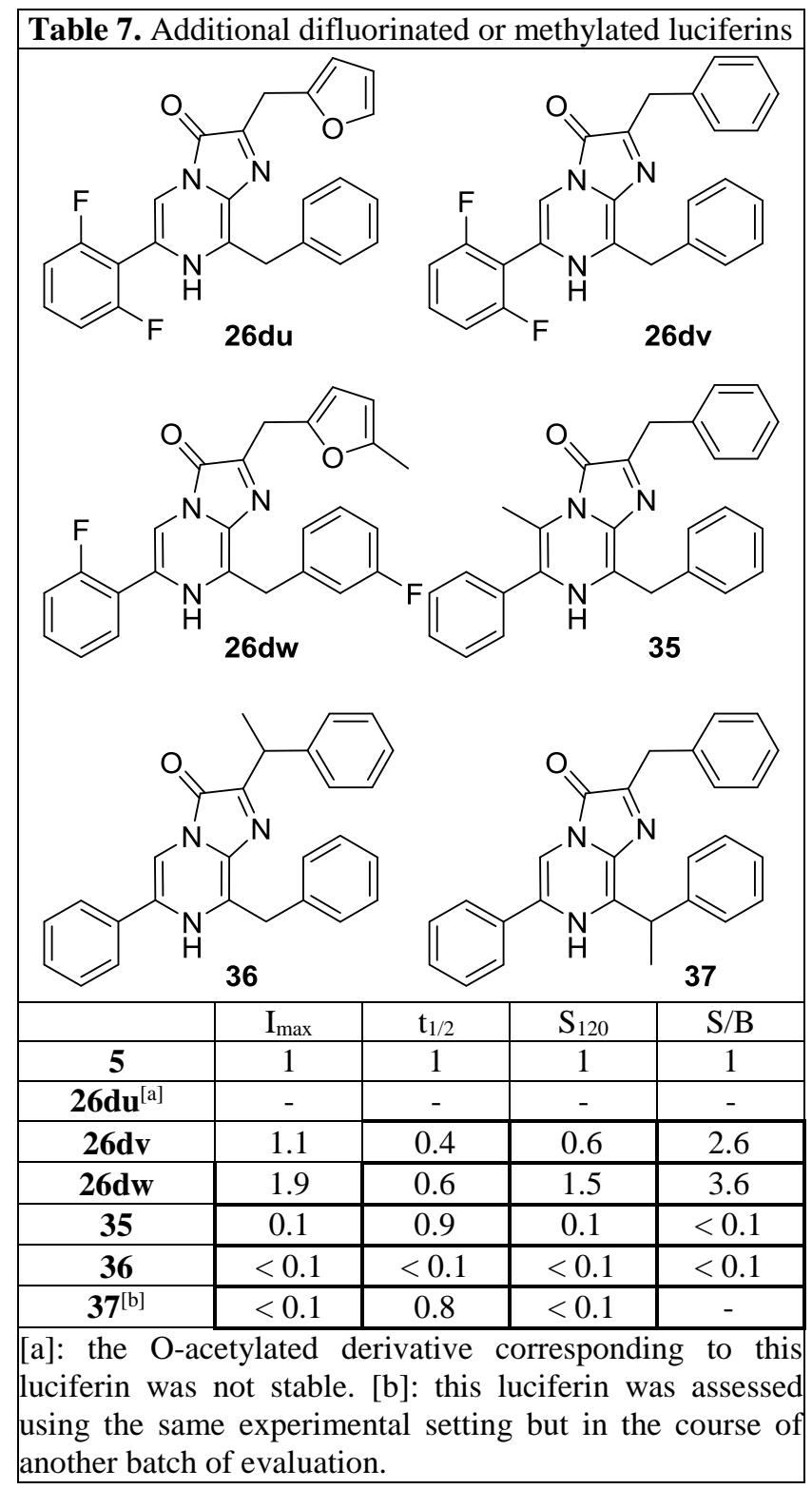

As detailed in table 8; in an attempt to understand the reasons behind the bioluminescence improvements observed, we undertook the extensive enzymatic study of a selection of 18 luciferins, including furimazine (5). These experiments were done using a Berthold Centro LB 960 luminometer and the delay between the automated injection of the enzyme and the initiation of the bioluminescence monitoring was of exactly one second, shaking included. For this reason, some of the signal maximum intensity ( $\mathrm{I}_{\max }$; in in RLU.s $\left.{ }^{-1}\right)$, half-life $\left(\mathrm{t}_{1 / 2}\right)$ and cumulated signal over two hours $\left(\mathrm{S}_{120}\right.$; in RLU) provided in table 8 may differ a bit from the normalized values listed in tables 1-7. Assuming again that the number of detected photons per consumed substrate molecule is constant whatever the substrate concentration, the use of a Michaelis-Menten model (and curve fitting calculations) as previously described, ${ }^{[11 b]}$ allowed us to determine the Michaelis constant $\left(\mathrm{K}_{\mathrm{M}}\right)$, the maximal reaction rate $\left(\mathrm{V}_{\max }\right)$ and the catalytic activity $\left(k_{c a t}\right)$ of nanoKAZ/NanoLuc for these substrates. A dissociation constant $\left(\mathrm{K}_{\mathrm{I}}\right)$ was required for these curve fitting and is illustrating the fact that there is an inhibition of the luciferase by an excess of substrate through the binding of a second luciferin to the Michaelis' complex luciferin-luciferase. Accordingly, we suggest 
the use of a $13 \mu \mathrm{M}$ substrate concentration in experiments in order to secure the highest signal intensity. Moreover, a slow (or fast) irreversible inactivation of the luciferase by a reaction product followed a first order rate constant $\left(k_{\text {inact }}\right)$ illustrating a stochastic mechanism. A far more extensive description of these experiments is available in the supplementary material. We could also, upon a complete consumption of the luciferin, ${ }^{[11 \mathrm{~b}]}$ determine the number of molecules consumed per photon detected by the luminometer in the condition of measurement (cpd·RLU $\left.{ }^{-1}\right)$. This actually allows a comparison of the light emission efficacy for each the enzyme/substrates couples, even if the value provided is intrinsically luminometer-dependent. As seen in table 8, the nature of the luciferin used had very little effect on the wavelength at maximum emission $\left(\lambda_{\max }\right)$, as these turned out to be always close to $460 \mathrm{~nm}$, as previously reported for furimazine (5). ${ }^{[9 \mathrm{~d}]}$ With these results, the luciferin could be classed in seven groups according to three criteria, namely the reaction rate $\left(k_{c a t}\right)$, the light emission efficiency $\left(\mathrm{cpd} \cdot \mathrm{RLU}^{-1} \cdot \mathrm{s}^{-1}\right)$ and the signal half-lifetime $\left(t_{1 / 2}\right)$. It turns out that analogue 26dl, is the only substrate featuring a high reaction rate $\left(k_{c a t}>\right.$ $1000 \mathrm{~mol} \cdot \mathrm{s}^{-1} \cdot \mathrm{mol}_{\text {luc }}{ }^{-1}$ ), a high light emission efficiency (<3000 cpd $\cdot \mathrm{RLU}^{-1}$ ) and a long half-lifetime (> $\left.30 \mathrm{~min}\right)$. Analogue 26dw is the only substrate studied with a high reaction rate, a low light emission efficiency and a long life whereas analogues 26ah, 6, and $\mathbf{1}$ are endowed with a high reaction rate, a low light emission efficiency and a short life. In this selection, quite a few substrates (furimazine (5), 26co, 26cu, 26cv, 26db, 26dc and 26bh) are displaying a low reaction rate, a high light emission efficiency and a long life. The analogs $\mathbf{2 6 g}$ and $\mathbf{2 6} \mathbf{c n}$ also have a low combustion rate and a high light emission efficiency but a rather short life. This in a relative opposition with the "isocoelenterazine" 26bj which has low reaction rate and low light emission efficiency but a long life. Finally, the substrates bis-coelenterazine (7), 26bf and 26bg turn out to have a low reaction rate, a low light emission and a short life. Interestingly, none of the substrates studied here combined a high reaction rate, a high light emission and a short life. In any case, an actual molecular mechanism accounting for all these previously unreported behaviors remains elusive. 


\begin{tabular}{|c|c|c|c|c|c|c|c|c|c|}
\hline $\mathrm{R}^{1}$ & 14 & & & $\mathrm{R}^{2}$ & 3 & 42 & $\mathrm{R}^{3}$ & le & \\
\hline $\mathbf{n b}\left\{R^{l}, R^{2}, R^{3}\right\}$ & $\begin{array}{c}\mathrm{I}_{\max } \\
\left(10^{6}\right. \\
\left.\mathrm{RLU} \cdot \mathrm{s}^{-1}\right)\end{array}$ & $\begin{array}{c}\mathrm{t}_{1 / 2} \\
(\mathrm{~min})\end{array}$ & $\begin{array}{c}\mathrm{S}_{120} \\
\left(10^{6}\right. \\
\text { RLU })\end{array}$ & $\mathrm{cpd} \cdot \mathrm{RLU}^{-1}$ & $\begin{array}{c}\mathrm{K}_{\mathrm{I}} \\
(\mu \mathrm{M})\end{array}$ & $\begin{array}{c}\mathrm{K}_{\mathrm{M}} \\
(\mu \mathrm{M})\end{array}$ & $\begin{array}{c}k_{\text {cat }} \\
\left(\mathrm{mol} \cdot \mathrm{s}^{-1}\right. \\
\cdot \mathrm{mol}_{\text {luc }} \\
\left.{ }^{1}\right)\end{array}$ & $\begin{array}{c}k_{\text {inact }} \\
\left(10^{-4}\right. \\
\left.\mathrm{s}^{-1}\right)\end{array}$ & $\begin{array}{c}\lambda_{\max } \\
(\mathrm{nm}) \\
\pm 3 \\
\mathrm{~nm}\end{array}$ \\
\hline $\mathbf{5}\{1,1,37\}$ & 1.7 & 74 & 85 & 1775 & 109 & 2.22 & 106 & 1.5 & 455 \\
\hline $7\{1,1,1\}$ & 3.3 & 19 & 81 & 4581 & 115 & 3.02 & 534 & 5.2 & 453 \\
\hline $\operatorname{26ah}\{1,1,42\}$ & 5.9 & 24 & 151 & 4483 & 58 & 6.73 & 1200 & 4.4 & 453 \\
\hline $\mathbf{2 6 g}\{1,1,12\}$ & 3.0 & 28 & 82 & 2450 & 167 & 4.30 & 270 & 2.1 & 453 \\
\hline $\mathbf{6}\{14,1,1\}$ & 2.0 & 3 & 6 & 12067 & 101 & 5.90 & 932 & 425 & 458 \\
\hline 26bf $\{14,1,37\}$ & 3.1 & 0.3 & 12 & 4463 & 101 & 3.88 & 535 & 375 & 462 \\
\hline $\operatorname{26cn}\{1,2,42\}$ & 6.0 & 38 & 216 & 2912 & 60 & 5.19 & 769 & 3.0 & 453 \\
\hline $\mathbf{1}\{14,1,63\}$ & 0.2 & 21 & 6 & 101169 & 22 & 6.80 & 1500 & 3.8 & 465 \\
\hline $\operatorname{26co}\{1,2,44\}$ & 4.2 & 68 & 128 & 1962 & 95 & 6.86 & 350 & 2.6 & 453 \\
\hline $\operatorname{26cu}\{1,3,42\}$ & 3.4 & 94 & 173 & 2106 & 40 & 6.06 & 565 & 4.0 & 453 \\
\hline $\mathbf{2 6 c v}\{1,3,44\}$ & 3.4 & 101 & 177 & 2095 & 60 & 5.80 & 319 & 2.4 & 453 \\
\hline $\mathbf{2 6 d b}\{1,22,37\}$ & 0.5 & 156 & 36 & 1836 & 140 & 2.20 & 30.8 & 1.3 & 463 \\
\hline $\operatorname{26dw}\{2,3,42\}$ & 3.4 & 94 & 146 & 22032 & 63 & 5.53 & 3380 & 1.8 & 453 \\
\hline $\operatorname{26dl}\{1,24,42\}$ & 5.3 & 39 & 178 & 2664 & 80 & 5.52 & 1420 & 4.5 & 455 \\
\hline 26dc $\{1,22,42\}$ & 0.8 & 157 & 58 & 2834 & 64 & 3.30 & 96.1 & 1.4 & 458 \\
\hline $\operatorname{26bh}\{13,1,37\}$ & 2.0 & 32 & 63 & 1804 & 84 & 3.30 & 140 & 4.5 & 458 \\
\hline $\mathbf{2 6} \mathbf{b j}\{13,1,63\}$ & 0.1 & 148 & 6 & 70020 & 35 & 2.94 & 362 & 3.1 & 460 \\
\hline $\operatorname{26bg}\{14,1,42\}$ & 3.0 & 1.5 & 11 & 4677 & 121 & 3.88 & 874 & 280 & 460 \\
\hline
\end{tabular}

\section{Conclusion}

This structure-bioluminescence activity investigation, very much reminiscent of a classic medicinal chemistry approach, involved the design and synthesis of many luciferins analogues in an iterative manner. Aside from the very intense but very short-lived bioluminescence profile we previously reported ${ }^{[11 b]}$ for a group of hydroxy-

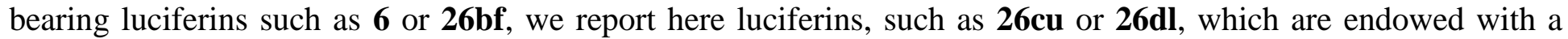
lasting as well as a much improved signal intensity in comparison with furimazine (5). Moreover, a third type of luciferins such as $\mathbf{2 6 d b}$, which displays a more modest signal intensity but an inordinate stability over time, also emerged from this work. Concerning the emission wavelength observed with these compounds, all of them turned out to emit blue photon. However, the Gauss Gaussian nature of these photon emission wavelengths lead to a substantial wavelength spreading. Indeed, the strong intensity provided by furimazine (5), although not centered on a red wavelength, still provided an increased number of red photons which was found sufficient for in vivo studies. ${ }^{[19]}$ Thus, the enhanced intensity observed here with the luciferin analogue 26dl could provide some more advantage in comparison with furimazine (5) or with approaches based on "red-shifting" marine-based bioluminescence reporting systems. ${ }^{[20]}$ Amongst avenues for further work, assessment of the signal profiles of this library of luciferins with other coelenterazine-using luciferases or the corresponding near infinite number of mutants, should provide many additional insights and further intensive for more structure-bioluminescence relationship studies. 
This work was supported by the Agence Nationale de la Recherche (ANR), grant ANR-11-CRNT-0004, in the context of the investment program 'GLOBAL CARE', an association of the Instituts Carnot 'Pasteur-Maladies Infectieuses', 'Curie-Cancer', 'Voir et Entendre', 'Institut du Cerveau et de la moelle Épinière' and the 'Consortium pour l'Accélération de l'Innovation et de son Transfert dans le domaine du Lymphome' (CALYM). E. P. C. was supported by a fellowship from Global Care and G.G. acknowledges a PhD fellowship from the Université Paris Descartes, Sorbonne Paris Cité. This project also benefited from the Valoexpress funding call of the Institut Pasteur. Prof. Christian Bréchot and Drs. Muriel Delepierre and Daniel Larzul are acknowledged for their interest and support. Dr. Daniel Dauzonne from the Institut Curie is acknowledged for his generous gift of some nitrostyrenes. Finally, Sarah Desmons, Mathilde Manuali, Candice Ford (who benefitted from an AMGEN scholars fellowship), Victor Monnot and Marylou Le Blanc-Gouverneur are acknowledged for their help in the syntheses of some of the compounds described here.

\section{Bibliography}

[1] O. Shimomura, Bioluminescence: Chemical Principles And Methods Revised Edition, World Scientific, Singapore, 2012, p. 1-468.

[2] a) P. J. Herring, J. Biolumin. Chemilumin. 1987, 1, 147-163; b) S. H. D. Haddock, M. A. Moline and J. F. Case, Annu. Rev. Mar. Sci. 2010, 2, 443-493.

[3] S. V. Markova and E. S. Vysotski, Biochemistry (Mosc) 2015, 80, 714-732.

[4] J. F. Head, S. Inouye, K. Teranishi and O. Shimomura, Nature 2000, 405, 372-376.

[5] M. Vacher, I. Fdez Galván, B. W. Ding, S. Schramm, R. Berraud-Pache, P. Naumov, N. Ferré, Y. J. Liu, I. Navizet, D. Roca-Sanjuán, W. J. Baader and R. Lindh, Chem. Rev. 2018, 118, 6927-6974.

[6] a) H. Hoshino, Expert Opin. Drug Dis. 2009, 9, 374-389; b) A. Roda, M. Guardigli, E. Michelini and M. Mirasoli, Trends Analyt. Chem. 2009, 28, 307-322; c) T. Ozawa, H. Yoshimura and S. B. Kim, Anal. Chem. 2013, 85, 590-609; d) E. Michelini, L. Cevenini, M. M. Calabretta and A. Roda, Anal. Bioanal. Chem. 2014, 406, 5531-5539; e) G. Thouand and R. Marks, Bioluminescence: Fundamentals and Applications in Biotechnology - Volume 1-3 (Advances in Biochemical Engineering/Biotechnology), 2014, p; f) C. G. England, E. B. Ehlerding and W. Cai, Bioconjug. Chem. 2016, 27, 1175-1187; g) Z. M. Kaskova, A. S. Tsarkova and I. V. Yampolsky, Chem. Soc. Rev. 2016, 45, 6048-6077; h) T. Jiang, L. Du and M. Li, Photochem. Photobiol. Sci. 2016, 15, 466-480; i) L. Mezzanotte, M. van 't Root, H. Karatas, E. A. Goun and C. W. G. M. Löwik, Trends Biotechnol. 2017, 35, 640-652; j) C. M. Rathbun and J. A. Prescher, Biochemistry 2017, 56, 5178-5184; k) H. W. Yeh and H. W. Ai, Annu. Rev. Anal. Chem. 2019, 12, 129-150; 1) H. W. Yeh, T. Wu, M. Chen and H. W. Ai, Biochemistry 2019, 58, 1689-1697; m) S. J. Williams and J. A. Prescher, Acc. Chem. Res. 2019, doi: 10.1021/acs.accounts.1029b00391.

[7] S. Inouye and O. Shimomura, Biochem. Biophys. Res. Commun. 1997, 233, 349-353.

[8] a) S. Inouye, K. Watanabe, H. Nakamura and O. Shimomura, FEBS Lett. 2000, 481, 19-25; b) S. Inouye, (Chisso Corporation, Japan), EP 1156103, 2001

[9] a) B. Binkowski, L. P. Encell, M. Hall, M. B. Robers, M. R. Slater, K. V. Wodd and M. G. Wood, (Promega), WO 2012061530, 2012; b) D. H. Klaubert, P. Meisenheimer and J. Unch, (Promega), WO 2012061529, 2012; c) L. P. Encell, K. V. Wood, M. G. Wood, M. Hall, P. Otto, V. Gediminas and K. Zimmerman, (Promega), US 8557970, 2013; d) M. P. Hall, J. Unch, B. F. Binkowski, M. P. Valley, B. L. Butler, M. G. Wood, P. Otto, K. Zimmerman, G. Vidugiris, T. Machleidt, M. B. Robers, H. A. Benink, C. T. Eggers, M. R. Slater, P. L. Meisenheimer, D. H. Klaubert, F. Fan, L. P. Encell and K. V. Wood, ACS Chem. Biol. 2012, 7 , 1848-1857.

[10] a) S. Inouye, J. Sato, Y. Sahara-Miura, S. Yoshida, H. Kurakata and T. Hosoya, Biochem. Biophys. Res. Commun. 2013, 437, 23-28; b) S. Inouye, J. Sato, Y. Sahara-Miura, S. Yoshida and T. Hosoya, Biochem. Biophys. Res. Commun. 2014, 445, 157-162; c) S. Inouye, J. Sato and Y. Miura, (JNC Corporation), US 20140302539, 2014; d) S. Inouye, Y. Sahara, R. Iimori and T. Hosoya, (JNC Corporation, Tokyo Institute of 
Technology), US 20150344936, 2015; e) S. Inouye and J. Sato, (JNC Corporation), US 20150259652, 2015; f) S. Inouye, Y. Miura and J. Sato, (JNC Corporation), US 20150299675, 2015; g) S. Inouye and J. Sato, (JNC Corporation), US 20150184134, 2015; h) S. Inouye and J. Sato, (JNC Corporation), US 20150111233 , 2015; i) A. Shakhmin, M. P. Hall, J. R. Walker, T. Machleidt, B. F. Binkowski, K. V. Wood and T. A. Kirkland, Chem. Eur. J. 2016, 22, 10369-10375.

[11] a) Y. L. Janin, E. P. Coutant, V. Hervin, G. Gagnot, Y. Jacob, S. Goyard and T. Rose, (Institut Pasteur, CNRS), WO 2018197727, 2018; b) E. P. Coutant, S. Goyard, V. Hervin, G. Gagnot, R. Baatallah, T. Rose, Y. Jacob and Y. L. Janin, Org. Biomol. Chem. 2019, 17, 3709-3713.

[12] D. Andreotti, S. Bacchi, M. Delpogetto, S. Guelfi, A. Perboni, A. Ribecai, S. Spada, P. Stabile and M. Tampieri, (SB Pharmco Puerto Rico), WO 2006108689, 2006

[13] a) R. G. Jones, J. Am. Chem. Soc. 1949, 71, 78-81; b) G. Karmas and P. E. Spoerri, J. Am. Chem. Soc. 1952, 74, 1580-1584.

[14] D. E. Jones and M. S. South, Tetrahedron 2010, 66, 2570-2581.

[15] a) D. E. Worrall, Organic Synthesis Coll. Vol 1 1941, 405-406; b) C. B. Gairaud and G. R. Lappin, J. Org. Chem. 1953, 18, 1-3.

[16] a) V. Hervin, E. P. Coutant, G. Gagnot and Y. L. Janin, Synthesis 2017, 49, 4093-4110; b) G. Gagnot, V. Hervin, E. P. Coutant, S. Desmons, R. Baatallah, V. Monnot and Y. L. Janin, Beilstein J. Org. Chem. 2018, 14, 2846-2852; c) E. P. Coutant, V. Hervin, G. Gagnot, C. Ford, R. Baatallah and Y. L. Janin, Beilstein J. Org. Chem. 2018, 2853-2859.

[17] A. Shakhmin, T. Kirkland, J. Walker, T. Machleidt, M. Hall and K. W. Wood, (Promega), US 20170233789, 2017

[18] B. Binkowski, M. P. Hall, T. Machleidt, J. Walker and W. Zhou, (Promega Corp.), US 20190337939, 2019

[19] a) M. Hattori, G. Kawamura, R. Kojima, M. Kamiya, Y. Urano and T. Ozawa, Anal. Chem. 2016, 88, 62316238; b) E. Silberstein, C. Serna, S. P. Fragoso, R. Nagarkatti and A. Debrabant, PLoS One 2018, 13, e0195879; c) E. Belarbi, V. Legros, J. Basset, P. Desprès, P. Roques and V. Choumet, Viruses 2019, 11, pii: E584.

[20] a) T. Curie, K. L. Rogers, C. Colasante and P. Brûlet, Mol. Imaging 2007, 6, 30-42; b) A. M. Loening, A. M. Wu and S. S. Gambhir, Nat. Methods 2007, 8, 641-643; c) G. Giuliani, P. Molinari, G. Ferretti, A. Cappelli, M. Anzini, S. Vomero and T. Costa, Tetrahedron Lett. 2012, 53, 5114-5118; d) R. Nishihara, H. Suzuki, E. Hoshino, S. Suganuma, M. Sato, T. Saitoh, S. Nishiyama, N. Iwasawa, D. Citterio and K. Suzuki, Chem. Commun. 2015, 51, 391-394; e) A. Takai, M. Nakano, K. Saito, R. Haruno, T. M. Watanabe, T. Ohyanagi, T. Jin, Y. Okada and T. Nagai, Proc. Natl. Acad. Sci. U.S.A. 2015, 112, 4352-4356; f) K. A. Rumyantsev, K. K. Turoverov and V. V. Verkhusha, Sci. Rep. 2016, 6, 36588; g) K. M. Grinstead, L. Rowe, C. M. Ensor, S. Joel, P. Daftarian, E. Dikici, J.-M. Zingg and S. Daunert, PLoS One 2016, 11, e0158579; h) A. Shakhmin, M. P. Hall, T. Machleidt, J. R. Walker, K. V. Wood and T. A. Kirkland, Org. Biomol. Chem. 2017, 15, 85598567 ; i) H. W. Yeh, O. Karmach, A. Ji, D. Carter, M. M. Martins-Green and H. W. Ai, Nature Methods 2017, 14, 971-974; j) A. Taylor, J. Sharkey, A. Plagge, B. Wilm and P. Murray, Contrast Media Mol. Imaging 2018, 2514796; k) D. C. Alcobia, A. I. Ziegler, A. Kondrashov, E. Comeo, S. Mistry, B. Kellam, A. Chang, J. Woolard, S. J. Hill and E. K. Sloan, iScience 2018, 6, 280-288; 1) R. Nishihara, R. Paulmurugan, T. Nakajima, E. Yamamoto, A. Natarajan, R. Afjei, Y. Hiruta, N. Iwasawa, S. Nishiyama, D. Citterio, M. Sato, S. B. Kim and K. Suzuki, Theranostics 2019, 13, 2646-2661; m) R. Nishihara, E. Hoshino, Y. Kakudate, S. Kishigami, N. Iwasawa, S. Sasaki, T. Nakajima, M. Sato, S. Nishiyama, D. Citterio, K. Suzuki and S. B. Kim, Bioconjug. Chem. 2019, 29, 1922-1931; n) M. Abe, R. Nishihara, Y. Ikeda, T. Nakajima, M. Sato, N. Iwasawa, S. Nishiyama, R. Paulmurugan, D. Citterio, S. Kim and K. Suzuki, Chembiochem 2019, 20, 19191923; o) H. W. Yeh, Y. Xiong, T. Wu, M. Chen, A. Ji, L. X. and H. W. Ai, ACS Chem. Biol. 2019, 17, 959965; p) F. Weihs and H. Dacres, Trends Analyt. Chem. 2019, 116, 61-73; q) H. Kobayashi, L. P. Picard, A. M. Schönegge and M. Bouvier, Nat. Protoc. 2019, 1084-1107. 
Table of content

Chemistry

HPLC/MS monitoring of the hydrolysis products of selected $\mathbf{O}$-acetylated luciferins 25

Biology

Bioluminescence assay, kinetic analysis and $\lambda_{\max }$ measurement

References for the supporting information 


\section{General}

${ }^{1} \mathrm{H}$ NMR and ${ }^{13} \mathrm{C}$ NMR spectra were recorded on a Bruker Avance 400 spectrometer at $400 \mathrm{MHz}$ and $100 \mathrm{MHz}$, respectively. Shifts $(\delta)$ are given in ppm with respect to the TMS signal and cross-coupling constants $(J)$ are given in Hertz. Column chromatography were performed either on Merck silica gel $60(0.035-0.070 \mathrm{~mm})$ or neutral alumina containing $1.5 \%$ of added water using a solvent pump and an automated collecting system driven by a UV detector set to $254 \mathrm{~nm}$ unless required otherwise. Sample deposition was carried out by absorption of the mixture to be purified on a small amount of the solid phase followed by its deposition of the top of the column. The low resolution mass spectra were obtained on an Agilent 1100 series LC/MSD system using an atmospheric electrospray ionization system or an Agilent 1200 series LC/MSD system using an Agilent Jet-Stream atmospheric electrospray ionization system and the high resolution mass spectra (HRMS) were obtained using a Waters Micromass Q-Tof with an electrospray ion source. As specified below, a Berthold Centro $\mathrm{XS}^{3}$ luminometer or a Berthold Centro LB 960 were used for the bioluminescence experiments. The wavelengths at maximum emission $\left(\lambda_{\max }\right)$ were determined using a JASCO FP-6300 spectrofluorometer. When specified, the anhydrous solvents used were purchased. Unless stated otherwise, a purity of at least $95 \%$ was obtained for all the compounds by means of chromatography, recrystallization or distillation and this level of purity was established by TLC, LC/MS and NMR spectroscopy.

\section{Numbering}

The chemset numbering system recommended by the ACS for the description of chemical libraries prepared by combinatorial chemistry was adopted here to number most of the compounds described. The following tables are mirroring the tables 1-8 of in the main text and are featuring an additional column in order to provide a correspondence between this numbering system and the numbers used for the description of the luciferins in the main text. 


\begin{tabular}{|c|c|c|c|c|c|c|}
\hline & \multicolumn{6}{|c|}{ Table 1. Variations on $\mathrm{R}^{3}$} \\
\hline & & $\mathrm{R}^{3}$ & $I_{\max }$ & $\mathrm{t}_{1 / 2}$ & $S_{120}$ & S/B \\
\hline $\mathbf{2 6}\{1,1,37\}$ & 5 & furan-2-yl & 1 & 1 & 1 & 1 \\
\hline $\mathbf{2 6}\{1,1,1\}$ & 7 & $\mathrm{C}_{6} \mathrm{H}_{5}$ & 1.25 & 0.44 & 0.77 & 1.37 \\
\hline $\mathbf{2 6}\{1,1,2\}$ & $26 a$ & $2-\mathrm{FC}_{6} \mathrm{H}_{4}$ & 0.40 & 1.03 & 0.41 & 0.58 \\
\hline $\mathbf{2 6}\{1,1,3\}$ & $26 b$ & $3-\mathrm{FC}_{6} \mathrm{H}_{4}$ & 0.70 & 0.73 & 0.59 & 0.91 \\
\hline $\mathbf{2 6}\{1,1,4\}$ & 8 & $4-\mathrm{FC}_{6} \mathrm{H}_{4}$ & 1.17 & 0.37 & 0.61 & 1.50 \\
\hline $\mathbf{2 6}\{1,1,25\}$ & $26 c$ & $2,4-\mathrm{F}_{2} \mathrm{C}_{6} \mathrm{H}_{3}$ & 1.02 & 0.32 & 0.50 & 1.52 \\
\hline $\mathbf{2 6}\{1,1,7\}$ & 26d & $4-\mathrm{ClC}_{6} \mathrm{H}_{4}$ & 0.38 & 0.36 & 0.19 & 0.50 \\
\hline $\mathbf{2 6}\{1,1,20\}$ & $26 e$ & $4-\mathrm{BrC}_{6} \mathrm{H}_{4}$ & 0.34 & 0.24 & 0.13 & 0.46 \\
\hline $\mathbf{2 6}\{1,1,11\}$ & $26 f$ & $2-\mathrm{MeC}_{6} \mathrm{H}_{4}$ & 0.02 & 1.41 & 0.02 & 0.03 \\
\hline $26\{1,1,12\}$ & $26 \mathrm{~g}$ & $3-\mathrm{MeC}_{6} \mathrm{H}_{4}$ & 1.29 & 0.62 & 0.97 & 1.60 \\
\hline $\mathbf{2 6}\{1,1,13\}$ & $26 \mathrm{~h}$ & $4-\mathrm{MeC}_{6} \mathrm{H}_{4}$ & 0.27 & 0.33 & 0.12 & 0.31 \\
\hline $\mathbf{2 6}\{1,1,8\}$ & $26 \mathbf{i}$ & $2-\mathrm{CF}_{3} \mathrm{C}_{6} \mathrm{H}_{4}$ & 0.07 & 0.94 & 0.06 & 0.17 \\
\hline $\mathbf{2 6}\{1,1,9\}$ & $26 \mathbf{j}$ & $3-\mathrm{CF}_{3} \mathrm{C}_{6} \mathrm{H}_{4}$ & 0.38 & 1.09 & 0.39 & 0.55 \\
\hline $26\{1,1,10\}$ & $26 \mathbf{k}$ & $4-\mathrm{CF}_{3} \mathrm{C}_{6} \mathrm{H}_{4}$ & 0.16 & 0.44 & 0.09 & 0.22 \\
\hline $\mathbf{2 6}\{1,1,14\}$ & 261 & $2-\mathrm{MeOC}_{6} \mathrm{H}_{4}$ & 0.40 & 0.47 & 0.24 & 0.39 \\
\hline $26\{1,1,15\}$ & $26 \mathrm{~m}$ & $3-\mathrm{MeOC}_{6} \mathrm{H}_{4}$ & 1.15 & 0.68 & 0.93 & 1.26 \\
\hline $\mathbf{2 6}\{1,1,16\}$ & $26 n$ & $4-\mathrm{MeOC}_{6} \mathrm{H}_{4}$ & 0.74 & 0.33 & 0.34 & 0.86 \\
\hline $\mathbf{2 6}\{1,1,62\}$ & $260^{a}$ & $3-\mathrm{HOC}_{6} \mathrm{H}_{4}$ & 0.22 & 0.05 & 0.03 & - \\
\hline $\mathbf{2 6}\{1,1,63\}$ & $26 p^{a}$ & $4-\mathrm{HOC}_{6} \mathrm{H}_{4}$ & 0.09 & 0.10 & 0.03 & - \\
\hline $\mathbf{2 6}\{1,1,27\}$ & $26 q$ & 3-n-PrC $6{ }_{6} \mathrm{H}_{4}$ & 0.49 & 1.01 & 0.49 & 0.60 \\
\hline $\mathbf{2 6}\{1,1,29\}$ & $26 r$ & $3-c-\mathrm{PrC}_{6} \mathrm{H}_{4}$ & 0.55 & 0.85 & 0.50 & 0.62 \\
\hline $26\{1,1,28\}$ & $26 s$ & $4-n-\operatorname{PrC}_{6} \mathrm{H}_{4}$ & 0.23 & 0.24 & 0.08 & 0.24 \\
\hline $\mathbf{2 6}\{1,1,31\}$ & $26 t$ & $4-i-\operatorname{PrC}_{6} \mathrm{H}_{4}$ & 0.27 & 0.41 & 0.13 & 0.26 \\
\hline $\mathbf{2 6}\{1,1,30\}$ & $26 u$ & 4-c- $\mathrm{PrC}_{6} \mathrm{H}_{4}$ & 0.31 & 0.30 & 0.12 & 0.35 \\
\hline
\end{tabular}




\begin{tabular}{|c|c|c|c|c|c|c|}
\hline & \multicolumn{6}{|c|}{ Table 2. Further alterations of $\mathrm{R}^{3}$} \\
\hline & & $\mathrm{R}^{3}$ & $I_{\max }$ & $\mathrm{t}_{1 / 2}$ & $S_{120}$ & S/B \\
\hline $\mathbf{2 6}\{1,1,37\}$ & 5 & furan-2-yl & 1 & 1 & 1 & 1 \\
\hline $2 \mathbf{6}\{1,1,38\}$ & $26 v$ & tetrahydrofuran-2-yl & 0.04 & 1.83 & 0.05 & 0 \\
\hline $\mathbf{2 6}\{1,1,39\}$ & $26 w$ & 1,3-dioxolan-2-yl & 0.03 & 1.73 & 0.04 & 0.01 \\
\hline $\mathbf{2 6}\{1,1,33\}$ & $26 x$ & cyclohexyl & 0.01 & 1.83 & 0.02 & 0.01 \\
\hline $\mathbf{2 6}\{1,1,36\}$ & $26 y$ & cyclopentyl & 0.01 & 1.83 & 0.02 & 0.01 \\
\hline $\mathbf{2 6}\{1,1,32\}$ & $26 z$ & benzyl & 0.14 & 0.55 & 0.09 & 0.12 \\
\hline $26\{1,1,34\}$ & $26 \mathbf{a a}^{\mathrm{a}}$ & 2-pyridyl & - & - & - & - \\
\hline $26\{1,1,40\}$ & $26 a b$ & furan-3-yl & 0.47 & 0.94 & 0.46 & 0.14 \\
\hline $\mathbf{2 6}\{1,1,41\}$ & $26 a c$ & thiophene-2-yl & 1.24 & 0.47 & 0.85 & 1.80 \\
\hline $26\{1,1,56\}$ & 26ad & 3-Meisoxazol-5-yl & 0.18 & 0.22 & 0.08 & 0.01 \\
\hline $\mathbf{2 6}\{1,1,57\}$ & $26 a e$ & 3-Etisoxazol-5-yl & 0.33 & 0.15 & 0.10 & 0.01 \\
\hline $26\{1,1,58\}$ & $26 \mathbf{f a f}^{\mathrm{b}}$ & 5-Meoxazol-2-yl & 0.20 & 1.76 & 0.24 & - \\
\hline $2 \mathbf{6}\{1,1,59\}$ & $26 a g$ & 4,5-Me 2 oxazol-2-yl & 0.23 & 0.92 & 0.22 & 0.15 \\
\hline
\end{tabular}




\begin{tabular}{|c|c|c|c|c|c|c|}
\hline & \multicolumn{6}{|c|}{ Table 3. few analogues related to furimazine (5) } \\
\hline & & $\mathrm{R}^{3}$ & $\mathrm{I}_{\max }$ & $\mathrm{t}_{1 / 2}$ & $\mathrm{~S}_{120}$ & $\mathrm{~S} / \mathrm{B}$ \\
\hline $\mathbf{2 6}\{1,1,37\}$ & 5 & furan-2-yl & 1 & 1 & 1 & 1 \\
\hline $\mathbf{2 6}\{1,1,42\}$ & 26ah & 5-Mefuran-2-yl & 1.96 & 0.50 & 1.26 & 3.15 \\
\hline $26\{1,1,44\}$ & 26ai & 5-Etfuran-2-yl & 1.89 & 0.59 & 1.34 & 3.82 \\
\hline $\mathbf{2 6}\{1,1,47\}$ & 26aj & 5-c-Prfuran-2-yl & 1.26 & 0.62 & 0.91 & 2.92 \\
\hline $26\{1,1,45\}$ & 26ak & 5-n-Prfuran-2-yl & 1.43 & 1.03 & 1.47 & 3.43 \\
\hline $26\{1,1,46\}$ & 26al & 5- $n$-Pentfuran-2-yl & 0.10 & 0.78 & 0.09 & 0.17 \\
\hline $\mathbf{2 6}\{1,1,43\}$ & 26am & 5-CF $\mathrm{CF}_{3}$ furan-2-yl & 0.20 & 1.83 & 0.27 & 0.21 \\
\hline $26\{1,1,48\}$ & 26an & 4,5-Me ${ }_{2}$ furan-2-yl & 1.40 & 0.47 & 0.85 & 2.85 \\
\hline $\mathbf{2 6}\{1,1,49\}$ & $26 a 0$ & tetrahydrobenzofuran-2-yl & 0.59 & 0.51 & 0.35 & 1.16 \\
\hline $26\{1,1,50\}$ & 26ap & 4-Me-5-Etfuran-2-yl & 0.86 & 0.78 & 0.74 & 1.75 \\
\hline $\mathbf{2 6}\{1,1,51\}$ & $26 a q$ & 4-Et-5-Mefuran-2-yl & 0.86 & 0.41 & 0.43 & 2.15 \\
\hline $26\{1,1,52\}$ & 26ar & 4-Me-5-i-Prfuran-2-yl & 0.36 & 0.98 & 0.36 & 0.83 \\
\hline $\mathbf{2 6}\{1,1,41\}$ & $26 a s$ & thiophene-2-yl & 1.24 & 0.47 & 0.85 & 1.80 \\
\hline $\mathbf{2 6}\{1,1,53\}$ & 26at & 5-Etthiophen-2-yl & 1.11 & 0.42 & 0.56 & 2.03 \\
\hline $26\{1,1,54\}$ & 26au & 3-Methiophen-2-yl & 0.15 & 1.83 & 0.20 & 0.22 \\
\hline $26\{1,1,55\}$ & $26 a v$ & 4,5- $\mathrm{Me}_{2}$ thiophen-2-yl & 0.54 & 1.11 & 0.55 & 1.04 \\
\hline
\end{tabular}




\begin{tabular}{|c|c|c|c|c|c|c|c|}
\hline & \multicolumn{7}{|c|}{ Table 4. More elaborated analogues of furimazine } \\
\hline & & $\mathrm{R}^{1}$ & $\mathrm{R}^{3}$ & $I_{\max }$ & $\mathrm{t}_{1 / 2}$ & $S_{120}$ & S/B \\
\hline $\mathbf{2 6}\{1,1,37\}$ & 5 & $\mathrm{H}$ & furan-2-yl & 1 & 1 & 1 & 1 \\
\hline $\mathbf{2 6}\{1,1,42\}$ & 26ah & $\mathrm{H}$ & 5-Mefuran-2-yl & 1.96 & 0.50 & 1.26 & 3.15 \\
\hline $\mathbf{2 6}\{2,1,42\}$ & $26 \mathbf{w w}^{\mathrm{a}}$ & $2-\mathrm{F}$ & 5-Mefuran-2-yl & 2.17 & 0.39 & 1.89 & - \\
\hline $\mathbf{2 6}\{2,1,37\}$ & 26ax & $2-\mathrm{F}$ & furan-2-yl & 1.35 & 0.80 & 1.18 & 1.15 \\
\hline $\mathbf{2 6}\{3,1,42\}$ & 26ay & $3-\mathrm{F}$ & 5-Mefuran-2-yl & 1.65 & 0.49 & 1.11 & 3.62 \\
\hline $\mathbf{2 6}\{4,1,42\}$ & $26 a z$ & $4-\mathrm{F}$ & 5-Mefuran-2-yl & 0.07 & 1.83 & 0.09 & 0.08 \\
\hline $\mathbf{2 6}\{5,1,42\}$ & $26 \mathrm{ba}$ & $3-\mathrm{Me}$ & 5-Mefuran-2-yl & 0.41 & 1.15 & 0.44 & 0.71 \\
\hline $\mathbf{2 6}\{6,1,42\}$ & $26 b b$ & $4-\mathrm{Me}$ & 5-Mefuran-2-yl & 0 & 1.39 & 0 & 0 \\
\hline $\mathbf{2 6}\{7,1,37\}$ & $26 b^{c^{a}}$ & $2-\mathrm{MeO}$ & furan-2-yl & 0.02 & 1.83 & 0.04 & 0 \\
\hline $\mathbf{2 6}\{8,1,42\}$ & 26bd & $3-\mathrm{MeO}$ & 5-Mefuran-2-yl & 0.04 & 2.18 & 0.04 & - \\
\hline $\mathbf{2 6}\{9,1,42\}$ & 26be & $4-\mathrm{MeO}$ & 5-Mefuran-2-yl & 0.01 & 1.83 & 0.01 & 0 \\
\hline $\mathbf{2 6}\{14,1,63\}$ & 1 & 4-HO & $4-\mathrm{HOC}_{6} \mathrm{H}_{5}$ & 0.09 & 0.64 & 0.08 & 0.01 \\
\hline $\mathbf{2 6}\{14,1,37\}$ & $26 \mathrm{bf}$ & $4-\mathrm{HO}$ & furan-2-yl & 3.06 & 0.05 & 0.14 & 0.12 \\
\hline $26\{14,1,42\}$ & $26 b^{b}$ & $4-\mathrm{HO}$ & 5-Mefuran-2-yl & 2.58 & 0 & 0.09 & - \\
\hline $\mathbf{2 6}\{14,1,1\}$ & 6 & $4-\mathrm{HO}$ & $\mathrm{C}_{6} \mathrm{H}_{5}$ & 0.52 & 0.03 & 0.04 & 0.09 \\
\hline $26\{13,1,37\}$ & 26bh & $3-\mathrm{HO}$ & furan-2-yl & 0.92 & 0.80 & 0.80 & 1.19 \\
\hline $\mathbf{2 6}\{13,1,42\}$ & 26bi & $3-\mathrm{HO}$ & 5-Mefuran-2-yl & 1.15 & 0.53 & 0.73 & 2.06 \\
\hline $\mathbf{2 6}\{13,1,63\}$ & 26bj & $3-\mathrm{HO}$ & $4-\mathrm{HOC}_{6} \mathrm{H}_{5}$ & 0.04 & 1.93 & 0.05 & 0.04 \\
\hline $\mathbf{2 6}\{2,1,1\}$ & $26 \mathrm{bk}$ & $2-\mathrm{F}$ & $\mathrm{C}_{6} \mathrm{H}_{5}$ & 1.32 & 0.41 & 0.75 & 2.55 \\
\hline $\mathbf{2 6}\{2,1,4\}$ & $26 \mathrm{bl}$ & $2-\mathrm{F}$ & $4-\mathrm{FC}_{6} \mathrm{H}_{4}$ & 1.28 & 0.24 & 0.47 & 2.16 \\
\hline $\mathbf{2 6}\{2,1,44\}$ & $26 b^{a}$ & $2-\mathrm{F}$ & 5-Etfuran-2-yl & 2.59 & 0.35 & 2.33 & - \\
\hline $\mathbf{2 6}\{2,1,48\}$ & $26 b^{a}$ & $2-\mathrm{F}$ & 4,5-Me $\mathrm{Me}_{2}$ uran-2-yl & 2.13 & 0.28 & 1.97 & - \\
\hline & $\begin{array}{l}\text { a The corre } \\
\text { results only } \\
\text { another bat }\end{array}$ & $\begin{array}{l}\text { S lucite } \\
\text { valuatio }\end{array}$ & $\begin{array}{l}\text { lerivatives } \mathbf{2 5} \text { were } \\
\text { sessed using the sa }\end{array}$ & e over & ne, ini & $\begin{array}{l}\text { biolu } \\
\text { in the }\end{array}$ & iescenc \\
\hline
\end{tabular}




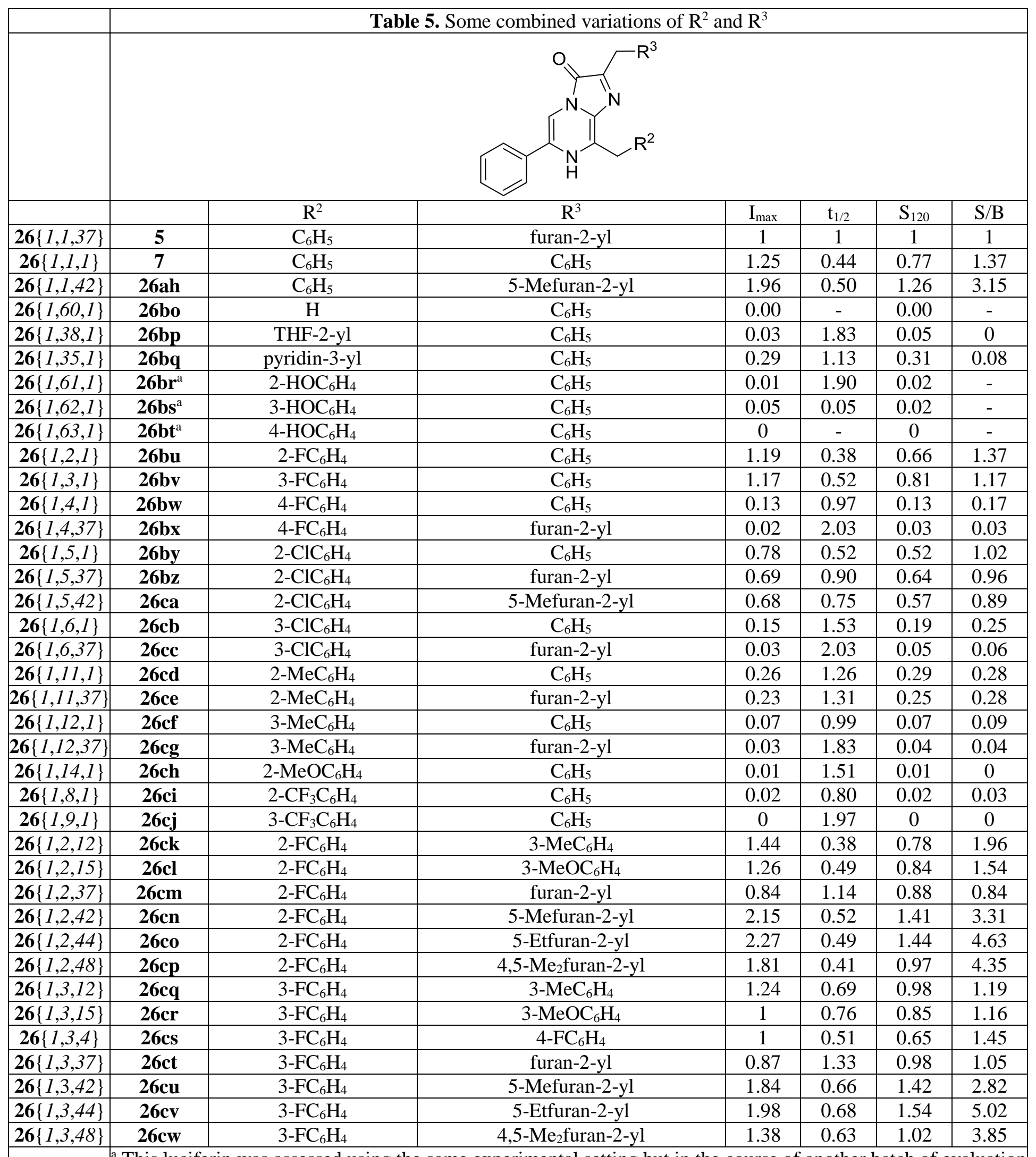

${ }^{\mathrm{a}}$ This luciferin was assessed using the same experimental setting but in the course of another batch of evaluation 


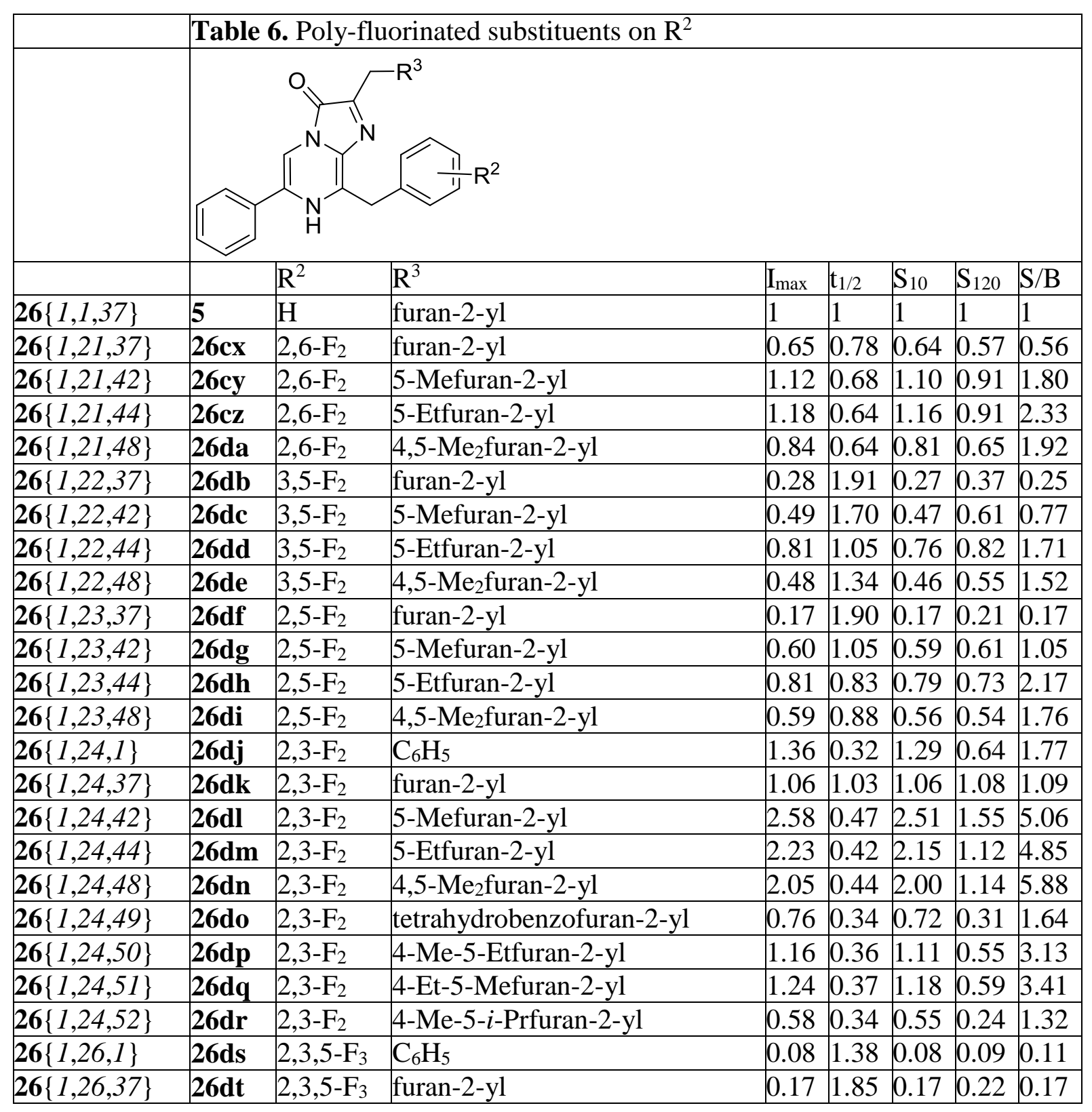




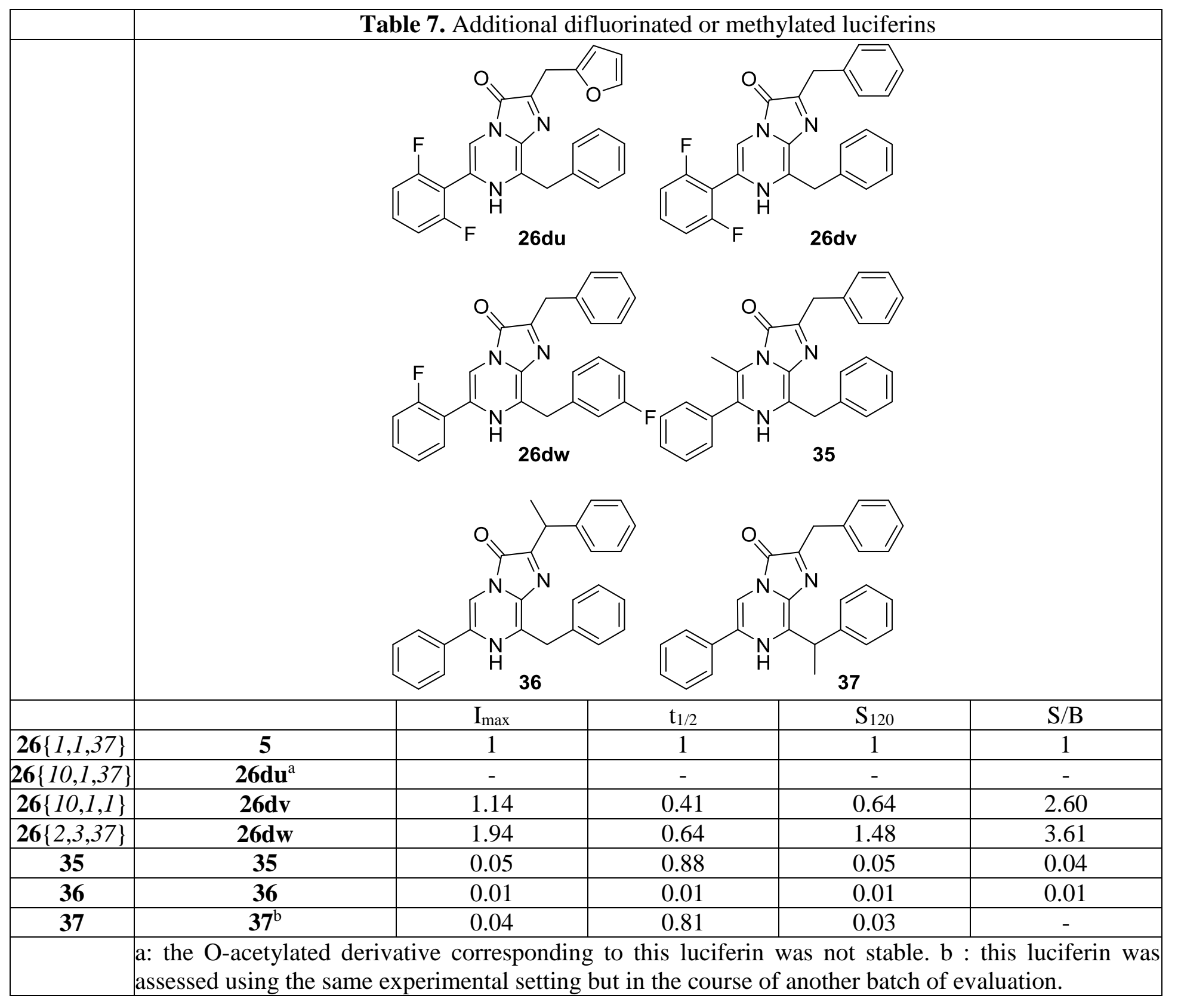




\begin{tabular}{|c|c|c|c|c|c|c|c|c|c|c|}
\hline & \multicolumn{10}{|c|}{ Table 8. bioluminescence profiles, kinetic parameters and $\lambda_{\max }$ for a selection of luciferins } \\
\hline & 1 & & $\left\{R^{1}\right.$, & & $\mathrm{R}^{2}$ & $\mathrm{~F}^{3}$ & & (1) & $\mathrm{Me}$ & - \\
\hline $\begin{array}{l}\text { Names }^{\circledR} \text { of } \\
\text { the O- } \\
\text { acetylated } \\
\text { precursor }\end{array}$ & $\left\{R^{l}, R^{2}, R^{3}\right\}$ & $\begin{array}{l}\mathrm{I}_{\max } \\
\left(10^{6}\right. \\
\text { RLU/s })\end{array}$ & $\begin{array}{l}\mathrm{t}_{1 / 2} \\
(\mathrm{~min})\end{array}$ & $\begin{array}{l}\mathrm{S}_{120} \\
\left(10^{6}\right. \\
\mathrm{RLU})\end{array}$ & $\mathrm{cpd} / \mathrm{RLU}$ & $\begin{array}{l}\mathrm{K}_{\mathrm{I}} \\
\left(10^{-6}\right. \\
\mathrm{M})\end{array}$ & $\begin{array}{l}\mathrm{K}_{\mathrm{M}} \\
\left(10^{-6}\right. \\
\mathrm{M})\end{array}$ & $\begin{array}{l}k_{\text {cat }} \\
\left(\mathrm{mol} \mathrm{s}^{-1}\right. \\
\left.\operatorname{mol}_{\mathrm{luc}^{-1}}\right)\end{array}$ & $\begin{array}{l}k_{\text {inact }} \\
\left(10^{-}\right. \\
4 / \mathrm{s})\end{array}$ & $\begin{array}{l}\lambda_{\max } \\
(\mathrm{nm})\end{array}$ \\
\hline $\begin{array}{l}\text { Hikarazine- } \\
1\end{array}$ & $\mathbf{5}\{1,1,37\}$ & 1.7 & 74 & 85 & 1775 & 109 & 2.22 & 106 & 1.5 & \\
\hline $\begin{array}{l}\text { Hikarazine- } \\
2\end{array}$ & $7\{1,1,1\}$ & 3.3 & 19 & 81 & 4581 & 115 & 3.02 & 534 & 5.2 & \\
\hline $\begin{array}{l}\text { Hikarazine- } \\
3\end{array}$ & $\mathbf{2 6 a h}\{1,1,42\}$ & 5.9 & 24 & 151 & 4483 & 58 & 6.73 & 1200 & 4.4 & \\
\hline $\begin{array}{l}\text { Hikarazine- } \\
14\end{array}$ & $\mathbf{2 6 g}\{1,1,12\}$ & 3.0 & 28 & 82 & 2450 & 167 & 4.30 & 270 & 2.1 & \\
\hline $\begin{array}{l}\text { Hikarazine- } \\
75\end{array}$ & $\mathbf{6}\{14,1,1\}$ & 2.0 & 3 & 6 & 12067 & 101 & 5.90 & 932 & 425 & \\
\hline $\begin{array}{l}\text { Hikarazine- } \\
80\end{array}$ & $\mathbf{2 6 b f}\{14,1,37\}$ & 3.1 & 0.3 & 12 & 4463 & 101 & 3.88 & 535 & 375 & \\
\hline $\begin{array}{l}\text { Hikarazine- } \\
85\end{array}$ & $26 \mathbf{c n}\{1,2,42\}$ & 6.0 & 38 & 216 & 2912 & 60 & 5.19 & 769 & 3.0 & \\
\hline $\begin{array}{l}\text { Hikarazine- } \\
86\end{array}$ & $\mathbf{1}\{14,1,63\}$ & 0.2 & 21 & 6 & 101169 & 22 & 6.80 & 1500 & 3.8 & \\
\hline $\begin{array}{l}\text { Hikarazine- } \\
96\end{array}$ & $\mathbf{2 6 c o}\{1,2,44\}$ & 4.2 & 68 & 128 & 1962 & 95 & 6.86 & 350 & 2.6 & \\
\hline $\begin{array}{l}\text { Hikarazine- } \\
97\end{array}$ & $\mathbf{2 6 c u}\{1,3,42\}$ & 3.4 & 94 & 173 & 2106 & 40 & 6.06 & 565 & 4.0 & \\
\hline $\begin{array}{l}\text { Hikarazine- } \\
99\end{array}$ & $\mathbf{2 6 c v}\{1,3,44\}$ & 3.4 & 101 & 177 & 2095 & 60 & 5.80 & 319 & 2.4 & \\
\hline $\begin{array}{l}\text { Hikarazine- } \\
103\end{array}$ & $\mathbf{2 6} \mathbf{d} \mathbf{b}\{1,22,37\}$ & 0.5 & 156 & 36 & 1836 & 140 & 2.20 & 30.8 & 1.3 & \\
\hline $\begin{array}{l}\text { Hikarazine- } \\
105\end{array}$ & $\mathbf{2 6 d w}\{2,3,37\}$ & 3.4 & 94 & 146 & 22032 & 63 & 5.53 & 3380 & 1.8 & \\
\hline $\begin{array}{l}\text { Hikarazine- } \\
108\end{array}$ & $\mathbf{2 6 d l}\{1,24,42\}$ & 5.3 & 39 & 178 & 2664 & 80 & 5.52 & 1420 & 4.5 & \\
\hline $\begin{array}{l}\text { Hikarazine- } \\
113\end{array}$ & $\mathbf{2 6 d c}\{1,22,42\}$ & 0.8 & 157 & 58 & 2834 & 64 & 3.30 & 96.1 & 1.4 & \\
\hline $\begin{array}{l}\text { Hikarazine- } \\
149\end{array}$ & $\mathbf{2 6} \mathbf{b h}\{13,1,37\}$ & 2.0 & 32 & 63 & 1804 & 84 & 3.30 & 140 & 4.5 & \\
\hline $\begin{array}{l}\text { Hikarazine- } \\
150\end{array}$ & $\mathbf{2 6} \mathbf{b j}\{13,1,63\}$ & 0.1 & 148 & 6 & 70020 & 35 & 2.94 & 362 & 3.1 & \\
\hline $\begin{array}{l}\text { Hikarazine- } \\
162\end{array}$ & $\operatorname{26bg}\{14,1,42\}$ & 3.0 & 1.5 & 11 & 4677 & 121 & 3.88 & 874 & 280 & \\
\hline
\end{tabular}




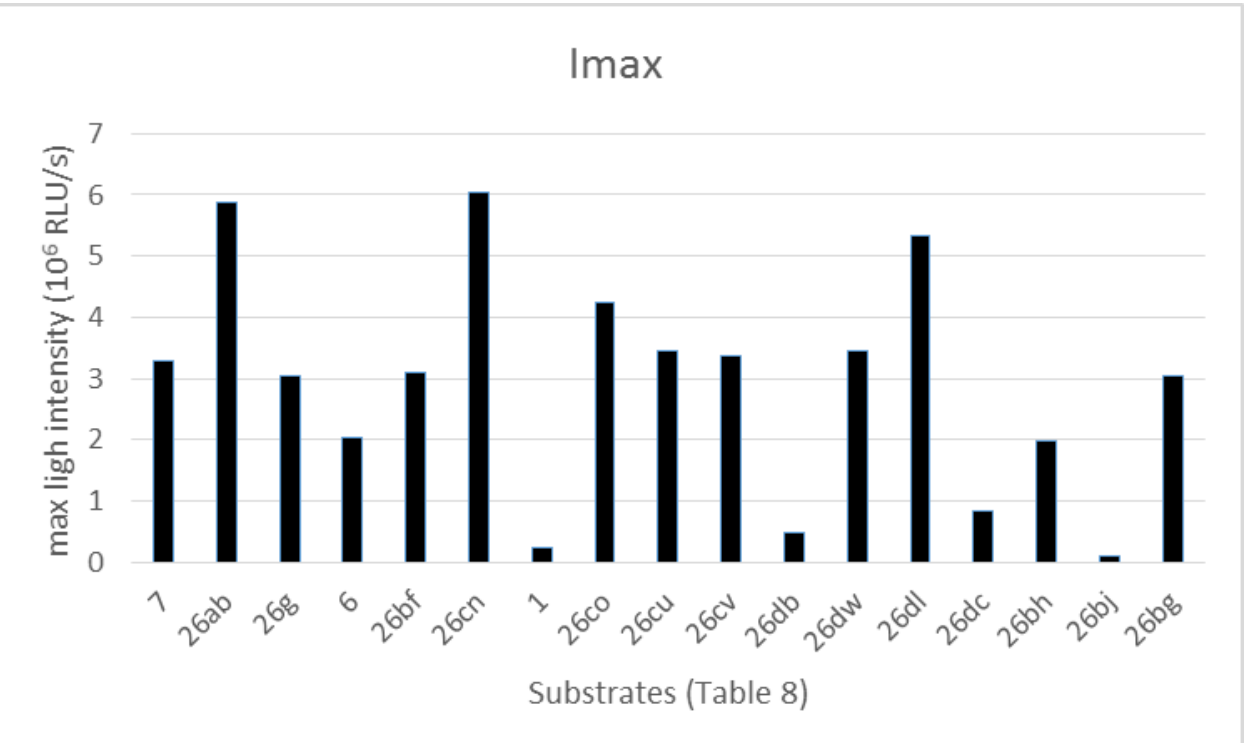

Bar graphs comparison of Imax for all the compounds listed in table 8 . 
General synthetic pathway used for synthesis of the $\mathbf{O}$-acylated luciferins 25 (same numbering than in the main text):<smiles>[R]C=C(C[Y5])NC([R])C[N+](=O)[O-]</smiles><smiles>[R]Cc1nc([R])cnc1Br</smiles>

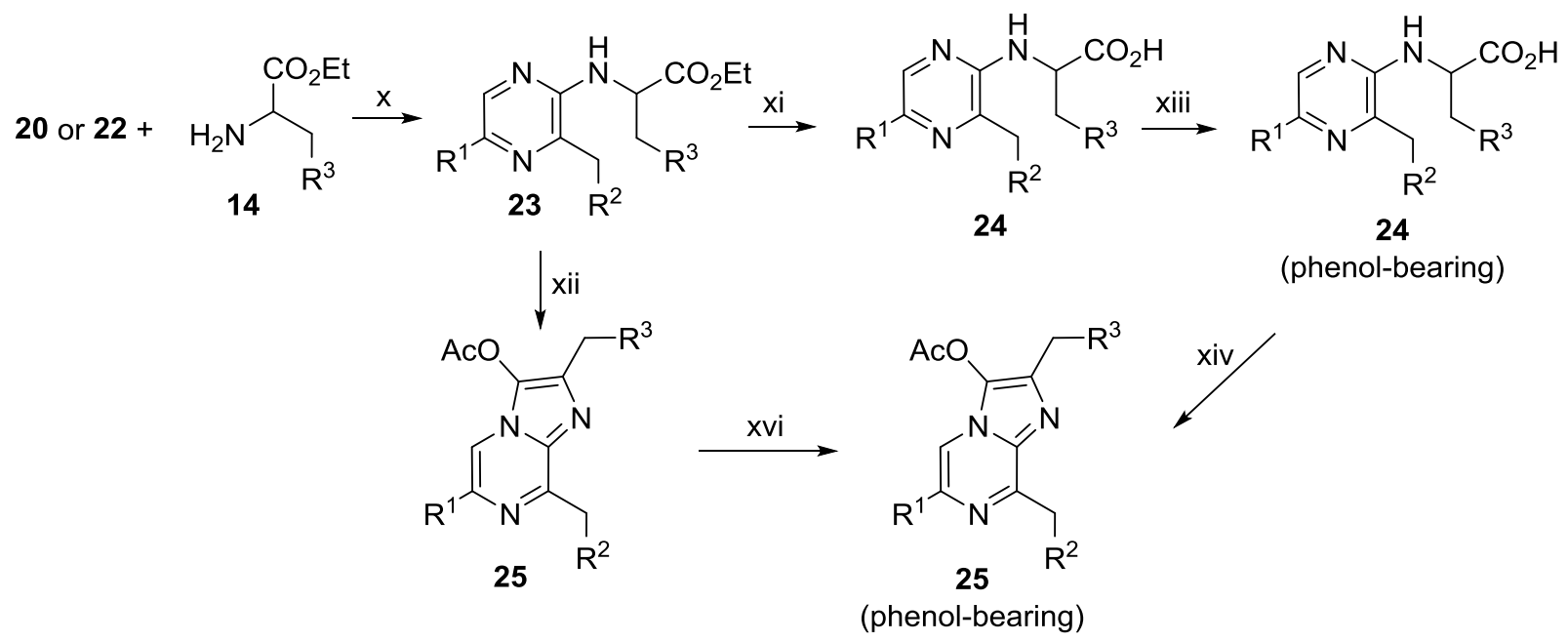

Scheme 1. i: neat, $20{ }^{\circ} \mathrm{C}, 10$ min- 12 h. ii: $\mathrm{Zn}, 37 \% \mathrm{H}_{3} \mathrm{O}^{+} \mathrm{Cl}^{-}$, dioxane, $0-20{ }^{\circ} \mathrm{C}, 2 \mathrm{~h}$. iii: neat, $140{ }^{\circ} \mathrm{C}, 3 \mathrm{~h}$. iv: $\mathrm{S}_{8}$, 1,3- $\mathrm{Cl}_{2} \mathrm{C}_{6} \mathrm{H}_{4}$ or decaline, reflux, $10 \mathrm{~h}$. v: $\mathrm{AcOOH}$, AcOEt, $20^{\circ} \mathrm{C}, 12 \mathrm{~h}$. vi: $\mathrm{NaOH}, \mathrm{EtOH}, 65^{\circ} \mathrm{C}, 1 \mathrm{~h}$. vii: $\mathrm{PhPOCl}_{2}$, $100{ }^{\circ} \mathrm{C}, 12$ h. viii: $\mathrm{Tf}_{2} \mathrm{O}, \mathrm{NEt}_{3}, \mathrm{CH}_{2} \mathrm{Cl}_{2}, 20^{\circ} \mathrm{C}, 40$ min. ix: $\mathrm{NaBr}$, TfOH, DMF, $120^{\circ} \mathrm{C}, 12 \mathrm{~h} . \mathrm{x}: \mathrm{Cs}_{2} \mathrm{CO}_{3}, \mathrm{Pd}(\mathrm{OAc})_{2}$, BINAP, MeCN, $60{ }^{\circ} \mathrm{C}$ or toluene, $90{ }^{\circ} \mathrm{C}, 12$ h. xi: a) $\mathrm{NaOH}$, THF, $20^{\circ} \mathrm{C}, 12 \mathrm{~h}$, b) $\mathrm{NH}_{4} \mathrm{Cl}, \mathrm{H}_{2} \mathrm{O}$. xii: a) $\mathrm{NaOH}, \mathrm{THF}$, $20{ }^{\circ} \mathrm{C}, 12 \mathrm{~h}$, b) $\mathrm{Ac}_{2} \mathrm{O}, 20^{\circ} \mathrm{C}, 2$ h. xiii: $\mathrm{Pd} / \mathrm{C}, \mathrm{NH}_{4}{ }^{+} \mathrm{HCO}_{2}{ }^{-}$, EtOH, reflux, 90 min. xiv: $\mathrm{Ac} 2 \mathrm{O}$, AcOEt, reflux, 30 min. xvi: $\mathrm{H}_{2}, \mathrm{Pd} / \mathrm{C}, \mathrm{AcOEt}, \mathrm{AcOH}, \mathrm{EtOH}, 20^{\circ} \mathrm{C}, 12 \mathrm{~h}$. 
Structure and numbering of the 12 nitrostyrenes 13 used. The last two were not used in the synthetic scheme depicted above but are provided for the convenient numbering of the phenol-bearing substrates prepared:<smiles>O=NC=Cc1ccccc1</smiles>

1<smiles>O=[N+]([O-])C=Cc1c(F)cccc1F</smiles>

10<smiles>Cc1ccc(F)cc1</smiles>

o, $m, p$

2, 3, 4<smiles>O=[N+]([O-])C=Cc1cccc(OCc2ccccc2)c1</smiles>

$m, p$

$m, p$

5,6<smiles>COc1ccc(/C=C/[N+](=O)[O-])cc1/C=C/c1ccc(C)cc1</smiles>

$0, m, p$

7, 8,9<smiles>O=[N+]([O-])C=Cc1ccccc1</smiles>

$m, p$

Note: these nitrostyrenes are commercially available and/or their preparations has been reported previously in the case of compound $\mathbf{1 3}\{5\},{ }^{[1]}$ and $\mathbf{1 3}\{10\} .{ }^{[2]}$ 
Structure and numbering of the $\alpha$-amino esters ${ }^{[3]} 14$ used. The last four were not used in the synthetic scheme depicted above but are provided for the convenient numbering of substrates prepared.<smiles>CCOC(=O)CCc1ccccc1</smiles>

1<smiles>CCOC(N)Cc1ccccc1</smiles>

$0, m, p$

$2,3,4$

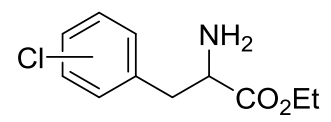

o, $m, p$

$5,6,7$

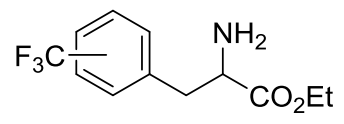

$0, m, p$

$8,9,10$<smiles>CCOC(=O)C(N)Cc1ccccc1</smiles>

o, $m, p$

$11,12,13$<smiles>CCOC(N)Cc1ccc2c(OC)c1CCC2OCC</smiles>

$0, m, p$

$14,15,16$<smiles>CCOC(=O)CCc1ccc2cc1[R9](Cc1ccccc1)=C2</smiles>

$17,18,19$<smiles>CCOC(=O)CCc1ccc(Br)cc1</smiles>

20<smiles>[Z17]C(Cc1cccc(F)c1F)Cc1cccc(F)c1CC(N)C(=O)OCC</smiles><smiles>CCOC(=O)C(N)Cc1cc(F)cc(F)c1F</smiles><smiles>CCOC(=O)C(N)Cc1ccccc1</smiles><smiles>CCOC(=O)C(N)Cc1cccc(C2CC2)c1</smiles>
m, p

$m, p$<smiles>CCOC(N)Cc1ccc(C(C)C)cc1</smiles>

27, 28

29,30

31<smiles>CCOC(=O)C(N)CCc1ccccc1</smiles>

32<smiles>CCOC(N)CC1CCCCC1</smiles><smiles>CCOC(=O)C(N)Cc1ccccn1</smiles><smiles>CCOC(=O)C(N)Cc1cccnc1</smiles><smiles>CCOC(=O)C(N)CC1CCCC1</smiles>

33

34

35

36

U1 $\mathrm{CO}_{2 \mathrm{Et}}^{\mathrm{NH}_{2}}$

37<smiles>CCOC(N)CC1CCCO1</smiles>

38<smiles>CCOC(=O)CC1OCCO1</smiles>

39<smiles>CCOC(=O)CCc1ccoc1</smiles>

40

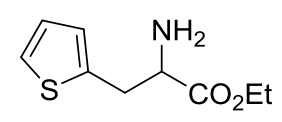

41<smiles>[R]OC(=O)C(N)Cc1ccc([R])o1</smiles>

42: $\mathrm{R}=\mathrm{Me} \quad$ 45: $\mathrm{R}=n \mathrm{Pr}$ 43: $\mathrm{R}=\mathrm{CF}_{3}$ 46: $\mathrm{R}=n$ Pent 44: $\mathrm{R}=\mathrm{Et} \quad$ 47: $\mathrm{R}=c \mathrm{Pr}$<smiles>CCOC(=O)C(N)Cc1cc(C)c(C)o1</smiles><smiles>CCOC(=O)C(N)Cc1cc2c(o1)CCCC2</smiles>

49<smiles>CCOC(=O)C(N)Cc1cc(C)c(CC)o1</smiles>

50<smiles>CCOC(=O)C(N)Cc1cc(CC(N)C(=O)OCC)c(CC(N)C(OCC)OCc2ccc(CC(N)C(OCC)OCc3cc(C)c(C(C)C)o3)s2)o1</smiles>
51

52 53<smiles>[R]c1cc(C[C@@H](N)OCC)on1</smiles>
56: $\mathrm{R}=\mathrm{Me}$ 57: $\mathrm{R}=\mathrm{Et}$<smiles>Cc1cnc(CC(N)C(=O)OCc2ccccc2)o1</smiles>

58<smiles>CCOC(C)N</smiles><smiles>CCOC(=O)C(N)Cc1ccccc1</smiles>

Note: by using the synthetic scheme depicted above, we have previously reported ${ }^{[4]}$ the preparations of the chloropyrazines $\mathbf{2 0}\{1,1\}, \mathbf{2 0}\{11,1\}$ and $\mathbf{2 0}\{12,1\}$ as well as the O-acetylated luciferins $\mathbf{2 5}\{1,1,1\}, \mathbf{2 5}\{1,1,37\}$, $\mathbf{2 5}\{13,1,37\}, \mathbf{2 5}\{13,1,63\}, \mathbf{2 5}\{14,1,1\}, \mathbf{2 5}\{14,1,37\}, \mathbf{2 5}\{14,1,63\}$ : 

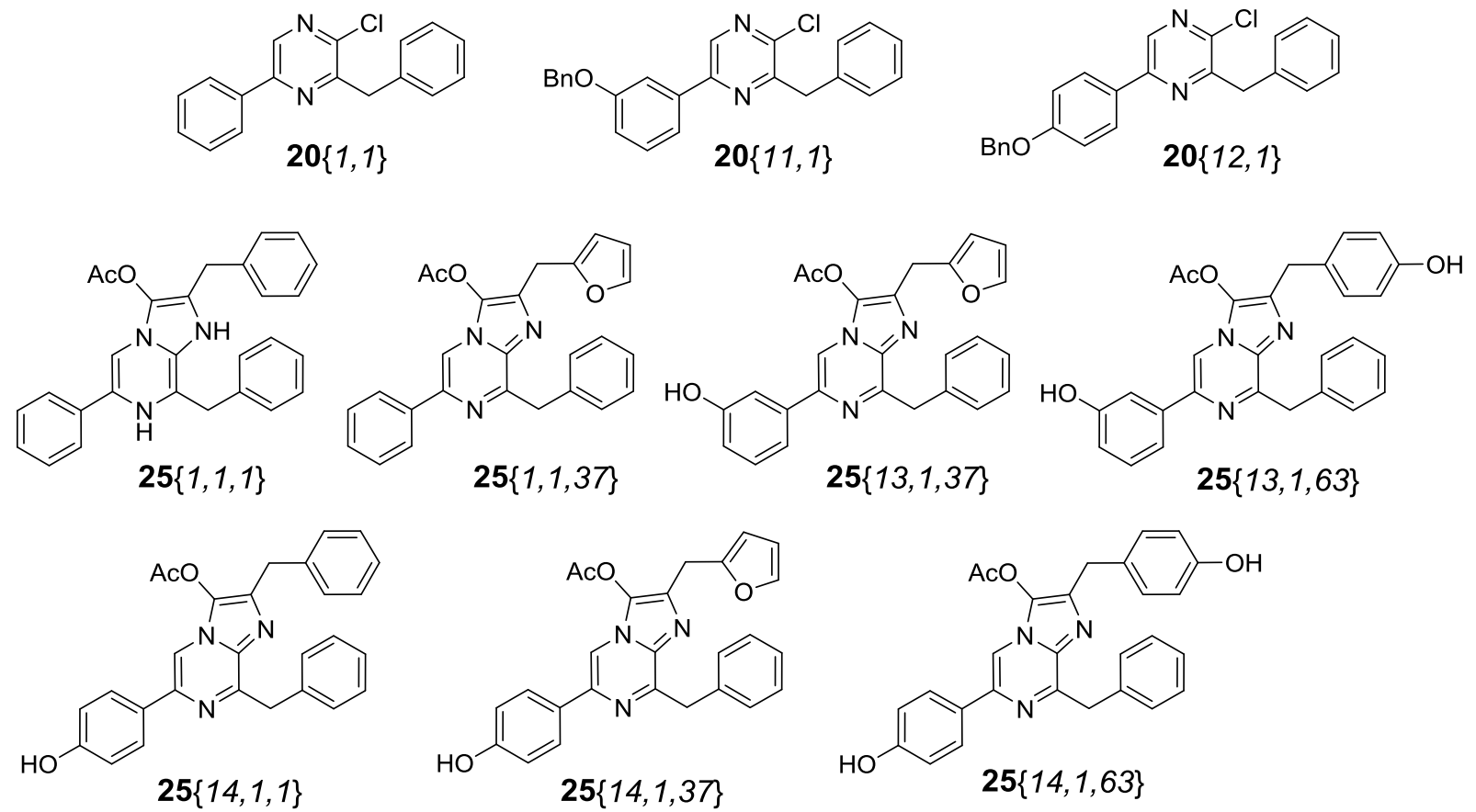

General preparation of piperazin-2-ones 17 via steps i-iii. Step i: the considered nitrostyrene 13 (0.1 mol) was added to a freshly extracted free base of $\alpha$-amino ester $14(0.1 \mathrm{~mol})$ (see notes 1 and 2$)$. Upon stirring on a rotatory evaporator at room temperature the suspension homogenized in sometime as quickly as 10 minutes to give the 1,4adduct 15 as an oil (see note 3). Step ii: this oil was dispersed in a cold solution of dioxane (300 mL) and 37\% hydrochloric acid $(110 \mathrm{~mL}, 1.2 \mathrm{~mol})$. Zinc dust $(34.4 \mathrm{~g}, 0.4 \mathrm{~mol}$, size $<10 \mu \mathrm{m})$ was promptly added portion-wise in the course of 10-15 minutes while cooling the reaction with an ice bath. The temperature was then allowed to rise back to room temperature and the suspension stirred for 2 hours. Most of the dioxane was removed (note 4) under vacuum and the residue was diluted in water, made basic with an excess of $22 \%$ ammonia and extracted with ethyl acetate. The organic layer was washed with $10 \%$ ammonia, brine, dried over sodium carbonate and concentrated to dryness to give the crude diamine 16 as an oil. Step iii: under an argon atmosphere, this oil was heated at $140{ }^{\circ} \mathrm{C}$ for 3 hours. The ethanol was removed under vacuum and the resulting solid was subjected to purification procedures as described below for each pair of diastereoisomers 17 (note 5). Concerning these diastereoisomers, their structure attribution was easily performed by checking for the existence (or not) of a nOe effect between $\mathrm{H}-3$ and H-5. Note 1: In the course of this work, it was found that for step i, dissolving the reagents in a small amount of dichloromethane followed by a concentration to dryness insured their proper mixing. Note 2: Mixing the nitrostyrene 13, the hydrochloride salt of the amino ester 14 and one equivalent of triethylamine in a small amount of dichloromethane prior to a concentration to dryness was also found possible, the resulting triethylamine hydrochloride did not interfere with the next step. Note 3: Ten minutes was found (by ${ }^{1} \mathrm{H}$ NMR) to be enough in some cases but the homogenous mixture was usually left to react overnight to ensure an almost complete 1,4addition (as seen by ${ }^{1} \mathrm{H}$ NMR). Note 4: This concentration is not required on a smaller scale. Note 5: In some cases (mentioned below) this separation was not performed and the crude mixture of diastereoisomers was directly used in the next step, fairly often via step v, to prepare the nitrones $\mathbf{1 8 .}$<smiles>O=C1NC[C@@H](c2ccccc2)NC1Cc1ccccc1F</smiles>

Cis-3-(2-fluorobenzyl)-5-phenylpiperazin-2-one 17\{1,2\} (YJ31070-020-5): This racemic isomer was obtained as a white powder $(2.85 \mathrm{~g}, 31 \%$ ) after a chromatography over silica gel (dichloromethane - ethanol 97/3 to 95/5) and a dispersion in boiling cyclohexane. ${ }^{1} \mathrm{H}$ NMR $\left(\mathrm{CDCl}_{3}\right)$ : $7.35(\mathrm{~m}, 6 \mathrm{H}), 7.21(\mathrm{~m}, 1 \mathrm{H}), 7.07(\mathrm{~m}, 2 \mathrm{H}), 6.60(\mathrm{~s}(\mathrm{br}), 1 \mathrm{H})$, $4.08(\mathrm{dd}, 1 \mathrm{H}, J=6.4,8.3 \mathrm{~Hz}), 3.92(\mathrm{dd}, 1 \mathrm{H}, J=3.1,9.6 \mathrm{~Hz}), 3.65(\mathrm{dd}, 1 \mathrm{H}, J=3.0,14.0 \mathrm{~Hz}), 3.34(\mathrm{~m}, 2 \mathrm{H}), 2.97$ $(\mathrm{dd}, 1 \mathrm{H}, J=9.6,14.0 \mathrm{~Hz}), 1.78(\mathrm{~s}(\mathrm{br}), 1 \mathrm{H}) .{ }^{13} \mathrm{C} \mathrm{NMR}\left(\mathrm{CDCl}_{3}\right): 170.9,161.6(246 \mathrm{~Hz}), 140.2,131.7(5 \mathrm{~Hz}), 128.7$, 
$128.4(8 \mathrm{~Hz}), 128.2,126.8,125.3(15 \mathrm{~Hz}), 124.1(3 \mathrm{~Hz}), 115.5(22 \mathrm{~Hz}), 59.6,57.7,49.9,31.9$. HRMS $(\mathrm{m} / z)$ : $[\mathrm{M}+\mathrm{H}]^{+}$calcd for $\mathrm{C}_{17} \mathrm{H}_{18} \mathrm{FN}_{2} \mathrm{O}: 285.1403$, found: 285.1412 .<smiles>O=C1NCC(c2ccccc2)NC1Cc1ccccc1F</smiles>

Trans-3-(2-fluorobenzyl)-5-phenylpiperazin-2-one 17 $\{1,2\}$ (YJ31070-020-6): This racemic isomer was obtained as a white powder (2.15 g, 23\%) after a chromatography over silica gel (dichloromethane - ethanol 97/3 to 95/5) and a recrystallization of a sample in cyclohexane for analytical purposes. ${ }^{1} \mathrm{H}$ NMR $\left(\mathrm{CDCl}_{3}\right): 7.40-7.26(\mathrm{~m}, 6 \mathrm{H}), 7.21(\mathrm{~m}$, $1 \mathrm{H}), 7.09(\mathrm{~m}, 1 \mathrm{H}), 7.03(\mathrm{~m}, 1 \mathrm{H}), 6.92(\mathrm{~s}(\mathrm{br}), 1 \mathrm{H}), 4.33(\mathrm{dd}, 1 \mathrm{H}, J=3.9,9.8 \mathrm{~Hz}), 3.92(\mathrm{dd}, 1 \mathrm{H}, J=3.9,10.6 \mathrm{~Hz})$, 3.51-3.33 (m, 3H), $3.27(\mathrm{dd}, 1 \mathrm{H}, J=4.0,14.0 \mathrm{~Hz}), 1.72(\mathrm{~s}(\mathrm{br}), 1 \mathrm{H}) .{ }^{13} \mathrm{C} \mathrm{NMR}\left(\mathrm{CDCl}_{3}\right): 171.7,161.7(244 \mathrm{~Hz})$, 140.0, $131.4(4 \mathrm{~Hz}), 128.7,128.6(8 \mathrm{~Hz}), 128.2,127.0,125.0(15 \mathrm{~Hz}), 124.5(3 \mathrm{~Hz}), 115.5(22 \mathrm{~Hz}), 58.8,51.7$, 49.2, 31.1. HRMS $(\mathrm{m} / \mathrm{z}):[\mathrm{M}+\mathrm{H}]^{+}$calcd for $\mathrm{C}_{17} \mathrm{H}_{18} \mathrm{FN}_{2} \mathrm{O}: 285.1403$, found: 285.1410 .<smiles>O=C1NC[C@@H](c2ccccc2)NC1Cc1cccc(F)c1</smiles>

Cis-3-(3-fluorobenzyl)-5-phenylpiperazin-2-one 17\{1,3\} (YJ31070-152-1): This isomer was obtained as a powder $(2.25 \mathrm{~g}, 13 \%)$ after a chromatography over silica gel (dichloromethane - ethanol 97.5/2.5) and a recrystallization in a mixture of toluene and cyclohexane. ${ }^{1} \mathrm{H}$ NMR $\left(\mathrm{CDCl}_{3}\right)$ : 7.40-7.24 (m, 6H), 7.10 (m, 1H), $7.05(\mathrm{~m}, 1 \mathrm{H}), 6.92(\mathrm{~m}$, $1 \mathrm{H}), 6.62(\mathrm{~s}(\mathrm{br}), 1 \mathrm{H}), 4.07(\mathrm{~m}, 1 \mathrm{H}), 3.85(\mathrm{dd}, 1 \mathrm{H}, J=2.9,10.0 \mathrm{~Hz}), 3.55(\mathrm{dd}, 1 \mathrm{H}, J=2.9,13.5 \mathrm{~Hz}), 3.34(\mathrm{~m}, 2 \mathrm{H})$, $2.93(\mathrm{dd}, 1 \mathrm{H}, J=10.0,13.5 \mathrm{~Hz}), 1.74$ (s (br), $1 \mathrm{H}) .{ }^{13} \mathrm{C} \mathrm{NMR}\left(\mathrm{CDCl}_{3}\right): 170.8,162.9(245 \mathrm{~Hz}), 140.9(7 \mathrm{~Hz}), 140.0$, $130.0(7 \mathrm{~Hz}), 128.7,128.3,126.8,125.1(2 \mathrm{~Hz}), 116.2(21 \mathrm{~Hz}), 113.5(21 \mathrm{~Hz}), 60.6,57.7,49.8,38.3 . \mathrm{HRMS}(\mathrm{m} / \mathrm{z})$ : $[\mathrm{M}+\mathrm{H}]^{+}$calcd for $\mathrm{C}_{17} \mathrm{H}_{19} \mathrm{FN}_{2} \mathrm{O}: 285.1403$, found: 285.1400 .

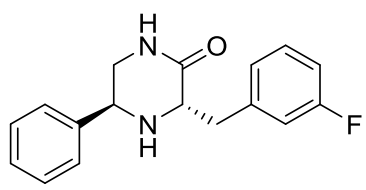

Trans-3-(3-fluorobenzyl)-5-phenylpiperazin-2-one 17\{1,3\} (YJ31070-151-5): This isomer was obtained as a powder $(2.49 \mathrm{~g}, 15 \%)$ after a chromatography over silica gel (dichloromethane - ethanol 97.5/2.5). ${ }^{1} \mathrm{H} \mathrm{NMR}$ $\left(\mathrm{CDCl}_{3}\right): 7.35(\mathrm{~m}, 5 \mathrm{H}), 7.26(\mathrm{~m}, 3 \mathrm{H}), 6.70(\mathrm{~s}(\mathrm{br}), 1 \mathrm{H}), 4.21(\mathrm{dd}, 1 \mathrm{H}, J=4.2,9.3 \mathrm{~Hz}), 3.87(\mathrm{dd}, 1 \mathrm{H}, J=3.8,10.3$ $\mathrm{Hz}), 3.54-3.41(\mathrm{~m}, 2 \mathrm{H}), 3.30(\mathrm{dd}, 1 \mathrm{H}, J=3.8,14.0 \mathrm{~Hz}), 3.30(\mathrm{dd}, 1 \mathrm{H}, J=10.3,14.0 \mathrm{~Hz}), 1.77(\mathrm{~s}(\mathrm{br}), 1 \mathrm{H}) .{ }^{13} \mathrm{C}$ NMR $\left(\mathrm{CDCl}_{3}\right): 171.5,163.0(245 \mathrm{~Hz}), 140.6(7 \mathrm{~Hz}), 139.6,130.2(7 \mathrm{~Hz}), 128.8,128.2,126.9,125.0(2 \mathrm{~Hz}), 116.2$ $(21 \mathrm{~Hz}), 113.7(21 \mathrm{~Hz}), 59.0,51.8,49.0,37.6$. HRMS $(\mathrm{m} / \mathrm{z}):[\mathrm{M}+\mathrm{H}]^{+}$calcd for $\mathrm{C}_{17} \mathrm{H}_{19} \mathrm{FN}_{2} \mathrm{O}: 285.1403$, found: 285.1409 .<smiles>O=C1NCC(c2ccccc2)NC1Cc1ccc(F)cc1</smiles>

3-(4-fluorobenzyl)-5-phenylpiperazin-2-one 17\{1,4\} (YJ31067-121-3): This compound was obtained as white powder $(0.9 \mathrm{~g}, 59 \%$ from nitrostyrene), as a mixture of diastereoisomers after a recrystallization in cyclohexane. HRMS (m/z): $[\mathrm{M}+\mathrm{H}]^{+}$calcd for $\mathrm{C}_{17} \mathrm{H}_{18} \mathrm{FN}_{2} \mathrm{O}$ : 285.1403, found: 285.1408.<smiles>O=C1NCC(c2ccccc2)NC1Cc1ccccc1Cl</smiles> 
3-(2-Chlorobenzyl)-5-phenylpiperazin-2-one 17\{1,5\} (EC31094-085-3): This compound was obtained a white powder containing a mixture of the two diastereoisomers after a recrystallization in cyclohexane $(2.21 \mathrm{~g}, 45 \%),{ }^{1} \mathrm{H}$ NMR $\left(\mathrm{CDCl}_{3}\right)$ : 7.42-7.29 (m, 7H), 7.25-7.14 (m, 2H), $6.85(\mathrm{~s}(\mathrm{br}), 0.4 \mathrm{H}), 6.69(\mathrm{~s}(\mathrm{br}), 0.6 \mathrm{H}), 4.37(\mathrm{dd}, 0.4 \mathrm{H}, J=$ 9.8, $4.2 \mathrm{~Hz}), 4.10-3.98(\mathrm{~m}, 1.4 \mathrm{H}), 3.83(\mathrm{dd}, 0.6 \mathrm{H}, J=13.8,3.4 \mathrm{~Hz}), 3.51-3.30(\mathrm{~m}, 3 \mathrm{H}), 3.02(\mathrm{dd}, 0.6 \mathrm{H}, J=13.8$, 9.8 Hz). HRMS (m/z): $[\mathrm{M}+\mathrm{H}]^{+}$calcd for $\mathrm{C}_{17} \mathrm{H}_{18} \mathrm{ClN}_{2} \mathrm{O}$ : 301.1108; found, 301.1117.

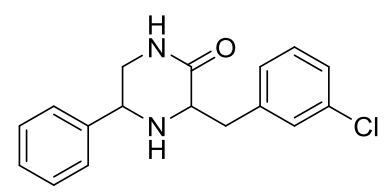

3-(3-Chlorobenzyl)-5-phenylpiperazin-2-one 17\{1,6\} (VM32021-015-1): This mixture of isomers was obtained as an oil and used directly in the N-oxidation step v.<smiles>O=C1NC[C@@H](c2ccccc2)N[C@H]1Cc1ccccc1C(F)(F)F</smiles>

Cis-5-phenyl-3-(2-(trifluoromethyl)benzyl)piperazin-2-one 17 1,8\} (YJ 33067-99-4): This isomer was obtained as a powder (3.51 g, 35\%) after a chromatography over silica gel (dichloromethane - ethanol 98/2 to 96.5/3.5) and a dispersion boiling cyclohexane. ${ }^{1} \mathrm{H}$ NMR (DMSO- $\left.d_{6}\right)$ : $7.84(\mathrm{~m}, 1 \mathrm{H}), 7.71(\mathrm{~m}, 1 \mathrm{H}), 7.66(\mathrm{~m}, 1 \mathrm{H}), 7.58(\mathrm{~m}, 1 \mathrm{H})$, 7.41-7.24 (m, 6H), $4.02(\mathrm{~m}, 1 \mathrm{H}), 3.74(\mathrm{~m}, 1 \mathrm{H}), 3.62(\mathrm{~m}, 1 \mathrm{H}), 3.30(\mathrm{dt}, 1 \mathrm{H}, J=4.1,11.3 \mathrm{~Hz}), 3.17(\mathrm{t}, 1 \mathrm{H}, J=11.5$ $\mathrm{Hz}), 2.93(\mathrm{dd}, 1 \mathrm{H}, J=9.8,14.9 \mathrm{~Hz}), 2.49(\mathrm{~m}, 1 \mathrm{H}) .{ }^{13} \mathrm{C}$ NMR (DMSO- $\left.d_{6}\right): 170.2,141.7,138.4(2 \mathrm{~Hz}), 132.5,132.3$, 128.7, $128.1(30 \mathrm{~Hz}), 127.8,127.0,126.9,126.1(6 \mathrm{~Hz}), 125.1(274 \mathrm{~Hz}), 59.4,56.6,49.3,34.2$. HRMS $(\mathrm{m} / z)$ : $[\mathrm{M}+\mathrm{H}]^{+}$calcd for $\mathrm{C}_{18} \mathrm{H}_{18} \mathrm{~F}_{3} \mathrm{~N}_{2} \mathrm{O}: 335.1371$, found: 335.1379 .

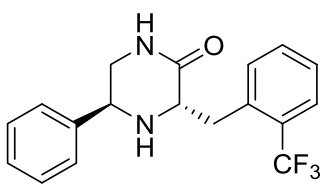

Trans-5-phenyl-3-(2-(trifluoromethyl)benzyl)piperazin-2-one 17 \{1,8\} (YJ 33067-99-3): This isomer was obtained as a powder $(1.83 \mathrm{~g}, 18 \%)$ after a chromatography over silica gel (dichloromethane - ethanol 98/2 to 96.5/3.5). ${ }^{1} \mathrm{H}$ NMR (DMSO- $\left.d_{6}\right): 7.89(\mathrm{~m}, 1 \mathrm{H}), 7.63(\mathrm{~m}, 1 \mathrm{H}), 7.55(\mathrm{~m}, 2 \mathrm{H}), 7.37(\mathrm{~m}, 3 \mathrm{H}), 7.31(\mathrm{~m}, 2 \mathrm{H}), 7.24(\mathrm{~m}, 1 \mathrm{H}), 4.25(\mathrm{~m}$, $1 \mathrm{H}), 3.59(\mathrm{~m}, 1 \mathrm{H}), 3.44(\mathrm{dt}, 1 \mathrm{H}, J=3.9,11.7 \mathrm{~Hz}), 3.35(\mathrm{~m}, 1 \mathrm{H}), 3.23(\mathrm{t}, 1 \mathrm{H}, J=10.9 \mathrm{~Hz}), 3.11(\mathrm{dd}, 1 \mathrm{H}, J=10.7$, $13.7 \mathrm{~Hz}$ ), 2.67 (dd, $1 \mathrm{H}, J=5.6,8.8 \mathrm{~Hz}) .{ }^{13} \mathrm{C}$ NMR (DMSO- $\left.d_{6}\right): 171.1,141.5,138.5(2 \mathrm{~Hz}), 133.1,132.3,128.7$, $128.0(30 \mathrm{~Hz}), 127.6,127.1,127.0,126.0(6 \mathrm{~Hz}), 125.1(274 \mathrm{~Hz}), 58.0,51.1,48.6,33.8$. HRMS $(\mathrm{m} / \mathrm{z}):[\mathrm{M}+\mathrm{H}]^{+}$ calcd for $\mathrm{C}_{18} \mathrm{H}_{18} \mathrm{~F}_{3} \mathrm{~N}_{2} \mathrm{O}$ : 335.1371, found: 335.1369 .

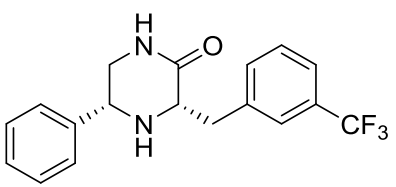

Cis-5-phenyl-3-(3-(trifluoromethyl)benzyl)piperazin-2-one 17\{1,9\} (YJ 33067-101-4): This isomer was obtained as a powder $(2.90 \mathrm{~g}, 32 \%)$ after a chromatography over silica gel (dichloromethane - ethanol 98/2 to 96.5/3.5) and a (slow) recrystallization in cyclohexane. ${ }^{1} \mathrm{H}$ NMR (DMSO- $\left.d_{6}\right): 7.75(\mathrm{~m}, 2 \mathrm{H}), 7.61(\mathrm{~m}, 1 \mathrm{H}), 7.51(\mathrm{~m}, 2 \mathrm{H}), 7.40-$ $7.24(\mathrm{~m}, 5 \mathrm{H}), 4.02(\mathrm{dt}, 1 \mathrm{H}, J=3.3,10.4 \mathrm{~Hz}), 3.75(\mathrm{~m}, 1 \mathrm{H}), 3.23(\mathrm{dd}, 1 \mathrm{H}, J=4.0,13.9 \mathrm{~Hz}), 3.62(\mathrm{~m}, 1 \mathrm{H}), 3.17(\mathrm{dt}$, $1 \mathrm{H}, J=3.6$ and $10.9 \mathrm{~Hz}), 3.04(\mathrm{dd}, 1 \mathrm{H}, J=6.6,14.4 \mathrm{~Hz}), 2.94(\mathrm{t}, 1 \mathrm{H}, J=11.4 \mathrm{~Hz}), 2.77(\mathrm{~m}, 1 \mathrm{H}) .{ }^{13} \mathrm{C} \mathrm{NMR}$ (DMSO- $\left.d_{6}\right)$ : 170.0, 141.8, 141.1, 134.4, 129.4, $128.9(30 \mathrm{~Hz}), 128.7,127.9,127.1,126.9(6 \mathrm{~Hz}), 124.9(271 \mathrm{~Hz})$, $123.0(3 \mathrm{~Hz}), 59.9,56.7,49.3,37.6$. HRMS $(\mathrm{m} / \mathrm{z}):[\mathrm{M}+\mathrm{H}]^{+}$calcd for $\mathrm{C}_{18} \mathrm{H}_{18} \mathrm{~F}_{3} \mathrm{~N}_{2} \mathrm{O}: 335.1371$, found: 335.1372 . 


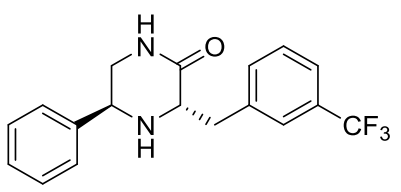

Trans-5-phenyl-3-(3-(trifluoromethyl)benzyl)piperazin-2-one 17\{1,9\} (YJ 33067-101-3): This isomer was obtained as a powder $(2.03 \mathrm{~g}, 22 \%)$ after a chromatography over silica gel (dichloromethane - ethanol 98/2 to 96.5/3.5). ${ }^{1} \mathrm{H}$ NMR (DMSO-d6): $7.86(\mathrm{~m}, 1 \mathrm{H}), 7.65(\mathrm{~m}, 1 \mathrm{H}), 7.55(\mathrm{~m}, 3 \mathrm{H}), 7.36-7.23(\mathrm{~m}, 5 \mathrm{H}), 4.07(\mathrm{~m}, 1 \mathrm{H}), 3.55$ $(\mathrm{m}, 1 \mathrm{H}), 3.36(\mathrm{dt}, 1 \mathrm{H}, J=3.7,11.6 \mathrm{~Hz}), 3.24(\mathrm{~m}, 1 \mathrm{H}), 3.14(\mathrm{~m}, 2 \mathrm{H}), 2.71(\mathrm{~m}, 1 \mathrm{H}) .{ }^{13} \mathrm{C}$ NMR $\left(\mathrm{DMSO}-d_{6}\right): 171.1$, 141.5, 133.9, 129.7, 129.2 (30 Hz), 128.6, 127.1, 126.0 (4 Hz), $124.9(274 \mathrm{~Hz}), 123.2(4 \mathrm{~Hz}), 58.3,51.2,48.1,37.5$ (two signals missing). HRMS $(\mathrm{m} / \mathrm{z})$ : $[\mathrm{M}+\mathrm{H}]^{+}$calcd for $\mathrm{C}_{18} \mathrm{H}_{18} \mathrm{~F}_{3} \mathrm{~N}_{2} \mathrm{O}: 335.1371$, found: 335.1371 .<smiles>Cc1ccccc1C[C@@H]1N[C@H](c2ccccc2)CNC1=O</smiles>

Cis-3-(2-methylbenzyl)-5-phenylpiperazin-2-one 17\{1,11\} (YJ31070-175-4): This compound was obtained as a white powder (4.04 g, 26\%) after a chromatography over silica gel (dichloromethane / ethanol 98/2 to 95/5) and a dispersion in boiling cyclohexane. ${ }^{1} \mathrm{H}$ NMR $\left(\mathrm{CDCl}_{3}\right)$ : $7.40-7.27(\mathrm{~m}, 6 \mathrm{H}), 7.14(\mathrm{~m}, 3 \mathrm{H}), 6.58(\mathrm{~s}(\mathrm{br}), 1 \mathrm{H}), 4.03$ (dd, $1 \mathrm{H}, J=4.1,10.5 \mathrm{~Hz}$ ), 3.82 (d (br), 1H, $J=10.5 \mathrm{~Hz}$ ), 3.68 (dd, $1 \mathrm{H}, J=2.7,14.0 \mathrm{~Hz}), 3.36(\mathrm{~m}, 2 \mathrm{H}), 2.92(\mathrm{dd}$, $1 \mathrm{H}, J=10.8,13.7 \mathrm{~Hz}), 2.45(\mathrm{~s}, 3 \mathrm{H}), 1.77(\mathrm{~s}, 1 \mathrm{H}) .{ }^{13} \mathrm{C} \mathrm{NMR}\left(\mathrm{CDCl}_{3}\right): 171.2,140.3,137.1,136.6,130.6,129.9$, 128.7, 128.2, 126.8, 126.7, 126.1, 60.0, 57.9, 50.0, 35.6, 19.7. HRMS $(m / z):[\mathrm{M}+\mathrm{H}]^{+}$calcd for $\mathrm{C}_{18} \mathrm{H}_{21} \mathrm{~N}_{2} \mathrm{O}$, 281.1654; found: 281.1652 .

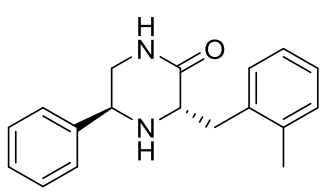

Trans-3-(2-methylbenzyl)-5-phenylpiperazin-2-one 17\{1,11\} (YJ31070-175-5): This compound was obtained as a white powder $(2.77 \mathrm{~g}, 17 \%)$ after a chromatography over silica gel (dichloromethane / ethanol 98/2 to 95/5) and a dispersion in boiling cyclohexane. ${ }^{1} \mathrm{H} \mathrm{NMR}\left(\mathrm{CDCl}_{3}\right)$ : $7.41-7.30(\mathrm{~m}, 5 \mathrm{H}), 7.14(\mathrm{~m}, 4 \mathrm{H}), 6.80(\mathrm{~s}(\mathrm{br}), 1 \mathrm{H}), 4.35$ $(\mathrm{dd}, 1 \mathrm{H}, J=5.0,9.8 \mathrm{~Hz}), 3.89(\mathrm{dd}, 1 \mathrm{H}, J=3.3,11.2 \mathrm{~Hz}), 3.43(\mathrm{~m}, 2 \mathrm{H}), 3.39$ (dd, 1H, $J=3.3,13.9 \mathrm{~Hz}), 3.16$ (dd, $1 \mathrm{H}, J=11.2,13.9 \mathrm{~Hz}), 2.40(\mathrm{~s}, 3 \mathrm{H}), 1.76(\mathrm{~s}, 1 \mathrm{H}) .{ }^{13} \mathrm{C} \mathrm{NMR}\left(\mathrm{CDCl}_{3}\right): 172.0,139.9,136.8,136.2,130.8,130.0$, 128.8, 128.2, 126.9, 126.8, 126.2, 58.2, 52.0, 49.4, 35.0, 19.5. HRMS $(m / z):[\mathrm{M}+\mathrm{H}]^{+}$calcd for $\mathrm{C}_{18} \mathrm{H}_{21} \mathrm{~N}_{2} \mathrm{O}$, 281.1654; found: 281.1652 .

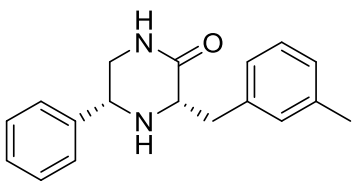

Cis-3-(3-methylbenzyl)-5-phenylpiperazin-2-one 17\{1,12\} (YJ31070-177-4): This compound was obtained as a white powder (3.70 g, 26\%) after a chromatography over silica gel (dichloromethane / ethanol 98/2 to 95/5) and a dispersion in boiling cyclohexane. ${ }^{1} \mathrm{H}$ NMR $\left(\mathrm{CDCl}_{3}\right)$ : $7.40-7.28(\mathrm{~m}, 5 \mathrm{H}), 7.20(\mathrm{~m}, 1 \mathrm{H}), 7.13(\mathrm{~m}, 2 \mathrm{H}), 7.04(\mathrm{~m}$, $1 \mathrm{H}), 6.72$ (s (br), 1H), 4.05 (dd, $1 \mathrm{H}, J=5.4,9.5 \mathrm{~Hz}), 3.83$ (d (br), $1 \mathrm{H}, J=10.4 \mathrm{~Hz}), 3.58(\mathrm{dd}, 1 \mathrm{H}, J=3.0,13.6 \mathrm{~Hz})$, $3.35(\mathrm{~m}, 2 \mathrm{H}), 2.86(\mathrm{dd}, 1 \mathrm{H}, J=10.4,13.6 \mathrm{~Hz}), 2.34(\mathrm{~s}, 3 \mathrm{H}), 1.80(\mathrm{~s}, 1 \mathrm{H}) .{ }^{13} \mathrm{C} \mathrm{NMR}\left(\mathrm{CDCl}_{3}\right): 171.3,140.2,138.3$, 138.2, 130.1, 128.7, 128.5, 128.1, 127.4, 126.8, 126.3, 60.9, 57.8, 49.9, 38.4, 21.4. HRMS (m/z): $[\mathrm{M}+\mathrm{H}]^{+}$calcd for $\mathrm{C}_{18} \mathrm{H}_{21} \mathrm{~N}_{2} \mathrm{O}$, 281.1654; found: 281.1649.

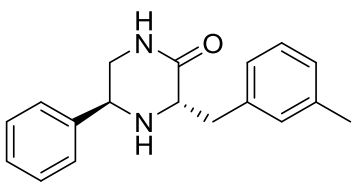


Trans-3-(3-methylbenzyl)-5-phenylpiperazin-2-one 17\{1,12\} (YJ31070-177-5): This compound was obtained as a white powder $(2.73 \mathrm{~g}, 19 \%)$ after a chromatography over silica gel (dichloromethane / ethanol 98/2 to 95/5) and a recrystallization in cyclohexane. ${ }^{1} \mathrm{H}$ NMR $\left(\mathrm{CDCl}_{3}\right)$ : $7.39-7.30(\mathrm{~m}, 5 \mathrm{H}), 7.19(\mathrm{~m}, 1 \mathrm{H}), 7.05(\mathrm{~m}, 3 \mathrm{H}), 6.81(\mathrm{~s}(\mathrm{br})$, $1 \mathrm{H}), 4.26(\mathrm{dd}, 1 \mathrm{H}, J=4.0,9.9 \mathrm{~Hz}), 3.87(\mathrm{dd}, 1 \mathrm{H}, J=3.8,10.7 \mathrm{~Hz}), 3.45(\mathrm{~m}, 2 \mathrm{H}), 3.28(\mathrm{dd}, 1 \mathrm{H}, J=3.5,13.7 \mathrm{~Hz})$, $3.14(\mathrm{dd}, 1 \mathrm{H}, J=10.7,13.7 \mathrm{~Hz}), 2.31$ (s, 3H), 1.81 (s, 1H). ${ }^{13} \mathrm{C} \mathrm{NMR}\left(\mathrm{CDCl}_{3}\right): 172.0,139.9,138.5,137.8,130.1$, 128.7 (two signals), 128.2, 127.5, 127.0, 126.3, 59.3, 51.7, 49.1, 37.6, 21.3. HRMS (m/z): $[\mathrm{M}+\mathrm{H}]^{+}$calcd for $\mathrm{C}_{18} \mathrm{H}_{21} \mathrm{~N}_{2} \mathrm{O}$, 281.1654; found: 281.1656.<smiles>COc1ccccc1C[C@H]1NC(=O)NC[C@H]1c1ccccc1</smiles>

Cis-3-(2-methoxybenzyl)-5-phenylpiperazin-2-one 17\{1,14\} (YJ31070-103-3): This isomer was obtained as a powder (4.03 g, still containing 5\% of the aminoester) after a chromatography over silica gel (dichloromethane ethanol 97.5/2.5). ${ }^{1} \mathrm{H}$ NMR $\left(\mathrm{CDCl}_{3}\right)$ : 7.39-7.27 (m, 6H), $7.21(\mathrm{~m}, 1 \mathrm{H}), 6.89(\mathrm{~m}, 2 \mathrm{H}), 6.38(\mathrm{~s}(\mathrm{br}), 1 \mathrm{H}), 4.05(\mathrm{dd}$, $1 \mathrm{H}, J=5.3,9.3 \mathrm{~Hz}), 3.92(\mathrm{~m}, 1 \mathrm{H}), 3.84(\mathrm{~s}, 3 \mathrm{H}), 3.75(\mathrm{dd}, 1 \mathrm{H}, J=3.3,13.7 \mathrm{~Hz}), 3.35(\mathrm{~m}, 2 \mathrm{H}), 2.88(\mathrm{dd}, 1 \mathrm{H}, J=$ 10.3, $13.7 \mathrm{~Hz}), 1.81$ (s (br), 1H). ${ }^{13} \mathrm{C}$ NMR $\left(\mathrm{CDCl}_{3}\right): 171.5,157.9,140.5,131.5,128.6,128.0,127.9,126.7,126.6$, 120.5, 110.4, 59.2, 57.8, 55.3, 50.1, 33.2. HRMS $(\mathrm{m} / z)$ : $[\mathrm{M}+\mathrm{H}]^{+}$calcd for $\mathrm{C}_{18} \mathrm{H}_{21} \mathrm{~N}_{2} \mathrm{O}: 297.1603$, found: 297.1607.<smiles>COc1ccccc1C[C@H]1NC(c2ccccc2)CNC1=O</smiles>

Trans-3-(2-methoxybenzyl)-5-phenylpiperazin-2-one 17\{1,14\} (YJ31070-103-4): This isomer was obtained as a powder $(2.23 \mathrm{~g}, 24 \%)$ after a chromatography over silica gel (dichloromethane - ethanol 97.5/2.5). ${ }^{1} \mathrm{H} \mathrm{NMR}$ $\left(\mathrm{CDCl}_{3}\right)$ : 7.39-7.30 (m, 5H), $7.21(\mathrm{~m}, 2 \mathrm{H}), 6.92(\mathrm{~m}, 1 \mathrm{H}), 6.82(\mathrm{~m}, 1 \mathrm{H}), 6.70(\mathrm{~s}(\mathrm{br}), 1 \mathrm{H}), 4.36(\mathrm{dd}, 1 \mathrm{H}, J=3.9,9.9$ $\mathrm{Hz}), 3.97(\mathrm{dd}, 1 \mathrm{H}, J=3.9,10.7 \mathrm{~Hz}), 3.68(\mathrm{~s}, 3 \mathrm{H}), 3.46-3.32(\mathrm{~m}, 4 \mathrm{H}), 3.26(\mathrm{dd}, 1 \mathrm{H}, J=3.8,13.5 \mathrm{~Hz}), 1.83$ (s (br), 1H). ${ }^{13} \mathrm{C} \mathrm{NMR}\left(\mathrm{CDCl}_{3}\right): 172.3,158.0,140.4,131.3,128.6,128.1,128.0,127.0,126.3,120.9,110.4,59.0,55.1$, 52.0, 49.5, 32.3. HRMS (m/z): $[\mathrm{M}+\mathrm{H}]^{+}$calcd for $\mathrm{C}_{18} \mathrm{H}_{21} \mathrm{~N}_{2} \mathrm{O}: 297.1603$, found: 297.1604.<smiles>O=C1NCC(c2ccccc2)NC1Cc1ccccc1OCc1ccccc1</smiles>

3-(2-(benzyloxy)benzyl)-5-phenylpiperazin-2-one 17\{1,17\} (RB 32489-041): This mixture of isomers was obtained as an oil and used directly in the $\mathrm{N}$-oxidation step v.

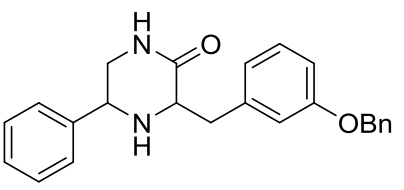

3-(3-(benzyloxy)benzyl)-5-phenylpiperazin-2-one 17\{1,18\} (RB 32489-039): This mixture of isomers was obtained as an oil and used directly in the N-oxidation step $\mathrm{v}$.<smiles>CCCCOc1ccc(CC2NC(c3ccccc3)CNC2=O)cc1</smiles>

3-(4-(benzyloxy)benzyl)-5-phenylpiperazin-2-one 17\{1,19\} (YJ 31776-031): This mixture of isomers was obtained as an oil and used directly in the N-oxidation step v. 


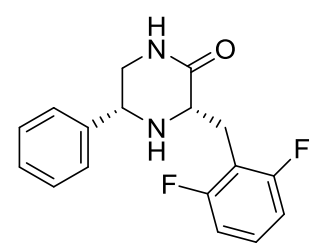

Cis-3-(2,6-difluorobenzyl)-5-phenylpiperazin-2-one 17 $\{1,21\}$ (EC31095-011-3): This isomer was obtained as a powder $(1.73 \mathrm{~g}, 38 \%)$ after a chromatography over silica gel (dichloromethane - ethanol 98/2 to 96.5/3.5). ${ }^{1} \mathrm{H}$ NMR $\left(\mathrm{CDCl}_{3}\right): 7.35(\mathrm{~m}, 5 \mathrm{H}), 7.18(\mathrm{~m}, 1 \mathrm{H}), 6.89(\mathrm{~m}, 2 \mathrm{H}), 6.55(\mathrm{~s}, 1 \mathrm{H}), 4.09(\mathrm{dd}, 1 \mathrm{H}, J=9.4,5.1 \mathrm{~Hz}), 3.95(\mathrm{~m}, 1 \mathrm{H}), 3.64$ $(\mathrm{m}, 1 \mathrm{H}), 3.38(\mathrm{~m}, 2 \mathrm{H}), 3.09(\mathrm{dd}, 1 \mathrm{H}, J=13.9,10.3 \mathrm{~Hz}), 1.73(\mathrm{~s}, 1 \mathrm{H}) .{ }^{13} \mathrm{C} \mathrm{NMR}\left(\mathrm{CDCl}_{3}\right): 170.7,162.0(248,9 \mathrm{~Hz})$, 140.2, 128.7, $128.3(10 \mathrm{~Hz}), 128.2,126.7,114.2(20 \mathrm{~Hz}), 111.2,58.8,57.6,50.0,25.8$. HRMS $(\mathrm{m} / z):[\mathrm{M}+\mathrm{H}]^{+}$calcd for $\mathrm{C}_{17} \mathrm{H}_{17} \mathrm{~F}_{2} \mathrm{~N}_{2} \mathrm{O}$ : 303.1309; found, 303.1301.

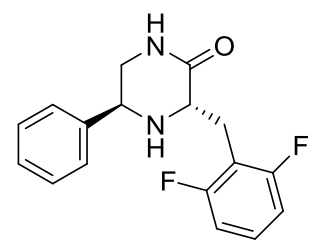

Trans-3-(2,6-difluorobenzyl)-5-phenylpiperazin-2-one 17\{1,21\} (EC31095-011-4): This isomer was obtained as a powder $(1.05 \mathrm{~g}, 23 \%)$ after a chromatography over silica gel (dichloromethane - ethanol 98/2 to 96.5/3.5). ${ }^{1} \mathrm{H}$ NMR $\left(\mathrm{CDCl}_{3}\right): 7.35(\mathrm{~m}, 5 \mathrm{H}), 7.18(\mathrm{~m}, 1 \mathrm{H}), 6.87(\mathrm{~m}, 2 \mathrm{H}), 6.58(\mathrm{~s}, 1 \mathrm{H}), 4.41(\mathrm{dd}, 1 \mathrm{H}, J=10.1,4.0 \mathrm{~Hz}), 3.96(\mathrm{dd}, 1 \mathrm{H}, J=$ 9.8, $5.4 \mathrm{~Hz}), 3.44(\mathrm{~m}, 4 \mathrm{H}), 1.63(\mathrm{~s}, 1 \mathrm{H}) .{ }^{13} \mathrm{C} \mathrm{NMR}\left(\mathrm{CDCl}_{3}\right): 171.3,162.0(247,9 \mathrm{~Hz}), 128.7,128.5(10 \mathrm{~Hz}), 128.2$, 127.0, $113.8(20 \mathrm{~Hz}), 111.3,57.6,51.5,49.3,25.3$. HRMS $(\mathrm{m} / \mathrm{z}):[\mathrm{M}+\mathrm{H}]^{+}$calcd for $\mathrm{C}_{17} \mathrm{H}_{17} \mathrm{~F}_{2} \mathrm{~N}_{2} \mathrm{O}: 303.1309$; found, 303.1306 .

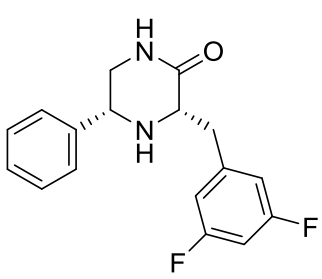

Cis-3-(3,5-difluorobenzyl)-5-phenylpiperazin-2-one 17\{1,22\} (EC31095-026-4): This isomer was obtained as a powder $(1.52 \mathrm{~g}, 36 \%)$ after a chromatography over silica gel (dichloromethane - ethanol 98/2 to 96/4). ${ }^{1} \mathrm{H}$ NMR $\left(\mathrm{CDCl}_{3}\right): 7.36(\mathrm{~m}, 5 \mathrm{H}), 6.87(\mathrm{~m}, 3 \mathrm{H}), 6.68(\mathrm{tt}, 1 \mathrm{H}, J=9.0,2.3 \mathrm{~Hz}), 4.09(\mathrm{dd}, 1 \mathrm{H}, J=8.4,6.0 \mathrm{~Hz}), 3.87(\mathrm{dd}, 1 \mathrm{H}, J=$ 9.4, $3.0 \mathrm{~Hz}), 3.50(\mathrm{dd}, 1 \mathrm{H}, J=13.8,3.2 \mathrm{~Hz}), 3.35(\mathrm{~m}, 2 \mathrm{H}), 2.94(\mathrm{dd}, 1 \mathrm{H}, J=13.8,9.5 \mathrm{~Hz}), 1.74(\mathrm{~s}, 1 \mathrm{H}) .{ }^{13} \mathrm{C}$ NMR $\left(\mathrm{CDCl}_{3}\right): 170.6,164.3(13 \mathrm{~Hz}), 161.8(13 \mathrm{~Hz}), 142.3(9 \mathrm{~Hz}), 139.9,128.8,128.3,126.8,112.3,102.2,60.3,57.7$, 49.7, 38.4. HRMS $(\mathrm{m} / \mathrm{z})$ : $[\mathrm{M}+\mathrm{H}]^{+}$calcd for $\mathrm{C}_{17} \mathrm{H}_{17} \mathrm{~F}_{2} \mathrm{~N}_{2} \mathrm{O}$ : 303.1309; found, 303.1302 .

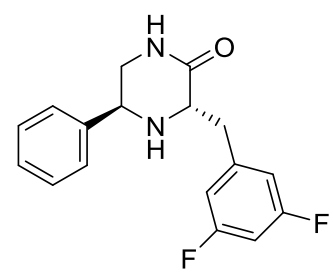

Trans-3-(3,5-difluorobenzyl)-5-phenylpiperazin-2-one 17\{1,22\} (EC31095-026-6): This isomer was obtained as a powder $(1.05 \mathrm{~g}, 25 \%)$ after a chromatography over silica gel (dichloromethane - ethanol 98/2 to 96/4). ${ }^{1} \mathrm{H}$ NMR $\left(\mathrm{CDCl}_{3}\right): 7.35(\mathrm{~m}, 5 \mathrm{H}), 7.02(\mathrm{~s}, 1 \mathrm{H}), 6.80(\mathrm{~m}, 2 \mathrm{H}), 6.69(\mathrm{tt}, 1 \mathrm{H}, J=9.0,2.3 \mathrm{~Hz}), 4.19(\mathrm{dd}, 1 \mathrm{H}, J=8.3,4.6 \mathrm{~Hz})$, $3.85(\mathrm{dd}, 1 \mathrm{H}, J=10.1,3.7 \mathrm{~Hz}), 3.49(\mathrm{~m}, 2 \mathrm{H}), 3.27(\mathrm{dd}, 1 \mathrm{H}, J=13.9,3.6 \mathrm{~Hz}), 3.15(\mathrm{dd}, 1 \mathrm{H}, J=13.9,10.1 \mathrm{~Hz})$, $1.76(\mathrm{~s}, 1 \mathrm{H}) .{ }^{13} \mathrm{C}$ NMR $\left(\mathrm{CDCl}_{3}\right): 171.4,164.4(13 \mathrm{~Hz}), 161.9(13 \mathrm{~Hz}), 142.2(9 \mathrm{~Hz}), 139.5,128.9,128.3,126.8$, 112.2, $102.3(25 \mathrm{~Hz}), 58.6,51.9,48.9,37.8$. HRMS $(\mathrm{m} / \mathrm{z})$ : $[\mathrm{M}+\mathrm{H}]^{+}$calcd for $\mathrm{C}_{17} \mathrm{H}_{17} \mathrm{~F}_{2} \mathrm{~N}_{2} \mathrm{O}: 303.1309$; found, 303.1306 . 


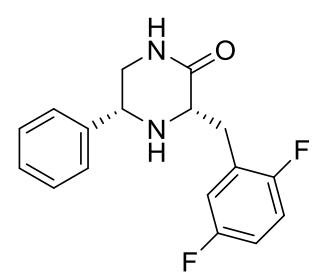

Cis-3-(2,5-difluorobenzyl)-5-phenylpiperazin-2-one 17 $\{1,23\}$ (EC31095-015-3): This isomer was obtained as a powder $(1.58 \mathrm{~g}, 35 \%)$ after a chromatography over silica gel (dichloromethane - ethanol 98/2 to 96/4). ${ }^{1} \mathrm{H}$ NMR $\left(\mathrm{CDCl}_{3}\right): 7.34(\mathrm{~m}, 5 \mathrm{H}), 7.05(\mathrm{~m}, 2 \mathrm{H}), 6.89(\mathrm{~m}, 1 \mathrm{H}), 6.66(\mathrm{~s}, 1 \mathrm{H}), 4.10(\mathrm{~m}, 1 \mathrm{H}), 3.93(\mathrm{~m}, 1 \mathrm{H}), 3.57(\mathrm{dd}, 1 \mathrm{H}, J=$ $14.0,2.4 \mathrm{~Hz}), 3.33(\mathrm{dd}, 2 \mathrm{H}, J=6.8,2.5 \mathrm{~Hz}), 2.99(\mathrm{ddd}, 1 \mathrm{H}, J=14.0,9.1,0.8 \mathrm{~Hz}), 1.77(\mathrm{~s}, 1 \mathrm{H}),{ }^{13} \mathrm{C} \mathrm{NMR}\left(\mathrm{CDCl}_{3}\right)$ : 170.6, $158.5(242,2 \mathrm{~Hz}), 157.6$ (241, $2 \mathrm{~Hz}), 140.1,128.8,128.3,127.1(18,8 \mathrm{~Hz}), 126.8,118.0(24,5 \mathrm{~Hz}), 116.2$ $(25,9 \mathrm{~Hz}), 114.6(24,9 \mathrm{~Hz}), 59.5,57.7,49.9,31.9$. HRMS $(\mathrm{m} / \mathrm{z}):[\mathrm{M}+\mathrm{H}]^{+}$calcd for $\mathrm{C}_{17} \mathrm{H}_{17} \mathrm{~F}_{2} \mathrm{~N}_{2} \mathrm{O}: 303.1309$; found, 303.1304 .

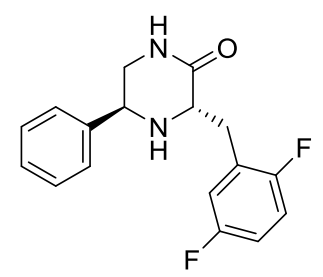

Trans-3-(2,5-difluorobenzyl)-5-phenylpiperazin-2-one 17\{1,23\} (EC31095-015-4): This isomer was obtained as a powder $(1.17 \mathrm{~g}, 26 \%)$ after a chromatography over silica gel (dichloromethane - ethanol 98/2 to 96/4). ${ }^{1} \mathrm{H}$ NMR $\left(\mathrm{CDCl}_{3}\right): 7.35(\mathrm{~m}, 5 \mathrm{H}), 6.96(\mathrm{~m}, 3 \mathrm{H}), 6.26(\mathrm{~s}, 1 \mathrm{H}), 4.31(\mathrm{dd}, 1 \mathrm{H}, J=9.7,4.1 \mathrm{~Hz}), 3.91(\mathrm{dd}, 1 \mathrm{H}, J=10.3,4.1 \mathrm{~Hz})$, $3.35(\mathrm{~m}, 4 \mathrm{H}), 1.70(\mathrm{~s}, 1 \mathrm{H}) .{ }^{13} \mathrm{C} \mathrm{NMR}\left(\mathrm{CDCl}_{3}\right): 171.0,158.7(240,2 \mathrm{~Hz}), 157.6(241,2 \mathrm{~Hz}), 139.7,128.8,128.2$, 127.0, $126.8(18,8 \mathrm{~Hz}), 117.6(24,5 \mathrm{~Hz}), 116.5(26,9 \mathrm{~Hz}), 114.9(24,9 \mathrm{~Hz}), 58.6,51.8,49.2,31.3$. HRMS $(\mathrm{m} / \mathrm{z})$ : $[\mathrm{M}+\mathrm{H}]^{+}$calcd for $\mathrm{C}_{17} \mathrm{H}_{17} \mathrm{~F}_{2} \mathrm{~N}_{2} \mathrm{O}$ : 303.1309; found, 303.1306.

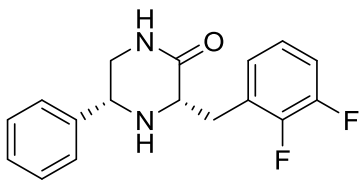

Cis-3-(2,3-difluorobenzyl)-5-phenylpiperazin-2-one 17\{1,24\} (EC31095-13-3): This isomer was obtained as a white powder $(1.57 \mathrm{~g}, 36 \%)$ after a chromatography over silica gel (dichloromethane - ethanol 98/2 to 96/4). ${ }^{1} \mathrm{H}$ NMR $\left(\mathrm{CDCl}_{3}\right): 7.34(\mathrm{~m}, 5 \mathrm{H}), 7.06(\mathrm{~m}, 3 \mathrm{H}), 6.66(\mathrm{~s}, 1 \mathrm{H}), 4.10(\mathrm{t}, 1 \mathrm{H}, J=7.2 \mathrm{~Hz}), 3.94(\mathrm{dd}, 1 \mathrm{H}, J=9.1,3.2 \mathrm{~Hz})$, $3.62(\mathrm{ddd}, 1 \mathrm{H}, J=13.9,3.4,1.4 \mathrm{~Hz}), 3.33(\mathrm{~d}, 2 \mathrm{H}, J=8.5 \mathrm{~Hz}), 3.04(\mathrm{dd}, 1 \mathrm{H}, J=14.0,9.3 \mathrm{~Hz}), 1.77(\mathrm{~s}, 1 \mathrm{H}) .{ }^{13} \mathrm{C}$ NMR $\left(\mathrm{CDCl}_{3}\right)$ : 170.6, $150.7(248,13 \mathrm{~Hz}), 149.6(247,13 \mathrm{~Hz}), 140.1,128.8,128.3,127.9(12 \mathrm{~Hz}), 126.8,126.3(3$ $\mathrm{Hz}), 123.9(7,5 \mathrm{~Hz}), 115.6(17 \mathrm{~Hz}), 59.5,57.7,49.9,31.7$. HRMS $(\mathrm{m} / z):[\mathrm{M}+\mathrm{H}]^{+}$calcd for $\mathrm{C}_{17} \mathrm{H}_{17} \mathrm{~F}_{2} \mathrm{~N}_{2} \mathrm{O}$ : 303.1309; found, 303.1308 .

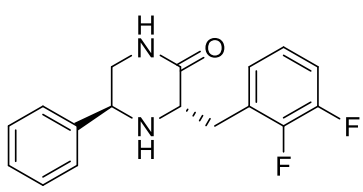

Trans-3-(2,3-difluorobenzyl)-5-phenylpiperazin-2-one 17\{1,24\} (EC31095-13-3): This isomer was obtained as a white powder $(1.21 \mathrm{~g}, 27 \%)$ after a chromatography over silica gel (dichloromethane - ethanol 98/2 to 96/4). ${ }^{1} \mathrm{H}$ NMR $\left(\mathrm{CDCl}_{3}\right): 7.36(\mathrm{~m}, 5 \mathrm{H}), 7.05(\mathrm{~m}, 3 \mathrm{H}), 6.58(\mathrm{~s}, 1 \mathrm{H}), 4.32(\mathrm{dd}, 1 \mathrm{H}, J=9.7,4.0 \mathrm{~Hz}), 3.92(\mathrm{dd}, 1 \mathrm{H}, J=10.2,4.2$ $\mathrm{Hz}), 3.38(\mathrm{~m}, 4 \mathrm{H}), 1.70(\mathrm{~s}, 1 \mathrm{H}) .{ }^{13} \mathrm{C}$ NMR $\left(\mathrm{CDCl}_{3}\right): 171.2,150.7(248,13 \mathrm{~Hz}), 149.7(247,13 \mathrm{~Hz}), 139.7,128.8$, 128.2, $127.7(12 \mathrm{~Hz}), 127.0,125.9(3 \mathrm{~Hz}), 124.3(7,5 \mathrm{~Hz}), 115.8(17 \mathrm{~Hz}), 58.6,51.8,49.2,31.0$. HRMS $(\mathrm{m} / \mathrm{z})$ : $[\mathrm{M}+\mathrm{H}]^{+}$calcd for $\mathrm{C}_{17} \mathrm{H}_{17} \mathrm{~F}_{2} \mathrm{~N}_{2} \mathrm{O}$ : 303.1309; found, 303.1304. 


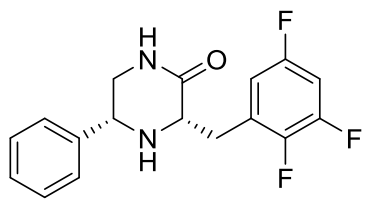

Cis-5-phenyl-3-(2,3,5-trifluorobenzyl)piperazin-2-one 17\{1,26\} (YJ 33067-97-4): This isomer was obtained as a powder (1.45 g, 41\%) after a chromatography over silica gel (dichloromethane - ethanol 97.5/2.5 to 96.5/3.5) and a dispersion in boiling cyclohexane. ${ }^{1} \mathrm{H}$ NMR (DMSO- $\left.d_{6}\right): 7.83(\mathrm{~m}, 1 \mathrm{H}), 7.39-7.24(\mathrm{~m}, 6 \mathrm{H}), 7.15(\mathrm{~m}, 1 \mathrm{H}), 4.05(\mathrm{~m}$, $1 \mathrm{H}), 3.74(\mathrm{~m}, 1 \mathrm{H}), 3.31(\mathrm{dd}, 1 \mathrm{H}, J=4.0,13.6 \mathrm{~Hz}), 3.22(\mathrm{dt}, 1 \mathrm{H}, J=4.0,11.5 \mathrm{~Hz}), 3.04(\mathrm{t}, 1 \mathrm{H}, J=11.5 \mathrm{~Hz}), 2.93$ (dd, $1 \mathrm{H}, J=7.9,14.1 \mathrm{~Hz}), 2.80(\mathrm{~m}, 1 \mathrm{H}) .{ }^{13} \mathrm{C}$ NMR (DMSO- $\left.d_{6}\right): 169.8,157.0(244,10$ and $3 \mathrm{~Hz}), 149.8(251,12$ and $14 \mathrm{~Hz}), 145.9$ (243, 13 and $4 \mathrm{~Hz}), 141.7,130.5$ (9 and 15 Hz), 128.7, 127.8, 127.1, 113.5 (3 and $23 \mathrm{~Hz}), 103.9$ $(21$ and $28 \mathrm{~Hz}), 59.1,56.6,49.3,31.0$.

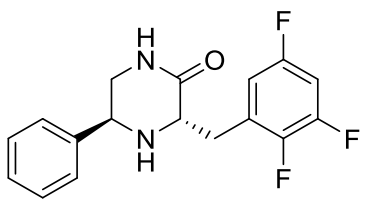

Trans-5-phenyl-3-(2,3,5-trifluorobenzyl)piperazin-2-one 17\{1,26\} (YJ 33067-97-3): This isomer was obtained as a powder $(0.77 \mathrm{~g}, 22 \%)$ after a chromatography over silica gel (dichloromethane - ethanol 97.5/2.5 to 96.5/3.5). ${ }^{1} \mathrm{H}$ NMR (DMSO-d $): 7.87(\mathrm{~m}, 1 \mathrm{H}), 7.38-7.23(\mathrm{~m}, 6 \mathrm{H}), 7.09(\mathrm{~m}, 1 \mathrm{H}), 4.18(\mathrm{~m}, 1 \mathrm{H}), 3.55(\mathrm{~m}, 1 \mathrm{H}), 3.40(\mathrm{dt}, 1 \mathrm{H}, J=$ 4.0, $11.0 \mathrm{~Hz}), 3.24(\mathrm{~m}, 1 \mathrm{H}), 3.16(\mathrm{dd}, 1 \mathrm{H}, J=4.6$ and $13.9 \mathrm{~Hz}), 3.07(\mathrm{dd}, 1 \mathrm{H}, J=10.3,13.6 \mathrm{~Hz}), 2.83(\mathrm{~m}, 1 \mathrm{H}) .{ }^{13} \mathrm{C}$ NMR (DMSO-d6): 170.7, 157.1 (244, 10 and $3 \mathrm{~Hz}), 149.7$ (251, 12 and 14 Hz), 145.9 (243, 13 and $4 \mathrm{~Hz}), 141.5$, 130.8 (9 and $15 \mathrm{~Hz}), 128.7,127.6,127.1,113.7$ (3 and $23 \mathrm{~Hz}), 103.9$ (21 and $28 \mathrm{~Hz}), 57.4,50.9,48.3,31.0$.<smiles>O=C1NC[C@@H](Cc2ccccc2)N[C@@H]1c1ccccc1F</smiles>

(3S,5R)-3-benzyl-5-(2-fluorophenyl)piperazin-2-one 17\{2,1\} (YJ31134-014-3): This isomer was obtained as a white powder (4.06 g, 39\%) after two chromatography over silica gel (dichloromethane - ethanol 96/4 to 94/6) and (cyclohexane - ethyl acetate $2 / 3$ to $1 / 3$ ) followed by a dispersion in boiling cyclohexane and extensive drying at 70 ${ }^{\circ} \mathrm{C}$ under vacuum. ${ }^{1} \mathrm{H}$ NMR $\left(\mathrm{CDCl}_{3}\right)$ : $7.49(\mathrm{~m}, 1 \mathrm{H}), 7.31(\mathrm{~m}, 4 \mathrm{H}), 7.26(\mathrm{~m}, 2 \mathrm{H}), 7.16(\mathrm{~m}, 1 \mathrm{H}), 7.02(\mathrm{~m}, 1 \mathrm{H}), 6.65(\mathrm{~s}$ (br), 1H), $4.42(\mathrm{~m}, 1 \mathrm{H}), 3.87(\mathrm{~m}, 1 \mathrm{H}), 3.57(\mathrm{dd}, 1 \mathrm{H}, J=3.3,13.8 \mathrm{~Hz}), 3.29(\mathrm{t}, 1 \mathrm{H}, J=10.9 \mathrm{~Hz}), 2.95$ (dd, $1 \mathrm{H}, J=$ 9.1, $13.8 \mathrm{~Hz}), 1.73$ (s (br), 1H). ${ }^{13} \mathrm{C} \mathrm{NMR}\left(\mathrm{CDCl}_{3}\right): 171.0,160.1(246 \mathrm{~Hz}), 138.2,129.4(7 \mathrm{~Hz}), 129.3,128.7,127.4$ (4 Hz), $127.1(13 \mathrm{~Hz}), 126.7,124.6(4 \mathrm{~Hz}), 115.4(22 \mathrm{~Hz}), 60.7,50.4(3 \mathrm{~Hz}), 48.4,38.3$. HRMS $(\mathrm{m} / z):[\mathrm{M}+\mathrm{H}]^{+}$ calcd for $\mathrm{C}_{17} \mathrm{H}_{18} \mathrm{FN}_{2} \mathrm{O}: 285.1403$, found: 285.1391 .

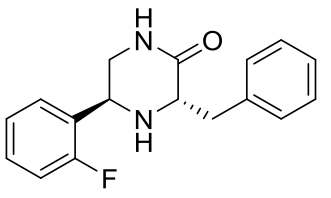

(3S,5S)-3-benzyl-5-(2-fluorophenyl)piperazin-2-one 17\{2,1\} (YJ31134-013-4): This isomer was obtained as a white powder $(2.16 \mathrm{~g}, 20 \%)$ after a chromatography over silica gel (dichloromethane - ethanol 96/4 to 94/6). ${ }^{1} \mathrm{H}$ NMR $\left(\mathrm{CDCl}_{3}\right): 7.44(\mathrm{~m}, 1 \mathrm{H}), 7.27(\mathrm{~m}, 6 \mathrm{H}), 7.13(\mathrm{~m}, 1 \mathrm{H}), 7.08(\mathrm{~m}, 1 \mathrm{H}), 6.74(\mathrm{~s}(\mathrm{br}), 1 \mathrm{H}), 4.65(\mathrm{dd}, 1 \mathrm{H}, J=4.3,9.1$ $\mathrm{Hz}), 3.84(\mathrm{dd}, 1 \mathrm{H}, J=3.5,10.8 \mathrm{~Hz}), 3.48(\mathrm{~m}, 2 \mathrm{H}), 3.34(\mathrm{dd}, 1 \mathrm{H}, J=3.8,13.7 \mathrm{~Hz})$, ), $3.15(\mathrm{dd}, 1 \mathrm{H}, J=10.7,13.7$ $\mathrm{Hz}), 1.78$ (s (br), 1H). ${ }^{13} \mathrm{C} \mathrm{NMR}\left(\mathrm{CDCl}_{3}\right): 171.8,160.5(247 \mathrm{~Hz}), 137.9,129.5(9 \mathrm{~Hz}), 129.3,128.8,127.9(3 \mathrm{~Hz})$, $126.8(13 \mathrm{~Hz}), 126.7,124.5(3 \mathrm{~Hz}), 115.6(22 \mathrm{~Hz}), 59.2,47.7,44.9(2 \mathrm{~Hz})$, 37.7. HRMS $(\mathrm{m} / z):[\mathrm{M}+\mathrm{H}]^{+}$calcd for $\mathrm{C}_{17} \mathrm{H}_{18} \mathrm{FN}_{2} \mathrm{O}$ : 285.1403, found: 285.1367. 


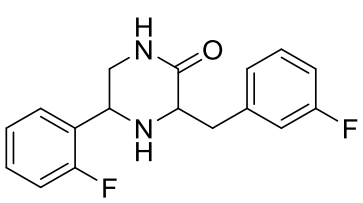

3-(3-Fluorobenzyl)-5-(2-fluorophenyl)piperazin-2-one 17\{2,3\} (YJ 31068-119-1): This crude mixture of isomers was obtained as an oil and used directly in the $\mathrm{N}$-oxidation step $\mathrm{v}$.<smiles>O=C1NCC(c2cccc(F)c2)NC1Cc1ccccc1</smiles>

3-benzyl-5-(3-fluorophenyl)piperazin-2-one 17\{3,1\} (YJ31067-006-2): This mixture of isomers was obtained as an oil, after washing it with cyclohexane to remove unreacted ethyl phenylalanine ester, and used directly in the aromatization step.<smiles>O=C1NC[C@@H](c2ccc(F)cc2)N[C@@H]1Cc1ccccc1</smiles>

(3S,5R)-3-benzyl-5-(4-fluorophenyl)piperazin-2-one 17\{4,1\} (YJ29794-192-7): This isomer was obtained as a white powder $(2.03 \mathrm{~g}, 42 \%)$ after two chromatography over silica gel (dichloromethane - ethanol 97/3 to 95/5) and (cyclohexane - ethyl acetate 1/2 to 1/3). ${ }^{1} \mathrm{H} \mathrm{NMR}\left(\mathrm{CDCl}_{3}\right): 7.28(\mathrm{~m}, 7 \mathrm{H}), 7.01(\mathrm{~m}, 2 \mathrm{H}), 6.61(\mathrm{~s}(\mathrm{br}), 1 \mathrm{H}), 4.05(\mathrm{t}$, $1 \mathrm{H}, J=7.3 \mathrm{~Hz}), 3.82(\mathrm{~d}, 1 \mathrm{H}, J=9.3 \mathrm{~Hz}), 3.58(\mathrm{dd}, 1 \mathrm{H}, J=3.0,13.6 \mathrm{~Hz}), 3.31(\mathrm{~m}, 2 \mathrm{H}), 2.89(\mathrm{dd}, 1 \mathrm{H}, J=9.8,13.6$ $\mathrm{Hz}), 1.72$ (s (br), 1H). ${ }^{13} \mathrm{C} \mathrm{NMR}\left(\mathrm{CDCl}_{3}\right): 173.2,164.7(246 \mathrm{~Hz}), 140.4,138.1,131.6,130.8,130.6(8 \mathrm{~Hz}), 128.8$, $117.7(21 \mathrm{~Hz}), 62.9,59.2,52.1,40.6$. HRMS $(\mathrm{m} / \mathrm{z}):[\mathrm{M}+\mathrm{H}]^{+}$calcd for $\mathrm{C}_{17} \mathrm{H}_{18} \mathrm{FN}_{2} \mathrm{O}$ : 285.1403, found: 285.1342 .<smiles>O=C1NCC(c2ccc(F)cc2)NC1Cc1ccccc1</smiles>

(3S,5S)-3-benzyl-5-(4-fluorophenyl)piperazin-2-one 17\{4,1\} (YJ29794-192-8): This isomer was obtained as a white powder (1.32 g, 27\%) after two chromatography over silica gel (dichloromethane - ethanol 97/3 to 95/5) and (ethyl acetate - ethanol 99/1). ${ }^{1} \mathrm{H}$ NMR $\left(\mathrm{CDCl}_{3}\right)$ : $7.29(\mathrm{~m}, 7 \mathrm{H}), 7.05(\mathrm{~m}, 2 \mathrm{H}), 6.79(\mathrm{~s}(\mathrm{br}), 1 \mathrm{H}), 4.21(\mathrm{dd}, 1 \mathrm{H}, J=$ 4.5, $9.5 \mathrm{~Hz}), 3.86(\mathrm{dd}, 1 \mathrm{H}, J=3.7,10.6 \mathrm{~Hz}), 3.40(\mathrm{~m}, 2 \mathrm{H}), 3.16(\mathrm{dd}, 1 \mathrm{H}, J=10.7,13.9 \mathrm{~Hz}), 1.74(\mathrm{~s}(\mathrm{br}), 1 \mathrm{H}) .{ }^{13} \mathrm{C}$ NMR $\left(\mathrm{CDCl}_{3}\right): 171.8,162.5(247 \mathrm{~Hz}), 137.8,135.6,129.3,128.9,128.6(8 \mathrm{~Hz}), 126.8,115.6(21 \mathrm{~Hz}), 59.2,51.1$, 49.2, 37.7. HRMS $(\mathrm{m} / z):[\mathrm{M}+\mathrm{H}]^{+}$calcd for $\mathrm{C}_{17} \mathrm{H}_{18} \mathrm{FN}_{2} \mathrm{O}: 285.1403$, found: 285.1315 .<smiles>Cc1cccc(C2CNC(=O)C(Cc3ccccc3)N2)c1</smiles>

3-Benzyl-5-(m-tolyl)piperazin-2-one 17\{5,1\} (YJ30367-057-3): An analytical sample of a mixture of the the two diastereoisomers was obtained by a recrystallization in cyclohexane but most of the solid and the filtrate were used directly in the in the aromatization step. HRMS $(\mathrm{m} / z)$ : $[\mathrm{M}+\mathrm{H}]^{+}$calcd for $\mathrm{C}_{18} \mathrm{H}_{20} \mathrm{~N}_{2} \mathrm{O}, 281.1654$; found: 281.1645 .<smiles>Cc1ccc(C2CNC(=O)C(Cc3ccccc3)N2)cc1</smiles>

3-Benzyl-5-(p-tolyl)piperazin-2-one 17\{6,1\} (YJ30367-027-3): This compound was obtained as a crude mixture of the two diastereoisomers $(5.40 \mathrm{~g})$ by dispersing the resulting solid in boiling cyclohexane and filtration at $20{ }^{\circ} \mathrm{C}$ 
and was used directly in the aromatization step. HRMS $(m / z)$ : $[\mathrm{M}+\mathrm{H}]^{+}$calcd for $\mathrm{C}_{18} \mathrm{H}_{20} \mathrm{~N}_{2} \mathrm{O}, 281.1654$; found: 281.1649 .

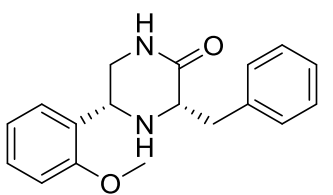

(3S,5R)-3-benzyl-5-(2-methoxyphenyl)piperazin-2-one 17\{7,1\} (YJ29589-082-2): This isomer was obtained as a glass (1.06 g, 25\%) after two chromatography over silica gel (dichloromethane - ethanol 96/4 to 95/5) and (cyclohexane - ethyl acetate 1/2 to 1/3). ${ }^{1} \mathrm{H}$ NMR $\left(\mathrm{CDCl}_{3}\right)$ : 7.40-7.22 (m, 7H), $6.98(\mathrm{~m}, 1 \mathrm{H}), 6.84(\mathrm{~m}, 1 \mathrm{H}), 6.69(\mathrm{~s}$ (br), 1H), 4.39 (dd, 1H, $J=3.7,10.7 \mathrm{~Hz}), 3.74(\mathrm{~s}, 3 \mathrm{H}), 3.85(\mathrm{dd}, 1 \mathrm{H}, J=3.2,9.2 \mathrm{~Hz}), 3.53(\mathrm{dd}, 1 \mathrm{H}, J=3.5,13.7$ $\mathrm{Hz}), 3.44$ (ddd, $1 \mathrm{H}, J=4.0,8.0,11.0 \mathrm{~Hz}), 3.28$ (t, 1H, $J=11.0 \mathrm{~Hz}), 3.01$ (dd, 1H, $J=8.3,13.7 \mathrm{~Hz}), 1.90$ (s (br), 1H). ${ }^{13} \mathrm{C} \mathrm{NMR}\left(\mathrm{CDCl}_{3}\right): 171.4,156.6,138.4,128.8,128.7,128.6,128.0,126.7,126.6,120.9,110.4,60.8,55.2$, 51.6, 47.8, 38.1. HRMS (m/z): $[\mathrm{M}+\mathrm{H}]^{+}$calcd for $\mathrm{C}_{18} \mathrm{H}_{21} \mathrm{~N}_{2} \mathrm{O}: 297.1603$, found: 297.1591 .

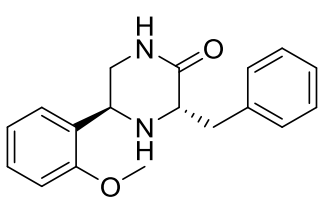

(3S,5S)-3-benzyl-5-(2-methoxyphenyl)piperazin-2-one 17\{7,1\} (YJ29589-082-3): This isomer was obtained as a white solid $(0.52 \mathrm{~g}, 12 \%)$ after two chromatography over silica gel (dichloromethane - ethanol 96/4 to 95) and (ethyl acetate - ethanol 1/0 to 99/1). ${ }^{1} \mathrm{H}$ NMR $\left(\mathrm{CDCl}_{3}\right)$ : 7.36-7.20 (m, 7H), $6.94(\mathrm{~m}, 1 \mathrm{H}), 6.88(\mathrm{~m}, 1 \mathrm{H}), 6.65(\mathrm{~s}(\mathrm{br})$, $1 \mathrm{H}), 4.64(\mathrm{dd}, 1 \mathrm{H}, J=4.2,8.1 \mathrm{~Hz}), 3.77(\mathrm{~s}, 3 \mathrm{H}), 3.71(\mathrm{dd}, 1 \mathrm{H}, J=3.3,10.8 \mathrm{~Hz}), 3.59(\mathrm{~m}, 1 \mathrm{H}), 3.47$ (ddd, $1 \mathrm{H}, J=$ 1.0, $11.2 \mathrm{~Hz}), 3.36(\mathrm{dd}, 1 \mathrm{H}, J=3.3,13.7 \mathrm{~Hz}), 3.09(\mathrm{dd}, 1 \mathrm{H}, J=10.8,13.7 \mathrm{~Hz}), 2.08(\mathrm{~s}(\mathrm{br}), 1 \mathrm{H}) .{ }^{13} \mathrm{C} \mathrm{NMR}$ $\left(\mathrm{CDCl}_{3}\right)$ : 171.9, 156.9, 138.2, 128.8, 128.6, 127.6, 129.3, 126.5, 127.2, 120.8, 110.4, 58.7, 55.2, 46.9, 46.0, 37.6. HRMS $(\mathrm{m} / \mathrm{z}):[\mathrm{M}+\mathrm{H}]^{+}$calcd for $\mathrm{C}_{18} \mathrm{H}_{21} \mathrm{~N}_{2} \mathrm{O}: 297.1603$, found: 297.1589 .<smiles>COc1cccc([C@@H]2CNC(=O)[C@H](Cc3ccccc3)N2)c1</smiles>

(3S,5R)-3-benzyl-5-(3-methoxyphenyl)piperazin-2-one 17\{8,1\} (YJ29589-084-3): This isomer was obtained as a glass (1.33 g, 30\%) after two chromatography over silica gel (dichloromethane - ethanol 97/3 to 95/5) and (cyclohexane - ethyl acetate 1/4). ${ }^{1} \mathrm{H}$ NMR $\left(\mathrm{CDCl}_{3}\right)$ : 7.34-7.20 (m, 6H), $6.94(\mathrm{~m}, 2 \mathrm{H}), 6.83(\mathrm{~m}, 1 \mathrm{H}), 6.72(\mathrm{~s}(\mathrm{br})$, $1 \mathrm{H}), 4.02(\mathrm{dd}, 1 \mathrm{H}, J=6.3,8.7 \mathrm{~Hz}), 3.83(\mathrm{~m}, 1 \mathrm{H}), 3.81(\mathrm{~s}, 3 \mathrm{H}), 3.58(\mathrm{dd}, 1 \mathrm{H}, J=3.3,13.4 \mathrm{~Hz}), 3.34$ (m, $2 \mathrm{H}), 2.91$ $(\mathrm{dd}, 1 \mathrm{H}, J=10.3,13.4 \mathrm{~Hz}), 1.90(\mathrm{~s}(\mathrm{br}), 1 \mathrm{H}) .{ }^{13} \mathrm{C} \mathrm{NMR}\left(\mathrm{CDCl}_{3}\right): 171.2,159.9,141.8,138.3,129.7,129.4,128.6$, 126.6, 119.1, 113.5, 112.5, 60.7, 57.6, 55.2, 49.8, 38.4. HRMS $(m / z):[\mathrm{M}+\mathrm{H}]^{+}$calcd for $\mathrm{C}_{18} \mathrm{H}_{21} \mathrm{~N}_{2} \mathrm{O}: 297.1603$, found: 297.1582 .

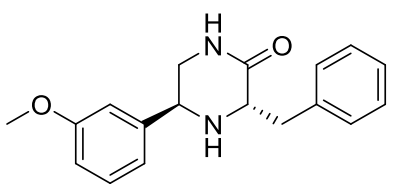

(3S,5S)-3-benzyl-5-(3-methoxyphenyl)piperazin-2-one 17\{8,1\} (YJ29589-084-4): This isomer was obtained as a glass $(0.84 \mathrm{~g}, 19 \%)$ after two chromatography over silica gel (dichloromethane - ethanol 97/3 to 95/5) and (ethyl acetate - ethanol 1/0 to 99/1). ${ }^{1} \mathrm{H}$ NMR $\left(\mathrm{CDCl}_{3}\right)$ : 7.33-7.22 (m, 6H), $6.94(\mathrm{~m}, 2 \mathrm{H}), 6.85(\mathrm{~m}, 1 \mathrm{H}), 6.76(\mathrm{~s}(\mathrm{br}), 1 \mathrm{H})$, $4.22(\mathrm{dd}, 1 \mathrm{H}, J=4.1,9.5 \mathrm{~Hz}), 3.88(\mathrm{dd}, 1 \mathrm{H}, J=3.6,10.6 \mathrm{~Hz}), 3.80(\mathrm{~s}, 3 \mathrm{H}), 3.45(\mathrm{~m}, 2 \mathrm{H}), 3.31(\mathrm{dd}, 1 \mathrm{H}, J=3.6$, $13.8 \mathrm{~Hz}), 3.16(\mathrm{dd}, 1 \mathrm{H}, J=10.7,13.8 \mathrm{~Hz}), 1.81$ (s (br), 1H). ${ }^{13} \mathrm{C} \mathrm{NMR}\left(\mathrm{CDCl}_{3}\right): 171.9,159.9,141.4,137.9,129.7$, 129.3, 128.9, 126.6, 119.2, 113.6, 112.5, 59.3, 55.2, 51.7, 49.1, 37.3. HRMS (m/z): $[\mathrm{M}+\mathrm{H}]^{+}$calcd for $\mathrm{C}_{18} \mathrm{H}_{21} \mathrm{~N}_{2} \mathrm{O}$ : 297.1603, found: 297.1584 . 
(1)

(3S,5R)-3-benzyl-5-(4-methoxyphenyl)piperazin-2-one 17\{9,1\} (YJ29589-122-1): This isomer was obtained as a glass (1.37 g, 27\%) after two chromatography over silica gel (dichloromethane - ethanol 97/3 to 95/5) and (cyclohexane - ethyl acetate 1/4). ${ }^{1} \mathrm{H}$ NMR $\left(\mathrm{CDCl}_{3}\right)$ : $7.28(\mathrm{~m}, 7 \mathrm{H}), 6.87(\mathrm{~m}, 2 \mathrm{H}), 6.38$ (s (br), 1H), $3.99(\mathrm{dd}, 1 \mathrm{H}, J$ $=4.3,10.3 \mathrm{~Hz}), 3.82(\mathrm{~m}, 1 \mathrm{H}), 3.80(\mathrm{~s}, 3 \mathrm{H}), 3.58(\mathrm{dd}, 1 \mathrm{H}, J=3.8,13.6 \mathrm{~Hz}), 3.31(\mathrm{~m}, 2 \mathrm{H}), 2.89(\mathrm{dd}, 1 \mathrm{H}, J=10.2$, $13.6 \mathrm{~Hz}), 1.90$ (s (br), $1 \mathrm{H}) .{ }^{13} \mathrm{C} \mathrm{NMR}\left(\mathrm{CDCl}_{3}\right): 171.1,159.5,138.4,132.2,129.4,128.6,127.9,126.6,114.1,60.9$, 57.1, 55.3, 50.0, 38.4. HRMS $(\mathrm{m} / z)$ : $[\mathrm{M}+\mathrm{H}]^{+}$calcd for $\mathrm{C}_{18} \mathrm{H}_{21} \mathrm{~N}_{2} \mathrm{O}: 297.1603$, found: 297.1585 .

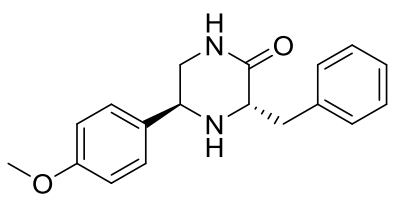

(3S,5S)-3-benzyl-5-(4-methoxyphenyl)piperazin-2-one 17\{9,1\} (YJ29589-122-2): This isomer was obtained as a white powder $(0.81 \mathrm{~g}, 16 \%)$ after two chromatography over silica gel (dichloromethane - ethanol 97/3 to 95/5) and (ethyl acetate - ethanol 1/0 to 99/1). ${ }^{1} \mathrm{H}$ NMR $\left(\mathrm{CDCl}_{3}\right)$ : $7.28(\mathrm{~m}, 7 \mathrm{H}), 6.81(\mathrm{~m}, 2 \mathrm{H}), 6.73$ (s (br), 1H), 4.19 (dd, $1 \mathrm{H}$, $J=4.0,9.8 \mathrm{~Hz}), 3.86(\mathrm{dd}, 1 \mathrm{H}, J=3.6,10.6 \mathrm{~Hz}), 3.80(\mathrm{~s}, 3 \mathrm{H}), 3.44(\mathrm{~m}, 1 \mathrm{H}), 3.31(\mathrm{~m}, 2 \mathrm{H}), 3.36(\mathrm{td}, 1 \mathrm{H}, J=3.8$, $11.5 \mathrm{~Hz}), 3.30(\mathrm{dd}, 1 \mathrm{H}, J=3.8,13.9 \mathrm{~Hz}), 3.17(\mathrm{dd}, 1 \mathrm{H}, J=10.6,13.9 \mathrm{~Hz}), 1.85(\mathrm{~s}(\mathrm{br}), 1 \mathrm{H}) .{ }^{13} \mathrm{C} \mathrm{NMR}\left(\mathrm{CDCl}_{3}\right)$ : $171.9,159.5,137.9,131.8,129.3,128.9,127.1,126.8,114.1,59.4,55.3,51.1,49.2,37.7$. HRMS $(\mathrm{m} / z):[\mathrm{M}+\mathrm{H}]^{+}$ calcd for $\mathrm{C}_{18} \mathrm{H}_{21} \mathrm{~N}_{2} \mathrm{O}: 297.1603$, found: 297.1533 .

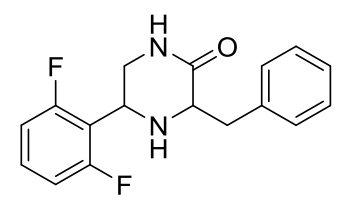

3-Benzyl-5-(2,6-difluorophenyl)piperazin-2-one 17\{10,1\} (MM34284-016-1): This compound was obtained as a $1 / 1$ mixture of diastereoisomers after a recrystallization in cyclohexane $(1.97 \mathrm{~g}, 62 \%)$ and was used directly in the next step. HRMS (m/z): $[\mathrm{M}+\mathrm{H}]^{+}$calcd for $\mathrm{C}_{17} \mathrm{H}_{17} \mathrm{~F}_{2} \mathrm{~N}_{2} \mathrm{O}: 303.1309$, found: 303.1323 .

General preparation of pyrazin-2-ol 19 via steps iv. The considered piperazin-2-one $17(0.011$ mol, note 1$)$ and sulfur ( $0.72 \mathrm{~g}, 0.0225 \mathrm{~mol}$, note 2$)$ were heated to reflux in 1,3-dichlorobenzene ( $40 \mathrm{~mL}$, note 3 ) for 10 hours. This was concentrated to dryness and the residue purified as described below. Note 1: no difference was observed when starting from the pure diastereoisomers or from a mixture of both. Note: 2: an excess of sulfur is detrimental to the reaction yield, hence the importance of starting from purified piperazin-2-ones. Note 3: decahydronaphthalene can also be used as solvent.

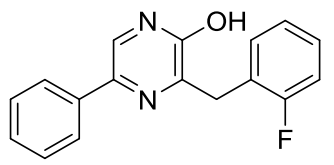

3-(2-Fluorobenzyl)-5-phenylpyrazin-2-ol 19\{1,2\} (YJ31070-025-2): Obtained as a white powder (3.66 g, 88\%) after a chromatography over silica gel (dichloromethane / ethanol 97.7:2.5). ${ }^{1} \mathrm{H}$ NMR (DMSO-d6) 12.45 (s, 1H), $7.89(\mathrm{~s}, 1 \mathrm{H}), 7.73(\mathrm{~m}, 2 \mathrm{H}), 7.38-7.22(\mathrm{~m}, 5 \mathrm{H}), 7.19-7.12(\mathrm{~m}, 2 \mathrm{H}), 4.12(\mathrm{~s}, 2 \mathrm{H}) .{ }^{13} \mathrm{C}$ NMR (DMSO-d $): 161.2(243$ $\mathrm{Hz}), 156.1,155.3,136.4,132.1(5 \mathrm{~Hz}), 131.2,129.0,128.8(8.2 \mathrm{~Hz}), 127.6,125.1(15 \mathrm{~Hz}), 124.8,124.5$ (3 Hz), 122.8, $115.4(22 \mathrm{~Hz}), 32.4(2 \mathrm{~Hz})$. HRMS (m/z): [M+H] $]^{+}$calcd for $\mathrm{C}_{17} \mathrm{H}_{14} \mathrm{FN}_{2} \mathrm{O}$ : 281.1090; found, 281.1087.

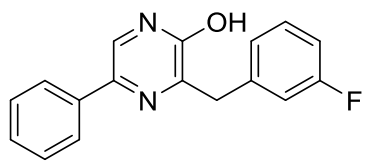


3-(3-Fluorobenzyl)-5-phenylpyrazin-2-ol 19\{1,3\} (YJ31070-179-3): Obtained as a white powder (3.92 g, 88\%) after a chromatography over silica gel (dichloromethane / ethanol 98:2). A sample was recrystallized in a mixture of cyclohexane and toluene for analytical purposes. ${ }^{1} \mathrm{H}$ NMR (DMSO- $\left.d_{6}\right) 12.45(\mathrm{~s}(\mathrm{br}), 1 \mathrm{H}), 7.89(\mathrm{~s}, 1 \mathrm{H}), 7.82(\mathrm{~m}$, 2H), 7.41-7.26 (m, 4H), $7.17(\mathrm{~m}, 2 \mathrm{H}), 7.04(\mathrm{~m}, 1 \mathrm{H}), 4.10(\mathrm{~s}, 2 \mathrm{H}) .{ }^{13} \mathrm{C}$ NMR (DMSO- $\left.d_{6}\right): 162.5(242 \mathrm{~Hz}), 156.8$, 155.4, $141.1(7 \mathrm{~Hz}), 136.4,131.4,130.4(8 \mathrm{~Hz}), 129.1,127.7,125.7(3 \mathrm{~Hz}), 124.9,123.1,116.4(21 \mathrm{~Hz}), 113.4(21$ $\mathrm{Hz})$, 38.9. HRMS $(\mathrm{m} / \mathrm{z})$ : $[\mathrm{M}+\mathrm{H}]^{+}$calcd for $\mathrm{C}_{17} \mathrm{H}_{14} \mathrm{FN}_{2} \mathrm{O}: 281.1090$; found, 281.1650.

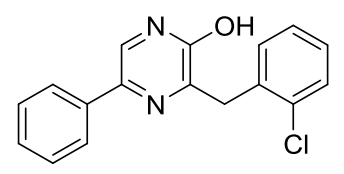

3-(2-Chlorobenzyl)-5-phenylpyrazin-2-ol 19\{1,5\} (EC31094-089-3): Obtained as a powder (2.06 g, 68\%) after a chromatography over silica gel (dichloromethane / ethanol 98:2) and a dispersion in a boiling mixture of toluene and cyclohexane. ${ }^{1} \mathrm{H}$ NMR (DMSO- $\left.d_{6}\right): 12.47(\mathrm{bs}, 1 \mathrm{H}), 7.90(\mathrm{~s}, 1 \mathrm{H}), 7.70(\mathrm{~m}, 2 \mathrm{H}), 7.46(\mathrm{~m}, 1 \mathrm{H}), 7.32(\mathrm{~m}, 5 \mathrm{H})$, $7.25(\mathrm{~m}, 1 \mathrm{H}), 4.22(\mathrm{~s}, 2 \mathrm{H}) .{ }^{13} \mathrm{C}$ NMR (DMSO- $\left.d_{6}\right): 156.1,155.3,136.4,136.3,134.1,132.2,131.2,129.5,129.0$, 128.7, 127.6, 127.4, 124.8, 122.7, 36.8. HRMS $(\mathrm{m} / \mathrm{z})$ : $[\mathrm{M}+\mathrm{H}]^{+}$calcd for $\mathrm{C}_{17} \mathrm{H}_{14} \mathrm{ClN}_{2} \mathrm{O}: 297.0795$; found, 297.0795 .

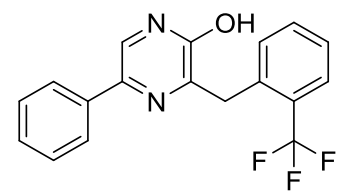

5-Phenyl-3-(2-(trifluoromethyl)benzyl)pyrazin-2-ol 19\{1,8\} (YJ 33067-105-1): This compound was obtained as a powder $(2.99 \mathrm{~g}, 60 \%)$ after two dispersions in boiling cyclohexane $\left(100 \mathrm{~mL}\right.$ each). ${ }^{1} \mathrm{H}$ NMR (DMSO- $\left.d_{6}\right): 12.49$ (s, $1 \mathrm{H}), 7.90(\mathrm{~s}, 1 \mathrm{H}), 7.3(\mathrm{~m}, 1 \mathrm{H}), 7.66-7.61(\mathrm{~m}, 3 \mathrm{H}), 7.48(\mathrm{~m}, 2 \mathrm{H}), 7.24(\mathrm{~m}, 2 \mathrm{H}), 7.20(\mathrm{~m}, 1 \mathrm{H}), 4.29(\mathrm{~s}, 2 \mathrm{H}) .{ }^{13} \mathrm{C}$ NMR (DMSO-d $\left.d_{6}\right)$ 156.6, 155.2, $136.7(2 \mathrm{~Hz}), 136.3,133.1,132.5,131.1,129.0128 .1(30 \mathrm{~Hz}), 127.6,127.4,126.2$ $(6 \mathrm{~Hz}), 125.0(273 \mathrm{~Hz}), 122.7,35.8$. HRMS $(\mathrm{m} / \mathrm{z}):[\mathrm{M}+\mathrm{H}]^{+}$calcd for $\mathrm{C}_{18} \mathrm{H}_{14} \mathrm{~F}_{3} \mathrm{~N}_{2} \mathrm{O}: 331.1058$; found, 331.1054.

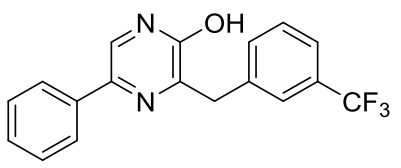

5-Phenyl-3-(3-(trifluoromethyl)benzyl)pyrazin-2-ol 19\{1,9\} (YJ 33067-107-3): Despite our efforts, was obtained as a solid $(5.50 \mathrm{~g})$ still containing some unidentified material out of which only the ${ }^{1} \mathrm{H}$ NMR could be properly described. ${ }^{1} \mathrm{H}$ NMR (DMSO- $\left.d_{6}\right): 12.47(\mathrm{~s}, 1 \mathrm{H}), 7.90(\mathrm{~s}, 1 \mathrm{H}), 7.81(\mathrm{~m}, 2 \mathrm{H}), 7.74(\mathrm{~s}, 1 \mathrm{H}), 7.65(\mathrm{~m}, 1 \mathrm{H}), 7.55(\mathrm{~m}$, $2 \mathrm{H}), 7.39(\mathrm{~m}, 2 \mathrm{H}), 7.26(\mathrm{~m}, 1 \mathrm{H}), 4.19(\mathrm{~s}, 2 \mathrm{H})$. HRMS $(\mathrm{m} / \mathrm{z})$ : $[\mathrm{M}+\mathrm{H}]^{+}$calcd for $\mathrm{C}_{18} \mathrm{H}_{14} \mathrm{~F}_{3} \mathrm{~N}_{2} \mathrm{O}: 331.1058$; found, 331.1053. This compound was then directly used in the next step.

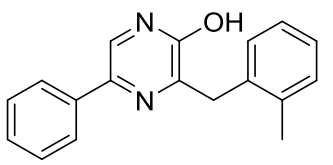

3-(2-Methylbenzyl)-5-phenylpyrazin-2-ol 19\{1,11\} (YJ31070-183-3): This compound was obtained as a powder $(5.65 \mathrm{~g}, 88 \%)$ after a chromatography over silica gel (dichloromethane - ethanol 99/1 - 98/2). ${ }^{1} \mathrm{H}$ NMR (DMSO- $d_{6}$ ) $12.40(\mathrm{~s}, 1 \mathrm{H}), 7.87(\mathrm{~s}, 1 \mathrm{H}), 7.77(\mathrm{~m}, 1 \mathrm{H}), 7.37(\mathrm{~m}, 2 \mathrm{H}), 7.24(\mathrm{~m}, 2 \mathrm{H}), 7.16(\mathrm{~m}, 1 \mathrm{H}), 7.10(\mathrm{~m}, 1 \mathrm{H}), 4.08(\mathrm{~s}, 2 \mathrm{H})$, 2.37 (s, 3H). ${ }^{13} \mathrm{C}$ NMR (DMSO- $\left.d_{6}\right): 157.3,155.4,137.0,136.8,136.4,131.3,130.3,130.2,129.0,127.6,126.8$, 126.0, 124.8, 122.7, 36.6, 20.0. HRMS $(\mathrm{m} / \mathrm{z})$ : $[\mathrm{M}+\mathrm{H}]^{+}$calcd for $\mathrm{C}_{18} \mathrm{H}_{17} \mathrm{~N}_{2} \mathrm{ON}$ : 277.1341; found, 277.1392.

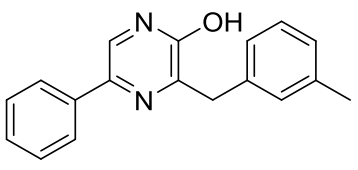

3-(3-Methylbenzyl)-5-phenylpyrazin-2-ol 19\{1,12\} (YJ31070-185-3): This compound was obtained as a powder $(5.52 \mathrm{~g}, 88 \%)$ after a chromatography over silica gel (dichloromethane - ethanol 99/1 - 98/2) and a sample $(0.29 \mathrm{~g})$ 
was recrystallized in a mixture of toluene and cyclohexane for analytical purposes $(0.27 \mathrm{~g}) .{ }^{1} \mathrm{H}$ NMR (DMSO- $\left.d_{6}\right)$ $12.38(\mathrm{~s}, 1 \mathrm{H}), 7.87(\mathrm{~s}, 1 \mathrm{H}), 7.84(\mathrm{~m}, 2 \mathrm{H}), 7.40(\mathrm{~m}, 2 \mathrm{H}), 7.28(\mathrm{~m}, 1 \mathrm{H}), 7.16(\mathrm{~m}, 3 \mathrm{H}), 7.01(\mathrm{~m}, 1 \mathrm{H}), 4.03(\mathrm{~s}, 2 \mathrm{H})$, $2.26(\mathrm{~s}, 3 \mathrm{H}) .{ }^{13} \mathrm{C}$ NMR (DMSO- $\left.d_{6}\right): 157.4,155.4,138.3,137.7,136.4,131.4,130.1,129.1,128.6,127.7,127.3$, 126.6, 124.9, 122.9, 39.2, 21.5. HRMS $(\mathrm{m} / \mathrm{z})$ : $[\mathrm{M}+\mathrm{H}]^{+}$calcd for $\mathrm{C}_{18} \mathrm{H}_{17} \mathrm{~N}_{2} \mathrm{ON}$ : 277.1341; found, 277.1395 .<smiles>COc1ccccc1Cc1nc(-c2ccccc2)cnc1O</smiles>

3-(2-Methoxybenzyl)-5-phenylpyrazin-2-ol 19\{1,14\} (YJ31070-106-1): Obtained as a white powder (1.7 g, 30\%) after a chromatography over silica gel (dichloromethane / ethanol 98.5:1.5 to 97.5:2.5) and a dispersion in a boiling mixture of cyclohexane and toluene. ${ }^{1} \mathrm{H}$ NMR (DMSO-d $) 12.35(\mathrm{~s}(\mathrm{br}), 1 \mathrm{H}), 7.86(\mathrm{~s}, 1 \mathrm{H}), 7.73(\mathrm{~m}, 2 \mathrm{H}), 7.34(\mathrm{~m}$, 2H), $7.23(\mathrm{~m}, 2 \mathrm{H}), 7.11(\mathrm{~m}, 1 \mathrm{H}), 6.98(\mathrm{~m}, 1 \mathrm{H}), 6.87(\mathrm{~m}, 1 \mathrm{H}), 4.04(\mathrm{~s}, 2 \mathrm{H}), 3.75(\mathrm{~s}, 3 \mathrm{H}) .{ }^{13} \mathrm{C}$ NMR (DMSO-d6): $157.7,157.3,155.5,136.5,131.2,130.6,129.0,128.0,127.6,126.6,124.8,122.4,111.2,55.9,33.3$. HRMS (m/z): $[\mathrm{M}+\mathrm{H}]^{+}$calcd for $\mathrm{C}_{18} \mathrm{H}_{17} \mathrm{~N}_{2} \mathrm{O}_{2}: 293.1290$; found, 293.1280.

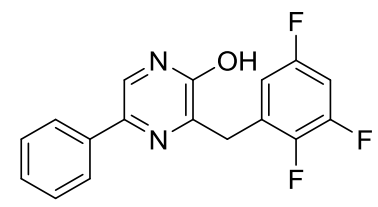

5-Phenyl-3-(2,3,5-trifluorobenzyl)pyrazin-2-ol 19\{1,26\} (YJ 33067-103-2): Obtained as a powder (1.72 g, 90\%) after a dispersion in boiling cyclohexane. ${ }^{1} \mathrm{H}$ NMR (DMSO- $\left.d_{6}\right): 12.54(\mathrm{~s}, 1 \mathrm{H}), 7.92(\mathrm{~s}, 1 \mathrm{H}), 7.70(\mathrm{~m}, 2 \mathrm{H}), 7.43$ $7.34(\mathrm{~m}, 3 \mathrm{H}), 7.25(\mathrm{~m}, 1 \mathrm{H}), 7.11(\mathrm{~m}, 1 \mathrm{H}), 4.17(\mathrm{~s}, 2 \mathrm{H}) .{ }^{13} \mathrm{C}$ NMR (DMSO-d $): 157.2(242,12$ and $3 \mathrm{~Hz}), 155.2$, 155.0, 149.9 (247 and $14 \mathrm{~Hz}), 145.7$ (242, 12 and $4 \mathrm{~Hz}$ ), 136.2, 131.2, 129.0, 128.9 (br), 127.7, 113.7 (3 and 24 $\mathrm{Hz}), 104.4$ (21 and $28 \mathrm{~Hz}), 32.6$. HRMS $(\mathrm{m} / \mathrm{z})$ : $[\mathrm{M}+\mathrm{H}]^{+}$calcd for $\mathrm{C}_{17} \mathrm{H}_{12} \mathrm{~F}_{3} \mathrm{~N}_{2} \mathrm{O}$ : 317.0902; found, 317.0910.

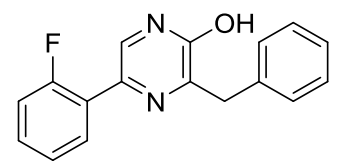

3-Benzyl-5-(2-fluorophenyl)pyrazin-2-ol 19\{2,1\} (YJ31134-027-2): This compound was as a white powder (3.38 $\mathrm{g}, 62 \%$ ) after a chromatography over silica gel (dichloromethane - ethanol 975/25) and a recrystallization in toluene. ${ }^{1} \mathrm{H}$ NMR (DMSO-d $\left.d_{6}\right) 12.38(\mathrm{~s}, 1 \mathrm{H}), 7.88(\mathrm{~m}, 1 \mathrm{H}), 7.68(\mathrm{~s}, 1 \mathrm{H}), 7.28(\mathrm{~m}, 8 \mathrm{H}), 4.06(\mathrm{~s}, 2 \mathrm{H}) .{ }^{13} \mathrm{C} \mathrm{NMR}$ (DMSO-d $): 159.5$ (246 Hz), 158.1, 155.0, 138.2, 129.7 (9 Hz), 129.6, 129.4 (3 Hz), 129.3, 128.7, 128.6, 126.7, $126.4(14 \mathrm{~Hz}), 125.1(3 \mathrm{~Hz}), 116.5(23 \mathrm{~Hz}), 39.2$. HRMS $(\mathrm{m} / \mathrm{z}):[\mathrm{M}+\mathrm{H}]^{+}$calcd for $\mathrm{C}_{17} \mathrm{H}_{14} \mathrm{FN}_{2} \mathrm{O}: 281.1090$; found, 281.1050 .<smiles>Oc1ncc(-c2cccc(F)c2)nc1Cc1ccccc1</smiles>

3-Benzyl-5-(3-fluorophenyl)pyrazin-2-ol 19\{3,1\} (YJ31067-011-2): This compound was obtained as a 90\% pure orange powder (1.69 g) after a chromatography over silica gel (dichloromethane - ethanol 98/2). ${ }^{1} \mathrm{H}$ NMR $\left(\mathrm{DMSO}-d_{6}\right) 12.50(\mathrm{~s}, 1 \mathrm{H}), 7.98(\mathrm{~s}, 1 \mathrm{H}), 7.69(\mathrm{~m}, 1 \mathrm{H}), 7.64(\mathrm{~m}, 1 \mathrm{H}), 7.42-7.17(\mathrm{~m}, 6 \mathrm{H}), 7.08(\mathrm{~m}, 1 \mathrm{H}), 4.07(\mathrm{~s}, 2 \mathrm{H})$. ${ }^{13} \mathrm{C}$ NMR (DMSO- $\left.d_{6}\right): 163.2(244 \mathrm{~Hz}), 157.6,155.5,139.1(8 \mathrm{~Hz}), 138.2,131.0(8 \mathrm{~Hz}), 129.6,129.0,128.7,126.7$, 123.7, $120.8(3 \mathrm{~Hz}), 114.2(22 \mathrm{~Hz}), 111.4(22 \mathrm{~Hz}), 39.3 . \mathrm{MS}(\mathrm{m} / z)=281$.

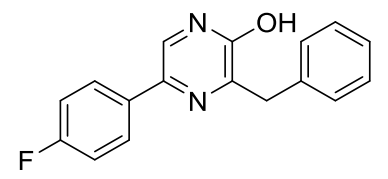

3-Benzyl-5-(4-fluorophenyl)pyrazin-2-ol 19\{4,1\} (YJ29589-041-3): This compound was as a white powder (0.43 g, 72\%) after a chromatography over silica gel (cyclohexane - ethyl acetate 1/1). ${ }^{1} \mathrm{H}$ NMR (DMSO- $\left.d_{6}\right) 12.4$ (s, 
1H), $7.87(\mathrm{~m}, 3 \mathrm{H}), 7.22(\mathrm{~m}, 7 \mathrm{H}), 4.06(\mathrm{~s}, 2 \mathrm{H}) .{ }^{13} \mathrm{C}$ NMR (DMSO- $\left.d_{6}\right): 162.2(244 \mathrm{~Hz}), 157.4,155.3,138.3,132.9(9$ $\mathrm{Hz}), 130.6,129.5,128.7,128.7,126.9(8 \mathrm{~Hz}), 126.7,122.9,115.8(21 \mathrm{~Hz}), 39.2$. HRMS $(\mathrm{m} / \mathrm{z}):[\mathrm{M}+\mathrm{H}]^{+}$calcd for $\mathrm{C}_{17} \mathrm{H}_{14} \mathrm{FN}_{2} \mathrm{O}$ : 281.1090; found, 281.1030.<smiles>Cc1cccc(-c2cnc(O)c(Cc3ccccc3)n2)c1</smiles>

3-Benzyl-5-(m-tolyl)pyrazin-2-ol 19\{5,1\} (YJ30367-063-3): A crude fraction was obtained by a filtration of the insoluble material formed in the course of the reaction followed by washing it with toluene. An analytical sample was obtained by a recrystallization in toluene of the concentrated filtrate. ${ }^{1} \mathrm{H}$ NMR (DMSO- $\left.d_{6}\right) 12.35(\mathrm{~s}, 1 \mathrm{H}), 7.85$ (s, 1H), $7.66(\mathrm{~m}, 1 \mathrm{H}), 7.62(\mathrm{~m}, 2 \mathrm{H}), 7.33-7.17(\mathrm{~m}, 5 \mathrm{H}), 7.08(\mathrm{~m}, 1 \mathrm{H}), 4.07(\mathrm{~s}, 2 \mathrm{H}), 2.33(\mathrm{~s}, 3 \mathrm{H}) .{ }^{13} \mathrm{C}$ NMR (DMSO$\left.d_{6}\right): 157.3,155.4,138.4,138.1,136.4,131.6,129.5,129.0,128.7,128.3,126.6,125.5,122.9,122.1,39.3,21.6$. HRMS $(m / z):[\mathrm{M}+\mathrm{Na}]^{+}$calcd for $\mathrm{C}_{18} \mathrm{H}_{16} \mathrm{~N}_{2} \mathrm{ONa}$ : 299.1160; found, 299.1172.

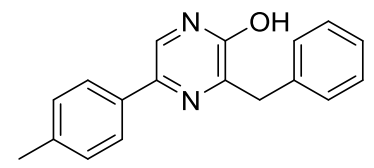

3-Benzyl-5-(p-tolyl)pyrazin-2-ol 19\{6,1\} (YJ30367-029-2): A pure sample (1.48 g) of this compound was obtained as a white solid by a dispersion of the crude mixture in dichloromethane. The concentrated filtrate and most of this solid were used directly in the next step. ${ }^{1} \mathrm{H}$ NMR (DMSO- $\left.d_{6}\right) 12.35(\mathrm{~s}, 1 \mathrm{H}), 7.82(\mathrm{~s}, 1 \mathrm{H}), 7.72(\mathrm{~m}$, 2H), 7.40-7.17 (m, 7H), 4.06 (s, 2H), 2.30 (s, 3H). ${ }^{13} \mathrm{C}$ NMR (DMSO- $\left.d_{6}\right): 157.2,155.4,138.4,136.9,133.7,131.7$, 129.6, 129.5, 128.7, 126.6, 124.9, 122.4, 39.3, 21.2. HRMS $(\mathrm{m} / \mathrm{z})$ : $[\mathrm{M}+\mathrm{Na}]^{+}$calcd for $\mathrm{C}_{18} \mathrm{H}_{16} \mathrm{~N}_{2} \mathrm{ONa}$ : 299.1160; found, 299.1177.

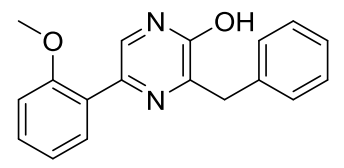

3-Benzyl-5-(2-methoxyphenyl)pyrazin-2-ol 19\{7,1\} (YJ31134-039-2): This compound was obtained as a powder $(0.54 \mathrm{~g}, 79 \%)$ after a chromatography over silica gel (dichloromethane - ethanol 97/3). ${ }^{1} \mathrm{H}$ NMR $\left(\mathrm{CDCl}_{3}\right): 13.33$ (s(br), 1H), $8.05(\mathrm{~s}, 1 \mathrm{H}), 8.02(\mathrm{dd}, 1 \mathrm{H}, J=7.7,1.7 \mathrm{~Hz}), 7.51-7.42(\mathrm{~m}, 2 \mathrm{H}), 7.37-7.28(\mathrm{~m}, 3 \mathrm{H}), 7.25-7.19(\mathrm{~m}$, $1 \mathrm{H}), 7.08(\mathrm{td}, 1 \mathrm{H}, J=7.7,1.0 \mathrm{~Hz}), 7.02-6.94(\mathrm{~m}, 1 \mathrm{H}), 4.27(\mathrm{~s}, 2 \mathrm{H}), 3.92(\mathrm{~s}, 3 \mathrm{H}) .{ }^{13} \mathrm{C} \mathrm{NMR}\left(\mathrm{CDCl}_{3}\right): 157.1$, 156.8, 156.5, 137.8, 131.2, 129.6 (2 signals), 129.1, 128.4, 126.5, 125.2, 124.6, 121.2, 111.3, 55.6, 39.5. HRMS $(\mathrm{m} / z):[\mathrm{M}+\mathrm{H}]^{+}$calcd for $\mathrm{C}_{18} \mathrm{H}_{17} \mathrm{~N}_{2} \mathrm{O}_{2}, 293.1290$; found, 293.1253.

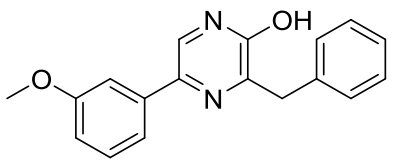

3-Benzyl-5-(3-methoxyphenyl)pyrazin-2-ol 19\{8,1\} (YJ30367-079-1): An analytical sample of this compound was obtained as a white powder after a recrystallization of the resulting residue in toluene. ${ }^{1} \mathrm{H}$ NMR (DMSO- $d_{6}$ ) 12.41 $(\mathrm{s}, 1 \mathrm{H}), 7.90(\mathrm{~s}, 1 \mathrm{H}), 7.41(\mathrm{~m}, 8 \mathrm{H}), 6.85(\mathrm{~m}, 1 \mathrm{H}), 4.07(\mathrm{~s}, 2 \mathrm{H}), 3.77(\mathrm{~s}, 3 \mathrm{H}) .{ }^{13} \mathrm{C}$ NMR (DMSO- $\left.d_{6}\right): 160.1,157.3$, 155.5, 138.3, 137.9, 131.1, 130.1, 129.7, 128.6, 126.6, 123.1, 117.2, 113.4, 110.4, 55.5, 39.2. HRMS (m/z): $[\mathrm{M}+\mathrm{H}]^{+}$calcd for $\mathrm{C}_{18} \mathrm{H}_{17} \mathrm{~N}_{2} \mathrm{O}_{2}: 293.1290$; found, 293.1279.

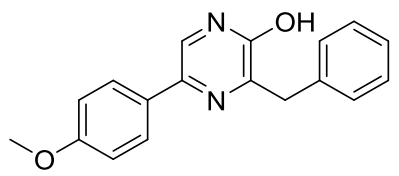

3-Benzyl-5-(4-methoxyphenyl)pyrazin-2-ol 19\{9,1\} (YJ29793-047-3): This compound was obtained as a powder $(0.9 \mathrm{~g}, 45 \%)$ after a chromatography over silica gel (dichloromethane - ethanol 98/2). ${ }^{1} \mathrm{H}$ NMR (DMSO- $\left.d_{6}\right) 12.30$ (s, 1H), $7.77(\mathrm{~m}, 3 \mathrm{H}), 7.30(\mathrm{~m}, 2 \mathrm{H}), 7.27(\mathrm{~m}, 2 \mathrm{H}), 7.21(\mathrm{~m}, 1 \mathrm{H}), 6.96(\mathrm{~m}, 2 \mathrm{H}), 4.06(\mathrm{~s}, 2 \mathrm{H}), 3.77(\mathrm{~s}, 3 \mathrm{H}) .{ }^{13} \mathrm{C}$ NMR 
(DMSO- $\left.d_{6}\right): 159.2,157.0,155.3,138.5,131.8,129.5,129.0,128.7,126.6,126.3,121.9,114.5,55.6,39.3$. HRMS $(\mathrm{m} / \mathrm{z}):[\mathrm{M}+\mathrm{H}]^{+}$calcd for $\mathrm{C}_{18} \mathrm{H}_{17} \mathrm{~N}_{2} \mathrm{O}_{2}: 293.1290$; found, 293.1284.

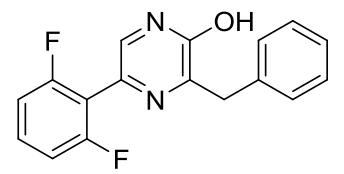

3-Benzyl-5-(2,6-difluorophenyl)pyrazin-2-ol 19\{10,1\} (MM34284-020-3): This compound was obtained as a white powder $(1.45 \mathrm{~g}, 83 \%)$ after a chromatography over silica gel (dichloromethane - ethanol 99/1). ${ }^{1} \mathrm{H}$ NMR (DMSO-d $\left.d_{6}\right): 12.49(\mathrm{~s}, 1 \mathrm{H}), 7.57(\mathrm{~s}, 1 \mathrm{H}), 7.47(\mathrm{~m}, 1 \mathrm{H}), 7.27(\mathrm{~m}, 4 \mathrm{H}), 7.19(\mathrm{~m}, 3 \mathrm{H}), 4.01(\mathrm{~s}, 2 \mathrm{H}) .{ }^{13} \mathrm{C}$ NMR (DMSO$\left.d_{6}\right): 162.4(7,244 \mathrm{~Hz}), 158.0,138.1,130.8$ (10 Hz), 129.4, 128.7, 128.3, 126.7, 122.2, $114.8(18 \mathrm{~Hz}), 112.3$ (7, 18 $\mathrm{Hz})$, 39.2. HRMS (m/z): $[\mathrm{M}+\mathrm{H}]^{+}$calcd for $\mathrm{C}_{17} \mathrm{H}_{13} \mathrm{~F}_{2} \mathrm{~N}_{2} \mathrm{O}$ : 299.0996; found, 299.0994.

General preparation of 5-oxo-2,3,4,5-tetrahydropyrazine 1-oxides 18 via steps v. The considered piperazin-2one $17(0.015 \mathrm{~mol})$ was dissolved in ethylacetate $(80 \mathrm{~mL})$ and the solution cooled to $0{ }^{\circ} \mathrm{C}$ with an ice bath. A $38 \%$ solution of peracetic acid in acetic acid $(5.3 \mathrm{~mL}, 0.03 \mathrm{~mol})$ was added (note 1 and 2$)$. The ice bath was removed and the solution stirred overnight (note 3). This was concentrated to dryness and the residue purified as described below (note 3). Note 1: when starting from crude piperazin-2-one 17, the amount of peracetic acid added was calculated as if the starting material was pure and was thus certainly larger than 2.0 equivalents but this had no ill effect on the reaction yield as 5-oxo-2,3,4,5-tetrahydropyrazine 1-oxides are quite resilient to further oxidation under these conditions. Note 2: since the title of a $36 \%$ peracetic acid solution in acetic acid steadily drops over time the use of aged solutions should take this into account to achieve a complete transformation. Note 3: the resulting 5-oxo-2,3,4,5-tetrahydropyrazine 1-oxide 18 can sometime precipitate in the course of the reaction. Note 4: initially, when possible, the resulting solutions were washed with water. Later on, we found out that most of the peracetic acid and acetic acid could be removed under vacuum.

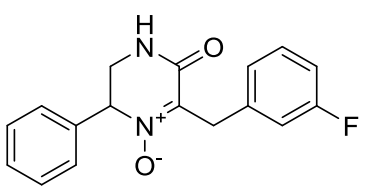

6-(3-fluorobenzyl)-5-oxo-2-phenyl-2,3,4,5-tetrahydropyrazine 1-oxide 18\{1,3\} (YJ 31068-153-3): Obtained as a solid (6.70 g, 48\% from nitrostyrene) after a chromatography over silica gel (cyclohexane - ethyl acetate $2 / 3$ ). ${ }^{1} \mathrm{H}$ NMR (DMSO-d $): 8.36$ (d (br), 1H, J = 3.7 Hz), 7.35-7.30 (m, 4H), 7.21-7.01 (m, 5H), 5.27 (t(br), 1H, J = 3.9 Hz), $3.99(\mathrm{~m}, 3 \mathrm{H}), 3.57(\mathrm{~m}, 1 \mathrm{H}) .{ }^{13} \mathrm{C}$ NMR (DMSO- $\left.d_{6}\right): 162.5(242 \mathrm{~Hz}), 160.6,140.6,139.9(8 \mathrm{~Hz}), 135.9,130.4(8$ $\mathrm{Hz}), 128.9,128.8,127.1,125.4(3 \mathrm{~Hz}), 116.1(21 \mathrm{~Hz}), 113.6(21 \mathrm{~Hz}), 71.5,42.6,30.0 . \mathrm{HRMS}(\mathrm{m} / z):[\mathrm{M}+\mathrm{Na}]^{+}$ calcd for $\mathrm{C}_{17} \mathrm{H}_{15} \mathrm{FN}_{2} \mathrm{O}_{2} \mathrm{Na}$ : 321.1015; found, 321.1015 .<smiles></smiles>

6-(4-Fluorobenzyl)-5-oxo-2-phenyl-2,3,4,5-tetrahydropyrazine 1-oxide 18\{1,4\} (YJ 33069-161-2): Obtained as a white powder (1.94 g, 36\% from nitrostyrene) after a chromatography over silica gel (cyclohexane - ethyl acetate $3 / 2$ to $1 / 1)$. ${ }^{1} \mathrm{H}$ NMR (DMSO- $\left.d_{6}\right) 8.32(\mathrm{~s}(\mathrm{br}), 1 \mathrm{H}), 7.35(\mathrm{~m}, 5 \mathrm{H}), 7.16(\mathrm{~m}, 2 \mathrm{H}), 7.10(\mathrm{~m}, 2 \mathrm{H}), 5.25(\mathrm{t}(\mathrm{br}), 1 \mathrm{H}, J=$ $3.7 \mathrm{~Hz}), 3.97(\mathrm{~m}, 1 \mathrm{H}), 3.95(\mathrm{~s}, 2 \mathrm{H}), 3.55(\mathrm{ddd}, 1 \mathrm{H}, J=3.6,4.7,13.9 \mathrm{~Hz}) .{ }^{13} \mathrm{C}$ NMR (DMSO-d6): $161.5(242 \mathrm{~Hz})$, 160.7, 141.0, 135.9, 133.2 (3 Hz), $131.3(8 \mathrm{~Hz}), 128.9,128.8,127.0,115.3(21 \mathrm{~Hz}), 71.5,42.7,29.5$. HRMS $(\mathrm{m} / \mathrm{z})$ : $[\mathrm{M}+\mathrm{H}]^{+}$calcd for $\mathrm{C}_{17} \mathrm{H}_{16} \mathrm{FN}_{2} \mathrm{O}_{2}: 299.1196$; found, 299.1202 .<smiles>O=C1NCC(c2ccccc2)[N+]([O-])=C1Cc1ccccc1Cl</smiles> 
6-(2-Chlorobenzyl)-5-oxo-2-phenyl-2,3,4,5-tetrahydropyrazine 1-oxide 18\{1,5\} (YJ 33069-159-1): Obtained as a white powder (2.92 g, $50 \%$ from nitrostyrene) after a chromatography over silica gel (cyclohexane - ethyl acetate 3/2 to $1 / 1$ ). ${ }^{1} \mathrm{H}$ NMR (DMSO-d $) 8.41$ (s (br), 1H), $7.44-7.37(\mathrm{~m}, 4 \mathrm{H}), 7.31(\mathrm{~m}, 2 \mathrm{H}), 7.24(\mathrm{~m}, 2 \mathrm{H}), 7.17(\mathrm{~m}, 1 \mathrm{H})$, $5.31(\mathrm{t}(\mathrm{br}), 1 \mathrm{H}, J=4.0 \mathrm{~Hz}), 4.11(\mathrm{~d}, 1 \mathrm{H}, J=15.0 \mathrm{~Hz}), 4.02(\mathrm{~m}, 2 \mathrm{H}), 3.64(\mathrm{dt}, 1 \mathrm{H}, J=14.0,4.1 \mathrm{~Hz}) .{ }^{13} \mathrm{C} \mathrm{NMR}$ (DMSO-d6): 160.6, 139.9, 135.7, 134.6, 133.4, 130.1, 129.6, 129.0, 128.9, 128.5, 127.5, 127.3, 71.8, 42.6, 28.3. $\operatorname{HRMS}(\mathrm{m} / z)$ : $[\mathrm{M}+\mathrm{H}]^{+}$calcd for $\mathrm{C}_{17} \mathrm{H}_{16} \mathrm{ClN}_{2} \mathrm{O}_{2}$ : 315.0900; found, 315.0923 .

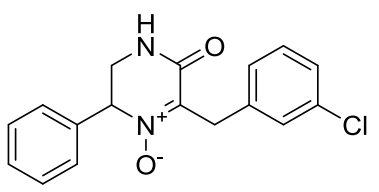

6-(3-Chlorobenzyl)-5-oxo-2-phenyl-2,3,4,5-tetrahydropyrazine 1-oxide 18\{1,6\} (YJ 33069-187-2): Obtained as a glass (3.12 g, 53\% from nitrostyrene) after a chromatography over silica gel (cyclohexane - ethyl acetate $2 / 3$ ). ${ }^{1} \mathrm{H}$ NMR (DMSO-d6): 7.42 (s (br), 1H), 7.44 - 7.37 (m, 4H), 7.19 (m, 4H), 7.01 (s (br), 1H), 5.13 (t (br), J=4.8 Hz), $4.08(\mathrm{~m}, 2 \mathrm{H}), 4.02(\mathrm{~m}, 1 \mathrm{H}), 3.68(\mathrm{dt}, 1 \mathrm{H}, J=13.5,4.2 \mathrm{~Hz}) .{ }^{13} \mathrm{C}$ NMR (DMSO-d $): 161.1,140.9,137.9,134.1$, $134.0,129.6,129.5,129.2,129.0,127.9,127.0,126.7,71.9,43.0,30.0$. HRMS $(\mathrm{m} / z):[\mathrm{M}+\mathrm{H}]^{+}$calcd for $\mathrm{C}_{17} \mathrm{H}_{16} \mathrm{ClN}_{2} \mathrm{O}_{2}$ : 315.0900; found, 315.0892.

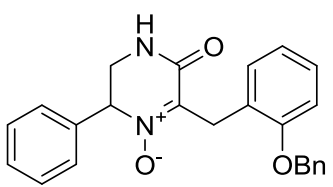

6-(2-(benzyloxy)benzyl)-5-oxo-2-phenyl-2,3,4,5-tetrahydropyrazine 1-oxide 18\{1,17\} (RB32489-045-3): This compound was obtained as a pale yellow oil (3.51 g, 36\% from nitrostyrene) after two chromatography over silica gel (cyclohexane - ethyl acetate 2/3) and (dichloromethane - ethanol 98/2). ${ }^{1} \mathrm{H}\left(\mathrm{CDCl}_{3}\right): 7.45-7.29(\mathrm{~m}, 8 \mathrm{H}), 7.22$ $(\mathrm{m}, 3 \mathrm{H}), 7.09(\mathrm{~m}, 1 \mathrm{H}), 7.04(\mathrm{~m}, 1 \mathrm{H}), 6.85(\mathrm{~m}, 1 \mathrm{H}), 5.10(\mathrm{~m}, 1 \mathrm{H}), 5.02(\mathrm{~s}, 2 \mathrm{H}), 4.11(\mathrm{~m}, 2 \mathrm{H}), 3.95(\mathrm{dd}, 1 \mathrm{H}, J=$ 13.5, $5.0 \mathrm{~Hz}), 3.60(\mathrm{dd}, 1 \mathrm{H}, J=13.5,3.8 \mathrm{~Hz}) .{ }^{13} \mathrm{C}\left(\mathrm{CDCl}_{3}\right): 161.2,158.9,141.5,137.6,137.1,134.2,129.4,129.1$, 129.0, 128.5, 127.9, 127.6, 126.7, 122.2, 115.9, 113.4, 71.8, 69.9, 42.9, 30.2. HRMS (m/z): $[\mathrm{M}+\mathrm{Na}]^{+}$calcd for $\mathrm{C}_{24} \mathrm{H}_{22} \mathrm{~N}_{2} \mathrm{O}_{3} \mathrm{Na}: 409.1528$; found, 409.1524 .

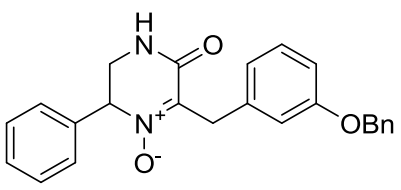

6-(3-(benzyloxy)benzyl)-5-oxo-2-phenyl-2,3,4,5-tetrahydropyrazine 1-oxide $\mathbf{1 8}\{1,18\}$ (RB32489-043-3): This compound was obtained as a pale yellow oil (4.49 g, 24\% from nitrostyrene) after two chromatography over silica gel (cyclohexane - ethyl acetate 2/3) and (dichloromethane - ethanol 98/2). ${ }^{1} \mathrm{H}\left(\mathrm{CDCl}_{3}\right): 7.49(\mathrm{~m}, 2 \mathrm{H}), 7.40-7.16$ $(\mathrm{m}, 10 \mathrm{H}), 6.91(\mathrm{~m}, 2 \mathrm{H}), 6.28(\mathrm{~m}, 1 \mathrm{H}), 5.09(\mathrm{~s}, 2 \mathrm{H}), 5.02(\mathrm{t}, 1 \mathrm{H}, J=3.9 \mathrm{~Hz}), 4.30(\mathrm{~d}, 1 \mathrm{H}, J=14.7 \mathrm{~Hz}), 4.12(\mathrm{~d}, 1 \mathrm{H}$, $J=14.7 \mathrm{~Hz}), 3.81(\mathrm{dd}, 1 \mathrm{H}, J=13.5,4.8 \mathrm{~Hz}), 3.48(\mathrm{dt}, 1 \mathrm{H},, J=13.3,8.0,3.7 \mathrm{~Hz}) .{ }^{13} \mathrm{C}\left(\mathrm{CDCl}_{3}\right): 161.0,156.5$, $141.9,137.2,134.3,130.3,129.0,128.9,128.4,127.9,127.8,127.7,126.8,125.0,120.9,111.8,72.0,70.3,42.9$, 26.1. HRMS (m/z): $[\mathrm{M}+\mathrm{Na}]^{+}$calcd for $\mathrm{C}_{24} \mathrm{H}_{22} \mathrm{~N}_{2} \mathrm{O}_{3} \mathrm{Na}$ : 409.1528; found, 409.1526 .

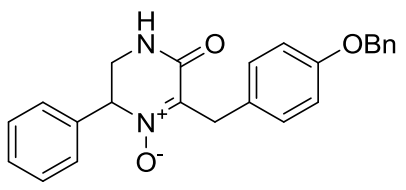

6-(4-(Benzyloxy)benzyl)-5-oxo-2-phenyl-2,3,4,5-tetrahydropyrazine $\quad$ 1-oxide $\quad \mathbf{1 8}\{1,19\} \quad$ (YJ 31776-033-2): Obtained as a white powder (3.40 g, 53\% from nitrostyrene) after a chromatography over silica gel (cyclohexane ethyl acetate 2/3). ${ }^{1} \mathrm{H}$ NMR $\left(\mathrm{CDCl}_{3}\right) 7.46-7.28(\mathrm{~m}, 10 \mathrm{H}), 7.16(\mathrm{~m}, 2 \mathrm{H}), 6.90(\mathrm{~m}, 2 \mathrm{H}), 6.77(\mathrm{~s}(\mathrm{br}), 1 \mathrm{H}), 5.12(\mathrm{t}$ (br), $J=4.2 \mathrm{~Hz}), 5.05(\mathrm{~s}, 2 \mathrm{H}), 4.06(\mathrm{~m}, 2 \mathrm{H}), 4.01(\mathrm{dd}, 1 \mathrm{H}, J=5.3,13.3 \mathrm{~Hz}), 3.68(\mathrm{dt}, 1 \mathrm{H}, J=4.0,13.3 \mathrm{~Hz}) .{ }^{13} \mathrm{C}$ NMR $\left(\mathrm{CDCl}_{3}\right)$ : 161.2, 157.7, 142.0, 137.2, 130.7, 129.1, 129.0, 128.5, 128.4, 127.9, 127.4, 126.6, 114.8, 71.8, 70.0, 43.1, 29.4. HRMS (m/z): $[\mathrm{M}+\mathrm{H}]^{+}$calcd for $\mathrm{C}_{24} \mathrm{H}_{23} \mathrm{~N}_{2} \mathrm{O}_{3}: 387.1709$; found, 387.1710. 


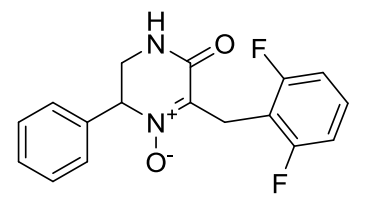

6-(2,6-Difluorobenzyl)-5-oxo-2-phenyl-2,3,4,5-tetrahydropyrazine 1-oxide 18\{1,21\} (EC31095-167-3): Obtained as a white solid (8.58 g, 30\% from nitrostyrene) after chromatography over silica gel (cyclohexane-ethyl acetate 1:1). ${ }^{1} \mathrm{H}$ NMR (DMSO-d6): $8.32(\mathrm{~d}, 1 \mathrm{H}, J=4.4 \mathrm{~Hz}), 7.38(\mathrm{~m}, 3 \mathrm{H}), 7.28(\mathrm{~m}, 3 \mathrm{H}), 7.03(\mathrm{~m}, 2 \mathrm{H}), 5.24(\mathrm{t}, 1 \mathrm{H}, J=4.1$ $\mathrm{Hz}), 4.03$ (s, 2H), 3.90 (ddd, $1 \mathrm{H}, J=13.9,4.8,1.6 \mathrm{~Hz}), 3.55(\mathrm{ddd}, 1 \mathrm{H}, J=13.9,4.7,3.4 \mathrm{~Hz}) .{ }^{13} \mathrm{C}$ NMR (DMSO$\left.d_{6}\right): 161.5$ (247, $\left.8 \mathrm{~Hz}\right), 160.1,139.3,135.7,129.0,128.9,128.8,127.1,112.8$ (19 Hz), 111.7 (br), $71.8,42.8,18.9$ $(3 \mathrm{~Hz})$. HRMS $(\mathrm{m} / z)$ : $[\mathrm{M}+\mathrm{H}]^{+}$calcd for $\mathrm{C}_{17} \mathrm{H}_{15} \mathrm{~F}_{2} \mathrm{~N}_{2} \mathrm{O}_{2}: 317.1102$; found, 317.1105.

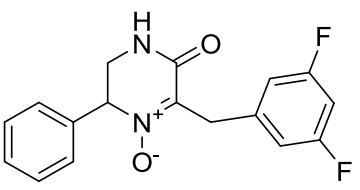

6-(3,5-difluorobenzyl)-5-oxo-2-phenyl-2,3,4,5-tetrahydropyrazine 1-oxide 18\{1,22\} (EC31095-167-3): Obtained as a white solid (4.82 g, 17\% from nitrostyrene) after chromatography over silica gel (cyclohexane-ethyl acetate 1:1 to $1: 2) .{ }^{1} \mathrm{H}$ NMR (DMSO- $\left.d_{6}\right): 8.39(\mathrm{~d}, 1 \mathrm{H}, J=4.3 \mathrm{~Hz}), 7.35(\mathrm{~m}, 3 \mathrm{H}), 7.20(\mathrm{~m}, 2 \mathrm{H}), 7.07(\mathrm{tt}, 1 \mathrm{H}, J=9.5,2.4$ $\mathrm{Hz}), 6.99(\mathrm{~m}, 2 \mathrm{H}), 5.29(\mathrm{t}, 1 \mathrm{H}, J=4.2 \mathrm{~Hz}), 4.00(\mathrm{~m}, 3 \mathrm{H}), 3.60(\mathrm{ddd}, 1 \mathrm{H}, J=13.9,4.6,3.7 \mathrm{~Hz}) .{ }^{13} \mathrm{C}$ NMR (DMSO$\left.d_{6}\right)$ : $162.6(246,13 \mathrm{~Hz}), 160.5,141.5(10 \mathrm{~Hz}), 140.0,135.8,128.9,128.9,127.1,112.5$ (br), 102.4 (26 Hz), 71.5, 42.6, 30.0. HRMS (m/z): $[\mathrm{M}+\mathrm{H}]^{+}$calcd for $\mathrm{C}_{17} \mathrm{H}_{15} \mathrm{~F}_{2} \mathrm{~N}_{2} \mathrm{O}_{2}$ : 317.1102; found, 317.1100.<smiles>O=C1NCC(c2ccccc2)[N+](=O)C1Cc1cc(F)ccc1F</smiles>

6-(2,5-difluorobenzyl)-5-oxo-2-phenyl-2,3,4,5-tetrahydropyrazine 1-oxide 18\{1,23\} (YJ 31069-005-4): Obtained as a white solid $(0.49 \mathrm{~g}, 11 \%$ from nitrostyrene) after chromatography over silica gel (cyclohexane-ethyl acetate 1:1). ${ }^{1} \mathrm{H} \mathrm{NMR}\left(\mathrm{CDCl}_{3}\right) 7.38(\mathrm{~m}, 3 \mathrm{H}), 7.26(\mathrm{~m}, 2 \mathrm{H}), 7.21(\mathrm{~m}, 1 \mathrm{H}), 6.98(\mathrm{~m}, 2 \mathrm{H}), 6.88(\mathrm{~m}, 1 \mathrm{H}), 5.15(\mathrm{t}(\mathrm{br}), 1 \mathrm{H}, \mathrm{J}=$ $4.8 \mathrm{~Hz}), 4.19(\mathrm{~d}, 1 \mathrm{H}, \mathrm{J}=14.4 \mathrm{~Hz}), 4.06(\mathrm{~m}, 2 \mathrm{H}), 3.73(\mathrm{~m}, 1 \mathrm{H}) .{ }^{13} \mathrm{C} \mathrm{NMR}\left(\mathrm{CDCl}_{3}\right): 161.1,158.5(242 \mathrm{and} 2 \mathrm{~Hz})$, $157.0(242$ and $2 \mathrm{~Hz}), 154.4,140.0,133.9,129.3,129.0,126.9,126.0,124.6(18$ and $8 \mathrm{~Hz}), 117.2(21 \mathrm{and} 4 \mathrm{~Hz})$, $116.1(25$ and $8 \mathrm{~Hz}), 114.6(24$ and $9 \mathrm{~Hz}), 72.1,42.9,24.0$. HRMS $(m / z)$ : $[\mathrm{M}+\mathrm{H}]^{+}$calcd for $\mathrm{C}_{17} \mathrm{H}_{15} \mathrm{~F}_{2} \mathrm{~N}_{2} \mathrm{O}_{2}$ : 317.1102; found, 317.1101.<smiles>O=C1NCC(c2ccccc2)[N+](=O)C1Cc1cccc(F)c1F</smiles>

6-(2,3-difluorobenzyl)-5-oxo-2-phenyl-2,3,4,5-tetrahydropyrazine 1-oxide 18\{1,24\} (YJ 31069-011-4): Obtained as a white solid (2.56 g, 46\% from nitrostyrene) after chromatography over silica gel (cyclohexane-ethyl acetate 1:2). ${ }^{1} \mathrm{H} \mathrm{NMR}\left(\mathrm{CDCl}_{3}\right) 7.38(\mathrm{~m}, 3 \mathrm{H}), 7.26(\mathrm{~m}, 2 \mathrm{H}), 7.11-6.93(\mathrm{~m}, 4 \mathrm{H}), 5.15$ (t (br), 1H, J = 4.5 Hz), 4.19 (d, $1 \mathrm{H}, J$ $=14.6 \mathrm{~Hz}), 4.06(\mathrm{~m}, 2 \mathrm{H}), 3.73(\mathrm{~m}, 1 \mathrm{H}) .{ }^{13} \mathrm{C} \mathrm{NMR}\left(\mathrm{CDCl}_{3}\right): 161.1,150.7(248$ and $13 \mathrm{~Hz}), 149.2(248 \mathrm{and} 13 \mathrm{~Hz})$, 140.0, 133.9, 129.3, 129.0, 126.8, 125.6 (22 and $6 \mathrm{~Hz}), 123.7$ (7 and 5 Hz), 115.7, 115.5, 72.1, 42.9, 23.8. HRMS $(\mathrm{m} / \mathrm{z}):[\mathrm{M}+\mathrm{H}]^{+}$calcd for $\mathrm{C}_{17} \mathrm{H}_{15} \mathrm{~F}_{2} \mathrm{~N}_{2} \mathrm{O}_{2}:$ 317.1102; found, 317.1100. HRMS $(\mathrm{m} / z)$ : $[\mathrm{M}+\mathrm{H}]^{+}$calcd for $\mathrm{C}_{17} \mathrm{H}_{15} \mathrm{~F}_{2} \mathrm{~N}_{2} \mathrm{O}_{2}$ : 317.1102; found, 317.1101.<smiles>O=C1NCC(c2ccccc2F)[N+]([O-])=C1Cc1cccc(F)c1</smiles> 
6-(3-fluorobenzyl)-2-(2-fluorophenyl)-5-oxo-2,3,4,5-tetrahydropyrazine 1-oxide $\mathbf{1 8}\{2,3\} \quad$ (YJ 31068-149-2): Obtained as a solid ( $2.68 \mathrm{~g}, 50 \%$ from the nitrostyrene) after a chromatography over silica gel (cyclohexane - ethyl acetate $3 / 2$ to $1 / 1)$. ${ }^{1} \mathrm{H}$ NMR (DMSO- $\left.d_{6}\right): 8.45(\mathrm{~s}(\mathrm{br}), 1 \mathrm{H}), 7.43(\mathrm{~m}, 1 \mathrm{H}), 7.30(\mathrm{~m}, 2 \mathrm{H}), 7.18-7.01(\mathrm{~m}, 5 \mathrm{H}), 5.55$ $(\mathrm{t}(\mathrm{br}), 1 \mathrm{H}, J=5.0 \mathrm{~Hz}), 3.99(\mathrm{~m}, 2 \mathrm{H}), 3.91(\mathrm{~m}, 1 \mathrm{H}), 3.61(\mathrm{~m}, 1 \mathrm{H}) .{ }^{13} \mathrm{C}$ NMR (DMSO-d $): 162.5(240 \mathrm{~Hz}), 160.6$, $160.5(247 \mathrm{~Hz}), 140.8,139.8(8 \mathrm{~Hz}), 131.3(8 \mathrm{~Hz}), 130.5(8 \mathrm{~Hz}), 129.1(3 \mathrm{~Hz}), 125.4(3 \mathrm{~Hz}), 125.0(3 \mathrm{~Hz}), 122.5$ $(13 \mathrm{~Hz}), 116.3(21 \mathrm{~Hz}), 116.1(21 \mathrm{~Hz}), 113.6(21 \mathrm{~Hz}), 66.8,41.7,30.1$. HRMS $(\mathrm{m} / \mathrm{z}):[\mathrm{M}+\mathrm{H}]^{+}$calcd for $\mathrm{C}_{17} \mathrm{H}_{15} \mathrm{~F}_{2} \mathrm{~N}_{2} \mathrm{O}_{2}$ : 317.1102 ; found, 317.1107 .

General preparation of pyrazin-2-ols 19 by dehydration of 5-oxo-2,3,4,5-tetrahydropyrazine 1-oxides 18 via step vi. The considered 5-oxo-2,3,4,5-tetrahydropyrazine 1-oxide $\mathbf{1 8}(0.09 \mathrm{~mol})$ and sodium hydroxide $(10.8 \mathrm{~g}$, $0.27 \mathrm{~mol})$ were dispersed in ethanol $(40 \mathrm{~mL})$ and stirred at $65^{\circ} \mathrm{C}$ for one hour. This was diluted in water $(200 \mathrm{~mL})$ and made acid with $1 \mathrm{~N}$ hydrochloric acid. The resulting precipitate was filtrated, washed with water and dried under vaccum at $60^{\circ} \mathrm{C}$ to yield the hydroxypyrazine 19 as described below.

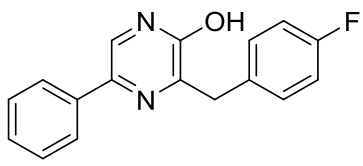

3-(4-Fluorobenzyl)-5-phenylpyrazin-2-ol 19\{1,4\} (YJ 33069-169-2): Obtained as a white powder (1.50 g, 93\%). ${ }^{1} \mathrm{H}$ NMR (CDCl3) $12.43(\mathrm{~s}(\mathrm{br}), 1 \mathrm{H}), 7.88(\mathrm{~s}, 1 \mathrm{H}), 7.83(\mathrm{~m}, 2 \mathrm{H}), 7.38(\mathrm{~m}, 4 \mathrm{H}), 7.29(\mathrm{~m}, 1 \mathrm{H}), 7.11(\mathrm{~m}, 2 \mathrm{H}), 4.06(\mathrm{~s}$, 2H). ${ }^{13} \mathrm{C}$ NMR (CDCl3): $161.4(242 \mathrm{~Hz}), 157.2,155.4,136.4,134.4(3 \mathrm{~Hz}), 131.4(8 \mathrm{~Hz}), 129.0,127.7,124.9$, 123.0, $115.3(21 \mathrm{~Hz}), 38.4$. HRMS $(\mathrm{m} / \mathrm{z}):[\mathrm{M}+\mathrm{H}]^{+}$calcd for $\mathrm{C}_{17} \mathrm{H}_{14} \mathrm{FN}_{2} \mathrm{O}_{2}: 281.1085$; found, 281.1096.

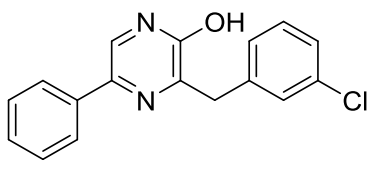

3-(3-Chlorobenzyl)-5-phenylpyrazin-2-ol 19\{1,6\} (YJ 33069-189-1): Obtained as a white powder (2.60 g, 88\%). ${ }^{1} \mathrm{H}$ NMR (DMSO-d6) 12.46 (s (br), 1H), 7.90 (s, 1H), 7.83 (m, 2H), $7.42-7.26(\mathrm{~m}, 6 \mathrm{H}), 4.09(\mathrm{~s}, 2 \mathrm{H}) .{ }^{13} \mathrm{C}$ NMR (DMSO-d6): 156.7, 155.4, 140.8, 136.3, 133.2, 131.4, 130.5, 129.5, 129.1, 128.4, 127.7, 126.7, 124.9, 123.1, 38.9. HRMS $(\mathrm{m} / z)$ : $[\mathrm{M}+\mathrm{H}]^{+}$calcd for $\mathrm{C}_{17} \mathrm{H}_{14} \mathrm{ClN}_{2} \mathrm{O}_{2}: 297.0795$; found, 297.0794.

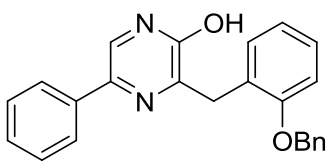

3-(2-(Benzyloxy)benzyl)-5-phenylpyrazin-2-ol 19 $\{1,17\}$ (RB32489-049-1): Obtained as a white powder (3.01 g, 90\%). ${ }^{1} \mathrm{H}$ NMR (DMSO- $\left.d_{6}\right): 12.41(\mathrm{~s}(\mathrm{br}), 1 \mathrm{H}), 7.89(\mathrm{~s}, 1 \mathrm{H}), 7.85(\mathrm{~m}, 2 \mathrm{H}), 7.43-7.26(\mathrm{~m}, 8 \mathrm{H}), 7.21(\mathrm{t}, 1 \mathrm{H}, J=7.9$ $\mathrm{Hz}), 7.01(\mathrm{~m}, 1 \mathrm{H}), 6.93(\mathrm{~m}, 1 \mathrm{H}), 6.85(\mathrm{~m}, 1 \mathrm{H}), 5.06(\mathrm{~s}, 2 \mathrm{H}), 4.05(\mathrm{~s}, 2 \mathrm{H}) .{ }^{13} \mathrm{C}$ NMR (DMSO- $\left.d_{6}\right): 158.8,157.2$, 155.4, 139.9, 137.6, 136.4, 131.5, 129.7, 129.1, 128.8, 128.2, 128.1, 127.7, 124.9, 123.0, 122.1, 116.2, 112.9, 69.6, 39.2. $\operatorname{HRMS}(\mathrm{m} / \mathrm{z})$ : $[\mathrm{M}+\mathrm{Na}]^{+}$calcd for $\mathrm{C}_{24} \mathrm{H}_{20} \mathrm{~N}_{2} \mathrm{O}_{2} \mathrm{Na}$ : 391.1422; found, 391.1422 .

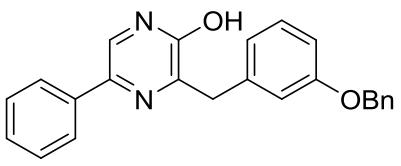

3-(2-(Benzyloxy)benzyl)-5-phenylpyrazin-2-ol 19 $\{1,18\}$ (RB32489-047-1): Obtained as a white powder (4.00 g, 94\%). ${ }^{1} \mathrm{H}$ NMR (DMSO- $\left.d_{6}\right): 12.36(\mathrm{~s}(\mathrm{br}), 1 \mathrm{H}), 7.86(\mathrm{~s}, 1 \mathrm{H}), 7.72(\mathrm{~m}, 2 \mathrm{H}), 7.37-7.18(\mathrm{~m}, 10 \mathrm{H}), 7.03(\mathrm{~m}, 1 \mathrm{H})$, $6.90(\mathrm{~m}, 1 \mathrm{H}), 5.10(\mathrm{~s}, 2 \mathrm{H}), 4.13(\mathrm{~s}, 2 \mathrm{H}) .{ }^{13} \mathrm{C}$ NMR (DMSO- $\left.d_{6}\right): 157.4,156.7,155.5,137.8,136.5,136.4,131.1$, 129.0, 128.7, 128.0, 127.9, 127.5, 127.4, 127.0, 124.8, 122.4, 120.8, 112.5, 69.6, 33.8. HRMS $(\mathrm{m} / \mathrm{z}):[\mathrm{M}+\mathrm{H}]^{+} \mathrm{calcd}$ for $\mathrm{C}_{24} \mathrm{H}_{21} \mathrm{~N}_{2} \mathrm{O}_{2}: 369.1603$; found, 369.1605 . 


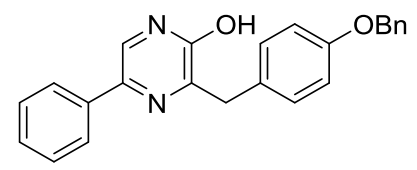

3-(4-(Benzyloxy)benzyl)-5-phenylpyrazin-2-ol 19\{1,19\} (YJ 31776-035-1): Obtained as a white powder (2.88 g, 93\%). ${ }^{1} \mathrm{H}$ NMR (DMSO-d $): 12.36(\mathrm{~s}(\mathrm{br}), 1 \mathrm{H}), 7.86(\mathrm{~s}, 1 \mathrm{H}), 7.84(\mathrm{~m}, 2 \mathrm{H}), 7.39(\mathrm{~m}, 6 \mathrm{H}), 7.28(\mathrm{~m}, 4 \mathrm{H}), 6.93(\mathrm{~m}$, 2H), 5.06 (s, 2H), $4.00(\mathrm{~s}, 2 \mathrm{H}) .{ }^{13} \mathrm{C}$ NMR (DMSO-d6): 157.7, 157.4, 155.4, 137.7, 136.5, 131.4, 130.6, 130.5, 129.1, 128.9, 128.2 , 128.0, 127.7, 124.9, 122.8, 115.1, 69.7, 31.1. HRMS (m/z): [M+Na] calcd for $\mathrm{C}_{24} \mathrm{H}_{20} \mathrm{~N}_{2} \mathrm{O}_{2} \mathrm{Na}^{+}$ 391.1422; found, 391.1421.

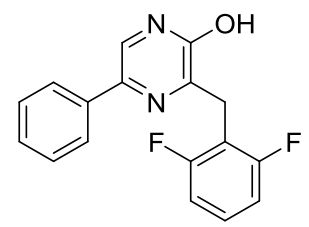

3-(2,6-Difluorobenzyl)-5-phenylpyrazin-2-ol 19\{1,21\} (EC31095-171-1): Obtained as a white powder (8.27 g, 90\%). ${ }^{1} \mathrm{H}$ NMR (DMSO-d6): $12.51(\mathrm{~s}, 1 \mathrm{H}), 7.90(\mathrm{~s}, 1 \mathrm{H}), 7.63(\mathrm{~m}, 2 \mathrm{H}), 7.40(\mathrm{~m}, 1 \mathrm{H}), 7.31(\mathrm{~m}, 2 \mathrm{H}), 7.17(\mathrm{~m}, 4 \mathrm{H})$, 4.14 (s, 2H). ${ }^{13} \mathrm{C}$ NMR (DMSO-d6): 161.7 (246, $\left.9 \mathrm{~Hz}\right), 155.2,154.8,136.3,131.0,129.3$ (10 Hz), 129.0, 127.7, 124.6, 122.8, $113.7(20 \mathrm{~Hz}), 111.5,26.4$. HRMS $(\mathrm{m} / \mathrm{z}):[\mathrm{M}+\mathrm{H}]^{+}$calcd for $\mathrm{C}_{17} \mathrm{H}_{13} \mathrm{~F}_{2} \mathrm{~N}_{2} \mathrm{O}$ : 299.0996; found, 299.1000 .

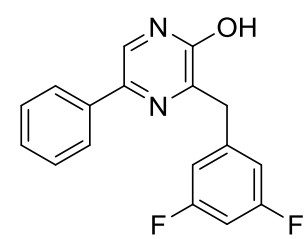

3-(3,5-difluorobenzyl)-5-phenylpyrazin-2-ol 19\{1,22\} (EC31095-169-1): Obtained as a white powder (3.91 g, 86\%). ${ }^{1} \mathrm{H}$ NMR (DMSO-d6): $12.50(\mathrm{~s}, 1 \mathrm{H}), 7.91(\mathrm{~s}, 1 \mathrm{H}), 7.81(\mathrm{~m}, 2 \mathrm{H}), 7.39(\mathrm{~m}, 2 \mathrm{H}), 7.28(\mathrm{~m}, 1 \mathrm{H}), 7.07(\mathrm{~m}, 3 \mathrm{H})$, 4.11 (s, 2H). ${ }^{13} \mathrm{C}$ NMR (DMSO-d6): 162.6 (245, $\left.13 \mathrm{~Hz}\right), 156.2,155.4,142.8$ (10 Hz), 136.3, 131.4, 129.1, 127.7, 124.9, 123.3, 112.8, $102.2(26 \mathrm{~Hz}), 38.8$. HRMS $(\mathrm{m} / \mathrm{z}):[\mathrm{M}+\mathrm{H}]^{+}$calcd for $\mathrm{C}_{17} \mathrm{H}_{13} \mathrm{~F}_{2} \mathrm{~N}_{2} \mathrm{O}$ : 299.0996; found, 299.1010.

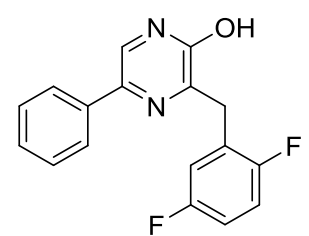

3-(2,5-difluorobenzyl)-5-phenylpyrazin-2-ol 19\{1,23\} (YJ31069-007-1): Obtained as a white powder (0.37 g, 83\%). ${ }^{1} \mathrm{H}$ NMR (DMSO-d $) 12.50(\mathrm{~s}(\mathrm{br}), 1 \mathrm{H}), 7.91(\mathrm{~s}, 1 \mathrm{H}), 7.72(\mathrm{~m}, 2 \mathrm{H}), 7.35(\mathrm{~m}, 2 \mathrm{H}), 7.24(\mathrm{~m}, 3 \mathrm{H}), 7.14(\mathrm{~m}$, $1 \mathrm{H}), 4.11(\mathrm{~s}, 2 \mathrm{H}) .{ }^{13} \mathrm{C}$ NMR (DMSO- $\left.d_{6}\right): 158.5$ (240 and $\left.2 \mathrm{~Hz}\right), 157.2$ (240 and $\left.2 \mathrm{~Hz}\right), 155.5,155.2,136.3,131.2$, 129.0, 127.7, 127.2 (8 Hz), $129.6(19$ and $9 \mathrm{~Hz}), 124.7,123.0,118.4$ (25 and $5 \mathrm{~Hz}), 116.7(25 \mathrm{and} 9 \mathrm{~Hz}), 115.1(24$ and $9 \mathrm{~Hz}), 32.6$. HRMS ( $\mathrm{m} / \mathrm{z})$ : $[\mathrm{M}+\mathrm{H}]^{+}$calcd for $\mathrm{C}_{17} \mathrm{H}_{13} \mathrm{~F}_{2} \mathrm{~N}_{2} \mathrm{O}: 299.0996$; found, 299.1001.

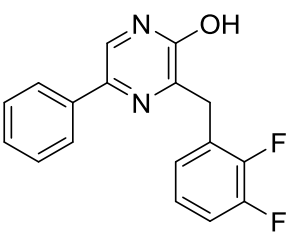

3-(2,3-difluorobenzyl)-5-phenylpyrazin-2-ol 19\{1,24\} (YJ31069-013-1): Obtained as a white powder (2.05 g, 85\%). ${ }^{1} \mathrm{H}$ NMR (DMSO-d6): $12.50(\mathrm{~s}, 1 \mathrm{H}), 7.91(\mathrm{~s}, 1 \mathrm{H}), 7.73(\mathrm{~m}, 2 \mathrm{H}), 7.24(\mathrm{~m}, 6 \mathrm{H}), 4.17(\mathrm{~s}, 2 \mathrm{H}) .{ }^{13} \mathrm{C} \mathrm{NMR}$ (DMSO-d $): 155.5,155.2,150.2(245,13 \mathrm{~Hz}), 148.9(245,13 \mathrm{~Hz}), 136.3,131.3,129.0,127.8(13 \mathrm{~Hz}), 127.7,127.2$ 
(3 Hz), 124.7 (2 signals), 123.0, 115.9 (17 Hz), 32.4. HRMS $(m / z):[\mathrm{M}+\mathrm{H}]^{+}$calcd for $\mathrm{C}_{17} \mathrm{H}_{13} \mathrm{~F}_{2} \mathrm{~N}_{2} \mathrm{O}: 299.0996$; found, 299.1006.

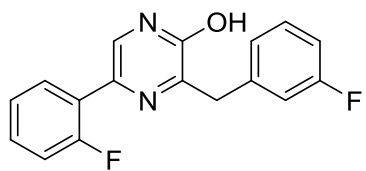

3-(3-Fluorobenzyl)-5-(2-fluorophenyl)pyrazin-2-ol 19\{2,3\} (YJ 31068-157-1): Obtained as a white powder (2.14 g, 90\%). ${ }^{1} \mathrm{H}$ NMR (DMSO-d 6$) 12.44(\mathrm{~s}(\mathrm{br}), 1 \mathrm{H}), 7.85(\mathrm{~m}, 1 \mathrm{H}), 7.43(\mathrm{~m}, 1 \mathrm{H}), 7.69(\mathrm{~s}, 1 \mathrm{H}), 7.34(\mathrm{~m}, 2 \mathrm{H}), 7.26(\mathrm{~m}$, 2H), $7.16(\mathrm{~m}, 1 \mathrm{H}), 7.04(\mathrm{~m}, 1 \mathrm{H}), 4.09(\mathrm{~s}, 2 \mathrm{H}) .{ }^{13} \mathrm{C}$ NMR (DMSO-d $): 162.5(240 \mathrm{~Hz}), 159.5(246 \mathrm{~Hz}), 157.5$, 155.0, 140.9 (8 Hz), 130.5 (8 Hz), 129.6 (8 Hz), 129.4 (3 Hz), 126.6, 126.5, 125.7 (3 Hz), 125.1 (3 Hz), 124.0 (13 $\mathrm{Hz}), 116.5(21 \mathrm{~Hz}), 116.3(21 \mathrm{~Hz}), 113.5(21 \mathrm{~Hz}), 38.9$. HRMS $(m / z):[\mathrm{M}+\mathrm{Na}]^{+}$calcd for $\mathrm{C}_{17} \mathrm{H}_{12} \mathrm{~F}_{2} \mathrm{~N}_{2} \mathrm{O}: 321.0815$; found, 321.0810 .

General procedure for the synthesis 2-chloropyrazines 20 via step vii. Under a calcium-protected atmosphere, the considered 2-hydroxypyrazine $19(0.02 \mathrm{~mol})$ was dispersed in phenylphosphonic dichloride $(10 \mathrm{~mL})$ and the suspension was heated at $100{ }^{\circ} \mathrm{C}$ for the indicated time. The resulting solution was diluted in ethyl acetate and poured onto an excess of crushed ice and stirred for 15 min (note 1). This was made basic with $22 \%$ ammonia and extracted with ethyl acetate. The organic layer was washed with water, brine, dried over magnesium sulfate and concentrated to dryness. The resulting residue was purified has described below. Note 1: stirring for a shorter time leads to the risk of generating a lot of an insoluble substance (probably $P$-phenylphosphonamidic acid) occurring upon the addition of ammonia on not yet fully hydrolyzed reactant.

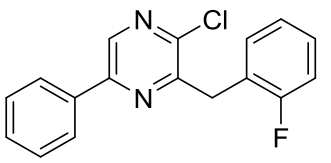

2-Chloro-3-(2-fluorobenzyl)-5-phenylpyrazine 20\{1,2\} (GG30532-114-3): Obtained as a white solid (3.01 g, 79\%) after heating for 10 hours and a chromatography over silica gel (cyclohexane - ethyl acetate 94/6). ${ }^{1} \mathrm{H}$ NMR $\left(\mathrm{CDCl}_{3}\right):{ }^{1} \mathrm{H} \mathrm{NMR}\left(\mathrm{CDCl}_{3}\right)$ : $8.70(\mathrm{~s}, 1 \mathrm{H}), 7.98-7.94(\mathrm{~m}, 2 \mathrm{H}), 7.53-7.45(\mathrm{~m}, 3 \mathrm{H}), 7.31-7.25(\mathrm{~m}, 2 \mathrm{H})$, 7.14-7.09 (m, 2H), $4.44(\mathrm{~s}, 2 \mathrm{H}) .{ }^{13} \mathrm{C} \mathrm{NMR}\left(\mathrm{CDCl}_{3}\right): 161.1(247 \mathrm{~Hz}), 152.5,150.2,146.8,138.6,135.2,131.1(4.2 \mathrm{~Hz}), 130.0$, 129.0, $128.6(8.2 \mathrm{~Hz}), 126.8,124.1(15.8 \mathrm{~Hz}), 124.0(3.4 \mathrm{~Hz}), 115.4(22.0 \mathrm{~Hz}), 34.2(3.0 \mathrm{~Hz})$. HRMS (m/z): $[\mathrm{M}+\mathrm{H}]^{+}$calcd for $\mathrm{C}_{17} \mathrm{H}_{13} \mathrm{ClFN}_{2}, 299.0751$; found, 299.0749.

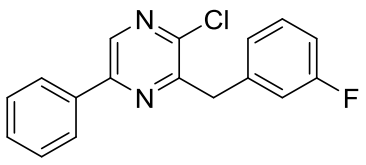

2-Chloro-3-(3-fluorobenzyl)-5-phenylpyrazine 20\{1,3\} (YJ31067-131-2): Obtained as a white solid (0.71 g, 38\% from 3-(3-fluorobenzyl)-5-phenylpiperazin-2-one) after heating for 12 hours and a chromatography over silica gel (cyclohexane - ethyl acetate 97/3). ${ }^{1} \mathrm{H}$ NMR $\left(\mathrm{CDCl}_{3}\right): 8.69(\mathrm{~s}, 1 \mathrm{H}), 8.03(\mathrm{~m}, 2 \mathrm{H}), 7.52(\mathrm{~m}, 3 \mathrm{H}), 7.29(\mathrm{~m}, 1 \mathrm{H}), 7.18$ $(\mathrm{m}, 1 \mathrm{H}), 7.12(\mathrm{~m}, 1 \mathrm{H}), 6.96(\mathrm{~m}, 1 \mathrm{H}), 4.39(\mathrm{~s}, 2 \mathrm{H}) .{ }^{13} \mathrm{C}$ NMR $\left(\mathrm{CDCl}_{3}\right): 162.9(243 \mathrm{~Hz}), 152.9,150.5,146.8,139.4$ (7Hz), 138.9, 135.2, 130.1, 129.9 (8 Hz), 129.1, 126.9, 124.8 (2 Hz), 116.1 (21 Hz), $113.7(21 \mathrm{~Hz}), 40.9(2 \mathrm{~Hz})$. HRMS (m/z): $[\mathrm{M}+\mathrm{H}]^{+}$calcd for $\mathrm{C}_{17} \mathrm{H}_{13} \mathrm{ClFN}_{2}$, 299.0751; found, 299.0749.

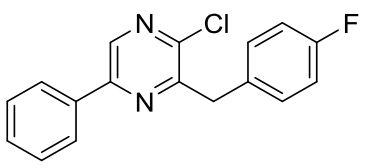

2-Chloro-3-(4-fluorobenzyl)-5-phenylpyrazine 20\{1,4\} (YJ31067-133-2): Obtained as a solid (1.2 g, 77\%) after heating for 10 hours and a chromatography over silica gel (cyclohexane - ethyl acetate 96/4). ${ }^{1} \mathrm{H} \mathrm{NMR}\left(\mathrm{CDCl}_{3}\right)$ : $8.68(\mathrm{~s}, 1 \mathrm{H}), 8.02(\mathrm{~m}, 2 \mathrm{H}), 7.52(\mathrm{~m}, 3 \mathrm{H}), 7.36(\mathrm{~m}, 2 \mathrm{H}), 7.02(\mathrm{~m}, 2 \mathrm{H}), 4.36(\mathrm{~s}, 2 \mathrm{H}) .{ }^{13} \mathrm{C} \mathrm{NMR}\left(\mathrm{CDCl}_{3}\right): 161.8(246$ 
$\mathrm{Hz}$ ), 153.4, 150.4, 146.7, 138.7, 135.2, 132.7 (3 Hz), 132.6 (8 Hz), 130.1, 129.1 (two signals), 126.8, 115.3 (21 $\mathrm{Hz})$, 40.4. HRMS $(\mathrm{m} / z)$ : $[\mathrm{M}+\mathrm{H}]^{+}$calcd for $\mathrm{C}_{17} \mathrm{H}_{13} \mathrm{ClFN}_{2}$, 299.0751; found, 299.0746.<smiles>Clc1ccccc1Cc1nc(-c2ccccc2)cnc1Cl</smiles>

2-Chloro-3-(2-chlorobenzyl)-5-phenylpyrazine 20\{1,5\} (EC31094-093-1): Obtained as a white solid (1.58 g, 73\%) after heating for 12 hours and a recrystallization in ethanol. ${ }^{1} \mathrm{H} \mathrm{NMR}\left(\mathrm{CDCl}_{3}\right)$ : $8.70(\mathrm{~s}, 1 \mathrm{H}), 7.93(\mathrm{~m}, 2 \mathrm{H}), 7.46(\mathrm{~m}$, 4H), $7.22(\mathrm{~m}, 3 \mathrm{H}), 4.52(\mathrm{~s}, 2 \mathrm{H}) .{ }^{13} \mathrm{C} \mathrm{NMR}\left(\mathrm{CDCl}_{3}\right)$ : 152.4, 150.2, 147.0, 138.5, 135.2, 134.6, 131.0, 130.0, 129.5, 129.0, 128.2, 126.8, 126.7, 38.6. (One signal missing). HRMS $(m / z)$ : $[\mathrm{M}+\mathrm{H}]^{+}$calcd for $\mathrm{C}_{17} \mathrm{H}_{13} \mathrm{Cl}_{2} \mathrm{~N}_{2}: 315.0456$; found, 315.0466.

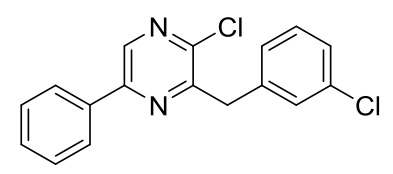

2-Chloro-3-(3-chlorobenzyl)-5-phenylpyrazine 20\{1,6\} (YJ 31776-007-1): Obtained as a solid (2.28 g, 85\%) after heating for 12 hours and a chromatography over silica gel (cyclohexane - ethyl acetate 97/3). ${ }^{1} \mathrm{H} \mathrm{NMR}\left(\mathrm{CDCl}_{3}\right)$ : $8.70(\mathrm{~s}, 1 \mathrm{H}), 8.02(\mathrm{~m}, 2 \mathrm{H}), 7.52(\mathrm{~m}, 3 \mathrm{H}), 7.40(\mathrm{~m}, 1 \mathrm{H}), 7.26(\mathrm{~m}, 3 \mathrm{H}), 4.37(\mathrm{~s}, 2 \mathrm{H}) .{ }^{13} \mathrm{C} \mathrm{NMR}\left(\mathrm{CDCl}_{3}\right): 152.8$, $150.5,146.8,139.0,138.9,135.2,134.3,130.1,129.7,129.3,129.1,127.4,127.0,126.9,40.8$. HRMS $(\mathrm{m} / \mathrm{z})$ : $[\mathrm{M}+\mathrm{H}]^{+}$calcd for $\mathrm{C}_{17} \mathrm{H}_{13} \mathrm{Cl}_{2} \mathrm{~N}_{2}$ : 315.0456; found, 315.0452 .

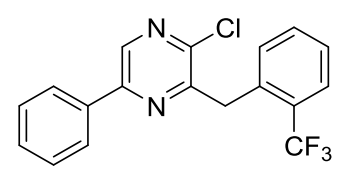

2-Chloro-5-phenyl-3-(2-(trifluoromethyl)benzyl)pyrazine 20\{1,8\} (YJ 33067-109-2): Obtained as a solid (3.81 g, $68 \%$ ) after heating for 12 hours and a chromatography over silica gel (cyclohexane-ethyl acetate 97/3). ${ }^{1} \mathrm{H}$ NMR $\left(\mathrm{CDCl}_{3}\right): 8.72(\mathrm{~s}, 1 \mathrm{H}), 7.92(\mathrm{~m}, 2 \mathrm{H}), 7.75(\mathrm{~m}, 1 \mathrm{H}), 7.51-7.44(\mathrm{~m}, 3 \mathrm{H}), 7.40(\mathrm{~m}, 1 \mathrm{H}), 7.17(\mathrm{~m}, 1 \mathrm{H}), 4.61(\mathrm{~s}, 2 \mathrm{H})$. ${ }^{13} \mathrm{C} \mathrm{NMR}\left(\mathrm{CDCl}_{3}\right): 152.5,150.1,146.9,138.6,135.6(2 \mathrm{~Hz}), 135.1,131.7,131.3129 .1(30 \mathrm{~Hz}), 129.0,126.8$, 126.7, $126.1(4 \mathrm{~Hz}), 124.6(273 \mathrm{~Hz}), 37.7$. HRMS $(\mathrm{m} / z):[\mathrm{M}+\mathrm{H}]^{+}$calcd for $\mathrm{C}_{18} \mathrm{H}_{13} \mathrm{ClF}_{3} \mathrm{~N}_{2}$ : 349.0719; found, 349.0725 .

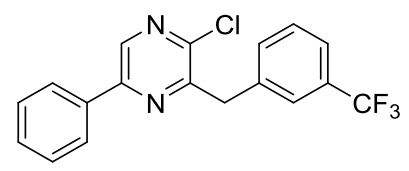

2-Chloro-5-phenyl-3-(3-(trifluoromethyl)benzyl)pyrazine 20\{1,9\} (YJ 33067-111-2): Obtained as a solid (1.83 g, 35\% from (YJ 33067-101-4) and (YJ 33067-101-3)) after heating for 12 hours and a chromatography over silica gel (cyclohexane-ethyl acetate 97/3). ${ }^{1} \mathrm{H}$ NMR $\left(\mathrm{CDCl}_{3}\right): 8.70(\mathrm{~s}, 1 \mathrm{H}), 8.01(\mathrm{~m}, 2 \mathrm{H}), 7.71(\mathrm{~s}, 1 \mathrm{H}), 7.58-7.45(\mathrm{~m}$, $6 \mathrm{H}), 4.45(\mathrm{~s}, 2 \mathrm{H}) .{ }^{13} \mathrm{C} \mathrm{NMR}\left(\mathrm{CDCl}_{3}\right): 152.6,150.5,146.7,138.9,137.8,135.1,132.6,130.9(30 \mathrm{~Hz}), 130.2 .0$, 129.1, 128.9, 128.8, $126.1(6 \mathrm{~Hz}), 124.2(273 \mathrm{~Hz}), 123.7(4 \mathrm{~Hz}), 40.8$. HRMS $(\mathrm{m} / z):[\mathrm{M}+\mathrm{H}]^{+}$calcd for $\mathrm{C}_{18} \mathrm{H}_{13} \mathrm{ClF}_{3} \mathrm{~N}_{2}$ : 349.0719; found, 349.0724 .

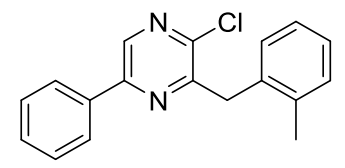

2-Chloro-3-(2-methylbenzyl)-5-phenylpyrazine 20\{1,11\} (YJ31070-187-2): This compound was obtained as a solid $(5.12 \mathrm{~g}, 89 \%)$ after heating for 12 hours and a chromatography over silica gel (cyclohexane-ethyl acetate 95/5). ${ }^{1} \mathrm{H}$ NMR $\left(\mathrm{CDCl}_{3}\right): 8.69(\mathrm{~s}, 1 \mathrm{H}), 7.98(\mathrm{~m}, 2 \mathrm{H}), 7.50(\mathrm{~m}, 3 \mathrm{H}), 7.20(\mathrm{~m}, 4 \mathrm{H}), 4.39(\mathrm{~s}, 2 \mathrm{H}), 2.46(\mathrm{~s}, 3 \mathrm{H}) .{ }^{13} \mathrm{C}$ NMR $\left(\mathrm{CDCl}_{3}\right)$ : 153.5, 150.2, 147.0, 138.4, 136.8, 135.7, 135.3, 130.3, 130.0, 129.8, 129.5, 129.0, 126.9, 126.8, 125.9, 38.4, 20.0. HRMS (m/z): $[\mathrm{M}+\mathrm{H}]^{+}$calcd for $\mathrm{C}_{18} \mathrm{H}_{16} \mathrm{ClN}_{2}, 295.1002$; found, 295.1009 . 


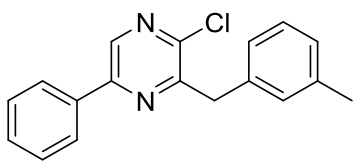

2-Chloro-3-(3-methylbenzyl)-5-phenylpyrazine 20 \{1,12\} (YJ31070-189-2): Obtained as a solid (5.03 g, 90\%) after heating for 12 hours and a chromatography over silica gel (cyclohexane-ethyl acetate $96 / 4) .{ }^{1} \mathrm{H}$ NMR $\left(\mathrm{CDCl}_{3}\right)$ : $8.68(\mathrm{~s}, 1 \mathrm{H}), 8.05(\mathrm{~m}, 2 \mathrm{H}), 7.51(\mathrm{~m}, 3 \mathrm{H}), 7.22(\mathrm{~m}, 3 \mathrm{H}), 7.08(\mathrm{~m}, 1 \mathrm{H}), 4.37(\mathrm{~s}, 2 \mathrm{H}), 2.36(\mathrm{~s}, 3 \mathrm{H}),{ }^{13} \mathrm{C} \mathrm{NMR}\left(\mathrm{CDCl}_{3}\right)$ : 153.7, 150.4, 146.9, 138.6, 138.2, 137.0, 135.4, 130.0, 129.9, 129.0 (two signals), 127.5, 126.9 (two signals), 126.2, 41.2, 21.4. HRMS (m/z): [M+H] ${ }^{+}$calcd for $\mathrm{C}_{18} \mathrm{H}_{16} \mathrm{ClN}_{2}$, 295.1002; found, 295.0997.

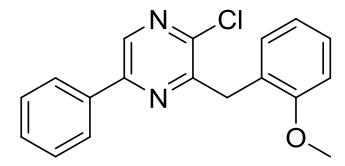

2-Chloro-3-(2-methoxybenzyl)-5-phenylpyrazine 20 $\{1,14\}$ (YJ31070-109-1): Obtained as a white solid (0.73 g, $63 \%)$ after heating for 13 hours and a recrystallization in ethanol. ${ }^{1} \mathrm{H}$ NMR $\left(\mathrm{CDCl}_{3}\right):{ }^{1} \mathrm{H} \mathrm{NMR}\left(\mathrm{CDCl}_{3}\right): 8.66(\mathrm{~s}$, $1 \mathrm{H}), 7.96(\mathrm{~m}, 2 \mathrm{H}), 7.48(\mathrm{~m}, 3 \mathrm{H}), 7.27(\mathrm{~m}, 1 \mathrm{H}), 7.14(\mathrm{~m}, 1 \mathrm{H}), 6.93(\mathrm{~m}, 2 \mathrm{H}), 4.40(\mathrm{~s}, 2 \mathrm{H}), 3.83(\mathrm{~s}, 2 \mathrm{H}) .{ }^{13} \mathrm{C}$ NMR $\left(\mathrm{CDCl}_{3}\right): 157.5,153.8,150.0,147.1,138.1,135.5,130.2,129.8,129.0,128.0,126.8,126.0,120.4,110.4,55.4$, 35.4. HRMS $(\mathrm{m} / z):[\mathrm{M}+\mathrm{H}]^{+}$calcd for $\mathrm{C}_{18} \mathrm{H}_{16} \mathrm{ClN}_{2} \mathrm{O}, 311.0951$; found, 311.0949 .

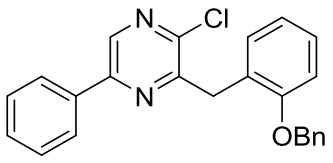

3-(2-(Benzyloxy)benzyl)-2-chloro-5-phenylpyrazine 20 $\{1,17\}$ (RB32489-053-2): Obtained as a light yellow powder $(2.67 \mathrm{~g}, 88 \%)$ after heating for 18 hours and a chromatography over silica gel (cyclohexane - ethyl acetate 96/4). ${ }^{1} \mathrm{H}$ NMR $\left(\mathrm{CDCl}_{3}\right): 8.68(\mathrm{~s}, 1 \mathrm{H}), 8.04(\mathrm{~m}, 2 \mathrm{H}), 7.53(\mathrm{~m}, 3 \mathrm{H}), 7.45-7.23(\mathrm{~m}, 6 \mathrm{H}), 7.00(\mathrm{~m}, 2 \mathrm{H}), 6.89(\mathrm{~m}, 1 \mathrm{H})$, 5.07 (s, 2H), 4.37 (s, 2H). ${ }^{13} \mathrm{C}$ NMR $\left(\mathrm{CDCl}_{3}\right): 158.9,153.4,150.4,146.9,138.7,138.6,137.0,135.3,130.0,129.5$, 129.1, 128.5, 127.9, 127.5, 126.9, 121.9, 116.0, 113.1, 70.1, 41.2. HRMS $(m / z):[\mathrm{M}+\mathrm{H}]^{+}$calcd for $\mathrm{C}_{24} \mathrm{H}_{20} \mathrm{ClN}_{2} \mathrm{O}$ 387.1264; found, 387.1270.

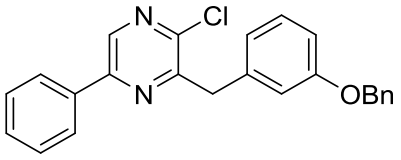

3-(3-(Benzyloxy)benzyl)-2-chloro-5-phenylpyrazine 20\{1,18\} (RB32489-051-2): Obtained as a light yellow powder $(3.67 \mathrm{~g}, 90 \%)$ after heating for 18 hours and a chromatography over silica gel (cyclohexane - ethyl acetate 96/4). ${ }^{1} \mathrm{H}$ NMR $\left(\mathrm{CDCl}_{3}\right): 8.65(\mathrm{~s}, 1 \mathrm{H}), 7.93(\mathrm{~m}, 2 \mathrm{H}), 7.47(\mathrm{~m}, 3 \mathrm{H}), 7.34-7.17(\mathrm{~m}, 7 \mathrm{H}), 6.97(\mathrm{~m}, 2 \mathrm{H}), 5.10(\mathrm{~s}, 2 \mathrm{H})$, 4.47 (s, 2H). ${ }^{13} \mathrm{C}$ NMR $\left(\mathrm{CDCl}_{3}\right): 156.6,153.8,150.0,147.1,138.1,137.1,135.4,130.6,129.8,128.9,128.4,128.0$, 127.7, 127.1, 126.8, 126.3, 120.7, 111.8, 70.1, 37.7. HRMS $(\mathrm{m} / \mathrm{z}):[\mathrm{M}+\mathrm{H}]^{+}$calcd for $\mathrm{C}_{24} \mathrm{H}_{20} \mathrm{ClN}_{2} \mathrm{O} 387.1264$; found, 387.1252 .

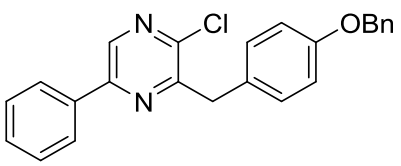

3-(4-(Benzyloxy)benzyl)-2-chloro-5-phenylpyrazine 20\{1,19\} (YJ31776-037-2): Obtained as a yellow powder $(2.00 \mathrm{~g}, 69 \%)$ after heating for 12 hours and a chromatography over silica gel (cyclohexane - ethyl acetate 96/4). ${ }^{1} \mathrm{H}$ NMR $\left(\mathrm{CDCl}_{3}\right): 8.67(\mathrm{~s}, 1 \mathrm{H}), 8.03(\mathrm{~m}, 2 \mathrm{H}), 7.53(\mathrm{~m}, 3 \mathrm{H}), 7.41(\mathrm{~m}, 4 \mathrm{H}), 7.33(\mathrm{~m}, 3 \mathrm{H}), 6.95(\mathrm{~m}, 2 \mathrm{H}), 5.07(\mathrm{~s}$, $2 \mathrm{H}), 4.34(\mathrm{~s}, 2 \mathrm{H}) .{ }^{13} \mathrm{C} \mathrm{NMR}\left(\mathrm{CDCl}_{3}\right): 157.8,153.9,150.4,146.8,138.5,137.1,135.4,130.2,130.0,129.4,129.1$, 128.6, 127.9, 127.5, 126.9, 114.9, 70.1, 40.4. HRMS $(\mathrm{m} / z):[\mathrm{M}+\mathrm{H}]^{+}$calcd for $\mathrm{C}_{24} \mathrm{H}_{20} \mathrm{ClN}_{2} \mathrm{O}$ 387.1264; found, 387.1247. 


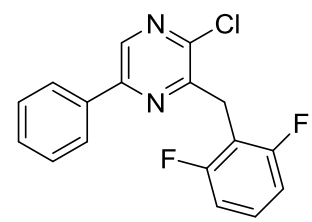

2-Chloro-3-(2,6-difluorobenzyl)-5-phenylpyrazine 20\{1,21\} (EC31095-047-1): Obtained as a solid (7.5 g, 90\%) after a heating for 12 hours and a chromatography over silica gel (cyclohexane-ethyl acetate 98:2). ${ }^{1} \mathrm{H}$ NMR $\left(\mathrm{CDCl}_{3}\right): 8.67(\mathrm{~m}, 1 \mathrm{H}), 7.85(\mathrm{~m}, 2 \mathrm{H}), 7.43(\mathrm{~m}, 3 \mathrm{H}), 7.28(\mathrm{~m}, 1 \mathrm{H}), 6.95(\mathrm{~m}, 2 \mathrm{H}), 4.43(\mathrm{~s}, 2 \mathrm{H}) .{ }^{13} \mathrm{C} \mathrm{NMR}\left(\mathrm{CDCl}_{3}\right)$ : $161.8(248,8 \mathrm{~Hz}), 151.2,150.0,146.4,138.3,135.2,129.9,128.9,128.6(10 \mathrm{~Hz}), 126.7,113.0(20 \mathrm{~Hz}), 111.0$, 28.1. HRMS $(\mathrm{m} / z):[\mathrm{M}+\mathrm{H}]^{+}$calcd for $\mathrm{C}_{17} \mathrm{H}_{12} \mathrm{ClF}_{2} \mathrm{~N}_{2}: 317.0657$; found, 317.0650 .

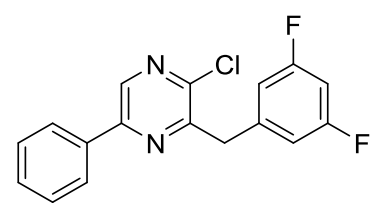

2-Chloro-3-(3,5-difluorobenzyl)-5-phenylpyrazine 20\{1,22\} (EC31095-049-1): Obtained as a white solid (3.5 g, 90\%) after heating for 12 hours and a chromatography over silica gel (cyclohexane-ethyl acetate 98:2). ${ }^{1} \mathrm{H}$ NMR $\left(\mathrm{CDCl}_{3}\right): 8.72(\mathrm{~s}, 1 \mathrm{H}), 8.02(\mathrm{~m}, 2 \mathrm{H}), 7.52(\mathrm{~m}, 3 \mathrm{H}), 6.91(\mathrm{~m}, 2 \mathrm{H}), 6.2(\mathrm{tt}, 1 \mathrm{H}, J=9.0,2.3 \mathrm{~Hz}), 4.36(\mathrm{~s}, 2 \mathrm{H}) .{ }^{13} \mathrm{C}$ NMR $\left(\mathrm{CDCl}_{3}\right): 163.0(\mathrm{dd}, J=248,13 \mathrm{~Hz}), 152.2,150.6,146.8,140.7(\mathrm{t}, J=9 \mathrm{~Hz}), 139.1,135.0,130.2,129.1$, 126.9, 112.1, $102.4(\mathrm{t}, J=25 \mathrm{~Hz}), 40.8$. $\operatorname{HRMS}(\mathrm{m} / \mathrm{z})$ : $[\mathrm{M}+\mathrm{H}]^{+}$calcd for $\mathrm{C}_{17} \mathrm{H}_{12} \mathrm{ClF}_{2} \mathrm{~N}_{2}$ : 317.0657; found, 317.0651 .

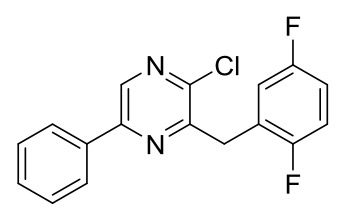

2-Chloro-3-(2,5-difluorobenzyl)-5-phenylpyrazine 20\{1,23\} (EC31095-049-1): Obtained as a white solid (1.16 g, $69 \%$ ) after heating for 12 hours and a chromatography over silica gel (cyclohexane-ethyl acetate 97:3). ${ }^{1} \mathrm{H}$ NMR $\left(\mathrm{CDCl}_{3}\right): 8.72(\mathrm{~s}, 1 \mathrm{H}), 7.96(\mathrm{~m}, 2 \mathrm{H}), 7.50(\mathrm{~m}, 3 \mathrm{H}), 7.07(\mathrm{~m}, 1 \mathrm{H}), 6.97(\mathrm{~m}, 2 \mathrm{H}), 4.40(\mathrm{~s}, 2 \mathrm{H}) .{ }^{13} \mathrm{C} \mathrm{NMR}\left(\mathrm{CDCl}_{3}\right)$ : 158.5 (dd, $J=242,2 \mathrm{~Hz}), 157.1(\mathrm{dd}, J=242,2 \mathrm{~Hz}), 151.7,150.4,146.7,138.9,135.1,130.1,129.1,126.8,125.7$ (dd, $J=19,8 \mathrm{~Hz}), 117.5(\mathrm{dd}, J=24,4 \mathrm{~Hz}), 116.2(\mathrm{dd}, J=25,9 \mathrm{~Hz}), 114.9(\mathrm{dd}, J=24,9 \mathrm{~Hz}), 34.2$. HRMS $(\mathrm{m} / z)$ : $[\mathrm{M}+\mathrm{H}]^{+}$calcd for $\mathrm{C}_{17} \mathrm{H}_{12} \mathrm{ClF}_{2} \mathrm{~N}_{2}: 317.0657$; found, 317.0658

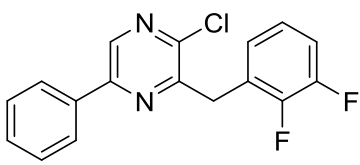

2-Chloro-3-(2,3-difluorobenzyl)-5-phenylpyrazine 20\{1,24\} (GG30533-126-1): Obtained as a white solid (1.91 g, $80 \%$ ) after a heating for 12 hours and a chromatography over silica gel (cyclohexane-ethyl acetate 97:3). ${ }^{1} \mathrm{H}$ NMR $\left(\mathrm{CDCl}_{3}\right): 8.71(\mathrm{~s}, 1 \mathrm{H}), 7.96(\mathrm{~m}, 2 \mathrm{H}), 7.50(\mathrm{~m}, 3 \mathrm{H}), 7.07(\mathrm{~m}, 3 \mathrm{H}), 4.45(\mathrm{~s}, 2 \mathrm{H}) .{ }^{13} \mathrm{C} \mathrm{NMR}\left(\mathrm{CDCl}_{3}\right): 151.8,150.7(\mathrm{dd}$, $J=248,13 \mathrm{~Hz}), 150.3,149.2(\mathrm{dd}, J=248,13 \mathrm{~Hz}), 146.7,138.8,135.1,130.1,129.1,126.8,126.6(\mathrm{~d}, J=13 \mathrm{~Hz})$, $125.8(\mathrm{t}, J=3 \mathrm{~Hz}), 123.8(\mathrm{dd}, J=7,5 \mathrm{~Hz}), 115.9(\mathrm{~d}, J=17 \mathrm{~Hz}), 34.1$. HRMS $(m / z):[\mathrm{M}+\mathrm{H}]^{+}$calcd for $\mathrm{C}_{17} \mathrm{H}_{12} \mathrm{ClF}_{2} \mathrm{~N}_{2}$ : 317.0657; found, 317.0651 .

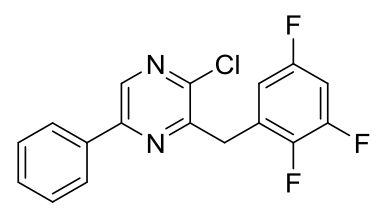

2-Chloro-5-phenyl-3-(2,3,5-trifluorobenzyl)pyrazine 20\{1,26\} (YJ 33067-113-2): Obtained as a white solid (1.51 $\mathrm{g}, 72 \%$ ) after a heating for 12 hours and a chromatography over silica gel (cyclohexane-ethyl acetate 97:3). ${ }^{1} \mathrm{H}$ NMR $\left(\mathrm{CDCl}_{3}\right): 8.73(\mathrm{~s}, 1 \mathrm{H}), 7.96(\mathrm{~m}, 2 \mathrm{H}), 7.50(\mathrm{~m}, 3 \mathrm{H}), 6.88(\mathrm{~m}, 1 \mathrm{H}), 6.79(\mathrm{~m}, 1 \mathrm{H}), 4.42(\mathrm{~s}, 2 \mathrm{H}) .{ }^{13} \mathrm{C}$ NMR 
$\left(\mathrm{CDCl}_{3}\right): 157.5(242,12$ and $3 \mathrm{~Hz}), 151.1,150.5,150.3(247,13$ and $15 \mathrm{~Hz}), 146.6,145.8(242,12$ and $4 \mathrm{~Hz})$, 139.1, 134.9, 130.2, 129.1, 127.3 (9 and $14 \mathrm{~Hz}), 126.8,112.2$ (3 and $24 \mathrm{~Hz}$ ), 104.2 (21 and $28 \mathrm{~Hz}$ ), 34.0. HRMS $(\mathrm{m} / \mathrm{z}):[\mathrm{M}+\mathrm{H}]^{+}$calcd for $\mathrm{C}_{17} \mathrm{H}_{11} \mathrm{ClF}_{3} \mathrm{~N}_{2}$ : 335.0563; found, 335.0556.

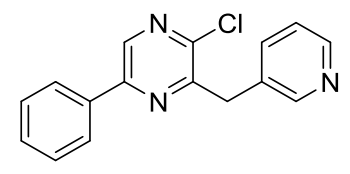

2-Chloro-5-phenyl-3-(pyridin-3-ylmethyl)pyrazine 20\{1,35\} (YJ30367-031-2): Obtained as an oil (0.42 g, 83\%) after heating for 10 hours and a chromatography over silica gel (dichloromethane - ethanol 98/2). ${ }^{1} \mathrm{H} \mathrm{NMR}$ $\left(\mathrm{CDCl}_{3}\right): 8.70(\mathrm{~m}, 1 \mathrm{H}), 8.69(\mathrm{~s}, 1 \mathrm{H}), 8.52(\mathrm{dd}, 1 \mathrm{H}, J=1.6,4.9 \mathrm{~Hz}), 7.99(\mathrm{~m}, 2 \mathrm{H}), 7.71(\mathrm{~m}, 1 \mathrm{H}), 7.50(\mathrm{~m}, 3 \mathrm{H}), 7.26$ $(\mathrm{ddd}, 1 \mathrm{H}, J=0.8,4.9,8.0 \mathrm{~Hz}), 4.38(\mathrm{~s}, 2 \mathrm{H}) .{ }^{13} \mathrm{C} \mathrm{NMR}\left(\mathrm{CDCl}_{3}\right): 152.5,150.6,150.5,148.3,146.6,139.0,136.6$, 135.0, 132.6, 130.2, 129.1, 126.8, 123.4, 38.4. HRMS $(\mathrm{m} / \mathrm{z}):[\mathrm{M}+\mathrm{H}]^{+}$calcd for $\mathrm{C}_{16} \mathrm{H}_{13} \mathrm{ClN}_{3}, 282.0798$; found, 282.0795 .<smiles>Cc1nc(-c2ccccc2)cnc1Cl</smiles>

2-Chloro-3-methyl-5-phenylpyrazine 20\{1,60\} (VHE30612-137-2): Obtained as a yellow solid (1.18 g, 74\%) after heating only at $80{ }^{\circ} \mathrm{C}$ for 12 hours and a chromatography over silica gel (cyclohexane - dichloromethane 1/1). ${ }^{1} \mathrm{H}$ NMR $\left(\mathrm{CDCl}_{3}\right): 8.63(\mathrm{~s}, 1 \mathrm{H}), 8.01(\mathrm{~m}, 2 \mathrm{H}), 7.50(\mathrm{~m}, 3 \mathrm{H}), 2.75(\mathrm{~s}, 3 \mathrm{H}) .{ }^{13} \mathrm{C} \mathrm{NMR}\left(\mathrm{CDCl}_{3}\right): 152.2,150.4,147.1$, 138.2, 135.5, 129.9, 129.0, 126.9, 22.4. HRMS $(\mathrm{m} / \mathrm{z}):[\mathrm{M}+\mathrm{H}]^{+}$calcd for $\mathrm{C}_{11} \mathrm{H}_{10} \mathrm{ClN}_{2}, 205.0533$; found, 205.0464.

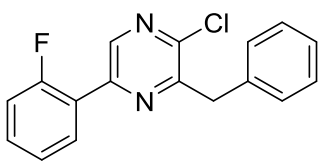

3-Benzyl-2-chloro-5-(2-fluorophenyl)pyrazine 20\{2,1\} (YJ31134-031-2): Obtained as a yellow solid (3.30 g, 67\%) after heating for 16 hours and a chromatography over silica gel (cyclohexane-dichloromethane 2/1). ${ }^{1} \mathrm{H}$ NMR $\left(\mathrm{CDCl}_{3}\right): 8.78(\mathrm{~d}, 1 \mathrm{H}, J=2.2 \mathrm{~Hz}), 8.04(\mathrm{dt}, 1 \mathrm{H}, J=7.6,1.8 \mathrm{~Hz}), 7.30(\mathrm{~m}, 8 \mathrm{H}), 4.40(\mathrm{~s}, 2 \mathrm{H}) .{ }^{13} \mathrm{C} \mathrm{NMR}\left(\mathrm{CDCl}_{3}\right)$ : $160.6(250 \mathrm{~Hz}), 153.9,147.1,146.3(3 \mathrm{~Hz}), 142.1(13 \mathrm{~Hz}), 137.0,131.6(9 \mathrm{~Hz}), 130.9(3 \mathrm{~Hz}), 129.2,128.5,126.8$, $124.8(3 \mathrm{~Hz}), 123.3(12 \mathrm{~Hz}), 111.4(22 \mathrm{~Hz}), 41.2$. HRMS $(\mathrm{m} / z):[\mathrm{M}+\mathrm{H}]^{+}$calcd for $\mathrm{C}_{17} \mathrm{H}_{13} \mathrm{ClFN}_{2}, 299.0751$; found, 299.0818.

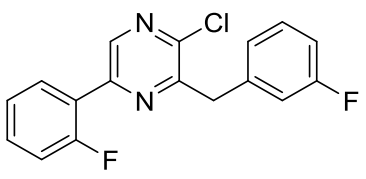

2-Chloro-3-(3-fluorobenzyl)-5-(2-fluorophenyl)pyrazine 20\{2,3\} (YJ 31068-167-2): Obtained as a white solid $(1.86 \mathrm{~g}, 84 \%)$ after heating for 14 hours and a chromatography over silica gel (cyclohexane-ethyl acetate 99/1 to 98.5/1.5). ${ }^{1} \mathrm{H}$ NMR $\left(\mathrm{CDCl}_{3}\right) 8.80(\mathrm{~d}, 1 \mathrm{H}, J=2.3 \mathrm{~Hz}), 8.04(\mathrm{~m}, 1 \mathrm{H}), 7.47(\mathrm{~m}, 1 \mathrm{H}), 7.30(\mathrm{~m}, 2 \mathrm{H}), 7.22(\mathrm{~m}, 1 \mathrm{H}), 7.16$ $(\mathrm{m}, 1 \mathrm{H}), 7.10(\mathrm{~m}, 1 \mathrm{H}), 6.96(\mathrm{~m}, 1 \mathrm{H}), 4.39(\mathrm{~s}, 2 \mathrm{H}) .{ }^{13} \mathrm{C} \mathrm{NMR}\left(\mathrm{CDCl}_{3}\right): 162.9(246 \mathrm{~Hz}), 160.7(250 \mathrm{~Hz}), 153.2$, $147.0(8 \mathrm{~Hz}), 142.3(8 \mathrm{~Hz}), 139.3(3 \mathrm{~Hz}), 131.6(3 \mathrm{~Hz}), 130.9,130.8,129.9(3 \mathrm{~Hz}), 124.9124 .8,123.2(11 \mathrm{~Hz})$, $116.4(21 \mathrm{~Hz}), 116.1(21 \mathrm{~Hz}), 113.7(21 \mathrm{~Hz}), 40.8$. HRMS $(m / z):[\mathrm{M}+\mathrm{H}]^{+}$calcd for $\mathrm{C}_{17} \mathrm{H}_{12} \mathrm{ClF}_{2} \mathrm{~N}_{2}, 317.0657$; found, 317.0653 .

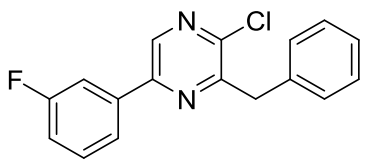

3-Benzyl-2-chloro-5-(3-fluorophenyl)pyrazine 20\{3,1\} (YJ30367-115-2): Obtained as a solid after heating for 12 hours (0.98 g, 8\% from 1-fluoro-3-(2-nitrovinyl)benzene). ${ }^{1} \mathrm{H}$ NMR $\left(\mathrm{CDCl}_{3}\right): 8.66(\mathrm{~s}, 1 \mathrm{H}), 7.78(\mathrm{~m}, 2 \mathrm{H}), 7.48(\mathrm{~m}$, $1 \mathrm{H}), 7.41-7.24(\mathrm{~m}, 4 \mathrm{H}), 7.18(\mathrm{~m}, 1 \mathrm{H}), 4.40(\mathrm{~s}, 2 \mathrm{H}) .{ }^{13} \mathrm{C} \mathrm{NMR}\left(\mathrm{CDCl}_{3}\right): 163.4(248 \mathrm{~Hz}), 153.9,149.0(2 \mathrm{~Hz}), 147.5$, 
138.5, 137.6, 137.5 136.9, 130.6 (9 Hz), 129.2, 128.6, 126.8, $122.3(3 \mathrm{~Hz}), 116.9(21 \mathrm{~Hz}), 113.9(23 \mathrm{~Hz}), 41.2$. HRMS (m/z): [M+H] $]^{+}$calcd for $\mathrm{C}_{17} \mathrm{H}_{13} \mathrm{ClFN}_{2}, 299.0751$; found, 299.0749.

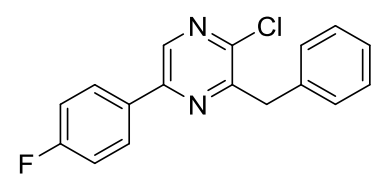

3-Benzyl-2-chloro-5-(4-fluorophenyl)pyrazine 20\{4,1\} (YJ30531-117-1): Obtained as a white solid (0.84 g, 52\%) after heating for 14 hours and a chromatography over silica gel (cyclohexane-dichloromethane $3 / 1$ ). ${ }^{1} \mathrm{H}$ NMR $\left(\mathrm{CDCl}_{3}\right): 8.63(\mathrm{~s}, 1 \mathrm{H}), 8.02(\mathrm{~m}, 2 \mathrm{H}), 7.39(\mathrm{~m}, 2 \mathrm{H}), 7.34(\mathrm{~m}, 2 \mathrm{H}), 7.28(\mathrm{~m}, 1 \mathrm{H}), 7.19(\mathrm{~m}, 2 \mathrm{H}), 4.39(\mathrm{~s}, 2 \mathrm{H}) .{ }^{13} \mathrm{C}$ NMR $\left(\mathrm{CDCl}_{3}\right): 164.1(250 \mathrm{~Hz}), 153.7,149.4,146.8,138.2137 .0,131.4(3 \mathrm{~Hz}), 129.2,128.9,128.8(8 \mathrm{~Hz}), 126.8$, $116.2(22 \mathrm{~Hz}), 41.2$. HRMS ( $/ z)$ : $[\mathrm{M}+\mathrm{H}]^{+}$calcd for $\mathrm{C}_{17} \mathrm{H}_{13} \mathrm{ClFN}_{2}, 299.0751$; found, 299.0763.

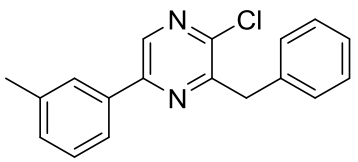

3-Benzyl-2-chloro-5-(m-tolyl)pyrazine 20\{5,1\} (YJ30367-065-2): This compound was obtained as a white solid (3.02 g, 33\% from 1-methyl-3-(2-nitrovinyl)benzene) after heating for 18 hours and a chromatography over silica gel (cyclohexane-ethyl acetate 96.5/3.5). ${ }^{1} \mathrm{H} \mathrm{NMR}\left(\mathrm{CDCl}_{3}\right): 8.66(\mathrm{~s}, 1 \mathrm{H}), 7.84(\mathrm{~m}, 1 \mathrm{H}), 7.82(\mathrm{~m}, 2 \mathrm{H}), 7.43-7.24$ $(\mathrm{m}, 6 \mathrm{H}), 4.40(\mathrm{~s}, 2 \mathrm{H}), 2.48(\mathrm{~s}, 3 \mathrm{H}) .{ }^{13} \mathrm{C} \mathrm{NMR}\left(\mathrm{CDCl}_{3}\right): 153.5,150.6,146.8,140.3,138.8,138.7,137.1,135.3$, 130.8, 129.2, 129.0, 128.5, 127.5, 126.7, 124.0, 41.2, 21.5. HRMS $(m / z):[\mathrm{M}+\mathrm{H}]^{+}$calcd for $\mathrm{C}_{18} \mathrm{H}_{16} \mathrm{ClN}_{2}, 295.1002$; found, 295.1014.

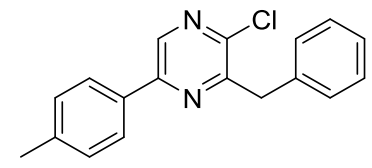

3-Benzyl-2-chloro-5-(p-tolyl)pyrazine 20\{6,1\} (YJ30367-035-2): Obtained as a yellow solid (1.83 g, 22\% from 1methyl-4-(2-nitrovinyl)benzene) after heating for 12 hours and a chromatography over silica gel (cyclohexanedichloromethane 3/2). ${ }^{1} \mathrm{H}$ NMR $\left(\mathrm{CDCl}_{3}\right): 8.64(\mathrm{~s}, 1 \mathrm{H}), 7.94(\mathrm{~m}, 2 \mathrm{H}), 7.48-7.23(\mathrm{~m}, 7 \mathrm{H}), 4.39(\mathrm{~s}, 2 \mathrm{H}), 2.45(\mathrm{~s}$, $3 \mathrm{H}) .{ }^{13} \mathrm{C}$ NMR $\left(\mathrm{CDCl}_{3}\right): 153.5,150.4,146.4,140.3,138.3,137.1,132.6,129.8,129.2, .128 .5,126.8,126.7,123.0$, 41.2, 21.3. HRMS (m/z): $[\mathrm{M}+\mathrm{H}]^{+}$calcd for $\mathrm{C}_{18} \mathrm{H}_{16} \mathrm{ClN}_{2}, 295.1002$; found, 295.0999.

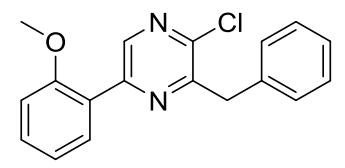

3-Benzyl-2-chloro-5-(2-methoxyphenyl)pyrazine 20\{7,1\} (EC31092-035-2): Obtained as a yellow solid (1.82 g, $81 \%$ ) after heating for 18 hours and a chromatography over silica gel (cyclohexane-dichloromethane $3 / 2) .{ }^{1} \mathrm{H} \mathrm{NMR}$ $\left(\mathrm{CDCl}_{3}\right): 8.86(\mathrm{~s}, 1 \mathrm{H}), 7.91(\mathrm{dd}, 1 \mathrm{H}, J=7.6,1.8 \mathrm{~Hz}), 7.48-7.36(\mathrm{~m}, 3 \mathrm{H}), 7.34-7.28(\mathrm{~m}, 2 \mathrm{H}), 7.26-7.18(\mathrm{~m}$, $1 \mathrm{H}), 7.11(\mathrm{td}, 1 \mathrm{H}, J=7.6,0.8 \mathrm{~Hz}), 7.03(\mathrm{~d}, 1 \mathrm{H}, J=8.3 \mathrm{~Hz}), 4.37(\mathrm{~s}, 2 \mathrm{H}), 3.90(\mathrm{~s}, 3 \mathrm{H}) .{ }^{13} \mathrm{C} \mathrm{NMR}\left(\mathrm{CDCl}_{3}\right): 157.4$, 153.4, 149.4, 146.2, 143.3, 137.5, 131.3 (two signals), 129.3, 128.6, 126.8, 124.9, 121.4, 111.6, 55.7, 41.4. HRMS $(\mathrm{m} / \mathrm{z}):[\mathrm{M}+\mathrm{H}]^{+}$calcd for $\mathrm{C}_{18} \mathrm{H}_{16} \mathrm{ClN}_{2} \mathrm{O}, 311.0951$; found, 311.0965 .

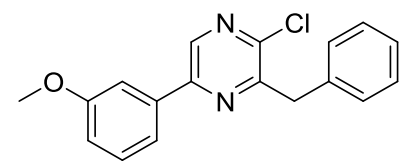

3-Benzyl-2-chloro-5-(3-methoxyphenyl)pyrazine 20\{8,1\} (YJ30367-083-2): This compound was obtained as a white powder $(0.4 \mathrm{~g}, 32 \%$ from piperazinone $17\{8,1\})$, after a chromatography over silica gel (cyclohexane-ethyl acetate 95/5). ${ }^{1} \mathrm{H}$ NMR $\left(\mathrm{CDCl}_{3}\right): 8.66(\mathrm{~s}, 1 \mathrm{H}), 7.97(\mathrm{~m}, 2 \mathrm{H}), 7.58(\mathrm{~m}, 2 \mathrm{H}), 7.41(\mathrm{~m}, 3 \mathrm{H}), 7.33(\mathrm{~m}, 2 \mathrm{H}), 7.25(\mathrm{~m}$, $1 \mathrm{H}), 7.03(\mathrm{~m}, 1 \mathrm{H}), 4.39$ (s, 2H), $3.90(\mathrm{~s}, 3 \mathrm{H}) .{ }^{13} \mathrm{C} \mathrm{NMR}\left(\mathrm{CDCl}_{3}\right): 160.2,153.5,150.1,146.9,138.6,137.1,136.7$, 
130.1, 129.2, 128.5, 126.7, 119.1, 115.8, 112.3, 55.4, 41.2. HRMS $(m / z):[\mathrm{M}+\mathrm{H}]^{+}$calcd for $\mathrm{C}_{18} \mathrm{H}_{16} \mathrm{ClN}_{2} \mathrm{O}$, 311.0951; found, 311.0962.

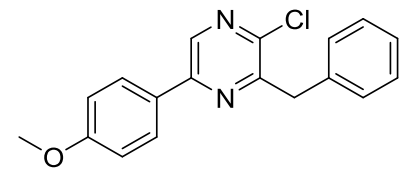

3-Benzyl-2-chloro-5-(4-methoxyphenyl)pyrazine 20\{9,1\} (YJ29793-127-2): Obtained as a yellow solid (0.33 g, $35 \%$ ) after heating for 12 hours and a chromatography over silica gel (cyclohexane-dichloromethane $3 / 2)$. ${ }^{1} \mathrm{H}$ NMR $\left(\mathrm{CDCl}_{3}\right): 8.61(\mathrm{~s}, 1 \mathrm{H}), 7.97(\mathrm{~m}, 2 \mathrm{H}), 7.40(\mathrm{~m}, 2 \mathrm{H}), 7.31(\mathrm{~m}, 2 \mathrm{H}), 7.27(\mathrm{~m}, 1 \mathrm{H}), 7.02(\mathrm{~m}, 2 \mathrm{H}), 4.37(\mathrm{~s}, 2 \mathrm{H}), 3.89(\mathrm{~s}$, $3 \mathrm{H}) .{ }^{13} \mathrm{C} \mathrm{NMR}\left(\mathrm{CDCl}_{3}\right): 161.3,153.3,150.2,145.8,137.9,137.2,129.2,128.5,128.3,127.9,127.7,114.5,55.4$, 41.2. HRMS $(\mathrm{m} / \mathrm{z})$ : $[\mathrm{M}+\mathrm{H}]^{+}$calcd for $\mathrm{C}_{18} \mathrm{H}_{16} \mathrm{ClN}_{2} \mathrm{O}, 311.0951$; found, 311.0955 .

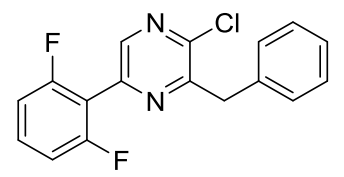

3-Benzyl-2-chloro-5-(2,6-difluorophenyl)pyrazine 20\{10,1\} (MM34284-021-2): Obtained as a white solid (0.75 g, $56 \%$ ) after heating for 12 hours and a chromatography over silica gel (cyclohexane-dichloromethane 3/1). ${ }^{1} \mathrm{H} \mathrm{NMR}$ $\left(\mathrm{CDCl}_{3}\right): 8.44(\mathrm{~m}, 1 \mathrm{H}), 7.43(\mathrm{~m}, 3 \mathrm{H}), 7.27(\mathrm{~m}, 2 \mathrm{H}), 7.25(\mathrm{~m}, 1 \mathrm{H}), 7.05(\mathrm{~m}, 2 \mathrm{H}), 4.40(\mathrm{~s}, 2 \mathrm{H}) .{ }^{13} \mathrm{C} \mathrm{NMR}\left(\mathrm{CDCl}_{3}\right)$ : $160.6(6,252 \mathrm{~Hz}), 154.3,148.0,143.2(5 \mathrm{~Hz}), 143.1,136.6,131.3(10 \mathrm{~Hz}), 129.2,128.5,126.8,114.1(18 \mathrm{~Hz})$, $112.1(6,19 \mathrm{~Hz}), 41.3$. HRMS $(\mathrm{m} / z)$ : $[\mathrm{M}+\mathrm{H}]^{+}$calcd for $\mathrm{C}_{17} \mathrm{H}_{12} \mathrm{ClF}_{2} \mathrm{~N}_{2}, 317.0657$; found, 317.0648.

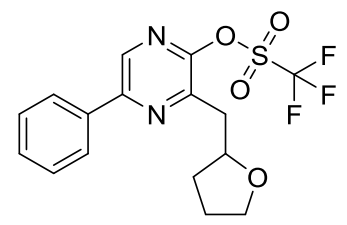

Synthesis, via step viii, of 5-phenyl-3-((tetrahydrofuran-2-yl)methyl)pyrazin-2-yl trifluoromethanesulfonate $21\{1,38\}$ (YJ31134-135-2): Compound 19 $\{1,38\}(0.56 \mathrm{~g}, 2.18 \mathrm{mmol})$ was dissolved in dry dichloromethane (30 $\mathrm{mL}$, stabilized by amylene, dried over $4 \AA$ molecular sieve). Triethylamine $(0.31 \mathrm{~mL}, 2.29 \mathrm{mmol})$ and triflic anhydride $(0.37 \mathrm{~mL}, 2.22 \mathrm{mmol})$ were then added. The resulting solution was stirred for 40 minutes, diluted in ethyl acetate, washed with water, brine, dried over magnesium sulfate and concentrated to dryness. The residue was purified by a chromatography over silica gel (cyclohexane-dichloromethane 1/3) to yield compound $\mathbf{2 1}\{1,38\}$ as an oil $(0.37 \mathrm{~g}, 43 \%) .{ }^{1} \mathrm{H}$ NMR $\left(\mathrm{CDCl}_{3}\right): 8.60(\mathrm{~s}, 1 \mathrm{H}), 8.01(\mathrm{~m}, 2 \mathrm{H}), 7.53(\mathrm{~m}, 3 \mathrm{H}), 4.51(\mathrm{~m}, 1 \mathrm{H}), 3.95(\mathrm{~m}, 1 \mathrm{H})$, $3.77(\mathrm{~m}, 1 \mathrm{H}), 3.27(\mathrm{dd}, 1 \mathrm{H}, J=7.8,14.1 \mathrm{~Hz}), 3.10(\mathrm{dd}, 1 \mathrm{H}, J=5.4,14.1 \mathrm{~Hz}), 2.17(\mathrm{~m}, 1 \mathrm{H}), 1.96(\mathrm{~m}, 2 \mathrm{H}), 1.76(\mathrm{~m}$, 1H). ${ }^{13} \mathrm{C} \mathrm{NMR}\left(\mathrm{CDCl}_{3}\right): 153.3,151.1,146.5,136.7,135.0,130.3,129.1,127.1,118.6(321 \mathrm{~Hz}), 77.3,67.9,38.2$, 26.9, 25.6. HRMS $(\mathrm{m} / z)$ : $[\mathrm{M}+\mathrm{H}]^{+}$calcd for $\mathrm{C}_{16} \mathrm{H}_{16} \mathrm{~F}_{3} \mathrm{~N}_{2} \mathrm{O}_{4} \mathrm{~S}, 389.0783$; found, 389.0808.

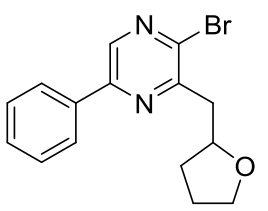

Synthesis, via step ix, of 2-bromo-5-phenyl-3-((tetrahydrofuran-2-yl)methyl)pyrazine 22\{1,38\} (YJ31134-145-4): In a tube featuring a Teflon-coated screw cap, compound $\mathbf{2 1}\{1,38\}(0.37 \mathrm{~g}, 0.95 \mathrm{mmol})$ and dry sodium bromide $(0.24 \mathrm{~g}, 2.38 \mathrm{mmol}$, flame-dried under vacuum) were dispersed in dry dimethylformamide $(4 \mathrm{~mL})$. Triflic acid $(0.07 \mathrm{~mL}, 0.86 \mathrm{mmol})$ was added, the tube was closed and heated at $120{ }^{\circ} \mathrm{C}$ overnight. The resulting solution was diluted in diluted in ethyl acetate, washed with water, brine, dried over magnesium sulfate and concentrated to dryness. The residue was purified by a chromatography over silica gel (cyclohexane-dichloromethane 1/4 to dichloromethane - ethanol 99/1) to yield compound $22\{1,38\}$ as an oil $(0.19 \mathrm{~g}, 62 \%) .{ }^{1} \mathrm{H} \mathrm{NMR}\left(\mathrm{CDCl}_{3}\right): 8.62(\mathrm{~s}$, $1 \mathrm{H}), 8.02(\mathrm{~m}, 2 \mathrm{H}), 7.50(\mathrm{~m}, 3 \mathrm{H}), 4.57(\mathrm{p}, 1 \mathrm{H}, J=6.7 \mathrm{~Hz}), 3.99(\mathrm{~m}, 1 \mathrm{H}), 3.80(\mathrm{~m}, 1 \mathrm{H}), 3.42(\mathrm{dd}, 1 \mathrm{H}, J=6.7,14.6$ $\mathrm{Hz}), 3.14(\mathrm{dd}, 1 \mathrm{H}, J=6.7,14.6 \mathrm{~Hz}), 2.12(\mathrm{~m}, 1 \mathrm{H}), 1.99(\mathrm{~m}, 2 \mathrm{H}), 1.76(\mathrm{~m}, 1 \mathrm{H}) .{ }^{13} \mathrm{C} \mathrm{NMR}\left(\mathrm{CDCl}_{3}\right): 154.3,150.5$, 
140.2, 138.9, 135.4, 130.0, 129.0, 126.9, 77.4, 67.9, 42.1, 31.2, 25.6. HRMS $(m / z):[\mathrm{M}+\mathrm{H}]^{+}$calcd for $\mathrm{C}_{15} \mathrm{H}_{16} \mathrm{BrN}_{2} \mathrm{O}$, 319.0446; found, 319.0464.

General procedure for the $\mathrm{N}$-arylation of $\alpha$-amino esters 14 by 2 -halogenopyrazine 20 or 22 , preparation of compounds 23 via steps x. In a $20 \mathrm{~mL}$ sealable vial (note 1), the considered 2-halogenopyrazine 20 or 22 (2.0 $\mathrm{mmol})$, the considered amino ester $14(2.05 \mathrm{mmol}$, either as a free base or as a hydrochloride salt), cesium carbonate $(1.98 \mathrm{~g} 6.04 \mathrm{mmol})$, palladium acetate $(0.022 \mathrm{~g}, 0.1 \mathrm{mmol})$ and 1,1'-binaphthalene-2,2'diyl)bis(diphenylphosphine) (BINAP) $(0.087 \mathrm{~g}, 0.14 \mathrm{~mol})$ were weighted. The air was replaced by argon and, under an inert atmosphere, dry acetonitrile $\left(8 \mathrm{~mL}\right.$ ) was injected (note 2). This was heated and stirred at $60{ }^{\circ} \mathrm{C}$ for 12 hours (note 3 ). The resulting dark red or black suspension was dispersed in ethyl acetate, filtered, rinsed with ethyl acetate and the filtrate was concentrated to dryness and the residue (note 4) subjected to further purification by a chromatography over silica gel as described below (note 5). Note 1: on larger scale, the use of standard glassware and rubber septum worked as fine. Note 2: as specified in some cases below, dry dimethylformamide at $60{ }^{\circ} \mathrm{C}$ or dry toluene at $90{ }^{\circ} \mathrm{C}$ were also used. Note 3: the occurrence of clumps of cesium hydrogen carbonate was observed to be detrimental to the reaction. A good stirring or an ultra sound bath (with a temperature regulation) were equally effective. Note 4: ${ }^{1} \mathrm{H}$ NMR spectra of this residue most often pointed out a complete or a welladvanced conversion. In some still unexplained (and fairly random) runs, no reaction occurred. However, starting the procedure described above again with this residue and fresh amount of cesium carbonate, palladium acetate and BINAP in the same solvent used often saved the day. Note 5: absorption of the residue on a small amount of silica gel prior to a chromatography was preferentially made under vacuum at $20{ }^{\circ} \mathrm{C}$.

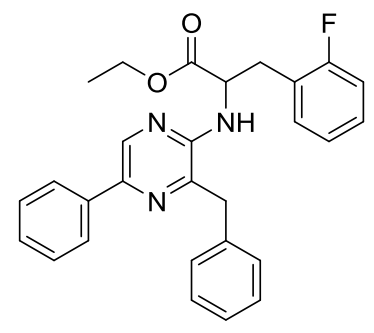

Ethyl 2-((3-benzyl-5-phenylpyrazin-2-yl)amino)-3-(2-fluorophenyl)propanoate $\quad 23\{1,1,2\} \quad$ (EC31093-155-2): Obtained as an oil $(0.32 \mathrm{~g}, 79 \%)$ after a chromatography over silica gel (cyclohexane-ethyl acetate 95:5). ${ }^{1} \mathrm{H}$ NMR $\left(\mathrm{CDCl}_{3}\right): 8.42(\mathrm{~s}, 1 \mathrm{H}), 7.96(\mathrm{~m}, 2 \mathrm{H}), 7.47(\mathrm{~m}, 2 \mathrm{H}), 7.37(\mathrm{~m}, 1 \mathrm{H}), 7.27(\mathrm{~m}, 6 \mathrm{H}), 6.98(\mathrm{~m}, 3 \mathrm{H}), 4.99(\mathrm{~m}, 2 \mathrm{H}), 4.15$ $(\mathrm{m}, 4 \mathrm{H}), 3.22(\mathrm{dd}, 1 \mathrm{H}, J=13.9,5.5 \mathrm{~Hz}), 3.16(\mathrm{dd}, 1 \mathrm{H}, J=13.9,6.3 \mathrm{~Hz}), 1.20(\mathrm{t}, 3 \mathrm{H}, J=7.1 \mathrm{~Hz}) .{ }^{13} \mathrm{C} \mathrm{NMR}$ $\left(\mathrm{CDCl}_{3}\right): 172.4,161.3(246 \mathrm{~Hz}), 150.4,141.2,141.1,137.5,136.7(25 \mathrm{~Hz}), 131.5(5 \mathrm{~Hz}), 128.8,128.7,128.6$, 128.6 (8 Hz), 127.8, 126.9, 125.7, 124.1, 124.0, 123.5 (16 Hz), 115.2 (22 Hz), 61.3, 54.3, 40.8, 31.2, 14.0. HRMS $(\mathrm{m} / \mathrm{z}):[\mathrm{M}+\mathrm{H}]^{+}$calcd for $\mathrm{C}_{28} \mathrm{H}_{27} \mathrm{FN}_{3} \mathrm{O}_{2}, 456.2087$; found, 456.2084.

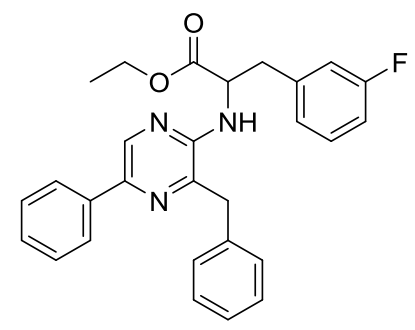

Ethyl 2-((3-benzyl-5-phenylpyrazin-2-yl)amino)-3-(3-fluorophenyl)propanoate $\quad \mathbf{2 3}\{1,1,3\} \quad$ (YJ30367-011-2): Obtained as an oil $(0.25 \mathrm{~g}, 64 \%)$ after a chromatography over silica gel (cyclohexane-ethyl acetate 95:5). ${ }^{1} \mathrm{H}$ NMR $\left(\mathrm{CDCl}_{3}\right): 8.44(\mathrm{~s}, 1 \mathrm{H}), 7.97(\mathrm{~m}, 2 \mathrm{H}), 7.48(\mathrm{~m}, 2 \mathrm{H}), 7.38(\mathrm{~m}, 1 \mathrm{H}), 7.32-7.23(\mathrm{~m}, 5 \mathrm{H}), 7.17(\mathrm{~m}, 1 \mathrm{H}), 6.92(\mathrm{~m}, 1 \mathrm{H})$, $6.73(\mathrm{~m}, 1 \mathrm{H}), 4.98(\mathrm{~m}, 2 \mathrm{H}), 4.16(\mathrm{~m}, 4 \mathrm{H}), 3.18(\mathrm{dd}, 1 \mathrm{H}, J=5.5,13.8 \mathrm{~Hz}), 3.03(\mathrm{dd}, 1 \mathrm{H}, J=5.1,13.8 \mathrm{~Hz}), 1.21(\mathrm{t}$, $3 \mathrm{H}, J=7.1 \mathrm{~Hz}) .{ }^{13} \mathrm{C} \mathrm{NMR}\left(\mathrm{CDCl}_{3}\right): 172.4,162.0(242 \mathrm{~Hz}), 150.2,141.2(5 \mathrm{~Hz}), 138.8(8 \mathrm{~Hz}), 137.4,136.8,136.4$, 129.8 (8 Hz), 128.9, 128.8, 128.6, 127.8, 127.0, 125.6, 124.9, 124.8, $116.2(21 \mathrm{~Hz}), 113.7$ (20 Hz), 61.3, 54.7, 41.0, 37.6, 14.1. HRMS $(\mathrm{m} / \mathrm{z})$ : $[\mathrm{M}+\mathrm{H}]^{+}$calcd for $\mathrm{C}_{28} \mathrm{H}_{27} \mathrm{FN}_{3} \mathrm{O}_{2}$, 456.2087; found, 456.2065. 


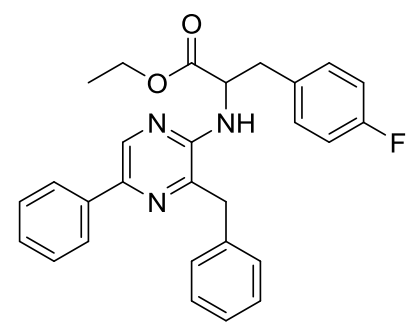

Ethyl 2-((3-benzyl-5-phenylpyrazin-2-yl)amino)-3-(4-fluorophenyl)propanoate $\quad \mathbf{2 3}\{1,1,4\} \quad$ (EC31092-091-2): Obtained as an oil $(0.45 \mathrm{~g}, 69 \%)$ after a chromatography over silica gel (cyclohexane-ethyl acetate 95:5). ${ }^{1} \mathrm{H}$ NMR $\left(\mathrm{CDCl}_{3}\right): 8.41(\mathrm{~s}, 1 \mathrm{H}), 7.99-7.91(\mathrm{~m}, 2 \mathrm{H}), 7.51-7.41(\mathrm{~m}, 2 \mathrm{H}), 7.40-7.32(\mathrm{~m}, 1 \mathrm{H}), 7.31-7.25(\mathrm{~m}, 3 \mathrm{H}), 7.23-$ $7.17(\mathrm{~m}, 2 \mathrm{H}), 6.91-6.79(\mathrm{~m}, 4 \mathrm{H}), 4.99-4.84(\mathrm{~m}, 2 \mathrm{H}), 4.21-4.06(\mathrm{~m}, 4 \mathrm{H}), 3.15(\mathrm{dd}, 1 \mathrm{H}, J=13.9,5.1 \mathrm{~Hz}), 3.03$ $(\mathrm{dd}, 1 \mathrm{H}, J=13.9,5.5 \mathrm{~Hz}), 1.20(\mathrm{t}, 3 \mathrm{H}, J=7.1 \mathrm{~Hz}) .{ }^{13} \mathrm{C} \mathrm{NMR}\left(\mathrm{CDCl}_{3}\right): 172.4,162.0(245 \mathrm{~Hz}), 150.4,141.3,141.3$, 137.6, 137.0, 136.6, $132.1(3 \mathrm{~Hz}), 130.8$ (8 Hz), 129.0, 128.9, 128.7, 128.0, 127.1, 125.8, $115.4(21 \mathrm{~Hz}), 61.4,55.0$, 41.1, 37.0, 14.3. HRMS ( $\mathrm{m} / \mathrm{z})$ : $[\mathrm{M}+\mathrm{H}]^{+}$calcd for $\mathrm{C}_{28} \mathrm{H}_{27} \mathrm{FN}_{3} \mathrm{O}_{2}$, 456.2087; found, 456.2110.

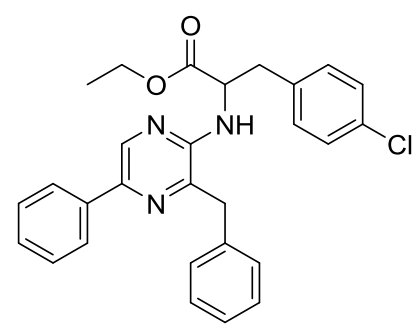

Ethyl 2-((3-benzyl-5-phenylpyrazin-2-yl)amino)-3-(4-chlorophenyl)propanoate $\quad \mathbf{2 3}\{1,1,7\} \quad$ (CF34391-030-1): Obtained as an oil (0.38 g, 64\%) after a chromatography over silica gel (cyclohexane-ethyl acetate 97:3 to 96:4). ${ }^{1} \mathrm{H}$ NMR $\left(\mathrm{CDCl}_{3}\right): 8.43(\mathrm{~s}, 1 \mathrm{H}), 7.97(\mathrm{~m}, 2 \mathrm{H}), 7.48(\mathrm{~m}, 2 \mathrm{H}), 7.37(\mathrm{~m}, 1 \mathrm{H}), 7.30(\mathrm{~m}, 3 \mathrm{H}) 7.22(\mathrm{~m}, 2 \mathrm{H}), 7.14(\mathrm{~m}, 2 \mathrm{H})$ $6.85(\mathrm{~m}, 2 \mathrm{H}), 4.95(\mathrm{~m}, 2 \mathrm{H}), 4.15(\mathrm{~m}, 4 \mathrm{H}), 3.16(\mathrm{~m}, 1 \mathrm{H}), 3.03(\mathrm{~m}, 1 \mathrm{H}), 1.23(\mathrm{t}, 3 \mathrm{H}, J=7.2 \mathrm{~Hz},) .{ }^{13} \mathrm{C}$ NMR $\left(\mathrm{CDCl}_{3}\right)$ : $172.1,150.2$, 141.2, 141.1, 137.4, 136.8, 136.4, 134.7, 132.7, 130.5, 128.9, 128.8, 128.6, 128.5, 127.9, 126.9, 125.7, 61.3, 54.6, 40.9, 37.0, 14.1. HRMS (m/z): $[\mathrm{M}+\mathrm{H}]^{+}$calcd for $\mathrm{C}_{28} \mathrm{H}_{27} \mathrm{ClN}_{3} \mathrm{O}_{2}, 472.1792$; found, 472.1782 .

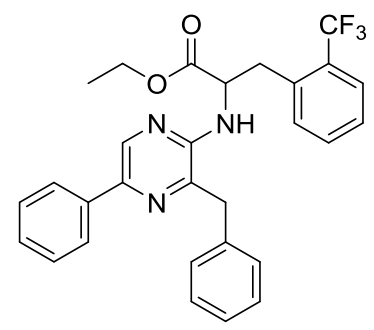

Ethyl 2-((3-benzyl-5-phenylpyrazin-2-yl)amino)-3-(2-(trifluoromethyl)phenyl)propanoate $\mathbf{2 3}\{1,1,8\}$ (YJ30531105-2): Obtained as an oil (0.08 g, 18\%) after a chromatography over silica gel (cyclohexane - ethyl acetate 95/5). ${ }^{1} \mathrm{H}$ NMR $\left(\mathrm{CDCl}_{3}\right): 8.36(\mathrm{~s}, 1 \mathrm{H}), 7.93(\mathrm{~m}, 2 \mathrm{H}), 7.62(\mathrm{~m}, 1 \mathrm{H}), 7.45(\mathrm{~m}, 2 \mathrm{H}), 7.37$ - $7.18(\mathrm{~m}, 9 \mathrm{H}), 5.01$ - $4.93(\mathrm{~m}$, 2H), $4.17(\mathrm{~s}, 2 \mathrm{H}), 4.12(\mathrm{~m}, 2 \mathrm{H}), 3.31(\mathrm{~m}, 1 \mathrm{H}), 3.15(\mathrm{~m}, 1 \mathrm{H}), 1.17(\mathrm{~m}, 3 \mathrm{H}) .{ }^{13} \mathrm{C} \mathrm{NMR}\left(\mathrm{CDCl}_{3}\right): 172.4,150.4,141.3$, 141.0, 137.4, 136.8, 136.6, 135.6, 131.6, 131.2, 128.9 (30 Hz), 128.8, 128.7, 128.6, 127.8, 126.9, 126.7, 125.9 (6 $\mathrm{Hz}), 125.6,124.5(275 \mathrm{~Hz}), 61.2,54.8,40.8,34.7,13.9$. HRMS $(\mathrm{m} / \mathrm{z}):[\mathrm{M}+\mathrm{H}]^{+}$calcd for $\mathrm{C}_{29} \mathrm{H}_{27} \mathrm{~F}_{3} \mathrm{~N}_{3} \mathrm{O}_{2}, 506.2055$; found, 506.2053.<smiles>CCOC(=O)C(Cc1cccc(C(F)(F)F)c1)Nc1ncc(-c2ccccc2)nc1Cc1ccccc1</smiles> 
Ethyl 2-((3-benzyl-5-phenylpyrazin-2-yl)amino)-3-(3-(trifluoromethyl)phenyl)propanoate 23\{1,1,9\} (YJ30531107-2): Obtained as an oil $(0.14 \mathrm{~g}, 31 \%)$ after a chromatography over silica gel (cyclohexane - ethyl acetate $95 / 5)$. ${ }^{1} \mathrm{H}$ NMR $\left(\mathrm{CDCl}_{3}\right): 8.44(\mathrm{~s}, 1 \mathrm{H}), 7.99(\mathrm{~m}, 2 \mathrm{H}), 7.62(\mathrm{~m}, 1 \mathrm{H}), 7.49(\mathrm{~m}, 3 \mathrm{H}), 7.40-7.26(\mathrm{~m}, 8 \mathrm{H}), 7.10(\mathrm{~m}, 1 \mathrm{H}), 5.02$ $(\mathrm{m}, 2 \mathrm{H}), 4.17(\mathrm{~s}, 2 \mathrm{H}), 4.13(\mathrm{~m}, 2 \mathrm{H}), 3.25(\mathrm{~m}, 1 \mathrm{H}), 3.14(\mathrm{~m}, 1 \mathrm{H}), 1.19(\mathrm{~m}, 3 \mathrm{H}) .{ }^{13} \mathrm{C} \mathrm{NMR}\left(\mathrm{CDCl}_{3}\right): 171.9,150.1$, 141.3, 141.2, 137.5, 137.4, 136.8, 136.5, 132.7, 130.6 (32 Hz), 128.9, 128.8 (two signals), 128.6, 127.9, 127.0, $126.1(6 \mathrm{~Hz}), 125.7,124.1(275 \mathrm{~Hz}), 123.6(6 \mathrm{~Hz}), 61.4,54.7,41.0,37.7,14.0$. HRMS $(\mathrm{m} / \mathrm{z}):[\mathrm{M}+\mathrm{H}]^{+}$calcd for $\mathrm{C}_{29} \mathrm{H}_{27} \mathrm{~F}_{3} \mathrm{~N}_{3} \mathrm{O}_{2}, 506.2055$; found, 506.2063.

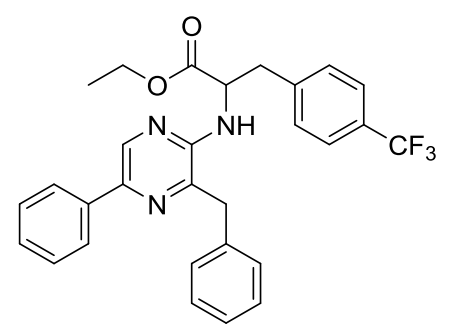

Ethyl 2-((3-benzyl-5-phenylpyrazin-2-yl)amino)-3-(4-(trifluoromethyl)phenyl)propanoate 23 $\{1,1,10\}$ (YJ30531109-2): Obtained as an oil $(0.12 \mathrm{~g}, 26 \%)$ after a chromatography over silica gel (cyclohexane - ethyl acetate 95/5). ${ }^{1} \mathrm{H} \mathrm{NMR}\left(\mathrm{CDCl}_{3}\right): 8.44(\mathrm{~s}, 1 \mathrm{H}), 7.98(\mathrm{~m}, 2 \mathrm{H}), 7.48(\mathrm{~m}, 2 \mathrm{H}), 7.40(\mathrm{~m}, 3 \mathrm{H}), 7.29-7.26(\mathrm{~m}, 5 \mathrm{H}), 7.03(\mathrm{~m}, 2 \mathrm{H}), 5.02$ $(\mathrm{m}, 2 \mathrm{H}), 4.19(\mathrm{~m}, 2 \mathrm{H}), 4.14(\mathrm{~s}, 2 \mathrm{H}), 3.26(\mathrm{dd}, 1 \mathrm{H}, J=5.6,13.6 \mathrm{~Hz}), 3.14(\mathrm{dd}, 1 \mathrm{H}, J=5.7,13.6 \mathrm{~Hz}), 1.22(\mathrm{t}, 3 \mathrm{H}, J$ $=7.0 \mathrm{~Hz}) .{ }^{13} \mathrm{C} \mathrm{NMR}\left(\mathrm{CDCl}_{3}\right): 171.9,150.1,141.3,141.2,137.4,136.9,136.4,129.5,129.2,128.9,128.9(32 \mathrm{~Hz})$, 128.8, 128.5, 127.9, 127.0, $126.9(275 \mathrm{~Hz}), 125.7,125.2(4 \mathrm{~Hz}), 61.4,54.6,41.0,37.4,14.1 . \mathrm{HRMS}(\mathrm{m} / \mathrm{z}):[\mathrm{M}+\mathrm{H}]^{+}$ calcd for $\mathrm{C}_{29} \mathrm{H}_{27} \mathrm{~F}_{3} \mathrm{~N}_{3} \mathrm{O}_{2}, 506.2055$; found, 506.2002.

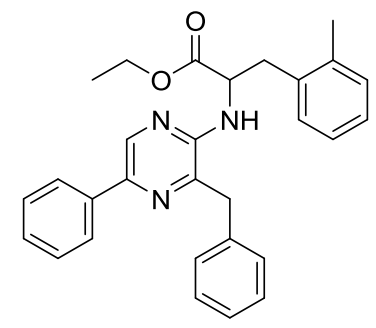

Ethyl 2-((3-benzyl-5-phenylpyrazin-2-yl)amino)-3-(o-tolyl)propanoate 23\{1,1,11\} (YJ30367-157-2): Obtained as an oil $(0.48 \mathrm{~g}, 90 \%)$ after a chromatography over silica gel (cyclohexane-ethyl acetate $95: 5) .{ }^{1} \mathrm{H} \mathrm{NMR}\left(\mathrm{CDCl}_{3}\right)$ : $8.40(\mathrm{~s}, 1 \mathrm{H}), 7.94(\mathrm{~m}, 2 \mathrm{H}), 7.48-7.42(\mathrm{~m}, 2 \mathrm{H}), 7.38-7.24(\mathrm{~m}, 6 \mathrm{H}), 7.15(\mathrm{~m}, 2 \mathrm{H}), 7.06(\mathrm{~m}, 1 \mathrm{H}), 6.93(\mathrm{~m}, 1 \mathrm{H})$, $4.92(\mathrm{~m}, 2 \mathrm{H}), 4.16(\mathrm{~m}, 4 \mathrm{H}), 3.14(\mathrm{dd}, 1 \mathrm{H}, J=6.1,14.0 \mathrm{~Hz}), 3.02(\mathrm{dd}, 1 \mathrm{H}, J=7.1,14.0 \mathrm{~Hz}), 2.29(\mathrm{~s}, 3 \mathrm{H}), 1.16(\mathrm{t}$, $3 \mathrm{H}, J=7.1 \mathrm{~Hz}) .{ }^{13} \mathrm{C} \mathrm{NMR}\left(\mathrm{CDCl}_{3}\right): 172.9,150.5,141.2,141.1,137.5,136.8,1367,136.5,134.7,130.5,129.8$, 128.9, 128.7, 128.6, 127.8, 126.9, 125.9, 125.7 (two signals), 61.1, 54.3, 40.9, 35.7, 19.4, 14.0. HRMS (m/z): $[\mathrm{M}+\mathrm{H}]^{+}$calcd for $\mathrm{C}_{29} \mathrm{H}_{29} \mathrm{~N}_{3} \mathrm{O}_{2}, 452.2338$; found, 452.2352 .

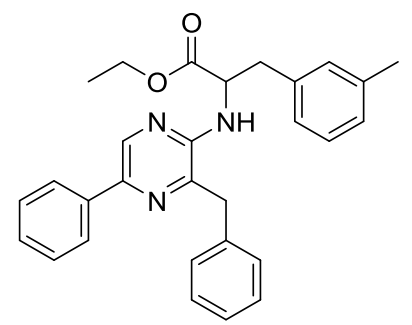

Ethyl 2-((3-benzyl-5-phenylpyrazin-2-yl)amino)-3-(m-tolyl)propanoate 23 1,1,12\} (EC31092-089-2): Obtained as an oil $(0.28 \mathrm{~g}, 61 \%)$ after a chromatography over silica gel (cyclohexane-ethyl acetate $95: 5) .{ }^{1} \mathrm{H} \mathrm{NMR}\left(\mathrm{CDCl}_{3}\right)$ : $8.41(\mathrm{~s}, 1 \mathrm{H}), 8.07-7.90(\mathrm{~m}, 2 \mathrm{H}), 7.48-7.42(\mathrm{~m}, 2 \mathrm{H}), 7.38-7.32(\mathrm{~m}, 1 \mathrm{H}), 7.30-7.26(\mathrm{~m}, 1 \mathrm{H}), 7.26-7.23(\mathrm{~m}$, $1 \mathrm{H}), 7.21-7.16(\mathrm{~m}, 2 \mathrm{H}), 7.15-7.08(\mathrm{~m}, 1 \mathrm{H}), 7.07-7.01(\mathrm{~m}, 1 \mathrm{H}), 6.88(\mathrm{~s}, 1 \mathrm{H}), 6.78(\mathrm{~d}, 1 \mathrm{H}, J=7.5 \mathrm{~Hz}), 4.93(\mathrm{t}$, $1 \mathrm{H}, J=5.9 \mathrm{~Hz}), 4.92(\mathrm{t}, 1 \mathrm{H}, J=6.5 \mathrm{~Hz}), 4.88-4.79(\mathrm{~m}, 2 \mathrm{H}), 4.11(\mathrm{q}, 2 \mathrm{H}, J=7.1 \mathrm{~Hz}), 4.11(\mathrm{q}, 2 \mathrm{H}, J=15.4 \mathrm{~Hz})$, $3.11(\mathrm{dd}, 1 \mathrm{H}, J=13.8,5.5 \mathrm{~Hz}), 3.03(\mathrm{dd}, 1 \mathrm{H}, J=13.8,6.1 \mathrm{~Hz}), 2.30(\mathrm{~s}, 3 \mathrm{H}), 1.17(\mathrm{t}, 3 \mathrm{H}, J=7.1 \mathrm{~Hz}) .{ }^{13} \mathrm{C} \mathrm{NMR}$ $\left(\mathrm{CDCl}_{3}\right)$ : 172.7, 150.6, 141.3, 141.3, 138.1, 137.7, 137.0, 136.7, 136.3, 130.2, 128.9, 128.9, 128.8, 128.5, 127.9, 
127.8, 127.0, 126.4, 125.8, 61.2, 55.1, 40.9, 38.0, 21.5, 14.2. HRMS $(m / z):[\mathrm{M}+\mathrm{H}]^{+}$calcd for $\mathrm{C}_{29} \mathrm{H}_{29} \mathrm{~N}_{3} \mathrm{O}_{2}$, 452.2338; found, 452.2408 .

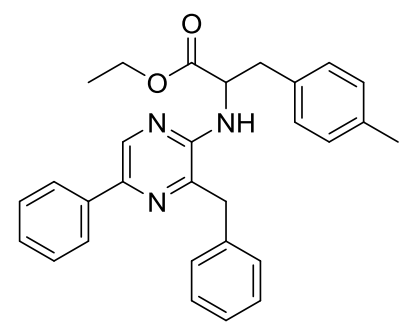

Ethyl 2-((3-benzyl-5-phenylpyrazin-2-yl)amino)-3-(p-tolyl)propanoate 23\{1,1,13\} (EC31093-153-2): Obtained as an oil $(0.35 \mathrm{~g}, 87 \%)$ after a chromatography over silica gel (cyclohexane-ethyl acetate $94: 6)$. ${ }^{1} \mathrm{H}$ NMR $\left(\mathrm{CDCl}_{3}\right)$ : $8.43(\mathrm{~s}, 1 \mathrm{H}), 7.97(\mathrm{~m}, 2 \mathrm{H}), 7.48(\mathrm{~m}, 2 \mathrm{H}), 7.37(\mathrm{~m}, 1 \mathrm{H}), 7.28(\mathrm{~m}, 3 \mathrm{H}), 7.22(\mathrm{~m}, 2 \mathrm{H}), 7.04(\mathrm{~m}, 2 \mathrm{H}), 6.89(\mathrm{~m}, 2 \mathrm{H})$, $4.93(\mathrm{~m}, 2 \mathrm{H}), 4.15(\mathrm{~m}, 4 \mathrm{H}), 3.15(\mathrm{dd}, 1 \mathrm{H}, J=13.8,5.2 \mathrm{~Hz}), 3.06(\mathrm{dd}, 1 \mathrm{H}, J=13.8,5.8 \mathrm{~Hz}), 2.35$ (s, 3H), $1.21(\mathrm{t}$, $3 \mathrm{H}, J=7.2 \mathrm{~Hz}) .{ }^{13} \mathrm{C} \mathrm{NMR}\left(\mathrm{CDCl}_{3}\right): 172.5,150.4,141.2,141.1,137.5,136.8,135.5,135.4,133.1,129.2,129.1$, $128.8,128.7,128.6,127.8,126.8,125.7,61.1,54.9,40.8,37.4,21.1,14.1$. HRMS $(\mathrm{m} / z):[\mathrm{M}+\mathrm{H}]^{+}$calcd for $\mathrm{C}_{29} \mathrm{H}_{29} \mathrm{~N}_{3} \mathrm{O}_{2}, 452.2338$; found, 452.2325 .

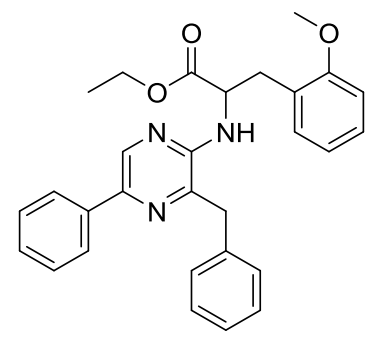

Ethyl 2-((3-benzyl-5-phenylpyrazin-2-yl)amino)-3-(2-methoxyphenyl)propanoate 23\{1,1,14\} (EC31093-157-2): Obtained as a yellow solid $(0.32 \mathrm{~g}, 77 \%)$ after a chromatography over silica gel (cyclohexane - ethyl acetate 95/5). ${ }^{1} \mathrm{H} \mathrm{NMR}\left(\mathrm{CDCl}_{3}\right): 8.38(\mathrm{~s}, 1 \mathrm{H}), 7.90(\mathrm{~m}, 2 \mathrm{H}), 7.44(\mathrm{~m}, 2 \mathrm{H}), 7.34(\mathrm{~m}, 1 \mathrm{H}), 7.26(\mathrm{~m}, 3 \mathrm{H}), 7.22(\mathrm{~m}, 6 \mathrm{H}), 7.04(\mathrm{dd}$, $1 \mathrm{H}, J=7.4,1.7 \mathrm{~Hz}), 6.88(\mathrm{td}, 1 \mathrm{H}, J=7.4,1.0 \mathrm{~Hz}), 6.83(\mathrm{~m}, 1 \mathrm{H}), 5.30(\mathrm{~d}, 1 \mathrm{H}, J=7.0 \mathrm{~Hz}), 4.85(\mathrm{~m}, 1 \mathrm{H}), 4.11(\mathrm{~m}$, $4 \mathrm{H}), 3.75(\mathrm{~s}, 3 \mathrm{H}), 3.20(\mathrm{dd}, 1 \mathrm{H}, J=13.6,5.6 \mathrm{~Hz}), 3.15(\mathrm{dd}, 1 \mathrm{H}, J=13.6,7.6 \mathrm{~Hz}), 1.20(\mathrm{t}, 3 \mathrm{H}, J=7.1 \mathrm{~Hz}) .{ }^{13} \mathrm{C}$ NMR $\left(\mathrm{CDCl}_{3}\right)$ : 173.0, 157.6, 150.7, 140.9, 140.8, 137.6, 136.8, 136.7, 131.0, 128.7 (two signals), 128.4, 127.6, 126.7, 125.6, 125.2, 120.8, 110.6, 60.9, 55.4, 55.2, 40.2, 32.4, 14.2, 14.1. HRMS (m/z): $[\mathrm{M}+\mathrm{H}]^{+}$calcd for $\mathrm{C}_{29} \mathrm{H}_{30} \mathrm{~N}_{3} \mathrm{O}_{3}, 468.2287$; found, 468.2281.

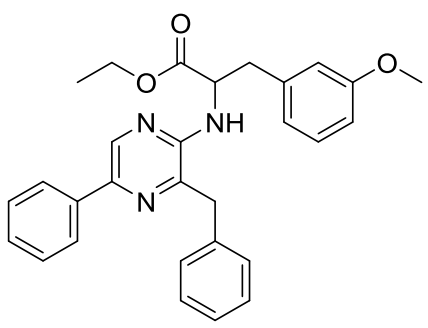

Ethyl 2-((3-benzyl-5-phenylpyrazin-2-yl)amino)-3-(3-methoxyphenyl)propanoate 23\{1,1,15\} (YJ31134-059-2): Obtained as an oil $(0.30 \mathrm{~g}, 51 \%)$ containing $5 \%$ of an unidentified impurity after a chromatography over silica gel (cyclohexane - ethyl acetate 95/5). ${ }^{1} \mathrm{H}$ NMR $\left(\mathrm{CDCl}_{3}\right): 8.42(\mathrm{~s}, 1 \mathrm{H}), 7.95(\mathrm{~m}, 2 \mathrm{H}), 7.46(\mathrm{~m}, 2 \mathrm{H}), 7.36(\mathrm{~m}, 1 \mathrm{H}), 7.30$ - $7.13(\mathrm{~m}, 6 \mathrm{H}), 6.79(\mathrm{~m}, 2 \mathrm{H}), 6.65(\mathrm{~m}, 1 \mathrm{H}), 6.58(\mathrm{~m}, 1 \mathrm{H}), 4.96-4.90(\mathrm{~m}, 2 \mathrm{H}), 4.14(\mathrm{~m}, 4 \mathrm{H}), 3.78(\mathrm{~s}, 3 \mathrm{H}), 3.15(\mathrm{dd}$, $1 \mathrm{H}, J=5.6,13.8 \mathrm{~Hz}), 3.07(\mathrm{dd}, 1 \mathrm{H}, J=6.0,13.8 \mathrm{~Hz}), 1.19(\mathrm{t}, 3 \mathrm{H}, J=7.1 \mathrm{~Hz}) .{ }^{13} \mathrm{C} \mathrm{NMR}\left(\mathrm{CDCl}_{3}\right): 172.4,159.7$, $150.4,141.2,141.1,137.8,137.5,136.8,136.5,129.5,129.3,128.8,128.6,127.8,126.8,125.6,121.6,115.0$, 112.3, 61.1, 55.1, 54.8, 40.8, 37.9, 14.1. HRMS (m/z): $[\mathrm{M}+\mathrm{H}]^{+}$calcd for $\mathrm{C}_{29} \mathrm{H}_{30} \mathrm{~N}_{3} \mathrm{O}_{3}, 468.2287$; found, 468.2301 . 


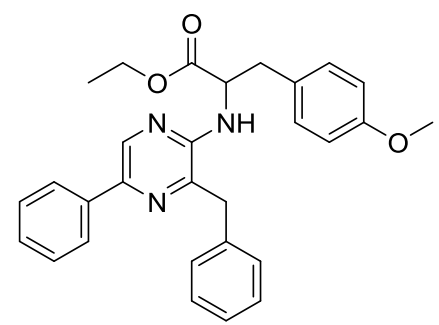

Ethyl 2-((3-benzyl-5-phenylpyrazin-2-yl)amino)-3-(4-methoxyphenyl)propanoate 23\{1,1,16\} (YJ30367-013-2): Obtained as an oil $(0.16 \mathrm{~g}, 35 \%)$ after a chromatography over silica gel (cyclohexane - ethyl acetate 93/7). ${ }^{1} \mathrm{H}$ NMR $\left(\mathrm{CDCl}_{3}\right): 8.42(\mathrm{~s}, 1 \mathrm{H}), 7.95(\mathrm{~m}, 2 \mathrm{H}), 7.46(\mathrm{~m}, 2 \mathrm{H}), 7.39-7.21(\mathrm{~m}, 6 \mathrm{H}), 6.89(\mathrm{~m}, 2 \mathrm{H}), 6.76(\mathrm{~m}, 1 \mathrm{H}), 4.96-$ $4.90(\mathrm{~m}, 2 \mathrm{H}), 4.15(\mathrm{~m}, 4 \mathrm{H}), 3.81(\mathrm{~s}, 3 \mathrm{H}), 3.12(\mathrm{dd}, 1 \mathrm{H}, J=4.8,13.7 \mathrm{~Hz}), 3.03(\mathrm{dd}, 1 \mathrm{H}, J=5.2,13.7 \mathrm{~Hz}), 1.21(\mathrm{t}$, $3 \mathrm{H}, J=7.1 \mathrm{~Hz}) .{ }^{13} \mathrm{C} \mathrm{NMR}\left(\mathrm{CDCl}_{3}\right): 172.4,159.7,150.4,141.2,141.1,137.8,137.5,136.8,136.5,129.5,129.3$, $128.8,128.6,127.8,126.8,125.6,121.6,115.0,112.3,61.1,55.1,54.8,40.8,37.9,14.1$. HRMS $(\mathrm{m} / \mathrm{z}):[\mathrm{M}+\mathrm{H}]^{+}$ calcd for $\mathrm{C}_{29} \mathrm{H}_{30} \mathrm{~N}_{3} \mathrm{O}_{3}, 468.2287$; found, 468.2271.

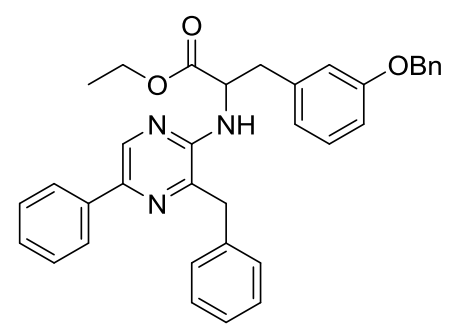

Ethyl 2-((3-benzyl-5-phenylpyrazin-2-yl)amino)-3-(3-(benzyloxy)phenyl)propanoate 23\{1,1,18\} (YJ 31776-1831): Obtained as an oil $(0.62 \mathrm{~g}, 77 \%)$ after a chromatography over silica gel (cyclohexane-ethyl acetate $95 / 5) .{ }^{1} \mathrm{H}$ NMR $\left(\mathrm{CDCl}_{3}\right): 8.43(\mathrm{~s}, 1 \mathrm{H}), 7.97(\mathrm{~m}, 2 \mathrm{H})$, 7.50-7.34 (m, 8H), $7.35(\mathrm{~m}, 4 \mathrm{H}), 7.26-7.16(\mathrm{~m}, 6 \mathrm{H}), 7.28-7.14(\mathrm{~m}, 6 \mathrm{H})$, $6.88(\mathrm{~m}, 1 \mathrm{H}), 6.74(\mathrm{~m}, 1 \mathrm{H}), 6.61(\mathrm{~m}, 1 \mathrm{H}), 5.04(\mathrm{~s}, 2 \mathrm{H}), 4.95(\mathrm{~m}, 2 \mathrm{H}), 4.13(\mathrm{~m}, 4 \mathrm{H}), 3.16(\mathrm{dd}, 1 \mathrm{H}, J=5.2,13.6 \mathrm{~Hz})$, $3.07(\mathrm{dd}, 1 \mathrm{H}, J=6.0,13.6 \mathrm{~Hz}), 1.20(\mathrm{t}, 3 \mathrm{H}, J=7.2 \mathrm{~Hz}) .{ }^{13} \mathrm{C}$ NMR $\left(\mathrm{CDCl}_{3}\right): 172.4,158.9,150.3,141.3,141.1$, 137.8, 137.4, 137.0, 136.6, 136.4, 129.5, 128.8, 128.7, 128.6, 128.5, 128.0, 127.8, 127.4, 126.9, 125.6, 121.9, 116.0, 113.2, 69.9, 61.2, 54.9, 40.8, 37.9, 14.1. HRMS $(m / z)$ : $[\mathrm{M}+\mathrm{H}]^{+}$calcd for $\mathrm{C}_{35} \mathrm{H}_{34} \mathrm{~N}_{3} \mathrm{O}_{3}, 544.2600$; found, 544.2607.

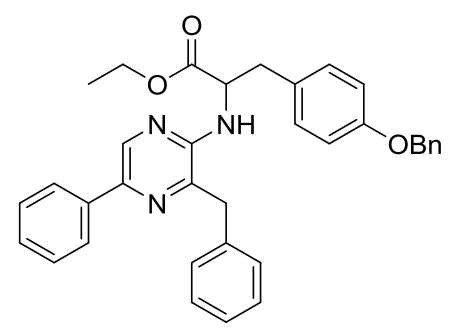

Ethyl 2-((3-benzyl-5-phenylpyrazin-2-yl)amino)-3-(4-(benzyloxy)phenyl)propanoate 23\{1,1,19\} (EC31093-0592): Obtained as an oil $(0.68 \mathrm{~g}, 70 \%)$ after a chromatography over silica gel (cyclohexane-ethyl acetate $95 / 5) .{ }^{1} \mathrm{H}$ NMR $\left(\mathrm{CDCl}_{3}\right): 8.41(\mathrm{~s}, 1 \mathrm{H}), 8.00-7.86(\mathrm{~m}, 2 \mathrm{H}), 7.50-7.16(\mathrm{~m}, 13 \mathrm{H}), 6.90-6.84(\mathrm{~m}, 2 \mathrm{H}), 6.84-6.79(\mathrm{~m}, 2 \mathrm{H})$, $5.05(\mathrm{~s}, 2 \mathrm{H}), 4.99-4.84(\mathrm{~m}, 2 \mathrm{H}), 4.12(\mathrm{qd}, 2 \mathrm{H}, J=7.1,0.6 \mathrm{~Hz}), 4.12(\mathrm{~s}, 2 \mathrm{H}), 3.11(\mathrm{dd}, 1 \mathrm{H}, J=13.9,5.0 \mathrm{~Hz}), 3.01$ (dd, $1 \mathrm{H}, J=13.9,5.5 \mathrm{~Hz}), 1.19(\mathrm{t}, 3 \mathrm{H}, J=7.1 \mathrm{~Hz}) .{ }^{13} \mathrm{C}$ NMR $\left(\mathrm{CDCl}_{3}\right): 172.6,157.9,150.5,141.2$ (two signals), 137.7, 137.2, 137.0, 136.7, 130.4, 128.9 (two signals), 128.8, 128.7, 128.6, 128.1, 127.9, 127.6, 127.0, 125.8, 115.0, 70.2, 61.3, 55.1, 41.0, 37.1, 14.3. HRMS (m/z): $[\mathrm{M}+\mathrm{H}]^{+}$calcd for $\mathrm{C}_{35} \mathrm{H}_{34} \mathrm{~N}_{3} \mathrm{O}_{3}, 544.2600$; found, 544.2654. 


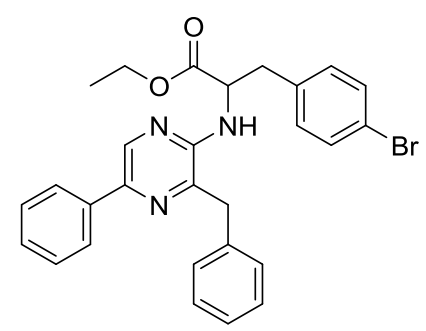

Ethyl 2-((3-benzyl-5-phenylpyrazin-2-yl)amino)-3-(4-bromophenyl)propanoate $\quad \mathbf{2 3}\{1,1,20\} \quad$ (CF34391-28-1): Obtained as an oil (0.37 g, 57\%) after a chromatography over silica gel (cyclohexane-ethyl acetate 97:3 to 96:4). ${ }^{1} \mathrm{H}$ NMR $\left(\mathrm{CDCl}_{3}\right): 8.43(\mathrm{~s}, 1 \mathrm{H}), 7.97(\mathrm{~m}, 2 \mathrm{H}), 7.48(\mathrm{~m}, 2 \mathrm{H}), 7.37(\mathrm{~m}, 1 \mathrm{H}), 7.29(\mathrm{~m}, 5 \mathrm{H}), 7.22(\mathrm{~m}, 2 \mathrm{H}), 6.79(\mathrm{~m}$, $2 \mathrm{H}), 4.95(\mathrm{~m}, 2 \mathrm{H}), 4.15(\mathrm{~m}, 4 \mathrm{H}), 3.16(\mathrm{~m}, 1 \mathrm{H}), 3.03(\mathrm{~m}, 1 \mathrm{H}), 1.23(\mathrm{t}, 3 \mathrm{H}, J=7.2 \mathrm{~Hz}) .{ }^{13} \mathrm{C} \mathrm{NMR}\left(\mathrm{CDCl}_{3}\right): 172.1$, 150.2, 141.2, 141.1, 137.4, 136.8, 136.4, 135.2, 131.5, 130.9, 128.9, 128.8, 128.6, 127.9, 126.9, 125.7, 120.8, 61.3, 54.6, 40.9, 37.0, 14.1. HRMS (m/z): [M+H] ${ }^{+}$calcd for $\mathrm{C}_{28} \mathrm{H}_{27} \mathrm{BrN}_{3} \mathrm{O}_{2}, 516.1287$; found, 516.1267.

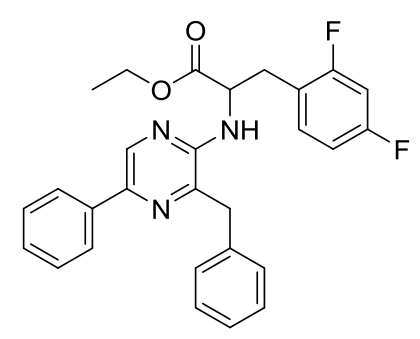

Ethyl 2-((3-benzyl-5-phenylpyrazin-2-yl)amino)-3-(2,4-difluorophenyl)propanoate $\mathbf{2 3}\{1,1,25\}$ (YJ31067-053-2): Obtained as an oil $(0.27 \mathrm{~g}, 61 \%)$ after a chromatography over silica gel (cyclohexane-ethyl acetate 96:4). ${ }^{1} \mathrm{H}$ NMR $\left(\mathrm{CDCl}_{3}\right): 8.42(\mathrm{~s}, 1 \mathrm{H}), 7.96(\mathrm{~m}, 2 \mathrm{H}), 7.47(\mathrm{~m}, 2 \mathrm{H}), 7.37(\mathrm{~m}, 1 \mathrm{H}), 7.27(\mathrm{~m}, 5 \mathrm{H}), 6.98(\mathrm{~m}, 1 \mathrm{H}), 6.70(\mathrm{~m}, 2 \mathrm{H}), 4.97$ $(\mathrm{m}, 2 \mathrm{H}), 4.16(\mathrm{~m}, 4 \mathrm{H}), 3.22(\mathrm{dd}, 1 \mathrm{H}, J=5.4,14.3 \mathrm{~Hz}), 3.10(\mathrm{dd}, 1 \mathrm{H}, J=6.1,14.3 \mathrm{~Hz}), 1.22(\mathrm{t}, 3 \mathrm{H}, J=7.1 \mathrm{~Hz}) .{ }^{13} \mathrm{C}$ NMR $\left(\mathrm{CDCl}_{3}\right)$ : 172.2, $161.9(11$ and $247 \mathrm{~Hz}), 161.2(11$ and $249 \mathrm{~Hz}), 150.2,141.2,141.1,137.4,136.8,136.4$, $132.0(6$ and $9 \mathrm{~Hz}), 128.8,128.7,128.6,127.8,126.9,119.3(4$ and $16 \mathrm{~Hz}), 111.1(3$ and $21 \mathrm{~Hz}), 103.6(25 \mathrm{~Hz})$, 61.4, 54.1, 40.9, 30.6, 14.0 (one signal missing). HRMS (m/z): $[\mathrm{M}+\mathrm{H}]^{+}$calcd for $\mathrm{C}_{28} \mathrm{H}_{26} \mathrm{~F}_{2} \mathrm{~N}_{3} \mathrm{O}_{2}, 474.1993$; found, 474.1988.

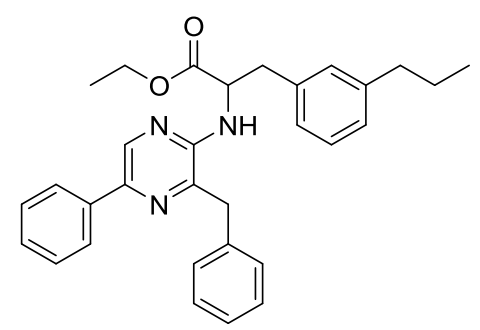

Ethyl 2-((3-benzyl-5-phenylpyrazin-2-yl)amino)-3-(3-propylphenyl)propanoate $\quad \mathbf{2 3}\{1,1,27\} \quad$ (CF34204-036-1): Obtained as an oil $(0.27 \mathrm{~g}, 79 \%)$ after a chromatography over silica gel (cyclohexane-ethyl acetate $95: 5) .{ }^{1} \mathrm{H}$ NMR $\left(\mathrm{CDCl}_{3}\right): 8.42(\mathrm{~s}, 1 \mathrm{H}), 7.95(\mathrm{~m}, 2 \mathrm{H}), 7.47(\mathrm{~m}, 2 \mathrm{H}), 7.37(\mathrm{~m}, 1 \mathrm{H}), 7.26(\mathrm{~m}, 5 \mathrm{H}), 7.16(\mathrm{~m}, 1 \mathrm{H}), 7.07(\mathrm{~m}, 1 \mathrm{H}), 6.92$ $(\mathrm{m}, 1 \mathrm{H}), 6.82(\mathrm{~m}, 1 \mathrm{H}), 4.93,(\mathrm{~m}, 2 \mathrm{H}), 4.14(\mathrm{~m}, 4 \mathrm{H}), 3.15(\mathrm{dd}, 1 \mathrm{H}, J=13.7,5.6 \mathrm{~Hz}), 3.07(\mathrm{dd}, 1 \mathrm{H}, J=13.7,6.1$ $\mathrm{Hz}), 2.56(\mathrm{~m}, 2 \mathrm{H}), 1.63(\mathrm{~m}, 2 \mathrm{H}), 1.18(\mathrm{t}, 3 \mathrm{H}, J=7.2 \mathrm{~Hz}), 0.95(\mathrm{t}, 3 \mathrm{H}, J=7.3 \mathrm{~Hz}) .{ }^{13} \mathrm{C}$ NMR $\left(\mathrm{CDCl}_{3}\right): 172.5$, 150.4, 142.8, 141.1 (2 signals), 137.5, 136.8, 136.5, 136.1, 129.4, 128.8, 128.7, 128.6, 128.4, 127.8, 127.1, 126.9, 126.6, 125.6, 61.1, 54.9, 40.8, 38.0, 37.9, 24.5, 14.1, 13.8. HRMS $(\mathrm{m} / \mathrm{z}):[\mathrm{M}+\mathrm{H}]^{+}$calcd for $\mathrm{C}_{31} \mathrm{H}_{34} \mathrm{~N}_{3} \mathrm{O}_{2}, 480.2651$; found, 480.2661 . 


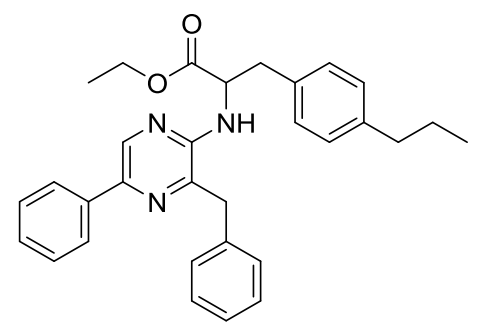

Ethyl 2-((3-benzyl-5-phenylpyrazin-2-yl)amino)-3-(4-propylphenyl)propanoate $\quad \mathbf{2 3}\{1,1,28\} \quad$ (CF34204-044-1): Obtained as an oil $(0.25 \mathrm{~g}, 73 \%)$ after a chromatography over silica gel (cyclohexane-ethyl acetate $95: 5) .{ }^{1} \mathrm{H}$ NMR $\left(\mathrm{CDCl}_{3}\right): 8.42(\mathrm{~s}, 1 \mathrm{H}), 7.96(\mathrm{~m}, 2 \mathrm{H}), 7.47(\mathrm{~m}, 2 \mathrm{H}), 7.37(\mathrm{~m}, 1 \mathrm{H}), 7.26(\mathrm{~m}, 5 \mathrm{H}), 7.05(\mathrm{~m}, 2 \mathrm{H}), 6.91(\mathrm{~m}, 2 \mathrm{H}), 4.93$, $(\mathrm{m}, 2 \mathrm{H}), 4.13(\mathrm{~m}, 4 \mathrm{H}), 3.14(\mathrm{dd}, 1 \mathrm{H}, J=13.9,5.5 \mathrm{~Hz}), 3.06(\mathrm{dd}, 1 \mathrm{H}, J=13.9,6.0 \mathrm{~Hz}), 2.59(\mathrm{~m}, 2 \mathrm{H}), 1.65(\mathrm{~m}, 2 \mathrm{H})$, $1.19(\mathrm{t}, 3 \mathrm{H}, J=7.2 \mathrm{~Hz}), 0.97(\mathrm{t}, 3 \mathrm{H}, J=7.3 \mathrm{~Hz}) .{ }^{13} \mathrm{C} \mathrm{NMR}\left(\mathrm{CDCl}_{3}\right): 172.5,150.4,141.2,141.1,141.0,137.5$, 136.9, 136.6, 133.3, 129.1, 128.8, 128.7, 128.6, 128.5, 127.8, 126.9, 125.6, 61.1, 54.9, 40.8, 37.7, 37.5, 24.5, 14.1, 13.8. HRMS $(\mathrm{m} / \mathrm{z}):[\mathrm{M}+\mathrm{H}]^{+}$calcd for $\mathrm{C}_{31} \mathrm{H}_{34} \mathrm{~N}_{3} \mathrm{O}_{2}, 480.2651$; found, 480.2671 .

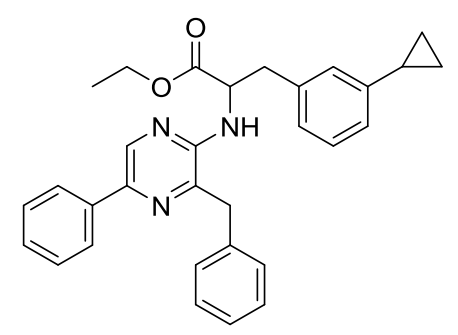

Ethyl 2-((3-benzyl-5-phenylpyrazin-2-yl)amino)-3-(3-cyclopropylphenyl)propanoate 23 1,1,29\} (YJ30367-103-2): Obtained as an oil $(0.46 \mathrm{~g}, 79 \%)$ after a chromatography over silica gel (cyclohexane-ethyl acetate 95:5). ${ }^{1} \mathrm{H}$ NMR $\left(\mathrm{CDCl}_{3}\right): 8.42(\mathrm{~s}, 1 \mathrm{H}), 7.95(\mathrm{~m}, 2 \mathrm{H}), 7.47(\mathrm{~m}, 2 \mathrm{H}), 7.36(\mathrm{~m}, 1 \mathrm{H}), 7.28-7.19(\mathrm{~m}, 5 \mathrm{H}), 7.13(\mathrm{~m}, 1 \mathrm{H}), 6.95(\mathrm{~m}, 1 \mathrm{H})$, $6.82(\mathrm{~m}, 1 \mathrm{H}), 6.77(\mathrm{~m}, 1 \mathrm{H}), 4.93,(\mathrm{~m}, 2 \mathrm{H}), 4.13(\mathrm{~m}, 4 \mathrm{H}), 3.13(\mathrm{dd}, 1 \mathrm{H}, J=5.5,13.7 \mathrm{~Hz}), 3.05(\mathrm{dd}, 1 \mathrm{H}, J=6.0$, $13.7 \mathrm{~Hz}), 1.86(\mathrm{~m}, 1 \mathrm{H}), 1.18(\mathrm{t}, 3 \mathrm{H}, J=7.2 \mathrm{~Hz}), 0.96(\mathrm{~m}, 2 \mathrm{H}), 0.67(\mathrm{~m}, 2 \mathrm{H}) .{ }^{13} \mathrm{C}$ NMR $\left(\mathrm{CDCl}_{3}\right): 172.5,150.4$, 141.2, 141.1, 137.5, 136.8, 136.5, 136.2, 128.8, 128.7, 128.6 (two signals), 128.4, 127.8, 126.8, 126.3, 125.6, 124.1, 61.1, 54.9, 40.8, 37.9, 15.3, 14.1, 9.2, 9.1. HRMS $(\mathrm{m} / z)$ : $[\mathrm{M}+\mathrm{H}]^{+}$calcd for $\mathrm{C}_{31} \mathrm{H}_{32} \mathrm{~N}_{3} \mathrm{O}_{2}, 478.2495$; found, 478.2480 .

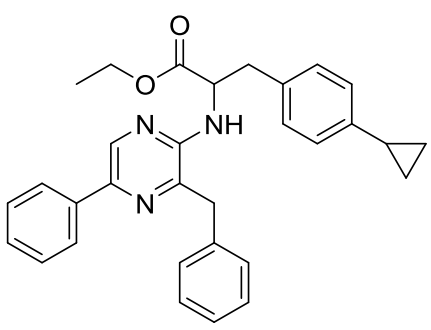

Ethyl 2-((3-benzyl-5-phenylpyrazin-2-yl)amino)-3-(4-cyclopropylphenyl)propanoate 23\{1,1,30\} (YJ30367-105-2): Obtained as an oil $(0.46 \mathrm{~g}, 76 \%)$ after a chromatography over silica gel (cyclohexane-ethyl acetate $95: 5) .{ }^{1} \mathrm{H}$ NMR $\left(\mathrm{CDCl}_{3}\right): 8.42(\mathrm{~s}, 1 \mathrm{H}), 7.96(\mathrm{~m}, 2 \mathrm{H}), 7.47(\mathrm{~m}, 2 \mathrm{H}), 7.36(\mathrm{~m}, 1 \mathrm{H}), 7.31-7.23(\mathrm{~m}, 3 \mathrm{H}), 7.20(\mathrm{~m}, 2 \mathrm{H}), 6.94(\mathrm{~m}, 2 \mathrm{H})$, $6.86(\mathrm{~m}, 2 \mathrm{H}), 4.93,(\mathrm{~m}, 2 \mathrm{H}), 4.13(\mathrm{~m}, 4 \mathrm{H}), 3.13(\mathrm{dd}, 1 \mathrm{H}, J=5.3,13.9 \mathrm{~Hz}), 3.04(\mathrm{dd}, 1 \mathrm{H}, J=5.9,13.9 \mathrm{~Hz}), 1.89(\mathrm{~m}$, $1 \mathrm{H}), 1.19(\mathrm{t}, 3 \mathrm{H}, J=7.2 \mathrm{~Hz}), 0.98(\mathrm{~m}, 2 \mathrm{H}), 0.69(\mathrm{~m}, 2 \mathrm{H}) .{ }^{13} \mathrm{C} \mathrm{NMR}\left(\mathrm{CDCl}_{3}\right): 172.5,150.4,142.5,141.1,141.0$, 137.5, 136.9, 136.5, 133.1, 129.1, 128.8, 128.7, 128.6, 127.7, 126.8, 125.8, 125.6, 61.1, 54.9, 40.8, 37.3, 15.1, 14.1, 9.1. HRMS $(\mathrm{m} / \mathrm{z})$ : $[\mathrm{M}+\mathrm{H}]^{+}$calcd for $\mathrm{C}_{31} \mathrm{H}_{32} \mathrm{~N}_{3} \mathrm{O}_{2}, 478.2495$; found, 478.2487 . 


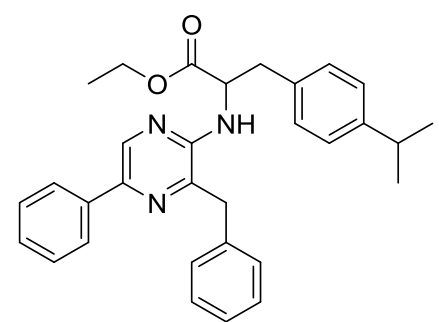

Ethyl 2-((3-benzyl-5-phenylpyrazin-2-yl)amino)-3-(4-isopropylphenyl)propanoate 23\{1,1,31\} (EC31093-091-2): Obtained as an oil $(0.25 \mathrm{~g}, 73 \%)$ after a chromatography over silica gel (cyclohexane-ethyl acetate $95: 5) .{ }^{1} \mathrm{H}$ NMR $\left(\mathrm{CDCl}_{3}\right): 8.40(\mathrm{~s}, 1 \mathrm{H}), 7.97-7.90(\mathrm{~m}, 2 \mathrm{H}), 7.49-7.40(\mathrm{~m}, 2 \mathrm{H}), 7.38-7.32(\mathrm{~m}, 1 \mathrm{H}), 7.32-7.23(\mathrm{~m}, 3 \mathrm{H}), 7.23-$ $7.17(\mathrm{~m}, 2 \mathrm{H}), 7.12-7.05(\mathrm{~m}, 2 \mathrm{H}), 6.94-6.88(\mathrm{~m}, 2 \mathrm{H}), 4.99-4.84(\mathrm{~m}, 2 \mathrm{H}), 4.18-4.06(\mathrm{~m}, 4 \mathrm{H}), 3.08(\mathrm{qd}, 2 \mathrm{H}, J=$ $13.8,5.7 \mathrm{~Hz}), 2.96-2.84(\mathrm{~m}, 1 \mathrm{H}), 1.25(\mathrm{~d}, 6 \mathrm{H}, J=6.9 \mathrm{~Hz}), 1.16(\mathrm{t}, 3 \mathrm{H}, J=7.1 \mathrm{~Hz}) .{ }^{13} \mathrm{C} \mathrm{NMR}\left(\mathrm{CDCl}_{3}\right): 172.7$, 150.6, 147.5, 141.3, 141.2, 137.7, 137.0, 136.7, 133.6, 129.3, 128.9, 128.9, 128.8, 127.9, 127.0, 126.6, 125.8, 61.2, 55.0, 41.0, 37.6, 33.9, 24.2, 24.1, 14.2. HRMS $(\mathrm{m} / z)$ : $[\mathrm{M}+\mathrm{H}]^{+}$calcd for $\mathrm{C}_{31} \mathrm{H}_{34} \mathrm{~N}_{3} \mathrm{O}_{2}, 480.2683$; found, 480.2690 .

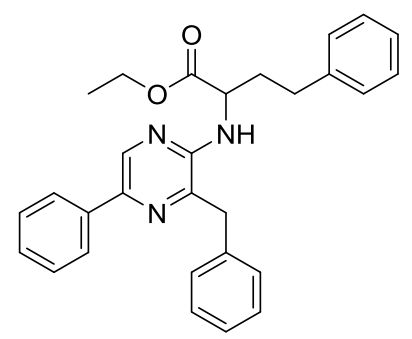

Ethyl 2-((3-benzyl-5-phenylpyrazin-2-yl)amino)-4-phenylbutanoate 23\{1,1,32\} (YJ31070-127-2): Obtained as an oil $(0.36 \mathrm{~g}, 80 \%)$ after a chromatography over silica gel (cyclohexane / ethyl acetate 95:5). ${ }^{1} \mathrm{H}$ NMR $\left(\mathrm{CDCl}_{3}\right): 8.42$ (s, 1H), $7.97(\mathrm{~m}, 2 \mathrm{H}), 7.48(\mathrm{~m}, 1 \mathrm{H}), 7.38(\mathrm{~m}, 5 \mathrm{H}), 7.28(\mathrm{~m}, 3 \mathrm{H}), 7.20(\mathrm{~m}, 1 \mathrm{H}), 7.06(\mathrm{~m}, 2 \mathrm{H}), 4.97(\mathrm{~d}(\mathrm{br}), 1 \mathrm{H}, J=$ $7.0 \mathrm{~Hz}), 4.73(\mathrm{~m}, 1 \mathrm{H}), 4.27(\mathrm{~d}, 1 \mathrm{H}, J=15.4 \mathrm{~Hz}), 4.21(\mathrm{~d}, 1 \mathrm{H}, J=15.4 \mathrm{~Hz}), 4.16(\mathrm{q}, 2 \mathrm{H}, J=7.1 \mathrm{~Hz}), 2.43(\mathrm{~m}, 2 \mathrm{H})$, $2.21(\mathrm{~m}, 1 \mathrm{H}), 1.99(\mathrm{~m}, 1 \mathrm{H}), 1.26(\mathrm{t}, 3 \mathrm{H}, J=7.1 \mathrm{~Hz}) .{ }^{13} \mathrm{C} \mathrm{NMR}\left(\mathrm{CDCl}_{3}\right): 173.0,150.5,141.1,141.0,140.9,137.5$, 136.9, 136.8, 129.0, 128.8, 128.7, 128.4, 127.8, 127.1, 126.1, 125.7, 61.2, 53.6, 41.2, 33.6, 31.2, 14.2. HRMS $(\mathrm{m} / \mathrm{z})$ : $[\mathrm{M}+\mathrm{H}]^{+}$calcd for $\mathrm{C}_{29} \mathrm{H}_{30} \mathrm{~N}_{3} \mathrm{O}_{2}, 452.2338$; found, 452.2335 .

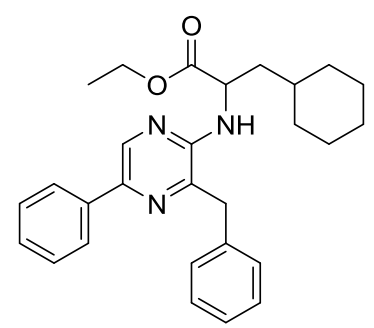

Ethyl 2-((3-benzyl-5-phenylpyrazin-2-yl)amino)-3-cyclohexylpropanoate 23\{1,1,33\} (YJ31067-065-2): Obtained as an oil $(0.36 \mathrm{~g}, 78 \%)$ after a chromatography over silica gel (cyclohexane - ethyl acetate 95/5). ${ }^{1} \mathrm{H}$ NMR $\left(\mathrm{CDCl}_{3}\right): 8.41(\mathrm{~s}, 1 \mathrm{H}), 7.95(\mathrm{~m}, 2 \mathrm{H}), 7.47(\mathrm{~m}, 2 \mathrm{H}), 7.38-7.32(\mathrm{~m}, 5 \mathrm{H}), 7.29-7.26(\mathrm{~m}, 1 \mathrm{H}), 4.60(\mathrm{~m}, 4 \mathrm{H}), 4.30(\mathrm{~d}$, $1 \mathrm{H}, J=15.5 \mathrm{~Hz}), 4.20(\mathrm{~d}, 1 \mathrm{H}, J=15.5 \mathrm{~Hz}), 4.16(\mathrm{~m}, 2 \mathrm{H}), 1.60(\mathrm{~m}, 6 \mathrm{H}), 1.48(\mathrm{~m}, 1 \mathrm{H}), 1.24(\mathrm{t}, 3 \mathrm{H}, J=7.2 \mathrm{~Hz})$, $1.08(\mathrm{~m}, 4 \mathrm{H}), 0.86(\mathrm{~m}, 2 \mathrm{H}) .{ }^{13} \mathrm{C} \mathrm{NMR}\left(\mathrm{CDCl}_{3}\right): 174.0,150.8,141.0,140.9,137.5,137.0,136.9,129.0,128.7$, 128.6, 127.7, 127.1, 125.7, 60.9, 51.9, 41.2, 39.9, 33.9, 33.6, 32.4, 26.3, 26.0, 25.9, 14.2. HRMS (m/z): $[\mathrm{M}+\mathrm{H}]^{+}$ calcd for $\mathrm{C}_{28} \mathrm{H}_{34} \mathrm{~N}_{3} \mathrm{O}_{2}$, 444.2651; found, 444.2647.

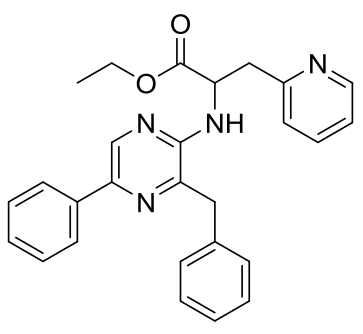


Ethyl 2-((3-benzyl-5-phenylpyrazin-2-yl)amino)-3-(pyridin-2-yl)propanoate $\quad \mathbf{2 3}\{1,1,34\} \quad$ (EC31092-107-3): Obtained as an oil $(0.45 \mathrm{~g}, 64 \%)$ after a chromatography over silica gel (cyclohexane-ethyl acetate 95:5 to 75:25). ${ }^{1} \mathrm{H}$ NMR $\left(\mathrm{CDCl}_{3}\right): 8.42-8.39(\mathrm{~m}, 1 \mathrm{H}), 8.38(\mathrm{~s}, 1 \mathrm{H}), 7.95-7.89(\mathrm{~m}, 2 \mathrm{H}), 7.52(\mathrm{td}, 1 \mathrm{H}, J=7.6,1.8 \mathrm{~Hz}), 7.47-$ $7.39(\mathrm{~m}, 2 \mathrm{H}), 7.36-7.29(\mathrm{~m}, 3 \mathrm{H}), 7.29-7.23(\mathrm{~m}, 2 \mathrm{H}), 7.23-7.18(\mathrm{~m}, 1 \mathrm{H}), 7.11(\mathrm{ddd}, 1 \mathrm{H}, J=7.6,4.9,1.0 \mathrm{~Hz})$, $7.01(\mathrm{~d}, 1 \mathrm{H}, J=7.8 \mathrm{~Hz}), 6.30(\mathrm{~d}, 1 \mathrm{H}, J=7.3 \mathrm{~Hz}), 5.03(\mathrm{dt}, 1 \mathrm{H}, J=7.3,5.4 \mathrm{~Hz}), 4.20(\mathrm{~s}, 2 \mathrm{H}), 4.08(\mathrm{qd}, 2 \mathrm{H}, J=7.1$, $1.1 \mathrm{~Hz}), 3.39-3.27(\mathrm{~m}, 2 \mathrm{H}), 1.11(\mathrm{t}, 3 \mathrm{H}, J=7.1 \mathrm{~Hz}) .{ }^{13} \mathrm{C} \mathrm{NMR}\left(\mathrm{CDCl}_{3}\right): 172.5,157.6,150.8,149.2,141.3,140.9$, 137.8, 137.1, 136.9, 136.6, 128.9, 128.8, 128.7, 127.7, 126.7, 125.7, 123.8, 121.9, 61.0, 54.0, 40.6, 39.1, 14.2. HRMS (m/z): $[\mathrm{M}+\mathrm{H}]^{+}$calcd for $\mathrm{C}_{27} \mathrm{H}_{27} \mathrm{~N}_{4} \mathrm{O}_{2}$, 439.2134; found, 439.2152.

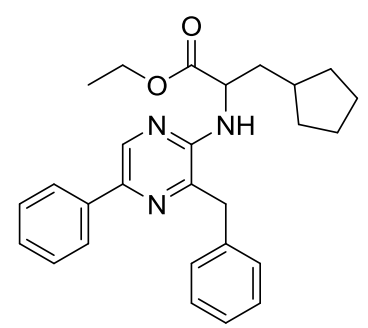

Ethyl 2-((3-benzyl-5-phenylpyrazin-2-yl)amino)-3-cyclopentylpropanoate 23\{1,1,36\} (YJ31067-067-2): Obtained as an oil $(0.26 \mathrm{~g}, 58 \%)$ after a chromatography over silica gel (cyclohexane - ethyl acetate $95 / 5)$. ${ }^{1} \mathrm{H}$ NMR $\left(\mathrm{CDCl}_{3}\right): 8.42(\mathrm{~s}, 1 \mathrm{H}), 7.95(\mathrm{~m}, 2 \mathrm{H}), 7.47(\mathrm{~m}, 2 \mathrm{H}), 7.38-7.32(\mathrm{~m}, 5 \mathrm{H}), 7.29-7.26(\mathrm{~m}, 1 \mathrm{H}), 4.78(\mathrm{~d}, 1 \mathrm{H}, J=7.4 \mathrm{~Hz})$, $4.60(\mathrm{~m}, 2 \mathrm{H}), 4.30(\mathrm{~d}, 1 \mathrm{H}, J=15.1 \mathrm{~Hz}), 4.21(\mathrm{~d}, 1 \mathrm{H}, J=15.1 \mathrm{~Hz}), 4.16(\mathrm{q}, 2 \mathrm{H}, J=7.2 \mathrm{~Hz}), 1.83(\mathrm{~m}, 1 \mathrm{H}), 1.70-$ $1.39(\mathrm{~m}, 7 \mathrm{H}), 1.24(\mathrm{t}, 3 \mathrm{H}, J=7.2 \mathrm{~Hz}), 1.01(\mathrm{~m}, 2 \mathrm{H}) .{ }^{13} \mathrm{C} \mathrm{NMR}\left(\mathrm{CDCl}_{3}\right): 173.8,150.7,141.0,140.9,137.6,137.0$, $136.9,128.9,128.7,128.6,127.7,127.0,125.6,60.9,53.7,41.2,38.3,36.5,32.8,32.4,25.0,24.8$, 14.2. HRMS $(\mathrm{m} / \mathrm{z}):[\mathrm{M}+\mathrm{H}]^{+}$calcd for $\mathrm{C}_{27} \mathrm{H}_{32} \mathrm{~N}_{3} \mathrm{O}_{2}, 430.2495$; found, 430.2485 .

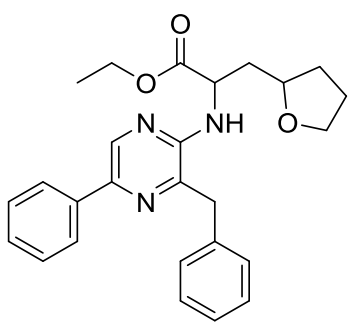

Ethyl 2-((3-benzyl-5-phenylpyrazin-2-yl)amino)-3-(tetrahydrofuran-2-yl)propanoate 23 1,1,38\} (YJ31134-043-3): Obtained as an oil $(0.50 \mathrm{~g}, 60 \%)$ after a chromatography over silica gel (cyclohexane - ethyl acetate $9 / 1) .{ }^{1} \mathrm{H}$ NMR (diastereoisomeric mixture, 0.53 / 0.47 ratio) $\left(\mathrm{CDCl}_{3}\right): 8.39(\mathrm{~s}, 1 \mathrm{H}), 7.96(\mathrm{~m}, 2 \mathrm{H}), 7.45(\mathrm{~m}, 2 \mathrm{H}), 7.35(\mathrm{~m}, 5 \mathrm{H}), 7.25$ $(\mathrm{m}, 1 \mathrm{H}), 4.78(\mathrm{~m}, 0.6 \mathrm{H}), 4.59(\mathrm{~m}, 0.4 \mathrm{H}), 4.20(\mathrm{~m}, 4 \mathrm{H}), 3.81(\mathrm{~m}, 1.6 \mathrm{H}), 3.70(\mathrm{~m}, 1.4 \mathrm{H}), 2.1(\mathrm{~m}, 1 \mathrm{H}), 1.88(\mathrm{~m}, 4 \mathrm{H})$, $1.46(\mathrm{~m}, 1 \mathrm{H}), 1.25(\mathrm{t}, 1.4 \mathrm{H}, J=7.2 \mathrm{~Hz}), 1.18(\mathrm{t}, 1.6 \mathrm{H}, J=7.2 \mathrm{~Hz}) .{ }^{13} \mathrm{C} \mathrm{NMR}\left(\mathrm{CDCl}_{3}\right): 173.2 / 173.0,150.9 / 150.5$, $141.2 / 141.0, \quad 140.7 / 140.6, \quad 137.7 / 137.6, \quad 137 / 137.0, \quad 136.8 / 136.7, \quad 128.9 / 128.8, \quad 128.7 / 128.65,128.6 / 128.5$, 127.6/127.5, 126.7/126.6, 125.6, 76.6/76.5, 68.0/67.8, 61.0/60.9, 53.6/53.1, 40.5/40.4, 37.0/36.5, 31.9/31.8, 25.4/25.3, 14.2/14.1. HRMS (m/z): $[\mathrm{M}+\mathrm{H}]^{+}$calcd for $\mathrm{C}_{26} \mathrm{H}_{30} \mathrm{~N}_{3} \mathrm{O}_{2}, 432.2287$; found, 432.2253 .

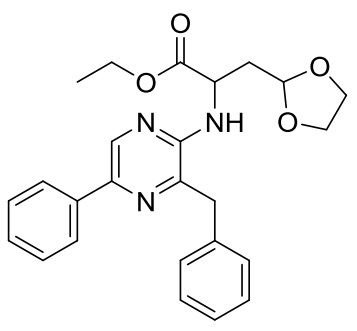

Ethyl 2-(3-benzyl-5-phenylpyrazin-2-ylamino)-3-(1,3-dioxolan-2-yl)propanoate 23\{1,1,39\} (VHE30855-183-4): Obtained as an oil $(0.61 \mathrm{~g}, 67 \%)$ after a chromatography over silica gel (cyclohexane - ethyl acetate $9 / 1$ to $7 / 3)$. ${ }^{1} \mathrm{H}$ NMR $\left(\mathrm{CDCl}_{3}\right): 8.41(\mathrm{~s}, 1 \mathrm{H}), 7.94(\mathrm{~m}, 2 \mathrm{H}), 7.46(\mathrm{~m}, 2 \mathrm{H}), 7.37-7.31(\mathrm{~m}, 5 \mathrm{H}), 7.25(\mathrm{~m}, 1 \mathrm{H}), 5.66(\mathrm{~m}, 1 \mathrm{H}), 4.85(\mathrm{t}$, $2 \mathrm{H}, J=4.3 \mathrm{~Hz}), 4.77(\mathrm{~m}, 1 \mathrm{H}), 4.23(\mathrm{~m}, 2 \mathrm{H}), 4.18(\mathrm{q}, 2 \mathrm{H}, J=7.1 \mathrm{~Hz}), 3.83(\mathrm{~m}, 2 \mathrm{H}), 3.75(\mathrm{~m}, 2 \mathrm{H}), 2.25(\mathrm{~m}, 2 \mathrm{H})$, $1.25(\mathrm{t}, 3 \mathrm{H}, J=7.1 \mathrm{~Hz}) .{ }^{13} \mathrm{C} \mathrm{NMR}\left(\mathrm{CDCl}_{3}\right): 172.5,150.6,141.0,137.6,136.9,136.8,128.8,128.7,128.6,127.7$, 
126.7, 125.6, 102.3, 64.9, 64.8, 61.1, 50.9, 40.5, 34.7, 26.9, 14.1. HRMS $(m / z):[\mathrm{M}+\mathrm{H}]^{+}$calcd for $\mathrm{C}_{25} \mathrm{H}_{28} \mathrm{~N}_{3} \mathrm{O}_{4}$, 434.2080; found, 434.2117.

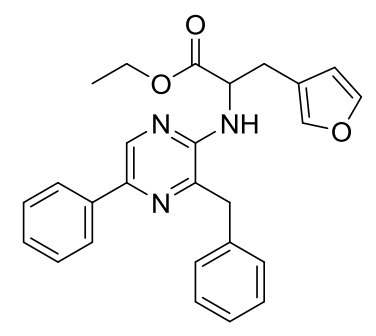

Ethyl 2-((3-benzyl-5-phenylpyrazin-2-yl)amino)-3-(furan-3-yl)propanoate $\quad \mathbf{2 3}\{1,1,40\} \quad$ (VHE30855-181-3): Obtained as an oil (1.2 g, 89\%) after a chromatography over silica gel (cyclohexane-ethyl acetate 95/5 to 9/1). ${ }^{1} \mathrm{H}$ NMR $\left(\mathrm{CDCl}_{3}\right): 8.43(\mathrm{~s}, 1 \mathrm{H}), 7.97(\mathrm{~m}, 2 \mathrm{H}), 7.48(\mathrm{~m}, 2 \mathrm{H}), 7.40-7.24(\mathrm{~m}, 7 \mathrm{H}), 6.96(\mathrm{~m}, 1 \mathrm{H}), 5.96(\mathrm{~m}, 1 \mathrm{H}), 5.00(\mathrm{~m}$, $1 \mathrm{H}), 4.93(\mathrm{~m}, 1 \mathrm{H}), 4.24-4.14(\mathrm{~m}, 4 \mathrm{H}), 3.02(\mathrm{dd}, 1 \mathrm{H}, J=5.1,14.7 \mathrm{~Hz}), 2.95(\mathrm{dd}, 1 \mathrm{H}, J=5.4,14.7 \mathrm{~Hz}), 1.22(\mathrm{t}$, $3 \mathrm{H}, J=7.2 \mathrm{~Hz}) .{ }^{13} \mathrm{C} \mathrm{NMR}\left(\mathrm{CDCl}_{3}\right): 172.3,150.3,142.9,141.2,141.1,140.3,137.5,136.9,136.5,128.9,128.7$, 128.6, 127.8, 127.0, 125.6, 119.0, 111.1, 61.3, 53.8, 41.0, 27.2, 14.1. HRMS (m/z): $[\mathrm{M}+\mathrm{H}]^{+}$calcd for $\mathrm{C}_{26} \mathrm{H}_{26} \mathrm{~N}_{3} \mathrm{O}_{3}$, 428.1974; found: 428.2006 .

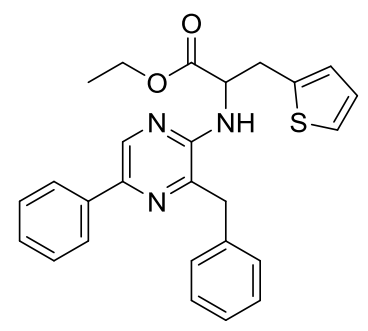

Ethyl 2-((3-benzyl-5-phenylpyrazin-2-yl)amino)-3-(thiophen-2-yl)propanoate $\quad \mathbf{2 3}\{1,1,41\} \quad$ (EC31092-087-2): Obtained as an oil $(0.51 \mathrm{~g}, 80 \%)$ after a chromatography over silica gel (cyclohexane-ethyl acetate 95:5). ${ }^{1} \mathrm{H}$ NMR $\left(\mathrm{CDCl}_{3}\right): 8.42(\mathrm{~s}, 1 \mathrm{H}), 8.00-7.89(\mathrm{~m}, 2 \mathrm{H}), 7.50-7.42(\mathrm{~m}, 2 \mathrm{H}), 7.40-7.32(\mathrm{~m}, 1 \mathrm{H}), 7.32-7.26(\mathrm{~m}, 3 \mathrm{H}), 7.26-$ $7.21(\mathrm{~m}, 2 \mathrm{H}), 7.14-7.08(\mathrm{~m}, 1 \mathrm{H}), 6.85(\mathrm{dd}, 1 \mathrm{H}, J=5.2,3.4 \mathrm{~Hz}), 6.58-6.50(\mathrm{~m}, 1 \mathrm{H}), 5.12(\mathrm{~d}, 1 \mathrm{H}, J=7.1 \mathrm{~Hz})$, $4.99(\mathrm{dt}, 1 \mathrm{H}, J=7.1,5.0 \mathrm{~Hz}), 4.17(\mathrm{~s}, 2 \mathrm{H}), 4.14$ (q, 2H, $J=7.1 \mathrm{~Hz}), 3.44$ (dd, 1H, $J=14.6,4.7 \mathrm{~Hz}), 3.37$ (dd, $1 \mathrm{H}, J$ $=14.9,4.9 \mathrm{~Hz}), 1.22(\mathrm{t}, 3 \mathrm{H}, J=7.1 \mathrm{~Hz}) .{ }^{13} \mathrm{C} \mathrm{NMR}\left(\mathrm{CDCl}_{3}\right): 171.9,150.3,141.5,141.3,137.8,137.6,137.0,136.7$, $129.0,128.9,128.9,128.0,127.0,127.0,126.7,125.8,124.7,61.6,54.7,41.1,32.0,14.3 . \mathrm{HRMS}(\mathrm{m} / \mathrm{z}):[\mathrm{M}+\mathrm{H}]^{+}$ calcd for $\mathrm{C}_{26} \mathrm{H}_{26} \mathrm{~N}_{3} \mathrm{O}_{2} \mathrm{~S}, 444.1746$; found, 444.1766 .

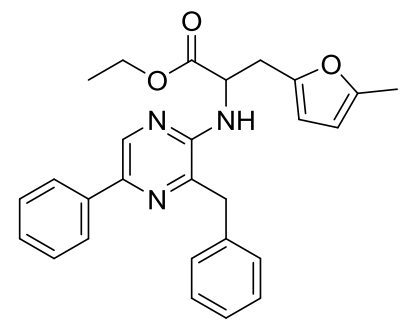

Ethyl 2-((3-benzyl-5-phenylpyrazin-2-yl)amino)-3-(5-methylfuran-2-yl)propanoate 23 1,1,42\} (EC29990-157-2): Obtained as a yellow oil $(0.69 \mathrm{~g}, 74 \%)$ after a chromatography over silica gel (dichloromethane). ${ }^{1} \mathrm{H}$ NMR $\left(\mathrm{CDCl}_{3}\right): 8.38(\mathrm{~s}, 1 \mathrm{H}), 7.96-7.89(\mathrm{~m}, 2 \mathrm{H}), 7.48-7.40(\mathrm{~m}, 2 \mathrm{H}), 7.38-7.26(\mathrm{~m}, 5 \mathrm{H}), 7.25-7.19(\mathrm{~m}, 1 \mathrm{H}), 5.83-$ $5.77(\mathrm{~m}, 1 \mathrm{H}), 5.72(\mathrm{~d}, 1 \mathrm{H}, J=3.0 \mathrm{~Hz}), 5.11(\mathrm{~d}, 1 \mathrm{H}, J=7.5 \mathrm{~Hz}), 4.89(\mathrm{dt}, 1 \mathrm{H}, J=7.5,5.3 \mathrm{~Hz}), 4.21-4.08(\mathrm{~m}, 4 \mathrm{H})$, $3.12(\mathrm{~d}, 2 \mathrm{H}, J=5.3 \mathrm{~Hz}), 2.20(\mathrm{~s}, 3 \mathrm{H}), 1.21(\mathrm{t}, 3 \mathrm{H}, J=7.1 \mathrm{~Hz}) .{ }^{13} \mathrm{C} \mathrm{NMR}\left(\mathrm{CDCl}_{3}\right): 172.2,151.6,150.5,148.7$, $141.3,141.2$, 137.7, 136.9, 136.8, 128.9, 128.9, 128.9, 127.9, 127.0, 125.8, 108.7, 106.3, 61.4, 53.4, 40.9, 30.7, 14.3, 13.6. HRMS (m/z): $[\mathrm{M}+\mathrm{H}]^{+}$calcd for $\mathrm{C}_{27} \mathrm{H}_{28} \mathrm{~N}_{3} \mathrm{O}_{3}, 442.2131$; found, 442.2121 . 


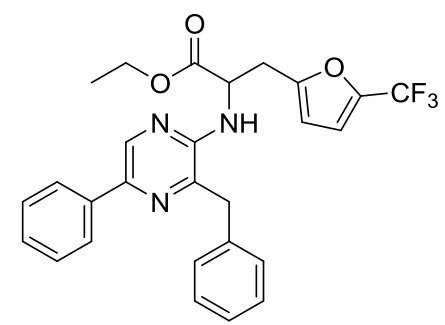

Ethyl 2-((3-benzyl-5-phenylpyrazin-2-yl)amino)-3-(5-(trifluoromethyl)furan-2-yl)propanoate $\quad \mathbf{2 3}\{1,1,43\}$ (EC31093-037-2): Obtained as an oil (0.17 g, 44\%) after a chromatography over silica gel (cyclohexane-ethyl acetate 93/7). ${ }^{1} \mathrm{H}$ NMR $\left(\mathrm{CDCl}_{3}\right): 8.40(\mathrm{~s}, 1 \mathrm{H}), 7.99-7.92(\mathrm{~m}, 2 \mathrm{H}), 7.50-7.42(\mathrm{~m}, 2 \mathrm{H}), 7.40-7.33(\mathrm{~m}, 1 \mathrm{H}), 7.33$ $-7.20(\mathrm{~m}, 5 \mathrm{H}), 6.55(\mathrm{dd}, 1 \mathrm{H}, J=3.3,1.2 \mathrm{~Hz}), 5.74(\mathrm{dd}, 1 \mathrm{H}, J=3.3,0.6 \mathrm{~Hz}), 5.12(\mathrm{~d}, 1 \mathrm{H}, J=7.1 \mathrm{~Hz}), 4.98(\mathrm{dt}, 1 \mathrm{H}$, $J=7.1,5.2 \mathrm{~Hz}), 4.26-4.09(\mathrm{~m}, 4 \mathrm{H}), 3.29(\mathrm{dd}, 1 \mathrm{H}, J=15.2,5.2 \mathrm{~Hz}), 3.19(\mathrm{dd}, 1 \mathrm{H}, J=15.2,5.2 \mathrm{~Hz}), 1.24(\mathrm{t}, 3 \mathrm{H}, J$ $=7.1 \mathrm{~Hz}) .{ }^{13} \mathrm{C} \mathrm{NMR}\left(\mathrm{CDCl}_{3}\right): 171.6,154.0,150.0,141.5(23 \mathrm{~Hz}), 141.4(15 \mathrm{~Hz}), 141.1(43 \mathrm{~Hz}), 137.6,136.9$, 136.7, 129.0, 128.9, 128.8, 128.0, 127.1, 125.8, 119.2 (267 Hz), 112.5 (3 Hz), 108.8, 61.9, 52.9, 41.1, 30.6, 14.1. HRMS (m/z): $[\mathrm{M}+\mathrm{H}]^{+}$calcd for $\mathrm{C}_{27} \mathrm{H}_{25} \mathrm{~F}_{3} \mathrm{~N}_{3} \mathrm{O}_{3}$, 496.1848; found, 496.1847.

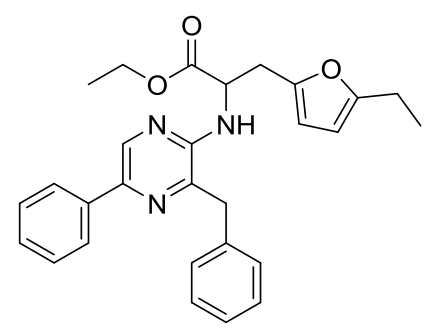

Ethyl 2-((3-benzyl-5-phenylpyrazin-2-yl)amino)-3-(5-ethylfuran-2-yl)propanoate $23\{1,1,44\} \quad$ (EC31092-085-2): Obtained as a yellow solid $(0.48 \mathrm{~g}, 74 \%)$ after a chromatography over silica gel (cyclohexane-ethyl acetate 95:5). ${ }^{1} \mathrm{H}$ NMR $\left(\mathrm{CDCl}_{3}\right): 8.39(\mathrm{~s}, 1 \mathrm{H}), 7.96-7.89(\mathrm{~m}, 2 \mathrm{H}), 7.50-7.40(\mathrm{~m}, 2 \mathrm{H}), 7.37-7.26(\mathrm{~m}, 4 \mathrm{H}), 7.26-7.20(\mathrm{~m}$, $2 \mathrm{H}), 5.80(\mathrm{dd}, 1 \mathrm{H}, J=2.0,1.0 \mathrm{~Hz}), 5.74(\mathrm{~d}, 1 \mathrm{H}, J=3.0 \mathrm{~Hz}), 5.09(\mathrm{~d}, 1 \mathrm{H}, J=7.5 \mathrm{~Hz}), 4.91(\mathrm{dt}, 1 \mathrm{H}, J=7.5,5.4$ $\mathrm{Hz}), 4.22-4.08(\mathrm{~m}, 4 \mathrm{H}), 3.14(\mathrm{~d}, 2 \mathrm{H}, J=5.3 \mathrm{~Hz}), 2.56(\mathrm{qd}, 2 \mathrm{H}, J=7.5,0.6 \mathrm{~Hz}), 1.21(\mathrm{t}, 3 \mathrm{H}, J=7.3 \mathrm{~Hz}), 1.19(\mathrm{t}$, $3 \mathrm{H}, J=7.3 \mathrm{~Hz}) .{ }^{13} \mathrm{C} \mathrm{NMR}\left(\mathrm{CDCl}_{3}\right): 172.3,157.3,150.5,148.6,141.3,141.2,137.7,137.0,136.8,128.9$ (three signals), 127.9, 127.0, 125.8, 108.5, 104.7, 61.4, 53.3, 40.9, 30.8, 21.5, 14.3, 12.2. HRMS ( $\mathrm{m} / \mathrm{z}):[\mathrm{M}+\mathrm{H}]^{+}$calcd for $\mathrm{C}_{28} \mathrm{H}_{30} \mathrm{~N}_{3} \mathrm{O}_{3}, 457.2319$; found, 457.2314.

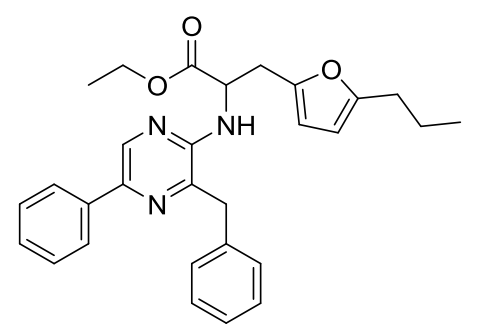

Ethyl 2-((3-benzyl-5-phenylpyrazin-2-yl)amino)-3-(5-propylfuran-2-yl)propanoate 23 1,1,45\} (EC32712-117-1): This compound was obtained (using toluene at $\left.90^{\circ} \mathrm{C}\right)$ as an oil $(0.30 \mathrm{~g}, 71 \%)$ after a chromatography over silica gel (cyclohexane-ethyl acetate 97:3). ${ }^{1} \mathrm{H}$ NMR $\left(\mathrm{CDCl}_{3}\right): 8.42(\mathrm{~s}, 1 \mathrm{H}), 7.95(\mathrm{~m}, 2 \mathrm{H}), 7.47(\mathrm{~m}, 2 \mathrm{H}), 7.31(\mathrm{~m}, 6 \mathrm{H})$, $5.83(\mathrm{~d}, 1 \mathrm{H}, J=3.0 \mathrm{~Hz}), 5.76(\mathrm{~d}, 1 \mathrm{H}, J=3.0 \mathrm{~Hz}), 5.12(\mathrm{~d}, 1 \mathrm{H}, J=7.5 \mathrm{~Hz}), 4.94(\mathrm{dt}, 1 \mathrm{H}, J=7.5,5.3 \mathrm{~Hz}), 4.18(\mathrm{~m}$, $4 \mathrm{H}), 3.16(\mathrm{~d}, 2 \mathrm{H}, J=5.3 \mathrm{~Hz}), 2.53(\mathrm{t}, 2 \mathrm{H}, J=7.6 \mathrm{~Hz}), 1.65(\mathrm{~h}, 2 \mathrm{H}, J=7.4 \mathrm{~Hz}), 1.24(\mathrm{t}, 3 \mathrm{H}, J=7.1 \mathrm{~Hz}), 0.97(\mathrm{t}$, $3 \mathrm{H}, J=7.4 \mathrm{~Hz}) .{ }^{13} \mathrm{C} \mathrm{NMR}\left(\mathrm{CDCl}_{3}\right): 172.1,155.7,150.3,148.4,141.1,141.0,137.5,136.8,136.6,128.8,128.7$, $127.7,126.8,125.6,108.3,105.5,61.2,53.2,40.7,30.6,30.0,21.3,14.1,13.7$, (one signal missing). HRMS $(\mathrm{m} / \mathrm{z})$ : $[\mathrm{M}+\mathrm{H}]^{+}$calcd for $\mathrm{C}_{29} \mathrm{H}_{32} \mathrm{~N}_{3} \mathrm{O}_{3}: 470.2444$; found, 470.2445 . 


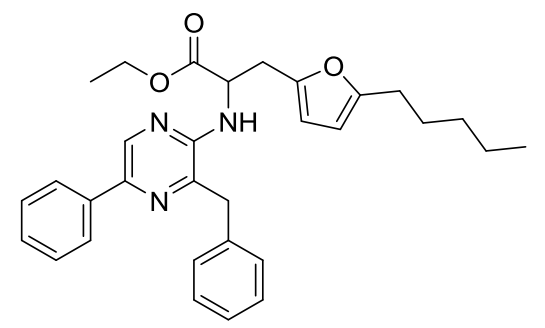

Ethyl 2-((3-benzyl-5-phenylpyrazin-2-yl)amino)-3-(5-pentylfuran-2-yl)propanoate propanoate $23\{1,1,46\}$ (YJ 31776-021-2): Obtained as an oil $(0.30 \mathrm{~g}, 60 \%)$ after two chromatography over silica gel (cyclohexane-ethyl acetate 97/3). ${ }^{1} \mathrm{H}$ NMR $\left(\mathrm{CDCl}_{3}\right): 8.41(\mathrm{~s}, 1 \mathrm{H}), 7.94(\mathrm{~m}, 2 \mathrm{H}), 7.47(\mathrm{~m}, 2 \mathrm{H}), 7.37(\mathrm{~m}, 1 \mathrm{H}), 7.38-7.23(\mathrm{~m}, 5 \mathrm{H}), 5.82$ $(\mathrm{m}, 1 \mathrm{H}), 5.75(\mathrm{~d}, 1 \mathrm{H}, J=3.1 \mathrm{~Hz}), 5.14(\mathrm{~d}(\mathrm{br}), J=7.3 \mathrm{~Hz}), 4.93(\mathrm{~m}, 1 \mathrm{H}), 4.17(\mathrm{~m}, 4 \mathrm{H}), 3.16(\mathrm{~d}, 2 \mathrm{H}, J=5.3 \mathrm{~Hz})$, $2.54(\mathrm{t}, 2 \mathrm{H}, J=7.6 \mathrm{~Hz}), 1.62(\mathrm{~m}, 2 \mathrm{H}), 1.34(\mathrm{~m}, 4 \mathrm{H}), 1.24(\mathrm{~d}, 3 \mathrm{H}, J=7.0 \mathrm{~Hz}), 0.92(\mathrm{t}, 3 \mathrm{H}, J=6.9 \mathrm{~Hz}) .{ }^{13} \mathrm{C} \mathrm{NMR}$ $\left(\mathrm{CDCl}_{3}\right): 172.0,156.0,150.2,148.3,141.3,141.0,137.4,136.6,136.5,128.8,128.7$ (two signals), 127.8, 126.8, 125.6, 108.4, 105.3, 61.2, 53.2, 40.7, 31.3, 30.6, 28.0, 27.7, 22.4, 14.1, 14.0. HRMS $(\mathrm{m} / \mathrm{z}):[\mathrm{M}+\mathrm{H}]^{+}$calcd for $\mathrm{C}_{31} \mathrm{H}_{36} \mathrm{~N}_{3} \mathrm{O}_{3}$ : 498.2557; found, 498.2743.

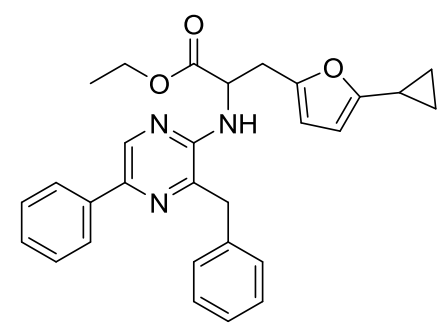

Ethyl 2-((3-benzyl-5-phenylpyrazin-2-yl)amino)-3-(5-cyclopropylfuran-2-yl)propanoate $23\{1,1,47\} \quad$ (EC31093075-2): Obtained as an oil (0.31 g, 67\%) after a chromatography over silica gel (cyclohexane-ethyl acetate 95/5). ${ }^{1} \mathrm{H}$ NMR $\left(\mathrm{CDCl}_{3}\right): 8.39(\mathrm{~s}, 1 \mathrm{H}), 7.97-7.90(\mathrm{~m}, 2 \mathrm{H}), 7.48-7.41(\mathrm{~m}, 2 \mathrm{H}), 7.38-7.20(\mathrm{~m}, 6 \mathrm{H}), 5.78(\mathrm{dd}, 1 \mathrm{H}, J=$ $3.1,0.5 \mathrm{~Hz}), 5.72(\mathrm{~d}, 1 \mathrm{H}, J=3.1 \mathrm{~Hz}), 5.08(\mathrm{~d}, 1 \mathrm{H}, J=7.6 \mathrm{~Hz}), 4.90(\mathrm{dt}, 1 \mathrm{H}, J=7.6,5.3 \mathrm{~Hz}), 4.20-4.11(\mathrm{~m}, 4 \mathrm{H})$, $3.12(\mathrm{~d}, 2 \mathrm{H}, J=5.3 \mathrm{~Hz}), 1.81(\mathrm{tt}, 1 \mathrm{H}, J=8.5,5.1 \mathrm{~Hz}), 1.22(\mathrm{t}, 3 \mathrm{H}, J=7.1 \mathrm{~Hz}), 0.88-0.80(\mathrm{~m}, 2 \mathrm{H}), 0.73-0.65$ $(\mathrm{m}, 2 \mathrm{H}) .{ }^{13} \mathrm{C}$ NMR $\left(\mathrm{CDCl}_{3}\right): 172.2,156.9,150.5,148.3,141.3,141.2,137.7,136.9,136.7,128.9$ (three signals), 127.9, 127.0, 125.8, 108.7, 104.3, 61.4, 53.2, 40.9, 30.8, 14.3, 8.9, 6.6.HRMS $(\mathrm{m} / \mathrm{z})$ : $[\mathrm{M}+\mathrm{H}]^{+}$calcd for $\mathrm{C}_{29} \mathrm{H}_{30} \mathrm{~N}_{3} \mathrm{O}_{3}$, 468.2287; found, 468.2291 .

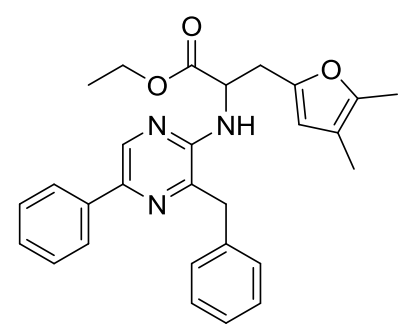

Ethyl 2-((3-benzyl-5-phenylpyrazin-2-yl)amino)-3-(4,5-dimethylfuran-2-yl)propanoate 23\{1,1,48\} (EC31092-1013): Obtained as an oil $(0.20 \mathrm{~g}, 49 \%)$ after a chromatography over silica gel (cyclohexane-ethyl acetate 95:5). ${ }^{1} \mathrm{H}$ NMR $\left(\mathrm{CDCl}_{3}\right): 8.39(\mathrm{~s}, 1 \mathrm{H}), 7.97-7.90(\mathrm{~m}, 2 \mathrm{H}), 7.50-7.40(\mathrm{~m}, 2 \mathrm{H}), 7.39-7.19(\mathrm{~m}, 6 \mathrm{H}), 5.65(\mathrm{~s}, 1 \mathrm{H}), 5.11(\mathrm{~d}$, $1 \mathrm{H}, J=7.5 \mathrm{~Hz}), 4.88(\mathrm{dt}, 1 \mathrm{H}, J=7.5,5.4 \mathrm{~Hz}), 4.19-4.12(\mathrm{~m}, 4 \mathrm{H}), 3.09(\mathrm{~d}, 2 \mathrm{H}, J=5.4 \mathrm{~Hz}), 2.12(\mathrm{~s}, 3 \mathrm{H}), 1.87(\mathrm{~s}$, $3 \mathrm{H}), 1.21(\mathrm{t}, 3 \mathrm{H}, J=7.1 \mathrm{~Hz}) .{ }^{13} \mathrm{C}$ NMR $\left(\mathrm{CDCl}_{3}\right): 172.3,150.6,147.4,146.8,141.3,141.2,137.7,137.0,136.8$, $128.9,128.8,127.9,126.9,125.8,114.5,111.2$ (two signals), 61.3, 53.4, 40.8, 30.7, 14.2, 11.4, 10.0. HRMS (m/z): $[\mathrm{M}+\mathrm{H}]^{+}$calcd for $\mathrm{C}_{28} \mathrm{H}_{30} \mathrm{~N}_{3} \mathrm{O}_{3}, 456.2287$; found, 456.2337. 


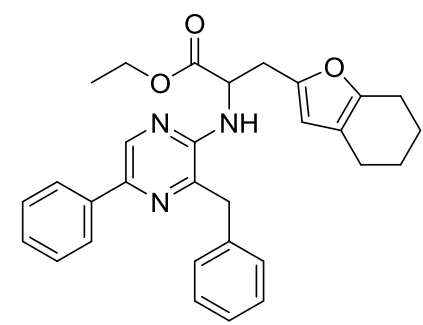

Ethyl 2-((3-benzyl-5-phenylpyrazin-2-yl)amino)-3-(4,5,6,7-tetrahydrobenzofuran-2-yl)propanoate 23 1,1,49\} (YJ 33068-073-2): Obtained as an oil $(0.38 \mathrm{~g}, 73 \%)$, using toluene at $90{ }^{\circ} \mathrm{C}$, after a chromatography over silica gel (cyclohexane-ethyl acetate 97:3). ${ }^{1} \mathrm{H}$ NMR $\left(\mathrm{CDCl}_{3}\right): 8.40(\mathrm{~s}, 1 \mathrm{H}), 7.95(\mathrm{~m}, 2 \mathrm{H}), 7.46(\mathrm{~m}, 2 \mathrm{H}), 7.36(\mathrm{~m}, 1 \mathrm{H}), 7.32$ $7.23(\mathrm{~m}, 5 \mathrm{H}), 5.68(\mathrm{~s}, 1 \mathrm{H}), 5.17(\mathrm{~d}(\mathrm{br}), J=7.6 \mathrm{~Hz}), 4.91(\mathrm{~m}, 1 \mathrm{H}), 4.17(\mathrm{~m}, 4 \mathrm{H}), 3.13(\mathrm{~m}, 2 \mathrm{H}), 2.52(\mathrm{~m}, 2 \mathrm{H}), 2.35$ $(\mathrm{m}, 2 \mathrm{H}), 1.82(\mathrm{~m}, 2 \mathrm{H}), 1.72(\mathrm{~m}, 2 \mathrm{H}), 1.22(\mathrm{t}, 3 \mathrm{H}, J=7.2 \mathrm{~Hz}) .{ }^{13} \mathrm{C} \mathrm{NMR}\left(\mathrm{CDCl}_{3}\right): 172.1,150.2,150.1,147.8$, 141.3, 141.0, 137.4, 136.6, 136.5, 128.7 (three signals), 127.8, 126.8, 125.6, 117.4, 108.8, 61.2, 53.4, 40.7, 30.7, 23.1, 23.0 (two signals), 22.1, 14.1. HRMS $\left(\mathrm{m} / \mathrm{z}\right.$ ): $[\mathrm{M}+\mathrm{H}]^{+}$calcd for $\mathrm{C}_{30} \mathrm{H}_{32} \mathrm{~N}_{3} \mathrm{O}_{3}, 482.2444$; found, 482.2428 .

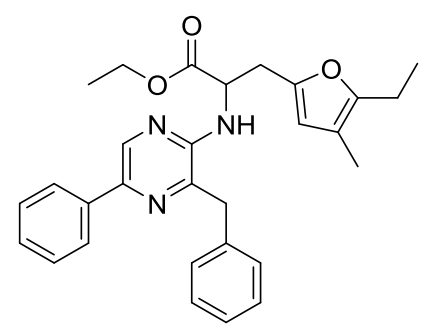

Ethyl 2-((3-benzyl-5-phenylpyrazin-2-yl)amino)-3-(5-ethyl-4-methylfuran-2-yl)propanoate 23 $\{1,1,50\}$ (YJ 33069007-1): Obtained as an oil $(0.63 \mathrm{~g}, 76 \%)$, using toluene at $90{ }^{\circ} \mathrm{C}$, after a chromatography over silica gel (cyclohexane-ethyl acetate 97:3). ${ }^{1} \mathrm{H}$ NMR $\left(\mathrm{CDCl}_{3}\right): 8.41(\mathrm{~s}, 1 \mathrm{H}), 7.95(\mathrm{~m}, 2 \mathrm{H}), 7.46(\mathrm{~m}, 2 \mathrm{H}), 7.37(\mathrm{~m}, 1 \mathrm{H}), 7.38$ $7.23(\mathrm{~m}, 5 \mathrm{H}), 5.66(\mathrm{~s}, 1 \mathrm{H}), 5.14(\mathrm{~d}(\mathrm{br}), J=7.4 \mathrm{~Hz}), 4.92(\mathrm{~m}, 1 \mathrm{H}), 4.18(\mathrm{~m}, 4 \mathrm{H}), 3.13(\mathrm{~m}, 2 \mathrm{H}), 2.52(\mathrm{q}, 2 \mathrm{H}, J=7.5$ $\mathrm{Hz}), 1.91(\mathrm{~s}, 3 \mathrm{H}), 1.24$ (t, $3 \mathrm{H} J=7.1 \mathrm{~Hz}), 1.20(\mathrm{t}, 3 \mathrm{H} J=7.5 \mathrm{~Hz}) .{ }^{13} \mathrm{C}$ NMR $\left(\mathrm{CDCl}_{3}\right): 172.1,151.9,150.3,147.2$, 141.2, 141.0, 137.5, 136.6, 128.7 (four signals), 127.8, 126.8, 125.6, 113.5, 111.1, 61.2, 53.2, 40.7, 30.6, 19.3, 14.1, 12.9, 9.7. HRMS $(m / z):[\mathrm{M}+\mathrm{H}]^{+}$calcd for $\mathrm{C}_{29} \mathrm{H}_{32} \mathrm{~N}_{3} \mathrm{O}_{3}, 470.2444$; found, 470.2450 .

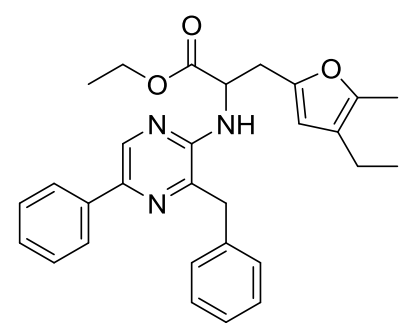

Ethyl 2-((3-benzyl-5-phenylpyrazin-2-yl)amino)-3-(4-ethyl-5-methylfuran-2-yl)propanoate 23 $\{1,1,51\}$ (YJ 33068077-2): Obtained as an oil $(0.32 \mathrm{~g}, 63 \%)$, using toluene at $90{ }^{\circ} \mathrm{C}$, after a chromatography over silica gel (cyclohexane-ethyl acetate 97:3). ${ }^{1} \mathrm{H}$ NMR $\left(\mathrm{CDCl}_{3}\right): 8.41(\mathrm{~s}, 1 \mathrm{H}), 7.95(\mathrm{~m}, 2 \mathrm{H}), 7.46(\mathrm{~m}, 2 \mathrm{H}), 7.37(\mathrm{~m}, 1 \mathrm{H}), 7.32$ $7.23(\mathrm{~m}, 5 \mathrm{H}), 5.76(\mathrm{~s}, 1 \mathrm{H}), 5.15(\mathrm{~d}(\mathrm{br}), J=7.7 \mathrm{~Hz}), 4.91(\mathrm{~m}, 1 \mathrm{H}), 4.17(\mathrm{~m}, 4 \mathrm{H}), 3.11(\mathrm{~m}, 2 \mathrm{H}), 2.30(\mathrm{q}, 2 \mathrm{H}, J=7.6$ $\mathrm{Hz}), 2.16(\mathrm{~s}, 3 \mathrm{H}), 1.22(\mathrm{~d}, 3 \mathrm{H} J=7.0 \mathrm{~Hz}), 1.11(\mathrm{~d}, 3 \mathrm{H} J=7.6 \mathrm{~Hz}) .{ }^{13} \mathrm{C} \mathrm{NMR}\left(\mathrm{CDCl}_{3}\right): 172.1,150.3,147.4,145.9$, 141.2, 141.0, 137.5, 136.6, 136.5, 128.7 (four signals), 127.8, 126.8, 125.6, 121.2, 109.5, 61.2, 53.3, 40.6, 30.7, 18.0, 14.9, 14.1, 11.4. HRMS $(\mathrm{m} / z)$ : $[\mathrm{M}+\mathrm{H}]^{+}$calcd for $\mathrm{C}_{29} \mathrm{H}_{32} \mathrm{~N}_{3} \mathrm{O}_{3}$, 470.2444; found, 470.2425.

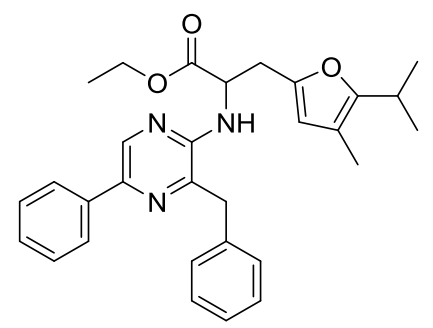


Ethyl 2-((3-benzyl-5-phenylpyrazin-2-yl)amino)-3-(5-isopropyl-4-methylfuran-2-yl)propanoate $\mathbf{2 3}\{1,1,52\}$ (YJ 33068-095-1): Obtained as an oil (0.47 g, 85\%), using toluene at $90{ }^{\circ} \mathrm{C}$, after a chromatography over silica gel (cyclohexane-ethyl acetate 97:3). ${ }^{1} \mathrm{H}$ NMR $\left(\mathrm{CDCl}_{3}\right)$ : $8.41(\mathrm{~s}, 1 \mathrm{H}), 7.94(\mathrm{~m}, 2 \mathrm{H}), 7.46(\mathrm{~m}, 2 \mathrm{H}), 7.37(\mathrm{~m}, 1 \mathrm{H}), 7.38$ $7.23(\mathrm{~m}, 5 \mathrm{H}), 5.63(\mathrm{~s}, 1 \mathrm{H}), 5.10(\mathrm{~d}(\mathrm{br}), J=7.4 \mathrm{~Hz}), 4.93(\mathrm{~m}, 1 \mathrm{H}), 4.18(\mathrm{~m}, 4 \mathrm{H}), 3.13(\mathrm{~m}, 2 \mathrm{H}), 2.94(\mathrm{sept}, 1 \mathrm{H}, J=$ $6.5 \mathrm{~Hz}), 1.91(\mathrm{~s}, 3 \mathrm{H}), 1.24(\mathrm{~d}, 3 \mathrm{H} J=7.0 \mathrm{~Hz}), 1.20(\mathrm{~d}, 3 \mathrm{H} J=6.5 \mathrm{~Hz}) .{ }^{13} \mathrm{C} \mathrm{NMR}\left(\mathrm{CDCl}_{3}\right): 172.1,154.9,150.3$, 146.8, 141.2, 141.0, 137.5, 136.5, 128.7 (four signals), 127.8, 126.8, 125.6, 112.3, 111.2, 61.2, 53.1, 40.6, 30.6, 26.1, 21.4, 21.3, 11.1, 9.7. HRMS (m/z): $[\mathrm{M}+\mathrm{H}]^{+}$calcd for $\mathrm{C}_{30} \mathrm{H}_{34} \mathrm{~N}_{3} \mathrm{O}_{3}$, 484.2600; found, 484.2609.

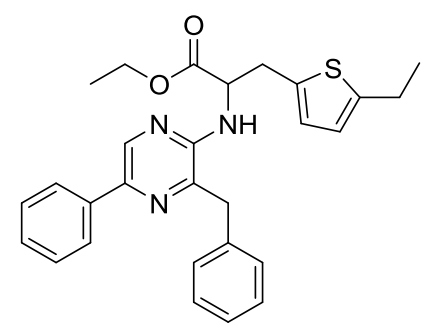

Ethyl 2-((3-benzyl-5-phenylpyrazin-2-yl)amino)-3-(5-ethylthiophen-2-yl)propanoate 23 1,1,53\} (YJ30367-165-2): Obtained as an oil $(0.47 \mathrm{~g}, 84 \%)$ after a chromatography over silica gel (cyclohexane-ethyl acetate $95: 5) .{ }^{1} \mathrm{H}$ NMR $\left(\mathrm{CDCl}_{3}\right): 8.43(\mathrm{~s}, 1 \mathrm{H}), 7.97(\mathrm{~m}, 2 \mathrm{H}), 7.47(\mathrm{~m}, 2 \mathrm{H}), 7.37(\mathrm{~m}, 1 \mathrm{H}), 7.33-7.23(\mathrm{~m}, 5 \mathrm{H}), 6.55(\mathrm{~m}, 1 \mathrm{H}), 6.39(\mathrm{~m}, 1 \mathrm{H})$, $5.13(\mathrm{~d}, 1 \mathrm{H}, J=7.1 \mathrm{~Hz}), 4.91(\mathrm{~m}, 1 \mathrm{H}), 4.17(\mathrm{~m}, 4 \mathrm{H}), 3.34(\mathrm{~m}, 2 \mathrm{H}), 2.79(\mathrm{qd}, 2 \mathrm{H}, J=0.8,7.5 \mathrm{~Hz}), 1.30(\mathrm{t}, 3 \mathrm{H}, J=$ $7.5 \mathrm{~Hz}), 1.24(\mathrm{t}, 3 \mathrm{H}, J=7.3 \mathrm{~Hz}) .{ }^{13} \mathrm{C} \mathrm{NMR}\left(\mathrm{CDCl}_{3}\right): 171.9,150.2,146.7,141.3,141.1,137.5,136.7,136.6,134.9$, 128.8, 128,7 (two signals), 127.8, 126.8, 126.2, 125.6, 123.0, 61.3, 54.6, 40.8, 32.2, 23.4, 15.8, 14.1. HRMS ( $\mathrm{m} / \mathrm{z})$ : $[\mathrm{M}+\mathrm{H}]^{+}$calcd for $\mathrm{C}_{28} \mathrm{H}_{30} \mathrm{~N}_{3} \mathrm{O}_{2} \mathrm{~S}, 472.2059$; found, 472.2055 .

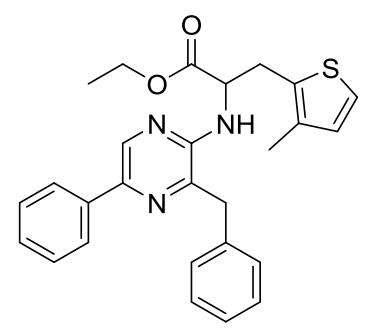

Ethyl 2-((3-benzyl-5-phenylpyrazin-2-yl)amino)-3-(3-methylthiophen-2-yl)propanoate 23 1,1,54\} (YJ30367-1672): Obtained as an oil $(0.41 \mathrm{~g}, 76 \%)$ after a chromatography over silica gel (cyclohexane-ethyl acetate $95: 5) .{ }^{1} \mathrm{H}$ NMR $\left(\mathrm{CDCl}_{3}\right): 8.44(\mathrm{~s}, 1 \mathrm{H}), 7.97(\mathrm{~m}, 2 \mathrm{H}), 7.47(\mathrm{~m}, 2 \mathrm{H}), 7.37(\mathrm{~m}, 1 \mathrm{H}), 7.33-7.23(\mathrm{~m}, 5 \mathrm{H}), 7.05(\mathrm{~d}, 1 \mathrm{H}, J=4.8$ $\mathrm{Hz}), 6.75(\mathrm{~d}, 1 \mathrm{H}, J=4.8 \mathrm{~Hz}), 5.15(\mathrm{~d}, 1 \mathrm{H}, J=7.1 \mathrm{~Hz}), 4.96(\mathrm{~m}, 1 \mathrm{H}), 4.15(\mathrm{~m}, 4 \mathrm{H}), 3.41(\mathrm{dd}, 1 \mathrm{H}, J=5.1,15.1 \mathrm{~Hz})$, $3.31(\mathrm{dd}, 1 \mathrm{H}, J=5.1,15.1 \mathrm{~Hz}), 2.0(\mathrm{~s}, 3 \mathrm{H}), 1.23(\mathrm{t}, 3 \mathrm{H}, J=7.3 \mathrm{~Hz}) .{ }^{13} \mathrm{C}$ NMR $\left(\mathrm{CDCl}_{3}\right): 172.0,150.3,141.4,141.1$, 137.5, 136.7, 136.5, 135.2, 131.2, 129.8, 128,8, 128.7 (two signals), 127.8, 126.8, 125.6, 122.8, 61.4, 54.7, 40.8, 29.8, 14.0, 13.6. HRMS (m/z): $[\mathrm{M}+\mathrm{H}]^{+}$calcd for $\mathrm{C}_{27} \mathrm{H}_{28} \mathrm{~N}_{3} \mathrm{O}_{2} \mathrm{~S}, 458.1902$; found, 458.1910.

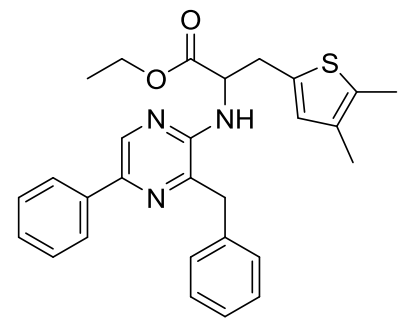

Ethyl 2-((3-benzyl-5-phenylpyrazin-2-yl)amino)-3-(4,5-dimethylthiophen-2-yl)propanoate 23\{1,1,55\} (YJ31067103-2): Obtained as an oil (0.24 g, 47\%) after a chromatography over silica gel (cyclohexane-ethyl acetate 96:4). ${ }^{1} \mathrm{H}$ NMR $\left(\mathrm{CDCl}_{3}\right): 8.42(\mathrm{~s}, 1 \mathrm{H}), 7.95(\mathrm{~m}, 2 \mathrm{H}), 7.47(\mathrm{~m}, 2 \mathrm{H}), 7.36(\mathrm{~m}, 1 \mathrm{H}), 7.28(\mathrm{~m}, 5 \mathrm{H}), 6.33(\mathrm{~s}, 1 \mathrm{H}), 5.12(\mathrm{~d}, 1 \mathrm{H}$, $J=7.3 \mathrm{~Hz}), 4.92(\mathrm{dt}, 1 \mathrm{H}, J=7.2,5.0 \mathrm{~Hz}), 4.18(\mathrm{~m}, 4 \mathrm{H}), 3.32(\mathrm{dd}, 1 \mathrm{H}, J=4.7,14.8 \mathrm{~Hz}), 3.26(\mathrm{dd}, 1 \mathrm{H}, J=5.4,14.8$ $\mathrm{Hz}), 2.28(\mathrm{~s}, 3 \mathrm{H}), 2.06(\mathrm{~s}, 3 \mathrm{H}), 1.24(\mathrm{t}, 3 \mathrm{H}, J=7.1 \mathrm{~Hz}) .{ }^{13} \mathrm{C} \mathrm{NMR}\left(\mathrm{CDCl}_{3}\right): 171.9,150.3,141.3,141.1,137.5$, 136.7, 136.6, 132.6, 132.5, 132.0, 129.6, 128.8, 128.7, 127.8, 126.8, 125.6, 61.3, 54.6, 40.7, 32.0, 14.1, $13.5,12.0$ (one signal missing). HRMS (m/z): $[\mathrm{M}+\mathrm{H}]^{+}$calcd for $\mathrm{C}_{28} \mathrm{H}_{30} \mathrm{~N}_{3} \mathrm{O}_{2} \mathrm{~S}, 472.2059$; found, 472.2054 . 


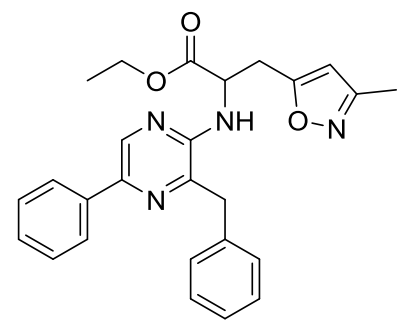

Ethyl 2-((3-benzyl-5-phenylpyrazin-2-yl)amino)-3-(3-methylisoxazol-5-yl)propanoate 23\{1,1,56\} (EC32712-0272): Obtained as a yellow oil $(0.17 \mathrm{~g}, 48 \%)$ after a chromatography over silica gel (cyclohexane-ethyl acetate 5:1).

${ }^{1} \mathrm{H} \mathrm{NMR}\left(\mathrm{CDCl}_{3}\right): 8.43(\mathrm{~s}, 1 \mathrm{H}), 7.97(\mathrm{~m}, 2 \mathrm{H}), 7.48(\mathrm{~m}, 2 \mathrm{H}), 7.33(\mathrm{~m}, 6 \mathrm{H}), 5.48(\mathrm{~s}, 1 \mathrm{H}), 5.20(\mathrm{~d}, 1 \mathrm{H}, J=6.9 \mathrm{~Hz})$, $5.01(\mathrm{dt}, 1 \mathrm{H}, J=6.9,5.2 \mathrm{~Hz}), 4.19(\mathrm{~m}, 4 \mathrm{H}), 3.41(\mathrm{dd}, 1 \mathrm{H}, J=15.4,5.1 \mathrm{~Hz}), 3.28(\mathrm{dd}, 1 \mathrm{H}, J=15.4,5.3 \mathrm{~Hz}), 2.22$ $(\mathrm{s}, 3 \mathrm{H}), 1.25(\mathrm{t}, 3 \mathrm{H}, J=7.1 \mathrm{~Hz}) .{ }^{13} \mathrm{C} \mathrm{NMR}\left(\mathrm{CDCl}_{3}\right): 171.2,167.9,159.6,149.9,141.5,141.4,137.3,136.7,136.5$, $128.9,128.8,128.7,127.9,126.9,125.7,103.2,61.8,52.4,41.0,29.0,14.1,11.4$. HRMS $(\mathrm{m} / z):[\mathrm{M}+\mathrm{H}]^{+}$calcd for $\mathrm{C}_{26} \mathrm{H}_{27} \mathrm{~N}_{4} \mathrm{O}_{3}$ : 443.2083; found, 443.2092.

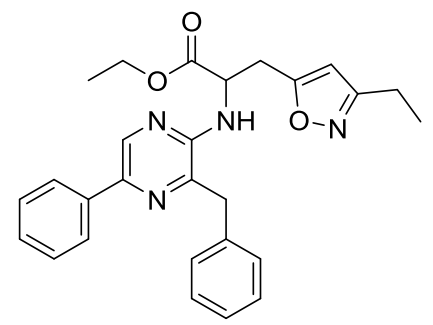

Ethyl 2-((3-benzyl-5-phenylpyrazin-2-yl)amino)-3-(3-ethylisoxazol-5-yl)propanoate 23 1,1,57\} (EC32712-027-2): Obtained as a yellow oil $(0.29 \mathrm{~g}, 50 \%)$ after a chromatography over silica gel (cyclohexane-ethyl acetate $5: 1)$. ${ }^{1} \mathrm{H}$ NMR $\left(\mathrm{CDCl}_{3}\right): 8.42(\mathrm{~s}, 1 \mathrm{H}), 7.96(\mathrm{~m}, 2 \mathrm{H}), 7.48(\mathrm{~m}, 2 \mathrm{H}), 7.33(\mathrm{~m}, 6 \mathrm{H}), 5.57(\mathrm{~s}, 1 \mathrm{H}), 5.20(\mathrm{~d}, 1 \mathrm{H}, J=7.0 \mathrm{~Hz}), 5.02$ $(\mathrm{dt}, 1 \mathrm{H}, J=7.0,5.3 \mathrm{~Hz}), 4.17(\mathrm{~m}, 4 \mathrm{H}), 3.40(\mathrm{dd}, 1 \mathrm{H}, J=15.3,5.1 \mathrm{~Hz}), 3.28(\mathrm{dd}, 1 \mathrm{H}, J=15.3,5.4 \mathrm{~Hz}), 2.62(\mathrm{qd}$, $2 \mathrm{H}, J=7.6,1.7 \mathrm{~Hz}), 1.23(\mathrm{~m}, 6 \mathrm{H}) .{ }^{13} \mathrm{C} \mathrm{NMR}\left(\mathrm{CDCl}_{3}\right): 171.3,167.8,165.0,149.9,141.5,141.4,137.3,136.7$, $136.5,128.8,128.8,128.7,127.9,126.9,125.7,101.9,61.8,52.5,40.9,29.2,19.5,14.1,12.5$. HRMS $(\mathrm{m} / \mathrm{z})$ : $[\mathrm{M}+\mathrm{H}]^{+}$calcd for $\mathrm{C}_{27} \mathrm{H}_{29} \mathrm{~N}_{4} \mathrm{O}_{3}: 457.2240$; found, 457.2244 .

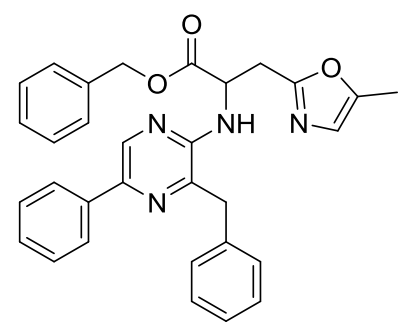

Benzyl 2-((3-benzyl-5-phenylpyrazin-2-yl)amino)-3-(5-methyloxazol-2-yl)propanoate 23 1,1,58\} (YJ31776-0872): Obtained a wax $(0.37 \mathrm{~g})$ still containing some BINAP after a chromatography over silica gel (cyclohexaneethyl acetate 99:1 to 3/2). ${ }^{1} \mathrm{H}$ NMR $\left(\mathrm{CDCl}_{3}\right): 8.37(\mathrm{~s}, 1 \mathrm{H}), 7.94(\mathrm{~m}, 2 \mathrm{H}), 7.48(\mathrm{~m}, 2 \mathrm{H}), 7.39-7.21(\mathrm{~m}, 13 \mathrm{H}), 6.58$ $(\mathrm{m}, 1 \mathrm{H}), 5.78(\mathrm{~d}(\mathrm{br}), 1 \mathrm{H}, \mathrm{J}=7.30 \mathrm{~Hz}), 5.16(\mathrm{~m}, 3 \mathrm{H}), 4.19(\mathrm{~s}, 2 \mathrm{H}), 3.37(\mathrm{dd}, 1 \mathrm{H}, J=5.6,15.8 \mathrm{~Hz}), 3.28(\mathrm{dd}, 1 \mathrm{H}, J=$ $5.2,15.8 \mathrm{~Hz}), 2.22(\mathrm{~d}, 3 \mathrm{H}, J=1.0 \mathrm{~Hz})$. This was used directly in the next step.<smiles>CCOC(=O)C(Cc1nc(C)c(C)o1)Nc1cc(Cc2ccccc2)cc(-c2ccccc2)n1</smiles> 
Ethyl 2-((3-benzyl-5-phenylpyrazin-2-yl)amino)-3-(4,5-dimethyloxazol-2-yl)propanoate 23\{1,1,59\} (YJ31067045-2): Obtained as an oil $(0.20 \mathrm{~g}, 49 \%)$ after a chromatography over silica gel (cyclohexane-ethyl acetate $97: 3$ to 3/1). ${ }^{1} \mathrm{H} \mathrm{NMR}\left(\mathrm{CDCl}_{3}\right): 8.39(\mathrm{~s}, 1 \mathrm{H}), 7.93(\mathrm{~m}, 2 \mathrm{H}), 7.46(\mathrm{~m}, 2 \mathrm{H}), 7.37-7.21(\mathrm{~m}, 6 \mathrm{H}), 5.75(\mathrm{~d}, 1 \mathrm{H}, J=7.8 \mathrm{~Hz})$, $5.07(\mathrm{~m}, 1 \mathrm{H}), 4.20(\mathrm{~m}, 2 \mathrm{H}), 4.15(\mathrm{q}, 2 \mathrm{H}, J=7.1 \mathrm{~Hz}), 3.30(\mathrm{dd}, 1 \mathrm{H}, J=5.6$ and $15.3 \mathrm{~Hz}), 3.22(\mathrm{dd}, 1 \mathrm{H}, J=5.4 \mathrm{and}$ $15.3 \mathrm{~Hz}), 2.17(\mathrm{~s}, 3 \mathrm{H}), 2.05(\mathrm{~s}, 3 \mathrm{H}), 1.18(\mathrm{t}, 3 \mathrm{H}, J=7.1 \mathrm{~Hz}) .{ }^{13} \mathrm{C}$ NMR $\left(\mathrm{CDCl}_{3}\right): 171.6,157.8,150.3,143.2,141.4$, 141.2, 137.5, 136.8, 136.7, 130.4, 128.8, 128.7, 128.6, 127.7, 126.7, 125.6, 61.4, 52.0, 40.5, 30.4, 14.0, 11.1, 9.8. HRMS (m/z): $[\mathrm{M}+\mathrm{H}]^{+}$calcd for $\mathrm{C}_{27} \mathrm{H}_{29} \mathrm{~N}_{4} \mathrm{O}_{3}, 457.2240$; found, 457.2234.

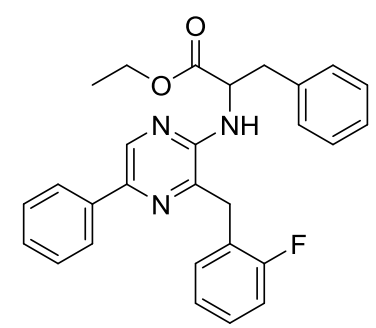

Ethyl (3-(2-fluorobenzyl)-5-phenylpyrazin-2-yl)phenylalaninate 23\{1,2,1\} (GG30532-117-1): Obtained as an oil $(0.19 \mathrm{~g}, 40 \%)$ after a chromatography over silica gel (cyclohexane-ethyl acetate 94/6). ${ }^{1} \mathrm{H}$ NMR $\left(\mathrm{CDCl}_{3}\right): 8.44(\mathrm{~s}$, $1 \mathrm{H})$, 7.94-7.91 (m, 2H), 7.48-7.44 (m, 2H), 7.38-7.34 (m, 1H), 7.28-7.22 (m, 5H), 7.11-7.05 (m, 4H), 5.10-5.08 (m, $1 \mathrm{H}), 5.04-4.99(\mathrm{~m}, 1 \mathrm{H}), 4.20-4.09(\mathrm{~m}, 4 \mathrm{H}), 3.28-3.23(\mathrm{~m}, 1 \mathrm{H}), 3.18-3.13(\mathrm{~m}, 1 \mathrm{H}), 1.23-1.20(\mathrm{t}, 3 \mathrm{H}, J=7.9 \mathrm{~Hz})$. ${ }^{13} \mathrm{C} \mathrm{NMR}\left(\mathrm{CDCl}_{3}\right)$ : 172.4, 161.9, 159.5, 150.1, 141.1, 140.0, 137.4, 136.9, 136.3, 130.8, 129.2, 128.7, 128.6, 128.5, $128.4,127.8,126.9,125.6,124.4,124.3,123.5,115.4,61.2,55.1,38.0,32.6,30.2,14.1 . \mathrm{HRMS}(\mathrm{m} / \mathrm{z}):[\mathrm{M}+\mathrm{H}]^{+}$ calcd for $\mathrm{C}_{28} \mathrm{H}_{27} \mathrm{FN}_{3} \mathrm{O}_{2}$, 456.2087; found, 456.2096 .

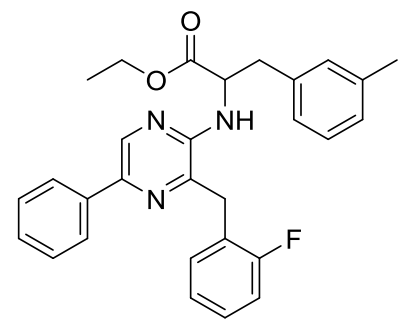

Ethyl 2-((3-(2-fluorobenzyl)-5-phenylpyrazin-2-yl)amino)-3-(m-tolyl)propanoate 23\{1,2,12\} (YJ31070-037-1): Obtained as an oil $(0.34 \mathrm{~g}, 69 \%)$ after a chromatography over silica gel (cyclohexane-ethyl acetate $95 / 5) .{ }^{1} \mathrm{H}$ NMR $\left(\mathrm{CDCl}_{3}\right): 8.42(\mathrm{~s}, 1 \mathrm{H}), 7.91(\mathrm{~m}, 2 \mathrm{H}), 7.45(\mathrm{~m}, 2 \mathrm{H}), 7.36(\mathrm{~m}, 1 \mathrm{H}), 7.23(\mathrm{~m}, 2 \mathrm{H}), 7.16-7.04(\mathrm{~m}, 4 \mathrm{H}), 6.95(\mathrm{~m}, 1 \mathrm{H})$, $6.88(\mathrm{~m}, 1 \mathrm{H}), 5.06(\mathrm{~d}(\mathrm{br}), 1 \mathrm{H}, J=7.3 \mathrm{~Hz}), 4.97(\mathrm{~m}, 1 \mathrm{H}), 4.15(\mathrm{~m}, 4 \mathrm{H}), 3.20(\mathrm{dd}, 1 \mathrm{H}, J=5.5,13.7 \mathrm{~Hz}), 3.10(\mathrm{dd}$, $1 \mathrm{H}, J=6.7,13.7 \mathrm{~Hz}), 2.31(\mathrm{~s}, 3 \mathrm{H}), 1.21(\mathrm{t}, 3 \mathrm{H}, J=7.1 \mathrm{~Hz}) .{ }^{13} \mathrm{C}$ NMR $\left(\mathrm{CDCl}_{3}\right): 172.5,160.7(244 \mathrm{~Hz}), 150.2$, $141.1,139.9,138.0,137.4,136.9,136.2,130.8$ (4 Hz), 130.0, 128.7, $128.5(8 \mathrm{~Hz}), 128.4,127.8,127.7,126.2$, 125.6, 124.3 (4 Hz), $123.5(15 \mathrm{~Hz}), 115.3(22 \mathrm{~Hz}), 61.1,55.2,37.9,32.6(3 \mathrm{~Hz}), 21.3,14.1 . \mathrm{HRMS}(\mathrm{m} / \mathrm{z}):[\mathrm{M}+\mathrm{H}]^{+}$ calcd for $\mathrm{C}_{29} \mathrm{H}_{29} \mathrm{FN}_{3} \mathrm{O}_{2}, 470.2244$, found, 470.2253.

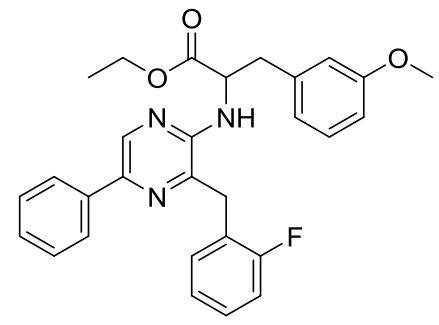

Ethyl 2-((3-(2-fluorobenzyl)-5-phenylpyrazin-2-yl)amino)-3-(3-methoxyphenyl)propanoate 23 1,2,15\} (YJ31070039-1): Obtained as an oil (0.32 g, 63\%) after a chromatography over silica gel (cyclohexane-ethyl acetate 94/6). ${ }^{1} \mathrm{H}$ NMR $\left(\mathrm{CDCl}_{3}\right): 8.42(\mathrm{~s}, 1 \mathrm{H}), 7.91(\mathrm{~m}, 2 \mathrm{H}), 7.45(\mathrm{~m}, 2 \mathrm{H}), 7.36(\mathrm{~m}, 1 \mathrm{H}), 7.23(\mathrm{~m}, 2 \mathrm{H}), 7.16(\mathrm{~m}, 1 \mathrm{H}), 7.06(\mathrm{~m}$, 2H), $6.78(\mathrm{~m}, 1 \mathrm{H}), 6.68(\mathrm{~m}, 2 \mathrm{H}), 5.08(\mathrm{~d}(\mathrm{br}), 1 \mathrm{H}, J=7.2 \mathrm{~Hz}), 4.99(\mathrm{~m}, 1 \mathrm{H}), 4.16(\mathrm{~m}, 4 \mathrm{H}), 3.77(\mathrm{~s}, 3 \mathrm{H}), 3.23(\mathrm{dd}$, $1 \mathrm{H}, J=5.6,13.9 \mathrm{~Hz}), 3.12(\mathrm{dd}, 1 \mathrm{H}, J=6.4,13.9 \mathrm{~Hz}), 1.22(\mathrm{t}, 3 \mathrm{H}, J=7.1 \mathrm{~Hz}) .{ }^{13} \mathrm{C} \mathrm{NMR}\left(\mathrm{CDCl}_{3}\right): 172.5,160.7$ (244 Hz), 159.6, 150.2, 141.1, 140.0, 137.9, 137.4, 136.9, 130.8 (4 Hz), 129.4, 128.7, 128.5 (8 Hz), 127.8, 127.8, 
125.6, 124.3 (4 Hz), 123.4 (15 Hz), 121.5, 115.3 (22 Hz), 114.8, 112.4, 61.2, 55.1 (two signals), 38.0, 32.6 (3 Hz), 14.1. HRMS $(\mathrm{m} / z):[\mathrm{M}+\mathrm{H}]^{+}$calcd for $\mathrm{C}_{29} \mathrm{H}_{29} \mathrm{FN}_{3} \mathrm{O}_{3}, 486.2193$, found, 486.2182 .

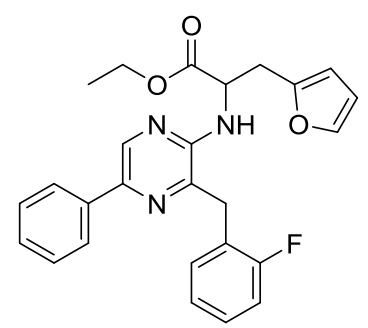

Ethyl 2-((3-(2-fluorobenzyl)-5-phenylpyrazin-2-yl)amino)-3-(furan-2-yl)propanoate 23 1,2,37\} (YJ31070-041-1): Obtained as an oil $(0.29 \mathrm{~g}, 62 \%)$ after a chromatography over silica gel (cyclohexane-ethyl acetate $95 / 5) .{ }^{1} \mathrm{H} \mathrm{NMR}$ $\left(\mathrm{CDCl}_{3}\right): 8.42(\mathrm{~s}, 1 \mathrm{H}), 7.92(\mathrm{~m}, 2 \mathrm{H}), 7.45(\mathrm{~m}, 2 \mathrm{H}), 7.36(\mathrm{~m}, 1 \mathrm{H}), 7.26(\mathrm{~m}, 3 \mathrm{H}), 7.09(\mathrm{~m}, 2 \mathrm{H}), 6.24(\mathrm{dd}, 1 \mathrm{H}, J=1.9$, $3.2 \mathrm{~Hz}), 5.95(\mathrm{~d}, 1 \mathrm{H}, J=3.1 \mathrm{~Hz}), 5.28(\mathrm{~d}(\mathrm{br}), 1 \mathrm{H}, J=7.6 \mathrm{~Hz}), 5.01(\mathrm{~m}, 1 \mathrm{H}), 4.19(\mathrm{~m}, 4 \mathrm{H}), 3.77(\mathrm{~s}, 3 \mathrm{H}), 3.26(\mathrm{~m}$, 2H), $1.24(\mathrm{t}, 3 \mathrm{H}, J=7.1 \mathrm{~Hz}) .{ }^{13} \mathrm{C} \mathrm{NMR}\left(\mathrm{CDCl}_{3}\right): 172.0,160.8(244 \mathrm{~Hz}), 150.6,150.1,142.0,141.1,140.0,137.4$, 136.8, 130.8 (4 Hz), 128.7, 128.5 (8 Hz), 127.8, 125.6, $124.3(4 \mathrm{~Hz}), 123.5$ (15 Hz), $115.3(22 \mathrm{~Hz}), 110.3,107.7$, 61.3, 53.3, $32.6(3 \mathrm{~Hz}), 30.5,14.1$. HRMS $(\mathrm{m} / z)$ : $[\mathrm{M}+\mathrm{H}]^{+}$calcd for $\mathrm{C}_{26} \mathrm{H}_{25} \mathrm{FN}_{3} \mathrm{O}_{3}, 446.1880$, found, 446.1873.

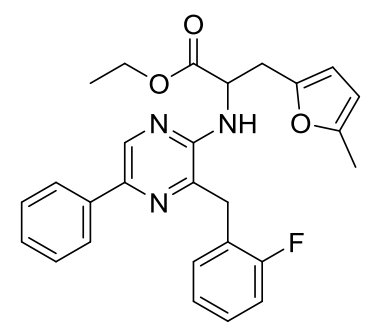

Ethyl 2-((3-(2-fluorobenzyl)-5-phenylpyrazin-2-yl)amino)-3-(5-methylfuran-2-yl)propanoate $\quad \mathbf{2 3}\{1,2,42\}$ (YJ31070-043-1): Obtained as an oil $(0.23 \mathrm{~g}, 48 \%)$ after a chromatography over silica gel (cyclohexane-ethyl acetate 95/5). ${ }^{1} \mathrm{H} \mathrm{NMR}\left(\mathrm{CDCl}_{3}\right): 8.42(\mathrm{~s}, 1 \mathrm{H}), 7.91(\mathrm{~m}, 2 \mathrm{H}), 7.45(\mathrm{~m}, 2 \mathrm{H}), 7.36(\mathrm{~m}, 1 \mathrm{H}), 7.25(\mathrm{~m}, 2 \mathrm{H}), 7.08(\mathrm{~m}$, 2H), $5.82(\mathrm{~m}, 2 \mathrm{H}), 5.27(\mathrm{~d}(\mathrm{br}), 1 \mathrm{H}, J=7.6 \mathrm{~Hz}), 4.97(\mathrm{~m}, 1 \mathrm{H}), 4.19(\mathrm{~m}, 4 \mathrm{H}), 3.77(\mathrm{~s}, 3 \mathrm{H}), 3.20(\mathrm{~d}, 2 \mathrm{H}, J=5.8 \mathrm{~Hz})$, $2.23(\mathrm{~s}, 3 \mathrm{H}), 1.24(\mathrm{t}, 3 \mathrm{H}, J=7.1 \mathrm{~Hz}) .{ }^{13} \mathrm{C} \mathrm{NMR}\left(\mathrm{CDCl}_{3}\right): 172.0,160.8(244 \mathrm{~Hz}), 151.5,150.1,148.6,141.1,139.9$, 137.4, 136.8, 130.8 (4 Hz), 128.7, 128.5 (8 Hz), 127.8, 125.6, 124.3 (4 Hz), $123.6(15 \mathrm{~Hz}), 115.3(22 \mathrm{~Hz}), 108.5$, 106.1, 61.2, 53.4, 32.5 (3 Hz), 30.6, 14.1, 13.4. HRMS $(\mathrm{m} / \mathrm{z})$ : $[\mathrm{M}+\mathrm{H}]^{+}$calcd for $\mathrm{C}_{27} \mathrm{H}_{27} \mathrm{FN}_{3} \mathrm{O}_{3}$, 460.2036, found, 460.2044 .

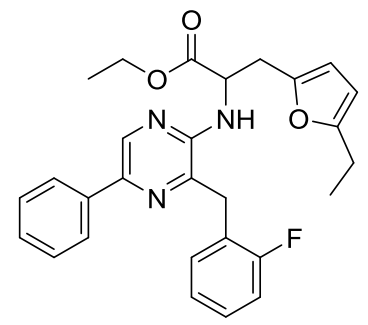

Ethyl 3-(5-ethylfuran-2-yl)-2-((3-(2-fluorobenzyl)-5-phenylpyrazin-2-yl)amino)propanoate 23\{1,2,44\} (YJ31068011-1): Obtained an oil $(0.19 \mathrm{~g}, 46 \%)$ after a chromatography over silica gel (cyclohexane-ethyl acetate $95 / 5) .{ }^{1} \mathrm{H}$ NMR $\left(\mathrm{CDCl}_{3}\right): 8.41(\mathrm{~s}, 1 \mathrm{H}), 7.91(\mathrm{~m}, 2 \mathrm{H}), 7.46(\mathrm{~m}, 2 \mathrm{H}), 7.34(\mathrm{~m}, 1 \mathrm{H}), 7.25(\mathrm{~m}, 2 \mathrm{H}), 7.07(\mathrm{~m}, 2 \mathrm{H}), 5.82(\mathrm{~m}, 2 \mathrm{H})$, $5.27(\mathrm{~d}(\mathrm{br}), 1 \mathrm{H}, J=7.3 \mathrm{~Hz}), 4.97(\mathrm{~m}, 1 \mathrm{H}), 4.20(\mathrm{~m}, 4 \mathrm{H}), 3.77(\mathrm{~s}, 3 \mathrm{H}), 3.20(\mathrm{~d}, 2 \mathrm{H}, J=5.1 \mathrm{~Hz}), 2.57$ (q, $2 \mathrm{H}, J=7.5$ $\mathrm{Hz}), 1.25(\mathrm{t}, 3 \mathrm{H}, J=7.1 \mathrm{~Hz}), 1.20(\mathrm{t}, 3 \mathrm{H}, J=7.5 \mathrm{~Hz}) .{ }^{13} \mathrm{C} \mathrm{NMR}\left(\mathrm{CDCl}_{3}\right): 172.0,160.8(244 \mathrm{~Hz}), 157.3,150.1$, 148.4, 141.1, 139.9, 137.4, 136.8, 130.8 (4 Hz), 128.7, 128.5 (8 Hz), 127.8, 125.6, $124.3(4 \mathrm{~Hz}), 123.6$ (15 Hz), $115.2(22 \mathrm{~Hz}), 108.3,104.5,61.2,53.4,32.5(3 \mathrm{~Hz}), 30.7,21.3,14.1,12.1$. HRMS $(\mathrm{m} / z):[\mathrm{M}+\mathrm{H}]^{+}$calcd for $\mathrm{C}_{28} \mathrm{H}_{29} \mathrm{FN}_{3} \mathrm{O}_{3}, 474.2193$, found, 474.2198. 


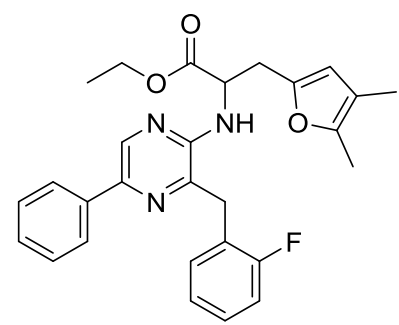

Ethyl 3-(4,5-dimethylfuran-2-yl)-2-((3-(2-fluorobenzyl)-5-phenylpyrazin-2-yl)amino)propanoate $\quad \mathbf{2 3}\{1,2,48\}$ (EC32712-113-1): Obtained an oil (0.32 g, 81\%) after a chromatography over silica gel (cyclohexane-ethyl acetate 97/3). ${ }^{1} \mathrm{H}$ NMR $\left(\mathrm{CDCl}_{3}\right): 8.41(\mathrm{~s}, 1 \mathrm{H}), 7.91(\mathrm{dd}, 2 \mathrm{H}, J=7.2,1.6 \mathrm{~Hz}), 7.44(\mathrm{~m}, 2 \mathrm{H}), 7.35(\mathrm{~m}, 1 \mathrm{H}), 7.25(\mathrm{~m}, 2 \mathrm{H})$, $7.08(\mathrm{~m}, 2 \mathrm{H}), 5.74(\mathrm{~s}, 1 \mathrm{H}, 1 \mathrm{H}), 5.26(\mathrm{~d}, J=7.4 \mathrm{~Hz}), 4.94(\mathrm{dt}, 1 \mathrm{H}, J=7.7,5.5 \mathrm{~Hz}), 4.20(\mathrm{~m}, 4 \mathrm{H}), 3.15(\mathrm{~d}, 2 \mathrm{H}, J=$ $5.5 \mathrm{~Hz}), 2.13(\mathrm{~s}, 3 \mathrm{H}), 1.88(\mathrm{~s}, 3 \mathrm{H}), 1.25(\mathrm{t}, 3 \mathrm{H}, J=7.1 \mathrm{~Hz}) .{ }^{13} \mathrm{C} \mathrm{NMR}\left(\mathrm{CDCl}_{3}\right): 172.1,160.8(244 \mathrm{~Hz}), 150.2$, 147.2, 146.7, 141.1, 139.9, 137.4, 136.9, 130.8 (4 Hz), 128.7, $128.4(8 \mathrm{~Hz}), 127.7,125.5,124.2(4 \mathrm{~Hz}), 123.7(16$ $\mathrm{Hz}), 115.2(22 \mathrm{~Hz}), 114.4,111.0,61.2,53.4,32.5(3 \mathrm{~Hz}), 30.6,14.1,11.2,9.8$. HRMS $(\mathrm{m} / \mathrm{z}):[\mathrm{M}+\mathrm{H}]^{+}$calcd for $\mathrm{C}_{28} \mathrm{H}_{29} \mathrm{FN}_{3} \mathrm{O}_{3}: 474.2193$; found, 474.2194 .

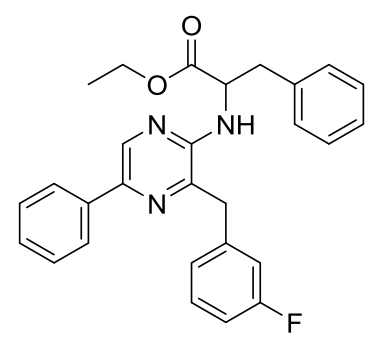

Ethyl (3-(3-fluorobenzyl)-5-phenylpyrazin-2-yl)phenylalaninate 23\{1,3,1\} (YJ31067-149-1): Obtained as an oil $(0.40 \mathrm{~g}, 57 \%)$ after a chromatography over silica gel (cyclohexane-ethyl acetate 94:6). ${ }^{1} \mathrm{H}$ NMR $\left(\mathrm{CDCl}_{3}\right)$ : 8.45 (s, $1 \mathrm{H}), 7.95(\mathrm{~m}, 2 \mathrm{H}), 7.48(\mathrm{~m}, 2 \mathrm{H}), 7.38(\mathrm{~m}, 1 \mathrm{H}), 7.36-7.21(\mathrm{~m}, 4 \mathrm{H}), 7.10-6.92(\mathrm{~m}, 5 \mathrm{H}), 5.02(\mathrm{~m}, 1 \mathrm{H}), 4.85(\mathrm{~d}(\mathrm{br})$, $1 \mathrm{H}, J=7.5 \mathrm{~Hz}), 4.16(\mathrm{q}, 2 \mathrm{H}, J=7.2 \mathrm{~Hz}), 4.11(\mathrm{~s}, 2 \mathrm{H}), 3.21(\mathrm{dd}, 1 \mathrm{H}, J=5.4,13.8 \mathrm{~Hz}), 3.13(\mathrm{dd}, 1 \mathrm{H}, J=5.9,13.8$ $\mathrm{Hz}), 1.22(\mathrm{t}, 3 \mathrm{H}, J=7.1 \mathrm{~Hz}) .{ }^{13} \mathrm{C}$ NMR $\left(\mathrm{CDCl}_{3}\right): 172.4,163.0(246 \mathrm{~Hz}), 150.3,141.3,140.3,139.1(7 \mathrm{~Hz}), 137.3$, 137.1, 136.1, $130.1(8 \mathrm{~Hz}), 129.2,129.0,128.4,127.9,127.0,125.6,124.2(2 \mathrm{~Hz}), 115.5(22 \mathrm{~Hz}), 113.9(21 \mathrm{~Hz})$, 61.2, 54.8, 40.3, 37.7, 14.1. HRMS $(\mathrm{m} / \mathrm{z})$ : $[\mathrm{M}+\mathrm{H}]^{+}$calcd for $\mathrm{C}_{28} \mathrm{H}_{27} \mathrm{FN}_{3} \mathrm{O}_{2}$, 456.2087; found, 456.2067.

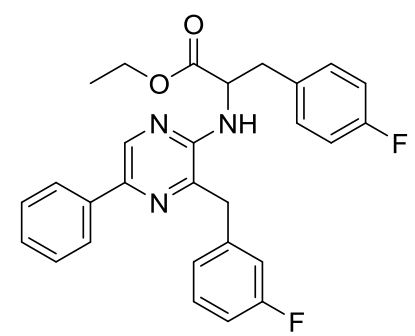

Ethyl 2-((3-(3-fluorobenzyl)-5-phenylpyrazin-2-yl)amino)-3-(4-fluorophenyl)propanoate $23\{1,3,4\}$ (YJ 31069025-3): Obtained as an oil (0.42 g, 58\%) after a chromatography over silica gel (cyclohexane-ethyl acetate 95:5). ${ }^{1} \mathrm{H}$ NMR $\left(\mathrm{CDCl}_{3}\right) 8.45(\mathrm{~s}, 1 \mathrm{H}), 7.95(\mathrm{~m}, 2 \mathrm{H}), 7.48(\mathrm{~m}, 2 \mathrm{H}), 7.38(\mathrm{~m}, 1 \mathrm{H}), 7.24(\mathrm{~m}, 1 \mathrm{H}), 7.01-6.86(\mathrm{~m}, 7 \mathrm{H}), 4.97$ $(\mathrm{m}, 1 \mathrm{H}), 4.19(\mathrm{~d}(\mathrm{br}), 1 \mathrm{H}, \mathrm{J}=6.9 \mathrm{~Hz}), 4.16(\mathrm{~m}, 4 \mathrm{H}), 3.20(\mathrm{dd}, 1 \mathrm{H}, \mathrm{J}=14.1$ and $5.5 \mathrm{~Hz}), 3.09(\mathrm{dd}, 1 \mathrm{H}, \mathrm{J}=14.1$ and $5.5 \mathrm{~Hz}), 1.24(\mathrm{t}, 3 \mathrm{H}, \mathrm{J}=7.0 \mathrm{~Hz}) .{ }^{13} \mathrm{C} \mathrm{NMR}\left(\mathrm{CDCl}_{3}\right): 172.2,163.0(246 \mathrm{~Hz}), 161.9(246 \mathrm{~Hz}), 150.1,141.3,139.0(7$ $\mathrm{Hz}), 137.2,137.0,131.8(3 \mathrm{~Hz}), 130.6(8 \mathrm{~Hz}), 130.3(8 \mathrm{~Hz}), 128.8,127.8,125.7,124.2(2 \mathrm{~Hz}), 115.5(21 \mathrm{~Hz})$, $115.2(21 \mathrm{~Hz}), 113.9(21 \mathrm{~Hz}), 61.4,54.8,40.5,36.8,14.1$. HRMS $(\mathrm{m} / \mathrm{z}):[\mathrm{M}+\mathrm{H}]^{+}$calcd for $\mathrm{C}_{28} \mathrm{H}_{26} \mathrm{~F}_{2} \mathrm{~N}_{3} \mathrm{O}_{2}$ : 474.1993; found, 474.1928. 


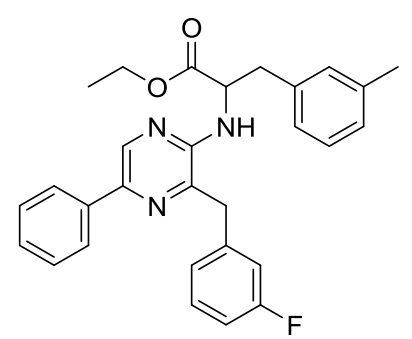

Ethyl 2-((3-(3-fluorobenzyl)-5-phenylpyrazin-2-yl)amino)-3-(m-tolyl)propanoate 23\{1,3,12\} (YJ31068-041-1): Obtained as an oil $(0.35 \mathrm{~g}, 74 \%)$ after a chromatography over silica gel (cyclohexane-ethyl acetate 95:5). ${ }^{1} \mathrm{H}$ NMR $\left(\mathrm{CDCl}_{3}\right): 8.45(\mathrm{~s}, 1 \mathrm{H}), 7.94(\mathrm{~m}, 2 \mathrm{H}), 7.47(\mathrm{~m}, 2 \mathrm{H}), 7.37(\mathrm{~m}, 1 \mathrm{H}), 7.23(\mathrm{~m}, 1 \mathrm{H}), 7.14(\mathrm{~m}, 1 \mathrm{H}), 7.07(\mathrm{~m}, 1 \mathrm{H}), 6.93$ $(\mathrm{m}, 3 \mathrm{H}), 6.81(\mathrm{~m}, 1 \mathrm{H}), 4.96(\mathrm{~m}, 1 \mathrm{H}), 4.82(\mathrm{~d}(\mathrm{br}), 1 \mathrm{H}, J=7.4), 4.18(\mathrm{~m}, 4 \mathrm{H}), 3.17(\mathrm{dd}, 1 \mathrm{H}, J=5.5,13.7), 3.09$ (dd, $1 \mathrm{H}, J=6.2,13.7), 2.32(\mathrm{~s}, 3 \mathrm{H}), 1.21(\mathrm{t}, 3 \mathrm{H} J=7.1)$. HRMS $(\mathrm{m} / \mathrm{z}):[\mathrm{M}+\mathrm{H}]^{+}$calcd for $\mathrm{C}_{29} \mathrm{H}_{29} \mathrm{FN}_{3} \mathrm{O}_{2}: 470.2244$; found, 470.2249 .

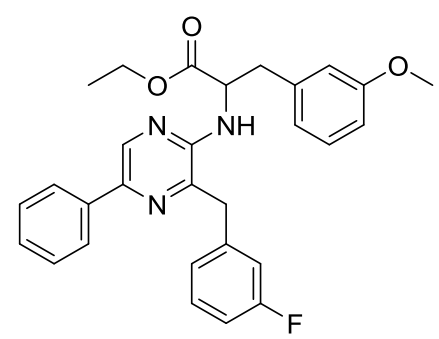

Ethyl 2-((3-(3-fluorobenzyl)-5-phenylpyrazin-2-yl)amino)-3-(3-methoxyphenyl)propanoate 23\{1,3,15\} (YJ31068043-1): Obtained as an oil (0.22 g, 45\%) after a chromatography over silica gel (cyclohexane-ethyl acetate 95:5). ${ }^{1} \mathrm{H}$ NMR $\left(\mathrm{CDCl}_{3}\right): 8.44(\mathrm{~s}, 1 \mathrm{H}), 7.94(\mathrm{~m}, 2 \mathrm{H}), 7.47(\mathrm{~m}, 2 \mathrm{H}), 7.36(\mathrm{~m}, 1 \mathrm{H}), 7.19(\mathrm{~m}, 2 \mathrm{H}), 6.93(\mathrm{~m}, 3 \mathrm{H}), 6.79(\mathrm{~m}$, $1 \mathrm{H}), 6.60(\mathrm{~m}, 2 \mathrm{H}), 4.97(\mathrm{~m}, 1 \mathrm{H}), 4.81(\mathrm{~d}(\mathrm{br}), 1 \mathrm{H}, J=7.7), 4.18(\mathrm{~m}, 4 \mathrm{H}), 3.77$ (s, 3H), 3.19 (dd, 1H, $J=5.7,13.8)$, $3.10(\mathrm{dd}, 1 \mathrm{H}, J=5.9,13.8), 1.21(\mathrm{t}, 3 \mathrm{H} J=7.1)$. HRMS $(\mathrm{m} / \mathrm{z}):[\mathrm{M}+\mathrm{H}]^{+}$calcd for $\mathrm{C}_{29} \mathrm{H}_{29} \mathrm{FN}_{3} \mathrm{O}_{3}$ : 486.2193; found, 486.2209 .

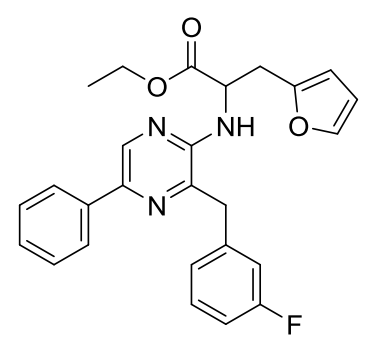

Ethyl 2-((3-(3-fluorobenzyl)-5-phenylpyrazin-2-yl)amino)-3-(furan-2-yl)propanoate 23 1,3,37\} (YJ31070-031-2): Obtained as an oil $(0.22 \mathrm{~g}, 57 \%)$ after a chromatography over silica gel (cyclohexane-ethyl acetate 95:5). ${ }^{1} \mathrm{H}$ NMR $\left(\mathrm{CDCl}_{3}\right): 8.44(\mathrm{~s}, 1 \mathrm{H}), 7.95(\mathrm{~m}, 2 \mathrm{H}), 7.48(\mathrm{~m}, 2 \mathrm{H}), 7.37(\mathrm{~m}, 1 \mathrm{H}), 7.28(\mathrm{~m}, 2 \mathrm{H}), 7.06(\mathrm{~m}, 1 \mathrm{H}), 6.96(\mathrm{~m}, 2 \mathrm{H}), 6.24$ $(\mathrm{dd}, 1 \mathrm{H}, J=2.0,3.2 \mathrm{~Hz}), 5.89(\mathrm{dd}, 1 \mathrm{H}, J=0.7,3.2 \mathrm{~Hz}), 5.08(\mathrm{~d}(\mathrm{br}), 1 \mathrm{H}, J=7.5 \mathrm{~Hz}), 4.87(\mathrm{~m}, 1 \mathrm{H}), 4.18(\mathrm{~m}, 4 \mathrm{H})$, $3.24(\mathrm{~d}, 2 \mathrm{H}, J=5.3 \mathrm{~Hz}), 1.23(\mathrm{t}, 3 \mathrm{H}, J=7.1 \mathrm{~Hz}) .{ }^{13} \mathrm{C} \mathrm{NMR}\left(\mathrm{CDCl}_{3}\right): 171.9,163.0(246 \mathrm{~Hz}), 150.5,150.2,142.0$, 141.3, 140.4, 139.1 (7 Hz), 137.3, 137.0, 130.1 (8 Hz), 128.7, 127.9, 125.6, $124.2(2 \mathrm{~Hz}), 115.6(22 \mathrm{~Hz}), 113.8(21$ $\mathrm{Hz}), 110.3,107.8,61.4,53.2,40.4,30.4,14.1$. HRMS $(\mathrm{m} / \mathrm{z}):[\mathrm{M}+\mathrm{H}]^{+}$calcd for $\mathrm{C}_{26} \mathrm{H}_{25} \mathrm{FN}_{3} \mathrm{O}_{3}$, 446.1880; found, 446.1883 .<smiles>CCOC(=O)C(Cc1ccc(C)o1)Nc1ncc(-c2ccccc2)nc1Cc1cccc(F)c1</smiles> 

acetate 95:5). ${ }^{1} \mathrm{H}$ NMR $\left(\mathrm{CDCl}_{3}\right): 8.43(\mathrm{~s}, 1 \mathrm{H}), 7.93(\mathrm{~m}, 2 \mathrm{H}), 7.47(\mathrm{~m}, 2 \mathrm{H}), 7.37(\mathrm{~m}, 1 \mathrm{H}), 7.28(\mathrm{~m}, 1 \mathrm{H}), 7.06(\mathrm{~m}$, $1 \mathrm{H}), 6.96(\mathrm{~m}, 2 \mathrm{H}), 5.82(\mathrm{~m}, 1 \mathrm{H}), 5.78(\mathrm{~m}, 1 \mathrm{H}), 5.06(\mathrm{~d}(\mathrm{br}), 1 \mathrm{H}, J=7.9 \mathrm{~Hz}), 4.93(\mathrm{~m}, 1 \mathrm{H}), 4.18(\mathrm{~m}, 4 \mathrm{H}), 3.20(\mathrm{~d}$, $2 \mathrm{H}, J=5.2 \mathrm{~Hz}), 2.22(\mathrm{~s}, 3 \mathrm{H}), 1.24(\mathrm{t}, 3 \mathrm{H}, J=7.1 \mathrm{~Hz}) .{ }^{13} \mathrm{C} \mathrm{NMR}\left(\mathrm{CDCl}_{3}\right): 172.0,163.0(244 \mathrm{~Hz}), 151.5,150.3$, 148.4, 141.2, 140.4, $139.1(7 \mathrm{~Hz}), 137.3,137.0,130.1(8 \mathrm{~Hz}), 128.8,127.9,125.6,124.3(2 \mathrm{~Hz}), 115.6(22 \mathrm{~Hz})$, $113.8(21 \mathrm{~Hz}), 108.6,106.2,61.3,53.2,40.3(2 \mathrm{~Hz}), 30.5,14.1,13.4$. HRMS $(\mathrm{m} / z):[\mathrm{M}+\mathrm{H}]^{+}$calcd for $\mathrm{C}_{27} \mathrm{H}_{27} \mathrm{FN}_{3} \mathrm{O}_{3}, 460.2036$; found, 460.2039 .

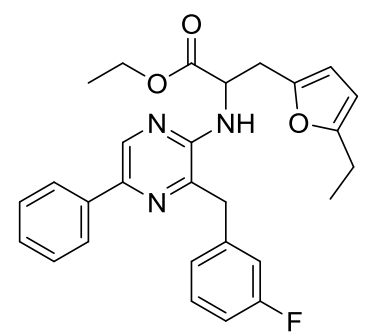

Ethyl 3-(5-ethylfuran-2-yl)-2-((3-(3-fluorobenzyl)-5-phenylpyrazin-2-yl)amino)propanoate 23\{1,3,44\} (YJ31068015-1): Obtained as an oil (0.20 g, 90\% pure) after a chromatography over silica gel (cyclohexane-ethyl acetate 95:5). ${ }^{1} \mathrm{H} \mathrm{NMR}\left(\mathrm{CDCl}_{3}\right): 8.43(\mathrm{~s}, 1 \mathrm{H}), 7.94(\mathrm{~m}, 2 \mathrm{H}), 7.47(\mathrm{~m}, 2 \mathrm{H}), 7.37(\mathrm{~m}, 1 \mathrm{H}), 7.27(\mathrm{~m}, 1 \mathrm{H}), 7.06(\mathrm{~m}, 1 \mathrm{H}), 6.96$ $(\mathrm{m}, 2 \mathrm{H}), 5.82(\mathrm{~m}, 1 \mathrm{H}), 5.78(\mathrm{~m}, 1 \mathrm{H}), 5.06(\mathrm{~d}(\mathrm{br}), 1 \mathrm{H}, J=7.7 \mathrm{~Hz}), 4.94(\mathrm{~m}, 1 \mathrm{H}), 4.18(\mathrm{~m}, 4 \mathrm{H}), 3.20(\mathrm{~d}, 2 \mathrm{H}, J=5.1$ $\mathrm{Hz}$ ), 2.57 (q, 2H, $J=7.6 \mathrm{~Hz}), 1.22(\mathrm{~m}, 6 \mathrm{H})$. HRMS $(\mathrm{m} / \mathrm{z}):[\mathrm{M}+\mathrm{H}]^{+}$calcd for $\mathrm{C}_{28} \mathrm{H}_{29} \mathrm{FN}_{3} \mathrm{O}_{3}$, 474.2193; found, 474.2199.

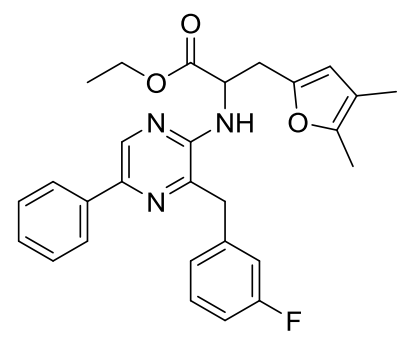

Ethyl 3-(4,5-dimethylfuran-2-yl)-2-((3-(3-fluorobenzyl)-5-phenylpyrazin-2-yl)amino)propanoate $\quad \mathbf{2 3}\{1,3,48\}$ (EC32712-115-1): Obtained as an oil $(0.35 \mathrm{~g}, 88 \%)$, using toluene at $90{ }^{\circ} \mathrm{C}$, after a chromatography over silica gel (cyclohexane-ethyl acetate 97:3). ${ }^{1} \mathrm{H}$ NMR $\left(\mathrm{CDCl}_{3}\right): 8.43(\mathrm{~s}, 1 \mathrm{H}), 7.94(\mathrm{~m}, 2 \mathrm{H}), 7.47(\mathrm{~m}, 2 \mathrm{H}), 7.37(\mathrm{~m}, 1 \mathrm{H}), 7.27$ $(\mathrm{m}, 1 \mathrm{H}), 7.07(\mathrm{~m}, 1 \mathrm{H}), 6.97(\mathrm{~m}, 2 \mathrm{H}), 5.70(\mathrm{~s}, 1 \mathrm{H}), 5.08(\mathrm{~d}, 1 \mathrm{H}, J=7.6 \mathrm{~Hz}), 4.91(\mathrm{dt}, 1 \mathrm{H}, J=7.6,5.3 \mathrm{~Hz}), 4.19(\mathrm{~m}$, $4 \mathrm{H}), 3.14(\mathrm{~m}, 2 \mathrm{H}), 2.13(\mathrm{~s}, 2 \mathrm{H}), 1.89(\mathrm{~s}, 2 \mathrm{H}), 1.24(\mathrm{t}, 3 \mathrm{H}, J=7.1 \mathrm{~Hz}) .{ }^{13} \mathrm{C} \mathrm{NMR}\left(\mathrm{CDCl}_{3}\right): 172.1,163.0(246 \mathrm{~Hz})$, 150.4, 147.1, 146.7, 141.2, 140.3, $139.2(7 \mathrm{~Hz}), 137.4,137.1,130.1(8 \mathrm{~Hz}), 128.7,127.8,125.6,124.3(3 \mathrm{~Hz})$, $115.6(22 \mathrm{~Hz}), 114.4,113.7(21 \mathrm{~Hz}), 111.1,61.2,53.2,40.2(2 \mathrm{~Hz}), 30.5,14.1,11.2,9.8 . \mathrm{HRMS}(\mathrm{m} / z):[\mathrm{M}+\mathrm{H}]^{+}$ calcd for $\mathrm{C}_{28} \mathrm{H}_{29} \mathrm{FN}_{3} \mathrm{O}_{3}$ : 474.2193; found, 474.2187 .

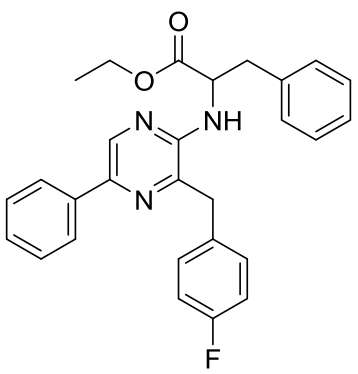

Ethyl (3-(4-fluorobenzyl)-5-phenylpyrazin-2-yl)phenylalaninate 23\{1,4,1\} (YJ31067-151-1): Obtained as an oil $(0.12 \mathrm{~g}, 34 \%)$ after a chromatography over silica gel (cyclohexane-ethyl acetate $94: 6) .{ }^{1} \mathrm{H} \mathrm{NMR}\left(\mathrm{CDCl}_{3}\right): 8.45(\mathrm{~s}$, $1 \mathrm{H}), 7.96(\mathrm{~m}, 2 \mathrm{H}), 7.48(\mathrm{~m}, 2 \mathrm{H}), 7.38(\mathrm{~m}, 1 \mathrm{H}), 7.27-7.22(\mathrm{~m}, 3 \mathrm{H}), 7.16(\mathrm{~m}, 2 \mathrm{H}), 7.01-6.94(\mathrm{~m}, 4 \mathrm{H}), 5.02(\mathrm{~m}$, $1 \mathrm{H}), 4.88$ (d(br), 1H, $J=7.5 \mathrm{~Hz}), 4.16$ (q, 2H, $J=7.0 \mathrm{~Hz}), 4.10$ (s, 2H), 3.24 (dd, 1H, $J=5.5,13.8 \mathrm{~Hz}), 3.14$ (dd, 
$1 \mathrm{H}, J=5.9,13.8 \mathrm{~Hz}), 1.24(\mathrm{t}, 3 \mathrm{H}, J=7.1 \mathrm{~Hz}) .{ }^{13} \mathrm{C} \mathrm{NMR}\left(\mathrm{CDCl}_{3}\right): 172.4,161.8(244 \mathrm{~Hz}), 150.3,141.2,140.9$, 137.4, 137.0, 136.1, 132.1 (3 Hz), 130.1 (8 Hz), 129.2, 128.8, 128.5, 127.9, 126.9, 125.6, $115.6(22 \mathrm{~Hz}), 61.2,54.8$, 39.9, 37.7, 14.1. HRMS (m/z): $[\mathrm{M}+\mathrm{H}]^{+}$calcd for $\mathrm{C}_{28} \mathrm{H}_{27} \mathrm{FN}_{3} \mathrm{O}_{2}, 456.2087$; found, 456.2072.

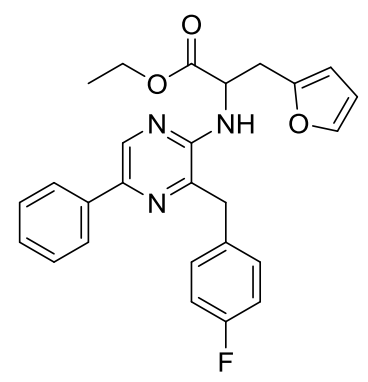

Ethyl 2-((3-(4-fluorobenzyl)-5-phenylpyrazin-2-yl)amino)-3-(furan-2-yl)propanoate 23 1,4,37\} (YJ 31776-005-1): Obtained as an oil $(0.30 \mathrm{~g}, 71 \%)$, using toluene at $90{ }^{\circ} \mathrm{C}$, after a chromatography over silica gel (cyclohexane-ethyl acetate 96:4). ${ }^{1} \mathrm{H}$ NMR $\left(\mathrm{CDCl}_{3}\right): 8.43(\mathrm{~s}, 1 \mathrm{H}), 7.95(\mathrm{~m}, 2 \mathrm{H}), 7.47(\mathrm{~m}, 2 \mathrm{H}), 7.37(\mathrm{~m}, 1 \mathrm{H}), 7.26(\mathrm{~m}, 3 \mathrm{H}), 6.99(\mathrm{~m}$, $2 \mathrm{H}), 6.24(\mathrm{~m}, 1 \mathrm{H}), 5.86(\mathrm{~d}(\mathrm{br}), 1 \mathrm{H}, J=5.8 \mathrm{~Hz}), 5.09(\mathrm{~d}(\mathrm{br}), 1 \mathrm{H}, J=7.8 \mathrm{~Hz}), 4.96(\mathrm{~m}, 1 \mathrm{H}), 4.20(\mathrm{~m}, 2 \mathrm{H}), 4.14(\mathrm{~s}$, $2 \mathrm{H}), 3.23(\mathrm{~m}, 2 \mathrm{H}), 1.25(\mathrm{t}, 3 \mathrm{H}, J=7.1 \mathrm{~Hz}) .{ }^{13} \mathrm{C} \mathrm{NMR}\left(\mathrm{CDCl}_{3}\right): 171.9,161.8(244 \mathrm{~Hz}), 150.5,150.0,141.9,141.2$, 141.0, 137.3, 136.7, 132.1 (3 Hz), 130.1 (8 Hz), 128.8, 127.9, 125.6, 115.6 (22 Hz), 110.3, 107.8, 61.4, 53.2, 39.9, 30.3, 14.1. HRMS $(\mathrm{m} / \mathrm{z})$ : $[\mathrm{M}+\mathrm{H}]^{+}$calcd for $\mathrm{C}_{26} \mathrm{H}_{25} \mathrm{FN}_{3} \mathrm{O}_{3}, 446.1880$; found, 446.1886.

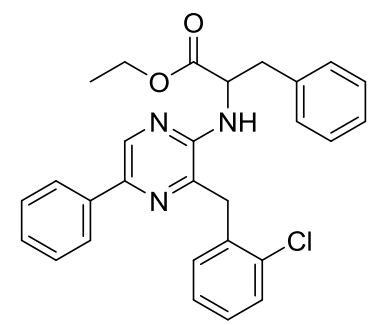

Ethyl (3-(2-chlorobenzyl)-5-phenylpyrazin-2-yl)phenylalaninate 23 1,5,1\} (EC31094-099-2): Obtained as an oil $(0.19 \mathrm{~g}, 68 \%)$ after a chromatography over silica gel (cyclohexane-ethyl acetate $96 / 4) .{ }^{1} \mathrm{H}$ NMR $\left(\mathrm{CDCl}_{3}\right): 8.44(\mathrm{~s}$, $1 \mathrm{H}), 7.91(\mathrm{~m}, 2 \mathrm{H}), 7.44(\mathrm{~m}, 3 \mathrm{H}), 7.35(\mathrm{~m}, 1 \mathrm{H}), 7.26-7.16(\mathrm{~m}, 6 \mathrm{H}), 7.05(\mathrm{~m}, 2 \mathrm{H}), 5.00(\mathrm{~m}, 2 \mathrm{H}), 4.24(\mathrm{~m}, 2 \mathrm{H}), 4.16$ $(\mathrm{dq}, 2 \mathrm{H}, J=7.2,1.0 \mathrm{~Hz}), 3.22(\mathrm{dd}, 1 \mathrm{H}, J=13.7,5.4 \mathrm{~Hz}), 3.13(\mathrm{dd}, 1 \mathrm{H}, J=13.7,6.3 \mathrm{~Hz}), 1.22(\mathrm{t}, 3 \mathrm{H}, J=7.2 \mathrm{~Hz})$. ${ }^{13} \mathrm{C} \mathrm{NMR}\left(\mathrm{CDCl}_{3}\right)$ : 172.4, 150.3, 141.2, 140.0, 137.4, 136.9, 136.3, 134.5, 134.0, 130.4, 129.5, 129.2, 128.7, 128.5, 128.2, 127.8, 127.0, 126.9, 125.6, 61.2, 55.0, 38.0, 37.1, 14.1. HRMS $(m / z):[\mathrm{M}+\mathrm{H}]^{+}$calcd for $\mathrm{C}_{28} \mathrm{H}_{27} \mathrm{ClN}_{3} \mathrm{O}_{2}$ : 472.1792; found, 472.1784 .

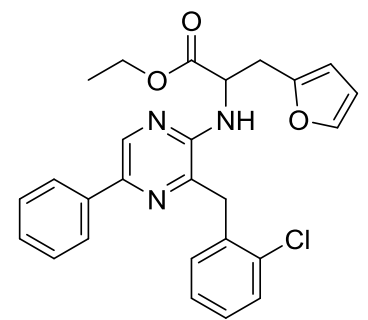

Ethyl 2-((3-(2-chlorobenzyl)-5-phenylpyrazin-2-yl)amino)-3-(furan-2-yl)propanoate 23 1,5,37\} (EC31094-097-2): Obtained as an oil $(0.44 \mathrm{~g}, 65 \%)$ after a chromatography over silica gel (cyclohexane-ethyl acetate $96 / 4) .{ }^{1} \mathrm{H}$ NMR $\left(\mathrm{CDCl}_{3}\right): 8.44(\mathrm{~s}, 1 \mathrm{H}), 7.91(\mathrm{~m}, 2 \mathrm{H}), 7.45(\mathrm{~m}, 3 \mathrm{H}), 7.35(\mathrm{~m}, 1 \mathrm{H}), 7.25-7.15(\mathrm{~m}, 4 \mathrm{H}), 6.22(\mathrm{~m}, 1 \mathrm{H}), 5.92(\mathrm{~m}, 1 \mathrm{H})$, $5.18(\mathrm{~m}, 1 \mathrm{H}), 5.00(\mathrm{~m}, 1 \mathrm{H}), 4.28(\mathrm{~m}, 2 \mathrm{H}), 4.19(\mathrm{q}, 2 \mathrm{H}, J=7.1 \mathrm{~Hz}), 3.24(\mathrm{~d}, 2 \mathrm{H}, J=5.5 \mathrm{~Hz}), 1.24(\mathrm{t}, 3 \mathrm{H}, J=7.1$ $\mathrm{Hz}) .{ }^{13} \mathrm{C} \mathrm{NMR}\left(\mathrm{CDCl}_{3}\right): 171.9,150.6,150.2,142.0,141.2,140.0,137.4,136.9,134.5,134.1,130.4,129.5,128.7$, $128.2,127.8,127.0,125.6,110.3,107.7,61.3,53.3,37.1,30.5,14.1$. HRMS $(\mathrm{m} / z):[\mathrm{M}+\mathrm{H}]^{+}$calcd for $\mathrm{C}_{26} \mathrm{H}_{25} \mathrm{ClN}_{3} \mathrm{O}_{3}$ : 462.1584; found, 462.1576. 


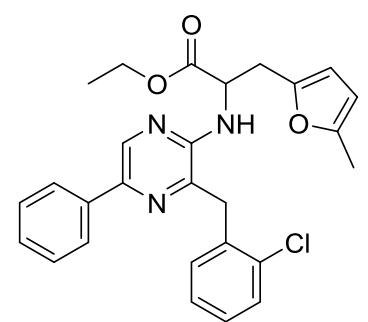

Ethyl 2-((3-(2-chlorobenzyl)-5-phenylpyrazin-2-yl)amino)-3-(5-methylfuran-2-yl)propanoate $\quad \mathbf{2 3}\{1,5,42\}$ (YJ31068-009-1): Obtained as an oil $(0.11 \mathrm{~g}, 36 \%)$ after a chromatography over silica gel (cyclohexane-ethyl acetate 95/5). ${ }^{1} \mathrm{H}$ NMR $\left(\mathrm{CDCl}_{3}\right): 8.43(\mathrm{~s}, 1 \mathrm{H}), 7.91(\mathrm{~m}, 2 \mathrm{H}), 7.43(\mathrm{~m}, 3 \mathrm{H}), 7.35(\mathrm{~m}, 1 \mathrm{H}), 7.20(\mathrm{~m}, 3 \mathrm{H}), 6.22(\mathrm{~m}$, $1 \mathrm{H}), 5.80(\mathrm{~m}, 2 \mathrm{H}), 5.19(\mathrm{~d}(\mathrm{br}), 1 \mathrm{H}, J=7.6 \mathrm{~Hz}), 4.98(\mathrm{~m}, 1 \mathrm{H}), 4.28(\mathrm{~m}, 2 \mathrm{H}), 4.20(\mathrm{~m}, 2 \mathrm{H}), 3.19(\mathrm{~m}, 2 \mathrm{H}), 2.21(\mathrm{~s}$, $3 \mathrm{H}), 1.25(\mathrm{t}, 3 \mathrm{H}, J=7.1 \mathrm{~Hz}) .{ }^{13} \mathrm{C} \mathrm{NMR}\left(\mathrm{CDCl}_{3}\right): 172.1,151.5,150.3,148.5,141.1,139.9,137.4,136.8,134.6$, $134.1,130.4,129.5,128.7,128.2$, 127.8, 127.0, 125.5, 108.6, 106.2, 61.2, 53.3, 37.0, 30.6, 14.1, 13.4. HRMS $(\mathrm{m} / \mathrm{z}):[\mathrm{M}+\mathrm{H}]^{+}$calcd for $\mathrm{C}_{27} \mathrm{H}_{27} \mathrm{ClN}_{3} \mathrm{O}_{3}: 476.1741$; found, 476.1744.

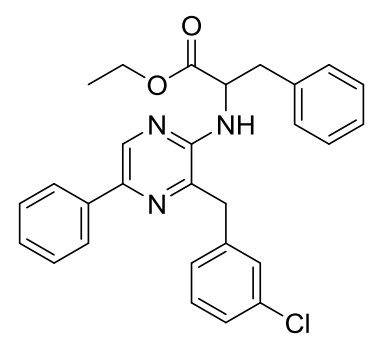

Ethyl (3-(3-chlorobenzyl)-5-phenylpyrazin-2-yl)phenylalaninate 23\{1,6,1\} (YJ 31776-015-1): Obtained as an oil $(0.23 \mathrm{~g}, 55 \%)$, using toluene at $90{ }^{\circ} \mathrm{C}$, after a chromatography over silica gel (cyclohexane-ethyl acetate $\left.96 / 4\right) .{ }^{1} \mathrm{H}$ NMR $\left(\mathrm{CDCl}_{3}\right): 8.44(\mathrm{~s}, 1 \mathrm{H}), 7.94(\mathrm{~m}, 2 \mathrm{H}), 7.47(\mathrm{~m}, 2 \mathrm{H}), 7.37(\mathrm{~m}, 1 \mathrm{H}), 7.22(\mathrm{~m}, 6 \mathrm{H}), 7.07(\mathrm{~m}, 1 \mathrm{H}), 7.00(\mathrm{~m}, 1 \mathrm{H})$, $5.01(\mathrm{~m}, 1 \mathrm{H}), 4.84(\mathrm{~d}(\mathrm{br}), 1 \mathrm{H}, J=7.6 \mathrm{~Hz}$ ), $4.18(\mathrm{q}, 2 \mathrm{H}, J=7.2 \mathrm{~Hz}), 4.09(\mathrm{~s}, 2 \mathrm{H}), 3.21(\mathrm{dd}, 1 \mathrm{H}, J=5.7,13.8 \mathrm{~Hz})$, $3.12(\mathrm{dd}, 1 \mathrm{H}, J=5.9,13.8 \mathrm{~Hz}), 1.22(\mathrm{t}, 3 \mathrm{H}, J=7.2 \mathrm{~Hz}) .{ }^{13} \mathrm{C} \mathrm{NMR}\left(\mathrm{CDCl}_{3}\right): 172.3,150.0,141.2,140.3,138.5$, 140.2, 138.7, 137.1, 136.7, 136.0, 134.6, 130.0, 129.2, 128.8, 128.7, 128.5, 128.0, 127.2, 127.0, 126.8, 125.7, 61.3, 54.9, 40.2, 37.7, 14.1. HRMS (m/z): $[\mathrm{M}+\mathrm{H}]^{+}$calcd for $\mathrm{C}_{28} \mathrm{H}_{27} \mathrm{ClN}_{3} \mathrm{O}_{2}$ : 472.1792; found, 472.1789 .

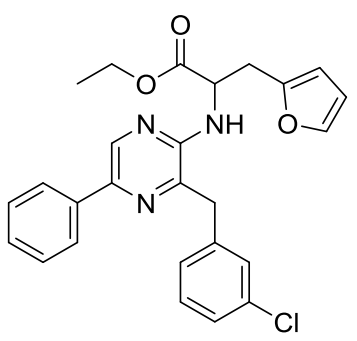

Ethyl 2-((3-(3-chlorobenzyl)-5-phenylpyrazin-2-yl)amino)-3-(furan-2-yl)propanoate 23 1,6,37\} (EC31095-083-3): Obtained as a yellow oil $(0.16 \mathrm{~g}, 36 \%)$ after a chromatography over silica gel (cyclohexane-ethyl acetate $97: 3) .{ }^{1} \mathrm{H}$ NMR $\left(\mathrm{CDCl}_{3}\right): 8.44(\mathrm{~s}, 1 \mathrm{H}), 7.94(\mathrm{~m}, 2 \mathrm{H}), 7.48(\mathrm{~m}, 2 \mathrm{H}), 7.38(\mathrm{~m}, 1 \mathrm{H}), 7.27(\mathrm{~m}, 2 \mathrm{H}), 7.24(\mathrm{~m}, 2 \mathrm{H}), 7.16(\mathrm{~m}, 1 \mathrm{H})$, $6.25(\mathrm{dd}, 1 \mathrm{H}, J=3.2,1.9 \mathrm{~Hz}), 5.89(\mathrm{dd}, 1 \mathrm{H}, J=3.2,0.7 \mathrm{~Hz}), 5.06(\mathrm{~d}, 1 \mathrm{H}, J=7.6 \mathrm{~Hz}), 4.95(\mathrm{~m}, 1 \mathrm{H}), 4.19(\mathrm{~m}, 2 \mathrm{H})$, $4.14(\mathrm{~d}, 2 \mathrm{H}, J=3.4 \mathrm{~Hz}), 3.24(\mathrm{~d}, 2 \mathrm{H}, J=5.2 \mathrm{~Hz}), 1.23(\mathrm{t}, 3 \mathrm{H}, J=7.1 \mathrm{~Hz}) .{ }^{13} \mathrm{C} \mathrm{NMR}\left(\mathrm{CDCl}_{3}\right): 171.9,150.5,150.2$, 142.0, 141.3, 140.2, 138.7, 137.3, 137.2, 134.6, 130.0, 128.8, 128.8, 127.9, 127.2, 126.9, 125.7, 110.3, 107.8, 61.4, 53.2, 40.3, 30.4, 14.1. HRMS (m/z): $[\mathrm{M}+\mathrm{H}]^{+}$calcd for $\mathrm{C}_{26} \mathrm{H}_{25} \mathrm{ClN}_{3} \mathrm{O}_{3}$ : 462.1584; found, 462.1597.

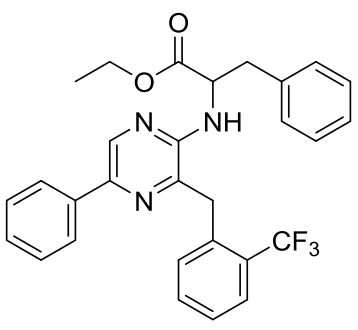


Ethyl (5-phenyl-3-(2-(trifluoromethyl)benzyl)pyrazin-2-yl)phenylalaninate 23\{1,8,1\} (YJ 33067-119-1): Obtained as an oil $(0.37 \mathrm{~g}, 85 \%)$, using toluene at $90{ }^{\circ} \mathrm{C}$, after a chromatography over silica gel (cyclohexane-ethyl acetate 97:3 to 96/4). ${ }^{1} \mathrm{H}$ NMR $\left(\mathrm{CDCl}_{3}\right): 8.47(\mathrm{~s}, 1 \mathrm{H}), 7.92(\mathrm{~m}, 2 \mathrm{H}), 7.72(\mathrm{~m}, 1 \mathrm{H}), 7.46-7.33(\mathrm{~m}, 5 \mathrm{H}), 7.18(\mathrm{~m}, 3 \mathrm{H}), 7.12$ $(\mathrm{m}, 1 \mathrm{H}), 6.98(\mathrm{~m}, 1 \mathrm{H}), 5.01(\mathrm{~m}, 1 \mathrm{H}), 4.79(\mathrm{~d}(\mathrm{br}), J=7.5 \mathrm{~Hz}), 4.32(\mathrm{~m}, 2 \mathrm{H}), 4.15(\mathrm{~m}, 2 \mathrm{H}), 3.18(\mathrm{dd}, 1 \mathrm{H}, J=5.5$ and $13.9 \mathrm{~Hz}), 3.08(\mathrm{dd}, 1 \mathrm{H}, J=6.6$ and $13.9 \mathrm{~Hz}), 1.20(\mathrm{t}, 3 \mathrm{H}, J=7.2 \mathrm{~Hz}) .{ }^{13} \mathrm{C} \mathrm{NMR}\left(\mathrm{CDCl}_{3}\right): 172.2,150.1,141.2$, 139.8, 137.2, 136.8, 136.1, 135.2, 132.0, 130.2, 129.0, 128.8, 128.7 (30 Hz), 128.4, 127.9, 126.9, $126.8,126.1$ (4 $\mathrm{Hz}), 125.5,124.5(273 \mathrm{~Hz}), 61.2,55.0,37.9,36.3,14.1$. HRMS $(m / z):[\mathrm{M}+\mathrm{H}]^{+}$calcd for $\mathrm{C}_{29} \mathrm{H}_{27} \mathrm{~F}_{3} \mathrm{~N}_{3} \mathrm{O}_{2}, 506.2055$; found, 506.2053.

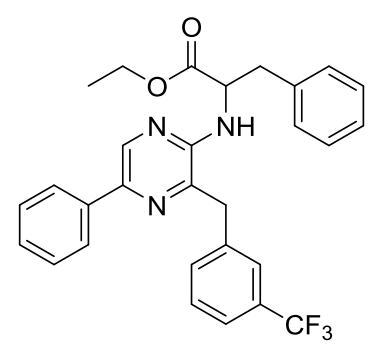

Ethyl (5-phenyl-3-(3-(trifluoromethyl)benzyl)pyrazin-2-yl)phenylalaninate 23\{1,9,1\} (YJ 33067-121-1): Obtained as an oil $(0.37 \mathrm{~g}, 85 \%)$, using toluene at $90{ }^{\circ} \mathrm{C}$, after a chromatography over silica gel (cyclohexane-ethyl acetate 97:3 to 96/4). ${ }^{1} \mathrm{H}$ NMR $\left(\mathrm{CDCl}_{3}\right): 8.46(\mathrm{~s}, 1 \mathrm{H}), 7.93(\mathrm{~m}, 2 \mathrm{H}), 7.53(\mathrm{~m}, 2 \mathrm{H}), 7.46(\mathrm{~m}, 2 \mathrm{H}), 7.41-7.35(\mathrm{~m}, 3 \mathrm{H}), 7.23$ (m, 3H), $7.00(\mathrm{~m}, 2 \mathrm{H}), 5.01(\mathrm{~m}, 1 \mathrm{H}), 4.84(\mathrm{~d}(\mathrm{br}), J=7.5 \mathrm{~Hz}), 4.17(\mathrm{q}, 2 \mathrm{H}, J=7.2 \mathrm{~Hz}), 4.14(\mathrm{~s}, 2 \mathrm{H}), 3.23(\mathrm{dd}, 1 \mathrm{H}$, $J=5.7$ and $13.9 \mathrm{~Hz}), 3.13(\mathrm{dd}, 1 \mathrm{H}, J=6.0$ and $13.9 \mathrm{~Hz}), 1.22(\mathrm{t}, 3 \mathrm{H}, J=7.2 \mathrm{~Hz}) .{ }^{13} \mathrm{C} \mathrm{NMR}\left(\mathrm{CDCl}_{3}\right): 172.4,150.1$, 141.3, 140.0, 137.5, 137.2, 137.0, 136.1, $132.0131 .0(31 \mathrm{~Hz}), 129.2,128.8,128.5,128.0,127.0,125.6,125.4(4$ $\mathrm{Hz}), 124.0(272 \mathrm{~Hz}), 123.8(3 \mathrm{~Hz}), 61.3,54.8,40.0,37.7,14.1$ (one signal missing). HRMS $(\mathrm{m} / \mathrm{z}):[\mathrm{M}+\mathrm{H}]^{+} \mathrm{calcd}$ for $\mathrm{C}_{29} \mathrm{H}_{27} \mathrm{~F}_{3} \mathrm{~N}_{3} \mathrm{O}_{2}, 506.2055$; found, 506.2058.

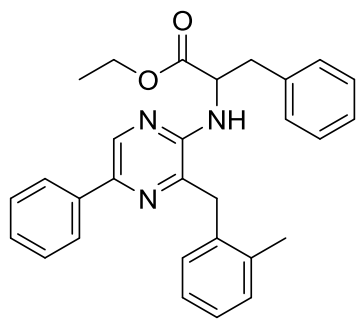

Ethyl (3-(2-methylbenzyl)-5-phenylpyrazin-2-yl)phenylalaninate 23 $\{1,11,1\}$ (YJ31068-017-1): Obtained as an oil $(0.40 \mathrm{~g}, 86 \%)$ after a chromatography over silica gel (cyclohexane-ethyl acetate 95:5). ${ }^{1} \mathrm{H}$ NMR $\left(\mathrm{CDCl}_{3}\right): 8.43(\mathrm{~s}$, 1H), $7.92(\mathrm{~m}, 2 \mathrm{H}), 7.43(\mathrm{~m}, 2 \mathrm{H}), 7.35(\mathrm{~m}, 1 \mathrm{H}), 7.20(\mathrm{~m}, 5 \mathrm{H}), 7.11(\mathrm{~m}, 1 \mathrm{H}), 6.98(\mathrm{~m}, 3 \mathrm{H}), 5.01(\mathrm{~m}, 1 \mathrm{H}), 4.86(\mathrm{~d}$ (br), $1 \mathrm{H}, J=7.1 \mathrm{~Hz}), 4.14(\mathrm{~m}, 4 \mathrm{H}), 3.17(\mathrm{dd}, 1 \mathrm{H}, J=5.6,13.8 \mathrm{~Hz}), 3.07$ (dd, 1H, $J=6.0,13.8 \mathrm{~Hz}), 2.33(\mathrm{~s}, 3 \mathrm{H})$, $1.21(\mathrm{t}, 3 \mathrm{H}, J=7.1 \mathrm{~Hz}) .{ }^{13} \mathrm{C}$ NMR $\left(\mathrm{CDCl}_{3}\right): 172.4,141.0,140.9,137.5,137.0,136.6,136.3,134.7,130.6,129.2$, 128.7, 128.6, 128.4, 127.8, 127.0, 126.8, 126.3, 125.6, 61.1, 54.8, 38.6, 37.9, 19.9, 14.1 (one signal missing). HRMS $(\mathrm{m} / z):[\mathrm{M}+\mathrm{H}]^{+}$calcd for $\mathrm{C}_{29} \mathrm{H}_{29} \mathrm{~N}_{3} \mathrm{O}_{2}, 452.2338$; found, 452.2338.

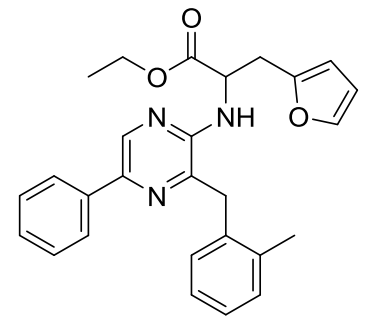

Ethyl 3-(furan-2-yl)-2-((3-(2-methylbenzyl)-5-phenylpyrazin-2-yl)amino)propanoate 23 1,11,37\} (YJ31068-0191): Obtained as an oil $(0.30 \mathrm{~g}, 71 \%)$ after a chromatography over silica gel (cyclohexane-ethyl acetate $95: 5) .{ }^{1} \mathrm{H}$ NMR $\left(\mathrm{CDCl}_{3}\right): 8.43(\mathrm{~s}, 1 \mathrm{H}), 7.93(\mathrm{~m}, 2 \mathrm{H}), 7.47(\mathrm{~m}, 2 \mathrm{H}), 7.35(\mathrm{~m}, 1 \mathrm{H}), 7.20(\mathrm{~m}, 3 \mathrm{H}), 7.13(\mathrm{~m}, 1 \mathrm{H}), 7.06(\mathrm{~m}, 1 \mathrm{H})$, $6.21(\mathrm{dd}, 1 \mathrm{H}, J=1.8,3.1 \mathrm{~Hz}), 5.85(\mathrm{~m}, 1 \mathrm{H}), 5.09(\mathrm{~d}(\mathrm{br}), 1 \mathrm{H}, J=7.6 \mathrm{~Hz}), 4.98(\mathrm{~m}, 1 \mathrm{H}), 4.18(\mathrm{~m}, 4 \mathrm{H}), 3.20(\mathrm{~m}$, 2H), $2.39(\mathrm{~s}, 3 \mathrm{H}), 1.23(\mathrm{t}, 3 \mathrm{H}, J=7.1 \mathrm{~Hz}) .{ }^{13} \mathrm{C} \mathrm{NMR}\left(\mathrm{CDCl}_{3}\right)$ : 172.0, 150.6, 150.4, 141.9, 141.1, 141.0, 137.4, 
137.0, 136.5, 134.7, 130.6, 128.8, 128.7, 127.8, 127.0, 126.3, 125.6, 110.3, 107.7, 61.3, 53.2, 38.5, 30.5, 20.0, 14.1. HRMS $(\mathrm{m} / \mathrm{z}):[\mathrm{M}+\mathrm{H}]^{+}$calcd for $\mathrm{C}_{27} \mathrm{H}_{28} \mathrm{~N}_{3} \mathrm{O}_{3}, 442.2131$; found, 442.2137 .

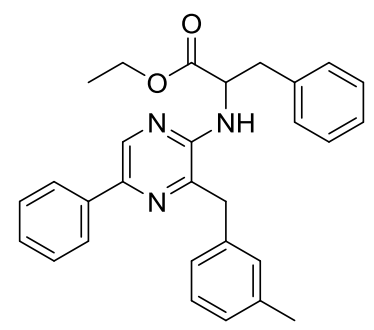

Ethyl (3-(3-methylbenzyl)-5-phenylpyrazin-2-yl)phenylalaninate 23 $\{1,12,1\}$ (YJ31068-021-1): Obtained as an oil $(0.41 \mathrm{~g}, 89 \%)$ after a chromatography over silica gel (cyclohexane-ethyl acetate 95:5). ${ }^{1} \mathrm{H}$ NMR $\left(\mathrm{CDCl}_{3}\right): 8.43(\mathrm{~s}$, 1H), $7.97(\mathrm{~m}, 2 \mathrm{H}), 7.48(\mathrm{~m}, 2 \mathrm{H}), 7.37(\mathrm{~m}, 1 \mathrm{H}), 7.21(\mathrm{~m}, 4 \mathrm{H}), 7.08-6.96(\mathrm{~m}, 4 \mathrm{H}), 4.97(\mathrm{~m}, 2 \mathrm{H}), 4.14(\mathrm{~m}, 4 \mathrm{H}), 3.17$ $(\mathrm{dd}, 1 \mathrm{H}, J=5.0,13.7 \mathrm{~Hz}), 3.07(\mathrm{dd}, 1 \mathrm{H}, J=5.7,13.7 \mathrm{~Hz}), 2.31(\mathrm{~s}, 3 \mathrm{H}), 1.20(\mathrm{t}, 3 \mathrm{H}, J=7.1 \mathrm{~Hz}) .{ }^{13} \mathrm{C} \mathrm{NMR}$ $\left(\mathrm{CDCl}_{3}\right): 172.3,150.4,141.3,141.0,138.5,137.5,136.8,136.4,136.3,129.3,129.2,128.7,128.4,127.7,127.6$, 126.8, 125.7, 61.1, 54.9, 40.9, 37.9, 21.4, 14.1 (two signals missing). HRMS (m/z): $[\mathrm{M}+\mathrm{H}]^{+}$calcd for $\mathrm{C}_{29} \mathrm{H}_{29} \mathrm{~N}_{3} \mathrm{O}_{2}$, 452.2338; found, 452.2337.

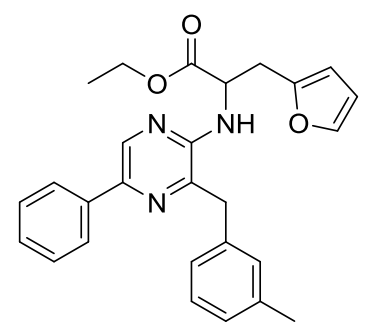

Ethyl 3-(furan-2-yl)-2-((3-(3-methylbenzyl)-5-phenylpyrazin-2-yl)amino)propanoate 23 1,12,37\} (YJ31068-0231): Obtained as an oil $(0.35 \mathrm{~g}, 75 \%)$ after a chromatography over silica gel (cyclohexane-ethyl acetate $95: 5) .{ }^{1} \mathrm{H}$ NMR $\left(\mathrm{CDCl}_{3}\right): 8.42(\mathrm{~s}, 1 \mathrm{H}), 7.96(\mathrm{~m}, 2 \mathrm{H}), 7.48(\mathrm{~m}, 2 \mathrm{H}), 7.38(\mathrm{~m}, 1 \mathrm{H}), 7.21(\mathrm{~m}, 2 \mathrm{H}), 7.08(\mathrm{~m}, 3 \mathrm{H}), 6.22(\mathrm{dd}, 1 \mathrm{H}, J$ $=2.0,3.3 \mathrm{~Hz}), 5.83(\mathrm{~m}, 1 \mathrm{H}), 5.16(\mathrm{~d}(\mathrm{br}), 1 \mathrm{H}, J=7.6 \mathrm{~Hz}), 4.96(\mathrm{~m}, 1 \mathrm{H}), 4.17(\mathrm{~m}, 4 \mathrm{H}), 3.21(\mathrm{~m}, 2 \mathrm{H}), 2.33(\mathrm{~s}, 3 \mathrm{H})$, $1.21(\mathrm{t}, 3 \mathrm{H}, J=7.1 \mathrm{~Hz}) .{ }^{13} \mathrm{C} \mathrm{NMR}\left(\mathrm{CDCl}_{3}\right): 172.0,150.6,150.3,141.8,141.3,141.1,138.4,137.5,136.7,136.4$, $129.4,128.7,128.6,127.8,127.7,125.8,125.7,110.2,107.6,61.3,53.2,40.9,30.4,21.4,14.1$. HRMS $(m / z)$ : $[\mathrm{M}+\mathrm{H}]^{+}$calcd for $\mathrm{C}_{27} \mathrm{H}_{28} \mathrm{~N}_{3} \mathrm{O}_{3}, 442.2131$; found, 442.2133 .

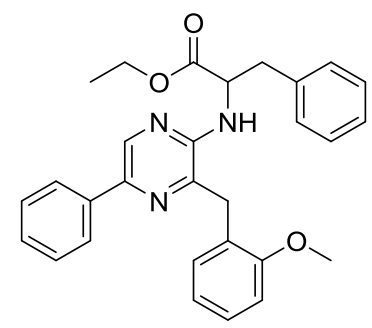

Ethyl (3-(2-methoxybenzyl)-5-phenylpyrazin-2-yl)phenylalaninate 23\{1,14,1\} (YJ31070-111-3): Obtained as an oil $(0.1 \mathrm{~g}, 28 \%)$ after a chromatography over silica gel (cyclohexane-ethyl acetate $96: 4) .{ }^{1} \mathrm{H}$ NMR $\left(\mathrm{CDCl}_{3}\right): 8.37(\mathrm{~s}$, 1H), $7.97(\mathrm{~m}, 2 \mathrm{H}), 7.47(\mathrm{~m}, 2 \mathrm{H}), 7.37(\mathrm{~m}, 2 \mathrm{H}), 7.21(\mathrm{~m}, 4 \mathrm{H}), 7.02(\mathrm{~m}, 2 \mathrm{H}), 6.93(\mathrm{~m}, 1 \mathrm{H}), 6.85(\mathrm{~m}, 1 \mathrm{H}), 6.03$ $(\mathrm{d}(\mathrm{br}), 1 \mathrm{H}, J=7.7 \mathrm{~Hz}), 5.08(\mathrm{~m}, 1 \mathrm{H}), 4.17(\mathrm{~m}, 4 \mathrm{H}), 3.72(\mathrm{~s}, 3 \mathrm{H}), 3.20(\mathrm{dd}, 1 \mathrm{H}, J=6.0,13.9 \mathrm{~Hz}), 3.12(\mathrm{dd}, 1 \mathrm{H}, J=$ $6.2,13.9 \mathrm{~Hz}), 1.21(\mathrm{t}, 3 \mathrm{H}, J=7.2 \mathrm{~Hz}) .{ }^{13} \mathrm{C} \mathrm{NMR}\left(\mathrm{CDCl}_{3}\right): 172.4,156.1,150.1,142.0,140.5,137.7,136.7,136.4$, $130.8,128.7,128.3,127.9,127.6,126.6,125.6,125.0,121.1,110.6,61.0,55.3,55.0,38.1,33.2$, 14.1. HRMS $(\mathrm{m} / \mathrm{z}):[\mathrm{M}+\mathrm{H}]^{+}$calcd for $\mathrm{C}_{29} \mathrm{H}_{29} \mathrm{~N}_{3} \mathrm{O}_{3}, 468.2287$; found, 468.2298 . 


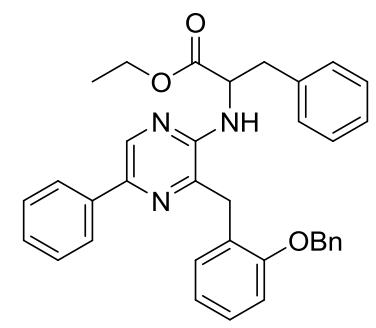

Ethyl (3-(2-(benzyloxy)benzyl)-5-phenylpyrazin-2-yl)phenylalaninate 23\{1,17,1\} (RB32489-61-2): Obtained as an oil $(0.54 \mathrm{~g}, 78 \%)$, using toluene at $90{ }^{\circ} \mathrm{C}$, after a chromatography over silica gel (cyclohexane - ethyl acetate 95/5). ${ }^{1} \mathrm{H}\left(\mathrm{CDCl}_{3}\right.$, traces of EtOAc): $8.44(\mathrm{~s}, 1 \mathrm{H}), 7.97(\mathrm{~m}, 2 \mathrm{H}), 7.48(\mathrm{~m}, 2 \mathrm{H}), 7.41-7.29(\mathrm{~m}, 6 \mathrm{H}), 7.19(\mathrm{~m}, 4 \mathrm{H}), 6.96(\mathrm{~m}$, $2 \mathrm{H}), 6.86(\mathrm{~m}, 3 \mathrm{H}), 5.01(\mathrm{~s}, 2 \mathrm{H}), 4.97(\mathrm{~m}, 2 \mathrm{H}), 4.13(\mathrm{q}, 2 \mathrm{H}, J=7.4 \mathrm{~Hz}), 4.12(\mathrm{~s}, 2 \mathrm{H}), 3.16(\mathrm{~m}, 1 \mathrm{H}), 3.07(\mathrm{~m}, 1 \mathrm{H})$, $1.20(\mathrm{t}, 3 \mathrm{H}, J=7.4 \mathrm{~Hz}) .{ }^{13} \mathrm{C}\left(\mathrm{CDCl}_{3}\right): 172.3,159.2,150.4,141.1,141.0,138.1,137.5,137.0,136.9,136.3,129.9$, 129.2, 128.7, 128.5, 128.4, 127.9, 127.8, 127.5, 126.8, 125.7, 121.3, 115.0, 113.6, 69.9, 61.1, 54.9, 41.0, $37.8,14.1$. HRMS (m/z): $[\mathrm{M}+\mathrm{H}]^{+}$calcd for $\mathrm{C}_{35} \mathrm{H}_{34} \mathrm{~N}_{3} \mathrm{O}_{3}, 544.2600$; found, 544.2605.

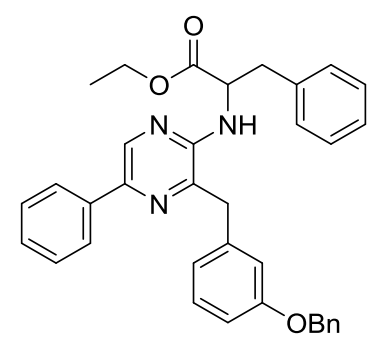

Ethyl (3-(3-(benzyloxy)benzyl)-5-phenylpyrazin-2-yl)phenylalaninate 23\{1,18,1\} (RB32489-59-2): Obtained as an oil $(0.57 \mathrm{~g}, 79 \%)$, using toluene at $90{ }^{\circ} \mathrm{C}$, after a chromatography over silica gel (cyclohexane - ethyl acetate 95/5). ${ }^{1} \mathrm{H}\left(\mathrm{CDCl}_{3}\right): 8.37(\mathrm{~s}, 1 \mathrm{H}), 7.93(\mathrm{~m}, 2 \mathrm{H}), 7.49-7.29(\mathrm{~m}, 9 \mathrm{H}), 7.23(\mathrm{~m}, 1 \mathrm{H}), 7.17(\mathrm{~m}, 3 \mathrm{H}), 6.96(\mathrm{~m}, 4 \mathrm{H}), 5.66(\mathrm{~d}$, $1 \mathrm{H}, J=7.5 \mathrm{~Hz}), 5.14(\mathrm{~d}, 1 \mathrm{H}, J=6.0,14.0 \mathrm{~Hz}), 5.07(\mathrm{~d}, 1 \mathrm{H}, J=7.0,12.4 \mathrm{~Hz}), 4.95(\mathrm{q}, 1 \mathrm{H}, J=8.5 \mathrm{~Hz}), 4.24(\mathrm{~m}$, 2H), $4.09(\mathrm{q}, 2 \mathrm{H}, J=7.3 \mathrm{~Hz}), 3.16(\mathrm{dd}, 1 \mathrm{H}, J=6.0,13.8 \mathrm{~Hz}), 3.07(\mathrm{dd}, 1 \mathrm{H}, J=7.1,13.8 \mathrm{~Hz}), 1.15(\mathrm{t}, 3 \mathrm{H}, J=7.3$ $\mathrm{Hz}) .{ }^{13} \mathrm{C}\left(\mathrm{CDCl}_{3}\right): 172.5,155.7,150.3,141.6,140.7,137.7,137.0,136.8,136.5,130.6,129.2,128.7,128.6,128.3$, 128.0, 127.9, 127.6, 127.3, 126.6, 125.6, 125.5, 121.4, 112.4, 70.3, 60.9, 55.1, 38.0, 33.8, 14.1. HRMS $(\mathrm{m} / \mathrm{z})$ : $[\mathrm{M}+\mathrm{H}]^{+}$calcd for $\mathrm{C}_{35} \mathrm{H}_{34} \mathrm{~N}_{3} \mathrm{O}_{3}, 544.2600$; found, 544.2609.

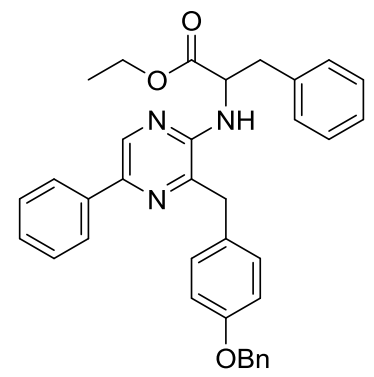

Ethyl (3-(2-(benzyloxy)benzyl)-5-phenylpyrazin-2-yl)phenylalaninate $23\{1,19,1\}$ (RB32489-063-2): Obtained as an oil $(0.57 \mathrm{~g}, 79 \%)$, using toluene at $90{ }^{\circ} \mathrm{C}$, after a chromatography over silica gel (cyclohexane - ethyl acetate 95/5). ${ }^{1} \mathrm{H}\left(\mathrm{CDCl}_{3}\right): 8.42(\mathrm{~s}, 1 \mathrm{H}), 7.98(\mathrm{~m}, 2 \mathrm{H}), 7.49-7.31(\mathrm{~m}, 8 \mathrm{H}), 7.22(\mathrm{~m}, 3 \mathrm{H}), 7.12(\mathrm{~m}, 2 \mathrm{H}), 6.96(\mathrm{~m}, 2 \mathrm{H}), 6.90$ $(\mathrm{m}, 2 \mathrm{H}), 5.07(\mathrm{~s}, 2 \mathrm{H}), 4.95(\mathrm{~m}, 2 \mathrm{H}), 4.15(\mathrm{q}, 2 \mathrm{H}, J=7.0 \mathrm{~Hz}), 4.08(\mathrm{~s}, 2 \mathrm{H}), 3.19(\mathrm{dd}, 1 \mathrm{H}, J=5.4,14.0 \mathrm{~Hz}), 3.09$ $(\mathrm{dd}, 1 \mathrm{H}, J=5.6,14.0 \mathrm{~Hz}), 1.20(\mathrm{t}, 3 \mathrm{H}, J=7.0 \mathrm{~Hz}) .{ }^{13} \mathrm{C}\left(\mathrm{CDCl}_{3}\right): 172.4,157.8,150.3,141.4,141.0,137.5,137.1$, $136.8,136.3,129.6,129.3,128.7,128.6,128.4,128.0,127.8,127.4,126.8,125.7,115.3,70.0,61.1,54.9,40.1$, 37.8, 14.1, (one signal missing). HRMS (m/z): $[\mathrm{M}+\mathrm{H}]^{+}$calcd for $\mathrm{C}_{35} \mathrm{H}_{34} \mathrm{~N}_{3} \mathrm{O}_{3}, 544.2600$; found, 544.2609. 


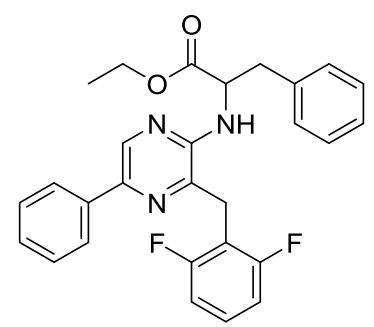

Ethyl (3-(2,6-difluorobenzyl)-5-phenylpyrazin-2-yl)phenylalaninate 23 1,21,1\} (YJ 33069-165-1): Obtained as an oil $(0.44 \mathrm{~g}, 91 \%)$, using toluene at $90^{\circ} \mathrm{C}$, after a chromatography over silica gel (cyclohexane - ethyl acetate 97/3). ${ }^{1} \mathrm{H}$ NMR $\left(\mathrm{CDCl}_{3}\right): 8.40(\mathrm{~s}, 1 \mathrm{H}), 7.80(\mathrm{~m}, 2 \mathrm{H}), 7.39(\mathrm{~m}, 2 \mathrm{H}), 7.39(\mathrm{~m}, 2 \mathrm{H}), 7.33-7.24(\mathrm{~m}, 5 \mathrm{H}), 7.17(\mathrm{~m}, 2 \mathrm{H}), 6.94$ $(\mathrm{m}, 2 \mathrm{H}), 5.17(\mathrm{~d}(\mathrm{br}), 1 \mathrm{H}, \mathrm{J}=7.0 \mathrm{~Hz}), 5.10(\mathrm{~m}, 1 \mathrm{H}), 4.21(\mathrm{q}, 2 \mathrm{H}, \mathrm{J}=7.0 \mathrm{~Hz}), 4.07(\mathrm{~s}, 2 \mathrm{H}), 3.33(\mathrm{dd}, 1 \mathrm{H}, \mathrm{J}=5.6$, $13.8 \mathrm{~Hz}), 3.23(\mathrm{dd}, 1 \mathrm{H}, \mathrm{J}=6.1,13.8 \mathrm{~Hz}), 1.26(\mathrm{t}, 3 \mathrm{H}, \mathrm{J}=7.0 \mathrm{~Hz}) .{ }^{13} \mathrm{C} \mathrm{NMR}\left(\mathrm{CDCl}_{3}\right): 172.5,161.8(248,8 \mathrm{~Hz})$, $149.5,140.9,138.7,137.1,136.3,136.0,129.3,128.6,128.5,128.3(10 \mathrm{~Hz}), 127.8,127.0,125.4,112.7(20 \mathrm{~Hz})$, $111.1(7,19 \mathrm{~Hz}), 61.3,55.1,37.9,26.6,14.1$. HRMS $(\mathrm{m} / z):[\mathrm{M}+\mathrm{H}]^{+}$calcd for $\mathrm{C}_{28} \mathrm{H}_{26} \mathrm{~F}_{2} \mathrm{~N}_{3} \mathrm{O}_{2}$ : 474.1993; found, 474.1995.

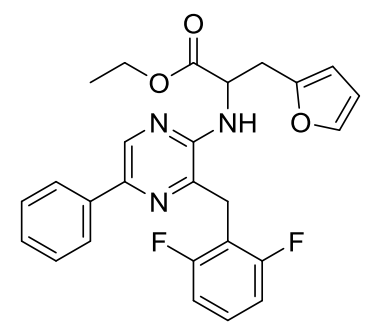

Ethyl 2-((3-(2,6-difluorobenzyl)-5-phenylpyrazin-2-yl)amino)-3-(furan-2-yl)propanoate $23\{1,21,37\}$ (EC31095077-2): Obtained as a white solid $(0.40 \mathrm{~g}, 90 \%)$ after a chromatography over silica gel (cyclohexane - ethyl acetate 97/3 to 96/4). ${ }^{1} \mathrm{H}$ NMR $\left(\mathrm{CDCl}_{3}\right): 8.40$ (s, 1H), 7.80 (m, 2H), 7.39 (m, 2H), 7.35 (dd, 1H, J = 1.9, 0.8 Hz), 7.28 (m, 2H), $6.94(\mathrm{~m}, 2 \mathrm{H}), 6.30(\mathrm{dd}, 1 \mathrm{H}, J=3.2,1.9 \mathrm{~Hz}), 6.07(\mathrm{dd}, 1 \mathrm{H}, J=3.2,0.6 \mathrm{~Hz}), 5.34(\mathrm{~d}, 1 \mathrm{H}, J=7.4 \mathrm{~Hz}), 5.08(\mathrm{dt}$, $1 \mathrm{H}, J=7.5,5.5 \mathrm{~Hz}), 4.25(\mathrm{~m}, 2 \mathrm{H}), 4.12(\mathrm{~s}, 2 \mathrm{H}), 3.34(\mathrm{~m}, 2 \mathrm{H}), 1.29(\mathrm{t}, 3 \mathrm{H}, J=7.1 \mathrm{~Hz}) .{ }^{13} \mathrm{C} \mathrm{NMR}\left(\mathrm{CDCl}_{3}\right): 172.1$, $161.8(248,8 \mathrm{~Hz}), 150.8,149.7,142.0,140.9,138.6,137.3,136.4,128.6,128.4(10 \mathrm{~Hz}), 127.7,125.4,112.8(20$ $\mathrm{Hz}), 111.1,110.3,107.8,61.4,53.4,30.6,26.6,14.1$. HRMS $(m / z):[\mathrm{M}+\mathrm{H}]^{+}$calcd for $\mathrm{C}_{26} \mathrm{H}_{24} \mathrm{~F}_{2} \mathrm{~N}_{3} \mathrm{O}_{3}: 464.1786$; found, 464.1798.

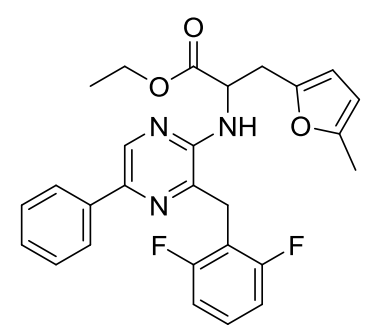

Ethyl 2-((3-(2,6-difluorobenzyl)-5-phenylpyrazin-2-yl)amino)-3-(5-methylfuran-2-yl)propanoate $\quad \mathbf{2 3}\{1,21,42\}$ (EC32712-083-2): Obtained as yellow solid (0.38 g, 83\%), using toluene at $90{ }^{\circ} \mathrm{C}$, after a chromatography over silica gel (cyclohexane - ethyl acetate 97/3). ${ }^{1} \mathrm{H}$ NMR $\left(\mathrm{CDCl}_{3}\right): 8.40(\mathrm{~s}, 1 \mathrm{H}), 7.80(\mathrm{~m}, 2 \mathrm{H}), 7.38(\mathrm{~m}, 2 \mathrm{H}), 7.29(\mathrm{~m}$, 2H), $6.95(\mathrm{~m}, 2 \mathrm{H}), 5.96(\mathrm{~d}, 1 \mathrm{H}, J=3.0 \mathrm{~Hz}), 5.88(\mathrm{dd}, 1 \mathrm{H}, J=3.0,1.0 \mathrm{~Hz}), 5.36(\mathrm{~d}, 1 \mathrm{H}, J=7.5 \mathrm{~Hz}), 5.04(\mathrm{dt}, 1 \mathrm{H}, J$ $=7.5,5.5 \mathrm{~Hz}), 4.25(\mathrm{~m}, 2 \mathrm{H}), 4.12(\mathrm{~s}, 2 \mathrm{H}), 3.27(\mathrm{~m}, 2 \mathrm{H}), 2.27(\mathrm{~d}, 3 \mathrm{H}, J=1.0 \mathrm{~Hz}), 1.30(\mathrm{t}, 3 \mathrm{H}, J=7.1 \mathrm{~Hz}) .{ }^{13} \mathrm{C}$ NMR $\left(\mathrm{CDCl}_{3}\right): 172.2,161.8(247,8 \mathrm{~Hz}), 151.6,149.8,148.8,140.8,138.5,137.3,136.4,128.6,128.4(10 \mathrm{~Hz})$, 127.7, 125.4, $112.8(20 \mathrm{~Hz}), 111.1$ (br), 108.6, 106.2, 61.3, 53.5, 30.7, $26.5(2 \mathrm{~Hz}), 14.1,13.5$. HRMS $(\mathrm{m} / \mathrm{z})$ : $[\mathrm{M}+\mathrm{H}]^{+}$calcd for $\mathrm{C}_{27} \mathrm{H}_{26} \mathrm{~F}_{2} \mathrm{~N}_{3} \mathrm{O}_{3}$ : 478.1942; found, 478.1951 . 


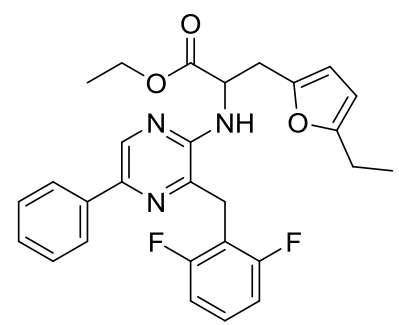

Ethyl 2-((3-(2,6-difluorobenzyl)-5-phenylpyrazin-2-yl)amino)-3-(5-ethylfuran-2-yl)propanoate $23\{1,21,44\}$ (EC32712-109-1): Obtained as an oil $(0.28 \mathrm{~g}, 72 \%)$, using toluene at $90{ }^{\circ} \mathrm{C}$, after a chromatography over silica gel (cyclohexane - ethyl acetate 97/3). ${ }^{1} \mathrm{H}$ NMR $\left(\mathrm{CDCl}_{3}\right): 8.40(\mathrm{~s}, 1 \mathrm{H}), 7.79(\mathrm{~m}, 2 \mathrm{H}), 7.39(\mathrm{~m}, 2 \mathrm{H}), 7.28(\mathrm{~m}, 2 \mathrm{H}), 6.95$ $(\mathrm{m}, 2 \mathrm{H}), 5.97(\mathrm{~d}, 1 \mathrm{H}, J=3.0 \mathrm{~Hz}), 5.88(\mathrm{~d}, 1 \mathrm{H}, J=3.0 \mathrm{~Hz}), 5.36(\mathrm{~d}, 1 \mathrm{H}, J=7.5 \mathrm{~Hz}), 5.05(\mathrm{dt}, 1 \mathrm{H}, J=7.5,5.5 \mathrm{~Hz})$, $4.26(\mathrm{~m}, 2 \mathrm{H}), 4.12(\mathrm{~s}, 2 \mathrm{H}), 3.28(\mathrm{~m}, 2 \mathrm{H}), 2.62(\mathrm{qd}, 2 \mathrm{H}, J=7.6,1.0 \mathrm{~Hz}), 1.30(\mathrm{t}, 3 \mathrm{H}, J=7.1 \mathrm{~Hz}), 1.23(\mathrm{t}, 3 \mathrm{H}, J=$ 7.6 Hz). ${ }^{13} \mathrm{C} \mathrm{NMR}\left(\mathrm{CDCl}_{3}\right): 172.3,161.8(248,8 \mathrm{~Hz}), 157.4,149.8,148.7,140.8,138.5,137.3,136.4,128.6,128.4$ $(10 \mathrm{~Hz}), 127.7,125.4,112.8(20 \mathrm{~Hz}), 111.1$ (br), 108.4, 104.6, 61.3, 53.5, 30.7, 26.5, 21.4, 14.1, 12.1. HRMS $(\mathrm{m} / z):[\mathrm{M}+\mathrm{H}]^{+}$calcd for $\mathrm{C}_{28} \mathrm{H}_{28} \mathrm{~F}_{2} \mathrm{~N}_{3} \mathrm{O}_{3}$ : 492.2099; found, 492.2103 .

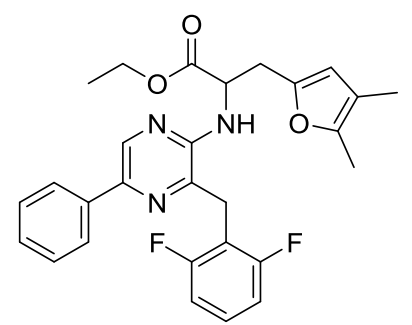

Ethyl 2-((3-(2,6-difluorobenzyl)-5-phenylpyrazin-2-yl)amino)-3-(4,5-dimethylfuran-2-yl)propanoate $\mathbf{2 3}\{1,21,48\}$ (EC32712-111-1): Obtained as an oil (0.33 g, 85\%), using toluene at $90{ }^{\circ} \mathrm{C}$, after a chromatography over silica gel (cyclohexane - ethyl acetate 97/3). ${ }^{1} \mathrm{H}$ NMR $\left(\mathrm{CDCl}_{3}\right): 8.39(\mathrm{~s}, 1 \mathrm{H}), 7.79(\mathrm{~d}, 1 \mathrm{H}, J=7.1 \mathrm{~Hz}), 7.38(\mathrm{~m}, 2 \mathrm{H}), 7.29$ $(\mathrm{m}, 3 \mathrm{H}), 6.94(\mathrm{~m}, 2 \mathrm{H}), 5.86(\mathrm{~s}, 1 \mathrm{H}), 5.37(\mathrm{~d}, 1 \mathrm{H}, J=7.4 \mathrm{~Hz}), 5.01(\mathrm{dt}, 1 \mathrm{H}, J=7.4,5.5 \mathrm{~Hz}), 4.25(\mathrm{qd}, 2 \mathrm{H}, J=7.1$, $4.6 \mathrm{~Hz}), 4.12(\mathrm{~s}, 2 \mathrm{H}), 3.23(\mathrm{~m}, 2 \mathrm{H}), 2.17(\mathrm{~s}, 3 \mathrm{H}), 1.91(\mathrm{~s}, 3 \mathrm{H}), 1.30(\mathrm{t}, 3 \mathrm{H}, J=7.1 \mathrm{~Hz}) .{ }^{13} \mathrm{C} \mathrm{NMR}\left(\mathrm{CDCl}_{3}\right): 172.3$, $161.8(248,8 \mathrm{~Hz}), 149.8,147.4,146.8,140.8,138.5,137.3,136.4,128.6,128.4$ (10 Hz), 127.6, 125.4, $114.5,112.8$ $(20 \mathrm{~Hz}), 111.1,111.0$ (br), 61.3, 53.6, 30.6, 26.5 (br), 14.1, 11.2, 9.8. HRMS (m/z): [M+H] ${ }^{+}$calcd for $\mathrm{C}_{28} \mathrm{H}_{28} \mathrm{~F}_{2} \mathrm{~N}_{3} \mathrm{O}_{3}$ : 492.2099; found, 492.2105.

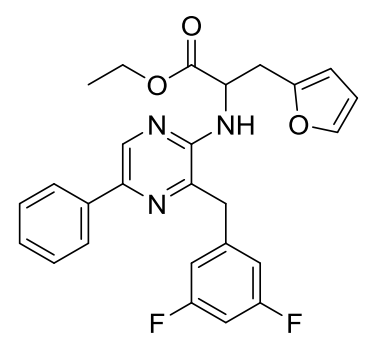

Ethyl 2-((3-(3,5-difluorobenzyl)-5-phenylpyrazin-2-yl)amino)-3-(furan-2-yl)propanoate 23\{1,22,37\} (EC31095081-3): Obtained as an oil (0.08 g, 18\%) after a chromatography over silica gel (cyclohexane - ethyl acetate 97/3). ${ }^{1} \mathrm{H}$ NMR $\left(\mathrm{CDCl}_{3}\right): 8.46(\mathrm{~s}, 1 \mathrm{H}), 7.95(\mathrm{~m}, 2 \mathrm{H}), 7.48(\mathrm{~m}, 2 \mathrm{H}), 7.39(\mathrm{~m}, 1 \mathrm{H}), 7.29(\mathrm{~m}, 1 \mathrm{H}), 6.81(\mathrm{~m}, 2 \mathrm{H}), 6.71(\mathrm{~m}$, $1 \mathrm{H}), 6.26(\mathrm{dd}, 1 \mathrm{H}, J=3.1,1.9 \mathrm{~Hz}), 5.93(\mathrm{~d}, 1 \mathrm{H}, J=3.2 \mathrm{~Hz}), 5.04(\mathrm{~d}, 1 \mathrm{H}, J=7.6 \mathrm{~Hz}), 4.97(\mathrm{dt}, 1 \mathrm{H}, J=7.5,5.0$ $\mathrm{Hz}), 4.20(\mathrm{q}, 2 \mathrm{H}, J=7.2 \mathrm{~Hz}), 4.14(\mathrm{~s}, 2 \mathrm{H}), 3.27(\mathrm{~d}, 2 \mathrm{H}, J=5.0 \mathrm{~Hz}), 1.25(\mathrm{t}, 3 \mathrm{H}, J=7.1 \mathrm{~Hz}) .{ }^{13} \mathrm{C} \mathrm{NMR}\left(\mathrm{CDCl}_{3}\right)$ : 171.9, $163.2(249,13 \mathrm{~Hz}), 150.5,150.2,142.1,141.4,140.6(9 \mathrm{~Hz}), 139.6,137.4,137.2,128.8,128.0,125.7$, 111.6, 110.3, 107.9, $102.5(25 \mathrm{~Hz}), 61.5,53.2,40.2,30.3,14.1$. HRMS $(m / z):[\mathrm{M}+\mathrm{H}]^{+}$calcd for $\mathrm{C}_{26} \mathrm{H}_{24} \mathrm{~F}_{2} \mathrm{~N}_{3} \mathrm{O}_{3}$ : 464.1786; found, 464.1788. 


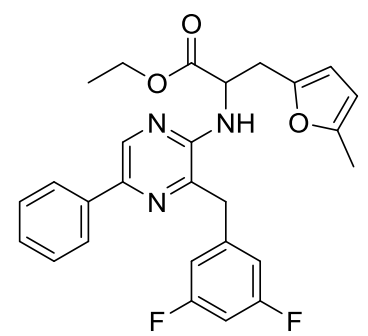

Ethyl 2-((3-(3,5-difluorobenzyl)-5-phenylpyrazin-2-yl)amino)-3-(5-methylfuran-2-yl)propanoate $23\{1,22,42\}$ (EC32712-005-2): Obtained as an oil $(0.33 \mathrm{~g}, 68 \%)$, using toluene at $90{ }^{\circ} \mathrm{C}$, after a chromatography over silica gel (cyclohexane - ethyl acetate 96/4). ${ }^{1} \mathrm{H}$ NMR $\left(\mathrm{CDCl}_{3}\right): 8.45(\mathrm{~s}, 1 \mathrm{H}), 7.93(\mathrm{~m}, 2 \mathrm{H}), 7.48(\mathrm{~m}, 2 \mathrm{H}), 7.38(\mathrm{~m}, 1 \mathrm{H}), 6.79$ $(\mathrm{m}, 2 \mathrm{H}), 6.70(\mathrm{tt}, 1 \mathrm{H}, J=8.9,2.3 \mathrm{~Hz}), 5.83(\mathrm{~m}, 1 \mathrm{H}), 5.81(\mathrm{~m}, 1 \mathrm{H}), 5.01(\mathrm{~d}, 1 \mathrm{H}, J=7.5 \mathrm{~Hz}), 4.93(\mathrm{dt}, 1 \mathrm{H}, J=7.5$, $5.1 \mathrm{~Hz}), 4.21(\mathrm{~m}, 2 \mathrm{H}), 4.13(\mathrm{~s}, 2 \mathrm{H}), 3.19(\mathrm{~m}, 2 \mathrm{H}), 2.22(\mathrm{~d}, 3 \mathrm{H}, J=0.9 \mathrm{~Hz}), 1.26(\mathrm{t}, 3 \mathrm{H}, J=7.1 \mathrm{~Hz}) .{ }^{13} \mathrm{C} \mathrm{NMR}$ $\left(\mathrm{CDCl}_{3}\right): 172.0,163.1(249,13 \mathrm{~Hz}), 151.7,150.2,148.3,141.3,140.6(9 \mathrm{~Hz}), 139.5,137.3,137.2,128.8,128.0$, 125.6, 111.6 (br), 108.7, 106.2, $102.4(25 \mathrm{~Hz}), 61.4,53.1,40.1(2 \mathrm{~Hz}), 30.5,14.1,13.4 . \mathrm{HRMS}(\mathrm{m} / \mathrm{z}):[\mathrm{M}+\mathrm{H}]^{+}$ calcd for $\mathrm{C}_{27} \mathrm{H}_{26} \mathrm{~F}_{2} \mathrm{~N}_{3} \mathrm{O}_{3}$ : 478.1942; found, 478.1945 .

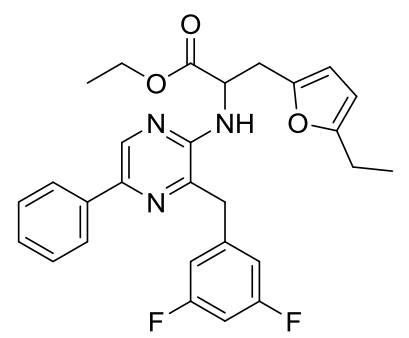

Ethyl 2-((3-(3,5-difluorobenzyl)-5-phenylpyrazin-2-yl)amino)-3-(5-ethylfuran-2-yl)propanoate $\quad \mathbf{2 3}\{1,22,44\}$ (EC32712-107-1): Obtained as an oil $(0.31 \mathrm{~g}, 80 \%)$, using toluene at $90{ }^{\circ} \mathrm{C}$, after a chromatography over silica gel (cyclohexane - ethyl acetate 97/3). ${ }^{1} \mathrm{H}$ NMR $\left(\mathrm{CDCl}_{3}\right): 8.45(\mathrm{~s}, 1 \mathrm{H}), 7.93(\mathrm{~m}, 2 \mathrm{H}), 7.47(\mathrm{~m}, 2 \mathrm{H}), 7.38(\mathrm{~m}, 1 \mathrm{H}), 6.79$ $(\mathrm{m}, 2 \mathrm{H}), 6.70(\mathrm{~m}, 1 \mathrm{H}), 5.83(\mathrm{~m}, 2 \mathrm{H}), 5.01(\mathrm{~d}, 1 \mathrm{H}, J=7.6 \mathrm{~Hz}), 4.95(\mathrm{dt}, 1 \mathrm{H}, J=7.5,5.1 \mathrm{~Hz}), 4.21(\mathrm{~m}, 2 \mathrm{H}), 4.12(\mathrm{~s}$, $2 \mathrm{H}), 3.22(\mathrm{~m}, 2 \mathrm{H}), 2.57(\mathrm{qd}, 2 \mathrm{H}, J=7.5,0.9 \mathrm{~Hz}), 1.27(\mathrm{t}, 3 \mathrm{H}, J=7.1 \mathrm{~Hz}), 1.21(\mathrm{t}, 3 \mathrm{H}, J=7.5 \mathrm{~Hz}) .{ }^{13} \mathrm{C} \mathrm{NMR}$ $\left(\mathrm{CDCl}_{3}\right)$ : 172.0, $163.1(249,13 \mathrm{~Hz}), 157.4,150.2,148.2,141.4,140.6(9 \mathrm{~Hz}), 139.5,137.3,137.2,128.8,127.9$, 125.6, 111.6 (br), 108.5, 104.5, $102.4(25 \mathrm{~Hz}), 61.4,53.1,40.0(2 \mathrm{~Hz}), 30.5,21.3,14.1,12.0$. HRMS (m/z): $[\mathrm{M}+\mathrm{H}]^{+}$calcd for $\mathrm{C}_{28} \mathrm{H}_{28} \mathrm{~F}_{2} \mathrm{~N}_{3} \mathrm{O}_{3}$ : 492.2099; found, 492.2103.

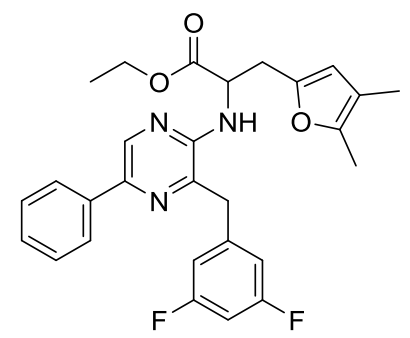

Ethyl 2-((3-(3,5-difluorobenzyl)-5-phenylpyrazin-2-yl)amino)-3-(4,5-dimethylfuran-2-yl)propanoate $23\{1,22,48\}$ (EC32712-091-2): Obtained as an oil (0.24 g, 73\%), using toluene at $90{ }^{\circ} \mathrm{C}$, after a chromatography over silica gel (cyclohexane - ethyl acetate 96/4). ${ }^{1} \mathrm{H}$ NMR $\left(\mathrm{CDCl}_{3}\right): 8.45(\mathrm{~s}, 1 \mathrm{H}), 7.93(\mathrm{~m}, 2 \mathrm{H}), 7.46(\mathrm{~m}, 2 \mathrm{H}), 7.38(\mathrm{~m}, 1 \mathrm{H}), 6.80$ $(\mathrm{m}, 2 \mathrm{H}), 6.70(\mathrm{~m}, 1 \mathrm{H}), 5.74(\mathrm{~s}, 1 \mathrm{H}), 5.04(\mathrm{~d}, 1 \mathrm{H}, J=7.6 \mathrm{~Hz}), 4.92(\mathrm{dt}, 1 \mathrm{H}, J=7.5,5.2 \mathrm{~Hz}), 4.21(\mathrm{~m}, 2 \mathrm{H}), 4.13(\mathrm{~s}$, 2H), $3.17(\mathrm{~m}, 2 \mathrm{H}), 2.14(\mathrm{~s}, 3 \mathrm{H}), 1.89(\mathrm{~s}, 3 \mathrm{H}), 1.26(\mathrm{t}, 3 \mathrm{H}, J=7.1 \mathrm{~Hz}) .{ }^{13} \mathrm{C} \mathrm{NMR}\left(\mathrm{CDCl}_{3}\right): 172.1,163.1(249,13$ Hz), 150.3, 147.1, 146.9, 141.4, 140.7 (9 Hz), 139.5, 137.3, 137.2, 128.8, 127.9, 125.6, 114.5, 111.6 (br), 111.2 , $102.3(25 \mathrm{~Hz}), 61.3,53.2,40.0$ (br), 30.4, 14.1, 11.2, 9.7. HRMS (m/z): $[\mathrm{M}+\mathrm{H}]^{+}$calcd for $\mathrm{C}_{28} \mathrm{H}_{28} \mathrm{~F}_{2} \mathrm{~N}_{3} \mathrm{O}_{3}$ : 492.2099; found, 492.2087. 


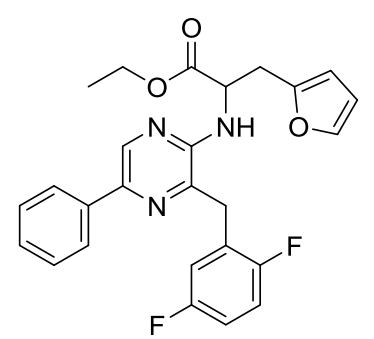

Ethyl 2-((3-(2,5-difluorobenzyl)-5-phenylpyrazin-2-yl)amino)-3-(furan-2-yl)propanoate $23\{1,23,37\}$ (EC32712015-3): Obtained as an oil $(0.32 \mathrm{~g}, 53 \%)$, using toluene at $90{ }^{\circ} \mathrm{C}$, after a chromatography over silica gel (cyclohexane - ethyl acetate 96/4). ${ }^{1} \mathrm{H}$ NMR $\left(\mathrm{CDCl}_{3}\right): 8.45(\mathrm{~s}, 1 \mathrm{H}), 7.91(\mathrm{~m}, 2 \mathrm{H}), 7.46(\mathrm{~m}, 2 \mathrm{H}), 7.37(\mathrm{~m}, 1 \mathrm{H}), 7.28$ $(\mathrm{m}, 1 \mathrm{H}), 7.06(\mathrm{~m}, 1 \mathrm{H}), 6.94(\mathrm{~m}, 2 \mathrm{H}), 6.25(\mathrm{dd}, 1 \mathrm{H}, J=3.2,1.9 \mathrm{~Hz}), 5.98(\mathrm{dd}, 1 \mathrm{H}, J=3.2,0.8 \mathrm{~Hz}), 5.22(\mathrm{~m}, 1 \mathrm{H})$, $5.01(\mathrm{dt}, 1 \mathrm{H}, J=7.5,5.4 \mathrm{~Hz}), 4.21(\mathrm{qd}, 2 \mathrm{H}, J=7.1,1.1 \mathrm{~Hz}), 4.14(\mathrm{~m}, 2 \mathrm{H}), 3.28(\mathrm{~m}, 2 \mathrm{H}), 1.25(\mathrm{t}, 3 \mathrm{H}, J=7.1 \mathrm{~Hz})$. ${ }^{13} \mathrm{C} \mathrm{NMR}\left(\mathrm{CDCl}_{3}\right): 172.0,158.7(242,2 \mathrm{~Hz}), 156.8(240,2 \mathrm{~Hz}), 150.6,150.0,142.0,141.3,139.2,137.2,137.2$, 128.8, 127.9, 125.6, 125.4 (10, 8 Hz), 117.1 (24, $4 \mathrm{~Hz}), 116.2$ (25, $9 \mathrm{~Hz}), 114.9$ (24, $9 \mathrm{~Hz}), 110.3,107.8,61.4$, 53.3, 32.5, 30.5, 14.1. HRMS $(m / z):[\mathrm{M}+\mathrm{H}]^{+}$calcd for $\mathrm{C}_{26} \mathrm{H}_{24} \mathrm{~F}_{2} \mathrm{~N}_{3} \mathrm{O}_{3}$ : 464.1786; found, 464.1783.

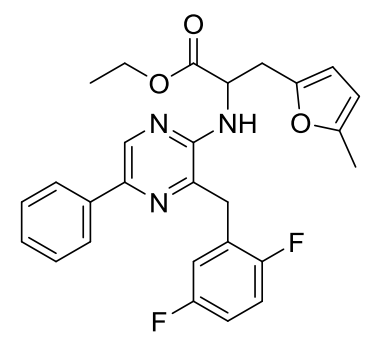

Ethyl 2-((3-(2,5-difluorobenzyl)-5-phenylpyrazin-2-yl)amino)-3-(5-methylfuran-2-yl)propanoate $\quad \mathbf{2 3}\{1,23,42\}$ (EC32712-041-2): Obtained as an oil (0.18 g, 47\%), using toluene at $90{ }^{\circ} \mathrm{C}$, after a chromatography over silica gel (cyclohexane - ethyl acetate 96/4). ${ }^{1} \mathrm{H}$ NMR $\left(\mathrm{CDCl}_{3}\right): 8.44(\mathrm{~s}, 1 \mathrm{H}), 7.90(\mathrm{~m}, 2 \mathrm{H}), 7.45(\mathrm{~m}, 2 \mathrm{H}), 7.36(\mathrm{~m}, 1 \mathrm{H}), 7.05$ $(\mathrm{td}, 1 \mathrm{H}, J=8.9,4.5 \mathrm{~Hz}), 6.94(\mathrm{~m}, 2 \mathrm{H}), 5.86(\mathrm{~m}, 1 \mathrm{H}), 5.82(\mathrm{~m}, 1 \mathrm{H}), 5.21(\mathrm{~d}, 1 \mathrm{H}, J=7.5 \mathrm{~Hz}), 4.97(\mathrm{dt}, 1 \mathrm{H}, J=7.5$, $5.5 \mathrm{~Hz}), 4.21(\mathrm{~m}, 2 \mathrm{H}), 4.13(\mathrm{~d}, 2 \mathrm{H}, J=1.5 \mathrm{~Hz}), 3.22(\mathrm{~d}, 2 \mathrm{H}, J=5.4 \mathrm{~Hz}), 2.23(\mathrm{~s}, 3 \mathrm{H}), 1.26(\mathrm{t}, 3 \mathrm{H}, J=7.1 \mathrm{~Hz}) .{ }^{13} \mathrm{C}$ NMR $\left(\mathrm{CDCl}_{3}\right)$ : 172.1, 158.7 (242, $\left.2 \mathrm{~Hz}\right), 156.7$ (240, $\left.3 \mathrm{~Hz}\right), 151.6,150.1,148.5,141.2,139.1,137.2,137.1,128.7$, 127.9, 125.5, 125.5 (19, $8 \mathrm{~Hz}), 117.1(24,4 \mathrm{~Hz}), 116.2(25,9 \mathrm{~Hz}), 114.9$ (24, $9 \mathrm{~Hz}), 108.6,106.1,61.3,53.4,32.4$, 30.6, 14.1, 13.4. HRMS (m/z): $[\mathrm{M}+\mathrm{H}]^{+}$calcd for $\mathrm{C}_{27} \mathrm{H}_{26} \mathrm{~F}_{2} \mathrm{~N}_{3} \mathrm{O}_{3}$ : 478.1942; found, 478.1940 .

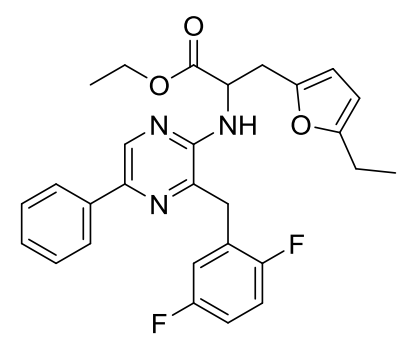

Ethyl 2-((3-(2,5-difluorobenzyl)-5-phenylpyrazin-2-yl)amino)-3-(5-ethylfuran-2-yl)propanoate $23\{1,23,44\}$ (EC32712-087-2): Obtained as an oil $(0.50 \mathrm{~g}, 75 \%)$, using toluene at $90{ }^{\circ} \mathrm{C}$, after a chromatography over silica gel (cyclohexane - ethyl acetate 96/4). ${ }^{1} \mathrm{H}$ NMR $\left(\mathrm{CDCl}_{3}\right): 8.44(\mathrm{~s}, 1 \mathrm{H}), 7.90(\mathrm{~m}, 2 \mathrm{H}), 7.45(\mathrm{~m}, 2 \mathrm{H}), 7.36(\mathrm{~m}, 1 \mathrm{H}), 7.04$ $(\mathrm{m}, 1 \mathrm{H}), 6.94(\mathrm{~m}, 2 \mathrm{H}), 5.88(\mathrm{~d}, 1 \mathrm{H}, J=3.1 \mathrm{~Hz}), 5.83(\mathrm{dt}, 1 \mathrm{H}, J=2.9,0.9 \mathrm{~Hz}), 5.21(\mathrm{~m}, 1 \mathrm{H}), 4.98(\mathrm{dt}, 1 \mathrm{H}, J=7.6$, $5.5 \mathrm{~Hz}), 4.21(\mathrm{~m}, 2 \mathrm{H}), 4.13(\mathrm{~d}, 2 \mathrm{H}, J=2.2 \mathrm{~Hz}), 3.23(\mathrm{~d}, 2 \mathrm{H}, J=5.4 \mathrm{~Hz}), 2.58(\mathrm{qd}, 2 \mathrm{H}, J=7.6,0.9 \mathrm{~Hz}), 1.26(\mathrm{t}, 3 \mathrm{H}$, $J=7.1 \mathrm{~Hz}), 1.21(\mathrm{t}, 3 \mathrm{H}, J=7.6 \mathrm{~Hz}) .{ }^{13} \mathrm{C} \mathrm{NMR}\left(\mathrm{CDCl}_{3}\right): 172.1,158.7(242,2 \mathrm{~Hz}), 157.3,156.8(240,2 \mathrm{~Hz}), 150.1$, 148.4, 141.2, 139.1, 137.2, 137.1, 128.7, 127.9, 125.5, 125.4 (dd, $J=18,8 \mathrm{~Hz}), 117.1(24,4 \mathrm{~Hz}), 116.2(25,9 \mathrm{~Hz})$, $114.9(24,9 \mathrm{~Hz}), 108.4,104.5,61.3,53.3,32.4$ (br), 30.6, 21.3, 14.1, 12.0. HRMS (m/z): $[\mathrm{M}+\mathrm{H}]^{+}$calcd for $\mathrm{C}_{28} \mathrm{H}_{28} \mathrm{~F}_{2} \mathrm{~N}_{3} \mathrm{O}_{3}$ : 492.2099; found, 492.2104. 


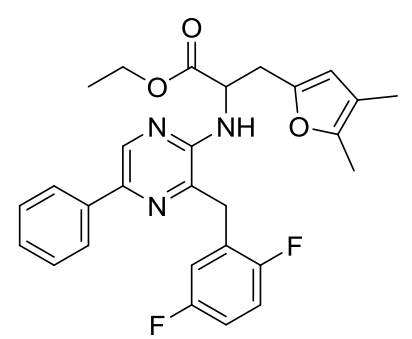

Ethyl 2-((3-(2,5-difluorobenzyl)-5-phenylpyrazin-2-yl)amino)-3-(4,5-dimethylfuran-2-yl)propanoate $23\{1,23,48\}$ (EC32712-085-2): Obtained as an oil $(0.25 \mathrm{~g}, 64 \%)$, using toluene at $90{ }^{\circ} \mathrm{C}$, after a chromatography over silica gel (cyclohexane - ethyl acetate 96/4). ${ }^{1} \mathrm{H}$ NMR $\left(\mathrm{CDCl}_{3}\right): 8.43(\mathrm{~s}, 1 \mathrm{H}), 7.90(\mathrm{~m}, 2 \mathrm{H}), 7.45(\mathrm{~m}, 2 \mathrm{H}), 7.36(\mathrm{~m}, 1 \mathrm{H}), 7.05$ $(\mathrm{m}, 1 \mathrm{H}), 6.94(\mathrm{~m}, 2 \mathrm{H}), 5.77(\mathrm{~s}, 1 \mathrm{H}), 5.22(\mathrm{~d}, 1 \mathrm{H}, J=7.5 \mathrm{~Hz}), 4.95(\mathrm{dt}, 1 \mathrm{H}, J=7.5,5.5 \mathrm{~Hz}), 4.21(\mathrm{~m}, 2 \mathrm{H}), 4.14(\mathrm{~d}$, $2 \mathrm{H}, J=2.7 \mathrm{~Hz}), 3.17(\mathrm{~d}, 2 \mathrm{H}, J=5.5 \mathrm{~Hz}), 2.13(\mathrm{~s}, 3 \mathrm{H}), 1.88(\mathrm{~s}, 3 \mathrm{H}), 1.26(\mathrm{t}, 3 \mathrm{H}, J=7.1 \mathrm{~Hz}) .{ }^{13} \mathrm{C} \mathrm{NMR}\left(\mathrm{CDCl}_{3}\right)$ : 172.1, 158.7 (241, $2 \mathrm{~Hz}), 156.8$ (241, $2 \mathrm{~Hz}), 150.2,147.2,146.8,141.2,139.0,137.2,137.1,128.7,127.9,125.5$, $125.5(19,8 \mathrm{~Hz}), 117.1(24,4 \mathrm{~Hz}), 116.1$ (25, $9 \mathrm{~Hz}), 114.8$ (24, $9 \mathrm{~Hz}), 114.4,61.3,53.4,32.4$ (br), 30.5, 14.1, 11.2, 9.8. HRMS $(\mathrm{m} / z):[\mathrm{M}+\mathrm{H}]^{+}$calcd for $\mathrm{C}_{28} \mathrm{H}_{28} \mathrm{~F}_{2} \mathrm{~N}_{3} \mathrm{O}_{3}$ : 492.2099; found, 492.2083 .

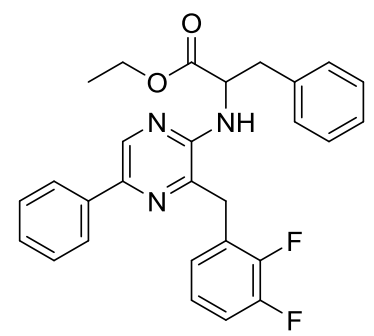

Ethyl (3-(2,3-difluorobenzyl)-5-phenylpyrazin-2-yl)phenylalaninate 23\{1,24,1\} (YJ 33067-117-1): Obtained as an oil $(0.37 \mathrm{~g}, 82 \%)$, using toluene at $90^{\circ} \mathrm{C}$, after a chromatography over silica gel (cyclohexane - ethyl acetate $97 / 3$ to $96 / 4) .{ }^{1} \mathrm{H}$ NMR $\left(\mathrm{CDCl}_{3}\right): 7.91(\mathrm{~s}, 1 \mathrm{H}), 7.90(\mathrm{~m}, 2 \mathrm{H}), 7.45(\mathrm{~m}, 2 \mathrm{H}), 7.35(\mathrm{~m}, 1 \mathrm{H}), 7.24(\mathrm{~m}, 3 \mathrm{H}), 7.07(\mathrm{~m}, 3 \mathrm{H})$, $6.95(\mathrm{~m}, 1 \mathrm{H}), 5.02(\mathrm{~m}, 2 \mathrm{H}), 4.18(\mathrm{q}, 2 \mathrm{H}, J=7.3 \mathrm{~Hz}), 4.12(\mathrm{~m}, 2 \mathrm{H}), 3.26(\mathrm{dd}, 1 \mathrm{H}, J=5.6$ and $14.1 \mathrm{~Hz}), 3.17(\mathrm{dd}$, $1 \mathrm{H}, J=6.0$ and $14.1 \mathrm{~Hz}), 1.23(\mathrm{t}, 3 \mathrm{H}, J=7.3 \mathrm{~Hz}) .{ }^{13} \mathrm{C} \mathrm{NMR}\left(\mathrm{CDCl}_{3}\right): 172.3,150.6(248$ and $13 \mathrm{~Hz}), 150.0,148.9$ (246, and $12 \mathrm{~Hz}), 141.3,139.3,137.1,136.9,136.1,129.1,128.7,128.5,127.9,127.0,126.0(12 \mathrm{~Hz}), 125.6,125.3$ $(3 \mathrm{~Hz}), 124.1$ (4 and $6 \mathrm{~Hz}), 115.8(18 \mathrm{~Hz}), 61.3,55.0,37.8,32.4,14.1$. HRMS $(\mathrm{m} / z):[\mathrm{M}+\mathrm{H}]^{+}$calcd for $\mathrm{C}_{28} \mathrm{H}_{26} \mathrm{~F}_{2} \mathrm{~N}_{3} \mathrm{O}_{2}$ : 474.1993; found, 474.1982.

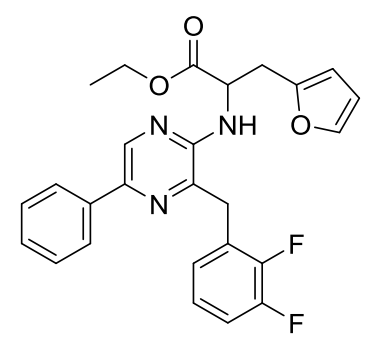

Ethyl 2-((3-(2,3-difluorobenzyl)-5-phenylpyrazin-2-yl)amino)-3-(furan-2-yl)propanoate 23\{1,24,37\} (EC31095125-4): Obtained as an oil $(0.36 \mathrm{~g}, 66 \%)$, using toluene at $90{ }^{\circ} \mathrm{C}$, after a chromatography over silica gel (cyclohexane - ethyl acetate 92/8). ${ }^{1} \mathrm{H}$ NMR $\left(\mathrm{CDCl}_{3}\right): 8.44(\mathrm{~s}, 1 \mathrm{H}), 7.91(\mathrm{~m}, 2 \mathrm{H}), 7.45(\mathrm{~m}, 2 \mathrm{H}), 7.37(\mathrm{~m}, 1 \mathrm{H}), 7.29$ $(\mathrm{dd}, 1 \mathrm{H}, J=1.9,0.8 \mathrm{~Hz}), 7.08(\mathrm{~m}, 1 \mathrm{H}), 7.00(\mathrm{~m}, 2 \mathrm{H}), 6.26(\mathrm{dd}, 1 \mathrm{H}, J=3.2,1.9 \mathrm{~Hz}), 5.98(\mathrm{dd}, 1 \mathrm{H}, J=3.2,0.8 \mathrm{~Hz})$, $5.18(\mathrm{~d}, 1 \mathrm{H}, J=7.5 \mathrm{~Hz}), 5.01(\mathrm{dt}, 1 \mathrm{H}, J=7.6,5.4 \mathrm{~Hz}), 4.20(\mathrm{~m}, 4 \mathrm{H}), 3.28(\mathrm{~d},, 2 \mathrm{H} J=5.4 \mathrm{~Hz}), 1.25(\mathrm{t}, 3 \mathrm{H}, J=7.1$ $\mathrm{Hz}) .{ }^{13} \mathrm{C}$ NMR $\left(\mathrm{CDCl}_{3}\right): 171.9,150.6(248,13 \mathrm{~Hz}), 150.5,150.1,148.9$ (246, $\left.13 \mathrm{~Hz}\right), 142.1,141.3,139.2(1 \mathrm{~Hz})$, 137.2, 137.1, 128.7, 127.9, 126.2 (d13 Hz), 125.6, 125.4 (3 Hz), 124.1 (7, 5 Hz), 115.7 (17 Hz), 110.3, 107.8, 61.4, 53.3, $32.4(3 \mathrm{~Hz}), 30.4,14.1$. HRMS (m/z): $[\mathrm{M}+\mathrm{H}]^{+}$calcd for $\mathrm{C}_{26} \mathrm{H}_{24} \mathrm{~F}_{2} \mathrm{~N}_{3} \mathrm{O}_{3}$ : 464.1786; found, 464.1780. 


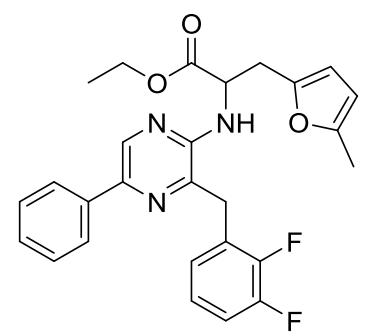

Ethyl 2-((3-(2,3-difluorobenzyl)-5-phenylpyrazin-2-yl)amino)-3-(5-methylfuran-2-yl)propanoate $\quad \mathbf{2 3}\{1,24,42\}$ (EC32712-039-2): Obtained as an oil $(0.31 \mathrm{~g}, 80 \%)$, using toluene at $90{ }^{\circ} \mathrm{C}$, after a chromatography over silica gel (cyclohexane - ethyl acetate 96/4). ${ }^{1} \mathrm{H}$ NMR $\left(\mathrm{CDCl}_{3}\right): 8.43(\mathrm{~s}, 1 \mathrm{H}), 7.90(\mathrm{~m}, 2 \mathrm{H}), 7.45(\mathrm{dd}, 2 \mathrm{H}, J=8.3,6.9 \mathrm{~Hz})$, $7.36(\mathrm{~m}, 1 \mathrm{H}), 7.07(\mathrm{~m}, 1 \mathrm{H}), 7.00(\mathrm{~m}, 2 \mathrm{H}), 5.87(\mathrm{~d}, 1 \mathrm{H}, J=3.1 \mathrm{~Hz}), 5.83(\mathrm{dd}, 1 \mathrm{H}, J=3.2,1.2 \mathrm{~Hz}), 5.17(\mathrm{~d}, 1 \mathrm{H}, J=$ $7.5 \mathrm{~Hz}$ ), $4.97(\mathrm{dt}, 1 \mathrm{H}, J=7.6,5.4 \mathrm{~Hz}), 4.19(\mathrm{~m}, 4 \mathrm{H}), 3.22(\mathrm{~d}, 2 \mathrm{H}, J=5.4 \mathrm{~Hz}), 2.23(\mathrm{~s}, 3 \mathrm{H}), 1.27(\mathrm{t}, 3 \mathrm{H}, J=7.2 \mathrm{~Hz})$. ${ }^{13} \mathrm{C} \mathrm{NMR}\left(\mathrm{CDCl}_{3}\right)$ : 172.0, 151.6, $150.6(248,13 \mathrm{~Hz}), 150.1,148.9(246,13 \mathrm{~Hz}), 148.4,141.2,139.2,137.2,137.0$, 128.7, 127.9, $126.2(12 \mathrm{~Hz}), 125.5,125.4(3 \mathrm{~Hz}), 124.0(7,5 \mathrm{~Hz}), 115.7(17 \mathrm{~Hz}), 108.6,106.1,61.3,53.3,32.3$, 30.6, 14.1, 13.4. HRMS (m/z): $[\mathrm{M}+\mathrm{H}]^{+}$calcd for $\mathrm{C}_{27} \mathrm{H}_{26} \mathrm{~F}_{2} \mathrm{~N}_{3} \mathrm{O}_{3}$ : 478.1942; found, 478.1946.

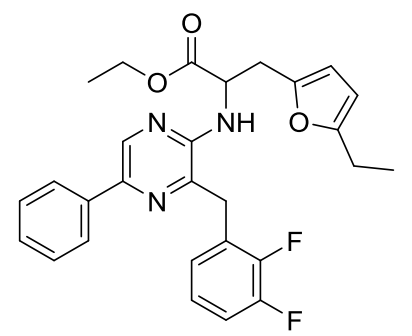

Ethyl 2-((3-(2,3-difluorobenzyl)-5-phenylpyrazin-2-yl)amino)-3-(5-ethylfuran-2-yl)propanoate $\quad \mathbf{2 3}\{1,24,44\}$ (EC32712-105-1): Obtained as an oil $(0.45 \mathrm{~g}, 74 \%)$, using toluene at $90{ }^{\circ} \mathrm{C}$, after a chromatography over silica gel (cyclohexane - ethyl acetate 96/4). ${ }^{1} \mathrm{H}$ NMR $\left(\mathrm{CDCl}_{3}\right): 8.43(\mathrm{~s}, 1 \mathrm{H}), 7.89(\mathrm{~m}, 2 \mathrm{H}), 7.44(\mathrm{~m}, 2 \mathrm{H}), 7.36(\mathrm{~m}, 1 \mathrm{H}), 7.07$ $(\mathrm{m}, 1 \mathrm{H}), 7.00(\mathrm{~m}, 2 \mathrm{H}), 5.86(\mathrm{~m}, 2 \mathrm{H}), 5.17(\mathrm{~d}, 1 \mathrm{H}, J=7.6 \mathrm{~Hz}), 4.98(\mathrm{dt}, 1 \mathrm{H} \mathrm{J}=7.6,5.4 \mathrm{~Hz}), 4.20(\mathrm{~m}, 4 \mathrm{H}), 3.23(\mathrm{~d}$, $2 \mathrm{H}, J=5.4 \mathrm{~Hz}), 2.57(\mathrm{qd}, 2 \mathrm{H}, J=7.6,1.0 \mathrm{~Hz}), 1.27(\mathrm{t}, 3 \mathrm{H}, J=7.1 \mathrm{~Hz}), 1.21(\mathrm{t}, 3 \mathrm{H}, J=7.6 \mathrm{~Hz}) .{ }^{13} \mathrm{C} \mathrm{NMR}$ $\left(\mathrm{CDCl}_{3}\right)$ : 172.0, 157.4, $150.6(248,13 \mathrm{~Hz}), 150.0,148.5(246,13 \mathrm{~Hz}), 148.3,141.2,139.2,137.2,137.0,128.7$, 127.9, $126.2(13 \mathrm{~Hz}), 125.5,125.4(3 \mathrm{~Hz}), 124.0(7,5 \mathrm{~Hz}), 115.7(17 \mathrm{~Hz}), 108.4,104.5,61.3,53.3,32.3,30.6$, 21.3, 14.1, 12.0. HRMS (m/z): $[\mathrm{M}+\mathrm{H}]^{+}$calcd for $\mathrm{C}_{28} \mathrm{H}_{28} \mathrm{~F}_{2} \mathrm{~N}_{3} \mathrm{O}_{3}$ : 492.2099; found, 492.2090 .

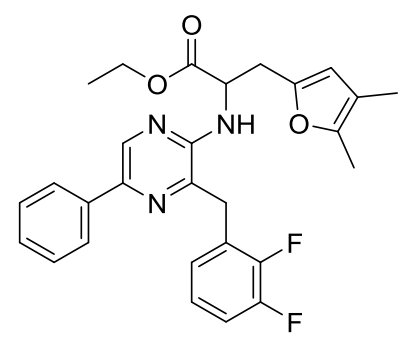

Ethyl 2-((3-(2,3-difluorobenzyl)-5-phenylpyrazin-2-yl)amino)-3-(4,5-dimethylfuran-2-yl)propanoate $\mathbf{2 3}\{1,24,48\}$ (EC32712-089-2): Obtained as an oil $(0.21 \mathrm{~g}, 46 \%)$, using toluene at $60{ }^{\circ} \mathrm{C}$, after a chromatography over silica gel (cyclohexane - ethyl acetate 96/4). ${ }^{1} \mathrm{H}$ NMR $\left(\mathrm{CDCl}_{3}\right): 8.43(\mathrm{~s}, 1 \mathrm{H}), 7.89(\mathrm{~m}, 2 \mathrm{H}), 7.44(\mathrm{~m}, 2 \mathrm{H}), 7.36(\mathrm{~m}, 1 \mathrm{H}), 7.08$ $(\mathrm{m}, 1 \mathrm{H}), 7.00(\mathrm{~m}, 2 \mathrm{H}), 5.77(\mathrm{~s}, 1 \mathrm{H}), 5.20(\mathrm{~d}, 1 \mathrm{H}, J=7.6 \mathrm{~Hz}), 4.95(\mathrm{dt}, 1 \mathrm{H}, J=7.6,5.4 \mathrm{~Hz}), 4.21(\mathrm{~m}, 4 \mathrm{H}), 3.18(\mathrm{~d}$, $2 \mathrm{H}, J=5.4 \mathrm{~Hz}), 2.13(\mathrm{~s}, 3 \mathrm{H}), 1.88(\mathrm{~s}, 3 \mathrm{H}), 1.27(\mathrm{t}, 3 \mathrm{H}, J=7.1 \mathrm{~Hz}) .{ }^{13} \mathrm{C} \mathrm{NMR}\left(\mathrm{CDCl}_{3}\right): 172.0,150.6(248,13 \mathrm{~Hz})$, 150.1, 148.9 (246, $13 \mathrm{~Hz}), 147.1,146.8,141.2,139.2,137.2,136.9,128.7,127.9,126.2(12 \mathrm{~Hz}), 125.5,125.4(\mathrm{~Hz})$, $124.0(7,5 \mathrm{~Hz}), 115.7(17 \mathrm{~Hz}), 114.4,111.1,61.3,53.4,32.3(3 \mathrm{~Hz}), 30.5,14.1,11.2,9.7 . \mathrm{HRMS}(\mathrm{m} / z):[\mathrm{M}+\mathrm{H}]^{+}$ calcd for $\mathrm{C}_{28} \mathrm{H}_{28} \mathrm{~F}_{2} \mathrm{~N}_{3} \mathrm{O}_{3}$ : 492.2099; found, 492.2111. 


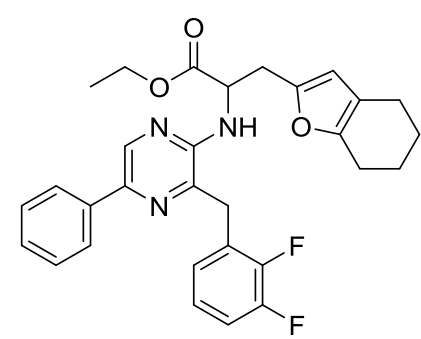

Ethyl 2-((3-(2,3-difluorobenzyl)-5-phenylpyrazin-2-yl)amino)-3-(4,5,6,7-tetrahydrobenzofuran-2-yl)propanoate $23\{1,24,49\}$ (YJ 33068-075-2): Obtained as an oil $(0.37 \mathrm{~g}, 56 \%)$, using toluene at $90{ }^{\circ} \mathrm{C}$, after a chromatography over silica gel (cyclohexane - ethyl acetate 97/3). ${ }^{1} \mathrm{H}$ NMR $\left(\mathrm{CDCl}_{3}\right): 8.42(\mathrm{~s}, 1 \mathrm{H}), 7.89(\mathrm{~m}, 2 \mathrm{H}), 7.44(\mathrm{~m}, 2 \mathrm{H}), 7.35$ $(\mathrm{m}, 1 \mathrm{H}), 7.12-6.99(\mathrm{~m}, 3 \mathrm{H}), 5.80(\mathrm{~s}, 1 \mathrm{H}), 5.22(\mathrm{~d}(\mathrm{br}), J=7.8 \mathrm{~Hz}), 4.96(\mathrm{~m}, 1 \mathrm{H}), 4.21(\mathrm{~m}, 4 \mathrm{H}), 3.20(\mathrm{~d}, 2 \mathrm{H}, J=$ $5.5 \mathrm{~Hz}), 2.52(\mathrm{~m}, 2 \mathrm{H}), 2.35(\mathrm{~m}, 2 \mathrm{H}), 1.82(\mathrm{~m}, 2 \mathrm{H}), 1.72(\mathrm{~m}, 2 \mathrm{H}), 1.26(\mathrm{t}, 3 \mathrm{H}, J=7.2 \mathrm{~Hz}) .{ }^{13} \mathrm{C} \mathrm{NMR}\left(\mathrm{CDCl}_{3}\right)$ : 172.1, 150.6 (13 and $248 \mathrm{~Hz}), 150.3,150.1,148.9$ (13 and $248 \mathrm{~Hz}), 147.8,141.2,139.2,137.2,136.9,128.7$, 127.9, $126.2(13 \mathrm{~Hz}), 125.5,125.4(4 \mathrm{~Hz}), 124.0(4$ and $7 \mathrm{~Hz}), 117.4,115.6(17 \mathrm{~Hz}), 108.8,61.3,53.5,32.3,30.7$, 23.1, 23.0 (two signals), 22.0, 14.1. HRMS $(\mathrm{m} / z)$ : $[\mathrm{M}+\mathrm{H}]^{+}$calcd for $\mathrm{C}_{30} \mathrm{H}_{30} \mathrm{~F}_{2} \mathrm{~N}_{3} \mathrm{O}_{3}$ : 518.2255; found, 518.2263 .

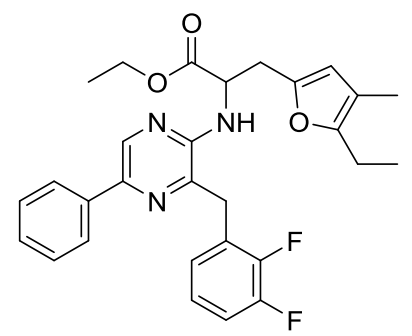

Ethyl 2-((3-(2,3-difluorobenzyl)-5-phenylpyrazin-2-yl)amino)-3-(5-ethyl-4-methylfuran-2-yl)propanoate $\mathbf{2 3}\{1,24,50\}$ (YJ 33069-009-1): Obtained as an oil (0.59 g, 77\%), using toluene at $90{ }^{\circ} \mathrm{C}$, after a chromatography over silica gel (cyclohexane - ethyl acetate 97/3). ${ }^{1} \mathrm{H}$ NMR $\left(\mathrm{CDCl}_{3}\right): 8.43(\mathrm{~s}, 1 \mathrm{H}), 7.89(\mathrm{~m}, 2 \mathrm{H}), 7.44(\mathrm{~m}, 2 \mathrm{H}), 7.35$ $(\mathrm{m}, 1 \mathrm{H}), 7.12-6.99(\mathrm{~m}, 3 \mathrm{H}), 5.77(\mathrm{~s}, 1 \mathrm{H}), 5.20(\mathrm{~d}(\mathrm{br}), J=7.8 \mathrm{~Hz}), 4.96(\mathrm{~m}, 1 \mathrm{H}), 4.22(\mathrm{~m}, 4 \mathrm{H}), 3.19(\mathrm{~d}, 2 \mathrm{H}, J=$ $5.5 \mathrm{~Hz}), 2.52(\mathrm{q}, 2 \mathrm{H}, J=7.7 \mathrm{~Hz}), 1.90(\mathrm{~s}, 3 \mathrm{H}), 1.27(\mathrm{~d}, 3 \mathrm{H} J=7.0 \mathrm{~Hz}), 1.15(\mathrm{~d}, 3 \mathrm{H} J=7.7 \mathrm{~Hz}) .{ }^{13} \mathrm{C} \mathrm{NMR}\left(\mathrm{CDCl}_{3}\right)$ : 172.1, 152.0, 150.6 (13 and $248 \mathrm{~Hz}), 150.1,148.9$ (13 and $248 \mathrm{~Hz}), 147.1,141.2,139.1,137.2,137.0,128.7$, 127.8, $126.2(13 \mathrm{~Hz}), 125.5,125.4(4 \mathrm{~Hz}), 124.0$ (4 and $7 \mathrm{~Hz}), 115.6(17 \mathrm{~Hz}), 113.5,111.2,61.3,53.3,32.3,30.6$, 19.3, 14.1, 12.8, 9.6. HRMS (m/z): $[\mathrm{M}+\mathrm{H}]^{+}$calcd for $\mathrm{C}_{29} \mathrm{H}_{30} \mathrm{~F}_{2} \mathrm{~N}_{3} \mathrm{O}_{3}: 505.2255$; found, 505.2260.

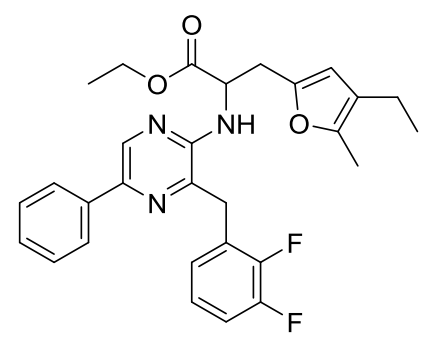

Ethyl 2-((3-(2,3-difluorobenzyl)-5-phenylpyrazin-2-yl)amino)-3-(4-ethyl-5-methylfuran-2-yl)propanoate $23\{1,24,51\}$ (YJ 33068-079-2): Obtained as an oil (0.45 g, 70\%), using toluene at $90{ }^{\circ} \mathrm{C}$, after a chromatography over silica gel (cyclohexane - ethyl acetate 97/3). ${ }^{1} \mathrm{H}$ NMR $\left(\mathrm{CDCl}_{3}\right): 8.43(\mathrm{~s}, 1 \mathrm{H}), 7.89(\mathrm{~m}, 2 \mathrm{H}), 7.44(\mathrm{~m}, 2 \mathrm{H}), 7.35$ $(\mathrm{m}, 1 \mathrm{H}), 7.12-6.99(\mathrm{~m}, 3 \mathrm{H}), 5.86(\mathrm{~s}, 1 \mathrm{H}), 5.23(\mathrm{~d}(\mathrm{br}), J=7.8 \mathrm{~Hz}), 4.97(\mathrm{~m}, 1 \mathrm{H}), 4.21(\mathrm{~m}, 4 \mathrm{H}), 3.18(\mathrm{~d}, 2 \mathrm{H}, J=$ $5.5 \mathrm{~Hz}), 2.29(\mathrm{q}, 2 \mathrm{H}, J=7.6), 2.15(\mathrm{~s}, 3 \mathrm{H}), 1.26(\mathrm{~d}, 3 \mathrm{H} J=7.0 \mathrm{~Hz}), 1.10(\mathrm{~d}, 3 \mathrm{H} J=7.6 \mathrm{~Hz}) .{ }^{13} \mathrm{C} \mathrm{NMR}\left(\mathrm{CDCl}_{3}\right)$ : 172.1, 150.6 (13 and $248 \mathrm{~Hz}$ ), 150.1, 148.9 (13 and $248 \mathrm{~Hz}), 147.3,146.2,139.1,137.2,136.9,128.7,127.8,126.2$ (13 Hz), 125.5, 125.4 (4 Hz), 124.0 (4 and $7 \mathrm{~Hz}), 121.2,115.6(17 \mathrm{~Hz}), 109.5,61.3,53.4,32.3,30.7,17.9,14.8$, 14.1, 11.2. HRMS $(m / z):[\mathrm{M}+\mathrm{H}]^{+}$calcd for $\mathrm{C}_{29} \mathrm{H}_{30} \mathrm{~F}_{2} \mathrm{~N}_{3} \mathrm{O}_{3}: 505.2255$; found, 505.2257. 


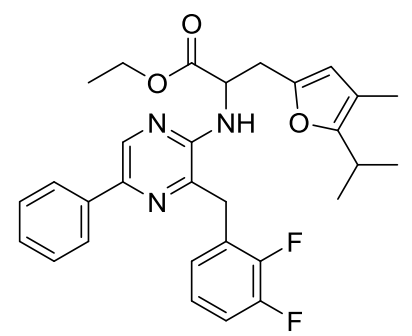

Ethyl 2-((3-(2,3-difluorobenzyl)-5-phenylpyrazin-2-yl)amino)-3-(5-isopropyl-4-methylfuran-2-yl)propanoate $\mathbf{2 3}\{1,24,52\}$ (YJ 33069-057-1): Obtained as an oil (0.54 g, 82\%), using toluene at $90{ }^{\circ} \mathrm{C}$, after a chromatography over silica gel (cyclohexane - ethyl acetate 97/3). ${ }^{1} \mathrm{H}$ NMR $\left(\mathrm{CDCl}_{3}\right): 8.43(\mathrm{~s}, 1 \mathrm{H}), 7.89(\mathrm{~m}, 2 \mathrm{H}), 7.44(\mathrm{~m}, 2 \mathrm{H}), 7.34$ (m, 1H), 7.12 - $6.97(\mathrm{~m}, 3 \mathrm{H}), 5.75(\mathrm{~s}, 1 \mathrm{H}), 5.16(\mathrm{~d}(\mathrm{br}), J=7.8 \mathrm{~Hz}), 4.99(\mathrm{~m}, 1 \mathrm{H}), 4.22(\mathrm{~m}, 4 \mathrm{H}), 3.21(\mathrm{~m}, 2 \mathrm{H}), 2.94$ (sept, $1 \mathrm{H}, J=7.1), 1.91(\mathrm{~s}, 3 \mathrm{H}), 1.28(\mathrm{~d}, 3 \mathrm{H} J=7.0 \mathrm{~Hz}), 1.19(\mathrm{~d}, 3 \mathrm{H} J=7.1 \mathrm{~Hz}), 1.18(\mathrm{~d}, 3 \mathrm{H} J=7.1 \mathrm{~Hz}) .{ }^{13} \mathrm{C}$ NMR $\left(\mathrm{CDCl}_{3}\right)$ : 172.1, 150.1, $150.6(13$ and $248 \mathrm{~Hz}), 150.1,148.9$ (13 and $\left.248 \mathrm{~Hz}\right), 146.7,141.1,139.2,137.2$, 136.8, 128.7, 127.9, $126.2(13 \mathrm{~Hz}), 125.5,125.4(4 \mathrm{~Hz}), 124.0$ (4 and 7 Hz), $115.6(17 \mathrm{~Hz}), 112.4,111.3,61.3$, 53.3, 32.3, 30.6, 26.1, 21.3, 21.2, .3, 14.1, 9.6. HRMS $(\mathrm{m} / \mathrm{z}):[\mathrm{M}+\mathrm{H}]^{+}$calcd for $\mathrm{C}_{30} \mathrm{H}_{32} \mathrm{~F}_{2} \mathrm{~N}_{3} \mathrm{O}_{3}$ : 520.2411; found, 520.2410 .

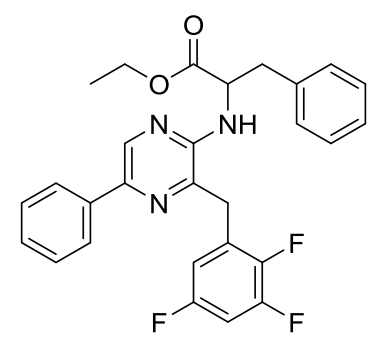

Ethyl (5-phenyl-3-(2,3,5-trifluorobenzyl)pyrazin-2-yl)phenylalaninate 23\{1,26,1\} (YJ 33067-123-2): Obtained as an oil $(0.13 \mathrm{~g}, 29 \%)$, using toluene at $60{ }^{\circ} \mathrm{C}$, after a chromatography over silica gel (cyclohexane - ethyl acetate 97/3 to 96/4). ${ }^{1} \mathrm{H}$ NMR $\left(\mathrm{CDCl}_{3}\right): 8.46(\mathrm{~s}, 1 \mathrm{H}), 7.89(\mathrm{~m}, 2 \mathrm{H}), 7.45(\mathrm{~m}, 2 \mathrm{H}), 7.36(\mathrm{~m}, 1 \mathrm{H}), 7.26(\mathrm{~m}, 3 \mathrm{H}), 7.08(\mathrm{~m}$, $2 \mathrm{H}), 6.83(\mathrm{~m}, 1 \mathrm{H}), 6.69(\mathrm{~m}, 1 \mathrm{H}), 5.03(\mathrm{~m}, 1 \mathrm{H}), 4.90(\mathrm{~d}(\mathrm{br}), J=7.3 \mathrm{~Hz}), 4.20(\mathrm{q}, 2 \mathrm{H}, J=7.1 \mathrm{~Hz}), 4.08(\mathrm{~m}, 2 \mathrm{H})$, $3.29(\mathrm{dd}, 1 \mathrm{H}, J=5.6$ and $13.8 \mathrm{~Hz}), 3.18(\mathrm{dd}, 1 \mathrm{H}, J=6.3$ and $13.8 \mathrm{~Hz}), 1.25(\mathrm{t}, 3 \mathrm{H}, J=7.1 \mathrm{~Hz}) .{ }^{13} \mathrm{C} \mathrm{NMR}\left(\mathrm{CDCl}_{3}\right)$ : 172.3, 157.5 (242, 12 and $3 \mathrm{~Hz}), 150.3$ (247, 13 and $15 \mathrm{~Hz}), 149.9,145.8$ (242, 12 and $4 \mathrm{~Hz}), 141.4,138.4,137.3$, 137.0, 136.1, 129.1, 128.8, 128.5, 128.0, 127.0, 126.9 (9 and $14 \mathrm{~Hz}), 125.5,111.8$ (3 and $24 \mathrm{~Hz}), 104.1$ (21 and 28 $\mathrm{Hz}), 61.3,55.0,37.7,32.2,14.1$. HRMS $(\mathrm{m} / \mathrm{z})$ : $[\mathrm{M}+\mathrm{H}]^{+}$calcd for $\mathrm{C}_{28} \mathrm{H}_{25} \mathrm{~F}_{3} \mathrm{~N}_{3} \mathrm{O}_{2}$ : 492.1899; found, 492.1893.

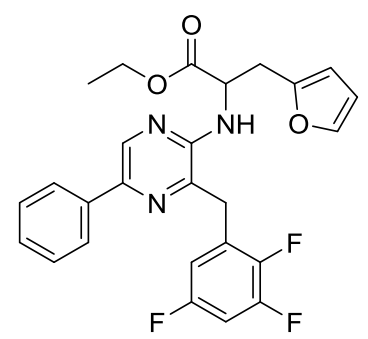

Ethyl 3-(furan-2-yl)-2-((5-phenyl-3-(2,3,5-trifluorobenzyl)pyrazin-2-yl)amino)propanoate 23 $\{1,26,37\}$ (YJ 33067125-2): Obtained as an oil (0.10 g, 23\%), using toluene at $60{ }^{\circ} \mathrm{C}$, after a chromatography over silica gel (cyclohexane - ethyl acetate 97/3). ${ }^{1} \mathrm{H}$ NMR $\left(\mathrm{CDCl}_{3}\right): 8.45(\mathrm{~s}, 1 \mathrm{H}), 7.91(\mathrm{~m}, 2 \mathrm{H}), 7.47(\mathrm{~m}, 2 \mathrm{H}), 7.35(\mathrm{~m}, 1 \mathrm{H}), 7.29$ $(\mathrm{m}, 1 \mathrm{H}), 6.87(\mathrm{~m}, 1 \mathrm{H}), 6.74(\mathrm{~m}, 1 \mathrm{H}), 6.26(\mathrm{~m}, 1 \mathrm{H}), 6.01(\mathrm{~m}, 1 \mathrm{H}), 5.15(\mathrm{~d}(\mathrm{br}), J=8.1 \mathrm{~Hz}), 5.03(\mathrm{~m}, 1 \mathrm{H}), 4.22(\mathrm{q}$, $2 \mathrm{H}, J=7.1 \mathrm{~Hz}), 4.16(\mathrm{~m}, 2 \mathrm{H}), 3.30(\mathrm{~m}, 2 \mathrm{H}), 1.26(\mathrm{t}, 3 \mathrm{H}, J=7.1 \mathrm{~Hz}) .{ }^{13} \mathrm{C} \mathrm{NMR}\left(\mathrm{CDCl}_{3}\right): 171.9,157.6(242,12$ and $3 \mathrm{~Hz}), 150.5,150.2$ (247, 13 and $15 \mathrm{~Hz}), 145.4$ (242, 12 and $4 \mathrm{~Hz}), 142.1,141.4,138.5,137.2,137.0,128.8,128.0$, 127.1 (9 and $14 \mathrm{~Hz}), 125.6,111.9$ (3 and $24 \mathrm{~Hz}), 110.3,107.8,104.1$ (21 and $28 \mathrm{~Hz}), 61.5,53.3,32.3,30.39,14.1$. $\operatorname{HRMS}(\mathrm{m} / \mathrm{z})$ : $[\mathrm{M}+\mathrm{H}]^{+}$calcd for $\mathrm{C}_{26} \mathrm{H}_{23} \mathrm{~F}_{3} \mathrm{~N}_{3} \mathrm{O}_{3}$ : 482.1692; found, 482.1682 . 


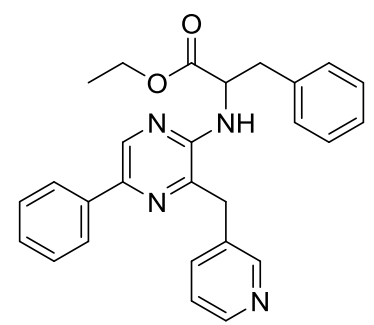

Ethyl (5-phenyl-3-(pyridin-3-ylmethyl)pyrazin-2-yl)phenylalaninate 23 $\{1,35,1\}$ (YJ30367-037-3): Obtained as an oil $(0.28 \mathrm{~g}, 62 \%)$ after a chromatography over silica gel (cyclohexane-ethyl acetate $2 / 1) .{ }^{1} \mathrm{H}$ NMR $\left(\mathrm{CDCl}_{3}\right): 8.52$ $(\mathrm{m}, 3 \mathrm{H}), 8.44(\mathrm{~s}, 1 \mathrm{H}), 7.90(\mathrm{~m}, 2 \mathrm{H}), 7.46(\mathrm{~m}, 3 \mathrm{H}), 7.35(\mathrm{~m}, 1 \mathrm{H}), 7.26(\mathrm{~m}, 3 \mathrm{H}), 7.19$ (ddd, $1 \mathrm{H}, J=0.9,4.9,5.6 \mathrm{~Hz})$, $7.03(\mathrm{~m}, 2 \mathrm{H}), 5.01(\mathrm{~m}, 1 \mathrm{H}), 4.84(\mathrm{~d}(\mathrm{br}, 1 \mathrm{H}, J=7.4 \mathrm{~Hz}), 4.17(\mathrm{q}, 2 \mathrm{H}, J=7.0 \mathrm{~Hz}), 4.08(\mathrm{~s}, 2 \mathrm{H}), 3.24(\mathrm{dd}, 1 \mathrm{H}, J=$ $5.9,13.5 \mathrm{~Hz}), 3.16(\mathrm{dd}, 1 \mathrm{H}, J=5.9,13.6 \mathrm{~Hz}), 1.23(\mathrm{t}, 3 \mathrm{H}, J=7.0 \mathrm{~Hz}) \cdot{ }^{13} \mathrm{C}$ NMR $\left(\mathrm{CDCl}_{3}\right): 172.4,150.1,150.0$, 148.3, 141.4, 139.7, 137.2, 137.1, 136.2, 136.1, 132.2, 129.2, 128.8, 128.5, 127.9, 127.0 125.6, 123.4, 61.3, 54.8, 37.7, 37.3, 14.1. HRMS (m/z): $[\mathrm{M}+\mathrm{H}]^{+}$calcd for $\mathrm{C}_{27} \mathrm{H}_{27} \mathrm{~N}_{4} \mathrm{O}_{2}, 439.2134$; found, 439.2137.

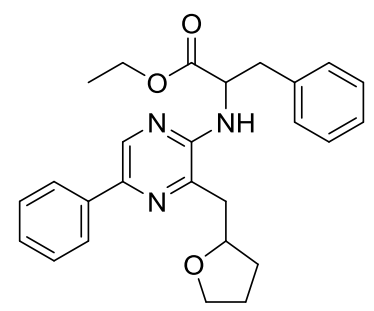

Ethyl (5-phenyl-3-((tetrahydrofuran-2-yl)methyl)pyrazin-2-yl)phenylalaninate $\quad \mathbf{2 3}\{1,38,1\} \quad$ (EC31093-017-3): Obtained as an oil $(0.06 \mathrm{~g}, 21 \%)$ after a chromatography over silica gel (cyclohexane - ethyl acetate 93/7). ${ }^{1} \mathrm{H}$ NMR $\left(\mathrm{CDCl}_{3} ; 1 / 1\right.$ mixture of diastereoisomers): $8.34(\mathrm{~s}, 0.5 \mathrm{H}), 8.33(\mathrm{~s}, 0.5 \mathrm{H}), 7.89-7.84(\mathrm{~m}, 2 \mathrm{H}), 7.45-7.40(\mathrm{~m}$, $2 \mathrm{H}), 7.35-7.21(\mathrm{~m}, 6 \mathrm{H}), 6.62(\mathrm{~d}, 0.5 \mathrm{H}, J=7.5 \mathrm{~Hz}), 6.44(\mathrm{~d}, 0.5 \mathrm{H}, J=7.1 \mathrm{~Hz}), 4.95(\mathrm{dd}, 0.5 \mathrm{H}, J=13.0,6.8 \mathrm{~Hz})$, $4.86(\mathrm{td}, 0.5 \mathrm{H}, J=7.4,5.9 \mathrm{~Hz}), 4.37-4.30(\mathrm{~m}, 0.5 \mathrm{H}), 4.30-4.23(\mathrm{~m}, 0.5 \mathrm{H}), 4.22-4.13(\mathrm{~m}, 2 \mathrm{H}), 3.89-3.79(\mathrm{~m}$, $0.5 \mathrm{H}), 3.77-3.58(\mathrm{~m}, 1.5 \mathrm{H}), 3.31-2.88(\mathrm{~m}, 4.5 \mathrm{H}), 2.17-2.00(\mathrm{~m}, 1 \mathrm{H}), 1.92-1.61(\mathrm{~m}, 3.5 \mathrm{H}), 1.22(\mathrm{t}, 1.5 \mathrm{H}, J=$ $7.1 \mathrm{~Hz}), 1.21(\mathrm{t}, 1.5 \mathrm{H}, J=7.1 \mathrm{~Hz}) .{ }^{13} \mathrm{C} \mathrm{NMR}\left(\mathrm{CDCl}_{3}\right): 173.4,173.1,152.2,152.0,141.5,141.2,141.0,140.9$, 137.9, 137.8, 137.2, 137.0, 136.7, 129.6, 129.5, 128.8, 128.5 (two signals), 127.7, 126.9 (two signals), 125.8, 125.7, 79.1, 79.0, 68.2 (two signals), 61.1, 61.0, 55.8, 55.5, 41.0, 40.5, 38.4, 38.3, 31.5, 30.3, 26.0, 25.8, 14.3. HRMS $(\mathrm{m} / z):[\mathrm{M}+\mathrm{H}]^{+}$calcd for $\mathrm{C}_{26} \mathrm{H}_{30} \mathrm{~N}_{3} \mathrm{O}_{3}, 432.2287$; found, 432.2273 .

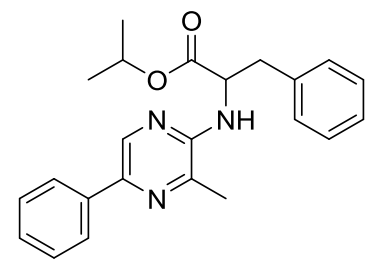

Isopropyl (3-methyl-5-phenylpyrazin-2-yl)phenylalaninate 23\{1,60,1\} (VHE30855-051-5): Obtained as a solid after a chromatography over silica gel (cyclohexane - ethyl acetate 6/1). ${ }^{1} \mathrm{H}$ NMR $\left(\mathrm{CDCl}_{3}\right): 8.37(\mathrm{~s}, 1 \mathrm{H}), 7.91(\mathrm{~m}$, $2 \mathrm{H}), 7.45(\mathrm{~m}, 2 \mathrm{H}),, 7.25-7.37(\mathrm{~m}, 4 \mathrm{H}), 7.20(\mathrm{~m}, 2 \mathrm{H}), 4.99-5.10(\mathrm{~m}, 2 \mathrm{H}), 4.89(\mathrm{~m}, 2 \mathrm{H}), 3.29(\mathrm{q}, 1 \mathrm{H}, J=13.8 \mathrm{~Hz})$, $3.27(\mathrm{q}, 1 \mathrm{H}, J=13.6 \mathrm{~Hz}), 2.43(\mathrm{~s}, 3 \mathrm{H}), 1.28(\mathrm{~d}, 3 \mathrm{H}, J=6.3 \mathrm{~Hz}), 1.23(\mathrm{~d}, 3 \mathrm{H}, J=6.3 \mathrm{~Hz}) .{ }^{13} \mathrm{C}$ NMR $\left(\mathrm{CDCl}_{3}\right)$ : 172.2, 150.5, 141.2, 139.6.2, 137.6, 136.4, 136.3, 129.4, 128.7, 128.5, 127.7, 127.0, 125.6, 69.1, 55.0, 37.8, 21.8, 21.7, 20.0. HRMS $(\mathrm{m} / \mathrm{z})$ : $[\mathrm{M}+\mathrm{H}]^{+}$calcd for $\mathrm{C}_{23} \mathrm{H}_{26} \mathrm{~N}_{3} \mathrm{O}_{2}, 376.2025$; found, 376.2027 .

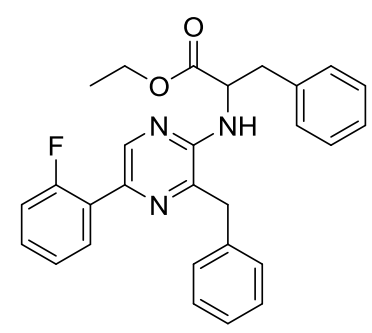


Ethyl (3-benzyl-5-(2-fluorophenyl)pyrazin-2-yl)phenylalaninate 23\{2,1,1\} (YJ31134-033-2): Obtained as an oil $(0.47 \mathrm{~g}, 81 \%)$ after a chromatography over silica gel (cyclohexane - ethyl acetate $91 / 9) .{ }^{1} \mathrm{H}\left(\mathrm{CDCl}_{3}\right): 8.53(\mathrm{~d}, 1 \mathrm{H}, J$ $=2.3 \mathrm{~Hz}), 8.04(\mathrm{dt}, 1 \mathrm{H}, J=1.9,7.9 \mathrm{~Hz}), 7.24(\mathrm{~m}, 11 \mathrm{H}), 6.99(\mathrm{~m}, 2 \mathrm{H}), 5.00(\mathrm{~m}, 2 \mathrm{H}), 4.13(\mathrm{~m}, 4 \mathrm{H}), 3.18(\mathrm{dd}, 1 \mathrm{H}, J$ $=5.1,13.7 \mathrm{~Hz}), 3.10(\mathrm{dd}, 1 \mathrm{H}, J=5.5,13.7 \mathrm{~Hz}), 1.20(\mathrm{t}, 3 \mathrm{H}, J=7.2 \mathrm{~Hz}) .{ }^{13} \mathrm{C} \mathrm{NMR}\left(\mathrm{CDCl}_{3}\right): 172.3,160.2(248 \mathrm{~Hz})$, 150.2, 141.4, 140.6 (13 Hz), 136.7 (3 Hz), 136.4, 136.2, 130.0 (3 Hz), 129.3, 129.1 (8 Hz), 128.9, 128.6, 128.4, 126.3, 126.8, $125.3(12 \mathrm{~Hz}), 124.5(3 \mathrm{~Hz}), 116.1(23 \mathrm{~Hz}), 61.2,54.8,40.8,37.8,14.1$. HRMS $(\mathrm{m} / \mathrm{z}):[\mathrm{M}+\mathrm{H}]^{+} \mathrm{calcd}$ for $\mathrm{C}_{28} \mathrm{H}_{27} \mathrm{FN}_{3} \mathrm{O}_{2}$, 456.2087; found, 456.2079.

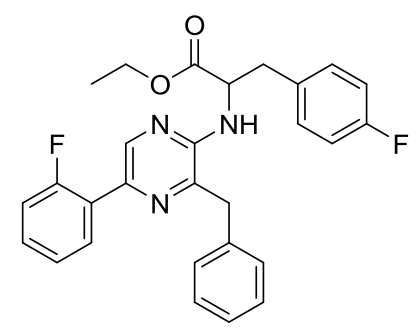

Ethyl 2-((3-benzyl-5-(2-fluorophenyl)pyrazin-2-yl)amino)-3-(4-fluorophenyl)propanoate 23 2,1,4\} (YJ30367-1192): Obtained as a $90 \%$ pure oil $(0.18 \mathrm{~g}, 59 \%)$ after a chromatography over silica gel (cyclohexane - ethyl acetate 95/5). ${ }^{1} \mathrm{H} \mathrm{NMR}\left(\mathrm{CDCl}_{3}\right): 8.53(\mathrm{~d}, 1 \mathrm{H}, J=2.3 \mathrm{~Hz}), 8.05(\mathrm{dt}, 1 \mathrm{H}, J=1.9,7.9 \mathrm{~Hz}), 7.37-7.14(\mathrm{~m}, 8 \mathrm{H}), 6.88(\mathrm{~m}, 2 \mathrm{H})$, $6.86(\mathrm{~m}, 2 \mathrm{H}), 4.99(\mathrm{~m}, 2 \mathrm{H}), 4.15(\mathrm{~m}, 4 \mathrm{H}), 3.16(\mathrm{~m}, 1 \mathrm{H}), 3.07(\mathrm{~m}, 1 \mathrm{H}), 1.20(\mathrm{t}, 3 \mathrm{H}, J=7.1 \mathrm{~Hz}) .{ }^{13} \mathrm{C} \mathrm{NMR}\left(\mathrm{CDCl}_{3}\right)$ : 172.1, 161.9 (245 Hz), $160.2(248 \mathrm{~Hz}), 150.1,141.4,140.5(13 \mathrm{~Hz}), 136.8(3 \mathrm{~Hz}), 136.4,131.8(3 \mathrm{~Hz}), 130.6(8$ Hz), 130.0 (3 Hz), 129.2 (8 Hz), 128.9, 128.6, 127.0, 124.5 (3 Hz), $116.1(23 \mathrm{~Hz}), 115.2(22 \mathrm{~Hz})$, 61.3, 54.8, 40.9, 36.8, 14.1, one signal missing. HRMS $(m / z)$ : $[\mathrm{M}+\mathrm{H}]^{+}$calcd for $\mathrm{C}_{28} \mathrm{H}_{26} \mathrm{~F}_{2} \mathrm{~N}_{3} \mathrm{O}_{2}$, 474.1993; found, 474.1991.

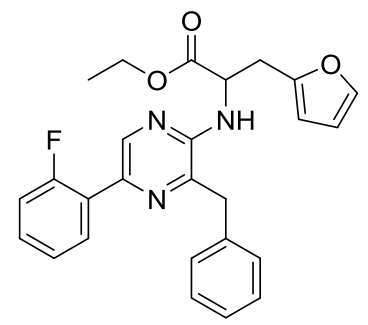

Ethyl 2-((3-benzyl-5-(2-fluorophenyl)pyrazin-2-yl)amino)-3-(furan-2-yl)propanoate 23\{2,1,37\} (YJ31134-087-2): Obtained as an oil $(0.42 \mathrm{~g}, 72 \%)$ after a chromatography over silica gel (cyclohexane - ethyl acetate $91 / 9) .{ }^{1} \mathrm{H}$ $\operatorname{NMR}\left(\mathrm{CDCl}_{3}\right): 8.52(\mathrm{~d}, 1 \mathrm{H}, J=2.3 \mathrm{~Hz}), 8.03(\mathrm{dt}, 1 \mathrm{H}, J=1.9,7.9 \mathrm{~Hz}), 7.41-7.24(\mathrm{~m}, 8 \mathrm{H}), 7.17(\mathrm{ddd}, 1 \mathrm{H}, J=1.3$, 8.0, $11.4 \mathrm{~Hz}), 6.22(\mathrm{dd}, 1 \mathrm{H}, J=1.8,3.0 \mathrm{~Hz}), 5.84(\mathrm{~m}, 1 \mathrm{H}), 5.19(\mathrm{~d}(\mathrm{br}), 1 \mathrm{H}, J=7.6 \mathrm{~Hz}), 4.97(\mathrm{~m}, 1 \mathrm{H}), 4.17(\mathrm{~m}$, $4 \mathrm{H}), 3.21(\mathrm{~m}, 2 \mathrm{H}), 1.22(\mathrm{t}, 3 \mathrm{H}, J=7.1 \mathrm{~Hz}) .{ }^{13} \mathrm{C} \mathrm{NMR}\left(\mathrm{CDCl}_{3}\right): 171.9,160.1(248 \mathrm{~Hz}), 150.5,150.2,141.9,141.4$, $140.5(13 \mathrm{~Hz}), 136.8$ (3 Hz), 136.5, 130.0 (3 Hz), 129.1 (8 Hz), 128.8, 128.7, 126.9, 125.3 (12 Hz), $124.4(3 \mathrm{~Hz})$, $116.1(23 \mathrm{~Hz}), 110.3,103.7,61.3,53.1,40.8,30.4$, 14.1. HRMS $(m / z):[\mathrm{M}+\mathrm{H}]^{+}$calcd for $\mathrm{C}_{26} \mathrm{H}_{25} \mathrm{FN}_{3} \mathrm{O}_{3}, 446.1880$; found, 446.1870 .

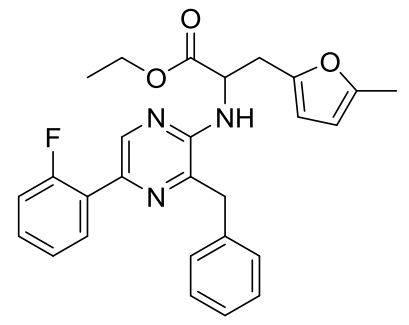

Ethyl 2-((3-benzyl-5-(2-fluorophenyl)pyrazin-2-yl)amino)-3-(5-methylfuran-2-yl)propanoate $\quad \mathbf{2 3}\{2,1,42\}$ (YJ30531-139-3): Obtained as an oil $(0.11 \mathrm{~g}, 30 \%)$ after a chromatography over silica gel (cyclohexane - ethyl acetate $98 / 2$ to $95 / 5) .{ }^{1} \mathrm{H}$ NMR $\left(\mathrm{CDCl}_{3}\right): 8.52(\mathrm{~d}, 1 \mathrm{H}, J=2.3 \mathrm{~Hz}), 8.03(\mathrm{dt}, 1 \mathrm{H}, J=1.9,7.8 \mathrm{~Hz}), 7.35-7.24(\mathrm{~m}, 7 \mathrm{H})$, $7.16(\mathrm{ddd}, 1 \mathrm{H}, J=1.2,8.1,11.1 \mathrm{~Hz}), 5.82(\mathrm{~m}, 1 \mathrm{H}), 5.75(\mathrm{~d}, 1 \mathrm{H}, J=3.0 \mathrm{~Hz}), 5.19(\mathrm{~d}(\mathrm{br}), 1 \mathrm{H}, J=7.5 \mathrm{~Hz}), 4.95(\mathrm{~m}$, $1 \mathrm{H}), 4.18(\mathrm{~m}, 4 \mathrm{H}), 3.16(\mathrm{~d}, 2 \mathrm{H}, J=5.4 \mathrm{~Hz}), 2.24(\mathrm{~s}, 3 \mathrm{H}), 1.24(\mathrm{t}, 3 \mathrm{H}, J=7.2 \mathrm{~Hz}) .{ }^{13} \mathrm{C} \mathrm{NMR}\left(\mathrm{CDCl}_{3}\right): 172.0,160.2$ (248 Hz), 151.4, 150.3, 148.5, 141.4, 140.6 (13 Hz), $136.8(3 \mathrm{~Hz}), 136.5,130.0(3 \mathrm{~Hz}), 129.2(8 \mathrm{~Hz}), 128.8,128.7$, 
126.9, $125.3(12 \mathrm{~Hz}), 124.4(3 \mathrm{~Hz}), 116.1(23 \mathrm{~Hz}), 108.6,106.2,61.3,53.2,40.7,30.6,14.1,13.5$. HRMS $(\mathrm{m} / z)$ : $[\mathrm{M}+\mathrm{H}]^{+}$calcd for $\mathrm{C}_{27} \mathrm{H}_{27} \mathrm{FN}_{3} \mathrm{O}_{3}, 460.2036$; found, 460.2039.

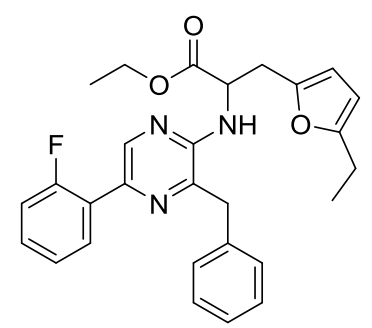

Ethyl 2-((3-benzyl-5-(2-fluorophenyl)pyrazin-2-yl)amino)-3-(5-ethylfuran-2-yl)propanoate 23\{2,1,44\} (YJ30367101-2): Obtained as an oil $(0.2 \mathrm{~g}, 46 \%)$ after a chromatography over silica gel (cyclohexane - ethyl acetate 95/5). ${ }^{1} \mathrm{H}$ NMR $\left(\mathrm{CDCl}_{3}\right): 8.51(\mathrm{~d}, 1 \mathrm{H}, J=2.3 \mathrm{~Hz}), 8.03(\mathrm{dt}, 1 \mathrm{H}, J=1.9,7.8 \mathrm{~Hz}), 7.35-7.23(\mathrm{~m}, 7 \mathrm{H}), 7.16(\mathrm{ddd}, 1 \mathrm{H}, J=$ $1.3,8.1,9.4 \mathrm{~Hz}), 5.82(\mathrm{~m}, 1 \mathrm{H}), 5.75(\mathrm{~d}, 1 \mathrm{H}, J=3.0 \mathrm{~Hz}), 5.19(\mathrm{~d}(\mathrm{br}), 1 \mathrm{H}, J=7.5 \mathrm{~Hz}), 4.95(\mathrm{~m}, 1 \mathrm{H}), 4.18(\mathrm{~m}, 4 \mathrm{H})$, $3.15(\mathrm{~d}, 2 \mathrm{H}, J=5.3 \mathrm{~Hz}), 2.58(\mathrm{q}, 2 \mathrm{H}, J=7.6 \mathrm{~Hz}), 1.24(\mathrm{t}, 3 \mathrm{H}, J=7.2 \mathrm{~Hz}), 1.21(\mathrm{t}, 3 \mathrm{H}, J=7.6 \mathrm{~Hz}) .{ }^{13} \mathrm{C} \mathrm{NMR}$ $\left(\mathrm{CDCl}_{3}\right): 172.0,160.1(248 \mathrm{~Hz}), 157.1,150.2,148.4,141.4,140.5(13 \mathrm{~Hz}), 136.8(3 \mathrm{~Hz}), 136.5,130.0(3 \mathrm{~Hz})$, 129.2 (8 Hz), 128.8, 128.7, 126.9, $125.3(12 \mathrm{~Hz}), 124.4(3 \mathrm{~Hz}), 116.1(23 \mathrm{~Hz}), 108.4,104.6,61.2,53.1,40.7,30.6$, 21.3, 14.1, 12.1. HRMS (m/z): [M+H] ${ }^{+}$calcd for $\mathrm{C}_{28} \mathrm{H}_{29} \mathrm{FN}_{3} \mathrm{O}_{3}, 474.2193$; found, 474.2186.

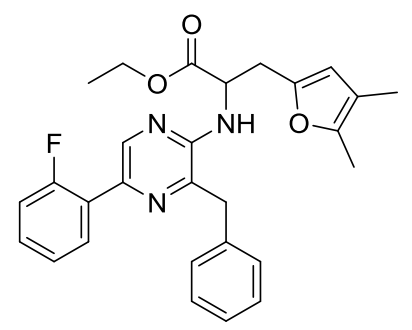

Ethyl 2-((3-benzyl-5-(2-fluorophenyl)pyrazin-2-yl)amino)-3-(4,5-dimethylfuran-2-yl)propanoate $\quad \mathbf{2 3}\{2,1,48\}$ (YJ30367-171-2): Obtained as an oil $(0.48 \mathrm{~g}, 86 \%)$ after a chromatography over silica gel (cyclohexane - ethyl acetate 95/5). ${ }^{1} \mathrm{H}$ NMR $\left(\mathrm{CDCl}_{3}\right): 8.51(\mathrm{~d}, 1 \mathrm{H}, J=2.4 \mathrm{~Hz}), 8.02(\mathrm{dt}, 1 \mathrm{H}, J=1.9,7.8 \mathrm{~Hz}), 7.35-7.23(\mathrm{~m}, 7 \mathrm{H}), 7.16$ (ddd, $1 \mathrm{H}, J=1.3,8.1,9.4 \mathrm{~Hz}), 5.65(\mathrm{~s}, 1 \mathrm{H}), 5.19(\mathrm{~d}(\mathrm{br}), 1 \mathrm{H}, J=7.5 \mathrm{~Hz}), 4.92(\mathrm{~m}, 1 \mathrm{H}), 4.16(\mathrm{~m}, 4 \mathrm{H}), 3.11(\mathrm{~d}, 2 \mathrm{H}, J$ $=5.1 \mathrm{~Hz}), 2.13(\mathrm{~s}, 3 \mathrm{H}), 1.88(\mathrm{~s}, 3 \mathrm{H}), 1.25(\mathrm{t}, 3 \mathrm{H}, J=7.2 \mathrm{~Hz}) \cdot{ }^{13} \mathrm{C} \mathrm{NMR}\left(\mathrm{CDCl}_{3}\right): 172.0,160.2(248 \mathrm{~Hz}), 150.2$, 147.1, 146.6, 141.4, $140.5(13 \mathrm{~Hz}), 136.7(3 \mathrm{~Hz}), 136.5,130.0(3 \mathrm{~Hz}), 129.2(8 \mathrm{~Hz}), 128.8,128.7,126.8,125.3(12$ $\mathrm{Hz}), 124.4(3 \mathrm{~Hz}), 116.1(23 \mathrm{~Hz}), 114.4,111.1,61.2,53.2,40.6,30.5,14.1,11.2,9.8$. HRMS $(\mathrm{m} / \mathrm{z}):[\mathrm{M}+\mathrm{H}]^{+} \mathrm{calcd}$ for $\mathrm{C}_{28} \mathrm{H}_{29} \mathrm{FN}_{3} \mathrm{O}_{3}, 474.2193$; found, 474.2203.

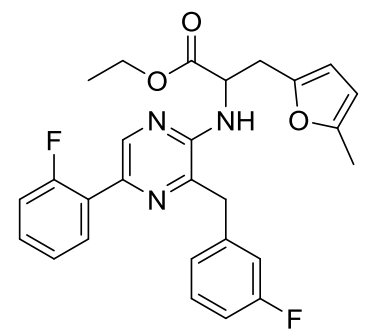

Ethyl 2-((3-(3-fluorobenzyl)-5-(2-fluorophenyl)pyrazin-2-yl)amino)-3-(5-methylfuran-2-yl)propanoate 23\{2,3,42\} (EC31095-109-2): Obtained as an oil $(0.53 \mathrm{~g}, 80 \%)$, after a chromatography over silica gel (cyclohexane - ethyl acetate $97 / 3) .{ }^{1} \mathrm{H}$ NMR $\left(\mathrm{CDCl}_{3}\right): 8.53(\mathrm{~d}, 1 \mathrm{H}, J=2.3 \mathrm{~Hz}), 8.00(\mathrm{td}, 1 \mathrm{H}, J=7.8,1.9 \mathrm{~Hz}), 7.33(\mathrm{~m}, 1 \mathrm{H}), 7.26(\mathrm{~m}$, $2 \mathrm{H}), 7.16(\mathrm{~m}, 1 \mathrm{H}), 7.06(\mathrm{dd}, 1 \mathrm{H}, J=7.6,0.6 \mathrm{~Hz}), 6.96(\mathrm{~m}, 2 \mathrm{H}), 5.82(\mathrm{~m}, 1 \mathrm{H}), 5.77(\mathrm{~d}, 1 \mathrm{H}, J=3.0 \mathrm{~Hz}), 5.11(\mathrm{~d}, 1 \mathrm{H}$, $J=7.5 \mathrm{~Hz}), 4.95(\mathrm{dt}, 1 \mathrm{H}, J=7.5,5.3 \mathrm{~Hz}), 4.20(\mathrm{~m}, 2 \mathrm{H}), 4.15(\mathrm{~s}, 2 \mathrm{H}, J=2.3 \mathrm{~Hz}), 3.18(\mathrm{~d}, 2 \mathrm{H}, J=5.3 \mathrm{~Hz}), 2.22(\mathrm{~d}$, $3 \mathrm{H}, J=0.7 \mathrm{~Hz}), 1.25(\mathrm{t}, 3 \mathrm{H}, J=7.1 \mathrm{~Hz}) .{ }^{13} \mathrm{C}$ NMR $\left(\mathrm{CDCl}_{3}\right): 172.0,163.1(246 \mathrm{~Hz}), 160.2(249 \mathrm{~Hz}), 151.5,150.2$, 148.4, $140.8(13 \mathrm{~Hz}), 140.6,139.1(7 \mathrm{~Hz}), 136.9(3 \mathrm{~Hz}), 130.2(8 \mathrm{~Hz}), 130.0(3 \mathrm{~Hz}), 129.2(8 \mathrm{~Hz}), 125.2(12 \mathrm{~Hz})$, $124.5(3 \mathrm{~Hz}), 124.3(3 \mathrm{~Hz}), 116.1(23 \mathrm{~Hz}), 115.6(22 \mathrm{~Hz}), 113.9(21 \mathrm{~Hz}), 108.7,106.2,61.3,53.1,40.2,30.5,14.1$, 13.4. HRMS $(\mathrm{m} / z)$ : $[\mathrm{M}+\mathrm{H}]^{+}$calcd for $\mathrm{C}_{27} \mathrm{H}_{26} \mathrm{~F}_{2} \mathrm{~N}_{3} \mathrm{O}_{3}: 478.1942$; found, 478.1935 . 


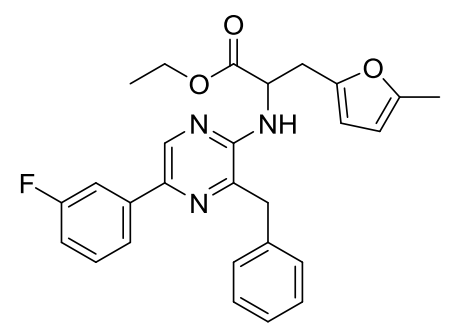

Ethyl 2-((3-benzyl-5-(3-fluorophenyl)pyrazin-2-yl)amino)-3-(5-methylfuran-2-yl)propanoate $\mathbf{2 3}\{3,1,42\}$ (YJ30367-133-2): Obtained as a 90\% pure oil (0.14 g, 36\%) after a chromatography over silica gel (cyclohexane ethyl acetate 95/5). ${ }^{1} \mathrm{H}$ NMR $\left(\mathrm{CDCl}_{3}\right): 8.73(\mathrm{~s}, 1 \mathrm{H}), 7.70(\mathrm{~m}, 2 \mathrm{H}), 7.45-7.23(\mathrm{~m}, 6 \mathrm{H}), 7.02(\mathrm{~m}, 1 \mathrm{H}), 5.82(\mathrm{~m}, 1 \mathrm{H})$, $5.74(\mathrm{~d}(\mathrm{br}), 1 \mathrm{H}, J=3.1 \mathrm{~Hz}), 5.18(\mathrm{~d}(\mathrm{br}), 1 \mathrm{H}, J=7.5 \mathrm{~Hz}), 4.92(\mathrm{~m}, 1 \mathrm{H}), 4.17(\mathrm{~m}, 4 \mathrm{H}), 3.15(\mathrm{~d}, 2 \mathrm{H}, J=5.5 \mathrm{~Hz}), 2.22$ $(\mathrm{m}, 3 \mathrm{H}), 1.24(\mathrm{t}, 3 \mathrm{H}, J=7.2 \mathrm{~Hz}) .{ }^{13} \mathrm{C}$ NMR $\left(\mathrm{CDCl}_{3}\right): 172.0,163.1(244 \mathrm{~Hz}), 151.4,150.6,148.4,141.3,139.8(8$ $\mathrm{Hz}), 139.6,139.5,136.9,136.4,130.1(8 \mathrm{~Hz}), 128.7(11 \mathrm{~Hz}), 126.9,120.9(2 \mathrm{~Hz}), 114.4(20 \mathrm{~Hz}), 112.5(23 \mathrm{~Hz})$, 108.6, 106.2, 61.2, 53.2, 40.7, 30.5, 14.1, 13.5. HRMS $(\mathrm{m} / \mathrm{z})$ : $[\mathrm{M}+\mathrm{H}]^{+}$calcd for $\mathrm{C}_{27} \mathrm{H}_{27} \mathrm{FN}_{3} \mathrm{O}_{3}, 460.2036$; found, 460.2040 .

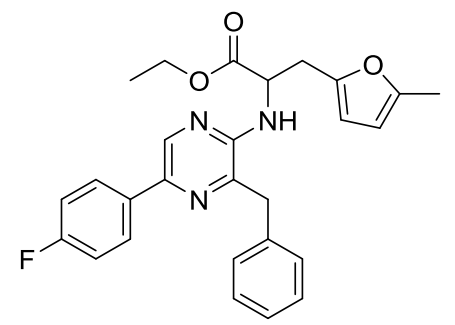

Ethyl 2-((3-benzyl-5-(4-fluorophenyl)pyrazin-2-yl)amino)-3-(5-methylfuran-2-yl)propanoate $\quad \mathbf{2 3}\{4,1,42\}$ (YJ30531-141-3): Obtained as an oil (0.12 g, 21\%) after a chromatography over silica gel (cyclohexane - ethyl acetate 96/4). ${ }^{1} \mathrm{H}$ NMR $\left(\mathrm{CDCl}_{3}\right): 8.36(\mathrm{~s}, 1 \mathrm{H}), 7.91(\mathrm{~m}, 2 \mathrm{H}), 7.34-7.23(\mathrm{~m}, 5 \mathrm{H}), 7.15(\mathrm{~m}, 2 \mathrm{H}), 5.82(\mathrm{~m}, 1 \mathrm{H}), 5.75$ $(\mathrm{d}, 1 \mathrm{H}, J=3.0 \mathrm{~Hz}), 5.13(\mathrm{~d}(\mathrm{br}), 1 \mathrm{H}, J=7.6 \mathrm{~Hz}), 4.92(\mathrm{~m}, 1 \mathrm{H}), 4.17(\mathrm{~m}, 4 \mathrm{H}), 3.15(\mathrm{~d}, 2 \mathrm{H}, J=5.3 \mathrm{~Hz}), 2.22(\mathrm{~s}, 3 \mathrm{H})$, $1.23(\mathrm{t}, 3 \mathrm{H}, J=7.2 \mathrm{~Hz}) .{ }^{13} \mathrm{C}$ NMR $\left(\mathrm{CDCl}_{3}\right): 172.1,162.8(246 \mathrm{~Hz}), 151.4,150.3,148.5,141.2,140.2,136.5,136.4$, $133.7(3 \mathrm{~Hz}), 128.8,128.7,127.3(8 \mathrm{~Hz}), 126.9,115.6(21 \mathrm{~Hz}), 108.6,106.2,61.2,53.2,40.7,30.6,14.1,13.5$. HRMS $(\mathrm{m} / z):[\mathrm{M}+\mathrm{H}]^{+}$calcd for $\mathrm{C}_{27} \mathrm{H}_{27} \mathrm{FN}_{3} \mathrm{O}_{3}, 460.2036$; found, 460.2039 .

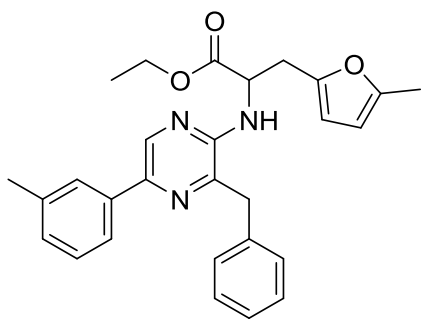

Ethyl 2-((3-benzyl-5-(m-tolyl)pyrazin-2-yl)amino)-3-(5-methylfuran-2-yl)propanoate 23\{5,1,42\} (YJ30367-0673): Obtained as an oil $(0.20 \mathrm{~g}, 26 \%)$ after a chromatography over silica gel (cyclohexane-ethyl acetate 96:4). ${ }^{1} \mathrm{H}$ NMR $\left(\mathrm{CDCl}_{3}\right): 8.39(\mathrm{~s}, 1 \mathrm{H}), 7.77(\mathrm{~m}, 1 \mathrm{H}), 7.72(\mathrm{~m}, 1 \mathrm{H}), 7.37-7.23(\mathrm{~m}, 6 \mathrm{H}), 7.17(\mathrm{~m}, 1 \mathrm{H}), 5.81(\mathrm{~m}, 1 \mathrm{H}), 5.74(\mathrm{~d}$, $1 \mathrm{H}, J=3.0 \mathrm{~Hz}), 5.09(\mathrm{~d}, 1 \mathrm{H}, J=7.5 \mathrm{~Hz}), 4.91(\mathrm{~m}, 1 \mathrm{H}), 4.17(\mathrm{~m}, 4 \mathrm{H}), 3.12(\mathrm{~d}, 2 \mathrm{H}, J=5.3 \mathrm{~Hz}), 2.45(\mathrm{~s}, 3 \mathrm{H}), 2.22$ (s, 3H), 1.22 (t, 3H, $J=7.2 \mathrm{~Hz}) .{ }^{13} \mathrm{C}$ NMR $\left(\mathrm{CDCl}_{3}\right)$ : 172.1, 151.4, 150.3, 148.6, 141.3, 141.1, 138.3, 137.5, 136.9, $136.7,128.8,128.7,128.6,128.5,126.8,126.4,122.8,108.5,106.2,61.2,53.2,40.8,30.6,21.6,14.1,13.5$. HRMS $(\mathrm{m} / \mathrm{z}):[\mathrm{M}+\mathrm{H}]^{+}$calcd for $\mathrm{C}_{28} \mathrm{H}_{30} \mathrm{~N}_{3} \mathrm{O}_{3}, 456.2287$; found, 456.2253. 


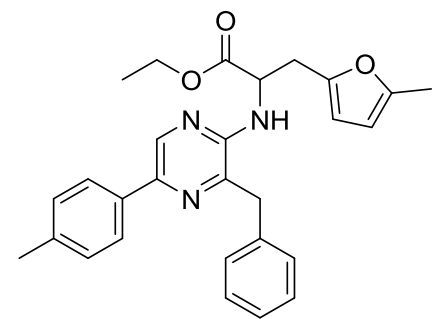

Ethyl 2-((3-benzyl-5-(p-tolyl)pyrazin-2-yl)amino)-3-(5-methylfuran-2-yl)propanoate 23\{6,1,42\} (YJ30367-043-2): Obtained as an oil $(0.23 \mathrm{~g}, 45 \%)$ after a chromatography over silica gel (cyclohexane-ethyl acetate $95: 5) .{ }^{1} \mathrm{H}$ NMR $\left(\mathrm{CDCl}_{3}\right): 8.38(\mathrm{~s}, 1 \mathrm{H}), 7.83(\mathrm{~m}, 2 \mathrm{H}), 7.28(\mathrm{~m}, 2 \mathrm{H}), 7.26-7.17(\mathrm{~m}, 7 \mathrm{H}), 5.81(\mathrm{~m}, 1 \mathrm{H}), 5.74(\mathrm{~d}, 1 \mathrm{H}, J=3.0 \mathrm{~Hz})$, $5.07(\mathrm{~d}, 1 \mathrm{H}, J=7.9 \mathrm{~Hz}), 4.91(\mathrm{~m}, 1 \mathrm{H}), 4.15(\mathrm{~m}, 4 \mathrm{H}), 3.13(\mathrm{~d}, 2 \mathrm{H}, J=5.3 \mathrm{~Hz}), 2.41(\mathrm{~s}, 3 \mathrm{H}), 2.22(\mathrm{~s}, 3 \mathrm{H}), 1.24(\mathrm{t}$, $3 \mathrm{H}, J=7.2 \mathrm{~Hz}) .{ }^{13} \mathrm{C} \mathrm{NMR}\left(\mathrm{CDCl}_{3}\right): 172.1,151.4,150.2,148.6,141.2,141.0,137.6,136.7,136.5,134.8,129.4$, $128.8,128.7,126.8,125.5,108.5,106.2,61.2,53.2,40.7,30.6,21.2,14.1,13.5$. HRMS $(\mathrm{m} / \mathrm{z}):[\mathrm{M}+\mathrm{H}]^{+}$calcd for $\mathrm{C}_{28} \mathrm{H}_{30} \mathrm{~N}_{3} \mathrm{O}_{3}, 456.2287$; found, 456.2274.

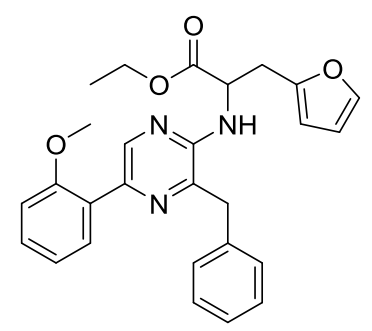

Ethyl 2-((3-benzyl-5-(2-methoxyphenyl)pyrazin-2-yl)amino)-3-(furan-2-yl)propanoate 23 7,1,37\} (EC31092-0492): Obtained as an oil $(0.40 \mathrm{~g}, 64 \%)$ after a chromatography over silica gel (cyclohexane-ethyl acetate 90:10). ${ }^{1} \mathrm{H}$ NMR $\left(\mathrm{CDCl}_{3}\right): 8.58(\mathrm{~s}, 1 \mathrm{H}), 7.87(\mathrm{dd}, 1 \mathrm{H}, J=7.7,1.8 \mathrm{~Hz}), 7.37-7.27(\mathrm{~m}, 5 \mathrm{H}), 7.25-7.19(\mathrm{~m}, 2 \mathrm{H}), 7.08(\mathrm{td}, 1 \mathrm{H}$, $J=7.5,1.0 \mathrm{~Hz}), 7.00(\mathrm{dd}, 1 \mathrm{H}, J=8.3,0.6 \mathrm{~Hz}), 6.20(\mathrm{dd}, 1 \mathrm{H}, J=3.1,1.8 \mathrm{~Hz}), 5.82(\mathrm{dd}, 1 \mathrm{H}, J=3.1,0.5 \mathrm{~Hz}), 5.07$ $(\mathrm{d}, 1 \mathrm{H}, J=7.6 \mathrm{~Hz}), 5.00-4.91(\mathrm{~m}, 1 \mathrm{H}), 4.15(\mathrm{~s}, 2 \mathrm{H}), 4.13(\mathrm{qd}, 2 \mathrm{H}, J=7.1,1.4 \mathrm{~Hz}), 3.89(\mathrm{~s}, 3 \mathrm{H}), 3.19(\mathrm{~d}, 2 \mathrm{H}, J=$ $5.3 \mathrm{~Hz}), 1.19$ (t, $3 \mathrm{H}, J=7.1 \mathrm{~Hz}) .{ }^{13} \mathrm{C}$ NMR $\left(\mathrm{CDCl}_{3}\right): 172.2,157.0,150.8,149.9,142.0,141.2,141.1,139.5,137.0$, 130.5, 129.1, 128.9 (two signals), 126.9, 121.3, 111.5, 110.4, 107.8, 61.4, 55.7, 41.0, 30.6, 27.1, 14.2. HRMS $(\mathrm{m} / \mathrm{z}):[\mathrm{M}+\mathrm{H}]^{+}$calcd for $\mathrm{C}_{27} \mathrm{H}_{28} \mathrm{~N}_{3} \mathrm{O}_{4}, 458.2080$; found, 458.2083 .

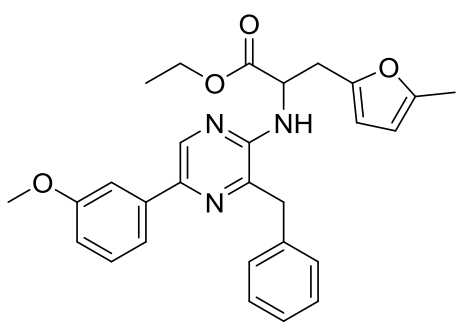

Ethyl 2-((3-benzyl-5-(3-methoxyphenyl)pyrazin-2-yl)amino)-3-(5-methylfuran-2-yl)propanoate $\quad \mathbf{2 3}\{8,1,42\}$ (YJ30367-093-3): Obtained as an oil (0.07 g, 20\%) after a chromatography over silica gel (cyclohexane - ethyl acetate $95 / 5$ to $94 / 6) .{ }^{1} \mathrm{H}$ NMR $\left(\mathrm{CDCl}_{3}\right): 8.41(\mathrm{~s}, 1 \mathrm{H}), 7.51(\mathrm{~m}, 2 \mathrm{H}), 7.40-7.23(\mathrm{~m}, 6 \mathrm{H}), 6.91(\mathrm{~m}, 1 \mathrm{H}), 5.82(\mathrm{~m}, 1 \mathrm{H})$, $5.75(\mathrm{~d}, 1 \mathrm{H}, J=2.9 \mathrm{~Hz}), 5.06(\mathrm{~d}(\mathrm{br}), 1 \mathrm{H}, J=7.3 \mathrm{~Hz}), 4.93(\mathrm{~m}, 1 \mathrm{H}), 4.17(\mathrm{~m}, 4 \mathrm{H}), 3.89(\mathrm{~s}, 3 \mathrm{H}), 3.14(\mathrm{~d}, 2 \mathrm{H}, J=5.4$ $\mathrm{Hz}), 2.23(\mathrm{~s}, 3 \mathrm{H}), 1.24(\mathrm{t}, 3 \mathrm{H}, J=7.2 \mathrm{~Hz}) .{ }^{13} \mathrm{C} \mathrm{NMR}\left(\mathrm{CDCl}_{3}\right): 172.1,160.1,151.4,150.4,148.5,141.1,140.8$, 139.0, 136.9, 136.6, 129.7, 128.8, 128.7, 126.8, 118.0, 113.6, 111.1, 108.6, 106.2, 61.2, 55.3, 53.2, 40.6, 30.6, 14.1, 13.5. HRMS $(\mathrm{m} / z):[\mathrm{M}+\mathrm{H}]^{+}$calcd for $\mathrm{C}_{28} \mathrm{H}_{30} \mathrm{~N}_{3} \mathrm{O}_{4}, 472.2236$; found, 472.2250 . 


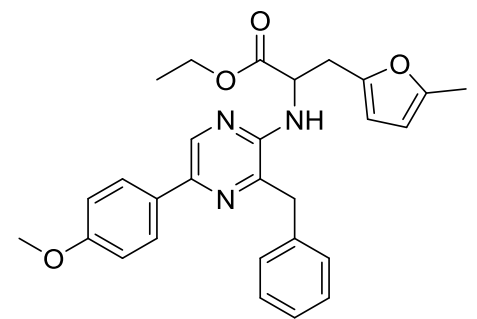

Ethyl 2-((3-benzyl-5-(4-methoxyphenyl)pyrazin-2-yl)amino)-3-(5-methylfuran-2-yl)propanoate $\quad \mathbf{2 3}\{9,1,42\}$ (YJ30531-175-3): Obtained, using DMF as the reaction solvant, as an oil (0.16 g, 44\%) after a chromatography over silica gel (cyclohexane - ethyl acetate 95/5 to 94/6). ${ }^{1} \mathrm{H}$ NMR $\left(\mathrm{CDCl}_{3}\right): 8.35$ (s, 1H), 7.90 (m, 2H), 7.33-7.23 $(\mathrm{m}, 5 \mathrm{H}), 7.01(\mathrm{~m}, 2 \mathrm{H}), 5.82(\mathrm{~m}, 1 \mathrm{H}), 5.75(\mathrm{~d}, 1 \mathrm{H}, J=2.6 \mathrm{~Hz}), 5.06(\mathrm{~d}(\mathrm{br}), 1 \mathrm{H}, J=7.5 \mathrm{~Hz}), 4.91(\mathrm{~m}, 1 \mathrm{H}), 4.21(\mathrm{~m}$, $4 \mathrm{H}), 3.87(\mathrm{~s}, 3 \mathrm{H}), 3.14(\mathrm{~d}, 2 \mathrm{H}, J=5.5 \mathrm{~Hz}), 2.22(\mathrm{~s}, 3 \mathrm{H}), 1.23(\mathrm{t}, 3 \mathrm{H}, J=7.2 \mathrm{~Hz}) .{ }^{13} \mathrm{C} \mathrm{NMR}\left(\mathrm{CDCl}_{3}\right): 172.2,159.6$, 151.4, 149.9, 148.6, 141.1, 140.1, 136.7, 136.1, 130.3, 128.8, 128.7, 126.9, 126.8, 114.2, 108.5, 106.2, 61.2, 55.3, 53.2, 40.7, 30.6, 14.1, 13.5. HRMS (m/z): $[\mathrm{M}+\mathrm{H}]^{+}$calcd for $\mathrm{C}_{28} \mathrm{H}_{30} \mathrm{~N}_{3} \mathrm{O}_{4}, 472.2236$; found, 472.2230.

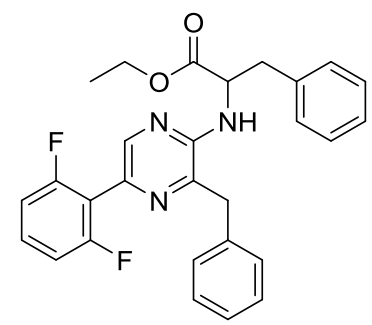

Ethyl (3-benzyl-5-(2,6-difluorophenyl)pyrazin-2-yl)phenylalaninate 23 10,1,1\} (MM34284-028-1): Obtained as an oil $(0.24 \mathrm{~g}, 65 \%)$ after a chromatography over silica gel (cyclohexane - ethyl acetate 9/1). ${ }^{1} \mathrm{H}$ NMR $\left(\mathrm{CDCl}_{3}\right): 8.14$ $(\mathrm{s}, 1 \mathrm{H}), 7.30-7.19(\mathrm{~m}, 9 \mathrm{H}), 7.04-6.96(\mathrm{~m}, 4 \mathrm{H}), 5.01(\mathrm{~m}, 2 \mathrm{H}), 4.12(\mathrm{~m}, 2 \mathrm{H}), 4.11(\mathrm{~s}, 2 \mathrm{H}), 3.16(\mathrm{dd}, 1 \mathrm{H}, J=5.2$, $13.8 \mathrm{~Hz}), 3.08(\mathrm{dd}, 1 \mathrm{H}, J=5.9,13.8 \mathrm{~Hz}), 1.18(\mathrm{t}, 3 \mathrm{H}, J=7.1 \mathrm{~Hz}) .{ }^{13} \mathrm{C} \mathrm{NMR}\left(\mathrm{CDCl}_{3}\right): 172.2,160.7(7,250 \mathrm{~Hz})$, 150.5, 150.2, $141.6(3 \mathrm{~Hz}), 141.5,136.2$ (3 Hz), 132.4, $129.5(10 \mathrm{~Hz}), 129.3,128.9,128.6,128.4,127.0,126.8$, $115.9(20 \mathrm{~Hz}), 111.7(7,19 \mathrm{~Hz}), 61.2,54.7,40.8,37.8,14.0$. HRMS $(m / z):[\mathrm{M}+\mathrm{H}]^{+}$calcd for $\mathrm{C}_{28} \mathrm{H}_{25} \mathrm{~F}_{2} \mathrm{~N}_{3} \mathrm{O}_{2}$, 474.1993; found, 474.2011.

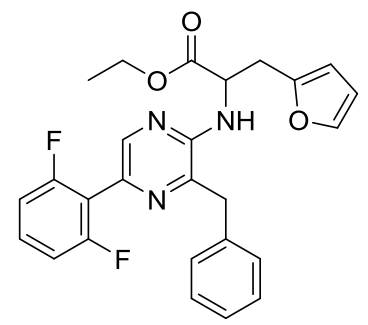

Ethyl 2-((3-benzyl-5-(2,6-difluorophenyl)pyrazin-2-yl)amino)-3-(furan-2-yl)propanoate 23 10,1,37\} (MM34284030-1): Obtained as an oil $(0.19 \mathrm{~g}, 52 \%)$ after a chromatography over silica gel (cyclohexane - ethyl acetate 9/1). ${ }^{1} \mathrm{H}$ NMR $\left(\mathrm{CDCl}_{3}\right): 8.13(\mathrm{~s}, 1 \mathrm{H}), 7.36-7.19(\mathrm{~m}, 7 \mathrm{H}), 7.04-6.98(\mathrm{~m}, 2 \mathrm{H}), 6.23(\mathrm{~m}, 1 \mathrm{H}), 5.84(\mathrm{~m}, 1 \mathrm{H}), 5.22(\mathrm{~d}, 1 \mathrm{H}, J$ $=7.3 \mathrm{~Hz}), 4.96(\mathrm{~m}, 1 \mathrm{H}), 4.16(\mathrm{~s}, 2 \mathrm{H}), 4.15(\mathrm{~m}, 2 \mathrm{H}), 3.20(\mathrm{~d}, 2 \mathrm{H}, J=5.3), 1.12(\mathrm{t}, 3 \mathrm{H}, J=7.0 \mathrm{~Hz}) .{ }^{13} \mathrm{C} \mathrm{NMR}$ $\left(\mathrm{CDCl}_{3}\right): 171.8,160.7(7,250 \mathrm{~Hz}), 150.5,150.4,141.9,141.6,141.5(3 \mathrm{~Hz}), 136.3132 .4,129.5(10 \mathrm{~Hz}), 128.9$, 128.6, 126.9, $115.8(20 \mathrm{~Hz}), 111.7(7,19 \mathrm{~Hz}), 110.3,107.7,61.3,53.0,40.8,30.4,14.0 . \mathrm{HRMS}(\mathrm{m} / \mathrm{z}):[\mathrm{M}+\mathrm{H}]^{+}$ calcd for $\mathrm{C}_{26} \mathrm{H}_{23} \mathrm{~F}_{2} \mathrm{~N}_{3} \mathrm{O}_{3}, 464.1786$; found, 464.1799 .

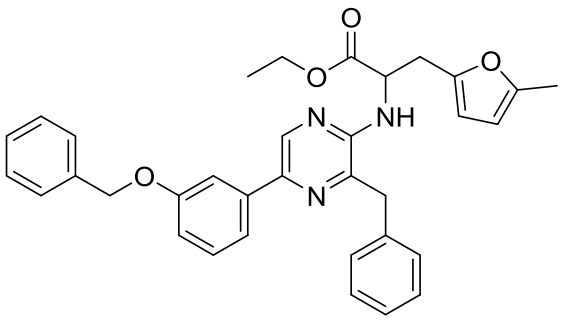


Ethyl 2-((3-benzyl-5-(3-(benzyloxy)phenyl)pyrazin-2-yl)amino)-3-(5-methylfuran-2-yl)propanoate $23\{11,1,42\}$ (RB32489-013-2): Obtained as an oil (0.50 g, 74\%) after a chromatography over silica gel (cyclohexane - ethyl acetate $97 / 3$ to $96 / 4) .{ }^{1} \mathrm{H}\left(\mathrm{CDCl}_{3}\right): 8.40(\mathrm{~s}, 1 \mathrm{H}), 7.65(\mathrm{~m}, 1 \mathrm{H}), 7.51(\mathrm{~m}, 3 \mathrm{H}), 7.44-7.26(\mathrm{~m}, 9 \mathrm{H}), 6.99(\mathrm{dd}, 1 \mathrm{H}, J=$ 8.1, $2.6 \mathrm{~Hz}), 5.82(\mathrm{~m}, 1 \mathrm{H}), 5.76(\mathrm{~d}, 1 \mathrm{H}, J=3 \mathrm{~Hz}), 5.16(\mathrm{~s}, 2 \mathrm{H}), 5.13(\mathrm{~m}, 1 \mathrm{H}), 4.94(\mathrm{~m}, 1 \mathrm{H}), 4.17(\mathrm{~m}, 4 \mathrm{H}), 3.16(\mathrm{~m}$, $2 \mathrm{H}), 2.23(\mathrm{~s}, 3 \mathrm{H}), 1.24(\mathrm{t}, 3 \mathrm{H}, J=7.3 \mathrm{~Hz}) .{ }^{13} \mathrm{C}\left(\mathrm{CDCl}_{3}\right) 172.1,159.4,151.4,150.4,148.5,141.1,140.7,139.0$, 137.2, 136.8, 136.6, 129.7, 128.8, 128.7, 128.6, 127.9, 127.6, 126.8, 118.2, 114.5, 112.1, 108.6, 106.2, 70.1, 61.2, 53.2, 40.7, 30.6, 14.1, 13.5. HRMS (m/z): $[\mathrm{M}+\mathrm{H}]^{+}$calcd for $\mathrm{C}_{34} \mathrm{H}_{34} \mathrm{~N}_{3} \mathrm{O}_{4}$, 548.2549; found, 548.2540.

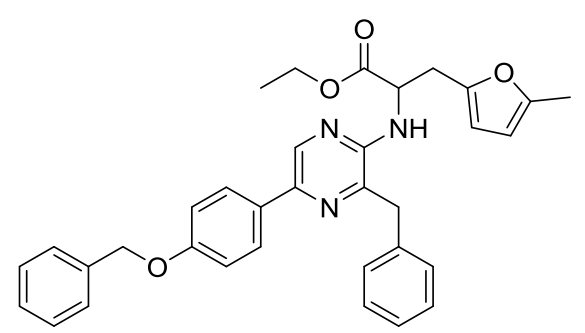

Ethyl 2-((3-benzyl-5-(4-(benzyloxy)phenyl)pyrazin-2-yl)amino)-3-(5-methylfuran-2-yl)propanoate $\mathbf{2 3}\{12,1,42\}$ (YJ 33069-163-1): Obtained as an oil (0.49 g, 82\%), using toluene at $90{ }^{\circ} \mathrm{C}$, after a chromatography over silica gel (cyclohexane - ethyl acetate 95/5). ${ }^{1} \mathrm{H}$ NMR $\left(\mathrm{CDCl}_{3}\right): 8.35(\mathrm{~s}, 1 \mathrm{H}), 7.88(\mathrm{~m}, 2 \mathrm{H}), 7.48(\mathrm{~m}, 2 \mathrm{H}), 7.42(\mathrm{~m}, 2 \mathrm{H}), 7.36$ $(\mathrm{m}, 1 \mathrm{H}), 7.32-7.20(\mathrm{~m}, 5 \mathrm{H}), 7.08(\mathrm{~m}, 2 \mathrm{H}), 5.82(\mathrm{~m}, 1 \mathrm{H}), 5.76(\mathrm{~d}(\mathrm{br}), 1 \mathrm{H}, J=3.0 \mathrm{~Hz}), 5.15(\mathrm{~s}, 2 \mathrm{H}), 5.07(\mathrm{~d}(\mathrm{br}), 1 \mathrm{H}$, $J=7.6 \mathrm{~Hz}), 4.94(\mathrm{~m}, 1 \mathrm{H}), 4.16(\mathrm{~m}, 4 \mathrm{H}), 3.15(\mathrm{~d}, 2 \mathrm{H}, J=5.3 \mathrm{~Hz}), 2.23(\mathrm{~s}, 3 \mathrm{H}), 1.23(\mathrm{t}, 3 \mathrm{H}, J=7.1 \mathrm{~Hz}) .{ }^{13} \mathrm{C} \mathrm{NMR}$ $\left(\mathrm{CDCl}_{3}\right)$ : 172.1, 158.9, 151.4, 149.7, 148.5, 141.2, 141.0, 137.0, 136.6, 135.8, 130.4, 128.8, 128.7, 128.6, 127.9, $127.4,126.9,126.8,115.2,108.6,106.2,70.1,61.2,53.2,40.7,30.5,14.1,13.5$. HRMS $(\mathrm{m} / z):[\mathrm{M}+\mathrm{H}]^{+}$calcd for $\mathrm{C}_{34} \mathrm{H}_{34} \mathrm{~N}_{3} \mathrm{O}_{4}, 548.2549$; found, 548.2556.

General procedure for the synthesis of the $\mathbf{O}$-acetylated luciferins $\mathbf{2 5}$ via step xii. In a sealable vessel, the considered N-pyrazyl aminoester $24(1.0 \mathrm{mmol})$ and sodium hydroxide $(0.16 \mathrm{~g}, 4 \mathrm{mmol})$ were weighted. The air was replaced with argon and anhydrous THF $(5 \mathrm{~mL})$ was injected. This was stirred at $20{ }^{\circ} \mathrm{C}$ under an inert atmosphere overnight and acetic anhydride $(1.41 \mathrm{~mL}, 15.0 \mathrm{mmol})$ was then injected. After stirring an additional two hours at room temperature, this was diluted in ethyl acetate, washed with water, brine and concentrated to dryness. The traces of acetic acid and acetic anhydride were removed by co-evaporation with toluene and then cyclohexane and the residue further purified as described below. Note: as mentioned in the main text, few of these compounds turned out to be unstable over at least a week. Most often an initial assessment of their bioluminescence properties could be made but this was not possible when, years later, we ran a second set of experiment with most of the compounds made. Although their analysis is provided in the following, these compounds are: $\mathbf{2 5}\{1,1,28\}, \mathbf{2 5}\{2,1,42, \mathbf{2 5}\{7,1,37\}, \mathbf{2 5}\{2,1,44\}, \mathbf{2 5}\{2,1,48\}$ and $\mathbf{2 5}\{10,1,37\}$.

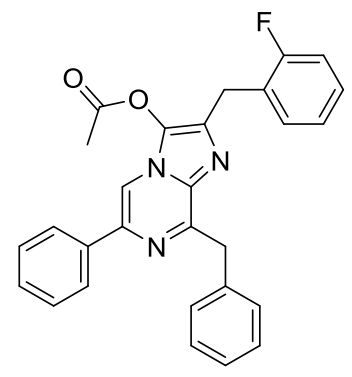

8-Benzyl-2-(2-fluorobenzyl)-6-phenylimidazo[1,2-a]pyrazin-3-yl acetate 25\{1,1,2\} (YJ30367-149-1): Obtained as a white solid $(0.22 \mathrm{~g}, 71 \%)$ after a recrystallization from $n$-heptane. ${ }^{1} \mathrm{H}$ NMR $\left(\mathrm{CDCl}_{3}\right): 7.90(\mathrm{~m}, 2 \mathrm{H}), 7.81(\mathrm{~s}, 1 \mathrm{H})$, $7.63(\mathrm{~m}, 2 \mathrm{H}), 7.48-7.21(\mathrm{~m}, 8 \mathrm{H}), 7.10(\mathrm{~m}, 2 \mathrm{H}), 4.63(\mathrm{~s}, 2 \mathrm{H}), 4.23(\mathrm{~s}, 2 \mathrm{H}), 2.29(\mathrm{~s}, 3 \mathrm{H}) .{ }^{13} \mathrm{C} \mathrm{NMR}\left(\mathrm{CDCl}_{3}\right): 167.1$, 160.9 (244 Hz), 153.0, 139.1, 137.9, 136.8, 133.9 (32 Hz), 131.3 (4 Hz), 129.7 (two signals?), 128.7, 128.5, 128.4, 128.3, 128.2, 126.5, 126.4, $125.1(16 \mathrm{~Hz}), 124.1(4 \mathrm{~Hz}), 115.1(21 \mathrm{~Hz}), 108.9,39.5,26.8(4 \mathrm{~Hz}), 19.9$. HRMS $(\mathrm{m} / \mathrm{z}):[\mathrm{M}+\mathrm{H}]^{+}$calcd for $\mathrm{C}_{28} \mathrm{H}_{23} \mathrm{FN}_{3} \mathrm{O}_{2}, 452.1774$; found, 452.1769 . 


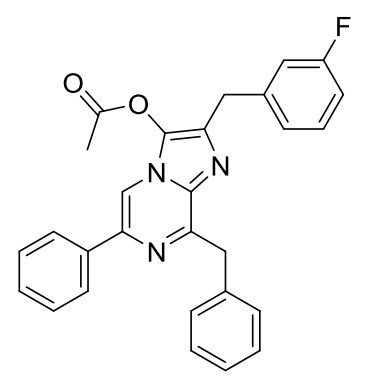

8-Benzyl-2-(3-fluorobenzyl)-6-phenylimidazo[1,2-a]pyrazin-3-yl acetate 25\{1,1,3\} (YJ30367-017-1): Obtained as a white solid $(0.13 \mathrm{~g}, 57 \%)$ after a recrystallization from $n$-heptane. ${ }^{1} \mathrm{H}$ NMR $\left(\mathrm{CDCl}_{3}\right): 7.92(\mathrm{~m}, 2 \mathrm{H}), 7.81(\mathrm{~s}, 1 \mathrm{H})$, $7.63(\mathrm{~m}, 2 \mathrm{H}), 7.47(\mathrm{~m}, 2 \mathrm{H}), 7.40(\mathrm{~m}, 1 \mathrm{H}), 7.33-7.22(\mathrm{~m}, 4 \mathrm{H}), 7.06(\mathrm{~m}, 2 \mathrm{H}), 6.95(\mathrm{~m}, 1 \mathrm{H}), 4.64(\mathrm{~s}, 2 \mathrm{H}), 4.19(\mathrm{~s}$, 2H), $2.29(\mathrm{~s}, 3 \mathrm{H}) .{ }^{13} \mathrm{C} \mathrm{NMR}\left(\mathrm{CDCl}_{3}\right): 167.0,162.8(245 \mathrm{~Hz}), 153.0,140.6(7 \mathrm{~Hz}), 139.2,137.8,136.8,134.4$, 133.7, 129.8 (8 Hz), 129.7, 128.8, 128.7, 128.6, 128.3, 126.5, 126.4, 124.6 (3 Hz), 115.9 (21 Hz), 113.4 (20 Hz), 108.9, 39.5, 33.7, 19.9. HRMS (m/z): $[\mathrm{M}+\mathrm{H}]^{+}$calcd for $\mathrm{C}_{28} \mathrm{H}_{23} \mathrm{FN}_{3} \mathrm{O}_{2}, 452.1774$; found, 452.1778 .

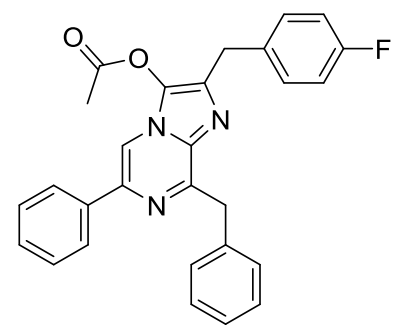

8-Benzyl-2-(4-fluorobenzyl)-6-phenylimidazo[1,2-a]pyrazin-3-yl acetate 25\{1,1,4\} (EC31092-099-2): Obtained as a beige solid $(0.31 \mathrm{~g}, 70 \%)$ after a recrystallization from $n$-heptane. ${ }^{1} \mathrm{H} \mathrm{NMR}\left(\mathrm{CDCl}_{3}\right): 7.94-7.86(\mathrm{~m}, 2 \mathrm{H}), 7.79(\mathrm{~s}$, $1 \mathrm{H}), 7.65-7.58(\mathrm{~m}, 2 \mathrm{H}), 7.50-7.36(\mathrm{~m}, 3 \mathrm{H}), 7.35-7.17(\mathrm{~m}, 5 \mathrm{H}), 7.00(\mathrm{dd}, 2 \mathrm{H}, J=9.8,7.7 \mathrm{~Hz}), 4.62(\mathrm{~s}, 2 \mathrm{H})$, $4.15(\mathrm{~s}, 2 \mathrm{H}), 2.25(\mathrm{~s}, 3 \mathrm{H}) .{ }^{13} \mathrm{C}$ NMR $\left(\mathrm{CDCl}_{3}\right): 167.2,161.8(\mathrm{~d}, J=244.6 \mathrm{~Hz}), 153.1,139.3,138.0,136.9,135.1$, $133.9(\mathrm{~d}, J=3.3 \mathrm{~Hz}), 133.8,130.6(\mathrm{~d}, J=7.9 \mathrm{~Hz}), 129.9,128.9,128.7,128.4,126.6,126.6,115.3(\mathrm{~d}, J=21.3 \mathrm{~Hz})$, 109.0, 39.6, 33.4, 20.1 (one signal missing). HRMS $(\mathrm{m} / \mathrm{z})$ : $[\mathrm{M}+\mathrm{H}]^{+}$calcd for $\mathrm{C}_{28} \mathrm{H}_{23} \mathrm{FN}_{3} \mathrm{O}_{2}$, 452.1774; found, 452.1776 .

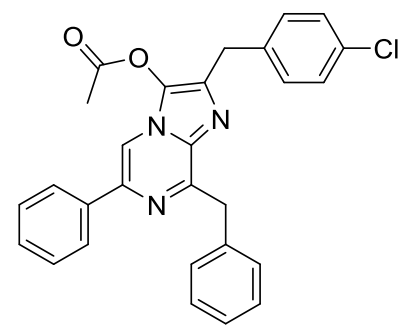

8-Benzyl-2-(4-chlorobenzyl)-6-phenylimidazo[1,2-a]pyrazin-3-yl acetate 25 $\{1,1,7\}$ (CF34391-037-1): Obtained as a white solid $(0.23 \mathrm{~g}, 62 \%)$ after a recrystallization from $n$-heptane. ${ }^{1} \mathrm{H}$ NMR $\left(\mathrm{CDCl}_{3}\right): 7.92(\mathrm{~m}, 2 \mathrm{H}), 7.81(\mathrm{~s}, 1 \mathrm{H})$, $7.62(\mathrm{~m}, 2 \mathrm{H}), 7.48(\mathrm{~m}, 2 \mathrm{H}), 7.40(\mathrm{~m}, 1 \mathrm{H}), 7.28(\mathrm{~m}, 7 \mathrm{H}), 4.63(\mathrm{~s}, 2 \mathrm{H}), 4.14(\mathrm{~s}, 2 \mathrm{H}), 2.27(\mathrm{~s}, 3 \mathrm{H}) .{ }^{13} \mathrm{C} \mathrm{NMR}\left(\mathrm{CDCl}_{3}\right)$ : $167.0,153.0,139.2,137.8,136.8,136.6,134.6,133.7,132.3,130.4,129.7,128.8,128.6,128.5,128.3,126.5$, 126.4, 108.9, 39.5, 33.4, 19.9. HRMS (m/z): $[\mathrm{M}+\mathrm{H}]^{+}$calcd for $\mathrm{C}_{28} \mathrm{H}_{23} \mathrm{ClN}_{3} \mathrm{O}_{2}$, 468.1479; found, 468.1485 .<smiles>CC(=O)Oc1c(Cc2ccccc2C(F)(F)F)nc2c(Cc3ccccc3)nc(-c3ccccc3)cn12</smiles> 
8-Benzyl-6-phenyl-2-(2-(trifluoromethyl)benzyl)imidazo[1,2-a]pyrazin-3-yl acetate 25\{1,1,8\} (YJ30531-123-1): Obtained as wax $(0.07 \mathrm{~g}, 95 \%) .{ }^{1} \mathrm{H}$ NMR $\left(\mathrm{CDCl}_{3}\right): 7.93(\mathrm{~m}, 2 \mathrm{H}), 7.80(\mathrm{~s}, 1 \mathrm{H}), 7.65(\mathrm{~m}, 3 \mathrm{H}), 7.45(\mathrm{~m}, 2 \mathrm{H}), 7.53-$ $7.21(\mathrm{~m}, 9 \mathrm{H}), 4.65(\mathrm{~s}, 2 \mathrm{H}), 4.38(\mathrm{~s}, 2 \mathrm{H}), 2.28(\mathrm{~s}, 3 \mathrm{H}) .{ }^{13} \mathrm{C} \mathrm{NMR}\left(\mathrm{CDCl}_{3}\right): 167.1,153.1,139.2,137.8,136.8,136.7$, 133.9, 133.8, 131.8, 131.7, 129.7, 128.9, 128.7 (30 Hz), 128.6, 128.3, 126.6, 126.5, 126.4, 126.0, 125.7 (6 Hz), $124.5(275 \mathrm{~Hz}), 109.1,39.6,29.7,19.8$. HRMS $(\mathrm{m} / \mathrm{z}):[\mathrm{M}+\mathrm{H}]^{+}$calcd for $\mathrm{C}_{29} \mathrm{H}_{23} \mathrm{~F}_{3} \mathrm{~N}_{3} \mathrm{O}_{2}, 502.1742$; found, 502.1722 .

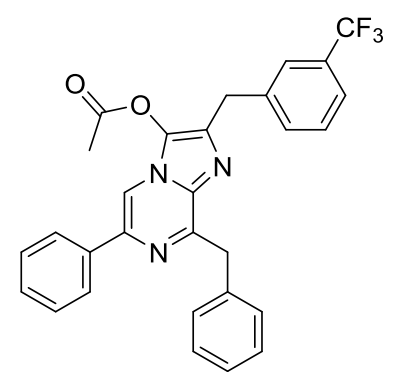

8-Benzyl-6-phenyl-2-(3-(trifluoromethyl)benzyl)imidazo[1,2-a]pyrazin-3-yl acetate 25\{1,1,9\} (YJ30531-125-1): Obtained as a white solid after a recrystallization in n-heptane $(0.08 \mathrm{~g}, 57 \%) .{ }^{1} \mathrm{H} \mathrm{NMR}\left(\mathrm{CDCl}_{3}\right): 7.91(\mathrm{~m}, 2 \mathrm{H}), 7.81$ $(\mathrm{s}, 1 \mathrm{H}), 7.63(\mathrm{~m}, 3 \mathrm{H}), 7.54-7.38(\mathrm{~m}, 6 \mathrm{H}), 7.34-7.21(\mathrm{~m}, 3 \mathrm{H}), 4.63(\mathrm{~s}, 2 \mathrm{H}), 4.25(\mathrm{~s}, 2 \mathrm{H}), 2.27(\mathrm{~s}, 3 \mathrm{H}) .{ }^{13} \mathrm{C} \mathrm{NMR}$ $\left(\mathrm{CDCl}_{3}\right): 166.9,153.1,139.3,139.1,137.8,136.8,134.2,133.8,132.5,130.7(31 \mathrm{~Hz}), 129.7,128.9,128.8,128.7$, 128.6, 128.3, 126.5, 126.4, 125.8 (6 Hz), 124.2 (275 Hz), $123.4(6 \mathrm{~Hz}), 108.9,39.5,33.7,19.9$. HRMS (m/z): $[\mathrm{M}+\mathrm{H}]^{+}$calcd for $\mathrm{C}_{29} \mathrm{H}_{23} \mathrm{~F}_{3} \mathrm{~N}_{3} \mathrm{O}_{2}, 502.1742$; found, 502.1795 .

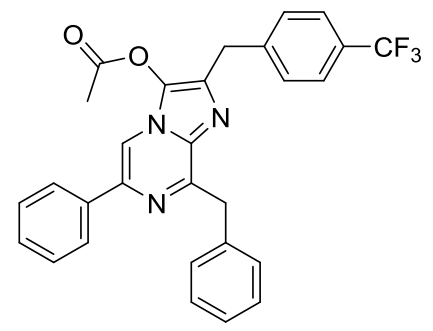

8-Benzyl-6-phenyl-2-(4-(trifluoromethyl)benzyl)imidazo[1,2-a]pyrazin-3-yl acetate 25 1,1,10\} (YJ30531-127-1): Obtained as a white solid after a recrystallization in n-heptane $(0.08 \mathrm{~g}, 66 \%) .{ }^{1} \mathrm{H}$ NMR $\left(\mathrm{CDCl}_{3}\right): 7.92(\mathrm{~m}, 2 \mathrm{H}), 7.81$ $(\mathrm{s}, 1 \mathrm{H}), 7.60(\mathrm{~m}, 4 \mathrm{H}), 7.49-7.38(\mathrm{~m}, 5 \mathrm{H}), 7.33-7.22(\mathrm{~m}, 3 \mathrm{H}), 4.63(\mathrm{~s}, 2 \mathrm{H}), 4.24(\mathrm{~s}, 2 \mathrm{H}), 2.27(\mathrm{~s}, 3 \mathrm{H}) .{ }^{13} \mathrm{C} \mathrm{NMR}$ $\left(\mathrm{CDCl}_{3}\right)$ : 167.0, 153.1, 142.3, 139.3, 137.7, 136.7, 134.1, 133.8, 129.7, 129.4, 128.9 (31 Hz), 128.8, 128.7, 128.6, 128.3, 126.5, 126.4, $125.3(6 \mathrm{~Hz}), 124.3(275 \mathrm{~Hz}), 108.9,39.5,33.7,19.9$. HRMS $(\mathrm{m} / \mathrm{z}):[\mathrm{M}+\mathrm{H}]^{+}$calcd for $\mathrm{C}_{29} \mathrm{H}_{23} \mathrm{~F}_{3} \mathrm{~N}_{3} \mathrm{O}_{2}, 502.1742$; found, 502.1730 .

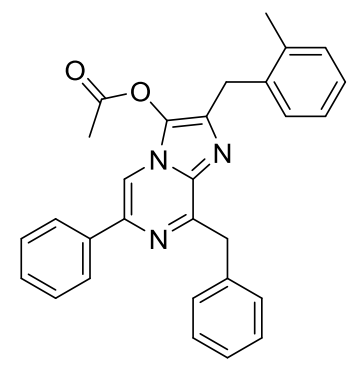

8-Benzyl-2-(2-methylbenzyl)-6-phenylimidazo[1,2-a]pyrazin-3-yl acetate 25\{1,1,11\} (YJ30367-179-1): Obtained as a white solid $(0.44 \mathrm{~g}, 96 \%):{ }^{1} \mathrm{H}$ NMR $\left(\mathrm{CDCl}_{3}\right): 7.91(\mathrm{~m}, 2 \mathrm{H}), 7.76(\mathrm{~s}, 1 \mathrm{H}), 7.64(\mathrm{~m}, 2 \mathrm{H}), 7.48-7.18(\mathrm{~m}, 10 \mathrm{H})$, $4.64(\mathrm{~s}, 2 \mathrm{H}), 4.24(\mathrm{~s}, 2 \mathrm{H}), 2.32(\mathrm{~s}, 3 \mathrm{H}), 2.06(\mathrm{~s}, 3 \mathrm{H}) .{ }^{13} \mathrm{C} \mathrm{NMR}\left(\mathrm{CDCl}_{3}\right): 167.1,152.8,139.0,137.9,137.2,136.9$, $135.9,135.5,133.6,130.3,130.0,129.7,128.9,128.8,128.5,128.3,126.9,126.5,126.4,125.9,108.8,39.4,32.6$, 26.9, 19.6. HRMS (m/z): $[\mathrm{M}+\mathrm{H}]^{+}$calcd for $\mathrm{C}_{29} \mathrm{H}_{26} \mathrm{~N}_{3} \mathrm{O}_{2}$, 448.2025; found, 448.2032. 


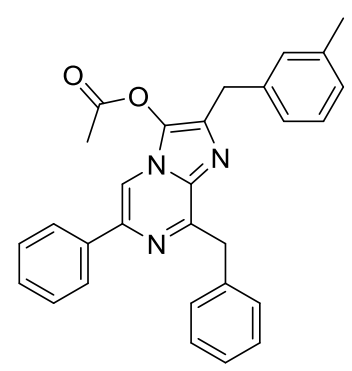

8-Benzyl-2-(3-methylbenzyl)-6-phenylimidazo[1,2-a]pyrazin-3-yl acetate 25\{1,1,12\} (EC31092-097-2): Obtained as a beige solid $(0.17 \mathrm{~g}, 61 \%)$ after a recrystallization from $n$-heptane. ${ }^{1} \mathrm{H} \mathrm{NMR}\left(\mathrm{CDCl}_{3}\right): 7.92-7.85(\mathrm{~m}, 2 \mathrm{H}), 7.77$ $(\mathrm{s}, 1 \mathrm{H}), 7.66-7.58(\mathrm{~m}, 2 \mathrm{H}), 7.49-7.35(\mathrm{~m}, 3 \mathrm{H}), 7.35-7.27(\mathrm{~m}, 2 \mathrm{H}), 7.24-7.16(\mathrm{~m}, 2 \mathrm{H}), 7.15-7.01(\mathrm{~m}, 3 \mathrm{H})$, $4.62(\mathrm{~s}, 2 \mathrm{H}), 4.16(\mathrm{~s}, 2 \mathrm{H}), 2.33(\mathrm{~s}, 3 \mathrm{H}), 2.17$ (s, 3H). ${ }^{13} \mathrm{C} \mathrm{NMR}\left(\mathrm{CDCl}_{3}\right): 167.2,153.0,139.2,138.2,138.1,137.0$, 135.5, 133.8, 130.0, 129.9, 129.0, 128.9, 128.7, 128.5, 128.4, 127.3, 126.6 (two signals), 126.2, 109.0, 39.6, 34.3, 21.5, 20.0 (one signal missing). HRMS (m/z): $[\mathrm{M}+\mathrm{H}]^{+}$calcd for $\mathrm{C}_{29} \mathrm{H}_{26} \mathrm{~N}_{3} \mathrm{O}_{2}$, 448.2025; found, 448.2053.

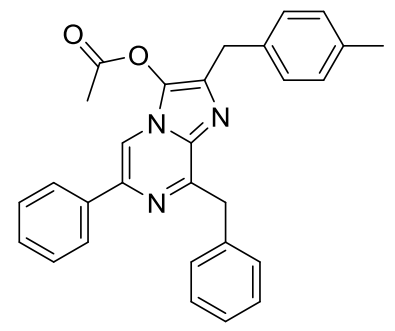

8-Benzyl-2-(4-methylbenzyl)-6-phenylimidazo[1,2-a]pyrazin-3-yl acetate 25 1,1,13\} (YJ30367-147-1): Obtained as a white solid $(0.21 \mathrm{~g}, 60 \%)$ after a recrystallization from $n$-heptane. ${ }^{1} \mathrm{H} \mathrm{NMR}\left(\mathrm{CDCl}_{3}\right): 7.91(\mathrm{~m}, 2 \mathrm{H}), 7.80(\mathrm{~s}$, 1H), $7.64(\mathrm{~m}, 2 \mathrm{H}), 7.49-7.13(\mathrm{~m}, 10 \mathrm{H}), 4.65(\mathrm{~s}, 2 \mathrm{H}), 4.18(\mathrm{~s}, 2 \mathrm{H}), 2.36(\mathrm{~s}, 3 \mathrm{H}), 2.20(\mathrm{~s}, 3 \mathrm{H}) .{ }^{13} \mathrm{C} \mathrm{NMR}(\mathrm{CDCl})$ : $167.1,152.9,139.0,137.9,136.9,135.9,135.5,134.9,133.6,129.8,129.1,128.9,128.8,128.7,128.5,128.3$, 126.5, 126.4, 108.8, 39.4, 33.8, 21.0, 19.9. HRMS $(\mathrm{m} / \mathrm{z}):[\mathrm{M}+\mathrm{H}]^{+}$calcd for $\mathrm{C}_{29} \mathrm{H}_{26} \mathrm{~N}_{3} \mathrm{O}_{2}, 448.2025$; found, 448.2034.

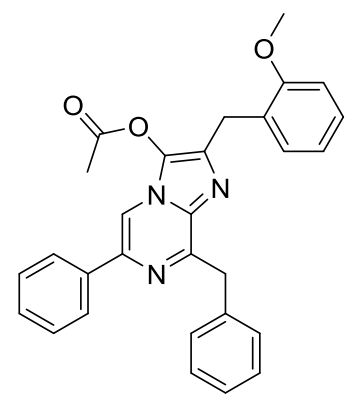

8-Benzyl-2-(2-methoxybenzyl)-6-phenylimidazo[1,2-a]pyrazin-3-yl acetate $\quad 25\{1,1,14\} \quad$ (YJ30367-151-1): Obtained as a white solid $(0.23 \mathrm{~g}, 72 \%)$ after a recrystallization from $n$-heptane. ${ }^{1} \mathrm{H} \mathrm{NMR}\left(\mathrm{CDCl}_{3}\right): 7.90(\mathrm{~m}, 2 \mathrm{H})$, $7.78(\mathrm{~s}, 1 \mathrm{H}), 7.64(\mathrm{~m}, 2 \mathrm{H}), 7.46(\mathrm{~m}, 2 \mathrm{H}), 7.39(\mathrm{~m}, 1 \mathrm{H}), 7.32(\mathrm{~m}, 2 \mathrm{H}), 7.24(\mathrm{~m}, 3 \mathrm{H}), 6.92(\mathrm{~m}, 2 \mathrm{H}), 4.63(\mathrm{~s}, 2 \mathrm{H})$, $4.22(\mathrm{~s}, 2 \mathrm{H}), 3.82(\mathrm{~s}, 3 \mathrm{H}), 2.16(\mathrm{~s}, 3 \mathrm{H}) .{ }^{13} \mathrm{C}$ NMR $\left(\mathrm{CDCl}_{3}\right): 167.2,157.6,152.8,138.9,138.0,137.0,135.1,133.6$, 130.6, 129.8, 128.8, 128.7, 128.4, 128.2, 127.9, 126.4 (two signals), 126.3, 120.5, 110.4, 108.9, 55.4, 39.4, 28.5, 19.8. HRMS $(m / z):[\mathrm{M}+\mathrm{H}]^{+}$calcd for $\mathrm{C}_{29} \mathrm{H}_{26} \mathrm{~N}_{3} \mathrm{O}_{3}, 464.1974$; found, 464.1978. 


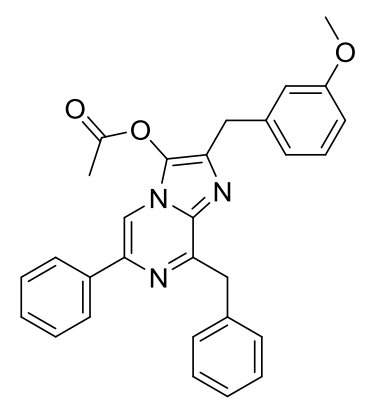

8-Benzyl-2-(3-methoxybenzyl)-6-phenylimidazo[1,2-a]pyrazin-3-yl acetate $\quad \mathbf{2 5}\{1,1,15\} \quad$ (EC31092-127-2): Obtained as a white solid $(0.20 \mathrm{~g}, 56 \%)$ after a recrystallization from $n$-heptane. ${ }^{1} \mathrm{H}$ NMR $\left(\mathrm{CDCl}_{3}\right)$ : $7.92-7.86(\mathrm{~m}$, 2H), $7.78(\mathrm{~s}, 1 \mathrm{H}), 7.65-7.58(\mathrm{~m}, 2 \mathrm{H}), 7.49-7.41(\mathrm{~m}, 2 \mathrm{H}), 7.41-7.34(\mathrm{~m}, 1 \mathrm{H}), 7.33-7.27(\mathrm{~m}, 2 \mathrm{H}), 7.25-7.18$ $(\mathrm{m}, 2 \mathrm{H}), 6.91-6.86(\mathrm{~m}, 1 \mathrm{H}), 6.86-6.83(\mathrm{~m}, 1 \mathrm{H}), 6.81-6.75(\mathrm{~m}, 1 \mathrm{H}), 4.63(\mathrm{~s}, 2 \mathrm{H}), 4.17(\mathrm{~s}, 2 \mathrm{H}), 3.77(\mathrm{~s}, 3 \mathrm{H})$, 2.21 (s, 3H). ${ }^{13} \mathrm{C}$ NMR $\left(\mathrm{CDCl}_{3}\right): 167.2,159.9,153.1,139.7,139.2,138.0,137.0,135.2,133.8,129.9,129.6,129.0$, 128.9, 128.7, 128.4, 126.6 (two signals), 121.6, 114.7, 112.3, 109.0, 55.3, 39.5, 34.4, 20.0. HRMS $(\mathrm{m} / \mathrm{z}):[\mathrm{M}+\mathrm{H}]^{+}$ calcd for $\mathrm{C}_{29} \mathrm{H}_{26} \mathrm{~N}_{3} \mathrm{O}_{3}, 464.1974$; found, 464.1982.

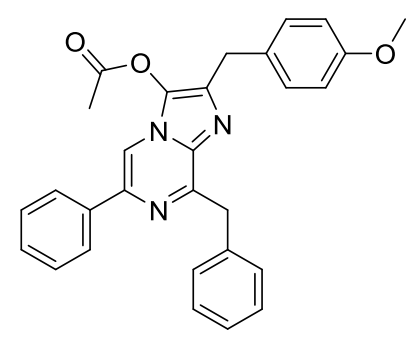

8-Benzyl-2-(4-methoxybenzyl)-6-phenylimidazo[1,2-a]pyrazin-3-yl acetate $\quad \mathbf{2 5}\{1,1,16\} \quad$ (YJ30367-019-1): Obtained as a white solid $(0.04 \mathrm{~g}, 28 \%)$ after a recrystallization from $n$-heptane. ${ }^{1} \mathrm{H}$ NMR $\left(\mathrm{CDCl}_{3}\right): 7.91(\mathrm{~m}, 2 \mathrm{H})$, $7.80(\mathrm{~s}, 1 \mathrm{H}), 7.63(\mathrm{~m}, 2 \mathrm{H}), 7.45(\mathrm{~m}, 2 \mathrm{H}), 7.40(\mathrm{~m}, 1 \mathrm{H}), 7.33(\mathrm{~m}, 3 \mathrm{H}), 7.23(\mathrm{~m}, 2 \mathrm{H}), 6.87(\mathrm{~m}, 2 \mathrm{H}), 4.63(\mathrm{~s}, 2 \mathrm{H})$, $4.17(\mathrm{~s}, 2 \mathrm{H}), 3.77(\mathrm{~s}, 3 \mathrm{H}), 2.21(\mathrm{~s}, 3 \mathrm{H}) .{ }^{13} \mathrm{C} \mathrm{NMR}\left(\mathrm{CDCl}_{3}\right)$ : 167.1, 158.3, 152.9, 139.0, 137.9, 136.9, 135.6, 133.6, 130.1, 130.0, 129.7, 128.7, 128.5, 128.3, 126.5, 126.4, 113.9, 108.4, 55.3, 39.4, 33.3, 19.9 (one signal missing). HRMS $(\mathrm{m} / \mathrm{z}):[\mathrm{M}+\mathrm{H}]^{+}$calcd for $\mathrm{C}_{29} \mathrm{H}_{26} \mathrm{~N}_{3} \mathrm{O}_{3}, 464.1974$; found, 464.1990 .

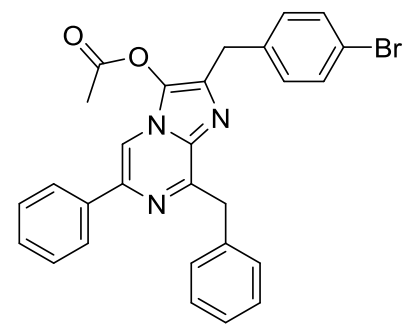

8-Benzyl-2-(4-bromobenzyl)-6-phenylimidazo[1,2-a]pyrazin-3-yl acetate 25 \{1,1,20\} (CF34391-035-1): Obtained as a white solid $(0.18 \mathrm{~g}, 49 \%)$ after a recrystallization from $n$-heptane. ${ }^{1} \mathrm{H}$ NMR $\left(\mathrm{CDCl}_{3}\right): 7.92(\mathrm{~m}, 2 \mathrm{H}), 7.80(\mathrm{~s}$, $1 \mathrm{H}), 7.62(\mathrm{~m}, 2 \mathrm{H}), 7.44(\mathrm{~m}, 5 \mathrm{H}), 7.32(\mathrm{~m}, 2 \mathrm{H}), 7.24(\mathrm{~m}, 1 \mathrm{H}), 7.18(\mathrm{~m}, 1 \mathrm{H}), 4.63(\mathrm{~s}, 2 \mathrm{H}), 4.14(\mathrm{~s}, 2 \mathrm{H}), 2.27(\mathrm{~s}, 3 \mathrm{H})$. ${ }^{13} \mathrm{C}$ NMR $\left(\mathrm{CDCl}_{3}\right): 167.0,153.0,139.2,137.8,137.1,136.8,134.5,133.7,131.5,130.8,129.7,128.8,128.6,128.3$, 126.5, 126.4, 120.4, 108.9, 39.5, 33.4, 19.9. HRMS $(\mathrm{m} / \mathrm{z})$ : $[\mathrm{M}+\mathrm{H}]^{+}$calcd for $\mathrm{C}_{28} \mathrm{H}_{23} \mathrm{BrN}_{3} \mathrm{O}_{2}, 512.0974$; found, 512.0950 .<smiles>CC(=O)Oc1c(Cc2ccc(F)cc2F)nc2c(Cc3ccccc3)nc(-c3ccccc3)cn12</smiles> 
8-Benzyl-2-(2,4-difluorobenzyl)-6-phenylimidazo[1,2-a]pyrazin-3-yl acetate $\quad \mathbf{2 5}\{1,1,25\} \quad$ (YJ31067-055-1): Obtained as a white solid $(0.13 \mathrm{~g}, 52 \%)$ after a recrystallization from $n$-heptane. ${ }^{1} \mathrm{H} \mathrm{NMR}\left(\mathrm{CDCl}_{3}\right): 7.90(\mathrm{~m}, 2 \mathrm{H})$, $7.81(\mathrm{~s}, 1 \mathrm{H}), 7.63(\mathrm{~m}, 2 \mathrm{H}), 7.47(\mathrm{~m}, 2 \mathrm{H}), 7.40(\mathrm{~m}, 1 \mathrm{H}), 7.32(\mathrm{~m}, 2 \mathrm{H}), 7.23(\mathrm{~m}, 1 \mathrm{H}), 6.84(\mathrm{~m}, 2 \mathrm{H}), 4.62(\mathrm{~s}, 2 \mathrm{H})$, $4.16(\mathrm{~s}, 2 \mathrm{H}), 2.36(\mathrm{~s}, 3 \mathrm{H}) .{ }^{13} \mathrm{C} \mathrm{NMR}\left(\mathrm{CDCl}_{3}\right): 167.1,161.8(11$ and $247 \mathrm{~Hz}), 160.7(11$ and $247 \mathrm{~Hz}), 153.0,139.2$, 137.8, 136.8, 133.7, 131.8 (5 and $9 \mathrm{~Hz}), 129.7,129.0,128.8,128.7,128.6,128.3,126.5,126.4,121.0$ (3 and 16 $\mathrm{Hz}), 111.1(3$ and $21 \mathrm{~Hz}), 108.9,103.5(26 \mathrm{~Hz}), 39.5,26.8(4 \mathrm{~Hz}), 19.9$. HRMS $(\mathrm{m} / z)$ : $[\mathrm{M}+\mathrm{H}]^{+}$calcd for $\mathrm{C}_{28} \mathrm{H}_{22} \mathrm{~F}_{2} \mathrm{~N}_{3} \mathrm{O}_{2}$, 470.1680; found, 470.1686.

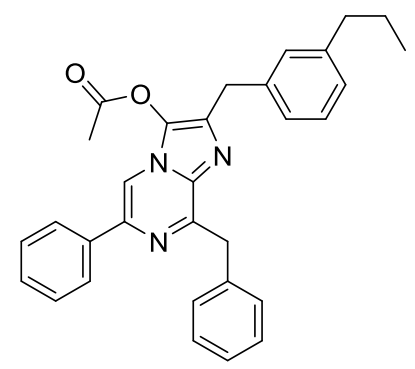

8-Benzyl-6-phenyl-2-(3-propylbenzyl)imidazo[1,2-a]pyrazin-3-yl acetate 25\{1,1,27\} (CF34204-038-1): Obtained as a white solid $(0.14 \mathrm{~g}, 54 \%)$ after a recrystallization from $n$-heptane. ${ }^{1} \mathrm{H} \mathrm{NMR}\left(\mathrm{CDCl}_{3}\right): 7.91(\mathrm{~m}, 2 \mathrm{H}), 7.80(\mathrm{~s}$, 1H), $7.64(\mathrm{~m}, 2 \mathrm{H}), 7.47(\mathrm{~m}, 2 \mathrm{H}), 7.40(\mathrm{~m}, 1 \mathrm{H}), 7.33(\mathrm{~m}, 2 \mathrm{H}), 7.24(\mathrm{~m}, 2 \mathrm{H}), 7.10(\mathrm{~m}, 3 \mathrm{H}), 4.65(\mathrm{~s}, 2 \mathrm{H}), 4.20(\mathrm{~s}$, $2 \mathrm{H}), 2.59(\mathrm{~m}, 2 \mathrm{H}), 2.17(\mathrm{~s}, 3 \mathrm{H}), 1.66(\mathrm{~m}, 2 \mathrm{H}), 0.97(\mathrm{t}, 3 \mathrm{H}, J=7.3 \mathrm{~Hz}) .{ }^{13} \mathrm{C} \mathrm{NMR}\left(\mathrm{CDCl}_{3}\right): 167.1,152.9,142.9$, $139.1,137.9,137.8,136.9,135.4,133.6,129.7,129.3,128.9,128.7,128.5,128.3,128.2,126.6,126.5,126.4$, 126.3, 108.9, 39.4, 38.0, 34.3, 24.6, 19.9, 13.9. HRMS $(\mathrm{m} / \mathrm{z}):[\mathrm{M}+\mathrm{H}]^{+}$calcd for $\mathrm{C}_{31} \mathrm{H}_{30} \mathrm{~N}_{3} \mathrm{O}_{2}, 476.2338$; found, 476.2346 .

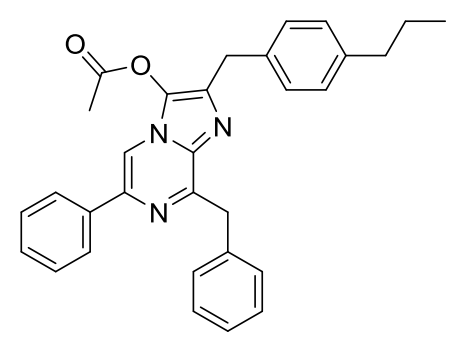

8-Benzyl-6-phenyl-2-(4-propylbenzyl)imidazo[1,2-a]pyrazin-3-yl acetate 25\{1,1,28\} (CF34204-046-1): Obtained as a white solid $(0.25 \mathrm{~g}, 73 \%)$ after a recrystallization from $n$-heptane. ${ }^{1} \mathrm{H}$ NMR $\left(\mathrm{CDCl}_{3}\right): 7.91(\mathrm{~m}, 2 \mathrm{H}), 7.80(\mathrm{~s}$, 1H), $7.64(\mathrm{~m}, 2 \mathrm{H}), 7.47(\mathrm{~m}, 2 \mathrm{H}), 7.40(\mathrm{~m}, 1 \mathrm{H}), 7.33(\mathrm{~m}, 2 \mathrm{H}), 7.24(\mathrm{~m}, 3 \mathrm{H}), 7.15(\mathrm{~m}, 2 \mathrm{H}), 4.65(\mathrm{~s}, 2 \mathrm{H}), 4.20(\mathrm{~s}$, 2H), $2.60(\mathrm{~m}, 2 \mathrm{H}), 2.18(\mathrm{~s}, 3 \mathrm{H}), 1.66(\mathrm{~m}, 2 \mathrm{H}), 0.98(\mathrm{t}, 3 \mathrm{H}, J=7.3 \mathrm{~Hz}) .{ }^{13} \mathrm{C} \mathrm{NMR}\left(\mathrm{CDCl}_{3}\right): 167.1,152.9,140.8$, 139.0, 137.9, 136.9, 135.5, 135.2, 133.6, 129.8, 128.9, 128.8, 128.7, 128.6, 128.5, 128.3, 126.4, 126.4, 108.8, 39.4, 37.7, 33.9, 24.6, 19.9, 13.8. HRMS (m/z): $[\mathrm{M}+\mathrm{H}]^{+}$calcd for $\mathrm{C}_{31} \mathrm{H}_{30} \mathrm{~N}_{3} \mathrm{O}_{2}$, 476.2338; found, 476.2332.

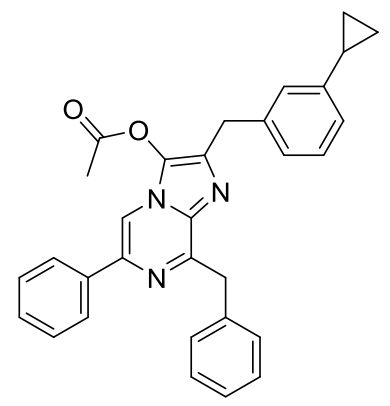

8-Benzyl-2-(3-cyclopropylbenzyl)-6-phenylimidazo[1,2-a]pyrazin-3-yl acetate 25\{1,1,29\} (YJ30367-109-1): Obtained as a white solid $(0.29 \mathrm{~g}, 65 \%)$ after a recrystallization from $n$-heptane. ${ }^{1} \mathrm{H} \mathrm{NMR}\left(\mathrm{CDCl}_{3}\right): 7.91(\mathrm{~m}, 2 \mathrm{H})$, $7.79(\mathrm{~s}, 1 \mathrm{H}), 7.64(\mathrm{~m}, 2 \mathrm{H}), 7.46(\mathrm{~m}, 2 \mathrm{H}), 7.39(\mathrm{~m}, 1 \mathrm{H}), 7.33(\mathrm{~m}, 2 \mathrm{H}), 7.23(\mathrm{~m}, 2 \mathrm{H}), 7.06(\mathrm{~m}, 2 \mathrm{H}), 6.96(\mathrm{~m}, 1 \mathrm{H})$, $4.64(\mathrm{~s}, 2 \mathrm{H}), 4.18(\mathrm{~s}, 2 \mathrm{H}), 2.18(\mathrm{~s}, 3 \mathrm{H}), 1.89(\mathrm{~m}, 1 \mathrm{H}), 0.95(\mathrm{~m}, 2 \mathrm{H}), 0.70(\mathrm{~m}, 2 \mathrm{H}) .{ }^{13} \mathrm{C} \mathrm{NMR}\left(\mathrm{CDCl}_{3}\right): 167.1,152.9$, 142.2 , 139.0, 137.9, 137.8, 136.9, 135.3, 133.6, 129.7, 128.7, 128.5, 128.4, 128.3, 126.6, 126.5, 126.4, 126.1, 123.7, 108.8, 39.4, 34.3, 19.8, 15.3, 9.1. HRMS (m/z): $[\mathrm{M}+\mathrm{H}]^{+}$calcd for $\mathrm{C}_{31} \mathrm{H}_{28} \mathrm{~N}_{3} \mathrm{O}_{2}, 474.2181$; found, 474.2189. 


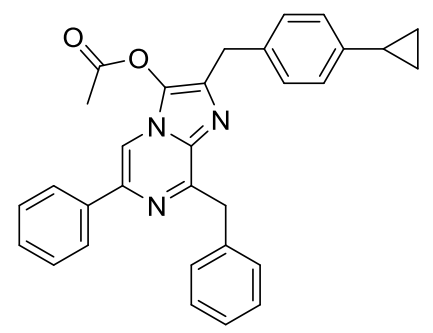

8-Benzyl-2-(4-cyclopropylbenzyl)-6-phenylimidazo[1,2-a]pyrazin-3-yl acetate $\quad \mathbf{2 5}\{1,1,30\} \quad$ (YJ30367-107-1): Obtained as a white solid $(0.29 \mathrm{~g}, 68 \%)$ after a recrystallization from $n$-heptane. ${ }^{1} \mathrm{H}$ NMR $\left(\mathrm{CDCl}_{3}\right): 7.91(\mathrm{~m}, 2 \mathrm{H})$, $7.80(\mathrm{~s}, 1 \mathrm{H}), 7.64(\mathrm{~m}, 2 \mathrm{H}), 7.46(\mathrm{~m}, 2 \mathrm{H}), 7.40(\mathrm{~m}, 1 \mathrm{H}), 7.33(\mathrm{~m}, 2 \mathrm{H}), 7.22(\mathrm{~m}, 3 \mathrm{H}), 7.04(\mathrm{~m}, 2 \mathrm{H}), 4.64(\mathrm{~s}, 2 \mathrm{H})$, 4.17 (s, 2H), $2.18(\mathrm{~s}, 3 \mathrm{H}), 1.92(\mathrm{~m}, 1 \mathrm{H}), 0.94(\mathrm{~m}, 2 \mathrm{H}), 0.68(\mathrm{~m}, 2 \mathrm{H}) .{ }^{13} \mathrm{C}$ NMR $\left(\mathrm{CDCl}_{3}\right): 167.1,152.9,142.1$, 139.0, 137.9, 136.9, 135.4, 134.9, 133.6, 129.7, 129.0, 128.8, 128.7, 128.5, 128.3, 126.5, 126.4, 125.8, 108.4, 39.4, 33.8, 19.9, , 15.0, 9.0. HRMS $(\mathrm{m} / \mathrm{z}):[\mathrm{M}+\mathrm{H}]^{+}$calcd for $\mathrm{C}_{31} \mathrm{H}_{28} \mathrm{~N}_{3} \mathrm{O}_{2}, 474.2181$; found, 474.2183.

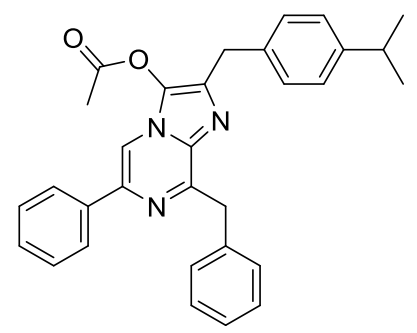

8-Benzyl-2-(4-isopropylbenzyl)-6-phenylimidazo[1,2-a]pyrazin-3-yl acetate 25 \{1,1,31\} (E31093-093-2): Obtained as a white solid $(0.16 \mathrm{~g}, 70 \%)$ after a recrystallization from $n$-heptane. ${ }^{1} \mathrm{H}$ NMR $\left(\mathrm{CDCl}_{3}\right): 7.93-7.84(\mathrm{~m}, 2 \mathrm{H}), 7.77$ (s, 1H), $7.65-7.59(\mathrm{~m}, 2 \mathrm{H}), 7.49-7.41(\mathrm{~m}, 2 \mathrm{H}), 7.41-7.33(\mathrm{~m}, 1 \mathrm{H}), 7.33-7.28(\mathrm{~m}, 2 \mathrm{H}), 7.24-7.14(\mathrm{~m}, 5 \mathrm{H})$, $4.62(\mathrm{~s}, 2 \mathrm{H}), 4.17(\mathrm{~s}, 2 \mathrm{H}), 2.90$ (hept, $1 \mathrm{H}, J=6.9 \mathrm{~Hz}), 2.13(\mathrm{~s}, 3 \mathrm{H}), 1.25(\mathrm{~d}, 6 \mathrm{H}, J=6.9 \mathrm{~Hz}) \cdot{ }^{13} \mathrm{C} \mathrm{NMR}\left(\mathrm{CDCl}_{3}\right)$ : 167.2, 153.0, 147.2, 139.2, 138.0, 137.0, 135.6, 135.4, 133.8, 129.9, 129.2, 129.0, 128.9, 128.7, 128.4, 126.7, 126.6, 126.6, 109.0, 39.5, 34.1, 33.9, 24.2, 20.0. HRMS $(\mathrm{m} / \mathrm{z})$ : $[\mathrm{M}+\mathrm{H}]^{+}$calcd for $\mathrm{C}_{31} \mathrm{H}_{30} \mathrm{~N}_{3} \mathrm{O}_{2}, 476.2338$; found, 476.2337.

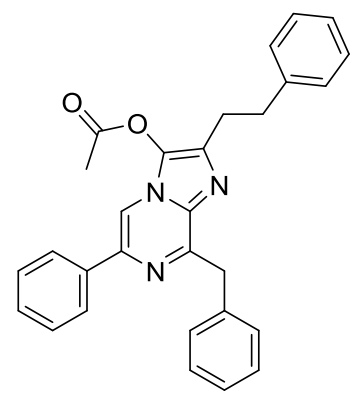

8-Benzyl-2-phenethyl-6-phenylimidazo[1,2-a]pyrazin-3-yl acetate 25\{1,1,32\} (YJ31070-131-1): Obtained as a white powder after a recrystallization from n-heptane $(0.22 \mathrm{~g}, 67 \%) .{ }^{1} \mathrm{H} N M R\left(\mathrm{CDCl}_{3}\right): 7.92(\mathrm{~m}, 2 \mathrm{H}), 7.81(\mathrm{~s}, 1 \mathrm{H})$, $7.64(\mathrm{~m}, 2 \mathrm{H}), 7.47(\mathrm{~m}, 2 \mathrm{H}), 7.40(\mathrm{~m}, 1 \mathrm{H}), 7.35-7.21(\mathrm{~m}, 8 \mathrm{H}), 4.65(\mathrm{~s}, 2 \mathrm{H}), 3.12(\mathrm{~m}, 4 \mathrm{H}), 2.42(\mathrm{~s}, 3 \mathrm{H}) .{ }^{13} \mathrm{C}$ NMR $\left(\mathrm{CDCl}_{3}\right): 167.0,141.5,139.0,138.0,136.9,136.0,137.0,133.7,129.7,128.8,128.5,128.4,128.3,128.2,126.5$, 126.4, 126.0, 108.9, 39.4, 34.7, 29.0, 20.1. HRMS $(\mathrm{m} / \mathrm{z})$ : $[\mathrm{M}+\mathrm{H}]^{+}$calcd for $\mathrm{C}_{29} \mathrm{H}_{26} \mathrm{~N}_{3} \mathrm{O}_{2}, 448.2025$; found, 448.2026 .<smiles>CC(=O)Oc1c(CC2CCCCC2)nc2c(Cc3ccccc3)nc(-c3ccccc3)cn12</smiles> 
8-Benzyl-2-(cyclohexylmethyl)-6-phenylimidazo[1,2-a]pyrazin-3-yl acetate $\quad 25\{1,1,33\} \quad$ (YJ31067-071-1): Obtained as a powder $(0.24 \mathrm{~g}, 72 \%)$ after a recrystallization in n-heptane. ${ }^{1} \mathrm{H}$ NMR $\left(\mathrm{CDCl}_{3}\right): 7.92(\mathrm{~m}, 2 \mathrm{H}), 7.80(\mathrm{~s}$, 1H), $7.65(\mathrm{~m}, 2 \mathrm{H}), 7.47(\mathrm{~m}, 2 \mathrm{H}), 7.39(\mathrm{~m}, 1 \mathrm{H}), 7.32(\mathrm{~m}, 2 \mathrm{H}), 7.23(\mathrm{~m}, 1 \mathrm{H}), 4.63(\mathrm{~s}, 2 \mathrm{H}), 2.78(\mathrm{~d}, 2 \mathrm{H}, J=7.2 \mathrm{~Hz})$, $2.50(\mathrm{~s}, 3 \mathrm{H}), 1.87(\mathrm{~m}, 1 \mathrm{H}), 1.75(\mathrm{~m}, 5 \mathrm{H}), 1.27(\mathrm{~m}, 3 \mathrm{H}), 1.05(\mathrm{~m}, 2 \mathrm{H}) .{ }^{13} \mathrm{C} \mathrm{NMR}\left(\mathrm{CDCl}_{3}\right): 167.3,152.7,138.9$, 138.0, 137.0, 136.0, 133.7, 129.8, 129.0, 128.7, 128.4, 128.2, 126.4, 126.3, 108.9, 39.4, 37.8, 34.5, 33.2, 26.5, 26.2, 20.2. HRMS (m/z): $[\mathrm{M}+\mathrm{H}]^{+}$calcd for $\mathrm{C}_{28} \mathrm{H}_{30} \mathrm{~N}_{3} \mathrm{O}_{2}, 440.2338$; found, 440.2326.

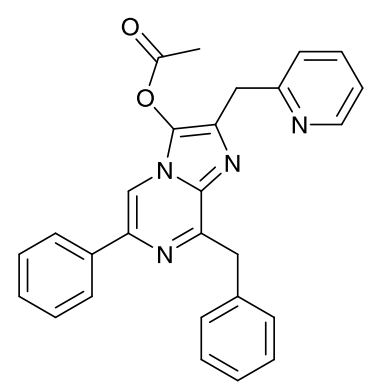

8-Benzyl-6-phenyl-2-(pyridin-2-ylmethyl)imidazo[1,2-a]pyrazin-3-yl acetate $\quad \mathbf{2 5}\{1,1,34\} \quad$ (YJ31134-089-1): Obtained as an oil $(0.41 \mathrm{~g}, 91 \%)$ which was evaluated without further purification. ${ }^{1} \mathrm{H} \mathrm{NMR}\left(\mathrm{CDCl}_{3}\right): 8.56(\mathrm{~m}$, 1H), $7.92(\mathrm{~m}, 1 \mathrm{H}), 7.90(\mathrm{~m}, 1 \mathrm{H}), 7.83(\mathrm{~s}, 1 \mathrm{H}), 7.62(\mathrm{~m}, 2 \mathrm{H}), 7.46(\mathrm{~m}, 2 \mathrm{H}), 7.40(\mathrm{~m}, 1 \mathrm{H}), 7.32(\mathrm{~m}, 3 \mathrm{H}), 7.17(\mathrm{~m}$, $3 \mathrm{H}), 4.63(\mathrm{~s}, 2 \mathrm{H}), 4.38(\mathrm{~s}, 2 \mathrm{H}), 2.35(\mathrm{~s}, 3 \mathrm{H}) .{ }^{13} \mathrm{C} \mathrm{NMR}\left(\mathrm{CDCl}_{3}\right): 167.2,158.4,153.0,149.1,139.1,137.8,136.8$, $136.5,133.8,133.7,129.7,129.2,129.0,128.8,128.5,128.2,126.4,123.4,121.5,108.9,39.4,36.6$, 20.1. HRMS $(\mathrm{m} / \mathrm{z}):[\mathrm{M}+\mathrm{H}]^{+}$calcd for $\mathrm{C}_{27} \mathrm{H}_{23} \mathrm{~N}_{4} \mathrm{O}_{2}, 435.1821$; found, 435.1823 .

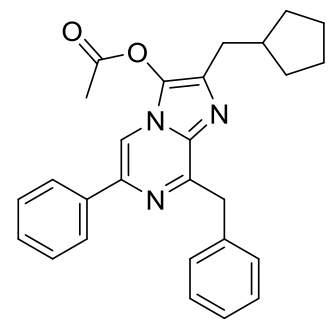

8-Benzyl-2-(cyclopentylmethyl)-6-phenylimidazo[1,2-a]pyrazin-3-yl acetate $\quad \mathbf{2 5}\{1,1,36\} \quad$ (YJ31067-073-1): Obtained as a powder $(0.21 \mathrm{~g}, 88 \%)$ after a concentration to dryness. ${ }^{1} \mathrm{H} \mathrm{NMR}\left(\mathrm{CDCl}_{3}\right): 7.92(\mathrm{~m}, 2 \mathrm{H}), 7.80(\mathrm{~s}, 1 \mathrm{H})$, $7.65(\mathrm{~m}, 2 \mathrm{H}), 7.47(\mathrm{~m}, 2 \mathrm{H}), 7.39(\mathrm{~m}, 1 \mathrm{H}), 7.32(\mathrm{~m}, 2 \mathrm{H}), 7.23(\mathrm{~m}, 1 \mathrm{H}), 4.63(\mathrm{~s}, 2 \mathrm{H}), 2.78(\mathrm{~d}, 2 \mathrm{H}, J=7.4 \mathrm{~Hz}), 2.50$ (s, 3H), $2.38(\mathrm{~m}, 1 \mathrm{H}), 1.80(\mathrm{~m}, 2 \mathrm{H}), 1.64(\mathrm{~m}, 4 \mathrm{H}), 1.29(\mathrm{~m}, 2 \mathrm{H}) .{ }^{13} \mathrm{C} \mathrm{NMR}\left(\mathrm{CDCl}_{3}\right): 167.3,152.7,138.9,138.0$, 137.0, 136.7, 133.6, 129.8, 128.7, 128.6, 128.5, 128.2, 126.4, 126.3, 108.9, 39.8, 38.4, 32.8, 32.5, 25.0, 20.2. HRMS $(\mathrm{m} / z):[\mathrm{M}+\mathrm{H}]^{+}$calcd for $\mathrm{C}_{27} \mathrm{H}_{28} \mathrm{~N}_{3} \mathrm{O}_{2}$, 426.2181; found, 426.2180.

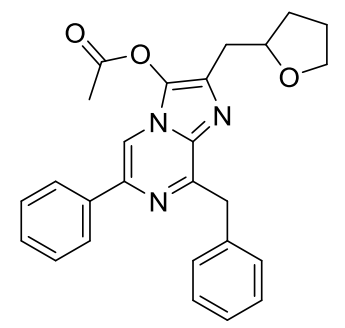

8-Benzyl-6-phenyl-2-((tetrahydrofuran-2-yl)methyl)imidazo[1,2-a]pyrazin-3-yl acetate 25 1,1,38\} (EC31092-1292): Obtained as an oil $(0.36 \mathrm{~g}, 81 \%)$ which was evaluated without further purification. ${ }^{1} \mathrm{H} \mathrm{NMR}\left(\mathrm{CDCl}_{3}\right): 7.93-$ $7.87(\mathrm{~m}, 2 \mathrm{H}), 7.82(\mathrm{~s}, 1 \mathrm{H}), 7.62-7.57(\mathrm{~m}, 2 \mathrm{H}), 7.48-7.41(\mathrm{~m}, 2 \mathrm{H}), 7.40-7.34(\mathrm{~m}, 1 \mathrm{H}), 7.32-7.27(\mathrm{~m}, 2 \mathrm{H})$, $7.23-7.17(\mathrm{~m}, 1 \mathrm{H}), 4.66-4.55(\mathrm{~m}, 2 \mathrm{H}), 4.32-4.23(\mathrm{~m}, 1 \mathrm{H}), 3.89-3.69(\mathrm{~m}, 2 \mathrm{H}), 3.10-2.95(\mathrm{~m}, 2 \mathrm{H}), 2.46(\mathrm{~s}$, $3 \mathrm{H}), 2.09-1.98(\mathrm{~m}, 1 \mathrm{H}), 1.92-1.82(\mathrm{~m}, 2 \mathrm{H}), 1.77-1.66(\mathrm{~m}, 1 \mathrm{H}) .{ }^{13} \mathrm{C} \mathrm{NMR}\left(\mathrm{CDCl}_{3}\right): 167.5,153.0,139.1,138.1$, 137.1, 133.9, 133.8, 129.9, 129.7, 128.9, 128.6, 128.4, 126.6 (two signals), 109.2, 78.3, 68.2, 39.5, 33.5, 31.0, 25.8, 20.5. HRMS $(m / z):[\mathrm{M}+\mathrm{H}]^{+}$calcd for $\mathrm{C}_{26} \mathrm{H}_{26} \mathrm{~N}_{3} \mathrm{O}_{3}$, 428.1974; found, 428.1956. 


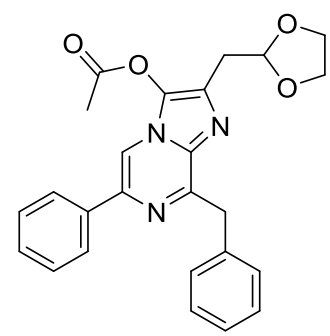

2-((1,3-Dioxolan-2-yl)methyl)-8-benzyl-6-phenylimidazo[1,2-a]pyrazin-3-yl acetate 25 1,1,39\} (VHE30855-1892): Obtained as a white solid $(0.46 \mathrm{~g}, 76 \%)$ after recrystallization from $n$-heptane. ${ }^{1} \mathrm{H} \mathrm{NMR}\left(\mathrm{CDCl}_{3}\right): 7.92(\mathrm{~m}, 2 \mathrm{H})$, $7.85(\mathrm{~s}, 1 \mathrm{H}), 7.62(\mathrm{~m}, 2 \mathrm{H}), 7.47(\mathrm{~m}, 2 \mathrm{H}), 7.40(\mathrm{~m}, 1 \mathrm{H}), 7.32(\mathrm{~m}, 2 \mathrm{H}), 7.23(\mathrm{~m}, 1 \mathrm{H}), 5.29(\mathrm{t}, 1 \mathrm{H}, J=4.5 \mathrm{~Hz}), 4.64$ $(\mathrm{s}, 2 \mathrm{H}), 3.97-3.86(\mathrm{~m}, 4 \mathrm{H}), 3.22(\mathrm{~d}, 2 \mathrm{H}, J=4.5 \mathrm{~Hz}), 2.49(\mathrm{~s}, 3 \mathrm{H}) .{ }^{13} \mathrm{C} \mathrm{NMR}\left(\mathrm{CDCl}_{3}\right): 137.3,153.0,139.0,137.9$, 136.9, 133.8, 131.1, 130.0, 129.7, 128.7, 128.5, 128.2, 126.4 (two signals), 109.0, 103.1, 65.1, 39.3, 32.9, 20.3. HRMS $(m / z):[\mathrm{M}+\mathrm{H}]^{+}$calcd for $\mathrm{C}_{25} \mathrm{H}_{24} \mathrm{~N}_{3} \mathrm{O}_{3}, 430.1767$; found, 430.1762.

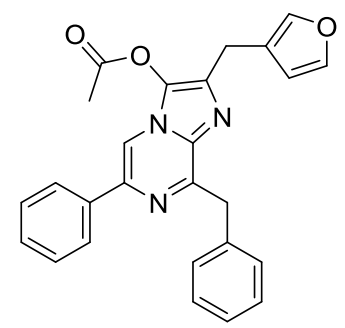

8-Benzyl-2-(furan-3-ylmethyl)-6-phenylimidazo[1,2-a]pyrazin-3-yl acetate $\quad \mathbf{2 5}\{1,1,40\} \quad$ (VHE30855-187-2): Obtained as a beige solid $(0.74 \mathrm{~g}, 67 \%)$ after a recrystallization from $n$-heptane. ${ }^{1} \mathrm{H}$ NMR $\left(\mathrm{CDCl}_{3}\right): 7.92(\mathrm{~m}, 2 \mathrm{H})$, $7.83(\mathrm{~s}, 1 \mathrm{H}), 7.63(\mathrm{~m}, 2 \mathrm{H}), 7.47(\mathrm{~m}, 2 \mathrm{H}), 7.40(\mathrm{~m}, 2 \mathrm{H}), 7.36-7.30(\mathrm{~m}, 3 \mathrm{H}), 7.24(\mathrm{~m}, 1 \mathrm{H}), 6.38(\mathrm{~m}, 1 \mathrm{H}), 4.64(\mathrm{~s}$, 2H), $4.00(\mathrm{~m}, 2 \mathrm{H}), 2.34(\mathrm{~s}, 3 \mathrm{H}) .{ }^{13} \mathrm{C} \mathrm{NMR}\left(\mathrm{CDCl}_{3}\right): 167.1,153.0,142.9,140.0,139.1,137.8,136.8,134.5,133.6$, $129.7,128.8,128.6,128.3,126.5,126.4,121.2,111.5,108.9,39.4,23.6,20.0 . \mathrm{HRMS}(\mathrm{m} / \mathrm{z}):[\mathrm{M}+\mathrm{H}]^{+}$calcd for $\mathrm{C}_{26} \mathrm{H}_{22} \mathrm{~N}_{3} \mathrm{O}_{3}, 424.1661$; found, 424.1688 .

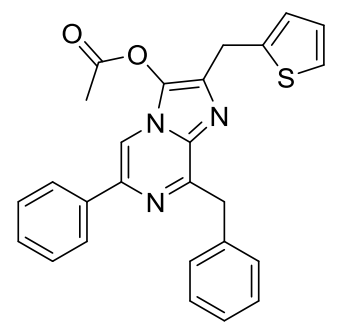

8-Benzyl-6-phenyl-2-(thiophen-2-ylmethyl)imidazo[1,2-a]pyrazin-3-yl acetate $\mathbf{2 5}\{1,1,41\}$ (EC31092-095-2): Obtained as a white solid $(0.37 \mathrm{~g}, 73 \%)$ after a recrystallization from $n$-heptane. ${ }^{1} \mathrm{H}$ NMR $\left(\mathrm{CDCl}_{3}\right): 7.92-7.87(\mathrm{~m}$, 2H), $7.80(\mathrm{~s}, 1 \mathrm{H}), 7.65-7.58(\mathrm{~m}, 2 \mathrm{H}), 7.49-7.42(\mathrm{~m}, 2 \mathrm{H}), 7.42-7.34(\mathrm{~m}, 1 \mathrm{H}), 7.34-7.27(\mathrm{~m}, 2 \mathrm{H}), 7.25-7.20$ $(\mathrm{m}, 1 \mathrm{H}), 7.19(\mathrm{dd}, 1 \mathrm{H}, J=5.1,1.3 \mathrm{~Hz}), 6.95(\mathrm{dd}, 1 \mathrm{H}, J=5.1,3.5 \mathrm{~Hz}), 6.93-6.88(\mathrm{~m}, 1 \mathrm{H}), 4.62(\mathrm{~s}, 2 \mathrm{H}), 4.38(\mathrm{~d}$, $2 \mathrm{H}, J=0.8 \mathrm{~Hz}), 2.30(\mathrm{~s}, 3 \mathrm{H}) .{ }^{13} \mathrm{C} \mathrm{NMR}\left(\mathrm{CDCl}_{3}\right): 167.0,153.1,140.3,139.2,137.8,136.8,134.4,133.5,129.8$, 128.8, 128.6 (two signals), 128.3, 126.8, 126.5, 126.4, 125.9, 124.3, 108.9, 39.4, 28.5, 20.0. HRMS $(\mathrm{m} / \mathrm{z}):[\mathrm{M}+\mathrm{H}]^{+}$ calcd for $\mathrm{C}_{26} \mathrm{H}_{22} \mathrm{~N}_{3} \mathrm{O}_{2} \mathrm{~S}$, 440.1433; found, 440.1486.

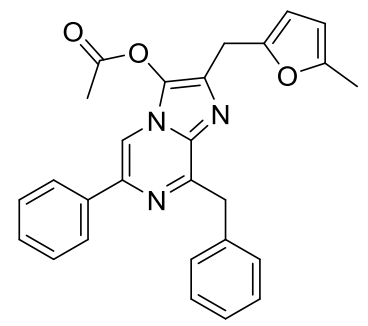

8-Benzyl-2-((5-methylfuran-2-yl)methyl)-6-phenylimidazo[1,2-a]pyrazin-3-yl acetate 25 1,1,42\} (EC29990-1641): Obtained as a white solid $(0.14 \mathrm{~g}, 24 \%)$ after a chromatography over silica gel (cyclohexane-ethyl acetate 5:1) and a recrystallization from cyclohexane. ${ }^{1} \mathrm{H} \mathrm{NMR}\left(\mathrm{CDCl}_{3}\right): 7.93-7.86(\mathrm{~m}, 2 \mathrm{H}), 7.82(\mathrm{~s}, 1 \mathrm{H}), 7.64-7.57(\mathrm{~m}$, 
2H), $7.49-7.41(\mathrm{~m}, 2 \mathrm{H}), 7.41-7.35(\mathrm{~m}, 1 \mathrm{H}), 7.35-7.27(\mathrm{~m}, 2 \mathrm{H}), 7.25-7.16(\mathrm{~m}, 1 \mathrm{H}), 6.03-5.97(\mathrm{~m}, 1 \mathrm{H}), 5.93$ $-5.86(\mathrm{~m}, 1 \mathrm{H}), 4.62(\mathrm{~s}, 2 \mathrm{H}), 4.17(\mathrm{~s}, 2 \mathrm{H}), 2.34(\mathrm{~s}, 3 \mathrm{H}), 2.26(\mathrm{~s}, 3 \mathrm{H}) .{ }^{13} \mathrm{C} \mathrm{NMR}\left(\mathrm{CDCl}_{3}\right)$ : 167.2, 153.1, 151.3, 149.7, 139.3, 138.0, 137.0, 133.7, 133.0, 129.9, 129.1, 128.9, 128.7, 128.4, 126.6 (two signals), 109.0, 107.6, 106.3, 39.5, 27.5, 20.2, 13.7. HRMS (m/z): $[\mathrm{M}+\mathrm{H}]^{+}$calcd for $\mathrm{C}_{27} \mathrm{H}_{24} \mathrm{~N}_{3} \mathrm{O}_{3}, 438.1818$; found, 438.1828.

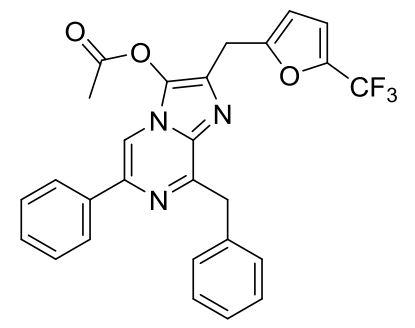

8-Benzyl-6-phenyl-2-((5-(trifluoromethyl)furan-2-yl)methyl)imidazo[1,2-a]pyrazin-3-yl $\quad$ acetate $\quad \mathbf{2 5}\{1,1,43\}$ (EC31093-039-1): Obtained as a white solid $(0.07 \mathrm{~g}, 43 \%)$ after a recrystallization from $n$-heptane. ${ }^{1} \mathrm{H}$ NMR $\left(\mathrm{CDCl}_{3}\right): 7.94-7.87(\mathrm{~m}, 2 \mathrm{H}), 7.83(\mathrm{~s}, 1 \mathrm{H}), 7.63-7.57(\mathrm{~m}, 2 \mathrm{H}), 7.49-7.42(\mathrm{~m}, 2 \mathrm{H}), 7.42-7.35(\mathrm{~m}, 1 \mathrm{H}), 7.33-$ $7.27(\mathrm{~m}, 2 \mathrm{H}), 7.24-7.17(\mathrm{~m}, 1 \mathrm{H}), 6.73(\mathrm{dd}, 1 \mathrm{H}, J=3.3,1.2 \mathrm{~Hz}), 6.21(\mathrm{dd}, 1 \mathrm{H}, J=3.4,0.7 \mathrm{~Hz}), 4.61(\mathrm{~s}, 2 \mathrm{H}), 4.23$ (s, 2H), 2.40 (s, 3H). ${ }^{13} \mathrm{C}$ NMR $\left(\mathrm{CDCl}_{3}\right): 166.9,154.9,153.2,140.9(43 \mathrm{~Hz}), 139.4,137.7,136.7,133.7,131.2$, 129.7, 129.0, 128.8, 128.7, 128.3, 126.5, 126.4, $119.2(267 \mathrm{~Hz}), 112.6(3 \mathrm{~Hz}), 109.0,107.9,39.5,27.0,20.0$. HRMS $(m / z):[\mathrm{M}+\mathrm{H}]^{+}$calcd for $\mathrm{C}_{27} \mathrm{H}_{21} \mathrm{~F}_{3} \mathrm{~N}_{3} \mathrm{O}_{3}, 492.1535$; found, 492.1566 .

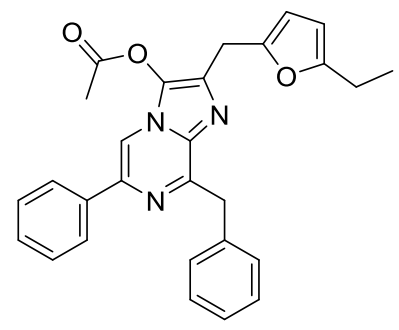

8-Benzyl-2-((5-ethylfuran-2-yl)methyl)-6-phenylimidazo[1,2-a]pyrazin-3-yl acetate 25\{1,1,44\} (EC31092-093-2): Obtained as a white solid $(0.27 \mathrm{~g}, 57 \%)$ after a recrystallization from $n$-heptane. ${ }^{1} \mathrm{H}$ NMR $\left(\mathrm{CDCl}_{3}\right): 7.93-7.86(\mathrm{~m}$, 2H), $7.81(\mathrm{~s}, 1 \mathrm{H}), 7.64-7.57(\mathrm{~m}, 2 \mathrm{H}), 7.49-7.41(\mathrm{~m}, 2 \mathrm{H}), 7.41-7.35(\mathrm{~m}, 1 \mathrm{H}), 7.33-7.27(\mathrm{~m}, 2 \mathrm{H}), 7.24-7.18$ $(\mathrm{m}, 1 \mathrm{H}), 6.01(\mathrm{~d}, 1 \mathrm{H}, J=3.0 \mathrm{~Hz}), 5.93-5.87(\mathrm{~m}, 1 \mathrm{H}), 4.62(\mathrm{~s}, 2 \mathrm{H}), 4.17(\mathrm{~s}, 2 \mathrm{H}), 2.61(\mathrm{q}, 2 \mathrm{H}, J=7.5 \mathrm{~Hz}), 2.33(\mathrm{~s}$, $3 \mathrm{H}), 1.22(\mathrm{t}, 3 \mathrm{H}, J=7.5 \mathrm{~Hz}) .{ }^{13} \mathrm{C}$ NMR $\left(\mathrm{CDCl}_{3}\right): 167.2,157.2,153.1,149.5,139.3,138.0,137.0,133.7,133.1$, 129.9, 129.1, 128.9, 128.7, 128.4, 126.6 (two signals), 109.0, 107.4, 104.7, 39.5, 27.5, 21.5, 20.2, 12.4. HRMS $(\mathrm{m} / \mathrm{z}):[\mathrm{M}+\mathrm{H}]^{+}$calcd for $\mathrm{C}_{28} \mathrm{H}_{26} \mathrm{~N}_{3} \mathrm{O}_{3}, 452.1974$; found, 452.2014 .

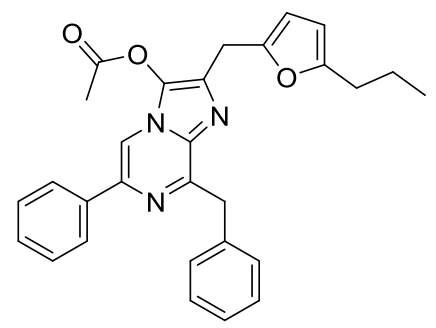

8-Benzyl-6-phenyl-2-((5-propylfuran-2-yl)methyl)imidazo[1,2-a]pyrazin-3-yl acetate 25\{1,1,45\} (EC32712-1332) Obtained as a white solid $(0.15 \mathrm{~g}, 54 \%)$ after a recrystallization in n-heptane. ${ }^{1} \mathrm{H}$ NMR $\left(\mathrm{CDCl}_{3}\right): 7.92(\mathrm{~m}, 2 \mathrm{H})$, $7.84(\mathrm{~s}, 1 \mathrm{H}), 7.63(\mathrm{~m}, 2 \mathrm{H}), 7.47(\mathrm{~m}, 2 \mathrm{H}), 7.41(\mathrm{~m}, 1 \mathrm{H}), 7.29(\mathrm{~m}, 3 \mathrm{H}), 6.03(\mathrm{~d}, 1 \mathrm{H}, J=3.0 \mathrm{~Hz}), 5.93(\mathrm{~d}, 1 \mathrm{H}, J=3.0$ $\mathrm{Hz}), 4.64(\mathrm{~s}, 2 \mathrm{H}), 4.20(\mathrm{~s}, 2 \mathrm{H}), 2.58(\mathrm{t}, 2 \mathrm{H}, J=7.4 \mathrm{~Hz}), 2.35(\mathrm{~s}, 3 \mathrm{H}), 1.67$ (hept, $2 \mathrm{H}, J=7.4 \mathrm{~Hz}), 0.99(\mathrm{t}, 3 \mathrm{H}, J=$ $7.4 \mathrm{~Hz}) .{ }^{13} \mathrm{C}$ NMR $\left(\mathrm{CDCl}_{3}\right): 167.0,155.6,153.0,149.3,139.1,137.8,136.8,133.5,132.9,129.7,128.9,128.7$, $128.5,128.3,126.4,126.4,108.8,107.2,105.4,39.3,30.1,27.4,21.4,20.1,13.7$. HRMS $(m / z):[\mathrm{M}+\mathrm{H}]^{+}$calcd for $\mathrm{C}_{29} \mathrm{H}_{28} \mathrm{~N}_{3} \mathrm{O}_{3}$ : 466.2131; found, 466.2141 . 


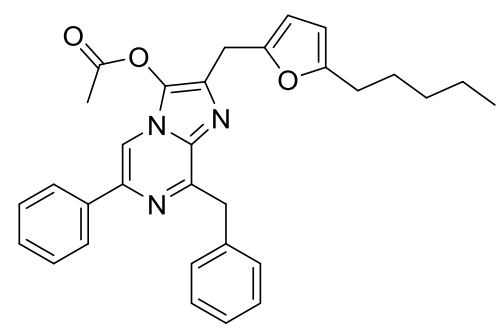

8-Benzyl-2-((5-pentylfuran-2-yl)methyl)-6-phenylimidazo[1,2-a]pyrazin-3-yl acetate 25\{1,1,46\} (YJ 31776-0291): Obtained as an oil which slowly solidified $(0.18 \mathrm{~g}, 95 \%) .{ }^{1} \mathrm{H}$ NMR $\left(\mathrm{CDCl}_{3}\right): 7.92(\mathrm{~m}, 2 \mathrm{H}), 7.84(\mathrm{~s}, 1 \mathrm{H}), 7.82(\mathrm{~s}$, 1H), $7.62(\mathrm{~m}, 2 \mathrm{H}), 7.47(\mathrm{~m}, 2 \mathrm{H}), 7.40(\mathrm{~m}, 1 \mathrm{H}), 7.32(\mathrm{~m}, 2 \mathrm{H}), 7.23(\mathrm{~m}, 1 \mathrm{H}), 6.03(\mathrm{~m}, 1 \mathrm{H}), 5.92(\mathrm{~d}, 1 \mathrm{H}, J=3.0 \mathrm{~Hz})$, $4.65(\mathrm{~s}, 2 \mathrm{H}), 4.21(\mathrm{~s}, 2 \mathrm{H}), 2.59(\mathrm{t}, 2 \mathrm{H}, J=7.5 \mathrm{~Hz}), 2.35(\mathrm{~s}, 3 \mathrm{H}), 1.63(\mathrm{~m}, 2 \mathrm{H}), 1.34(\mathrm{~m}, 4 \mathrm{H}), 0.91(\mathrm{~m}, 3 \mathrm{H}) .{ }^{13} \mathrm{C}$ NMR $\left(\mathrm{CDCl}_{3}\right): 166.9,155.9,152.8,149.0,139.4,137.6,136.6,133.4,133.1,132.6,129.7,128.9,128.8,128.7$, $128.3,126.5,126.4,108.8,107.4,105.3,39.3,31.4,28.0,27.7,27.2,22.4,20.1,14.0$. HRMS $(\mathrm{m} / \mathrm{z}):[\mathrm{M}+\mathrm{H}]^{+}$calcd for $\mathrm{C}_{31} \mathrm{H}_{32} \mathrm{~N}_{3} \mathrm{O}_{3}: 494.2444$; found, 494.2453 .

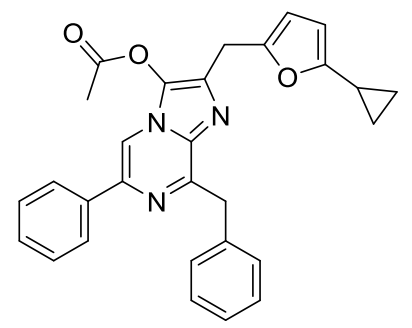

8-Benzyl-2-((5-cyclopropylfuran-2-yl)methyl)-6-phenylimidazo[1,2-a]pyrazin-3-yl acetate 25 1,1,47\} (EC31093079-2): Obtained as a white solid $(0.13 \mathrm{~g}, 42 \%)$ after a recrystallization in n-heptane. ${ }^{1} \mathrm{H}$ NMR $\left(\mathrm{CDCl}_{3}\right): 7.93-$ $7.86(\mathrm{~m}, 2 \mathrm{H}), 7.81(\mathrm{~s}, 1 \mathrm{H}), 7.64-7.57(\mathrm{~m}, 2 \mathrm{H}), 7.48-7.41(\mathrm{~m}, 2 \mathrm{H}), 7.41-7.35(\mathrm{~m}, 1 \mathrm{H}), 7.34-7.27(\mathrm{~m}, 2 \mathrm{H})$, $7.24-7.17(\mathrm{~m}, 1 \mathrm{H}), 5.98(\mathrm{~d}, 1 \mathrm{H}, J=3.1 \mathrm{~Hz}), 5.86(\mathrm{~d}, 1 \mathrm{H}, J=3.1 \mathrm{~Hz}), 4.61(\mathrm{~s}, 2 \mathrm{H}), 4.15(\mathrm{~s}, 2 \mathrm{H}), 2.34(\mathrm{~s}, 3 \mathrm{H}), 1.84$ (tt, $1 \mathrm{H}, J=8.4,5.1 \mathrm{~Hz}), 0.89-0.80(\mathrm{~m}, 2 \mathrm{H}), 0.76-0.69(\mathrm{~m}, 2 \mathrm{H}) .{ }^{13} \mathrm{C} \mathrm{NMR}\left(\mathrm{CDCl}_{3}\right): 167.2,156.8,153.1,149.3$, 139.3, 138.0, 137.0, 133.7, 133.0, 129.9, 129.1, 128.9, 128.7, 128.4, 126.6, 126.6, 109.0, 107.6, 104.3, 39.5, 27.6, 20.2, 8.9, 6.6. HRMS $(\mathrm{m} / \mathrm{z})$ : $[\mathrm{M}+\mathrm{H}]^{+}$calcd for $\mathrm{C}_{29} \mathrm{H}_{26} \mathrm{~N}_{3} \mathrm{O}_{3}, 464.1974$; found, 464.1990.

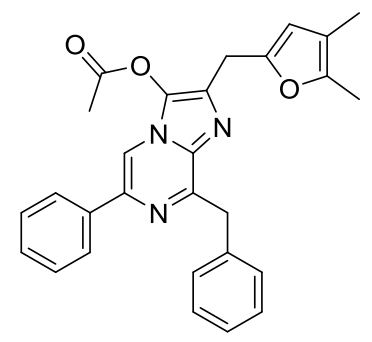

8-Benzyl-2-((4,5-dimethylfuran-2-yl)methyl)-6-phenylimidazo[1,2-a]pyrazin-3-yl acetate $25\{1,1,48\}$ (EC31092103-2): Obtained as a beige solid $(0.09 \mathrm{~g}, 45 \%)$ after a recrystallization from $n$-heptane. ${ }^{1} \mathrm{H}$ NMR $\left(\mathrm{CDCl}_{3}\right): 7.94-$ $7.86(\mathrm{~m}, 2 \mathrm{H}), 7.82(\mathrm{~s}, 1 \mathrm{H}), 7.65-7.58(\mathrm{~m}, 2 \mathrm{H}), 7.50-7.41(\mathrm{~m}, 2 \mathrm{H}), 7.41-7.35(\mathrm{~m}, 1 \mathrm{H}), 7.34-7.27(\mathrm{~m}, 2 \mathrm{H})$, $7.25-7.16(\mathrm{~m}, 1 \mathrm{H}), 5.90(\mathrm{~s}, 1 \mathrm{H}), 4.62(\mathrm{~s}, 2 \mathrm{H}), 4.13(\mathrm{~s}, 2 \mathrm{H}), 2.34(\mathrm{~s}, 3 \mathrm{H}), 2.17(\mathrm{~s}, 3 \mathrm{H}), 1.91(\mathrm{~s}, 3 \mathrm{H}) .{ }^{13} \mathrm{C}$ NMR $\left(\mathrm{CDCl}_{3}\right): 167.2,153.1,148.4,146.4,139.2,138.0,137.0,133.7,133.2,129.9,129.0,128.9,128.7,128.4,126.6$, 126.5, 114.7, 110.2, 109.0, 39.5, 27.4, 20.2, 11.4, 10.0. HRMS $(\mathrm{m} / \mathrm{z})$ : $[\mathrm{M}+\mathrm{H}]^{+}$calcd for $\mathrm{C}_{28} \mathrm{H}_{26} \mathrm{~N}_{3} \mathrm{O}_{3}, 452.1974$; found, 452.1990 .

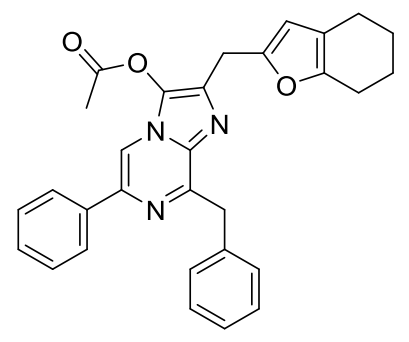


8-Benzyl-6-phenyl-2-((4,5,6,7-tetrahydrobenzofuran-2-yl)methyl)imidazo[1,2-a]pyrazin-3-yl acetate $\mathbf{2 5}\{1,1,49\}$ (YJ 33068-083-1): Obtained as a white solid (0.37 g, 47\%) after a recrystallization from $n$-heptane. ${ }^{1} \mathrm{H}$ NMR $\left(\mathrm{CDCl}_{3}\right): 7.91(\mathrm{~m}, 2 \mathrm{H}), 7.84(\mathrm{~s}, 1 \mathrm{H}), 7.82(\mathrm{~s}, 1 \mathrm{H}), 7.62(\mathrm{~m}, 2 \mathrm{H}), 7.47(\mathrm{~m}, 2 \mathrm{H}), 7.40(\mathrm{~m}, 1 \mathrm{H}), 7.32(\mathrm{~m}, 2 \mathrm{H}), 7.23(\mathrm{~m}$, $1 \mathrm{H}), 5.96(\mathrm{~s}, 1 \mathrm{H}), 4.64(\mathrm{~s}, 2 \mathrm{H}), 4.18(\mathrm{~s}, 2 \mathrm{H}), 2.58(\mathrm{~m}, 2 \mathrm{H}), 2.40(\mathrm{~m}, 2 \mathrm{H}), 2.37(\mathrm{~s}, 3 \mathrm{H}), 1.84(\mathrm{~m}, 2 \mathrm{H}), 1.73(\mathrm{~m}, 2 \mathrm{H})$. ${ }^{13} \mathrm{C} \mathrm{NMR}\left(\mathrm{CDCl}_{3}\right)$ : 167.0, 152.9, 149.8, 148.8, 139.2, 137.8, 136.7, 133.4, 132.9, 129.7, 128.9, 128.8, 128.6, 128.3, $126.5,126.4,117.5,108.9,107.8,39.3,31.9,27.3,23.2,23.1$ (two signals), 22.1. HRMS ( $\mathrm{m} / z)$ : $[\mathrm{M}+\mathrm{H}]^{+}$calcd for $\mathrm{C}_{30} \mathrm{H}_{28} \mathrm{~N}_{3} \mathrm{O}_{3}, 478.2131$; found, 478.2139.

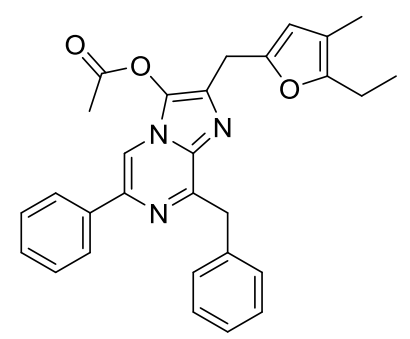

8-Benzyl-2-((5-ethyl-4-methylfuran-2-yl)methyl)-6-phenylimidazo[1,2-a]pyrazin-3-yl acetate $\mathbf{2 5}\{1,1,50\}$ (YJ 33069-011-1): Obtained as a white solid (0.41 g, 66\%) after a recrystallization from cyclohexane. ${ }^{1} \mathrm{H}$ NMR $\left(\mathrm{CDCl}_{3}\right): 7.92(\mathrm{~m}, 2 \mathrm{H}), 7.84(\mathrm{~s}, 1 \mathrm{H}), 7.63(\mathrm{~m}, 2 \mathrm{H}), 7.47(\mathrm{~m}, 2 \mathrm{H}), 7.40(\mathrm{~m}, 1 \mathrm{H}), 7.32(\mathrm{~m}, 2 \mathrm{H}), 7.24(\mathrm{~m}, 1 \mathrm{H}), 5.91(\mathrm{~s}$, $1 \mathrm{H}), 4.65(\mathrm{~s}, 2 \mathrm{H}), 4.16(\mathrm{~s}, 2 \mathrm{H}), 2.57(\mathrm{q}, 2 \mathrm{H}, J=7.3 \mathrm{~Hz}), 2.35(\mathrm{~s}, 3 \mathrm{H}), 1.94(\mathrm{~s}, 3 \mathrm{H}), 1.20(\mathrm{t}, 6 \mathrm{H}, J=7.3 \mathrm{~Hz}) .{ }^{13} \mathrm{C}$ NMR $\left(\mathrm{CDCl}_{3}\right)$ : 167.0, 152.9, 151.6, 148.1, 139.2, 137.8, 136.7, 133.3, 132.9, 129.7, 128.9, 128.8, 128.6, 128.3, 126.5, 126.4, 113.7, 110.2, 108.9, 39.3, 27.2, 20.0, 19.3, 13.1, 9.7. HRMS (m/z): $[\mathrm{M}+\mathrm{H}]^{+}$calcd for $\mathrm{C}_{29} \mathrm{H}_{28} \mathrm{~N}_{3} \mathrm{O}_{3}$, 466.2131; found, 466.2127.

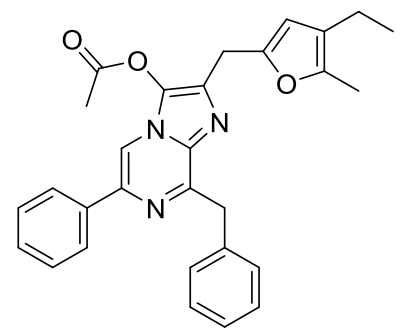

8-Benzyl-2-((4-ethyl-5-methylfuran-2-yl)methyl)-6-phenylimidazo[1,2-a]pyrazin-3-yl acetate $\quad \mathbf{2 5}\{1,1,51\} \quad$ (YJ 33068-087-1): Obtained as a white solid $(0.15 \mathrm{~g}, 52 \%)$ after a recrystallization from $n$-heptane. ${ }^{1} \mathrm{H} \mathrm{NMR}\left(\mathrm{CDCl}_{3}\right)$ : $7.91(\mathrm{~m}, 2 \mathrm{H}), 7.84(\mathrm{~s}, 1 \mathrm{H}), 7.82(\mathrm{~s}, 1 \mathrm{H}), 7.62(\mathrm{~m}, 2 \mathrm{H}), 7.47(\mathrm{~m}, 2 \mathrm{H}), 7.40(\mathrm{~m}, 1 \mathrm{H}), 7.32(\mathrm{~m}, 2 \mathrm{H}), 7.23(\mathrm{~m}, 1 \mathrm{H})$, $5.97(\mathrm{~s}, 1 \mathrm{H}), 4.64(\mathrm{~s}, 2 \mathrm{H}), 4.16(\mathrm{~s}, 2 \mathrm{H}), 2.36(\mathrm{~s}, 3 \mathrm{H}), 2.33(\mathrm{q}, 2 \mathrm{H}, J=7.4 \mathrm{~Hz}), 2.20(\mathrm{~s}, 3 \mathrm{H}), 1.13(\mathrm{t}, 3 \mathrm{H}, J=7.4 \mathrm{~Hz})$. ${ }^{13} \mathrm{C} \mathrm{NMR}\left(\mathrm{CDCl}_{3}\right)$ : 167.0, 152.9, 148.3, 145.5, 139.2, 137.8, 136.8, 133.4, 132.9, 129.7, 128.9, 128.8, 128.6, 128.3, 126.5, 126.4, 121.3, 108.9, 108.4, 39.3, 27.3, 20.1, 18.1, 14.9, 11.3. HRMS (m/z): $[\mathrm{M}+\mathrm{H}]^{+}$calcd for $\mathrm{C}_{29} \mathrm{H}_{28} \mathrm{~N}_{3} \mathrm{O}_{3}$, 466.2131; found, 466.2131 .

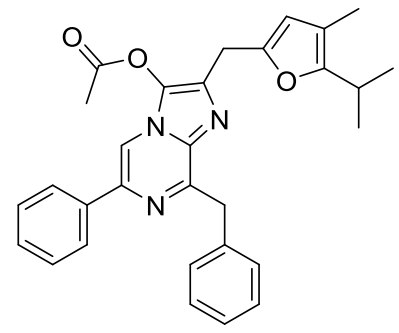

8-Benzyl-2-((5-isopropyl-4-methylfuran-2-yl)methyl)-6-phenylimidazo[1,2-a]pyrazin-3-yl acetate 25 $\{1,1,52\}$ (YJ 33068-097-1): Obtained as a white solid $(0.29 \mathrm{~g}, 62 \%)$ after a recrystallization from $n$-heptane. ${ }^{1} \mathrm{H} \mathrm{NMR}\left(\mathrm{CDCl}_{3}\right)$ : $7.92(\mathrm{~m}, 2 \mathrm{H}), 7.83(\mathrm{~s}, 1 \mathrm{H}), 7.82(\mathrm{~s}, 1 \mathrm{H}), 7.62(\mathrm{~m}, 2 \mathrm{H}), 7.47(\mathrm{~m}, 2 \mathrm{H}), 7.40(\mathrm{~m}, 1 \mathrm{H}), 7.32(\mathrm{~m}, 2 \mathrm{H}), 7.23(\mathrm{~m}, 1 \mathrm{H})$, $5.87(\mathrm{~s}, 1 \mathrm{H}), 4.64(\mathrm{~s}, 2 \mathrm{H}), 4.15(\mathrm{~s}, 2 \mathrm{H}), 2.97(\mathrm{sept}, 1 \mathrm{H}, J=7.0 \mathrm{~Hz}), 2.34(\mathrm{~s}, 3 \mathrm{H}), 1.95(\mathrm{~s}, 3 \mathrm{H}), 1.24(\mathrm{~d}, 6 \mathrm{H}, J=7.0$ $\mathrm{Hz}) .{ }^{13} \mathrm{C} \mathrm{NMR}\left(\mathrm{CDCl}_{3}\right)$ : 167.0, 154.6, 152.9, 147.8, 139.2, 137.8, 136.8, 133.4, 133.0, 129.7, 128.9, 128.8, 128.6, $128.3,126.5,126.4,112.5,110.2,108.9,39.3,31.9,27.2,21.4,14.1,9.8$. . HRMS (m/z): $[\mathrm{M}+\mathrm{H}]^{+}$calcd for $\mathrm{C}_{30} \mathrm{H}_{30} \mathrm{~N}_{3} \mathrm{O}_{3}, 480.2287$; found, 480.2280 . 


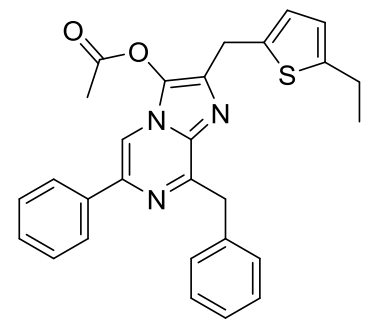

8-Benzyl-2-((5-ethylthiophen-2-yl)methyl)-6-phenylimidazo[1,2-a]pyrazin-3-yl acetate 25 \{1,1,53\} (YJ30367-1731): Obtained as a white solid $(0.25 \mathrm{~g}, 56 \%)$ after a recrystallization from $n$-heptane. ${ }^{1} \mathrm{H}$ NMR $\left(\mathrm{CDCl}_{3}\right): 7.93(\mathrm{~m}$, 2H), $7.83(\mathrm{~s}, 1 \mathrm{H}), 7.65(\mathrm{~m}, 2 \mathrm{H}), 7.47(\mathrm{~m}, 2 \mathrm{H}), 7.40(\mathrm{~m}, 1 \mathrm{H}), 7.32(\mathrm{~m}, 2 \mathrm{H}), 7.24(\mathrm{~m}, 1 \mathrm{H}), 6.72(\mathrm{~m}, 1 \mathrm{H}), 6.63(\mathrm{~m}$, $1 \mathrm{H}), 4.64(\mathrm{~s}, 2 \mathrm{H}), 4.32(\mathrm{~s}, 2 \mathrm{H}), 2.82(\mathrm{q}, 2 \mathrm{H}, J=7.6 \mathrm{~Hz}), 2.33(\mathrm{~s}, 3 \mathrm{H}), 1.31(\mathrm{t}, 3 \mathrm{H}, J=7.6 \mathrm{~Hz}),{ }^{13} \mathrm{C} \mathrm{NMR}\left(\mathrm{CDCl}_{3}\right)$ : $167.0,153.1,146.5,139.1,137.9,137.5,136.8,134.6,133.5,129.8,128.8,128.6,128.5,128.3,126.5,126.4$, 125.4, 122.8, 108.9, 39.4, 28.7, 23.5, 20.0, 16.0. HRMS $(\mathrm{m} / \mathrm{z}):[\mathrm{M}+\mathrm{H}]^{+}$calcd for $\mathrm{C}_{28} \mathrm{H}_{26} \mathrm{~N}_{3} \mathrm{O}_{2} \mathrm{~S}, 468.1746$; found, 468.1757.

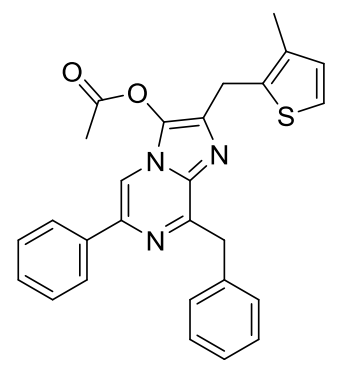

8-Benzyl-2-((3-methylthiophen-2-yl)methyl)-6-phenylimidazo[1,2-a]pyrazin-3-yl acetate 25 $\{1,1,54\}$ (YJ30367175-1): Obtained as a wax which solidified $(0.37 \mathrm{~g}, 95 \%) .{ }^{1} \mathrm{H} \mathrm{NMR}\left(\mathrm{CDCl}_{3}\right): 7.92(\mathrm{~m}, 2 \mathrm{H}), 7.79(\mathrm{~s}, 1 \mathrm{H}), 7.64(\mathrm{~m}$, $2 \mathrm{H}), 7.47(\mathrm{~m}, 2 \mathrm{H}), 7.39(\mathrm{~m}, 1 \mathrm{H}), 7.32(\mathrm{~m}, 2 \mathrm{H}), 7.23(\mathrm{~m}, 1 \mathrm{H}), 7.10(\mathrm{~d}, 1 \mathrm{H}, J=5.2 \mathrm{~Hz}), 6.83(\mathrm{~d}, 1 \mathrm{H}, J=5.2 \mathrm{~Hz})$, $4.64(\mathrm{~s}, 2 \mathrm{H}), 4.32(\mathrm{~s}, 2 \mathrm{H}), 2.27(\mathrm{~s}, 3 \mathrm{H}), 2.22(\mathrm{~s}, 3 \mathrm{H}) .{ }^{13} \mathrm{C} \mathrm{NMR}\left(\mathrm{CDCl}_{3}\right)$ : 167.0, 153.0, 139.1, 137.8, 136.8, 134.5, 134.3, 133.4, 130.0, 130.0, 129.7, 128.8, 128.5, 128.3, 126.5, 126.4, 122.3, 108.9, 39.5, 26.8, 19.9, 13.8 (one signal missing). HRMS (m/z): [M+H] $]^{+}$calcd for $\mathrm{C}_{27} \mathrm{H}_{24} \mathrm{~N}_{3} \mathrm{O}_{2} \mathrm{~S}, 454.1589$; found, 454.1591 .

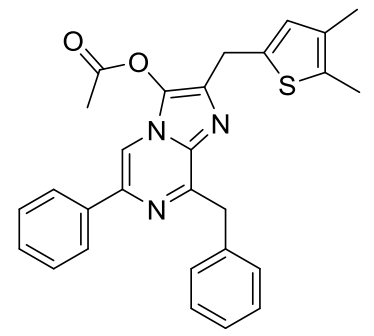

8-Benzyl-2-((4,5-dimethylthiophen-2-yl)methyl)-6-phenylimidazo[1,2-a]pyrazin-3-yl $\quad$ acetate $\quad 25\{1,1,55\}$ (YJ310697-113-1): Obtained as a white solid (0.14 g, 59\%) after a recrystallization from $n$-heptane. ${ }^{1} \mathrm{H}$ NMR $\left(\mathrm{CDCl}_{3}\right): 7.91(\mathrm{~m}, 2 \mathrm{H}), 7.82(\mathrm{~s}, 1 \mathrm{H}), 7.64(\mathrm{~m}, 2 \mathrm{H}), 7.47(\mathrm{~m}, 2 \mathrm{H}), 7.40(\mathrm{~m}, 1 \mathrm{H}), 7.33(\mathrm{~m}, 2 \mathrm{H}), 7.24(\mathrm{~m}, 1 \mathrm{H}), 6.59(\mathrm{~s}$, $1 \mathrm{H}), 4.64(\mathrm{~s}, 2 \mathrm{H}), 4.26(\mathrm{~s}, 2 \mathrm{H}), 2.35(\mathrm{~s}, 3 \mathrm{H}), 2.31(\mathrm{~s}, 3 \mathrm{H}), 2.09(\mathrm{~s}, 3 \mathrm{H}) .{ }^{13} \mathrm{C} \mathrm{NMR}\left(\mathrm{CDCl}_{3}\right): 167.0,153.1,139.1$, $137.9,136.8,135.2$, 134.6, 133.5, 132.5, 131.4, 129.8, 128.8, 128.7, 128.5, 128.3, 126.5, 126.4, 108.9, 39.4, 31.9, 28.4, 20.0, 13.5, 12.9. HRMS (m/z): $[\mathrm{M}+\mathrm{H}]^{+}$calcd for $\mathrm{C}_{28} \mathrm{H}_{26} \mathrm{~N}_{3} \mathrm{O}_{2} \mathrm{~S}, 468.1746$; found, 468.1758 .

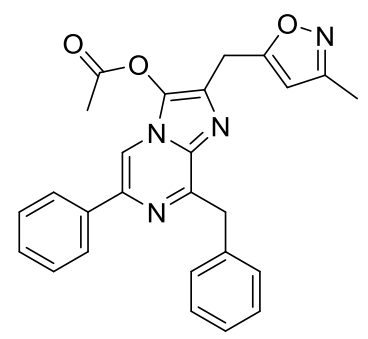


8-Benzyl-2-((3-methylisoxazol-5-yl)methyl)-6-phenylimidazo[1,2-a]pyrazin-3-yl acetate $\mathbf{2 5}\{1,1,56\}$ (EC32712033-1): Obtained as a white solid $(0.10 \mathrm{~g}, 59 \%)$ after a recrystallization from cyclohexane. ${ }^{1} \mathrm{H} \mathrm{NMR}\left(\mathrm{CDCl}_{3}\right): 7.93$ $(\mathrm{m}, 2 \mathrm{H}), 7.86(\mathrm{~s}, 1 \mathrm{H}), 7.61(\mathrm{~m}, 2 \mathrm{H}), 7.48(\mathrm{~m}, 2 \mathrm{H}), 7.42(\mathrm{~m}, 1 \mathrm{H}), 7.32(\mathrm{~m}, 2 \mathrm{H}), 7.25(\mathrm{~m}, 1 \mathrm{H}), 5.94(\mathrm{~s}, 1 \mathrm{H}), 4.63(\mathrm{~s}$, 2H), $4.27(\mathrm{~d}, 2 \mathrm{H}, J=0.9 \mathrm{~Hz}), 2.46(\mathrm{~s}, 3 \mathrm{H}), 2.29(\mathrm{~s}, 3 \mathrm{H}) .{ }^{13} \mathrm{C} \mathrm{NMR}\left(\mathrm{CDCl}_{3}\right): 168.9,167.0,160.0,153.2,139.4$, 137.7, 136.6, 133.7, 130.6, 129.7, 129.0, 128.8, 128.7, 128.3, 126.5, 126.4, 109.0, 103.1, 39.5, 25.7, 20.1, 11.4. $\operatorname{HRMS}(\mathrm{m} / \mathrm{z}):[\mathrm{M}+\mathrm{H}]^{+}$calcd for $\mathrm{C}_{26} \mathrm{H}_{23} \mathrm{~N}_{4} \mathrm{O}_{3}$ : 439.1770; found, 439.1778.

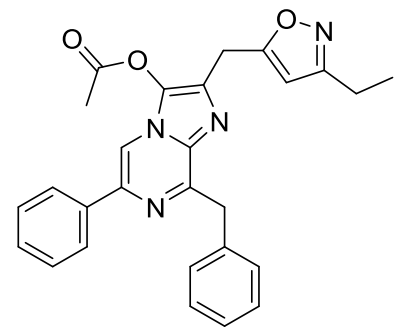

8-Benzyl-2-((3-ethylisoxazol-5-yl)methyl)-6-phenylimidazo[1,2-a]pyrazin-3-yl acetate 25 1,1,57\} (EC32712-0351): Obtained as a white solid $(0.12 \mathrm{~g}, 41 \%)$ after recrystallization in $n$-heptane. ${ }^{1} \mathrm{H}$ NMR $\left(\mathrm{CDCl}_{3}\right): 7.93(\mathrm{~m}, 2 \mathrm{H})$, $7.86(\mathrm{~s}, 1 \mathrm{H}), 7.61(\mathrm{~m}, 2 \mathrm{H}), 7.48(\mathrm{~m}, 2 \mathrm{H}), 7.42(\mathrm{~m}, 1 \mathrm{H}), 7.32(\mathrm{~m}, 2 \mathrm{H}), 7.25(\mathrm{~m}, 1 \mathrm{H}), 5.97(\mathrm{~s}, 1 \mathrm{H}), 4.63(\mathrm{~s}, 2 \mathrm{H}), 4.28$ $(\mathrm{d}, 2 \mathrm{H}, J=0.9 \mathrm{~Hz}), 2.68(\mathrm{q}, 2 \mathrm{H}, J=7.6 \mathrm{~Hz}), 2.45(\mathrm{~s}, 3 \mathrm{H}), 1.27(\mathrm{t}, 3 \mathrm{H}, J=7.6 \mathrm{~Hz}) .{ }^{13} \mathrm{C} \mathrm{NMR}\left(\mathrm{CDCl}_{3}\right): 168.8$, $167.0,165.4,153.2,139.4,137.7,136.6,133.7,130.6,129.7,129.0,128.8,128.7,128.3,126.5,126.4,109.0$, 101.8, 39.5, 25.7, 20.1, 19.6, 12.6. HRMS (m/z): $[\mathrm{M}+\mathrm{H}]^{+}$calcd for $\mathrm{C}_{27} \mathrm{H}_{25} \mathrm{~N}_{4} \mathrm{O}_{3}$ : 453.1927; found, 453.1938 .

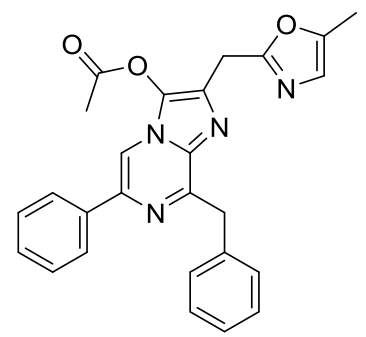

8-Benzyl-2-((5-methyloxazol-2-yl)methyl)-6-phenylimidazo[1,2-a]pyrazin-3-yl acetate 25 \{1,1,58\} (YJ 31776-0962): Obtained as a white solid (0.01 g, 1.6\% from 3-benzyl-2-chloro-5-phenylpyrazine) after a chromatography over silica gel (cyclohexane - ethyl acetate 1/1) and a recrystallization from cyclohexane. ${ }^{1} \mathrm{H}$ NMR $\left(\mathrm{CDCl}_{3}\right): 7.92(\mathrm{~m}$, 2H), $7.86(\mathrm{~s}, 1 \mathrm{H}), 7.62(\mathrm{~m}, 2 \mathrm{H}), 7.47(\mathrm{~m}, 2 \mathrm{H}), 7.40(\mathrm{~m}, 1 \mathrm{H}), 7.32(\mathrm{~m}, 2 \mathrm{H}), 7.23(\mathrm{~m}, 1 \mathrm{H}), 6.67(\mathrm{~m}, 1 \mathrm{H}), 4.64(\mathrm{~s}$, $2 \mathrm{H}), 4.33(\mathrm{~s}, 2 \mathrm{H}), 2.43(\mathrm{~s}, 3 \mathrm{H}), 2.30(\mathrm{~s}, 3 \mathrm{H}) .{ }^{13} \mathrm{C} \mathrm{NMR}\left(\mathrm{CDCl}_{3}\right): 166.9,159.4,153.2,149.2,139.3,137.7,136.6$, $133.5,130.3,129.7,129.2,128.8,128.7,128.3,126.5,126.4,122.8,108.9,39.3,27.7,20.2,10.8$. HRMS $(\mathrm{m} / \mathrm{z})$ : $[\mathrm{M}+\mathrm{H}]^{+}$calcd for $\mathrm{C}_{26} \mathrm{H}_{23} \mathrm{~N}_{4} \mathrm{O}_{3}$ : 439.1770; found, 439.1778 .

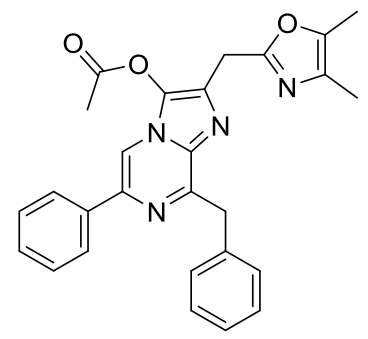

8-Benzyl-2-((4,5-dimethyloxazol-2-yl)methyl)-6-phenylimidazo[1,2-a]pyrazin-3-yl acetate 25\{1,1,59\} (YJ31067047-1): Obtained as a white crystals $(0.1 \mathrm{~g}, 34 \%)$ after a recrystallization from $n$-heptane. ${ }^{1} \mathrm{H}$ NMR $\left(\mathrm{CDCl}_{3}\right): 7.91$ $(\mathrm{m}, 2 \mathrm{H}), 7.85(\mathrm{~s}, 1 \mathrm{H}), 7.47(\mathrm{~m}, 2 \mathrm{H}), 7.39(\mathrm{~m}, 1 \mathrm{H}), 7.31(\mathrm{~m}, 2 \mathrm{H}), 7.24(\mathrm{~m}, 1 \mathrm{H}), 4.62(\mathrm{~s}, 2 \mathrm{H}), 4.28(\mathrm{~s}, 2 \mathrm{H}), 2.41(\mathrm{~s}$, $3 \mathrm{H}), 2.21(\mathrm{~s}, 3 \mathrm{H}), 2.08(\mathrm{~s}, 3 \mathrm{H}) .{ }^{13} \mathrm{C} \mathrm{NMR}\left(\mathrm{CDCl}_{3}\right): 166.9,157.9,153.2,143.5,139.2,137.8,136.7,133.6,130.5$, $130.4,129.7,129.2,128.8,128.6,128.3,126.5,126.4,108.9,39.4,27.7,20.2,11.1,9.9 . \mathrm{HRMS}(\mathrm{m} / z):[\mathrm{M}+\mathrm{H}]^{+}$ calcd for $\mathrm{C}_{27} \mathrm{H}_{25} \mathrm{~N}_{4} \mathrm{O}_{3}, 453.1927$; found, 453.1915 . 


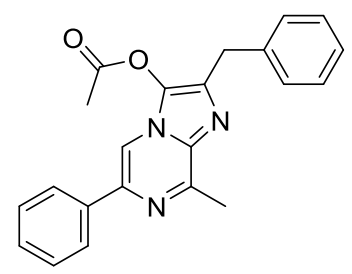

2-Benzyl-8-methyl-6-phenylimidazo[1,2-a]pyrazin-3-yl acetate 25\{1,60,1 $\}$ (VHE30448-007-1): Obtained as a yellow solid $(0.59 \mathrm{~g}, 88 \%)$ after a recrystallization from $n$-heptane. ${ }^{1} \mathrm{H} \mathrm{NMR}\left(\mathrm{CDCl}_{3} 7.91(\mathrm{~m}, 2 \mathrm{H}), 7.80(\mathrm{~m}, 1 \mathrm{H})\right.$, $7.48(\mathrm{~m}, 2 \mathrm{H}), 7.41(\mathrm{~m}, 1 \mathrm{H}), 7.35-7.23(\mathrm{~m}, 5 \mathrm{H}), 4.21(\mathrm{~s}, 2 \mathrm{H}), 2.96(\mathrm{~m}, 3 \mathrm{H}), 2.18(\mathrm{~s}, 3 \mathrm{H}) .{ }^{13} \mathrm{C} \mathrm{NMR}\left(\mathrm{CDCl}_{3}\right)$ : 167.1, 151.6, 139.2, 137.9, 16.9, 134.9, 137.0, 129.0, 128.8, 128.7, 128.6, 128.5, 126.5, 108.9, 34.3, 20.3, 19.8. $\operatorname{HRMS}(\mathrm{m} / \mathrm{z}):[\mathrm{M}+\mathrm{H}]^{+}$calcd for $\mathrm{C}_{22} \mathrm{H}_{20} \mathrm{~N}_{3} \mathrm{O}_{2}, 358.1556$; found, 358.1544 .

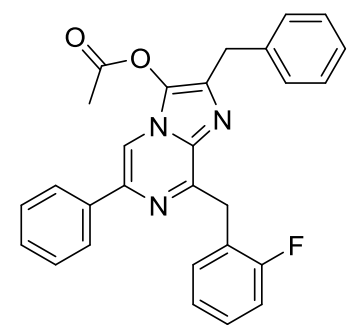

2-Benzyl-8-(2-fluorobenzyl)-6-phenylimidazo[1,2-a]pyrazin-3-yl acetate 25\{1,2,1\} (GG30532-121-1): Obtained as a white solid $(0.12 \mathrm{~g}, 61 \%)$ after a recrystallization in n-heptane. ${ }^{1} \mathrm{H}$ NMR $\left(\mathrm{CDCl}_{3}\right): 7.86-7.83(\mathrm{~m}, 3 \mathrm{H}), 7.52-7.48$ $(\mathrm{dt}, 1 \mathrm{H}, J=7.4,1.9 \mathrm{~Hz}), 7.47-7.23(\mathrm{~m}, 8 \mathrm{H}), 7.14-7.08(\mathrm{~m}, 2 \mathrm{H}), 4.72(\mathrm{~s}, 2 \mathrm{H}), 4.23(\mathrm{~s}, 2 \mathrm{H}), 2.19(\mathrm{~s}, 3 \mathrm{H}) .{ }^{13} \mathrm{C} \mathrm{NMR}$ $\left(\mathrm{CDCl}_{3}\right): 167.1,161.4(247.0 \mathrm{~Hz}), 151.6,138.9,138.0,136.7,135.2,133.7,131.9(4.3 \mathrm{~Hz}), 129.1,128.9,128.7$, 128.6, 128.5, $128.3(8.1 \mathrm{~Hz}), 126.5,126.3,124.7(15.9 \mathrm{~Hz}), 123.7(3.5 \mathrm{~Hz}), 115.2(22.4 \mathrm{~Hz}), 108.8,34.3,32.2(2.5$ $\mathrm{Hz})$, 19.9. HRMS $(\mathrm{m} / \mathrm{z})$ : $[\mathrm{M}+\mathrm{H}]^{+}$calcd for $\mathrm{C}_{28} \mathrm{H}_{23} \mathrm{FN}_{3} \mathrm{O}_{2}, 452.1774$, found, 452.1774 .

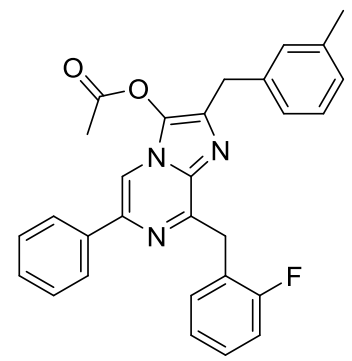

8-(2-Fluorobenzyl)-2-(3-methylbenzyl)-6-phenylimidazo[1,2-a]pyrazin-3-yl acetate 25 1,2,12\} (YJ31070-047-1): Obtained as a white solid after a recrystallization in $n$-heptane $(0.18 \mathrm{~g}, 58 \%) .{ }^{1} \mathrm{H}$ NMR $\left(\mathrm{CDCl}_{3}\right): 7.84(\mathrm{~m}, 2 \mathrm{H}), 7.83$ (s, 1H), $7.50(\mathrm{~m}, 1 \mathrm{H}), 7.42(\mathrm{~m}, 2 \mathrm{H}), 7.36(\mathrm{~m}, 1 \mathrm{H}), 7.23(\mathrm{~m}, 2 \mathrm{H}), 7.13-7.06(\mathrm{~m}, 5 \mathrm{H}), 4.72(\mathrm{~s}, 2 \mathrm{H}), 4.19(\mathrm{~s}, 2 \mathrm{H}), 2.35$ $(\mathrm{s}, 3 \mathrm{H}), 2.19(\mathrm{~s}, 3 \mathrm{H}) .{ }^{13} \mathrm{C} \mathrm{NMR}\left(\mathrm{CDCl}_{3}\right): 167.1,161.4(245 \mathrm{~Hz}), 151.6,138.9,138.1,137.8,136.7,135.3,133.6$, 131.8 (4 Hz), 129.8, 128.8, 128.7, 128.5, 128.4, 128.2 (8 Hz), 127.2, 126.2, 126.1, 124.7 (16 Hz), 123.7 (4 Hz), $115.1(22 \mathrm{~Hz}), 108.8,34.3,32.2(2 \mathrm{~Hz}), 21.3,19.8$. HRMS $(m / z):[\mathrm{M}+\mathrm{H}]^{+}$calcd for $\mathrm{C}_{29} \mathrm{H}_{25} \mathrm{FN}_{3} \mathrm{O}_{2}, 466.1931$, found, 466.1938 .

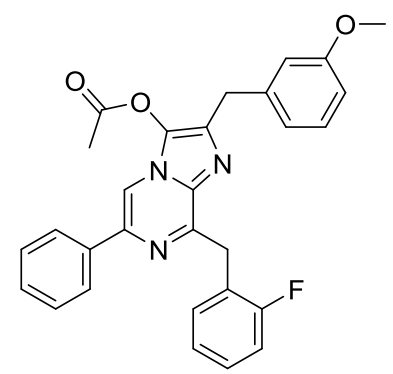

8-(2-Fluorobenzyl)-2-(3-methoxybenzyl)-6-phenylimidazo[1,2-a]pyrazin-3-yl acetate 25\{1,2,15\} (YJ31070-0491): Obtained as a white solid after a recrystallization in $n$-heptane $(0.26 \mathrm{~g}, 87 \%) .{ }^{1} \mathrm{H} \mathrm{NMR}\left(\mathrm{CDCl}_{3}\right): 7.85(\mathrm{~m}, 2 \mathrm{H})$, 
$7.83(\mathrm{~s}, 1 \mathrm{H}), 7.48(\mathrm{~m}, 1 \mathrm{H}), 7.42(\mathrm{~m}, 2 \mathrm{H}), 7.36(\mathrm{~m}, 1 \mathrm{H}), 7.25(\mathrm{~m}, 2 \mathrm{H}), 7.10(\mathrm{~m}, 2 \mathrm{H}), 6.90(\mathrm{~m}, 2 \mathrm{H}), 6.81(\mathrm{~m}, 1 \mathrm{H})$, $4.71(\mathrm{~s}, 2 \mathrm{H}), 4.19(\mathrm{~s}, 2 \mathrm{H}), 3.80(\mathrm{~s}, 3 \mathrm{H}), 2.23(\mathrm{~s}, 3 \mathrm{H}) .{ }^{13} \mathrm{C} \mathrm{NMR}\left(\mathrm{CDCl}_{3}\right): 167.1,161.4(245 \mathrm{~Hz}), 159.8,151.6$, 139.5, 138.9, 136.6, 135.1, 133.6, 131.8 (4 Hz), 129.4, 128.9, 128.7, 128.5, $128.3(8 \mathrm{~Hz}), 126.3,124.7$ (16 Hz), 123.7 (4 Hz), 121.4, $115.1(22 \mathrm{~Hz}), 114.6,112.2,108.8,55.2,34.2,32.2(2 \mathrm{~Hz}), 19.9$. HRMS $(\mathrm{m} / z):[\mathrm{M}+\mathrm{H}]^{+} \mathrm{calcd}$ for $\mathrm{C}_{29} \mathrm{H}_{25} \mathrm{FN}_{3} \mathrm{O}_{3}, 482.1880$, found, 482.1873 .

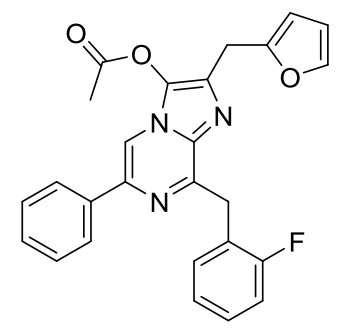

8-(2-Fluorobenzyl)-2-(furan-2-ylmethyl)-6-phenylimidazo[1,2-a]pyrazin-3-yl acetate 25\{1,2,37\} (YJ31070-0511): Obtained as a white solid after a recrystallization in $n$-heptane $(0.19 \mathrm{~g}, 73 \%) .{ }^{1} \mathrm{H} \mathrm{NMR}\left(\mathrm{CDCl}_{3}\right): 7.87(\mathrm{~s}, 1 \mathrm{H})$, $7.85(\mathrm{~m}, 2 \mathrm{H}), 7.50-7.34(\mathrm{~m}, 5 \mathrm{H}), 7.24(\mathrm{~m}, 1 \mathrm{H}), 7.10(\mathrm{~m}, 2 \mathrm{H}), 6.35(\mathrm{dd}, 1 \mathrm{H}, J=1.9,3.2 \mathrm{~Hz}), 6.17(\mathrm{dd}, 1 \mathrm{H}, J=0.7$, $3.2 \mathrm{~Hz}), 4.70(\mathrm{~s}, 2 \mathrm{H}), 4.24(\mathrm{~s}, 2 \mathrm{H}), 2.37(\mathrm{~s}, 3 \mathrm{H}) .{ }^{13} \mathrm{C} \mathrm{NMR}\left(\mathrm{CDCl}_{3}\right): 167.1,161.4(245 \mathrm{~Hz}), 151.7,151.5,141.7$, 139.0, 136.6, 133.5, 131.8 (4 Hz), 128.9, 128.7, 128.6, 128.3 (8 Hz), 126.3, $124.6(16 \mathrm{~Hz}), 123.7$ (4 Hz), $115.1(22$ $\mathrm{Hz}), 110.5,108.8,106.8,32.2(2 \mathrm{~Hz}), 27.2,20.1$ (one signal missing). HRMS $(\mathrm{m} / \mathrm{z})$ : $[\mathrm{M}+\mathrm{H}]^{+}$calcd for $\mathrm{C}_{26} \mathrm{H}_{21} \mathrm{FN}_{3} \mathrm{O}_{3}, 442.1567$, found, 442.1567.

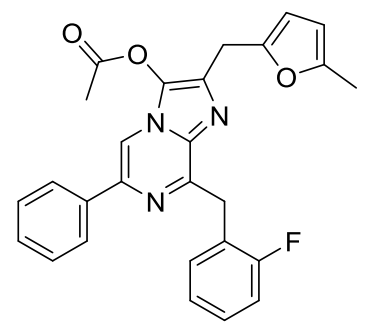

8-(2-Fluorobenzyl)-2-((5-methylfuran-2-yl)methyl)-6-phenylimidazo[1,2-a]pyrazin-3-yl acetate $\quad \mathbf{2 5}\{1,2,42\}$ (YJ31070-053-1): Obtained as a white solid after a recrystallization in $n$-heptane $(0.08 \mathrm{~g}, 38 \%) .{ }^{1} \mathrm{H} \mathrm{NMR}\left(\mathrm{CDCl}_{3}\right)$ : $7.87(\mathrm{~s}, 1 \mathrm{H}), 7.85(\mathrm{~m}, 2 \mathrm{H}), 7.50-7.34(\mathrm{~m}, 4 \mathrm{H}), 7.24(\mathrm{~m}, 1 \mathrm{H}), 7.10(\mathrm{~m}, 2 \mathrm{H}), 6.04(\mathrm{~d}, 1 \mathrm{H}, J=3.0 \mathrm{~Hz}), 5.91(\mathrm{~m}, 1 \mathrm{H})$, $4.70(\mathrm{~s}, 2 \mathrm{H}), 4.19$ (s, 2H), 2.37 (s, 3H), 2.28 (s, 3H). ${ }^{13} \mathrm{C} \mathrm{NMR}\left(\mathrm{CDCl}_{3}\right): 167.1,161.4(245 \mathrm{~Hz}), 151.7,151.2$, 149.5, 138.9, 136.6, 133.5, 132.8, 131.9 (4 Hz), 128.9, 128.7, 128.5, 128.2 (8 Hz), 126.2, 124.6 (16 Hz), 123.7 (4 $\mathrm{Hz}), 115.2(22 \mathrm{~Hz}), 108.3,107.5,106.2,32.2(2 \mathrm{~Hz}), 27.3,20.1,13.5$. HRMS $(\mathrm{m} / z):[\mathrm{M}+\mathrm{H}]^{+}$calcd for $\mathrm{C}_{27} \mathrm{H}_{23} \mathrm{FN}_{3} \mathrm{O}_{3}, 456.1723$, found, 456.1719 .

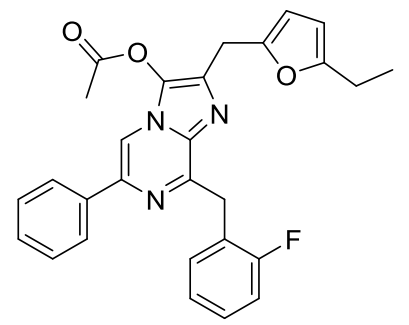

2-((5-Ethylfuran-2-yl)methyl)-8-(2-fluorobenzyl)-6-phenylimidazo[1,2-a]pyrazin-3-yl $\quad$ acetate $\quad \mathbf{2 5}\{1,2,44\}$ (YJ31068-027-1): Obtained as a white solid after a recrystallization in $n$-heptane $(0.05 \mathrm{~g}, 28 \%) .{ }^{1} \mathrm{H} \mathrm{NMR}\left(\mathrm{CDCl}_{3}\right)$ : $7.87(\mathrm{~s}, 1 \mathrm{H}), 7.85(\mathrm{~m}, 2 \mathrm{H}), 7.50-7.34(\mathrm{~m}, 4 \mathrm{H}), 7.24(\mathrm{~m}, 1 \mathrm{H}), 7.10(\mathrm{~m}, 2 \mathrm{H}), 6.04(\mathrm{~d}, 1 \mathrm{H}, J=3.1 \mathrm{~Hz}), 5.92(\mathrm{~m}, 1 \mathrm{H})$, $4.70(\mathrm{~s}, 2 \mathrm{H}), 4.20(\mathrm{~s}, 2 \mathrm{H}), 2.64(\mathrm{q}, 2 \mathrm{H}, J=7.2 \mathrm{~Hz}), 2.36(\mathrm{~s}, 3 \mathrm{H}), 1.24(\mathrm{t}, 3 \mathrm{H}, J=7.2 \mathrm{~Hz}) .{ }^{13} \mathrm{C} \mathrm{NMR}\left(\mathrm{CDCl}_{3}\right): 167.1$, $161.4(245 \mathrm{~Hz}), 151.7,151.7,149.3,138.9,136.6,133.5,132.8,131.9$ (4 Hz), 128.9, 128.7, 128.5, 128.2 (8 Hz), 126.3, $124.6(16 \mathrm{~Hz}), 123.7$ (4 Hz), $115.2(22 \mathrm{~Hz}), 108.8,107.5,104.6,32.2(2 \mathrm{~Hz}), 27.3,21.3,20.1$, 12.9. HRMS $(\mathrm{m} / \mathrm{z}):[\mathrm{M}+\mathrm{H}]^{+}$calcd for $\mathrm{C}_{28} \mathrm{H}_{25} \mathrm{FN}_{3} \mathrm{O}_{3}, 470.1880$, found, 470.1881 . 


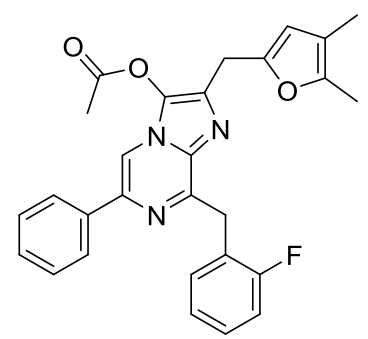

2-((4,5-Dimethylfuran-2-yl)methyl)-8-(2-fluorobenzyl)-6-phenylimidazo[1,2-a]pyrazin-3-yl acetate $\mathbf{2 5}\{1,2,48\}$ (EC32712-129-2): Obtained as a white solid after a recrystallization in $n$-heptane $(0.19 \mathrm{~g}, 64 \%) .{ }^{1} \mathrm{H} \mathrm{NMR}\left(\mathrm{CDCl}_{3}\right)$ : $7.87(\mathrm{~s}, 1 \mathrm{H}), 7.85(\mathrm{~m}, 2 \mathrm{H}), 7.43(\mathrm{~m}, 4 \mathrm{H}), 7.25(\mathrm{~m}, 1 \mathrm{H}), 7.10(\mathrm{~m}, 2 \mathrm{H}), 5.93(\mathrm{~s}, 1 \mathrm{H}), 4.70(\mathrm{~s}, 2 \mathrm{H}), 4.15(\mathrm{~s}, 2 \mathrm{H}), 2.38$ $(\mathrm{s}, 3 \mathrm{H}), 2.19(\mathrm{~s}, 3 \mathrm{H}), 1.93(\mathrm{~s}, 3 \mathrm{H}) .{ }^{13} \mathrm{C} \mathrm{NMR}\left(\mathrm{CDCl}_{3}\right): 167.0,161.4(246 \mathrm{~Hz}), 151.7,148.2,146.3,138.9,136.6$, 133.5, 132.9, 131.9 (4 Hz), 128.9, 128.7, 128.5, 128.2 (8 Hz), 126.2, $124.7(16 \mathrm{~Hz}), 123.7(\mathrm{~Hz}), 115.2(22 \mathrm{~Hz})$, 114.6, 110.1, 108.8, $32.2(3 \mathrm{~Hz}), 27.2,20.0,11.2$, 9.8. HRMS $(\mathrm{m} / \mathrm{z}):[\mathrm{M}+\mathrm{H}]^{+}$calcd for $\mathrm{C}_{28} \mathrm{H}_{25} \mathrm{FN}_{3} \mathrm{O}_{3}: 470.1880$; found, 470.1870 .

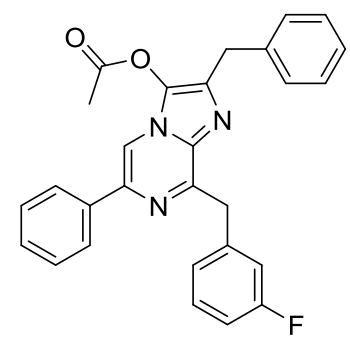

2-Benzyl-8-(3-fluorobenzyl)-6-phenylimidazo[1,2-a]pyrazin-3-yl acetate 25 $\{1,3,1\}$ (YJ31067-161-1): Obtained as a solid $(0.19 \mathrm{~g}, 50 \%)$ after a recrystallization from $n$-heptane. ${ }^{1} \mathrm{H} \mathrm{NMR}\left(\mathrm{CDCl}_{3}\right): 7.90(\mathrm{~m}, 2 \mathrm{H}), 7.82(\mathrm{~s}, 1 \mathrm{H}), 7.47$ $(\mathrm{m}, 2 \mathrm{H}), 7.42-7.25(\mathrm{~m}, 9 \mathrm{H}), 6.95(\mathrm{~m}, 1 \mathrm{H}), 4.63(\mathrm{~s}, 2 \mathrm{H}), 4.22(\mathrm{~s}, 2 \mathrm{H}), 2.20(\mathrm{~s}, 3 \mathrm{H}) .{ }^{13} \mathrm{C} \mathrm{NMR}\left(\mathrm{CDCl}_{3}\right): 167.1$, $162.8(244 \mathrm{~Hz}), 152.2,140.2(8 \mathrm{~Hz}), 139.1,137.9,136.7,135.4,129.6(8 \mathrm{~Hz}), 129.1,128.9,128.8,128.6,128.5$, 126.5, 126.4, $125.4(3 \mathrm{~Hz}), 116.6(22 \mathrm{~Hz}), 113.3(21 \mathrm{~Hz}), 109.0,39.1,34.2,19.9 . \mathrm{HRMS}(\mathrm{m} / z):[\mathrm{M}+\mathrm{H}]^{+}$calcd for $\mathrm{C}_{28} \mathrm{H}_{23} \mathrm{FN}_{3} \mathrm{O}_{2}, 452.1774$; found, 452.1775 .

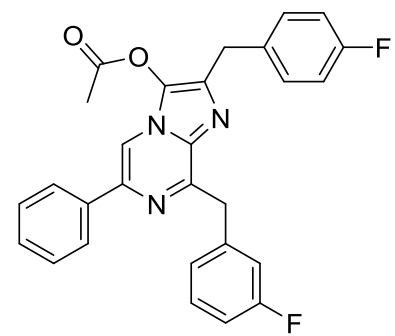

8-(3-Fluorobenzyl)-2-(4-fluorobenzyl)-6-phenylimidazo[1,2-a]pyrazin-3-yl acetate 25\{1,3,4\} (YJ31069-031-1): (YJ 31069-31-1): Obtained as a white solid after a recrystallization in $n$-heptane $(0.34 \mathrm{~g}, 85 \%) .{ }^{1} \mathrm{H} \mathrm{NMR}\left(\mathrm{CDCl}_{3}\right)$ $7.90(\mathrm{~m}, 2 \mathrm{H}), 7.83(\mathrm{~s}, 1 \mathrm{H}), 7.48(\mathrm{~m}, 2 \mathrm{H}), 7.48(\mathrm{~m}, 2 \mathrm{H}), 7.38(\mathrm{~m}, 3 \mathrm{H}), 7.27(\mathrm{~m}, 3 \mathrm{H}), 7.02(\mathrm{~m}, 2 \mathrm{H}), 6.93(\mathrm{~m}, 1 \mathrm{H})$, 4.63 (s, 2H), 4.17 (s, 2H), 2.28 (s, 3H). ${ }^{13} \mathrm{C} \mathrm{NMR}\left(\mathrm{CDCl}_{3}\right): 167.0,162.7$ (246 Hz), $161.7(244 \mathrm{~Hz}), 152.2,140.1$ (7 $\mathrm{Hz}), 139.3,136.6,133.6$ (3 Hz), 133.5, $130.4(8 \mathrm{~Hz}), 129.6(8 \mathrm{~Hz}), 128.8,128.7,126.4,125.3(2 \mathrm{~Hz}), 116.6(21$ $\mathrm{Hz}), 115.2(21 \mathrm{~Hz}), 113.4(21 \mathrm{~Hz}), 109.0,39.1,33.1,19.9$. HRMS $(m / z):[\mathrm{M}+\mathrm{H}]^{+}$calcd for $\mathrm{C}_{28} \mathrm{H}_{22} \mathrm{~F}_{2} \mathrm{~N}_{3} \mathrm{O}_{2}$, 470.1680, found, 470.1676 .

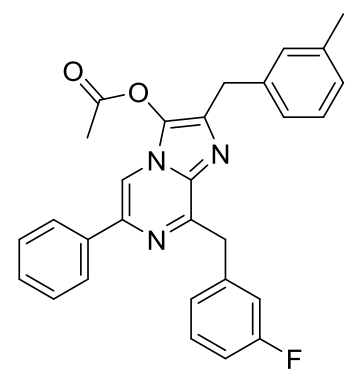


8-(3-Fluorobenzyl)-2-(3-methylbenzyl)-6-phenylimidazo[1,2-a]pyrazin-3-yl acetate 25 1,3,12\} (YJ31068-045-1): Obtained as a white solid after a recrystallization in $n$-heptane $(0.16 \mathrm{~g}, 50 \%) .{ }^{1} \mathrm{H} \mathrm{NMR}\left(\mathrm{CDCl}_{3}\right): 7.91(\mathrm{~m}, 2 \mathrm{H}), 7.82$ $(\mathrm{s}, 1 \mathrm{H}), 7.47(\mathrm{~m}, 2 \mathrm{H}), 7.38(\mathrm{~m}, 3 \mathrm{H}), 7.28(\mathrm{~m}, 1 \mathrm{H}), 7.22(\mathrm{~m}, 1 \mathrm{H}), 7.09(\mathrm{~m}, 3 \mathrm{H}), 6.95(\mathrm{~m}, 1 \mathrm{H}), 4.63(\mathrm{~s}, 2 \mathrm{H}), 4.18(\mathrm{~s}$, 2H), 2.35 (s, 3H), 2.20 (s, 3H). ${ }^{13} \mathrm{C}$ NMR $\left(\mathrm{CDCl}_{3}\right): 167.1,162.8(244 \mathrm{~Hz}), 152.2,140.2(8 \mathrm{~Hz}), 139.1,138.1,137.8$, 136.7, 135.6, 135.5, 129.8 129.6, (8 Hz), 128.9, 128.7, 128.6, 128.4, 127.2, 126.4, 126.1, 125.4 (3 Hz), 116.6 (22 $\mathrm{Hz}), 113.3(21 \mathrm{~Hz}), 109.0,39.1(2 \mathrm{~Hz}), 34.1,21.3,19.8$. HRMS $(\mathrm{m} / z):[\mathrm{M}+\mathrm{H}]^{+}$calcd for $\mathrm{C}_{29} \mathrm{H}_{25} \mathrm{FN}_{3} \mathrm{O}_{2}, 466.1931$, found, 466.1926.

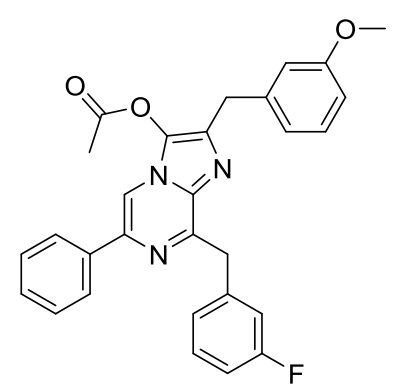

8-(3-Fluorobenzyl)-2-(3-methoxybenzyl)-6-phenylimidazo[1,2-a]pyrazin-3-yl acetate 25\{1,3,15\} (YJ31068-0471): Obtained as a white solid after a recrystallization in $n$-heptane $(0.12 \mathrm{~g}, 60 \%) .{ }^{1} \mathrm{H} \mathrm{NMR}\left(\mathrm{CDCl}_{3}\right): 7.90(\mathrm{~m}, 2 \mathrm{H})$, $7.82(\mathrm{~s}, 1 \mathrm{H}), 7.47(\mathrm{~m}, 2 \mathrm{H}), 7.38(\mathrm{~m}, 3 \mathrm{H}), 7.26(\mathrm{~m}, 1 \mathrm{H}), 6.95(\mathrm{~m}, 3 \mathrm{H}), 6.80(\mathrm{~m}, 1 \mathrm{H}), 4.63(\mathrm{~s}, 2 \mathrm{H}), 4.19(\mathrm{~s}, 2 \mathrm{H}), 3.79$ (s, 3H), 2.24 (s, 3H). ${ }^{13} \mathrm{C} \mathrm{NMR}\left(\mathrm{CDCl}_{3}\right): 167.1,162.8(244 \mathrm{~Hz}), 159.8,152.2,140.2(8 \mathrm{~Hz}), 139.5,139.1,136.7$, 135.3, 133.5, $129.6(8 \mathrm{~Hz}), 129.4,128.9,128.8,128.6,126.4,125.4(3 \mathrm{~Hz}), 121.4,116.6(22 \mathrm{~Hz}), 114.6,113.3(21$ $\mathrm{Hz}), 112.2$, 109.0, 55.2, 39.0, 34.2, 19.9. HRMS $(\mathrm{m} / \mathrm{z}):[\mathrm{M}+\mathrm{H}]^{+}$calcd for $\mathrm{C}_{29} \mathrm{H}_{25} \mathrm{FN}_{3} \mathrm{O}_{3}$, 482.1880, found, 482.1879 .

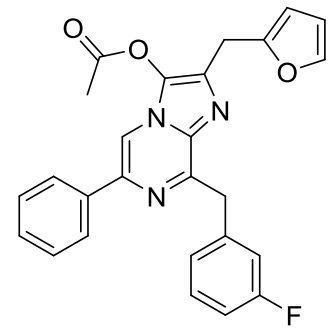

8-(3-Fluorobenzyl)-2-(furan-2-ylmethyl)-6-phenylimidazo[1,2-a]pyrazin-3-yl acetate 25\{1,3,37\} (YJ31070-0451): Obtained as a solid $(0.13 \mathrm{~g}, 59 \%)$ after a recrystallization from $n$-heptane. ${ }^{1} \mathrm{H}$ NMR $\left(\mathrm{CDCl}_{3}\right): 7.91(\mathrm{~m}, 2 \mathrm{H}), 7.85$ (s, 1H), $7.49(\mathrm{~m}, 2 \mathrm{H}), 7.42-7.33(\mathrm{~m}, 4 \mathrm{H}), 7.26(\mathrm{~m}, 1 \mathrm{H}), 6.93(\mathrm{~m}, 1 \mathrm{H}), 6.36(\mathrm{dd}, 1 \mathrm{H}, J=2.0,3.2 \mathrm{~Hz}), 6.17(\mathrm{dd}, 1 \mathrm{H}$, $J=0.8,3.2 \mathrm{~Hz}), 4.62(\mathrm{~s}, 2 \mathrm{H}), 4.24(\mathrm{~s}, 2 \mathrm{H}), 2.36(\mathrm{~s}, 3 \mathrm{H}) .{ }^{13} \mathrm{C} \mathrm{NMR}\left(\mathrm{CDCl}_{3}\right): 167.1,162.8(244 \mathrm{~Hz}), 152.3,151.4$, 141.7, 140.2 (8 Hz), 139.2, 136.6, 133.5, 132.7, 129.6 (8 Hz), 129.0, 128.8, 128.7, 126.4, 125.3 (3 Hz), 116.6 (22 $\mathrm{Hz}), 113.3(21 \mathrm{~Hz}), 110.5,109.0,106.9,39.0,27.2,20.1$. HRMS $(\mathrm{m} / z)$ : $[\mathrm{M}+\mathrm{H}]^{+}$calcd for $\mathrm{C}_{26} \mathrm{H}_{21} \mathrm{FN}_{3} \mathrm{O}_{3}, 442.1567$; found, 442.1549 .

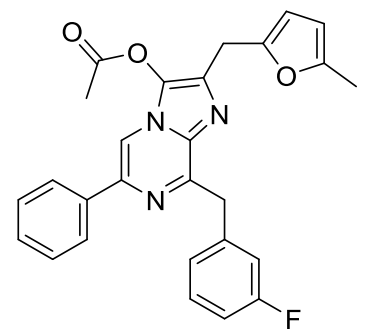

8-(3-Fluorobenzyl)-2-((5-methylfuran-2-yl)methyl)-6-phenylimidazo[1,2-a]pyrazin-3-yl acetate $\quad \mathbf{2 5}\{1,3,42\}$ (YJ31068-029-1): Obtained as a solid $(0.10 \mathrm{~g}, 45 \%)$ after a recrystallization from $n$-heptane. ${ }^{1} \mathrm{H}$ NMR $\left(\mathrm{CDCl}_{3}\right)$ : $7.91(\mathrm{~m}, 2 \mathrm{H}), 7.85(\mathrm{~s}, 1 \mathrm{H}), 7.47(\mathrm{~m}, 2 \mathrm{H}), 7.42-7.24(\mathrm{~m}, 4 \mathrm{H}), 6.92(\mathrm{~m}, 1 \mathrm{H}), 6.03(\mathrm{~d}, 1 \mathrm{H}, J=3.0 \mathrm{~Hz}), 5.91(\mathrm{~m}, 1 \mathrm{H})$, $4.62(\mathrm{~s}, 2 \mathrm{H}), 4.18(\mathrm{~s}, 2 \mathrm{H}), 2.37(\mathrm{~s}, 3 \mathrm{H}), 2.28(\mathrm{~s}, 3 \mathrm{H}) .{ }^{13} \mathrm{C} \mathrm{NMR}\left(\mathrm{CDCl}_{3}\right): 167.0,162.8(244 \mathrm{~Hz}), 152.2,151.2$, 149.4, 140.2 (8 Hz), 139.2, 136.6, 133.4, 133.0, 129.6 (8 Hz), 129.0, 128.8, 128.6, 126.4, 125.3 (3 Hz), 116.6 (22 
$\mathrm{Hz}), 113.3(21 \mathrm{~Hz}), 109.0,107.5,106.2,39.0,27.3,20.1,13.5$. HRMS $(m / z):[\mathrm{M}+\mathrm{H}]^{+}$calcd for $\mathrm{C}_{27} \mathrm{H}_{23} \mathrm{FN}_{3} \mathrm{O}_{3}$, 456.1723; found, 456.1732 .

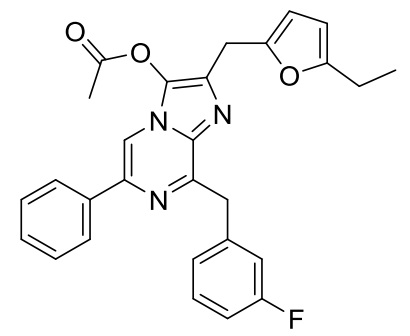

2-((5-Ethylfuran-2-yl)methyl)-8-(3-fluorobenzyl)-6-phenylimidazo[1,2-a]pyrazin-3-yl acetate $\quad \mathbf{2 5}\{1,3,44\}$ (YJ31068-031-1): Obtained as a white solid (0.05 g, 11\% from the 2-chloro-3-(3-fluorobenzyl)-5-phenylpyrazine) after a chromatography over silica gel (cyclohexane ethyl acetate 6/1) and recrystallization from $n$-heptane. ${ }^{1} \mathrm{H}$ NMR $\left(\mathrm{CDCl}_{3}\right): 7.91(\mathrm{~m}, 2 \mathrm{H}), 7.85(\mathrm{~s}, 1 \mathrm{H}), 7.48(\mathrm{~m}, 2 \mathrm{H}), 7.42-7.24(\mathrm{~m}, 4 \mathrm{H}), 6.92(\mathrm{~m}, 1 \mathrm{H}), 6.03(\mathrm{~d}, 1 \mathrm{H}, J=3.1$ $\mathrm{Hz}), 5.92(\mathrm{~m}, 1 \mathrm{H}), 4.62(\mathrm{~s}, 2 \mathrm{H}), 4.19(\mathrm{~s}, 2 \mathrm{H}), 2.63(\mathrm{q}, 2 \mathrm{H}, J=7.5 \mathrm{~Hz}), 2.36(\mathrm{~s}, 3 \mathrm{H}), 2.63(\mathrm{t}, 3 \mathrm{H}, J=7.5 \mathrm{~Hz}) .{ }^{13} \mathrm{C}$ NMR $\left(\mathrm{CDCl}_{3}\right): 167.0,162.8(244 \mathrm{~Hz}), 157.1,152.2,149.3,140.2(8 \mathrm{~Hz}), 139.1,136.7,133.4,133.1,129.6(8 \mathrm{~Hz})$, 129.0, 128.8, 128.6, 126.4, $125.3(3 \mathrm{~Hz}), 116.6(22 \mathrm{~Hz}), 113.3(21 \mathrm{~Hz}), 109.0,107.3,104.6,39.0,27.3,21.3,20.1$, 12.2. HRMS (m/z): $[\mathrm{M}+\mathrm{H}]^{+}$calcd for $\mathrm{C}_{28} \mathrm{H}_{25} \mathrm{FN}_{3} \mathrm{O}_{3}, 470.1880$; found, 470.1888 .

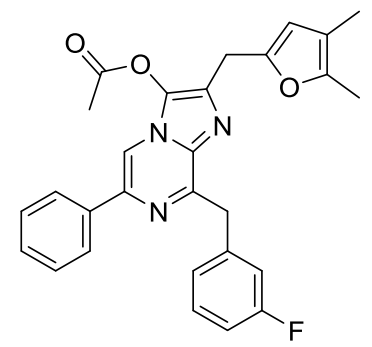

2-((4,5-Dimethylfuran-2-yl)methyl)-8-(3-fluorobenzyl)-6-phenylimidazo[1,2-a]pyrazin-3-yl acetate $\mathbf{2 5}\{1,3,48\}$ (EC32712-131-2): Obtained as a solid $(0.18 \mathrm{~g}, 55 \%)$ after a recrystallization from $n$-heptane. ${ }^{1} \mathrm{H}$ NMR $\left(\mathrm{CDCl}_{3}\right)$ : $7.91(\mathrm{~m}, 2 \mathrm{H}), 7.85(\mathrm{~s}, 1 \mathrm{H}), 7.47(\mathrm{~m}, 2 \mathrm{H}), 7.38(\mathrm{~m}, 3 \mathrm{H}), 7.27(\mathrm{~m}, 1 \mathrm{H}), 6.93(\mathrm{~m}, 1 \mathrm{H}), 5.92(\mathrm{~s}, 1 \mathrm{H}), 4.62(\mathrm{~s}, 2 \mathrm{H}), 4.14$ (s, 2H), 2.38 (s, 3H), 2.19 (s, 3H), $1.93(\mathrm{~s}, 3 \mathrm{H}) .{ }^{13} \mathrm{C} \mathrm{NMR}\left(\mathrm{CDCl}_{3}\right): 167.0,162.7(\mathrm{~d}, J=245 \mathrm{~Hz}), 152.2,148.2$, $146.3,140.2$ (d, $J=8 \mathrm{~Hz}), 139.1,136.7,133.4,133.2,129.6$ (d, $J=8 \mathrm{~Hz}), 128.9,128.8,128.6,126.4,125.4$ (d, $J=$ $3 \mathrm{~Hz}), 116.6(\mathrm{~d}, J=22 \mathrm{~Hz}), 114.6,113.4(\mathrm{~d}, J=21 \mathrm{~Hz}), 110.1,109.0,39.0$ (d, $J=2 \mathrm{~Hz}), 27.2$, 20.0, 11.2, 9.8. HRMS (m/z): $[\mathrm{M}+\mathrm{H}]^{+}$calcd for $\mathrm{C}_{28} \mathrm{H}_{25} \mathrm{FN}_{3} \mathrm{O}_{3}$ : 470.1880; found, 470.1880.

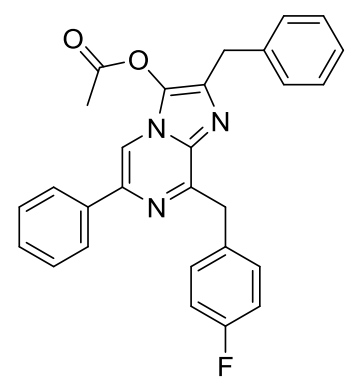

2-Benzyl-8-(4-fluorobenzyl)-6-phenylimidazo[1,2-a]pyrazin-3-yl acetate 25 1 1,4,1\} (YJ31067-163-1): Obtained as a solid in two crops $(0.04 \mathrm{~g}, 33 \%)$ after a recrystallization from $n$-heptane. ${ }^{1} \mathrm{H} \mathrm{NMR}\left(\mathrm{CDCl}_{3}\right): 7.90(\mathrm{~m}, 2 \mathrm{H}), 7.81(\mathrm{~s}$, 1H), $7.58(\mathrm{~m}, 2 \mathrm{H}), 7.47(\mathrm{~m}, 2 \mathrm{H}), 7.40(\mathrm{~m}, 1 \mathrm{H}), 7.36-7.24(\mathrm{~m}, 5 \mathrm{H}), 7.00(\mathrm{~m}, 2 \mathrm{H}), 4.60(\mathrm{~s}, 2 \mathrm{H}), 4.21(\mathrm{~s}, 2 \mathrm{H}), 2.19$ $(\mathrm{s}, 3 \mathrm{H}) .{ }^{13} \mathrm{C} \mathrm{NMR}\left(\mathrm{CDCl}_{3}\right): 167.1,161.7(244 \mathrm{~Hz}), 139.1,137.9,136.7,135.3,133.5,133.4(3 \mathrm{~Hz}), 131.1(7 \mathrm{~Hz})$, 129.1, 128.9, 128.8, 128.6, 128.5, 126.5, 126.4, $115.0(21 \mathrm{~Hz}), 108.9,38.6,34.2,19.9$ (one signal missing). HRMS $(\mathrm{m} / \mathrm{z}):[\mathrm{M}+\mathrm{H}]^{+}$calcd for $\mathrm{C}_{28} \mathrm{H}_{23} \mathrm{FN}_{3} \mathrm{O}_{2}, 452.1774$; found, 452.1789 . 


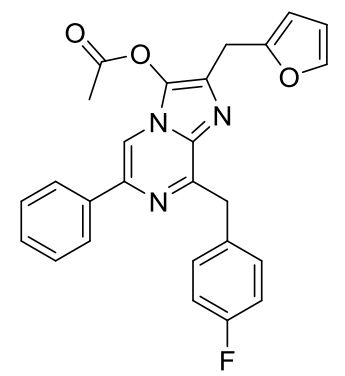

8-(4-Fluorobenzyl)-2-(furan-2-ylmethyl)-6-phenylimidazo[1,2-a]pyrazin-3-yl acetate 25\{1,4,37\} (YJ 31776-0111): Obtained as a solid $(0.28 \mathrm{~g}, 95 \%)$ after dispertion of the reaction media in water, a filtration and drying under vacuum at $40^{\circ} \mathrm{C} .{ }^{1} \mathrm{H} \mathrm{NMR}\left(\mathrm{CDCl}_{3}\right): 7.91(\mathrm{~m}, 2 \mathrm{H}), 7.85(\mathrm{~s}, 1 \mathrm{H}), 7.57(\mathrm{~m}, 2 \mathrm{H}), 7.47(\mathrm{~m}, 2 \mathrm{H}), 7.40(\mathrm{~m}, 2 \mathrm{H}), 7.00(\mathrm{~m}$, 2H), $6.36(\mathrm{~m}, 1 \mathrm{H}), 6.16(\mathrm{~m}, 1 \mathrm{H}), 4.60(\mathrm{~s}, 2 \mathrm{H}), 4.24(\mathrm{~s}, 2 \mathrm{H}), 2.37(\mathrm{~s}, 3 \mathrm{H}) .{ }^{13} \mathrm{C} \mathrm{NMR}\left(\mathrm{CDCl}_{3}\right): 167.0,161.7(244 \mathrm{~Hz})$, 152.7, 151.4, 141.7, 139.3, 136.6, 133.4 (3 Hz), 132.5, $131.1(7 \mathrm{~Hz}), 129.0,128.8,128.7,126.4,115.0(21 \mathrm{~Hz})$, $110.5,108.9,106.9,38.5,27.1,20.1$ (one signal missing).

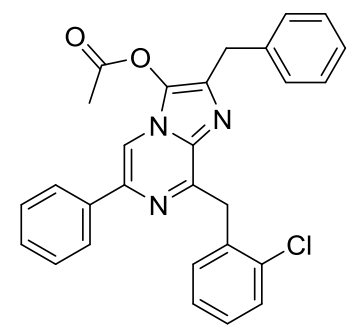

2-Benzyl-8-(2-chlorobenzyl)-6-phenylimidazo[1,2-a]pyrazin-3-yl acetate 25 1,5,1\} (EC31094-105-1): Obtained as a white solid $(0.12 \mathrm{~g}, 67 \%)$ after a recrystallization in n-heptane. ${ }^{1} \mathrm{H}$ NMR $\left(\mathrm{CDCl}_{3}\right): 7.84(\mathrm{~s}, 1 \mathrm{H}), 7.83(\mathrm{~m}, 2 \mathrm{H})$, 7.50-7.20 (m, 12H), $4.82(\mathrm{~s}, 2 \mathrm{H}), 4.23(\mathrm{~s}, 2 \mathrm{H}), 2.20(\mathrm{~s}, 3 \mathrm{H}) .{ }^{13} \mathrm{C} \mathrm{NMR}\left(\mathrm{CDCl}_{3}\right): 167.1,151.5,138.8,138.0,136.6$, 135.7, 135.1, 134.9, 133.7, 131.8, 129.3, 129.1, 128.8, 128.7, 128.5 (two signals), 127.9, 126.5 (two signals), 126.2, 108.8, 36.6, 34.3, 19.9. HRMS (m/z): $[\mathrm{M}+\mathrm{H}]^{+}$calcd for $\mathrm{C}_{28} \mathrm{H}_{23} \mathrm{ClN}_{3} \mathrm{O}_{2}$ : 468.1479; found, 468.1489.

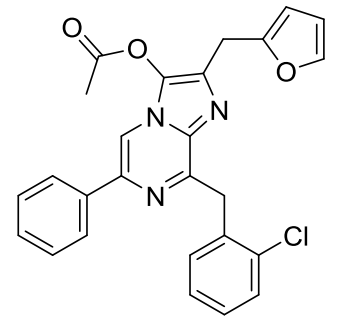

8-(2-Chlorobenzyl)-2-(furan-2-ylmethyl)-6-phenylimidazo[1,2-a]pyrazin-3-yl acetate 25 1,5,37\} (EC31094-1031): Obtained as a white solid after a recrystallization in $\mathrm{n}$-heptane $(0.27 \mathrm{~g}, 70 \%) .{ }^{1} \mathrm{H} \mathrm{NMR}\left(\mathrm{CDCl}_{3}\right): 7.88(\mathrm{~s}, 1 \mathrm{H})$, $7.83(\mathrm{~m}, 2 \mathrm{H}), 7.46-7.34(\mathrm{~m}, 6 \mathrm{H}), 7.23(\mathrm{~m}, 2 \mathrm{H}), 6.36(\mathrm{~m}, 1 \mathrm{H}), 6.18(\mathrm{~m}, 1 \mathrm{H}), 4.81(\mathrm{~s}, 2 \mathrm{H}), 4.25(\mathrm{~s}, 2 \mathrm{H}), 2.38(\mathrm{~s}, 3 \mathrm{H})$. ${ }^{13} \mathrm{C} \mathrm{NMR}\left(\mathrm{CDCl}_{3}\right)$ : 167.1, 151.7, 151.5, 141.7, 138.9, 136.6, 135.6, 134.9, 133.7, 132.4, 131.8, 129.3, 128.9, 128.7, 128.6, 127.9, 126.5, 126.2, 110.5, 108.8, 106.9, 36.6, 27.3, 20.1. HRMS $(m / z):[\mathrm{M}+\mathrm{H}]^{+}$calcd for $\mathrm{C}_{26} \mathrm{H}_{20} \mathrm{ClN}_{3} \mathrm{O}_{3}$ : 458.1271; found, 458.1266.<smiles>CC(=O)Oc1c(Cc2ccc(C)o2)nc2c(Cc3ccccc3Cl)nc(-c3ccccc3)cn12</smiles>

8-(2-Chlorobenzyl)-2-((5-methylfuran-2-yl)methyl)-6-phenylimidazo[1,2-a]pyrazin-3-yl acetate $\quad \mathbf{2 5}\{1,5,42\}$ (YJ31068-025-1): Obtained as a solid (0.03 g, 27\%) after a recrystallization in n-heptane. ${ }^{1} \mathrm{H}$ NMR $\left(\mathrm{CDCl}_{3}\right): 7.88$ $(\mathrm{s}, 1 \mathrm{H}), 7.83(\mathrm{~m}, 2 \mathrm{H}), 7.46-7.33(\mathrm{~m}, 5 \mathrm{H}), 7.21(\mathrm{~m}, 2 \mathrm{H}), 6.04(\mathrm{~m}, 1 \mathrm{H}), 5.91(\mathrm{~m}, 1 \mathrm{H}), 4.81(\mathrm{~s}, 2 \mathrm{H}), 4.19(\mathrm{~s}, 2 \mathrm{H}), 2.38$ 
(s, 3H), 2.28 (s, 3H). ${ }^{13} \mathrm{C}$ NMR $\left(\mathrm{CDCl}_{3}\right)$ : 167.1, 151.6, 151.2, 149.5, 138.9, 136.6, 135.6, 134.9, 133.6, 132.7, $131.8,129.3,128.9,128.7,128.5,127.9,126.5,126.2,108.8,107.5,106.2,36.6,27.3,20.1,13.5$. HRMS $(\mathrm{m} / \mathrm{z})$ : $[\mathrm{M}+\mathrm{H}]^{+}$calcd for $\mathrm{C}_{27} \mathrm{H}_{23} \mathrm{ClN}_{3} \mathrm{O}_{3}: 472.1428$; found, 472.1408 .

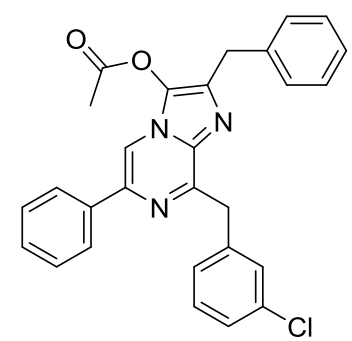

2-Benzyl-8-(3-chlorobenzyl)-6-phenylimidazo[1,2-a]pyrazin-3-yl acetate 25 \{1,6,1\} (YJ 31776-019-1): Obtained as a solid $(0.20 \mathrm{~g}, 90 \%)$ after dispertion of the reaction media in water, a filtration and drying under vacuum at $40^{\circ} \mathrm{C}$. ${ }^{1} \mathrm{H}$ NMR $\left(\mathrm{CDCl}_{3}\right)$ : $7.90(\mathrm{~m}, 2 \mathrm{H}), 7.81(\mathrm{~s}, 1 \mathrm{H}), 7.64(\mathrm{~m}, 1 \mathrm{H}), 7.47(\mathrm{~m}, 3 \mathrm{H}), 7.42-7.20(\mathrm{~m}, 8 \mathrm{H}), 4.62(\mathrm{~s}, 2 \mathrm{H}), 4.22$ (s, 2H). ${ }^{13} \mathrm{C}$ NMR $\left(\mathrm{CDCl}_{3}\right)$ : 167.0, 152.0, 139.7, 139.2, 137.8, 136.6, 135.3, 134.0, 129.8, 129.5, 129.1, 128.9, $128.8,128.7,128.5,128.0,126.7,126.5,126.4,109.3,39.0,34.1,19.9$. HRMS $(m / z):[\mathrm{M}+\mathrm{H}]^{+}$calcd for $\mathrm{C}_{28} \mathrm{H}_{23} \mathrm{ClN}_{3} \mathrm{O}_{2}: 468.1479$; found, 468.1480 .

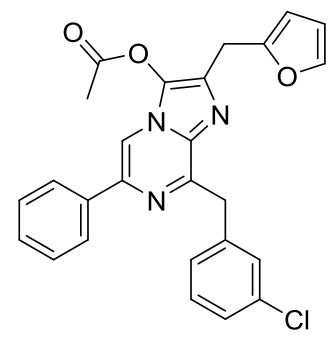

8-(3-Chlorobenzyl)-2-(furan-2-ylmethyl)-6-phenylimidazo[1,2-a]pyrazin-3-yl acetate 25\{1,6,37\} (EC31095-0892): Obtained as a white solid $(0.05 \mathrm{~g}, 30 \%)$ after a chromatography over silica gel (cyclohexane - ethyl acetate 6/1) and a recrystallization in $n$-heptane. ${ }^{1} \mathrm{H}$ NMR $\left(\mathrm{CDCl}_{3}\right): 7.91(\mathrm{~m}, 2 \mathrm{H}), 7.85(\mathrm{~s}, 1 \mathrm{H}), 7.62(\mathrm{~m}, 1 \mathrm{H}), 7.48(\mathrm{~m}, 3 \mathrm{H}), 7.40$ $(\mathrm{m}, 2 \mathrm{H}), 7.24(\mathrm{~m}, 2 \mathrm{H}), 6.36(\mathrm{dd}, 1 \mathrm{H}, J=3.2,1.9 \mathrm{~Hz}), 6.17(\mathrm{~m}, 1 \mathrm{H}), 4.60(\mathrm{~s}, 2 \mathrm{H}), 4.24(\mathrm{~s}, 2 \mathrm{H}), 2.37(\mathrm{~s}, 3 \mathrm{H}) .{ }^{13} \mathrm{C}$ NMR $\left(\mathrm{CDCl}_{3}\right): 167.0,152.2,151.4,141.7,139.7,139.2,136.6,134.0,133.5,132.7,129.8,129.5,129.0,128.8$, 128.7, 127.9, 126.7, 126.4, 110.5, 109.1, 106.9, 38.9, 27.2, 20.1. HRMS $(m / z):[\mathrm{M}+\mathrm{H}]^{+}$calcd for $\mathrm{C}_{26} \mathrm{H}_{21} \mathrm{ClN}_{3} \mathrm{O}_{3}$ : 458.1271; found, 458.1270 .

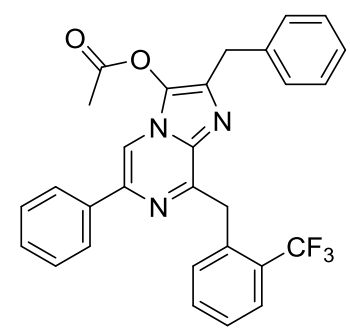

2-Benzyl-6-phenyl-8-(2-(trifluoromethyl)benzyl)imidazo[1,2-a]pyrazin-3-yl acetate 25\{1,8,1\} (YJ 33067-129-1): Obtained as a white solid $(0.24 \mathrm{~g}, 68 \%)$ after a recrystallization from $n$-heptane. ${ }^{1} \mathrm{H}$ NMR $\left(\mathrm{CDCl}_{3}\right): 7.85(\mathrm{~s}, 1 \mathrm{H})$, $7.79(\mathrm{~m}, 2 \mathrm{H}), 7.74(\mathrm{~m}, 1 \mathrm{H}), 7.52-7.33(\mathrm{~m}, 10 \mathrm{H}), 7.28(\mathrm{~m}, 1 \mathrm{H}), 4.90(\mathrm{~s}, 2 \mathrm{H}), 4.23(\mathrm{~s}, 2 \mathrm{H}), 2.22(\mathrm{~s}, 3 \mathrm{H}) .{ }^{13} \mathrm{C} \mathrm{NMR}$ $\left(\mathrm{CDCl}_{3}\right): 167.1,151.7,138.8,137.9,136.5,136.1(1 \mathrm{~Hz}), 135.1,133.7,132.6131 .4,129.4(30 \mathrm{~Hz}), 129.2,128.9$, 128.7, 128.6, 128.5, 126.5, 126.1, $125.9(4 \mathrm{~Hz}), 124.6(273 \mathrm{~Hz}), 108.7,35.7,34.2,19.8$ (one signal missing). HRMS $(\mathrm{m} / \mathrm{z}):[\mathrm{M}+\mathrm{H}]^{+}$calcd for $\mathrm{C}_{29} \mathrm{H}_{23} \mathrm{~F}_{3} \mathrm{~N}_{3} \mathrm{O}_{2}:$ 502.1742; found, 502.1740 . 


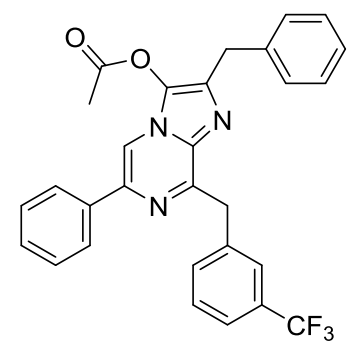

2-Benzyl-6-phenyl-8-(3-(trifluoromethyl)benzyl)imidazo[1,2-a]pyrazin-3-yl acetate 25\{1,9,1\} (YJ 33067-131-1): Obtained as a white solid $(0.12 \mathrm{~g}, 34 \%)$ after a recrystallization from $n$-heptane. ${ }^{1} \mathrm{H}$ NMR $\left(\mathrm{CDCl}_{3}\right): 7.96(\mathrm{~s}(\mathrm{br})$, $1 \mathrm{H}), 7.89(\mathrm{~m}, 2 \mathrm{H}), 7.82(\mathrm{~s}, 1 \mathrm{H}), 7.79(\mathrm{~m}, 1 \mathrm{H}), 7.52-7.23(\mathrm{~m}, 10 \mathrm{H}), 4.69(\mathrm{~s}, 2 \mathrm{H}), 4.22(\mathrm{~s}, 2 \mathrm{H}), 2.21(\mathrm{~s}, 3 \mathrm{H}) .{ }^{13} \mathrm{C}$ NMR $\left(\mathrm{CDCl}_{3}\right): 167.0,151.8,139.2,138.5,137.8,136.5,135.4,133.3,133.2,130.5(31 \mathrm{~Hz}), 129.1,128.8,128.7$, 128.6, 128.5, 128.3, $126.7(4 \mathrm{~Hz}), 126.6,126.4,124.3(272 \mathrm{~Hz}), 123.4(3 \mathrm{~Hz}), 109.0,39.0,34.1,19.9$. HRMS $(\mathrm{m} / \mathrm{z}):[\mathrm{M}+\mathrm{H}]^{+}$calcd for $\mathrm{C}_{29} \mathrm{H}_{23} \mathrm{~F}_{3} \mathrm{~N}_{3} \mathrm{O}_{2}: 502.1742$; found, 502.1735.

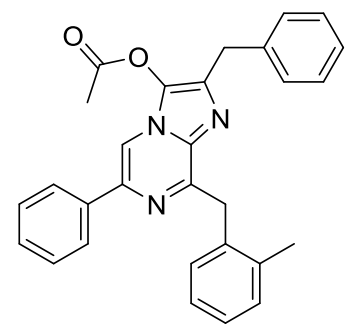

2-Benzyl-8-(2-methylbenzyl)-6-phenylimidazo[1,2-a]pyrazin-3-yl acetate 25 $11,11,1\}$ (YJ31068-033-1): Obtained as a white solid $(0.29 \mathrm{~g}, 77 \%)$ after a recrystallization from $n$-heptane. ${ }^{1} \mathrm{H} \mathrm{NMR}\left(\mathrm{CDCl}_{3}\right): 7.87(\mathrm{~m}, 2 \mathrm{H}), 7.82(\mathrm{~s}$, 1H), $7.57(\mathrm{~m}, 1 \mathrm{H}), 7.44(\mathrm{~m}, 2 \mathrm{H}), 7.37(\mathrm{~m}, 1 \mathrm{H}), 7.33(\mathrm{~m}, 4 \mathrm{H}), 7.27(\mathrm{~m}, 1 \mathrm{H}), 7.22(\mathrm{~m}, 1 \mathrm{H}), 7.17(\mathrm{~m}, 2 \mathrm{H}), 4.67(\mathrm{~s}$, 2H), $4.22(\mathrm{~s}, 2 \mathrm{H}), 2.61(\mathrm{~s}, 3 \mathrm{H}), 2.20(\mathrm{~s}, 3 \mathrm{H}) .{ }^{13} \mathrm{C} \mathrm{NMR}\left(\mathrm{CDCl}_{3}\right)$ : 167.1, 152.9, 138.9, 138.0, 137.4, 136.8, 136.3, 135.1, 133.7, 130.7, 130.1, 129.1, 128.8, 128.7, 128.5, 128.4, 126.6, 126.5, 126.2, 125.7, 108.7, 36.6, 34.2, 20.3, 19.9. HRMS $(m / z):[\mathrm{M}+\mathrm{H}]^{+}$calcd for $\mathrm{C}_{29} \mathrm{H}_{26} \mathrm{~N}_{3} \mathrm{O}_{2}: 448.2025$; found, 448.2031.

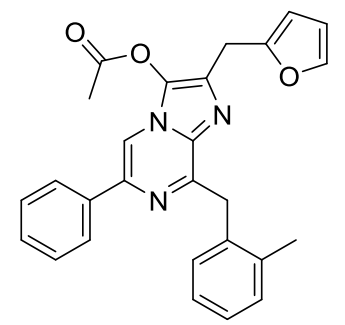

2-(furan-2-ylmethyl)-8-(2-methylbenzyl)-6-phenylimidazo[1,2-a]pyrazin-3-yl acetate 25\{1,11,37\} (YJ31068-0351): Obtained as a white solid $(0.18 \mathrm{~g}, 64 \%)$ after a recrystallization in $\mathrm{n}$-heptane. ${ }^{1} \mathrm{H}$ NMR $\left(\mathrm{CDCl}_{3}\right): 7.88(\mathrm{~m}, 2 \mathrm{H})$, $7.85(\mathrm{~s}, 1 \mathrm{H}), 7.54(\mathrm{~m}, 2 \mathrm{H}), 7.45(\mathrm{~m}, 2 \mathrm{H}), 7.38(\mathrm{~m}, 2 \mathrm{H}), 7.23(\mathrm{~m}, 1 \mathrm{H}), 7.16(\mathrm{~m}, 2 \mathrm{H}), 6.36(\mathrm{dd}, 1 \mathrm{H}, J=1.9,3.4 \mathrm{~Hz})$, $6.17(\mathrm{~m}, 1 \mathrm{H}), 4.66$ (s, 2H), $4.24(\mathrm{~s}, 2 \mathrm{H}), 2.60$ (s, 3H), 2.36 (s, 3H). ${ }^{13} \mathrm{C} \mathrm{NMR}\left(\mathrm{CDCl}_{3}\right):$ 167.1, 153.0, 151.6, 141.6, 139.0, 137.3, 136.7, 136.3, 133.7, 132.4, 130.6, 130.1, 128.8, 128.7, 128.5, 126.6, 126.3, 125.7, 110.5, 108.7, 106.8, 36.5, 27.2, 20.3, 20.1. HRMS $(\mathrm{m} / \mathrm{z})$ : $[\mathrm{M}+\mathrm{H}]^{+}$calcd for $\mathrm{C}_{27} \mathrm{H}_{24} \mathrm{~N}_{3} \mathrm{O}_{3}, 438.1818$; found, 438.1823 .

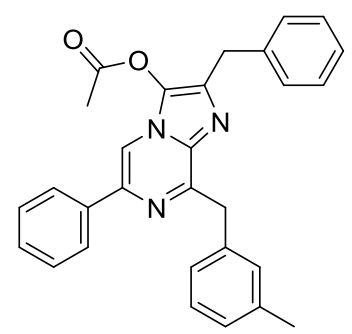

2-Benzyl-8-(3-methylbenzyl)-6-phenylimidazo[1,2-a]pyrazin-3-yl acetate 25\{1,12,1\} (YJ31068-037-1): Obtained as a white solid $(0.27 \mathrm{~g}, 70 \%)$ after a recrystallization from $n$-heptane. ${ }^{1} \mathrm{H}$ NMR $\left(\mathrm{CDCl}_{3}\right): 7.92(\mathrm{~m}, 2 \mathrm{H}), 7.80(\mathrm{~s}$, $1 \mathrm{H}), 7.57(\mathrm{~m}, 1 \mathrm{H}), 7.50-7.37(\mathrm{~m}, 5 \mathrm{H}), 7.32(\mathrm{~m}, 4 \mathrm{H}), 7.28-7.20(\mathrm{~m}, 2 \mathrm{H}), 7.05(\mathrm{~m}, 1 \mathrm{H}), 4.61(\mathrm{~s}, 2 \mathrm{H}), 4.22(\mathrm{~s}, 2 \mathrm{H})$, 
2.35 (s, 3H), 2.19 (s, 3H). ${ }^{13} \mathrm{C}$ NMR $\left(\mathrm{CDCl}_{3}\right): 167.1,153.0,139.1,138.0,137.8,137.7,136.9,135.2,133.7,130.5$, 129.1, 128.8, 128.7, 128.5, 128.4, 128.2, 127.2, 126.7, 126.5, 126.4, 108.8, 39.4, 34.2, 21.4, 19.9. HRMS (m/z): $[\mathrm{M}+\mathrm{H}]^{+}$calcd for $\mathrm{C}_{29} \mathrm{H}_{26} \mathrm{~N}_{3} \mathrm{O}_{2}$ : 448.2025; found, 448.2026.

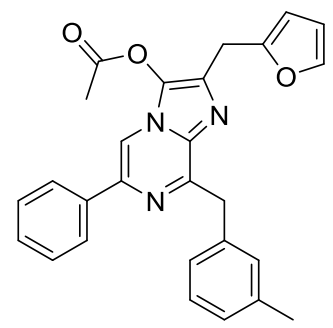

2-(Furan-2-ylmethyl)-8-(3-methylbenzyl)-6-phenylimidazo[1,2-a]pyrazin-3-yl acetate 25\{1,12,37\} (YJ31068-0391): Obtained as a white solid $(0.23 \mathrm{~g}, 70 \%)$ after a recrystallization in n-heptane. ${ }^{1} \mathrm{H}$ NMR $\left(\mathrm{CDCl}_{3}\right): 7.93(\mathrm{~m}, 2 \mathrm{H})$, $7.84(\mathrm{~s}, 1 \mathrm{H}), 7.49-7.37(\mathrm{~m}, 6 \mathrm{H}), 7.22(\mathrm{~m}, 1 \mathrm{H}), 7.05(\mathrm{~m}, 1 \mathrm{H}), 6.35(\mathrm{dd}, 1 \mathrm{H}, J=1.7,3.1 \mathrm{~Hz}), 6.16(\mathrm{~m}, 1 \mathrm{H}), 4.60(\mathrm{~s}$, $2 \mathrm{H}), 4.24(\mathrm{~s}, 2 \mathrm{H}), 2.36(\mathrm{~s}, 3 \mathrm{H}), 2.35$ (s, 3H). ${ }^{13} \mathrm{C} \mathrm{NMR}\left(\mathrm{CDCl}_{3}\right)$ : 167.1, 153.1, 151.6, 141.6, 139.2, 137.8, 137.7, 136.8, 133.6, 132.5, 130.5, 128.9, 128.8, 128.6, 128.2, 127.2, 126.7, 126.4, 110.5, 108.9, 106.8, 39.3, 27.2, 21.4, 20.1. HRMS $(m / z):[\mathrm{M}+\mathrm{H}]^{+}$calcd for $\mathrm{C}_{27} \mathrm{H}_{24} \mathrm{~N}_{3} \mathrm{O}_{3}, 438.1818$; found, 438.1820 .

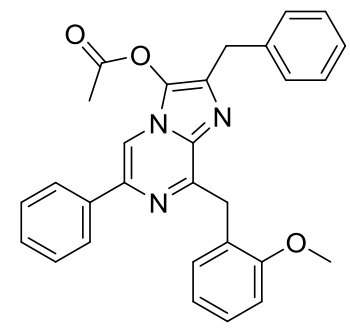

2-Benzyl-8-(2-methoxybenzyl)-6-phenylimidazo[1,2-a]pyrazin-3-yl acetate $\quad \mathbf{2 5}\{1,14,1\} \quad$ (YJ31070-117-1): Obtained as a white solid $(0.06 \mathrm{~g}, 70 \%)$ after a recrystallization from $n$-heptane. ${ }^{1} \mathrm{H}$ NMR $\left(\mathrm{CDCl}_{3}\right): 7.84(\mathrm{~m}, 2 \mathrm{H})$, $7.82(\mathrm{~s}, 1 \mathrm{H}), 7.43-7.23(\mathrm{~m}, 10 \mathrm{H}), 6.93(\mathrm{~m}, 2 \mathrm{H}), 4.69(\mathrm{~s}, 2 \mathrm{H}), 4.22(\mathrm{~s}, 2 \mathrm{H}), 3.83(\mathrm{~s}, 3 \mathrm{H}), 2.18(\mathrm{~s}, 3 \mathrm{H}) .{ }^{13} \mathrm{C}$ NMR $\left(\mathrm{CDCl}_{3}\right): 167.1,157.8,153.0,138.8,138.1,136.8,134.8,133.9,131.0,129.1,128.7,128.5,128.4,127.7,126.5$, 126.4, 126.3, 120.3, 110.6, 108.5, 55.6, 34.2, 33.2, 19.9. HRMS $(\mathrm{m} / \mathrm{z})$ : $[\mathrm{M}+\mathrm{H}]^{+}$calcd for $\mathrm{C}_{29} \mathrm{H}_{26} \mathrm{~N}_{3} \mathrm{O}_{3}: 464.1974$; found, 464.1983 .<smiles>CC(=O)Oc1c(Cc2ccccc2)nc(Cc2c(F)cccc2F)n1Cc1ccccc1-c1ccccc1F</smiles>

2-Benzyl-8-(2,6-difluorobenzyl)-6-phenylimidazo[1,2-a]pyrazin-3-yl acetate $25\{1,21,1\}$ (YJ 33069-173-1): Obtained as a solid $(0.40 \mathrm{~g}, 95 \%)$ after dispertion of the reaction media in water, a filtration and drying under vacuum at $40^{\circ} \mathrm{C} .{ }^{1} \mathrm{H}$ NMR $\left(\mathrm{CDCl}_{3}\right): 7.84(\mathrm{~s}, 1 \mathrm{H}), 7.75(\mathrm{~m}, 2 \mathrm{H}), 7.34(\mathrm{~m}, 9 \mathrm{H}), 6.97(\mathrm{~m}, 2 \mathrm{H}), 4.77(\mathrm{~s}, 2 \mathrm{H}), 4.24(\mathrm{~s}$, 2H), 2.19 (s, 3H). ${ }^{13} \mathrm{C} \mathrm{NMR}\left(\mathrm{CDCl}_{3}\right): 167.1,161.8(\mathrm{dd}, \mathrm{J}=248,8 \mathrm{~Hz}), 150.2,140.9,138.7,137.8,136.4,134.8$, 133.2, 129.1, 128.9, 128.6, 128.5, $128.3(10 \mathrm{~Hz}), 126.5,126.1,113.2(20 \mathrm{~Hz}), 110.8(7,19 \mathrm{~Hz}), 108.8,34.2,26.2$, 19.8. HRMS $(\mathrm{m} / z):[\mathrm{M}+\mathrm{H}]^{+}$calcd for $\mathrm{C}_{28} \mathrm{H}_{22} \mathrm{~F}_{2} \mathrm{~N}_{3} \mathrm{O}_{2}: 470.1680$; found, 470.1680 . 


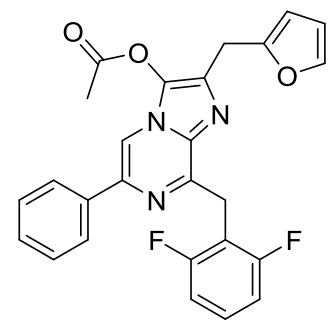

8-(2,6-Difluorobenzyl)-2-(furan-2-ylmethyl)-6-phenylimidazo[1,2-a]pyrazin-3-yl acetate 25 1,21,37\} (EC31095085-2): Obtained as a solid $(0.26 \mathrm{~g}, 69 \%)$ after a recrystallization from $n$-heptane. ${ }^{1} \mathrm{H}$ NMR $\left(\mathrm{CDCl}_{3}\right): 7.88(\mathrm{~s}, 1 \mathrm{H})$, $7.75(\mathrm{~m}, 2 \mathrm{H}), 7.35(\mathrm{~m}, 5 \mathrm{H}), 6.96(\mathrm{~m}, 2 \mathrm{H}), 6.36(\mathrm{dd}, 1 \mathrm{H}, J=3.2,1.9 \mathrm{~Hz}), 6.19(\mathrm{~m}, 1 \mathrm{H}), 4.75(\mathrm{~s}, 2 \mathrm{H}), 4.26(\mathrm{~s}, 2 \mathrm{H})$, 2.38 (s, 3H). ${ }^{13} \mathrm{C}$ NMR $\left(\mathrm{CDCl}_{3}\right): 167.2,162.1(248,8 \mathrm{~Hz}), 151.4,150.3,141.7,138.6,136.4,133.4,132.3,128.9$, 128.7, 128.5, $128.3(10 \mathrm{~Hz}), 126.1,113.1(20 \mathrm{~Hz}), 110.9,110.5,108.8,106.9,27.3,26.1,20.1$. HRMS $(\mathrm{m} / \mathrm{z})$ : $[\mathrm{M}+\mathrm{H}]^{+}$calcd for $\mathrm{C}_{26} \mathrm{H}_{20} \mathrm{~F}_{2} \mathrm{~N}_{3} \mathrm{O}_{3}: 460.1473$; found, 460.1469 .

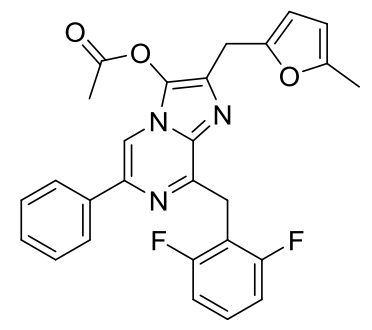

8-(2,6-Difluorobenzyl)-2-((5-methylfuran-2-yl)methyl)-6-phenylimidazo[1,2-a]pyrazin-3-yl acetate $\mathbf{2 5}\{1,21,42\}$ (EC32712-093-2): Obtained as a solid $(0.29 \mathrm{~g}, 82 \%)$ after a recrystallization from $n$-heptane. ${ }^{1} \mathrm{H}$ NMR $\left(\mathrm{CDCl}_{3}\right)$ : $7.88(\mathrm{~s}, 1 \mathrm{H}), 7.75(\mathrm{~m}, 2 \mathrm{H}), 7.34(\mathrm{~m}, 4 \mathrm{H}), 6.96(\mathrm{~m}, 2 \mathrm{H}), 6.05(\mathrm{~d}, 1 \mathrm{H}, J=3.0 \mathrm{~Hz}), 5.92(\mathrm{dd}, 1 \mathrm{H}, J=3.0,1.1 \mathrm{~Hz})$, $4.75(\mathrm{~s}, 2 \mathrm{H}), 4.21(\mathrm{~s}, 2 \mathrm{H}), 2.38(\mathrm{~s}, 3 \mathrm{H}), 2.29(\mathrm{~s}, 3 \mathrm{H}) .{ }^{13} \mathrm{C} \mathrm{NMR}\left(\mathrm{CDCl}_{3}\right): 167.0,162.1(248,8 \mathrm{~Hz}), 151.2,150.2$, 149.3, 138.7, 136.4, 133.1, 132.5, 128.9, 128.6, 128.5, $128.3(10 \mathrm{~Hz}), 126.1,113.1$ (20 Hz), 110.8 (br), 108.8, 107.5, 106.2, 27.3, $26.2(2 \mathrm{~Hz}), 20.1,13.5$. HRMS $(\mathrm{m} / \mathrm{z})$ : $[\mathrm{M}+\mathrm{H}]^{+}$calcd for $\mathrm{C}_{27} \mathrm{H}_{22} \mathrm{~F}_{2} \mathrm{~N}_{3} \mathrm{O}_{3}$ : 474.1629; found, 474.1620.

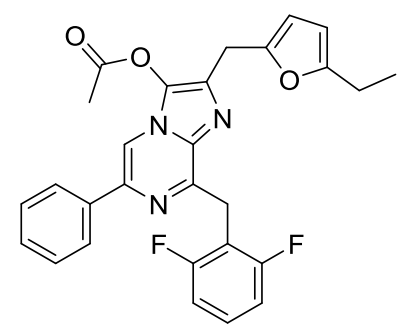

8-(2,6-Difluorobenzyl)-2-((5-ethylfuran-2-yl)methyl)-6-phenylimidazo[1,2-a]pyrazin-3-yl acetate $\mathbf{2 5}\{1,21,44\}$ (EC32712-125-2): Obtained as a solid $(0.18 \mathrm{~g}, 70 \%)$ after a recrystallization from $n$-heptane. ${ }^{1} \mathrm{H}$ NMR $\left(\mathrm{CDCl}_{3}\right)$ : $7.88(\mathrm{~s}, 1 \mathrm{H}), 7.75(\mathrm{~m}, 2 \mathrm{H}), 7.33(\mathrm{~m}, 4 \mathrm{H}), 6.97(\mathrm{~m}, 2 \mathrm{H}), 6.06(\mathrm{~d}, 1 \mathrm{H}, J=3.0 \mathrm{~Hz}), 5.93(\mathrm{~d}, 1 \mathrm{H}, J=3.0 \mathrm{~Hz}), 4.75(\mathrm{~s}$, $2 \mathrm{H}), 4.21(\mathrm{~s}, 2 \mathrm{H}), 2.64(\mathrm{q}, 2 \mathrm{H}, J=7.5 \mathrm{~Hz}), 2.37(\mathrm{~s}, 3 \mathrm{H}), 1.24(\mathrm{t}, 3 \mathrm{H}, J=7.5 \mathrm{~Hz}) .{ }^{13} \mathrm{C}$ NMR $\left(\mathrm{CDCl}_{3}\right): 167.0,162.1$ $(248,9 \mathrm{~Hz}), 157.0,150.3,149.3,138.5,136.5,133.3,132.6,128.9,128.6,128.5,128.3(10 \mathrm{~Hz}), 126.1,113.2(20$ $\mathrm{Hz}), 110.8(\mathrm{br}), 108.8,107.3,104.6,27.4,26.1$ (br), 21.4, 20.1, 12.2. HRMS $(\mathrm{m} / \mathrm{z}):[\mathrm{M}+\mathrm{H}]^{+}$calcd for $\mathrm{C}_{28} \mathrm{H}_{24} \mathrm{~F}_{2} \mathrm{~N}_{3} \mathrm{O}_{3}$ : 488.1786; found, 488.1785<smiles></smiles> 
$\mathbf{2 5}\{1,21,48\}$ (EC32712-127-2): Obtained as a solid $(0.23 \mathrm{~g}, 75 \%)$ after a recrystallization from $n$-heptane. ${ }^{1} \mathrm{H}$ NMR $\left(\mathrm{CDCl}_{3}\right): 7.88(\mathrm{~s}, 1 \mathrm{H}), 7.75(\mathrm{~m}, 2 \mathrm{H}), 7.34(\mathrm{~m}, 4 \mathrm{H}), 6.96(\mathrm{~m}, 2 \mathrm{H}), 5.95(\mathrm{~s}, 1 \mathrm{H}), 4.75(\mathrm{~s}, 2 \mathrm{H}), 4.16(\mathrm{~s}, 2 \mathrm{H}), 2.38(\mathrm{~s}$, $3 \mathrm{H}), 2.19(\mathrm{~s}, 3 \mathrm{H}), 1.93(\mathrm{~s}, 3 \mathrm{H}) .{ }^{13} \mathrm{C}$ NMR $\left(\mathrm{CDCl}_{3}\right): 167.0,162.1(248,9 \mathrm{~Hz}), 150.3,148.2,146.3,138.5,136.5$, 133.3, 132.7, 128.9, 128.6, 128.5, $128.2(10 \mathrm{~Hz}), 126.1,114.6,113.2(20 \mathrm{~Hz}), 110.8(\mathrm{br}), 110.1,108.8,27.2,26.1$ (2 Hz), 20.0, 11.3, 9.8. HRMS (m/z): $[\mathrm{M}+\mathrm{H}]^{+}$calcd for $\mathrm{C}_{28} \mathrm{H}_{24} \mathrm{~F}_{2} \mathrm{~N}_{3} \mathrm{O}_{3}: 488.1786$; found, 488.1787.

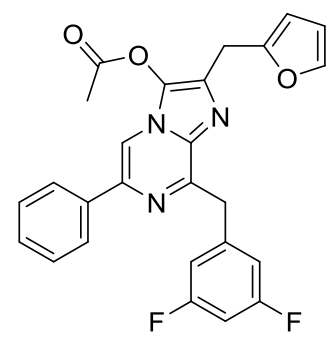

8-(3,5-Difluorobenzyl)-2-(furan-2-ylmethyl)-6-phenylimidazo[1,2-a]pyrazin-3-yl acetate 25\{1,22,37\} (EC32712007-1): Obtained as a solid $(0.29 \mathrm{~g}, 61 \%)$ after a recrystallization from a mixture of $n$-heptane and cyclohexane (two crops). ${ }^{1} \mathrm{H}$ NMR $\left(\mathrm{CDCl}_{3}\right): 7.90(\mathrm{~m}, 2 \mathrm{H}), 7.87(\mathrm{~s}, 1 \mathrm{H}), 7.48(\mathrm{~m}, 2 \mathrm{H}), 7.43(\mathrm{~m}, 1 \mathrm{H}), 7.38(\mathrm{dd}, 1 \mathrm{H}, J=1.9,0.9$ $\mathrm{Hz}), 7.14(\mathrm{~m}, 2 \mathrm{H}), 6.68(\mathrm{tt}, 1 \mathrm{H}, J=9.1,2.4 \mathrm{~Hz}), 6.36(\mathrm{dd}, 1 \mathrm{H}, J=3.2,1.9 \mathrm{~Hz}), 6.17(\mathrm{~m}, 1 \mathrm{H}), 4.59(\mathrm{~s}, 2 \mathrm{H}), 4.24(\mathrm{~s}$, 2H), $2.38(\mathrm{~s}, 3 \mathrm{H}) .{ }^{13} \mathrm{C}$ NMR $\left(\mathrm{CDCl}_{3}\right): 167.1,162.8(248,13 \mathrm{~Hz}), 151.6,151.3,141.7,141.4(9 \mathrm{~Hz}), 139.2,136.5$, 133.4, 132.9, 129.0, 128.9, 128.8, 126.4, 112.5 (br), 110.5, 109.2, 106.9, $102.0(25 \mathrm{~Hz}), 38.9$ (2 Hz), 27.2, 20.1. HRMS $(m / z):[\mathrm{M}+\mathrm{H}]^{+}$calcd for $\mathrm{C}_{26} \mathrm{H}_{20} \mathrm{~F}_{2} \mathrm{~N}_{3} \mathrm{O}_{3}: 460.1473$; found, 460.1473 .

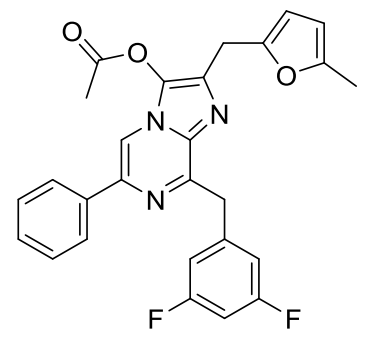

8-(3,5-Difluorobenzyl)-2-((5-methylfuran-2-yl)methyl)-6-phenylimidazo[1,2-a]pyrazin-3-yl acetate $\mathbf{2 5}\{1,22,42\}$ (EC32712-011-1): Obtained as a solid $(0.24 \mathrm{~g}, 81 \%)$ after a recrystallization from $n$-heptane. ${ }^{1} \mathrm{H}$ NMR $\left(\mathrm{CDCl}_{3}\right)$ : $7.90(\mathrm{~m}, 2 \mathrm{H}), 7.87(\mathrm{~s}, 1 \mathrm{H}), 7.48(\mathrm{~m}, 2 \mathrm{H}), 7.42(\mathrm{~m}, 1 \mathrm{H}), 7.14(\mathrm{~m}, 2 \mathrm{H}), 6.68(\mathrm{tt}, 1 \mathrm{H}, J=9.1,2.3 \mathrm{~Hz}), 6.03(\mathrm{~d}, 1 \mathrm{H}, J=$ $3.1 \mathrm{~Hz}), 5.92(\mathrm{~m}, 1 \mathrm{H}), 4.59(\mathrm{~s}, 2 \mathrm{H}), 4.18(\mathrm{~s}, 2 \mathrm{H}), 2.38(\mathrm{~s}, 3 \mathrm{H}), 2.28(\mathrm{~d}, 3 \mathrm{H}, J=1.0 \mathrm{~Hz}) .{ }^{13} \mathrm{C} \mathrm{NMR}\left(\mathrm{CDCl}_{3}\right): 167.0$, $162.8(248,13 \mathrm{~Hz}), 151.5,151.3,149.3,141.4(9 \mathrm{~Hz}), 139.2,136.5,133.3,133.2,129.0,128.9,128.7,126.4,112.6$ (br), 109.2, 107.5, 106.2, $102.0(25 \mathrm{~Hz}), 38.9,27.3,20.1,13.5$. HRMS $(\mathrm{m} / \mathrm{z}):[\mathrm{M}+\mathrm{H}]^{+}$calcd for $\mathrm{C}_{27} \mathrm{H}_{22} \mathrm{~F}_{2} \mathrm{~N}_{3} \mathrm{O}_{3}$ : 474.1629; found, 474.1631 .

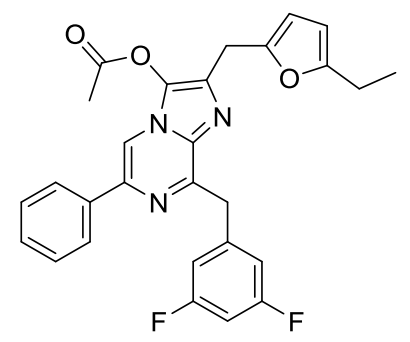

8-(3,5-Difluorobenzyl)-2-((5-ethylfuran-2-yl)methyl)-6-phenylimidazo[1,2-a]pyrazin-3-yl acetate $\mathbf{2 5}\{1,22,44\}$ (EC32712-123-2): Obtained as a solid $(0.13 \mathrm{~g}, 45 \%)$ after a recrystallization from $n$-heptane. ${ }^{1} \mathrm{H}$ NMR $\left(\mathrm{CDCl}_{3}\right)$ : $7.91(\mathrm{~m}, 2 \mathrm{H}), 7.87(\mathrm{~s}, 1 \mathrm{H}), 7.48(\mathrm{~m}, 2 \mathrm{H}), 7.42(\mathrm{~m}, 1 \mathrm{H}), 7.14(\mathrm{~m}, 2 \mathrm{H}), 6.68(\mathrm{tt}, 1 \mathrm{H}, J=9.1,2.4 \mathrm{~Hz}), 6.04(\mathrm{~d}, 1 \mathrm{H}, J=$ $3.2 \mathrm{~Hz}), 5.93(\mathrm{dt}, 1 \mathrm{H}, J=3.2,1.0 \mathrm{~Hz}), 4.60(\mathrm{~s}, 2 \mathrm{H}), 4.19(\mathrm{~s}, 2 \mathrm{H}), 2.63(\mathrm{qd}, 2 \mathrm{H}, J=7.6,1.0 \mathrm{~Hz}), 2.37(\mathrm{~s}, 3 \mathrm{H}), 1.24$ $(\mathrm{t}, 3 \mathrm{H}, J=7.6 \mathrm{~Hz}) .{ }^{13} \mathrm{C} \mathrm{NMR}\left(\mathrm{CDCl}_{3}\right): 167.0,162.8(248,13 \mathrm{~Hz}), 157.1,151.5,149.2,141.4(10 \mathrm{~Hz}), 139.2,136.5$, 133.4, 133.3, 129.0, 128.8, 128.7, 126.4, 112.6 (br), 109.2, 107.3, 104.6, $102.0(25 \mathrm{~Hz}), 38.9$ (2 Hz), 27.3, 21.3, 20.1, 12.2. HRMS $(\mathrm{m} / \mathrm{z}):[\mathrm{M}+\mathrm{H}]^{+}$calcd for $\mathrm{C}_{28} \mathrm{H}_{24} \mathrm{~F}_{2} \mathrm{~N}_{3} \mathrm{O}_{3}$ : 488.1786; found, 488.1776. 


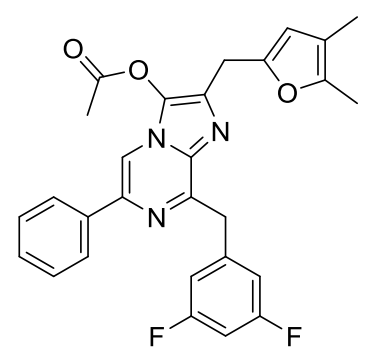

8-(3,5-difluorobenzyl)-2-((4,5-dimethylfuran-2-yl)methyl)-6-phenylimidazo[1,2-a]pyrazin-3-yl

acetate 25 $\{1,22,48\}$ (EC32712-101-2): Obtained as a solid (0.15 g, 72\%) after a chromatography over silica gel (cyclohexane - ethyl acetate 6/) and a recrystallization from $n$-heptane. ${ }^{1} \mathrm{H}$ NMR $\left(\mathrm{CDCl}_{3}\right): 7.91(\mathrm{~m}, 2 \mathrm{H}), 7.87(\mathrm{~s}$, $1 \mathrm{H}), 7.46(\mathrm{~m}, 3 \mathrm{H}), 7.15(\mathrm{~m}, 2 \mathrm{H}), 6.68(\mathrm{tt}, 1 \mathrm{H}, J=9.1,2.4 \mathrm{~Hz}), 5.93(\mathrm{~s}, 1 \mathrm{H}), 4.60(\mathrm{~s}, 2 \mathrm{H}), 4.14(\mathrm{~s}, 2 \mathrm{H}), 2.39(\mathrm{~s}, 3 \mathrm{H})$, 2.19 (s, 3H), 1.93 (s, 3H). ${ }^{13} \mathrm{C}$ NMR $\left(\mathrm{CDCl}_{3}\right): 167.0,162.8(247,13 \mathrm{~Hz}), 151.4,148.0,146.4,141.4(9 \mathrm{~Hz}), 139.3$, 136.5, 133.3, 129.0, 128.8, 128.7, 126.4, 114.6, 112.5 (br), 110.1, 109.2, 102.0 (25 Hz), 38.9, 27.1, 20.0, 11.2, 9.8, (one signal missing). HRMS (m/z): $[\mathrm{M}+\mathrm{H}]^{+}$calcd for $\mathrm{C}_{28} \mathrm{H}_{24} \mathrm{~F}_{2} \mathrm{~N}_{3} \mathrm{O}_{3}$ : 488.1786; found, 488.1781.

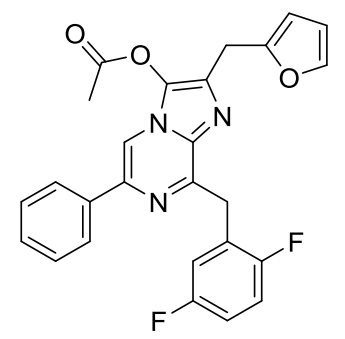

8-(2,5-Difluorobenzyl)-2-(furan-2-ylmethyl)-6-phenylimidazo[1,2-a]pyrazin-3-yl acetate 25\{1,23,37\} (EC31095141-1): Obtained as a solid (0.15 g, 54\%) after a chromatography over silica gel (cyclohexane - ethyl acetate 5/1). ${ }^{1} \mathrm{H}$ NMR $\left(\mathrm{CDCl}_{3}\right): 7.89(\mathrm{~s}, 1 \mathrm{H}), 7.85(\mathrm{~m}, 2 \mathrm{H}), 7.41(\mathrm{~m}, 4 \mathrm{H}), 7.20(\mathrm{ddd}, 1 \mathrm{H}, J=8.9,5.7,3.2 \mathrm{~Hz}), 7.05(\mathrm{td}, 1 \mathrm{H}, J=$ 8.9, $4.5 \mathrm{~Hz}), 6.93(\mathrm{~m}, 1 \mathrm{H}), 6.35(\mathrm{dd}, 1 \mathrm{H}, J=3.2,1.9 \mathrm{~Hz}), 6.18(\mathrm{dd}, 1 \mathrm{H}, J=3.1,0.9 \mathrm{~Hz}), 4.67(\mathrm{~s}, 2 \mathrm{H}), 4.24(\mathrm{~s}, 2 \mathrm{H})$, $2.38(\mathrm{~s}, 3 \mathrm{H}) .{ }^{13} \mathrm{C}$ NMR $\left(\mathrm{CDCl}_{3}\right): 167.1,158.4(241,2 \mathrm{~Hz}), 157.3(242,2 \mathrm{~Hz}), 151.3,151.0,141.7,139.1,136.4$, 133.5, 132.7, 128.8, 128.7, 126.3, $126.2(18,8 \mathrm{~Hz}), 118.2(24,4 \mathrm{~Hz}), 116.0(25,9 \mathrm{~Hz}), 114.5(24,8 \mathrm{~Hz}), 110.5$, 109.0, 106.9, 32.2, 27.1, 20.1. HRMS $(m / z):[\mathrm{M}+\mathrm{H}]^{+}$calcd for $\mathrm{C}_{26} \mathrm{H}_{20} \mathrm{~F}_{2} \mathrm{~N}_{3} \mathrm{O}_{3}$ : 460.1473; found, 460.1477 .

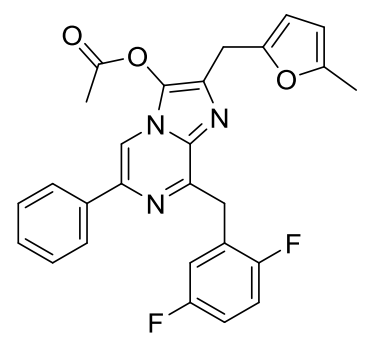

8-(2,5-Difluorobenzyl)-2-((5-methylfuran-2-yl)methyl)-6-phenylimidazo[1,2-a]pyrazin-3-yl acetate 25\{1,23,42\} (EC32712-045-1): Obtained as a solid $(0.11 \mathrm{~g}, 65 \%)$ after a recrystallization from $n$-heptane. ${ }^{1} \mathrm{H}$ NMR $\left(\mathrm{CDCl}_{3}\right)$ : $7.89(\mathrm{~s}, 1 \mathrm{H}), 7.85(\mathrm{~m}, 2 \mathrm{H}), 7.44(\mathrm{~m}, 2 \mathrm{H}), 7.39(\mathrm{~m}, 1 \mathrm{H}), 7.21(\mathrm{ddd}, 1 \mathrm{H}, J=8.9,5.7,3.2 \mathrm{~Hz}), 7.05(\mathrm{td}, 1 \mathrm{H}, J=8.9$, $4.6 \mathrm{~Hz}), 6.93(\mathrm{~m}, 1 \mathrm{H}), 6.04(\mathrm{~d}, 1 \mathrm{H}, J=3.1 \mathrm{~Hz}), 5.92(\mathrm{~m}, 1 \mathrm{H}), 4.67(\mathrm{~s}, 2 \mathrm{H}), 4.19(\mathrm{~s}, 2 \mathrm{H}), 2.38(\mathrm{~s}, 3 \mathrm{H}), 2.28(\mathrm{~s}, 3 \mathrm{H})$. ${ }^{13} \mathrm{C}$ NMR $\left(\mathrm{CDCl}_{3}\right)$ : 167.0, $158.4(241,2 \mathrm{~Hz}), 157.3(242,2 \mathrm{~Hz}), 151.2,151.0,149.4,139.0,136.5,133.4,133.0$, 129.0, 128.8, 128.6, $126.3(19,8 \mathrm{~Hz}), 126.2,118.2(24,5 \mathrm{~Hz}), 116.0(25,9 \mathrm{~Hz}), 114.5(24,8 \mathrm{~Hz}), 109.0,107.5$, 106.2, $32.1(\mathrm{~m}), 27.3,20.1,13.5$. HRMS $(\mathrm{m} / \mathrm{z}):[\mathrm{M}+\mathrm{H}]^{+}$calcd for $\mathrm{C}_{27} \mathrm{H}_{22} \mathrm{~F}_{2} \mathrm{~N}_{3} \mathrm{O}_{3}: 474.1629$; found, 474.1630. 


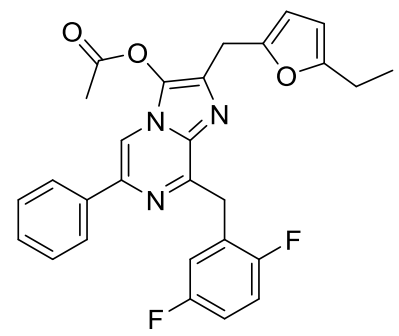

8-(2,5-Difluorobenzyl)-2-((5-ethylfuran-2-yl)methyl)-6-phenylimidazo[1,2-a]pyrazin-3-yl acetate $\mathbf{2 5}\{1,23,44\}$ (EC32712-097-2): Obtained as a solid (0.08 g, 57\%) after a chromatography over silica gel (cyclohexane - ethyl acetate 5/1). ${ }^{1} \mathrm{H}$ NMR $\left(\mathrm{CDCl}_{3}\right): 7.89(\mathrm{~s}, 1 \mathrm{H}), 7.85(\mathrm{~m}, 2 \mathrm{H}), 7.43(\mathrm{~m}, 3 \mathrm{H}), 7.21(\mathrm{ddd}, 1 \mathrm{H}, J=8.9,5.7,3.2 \mathrm{~Hz}), 7.05$ $(\mathrm{td}, 1 \mathrm{H}, J=8.9,4.5 \mathrm{~Hz}), 6.93(\mathrm{~m}, 1 \mathrm{H}), 6.05(\mathrm{~d}, 1 \mathrm{H}, J=3.0 \mathrm{~Hz}), 5.92(\mathrm{~d}, 1 \mathrm{H}, J=2.9 \mathrm{~Hz}), 4.67(\mathrm{~s}, 2 \mathrm{H}), 4.20(\mathrm{~s}, 2 \mathrm{H})$, $2.63(\mathrm{q}, 2 \mathrm{H}, J=7.5 \mathrm{~Hz}), 2.37(\mathrm{~s}, 3 \mathrm{H}), 1.24(\mathrm{t}, 3 \mathrm{H}, J=7.5 \mathrm{~Hz}) \cdot{ }^{13} \mathrm{C} \mathrm{NMR}\left(\mathrm{CDCl}_{3}\right): 167.0,158.4(241,2 \mathrm{~Hz}), 157.3$ (243, $3 \mathrm{~Hz}), 157.1,150.9,149.1,139.1,136.4,133.3,132.9,129.0,128.8,128.7,126.3,126.2(19,8 \mathrm{~Hz}), 118.2$ $(24,4 \mathrm{~Hz}), 116.0(25,9 \mathrm{~Hz}), 114.5(24,8 \mathrm{~Hz}), 109.0,107.4,104.6,32.2,27.3,21.3,20.1,12.2 . \mathrm{HRMS}(\mathrm{m} / \mathrm{z})$ : $[\mathrm{M}+\mathrm{H}]^{+}$calcd for $\mathrm{C}_{28} \mathrm{H}_{24} \mathrm{~F}_{2} \mathrm{~N}_{3} \mathrm{O}_{3}$ : 488.1786; found, 488.1786 .

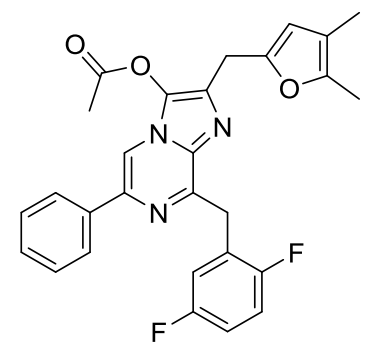

8-(2,5-Difluorobenzyl)-2-((4,5-dimethylfuran-2-yl)methyl)-6-phenylimidazo[1,2-a]pyrazin-3-yl acetate 25 $\{1,23,48\}$ (EC32712-095-2): Obtained as a solid $(0.17 \mathrm{~g}, 71 \%)$ after a chromatography over silica gel (cyclohexane - ethyl acetate 5/1). ${ }^{1} \mathrm{H}$ NMR $\left(\mathrm{CDCl}_{3}\right)$ : $7.89(\mathrm{~s}, 1 \mathrm{H}), 7.85(\mathrm{~m}, 2 \mathrm{H}), 7.42(\mathrm{~m}, 3 \mathrm{H}), 7.21(\mathrm{~m}, 1 \mathrm{H}), 7.05$ $(\mathrm{m}, 1 \mathrm{H}), 6.93(\mathrm{~m}, 1 \mathrm{H}), 5.94(\mathrm{~s}, 1 \mathrm{H}), 4.67(\mathrm{~s}, 3 \mathrm{H}), 4.14(\mathrm{~s}, 2 \mathrm{H}), 2.39(\mathrm{~s}, 3 \mathrm{H}), 2.19(\mathrm{~s}, 3 \mathrm{H}), 1.92(\mathrm{~s}, 4 \mathrm{H}) .{ }^{13} \mathrm{C} \mathrm{NMR}$ $\left(\mathrm{CDCl}_{3}\right)$ : 167.0, $158.4(241,2 \mathrm{~Hz}), 157.3$ (242, $\left.2 \mathrm{~Hz}\right), 150.9,148.0,146.3,139.0,136.4,133.3,133.0,129.0$, 128.8, 128.7, $126.3(19,8 \mathrm{~Hz}), 126.3,118.2$ (24, $5 \mathrm{~Hz}), 116.0(25,9 \mathrm{~Hz}), 114.6,114.5$ (24, $8 \mathrm{~Hz}), 110.1,109.0$, 32.2, 27.1, 20.0, 11.2, 9.8. HRMS (m/z): $[\mathrm{M}+\mathrm{H}]^{+}$calcd for $\mathrm{C}_{28} \mathrm{H}_{24} \mathrm{~F}_{2} \mathrm{~N}_{3} \mathrm{O}_{3}$ : 488.1786; found, 488.1785 .

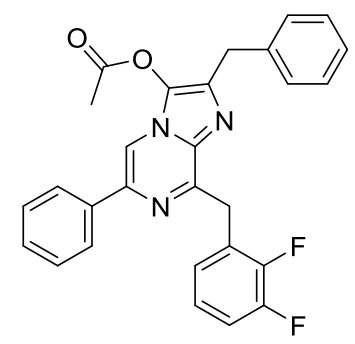

2-Benzyl-8-(2,3-difluorobenzyl)-6-phenylimidazo[1,2-a]pyrazin-3-yl acetate $\mathbf{2 5}\{1,24,1\}$ (YJ 33067-127-1): Obtained as a solid $(0.11 \mathrm{~g}, 65 \%)$ after a recrystallization from $n$-heptane. ${ }^{1} \mathrm{H}$ NMR $(\mathrm{CDCl} 3)$ : $7.85(\mathrm{~m}, 3 \mathrm{H}), 7.45-$ $722(\mathrm{~m}, 9 \mathrm{H}), 7.03(\mathrm{~m}, 3 \mathrm{H}), 4.73(\mathrm{~s}, 2 \mathrm{H}), 4.22(\mathrm{~s}, 2 \mathrm{H}), 2.02(\mathrm{~s}, 3 \mathrm{H}) .{ }^{13} \mathrm{C}$ NMR $(\mathrm{CDCl} 3): 167.1,150.9,150.7(248$ and $13 \mathrm{~Hz}), 149.5$ (246, and $12 \mathrm{~Hz}), 139.0,137.8,136.5,135.2,133.4,129.1,128.9,128.8,128.6,128.5,127.1(12$ $\mathrm{Hz}), 126.6,126.5(\mathrm{~m}), 126.2,123.5(4 \mathrm{and} 6 \mathrm{~Hz}), 115.5(18 \mathrm{~Hz}), 109.0,34.2,32.1,19.9 . \mathrm{HRMS}(\mathrm{m} / z):[\mathrm{M}+\mathrm{H}]^{+}$ calcd for $\mathrm{C}_{28} \mathrm{H}_{22} \mathrm{~F}_{2} \mathrm{~N}_{3} \mathrm{O}_{2}$ : 470.1680; found, 470.1661 . 


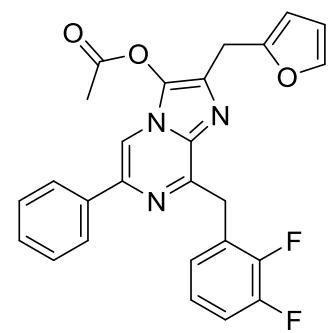

8-(2,3-Difluorobenzyl)-2-(furan-2-ylmethyl)-6-phenylimidazo[1,2-a]pyrazin-3-yl acetate 25 $\{1,24,37\}$ (EC32712021-1): Obtained as a solid $(0.24 \mathrm{~g}, 73 \%)$ after a recrystallization from $n$-heptane. ${ }^{1} \mathrm{H} \mathrm{NMR}\left(\mathrm{CDCl}_{3}\right): 7.88(\mathrm{~s}, 1 \mathrm{H})$, $7.85(\mathrm{~m}, 2 \mathrm{H}), 7.41(\mathrm{~m}, 4 \mathrm{H}), 7.22(\mathrm{tt}, 1 \mathrm{H}, J=6.1,1.7 \mathrm{~Hz}), 7.05(\mathrm{~m}, 2 \mathrm{H}), 6.36(\mathrm{dd}, 1 \mathrm{H}, J=3.2,1.9 \mathrm{~Hz}), 6.18(\mathrm{~m}$, $1 \mathrm{H}), 4.72(\mathrm{~s}, 2 \mathrm{H}), 4.25(\mathrm{~s}, 2 \mathrm{H}), 2.38(\mathrm{~s}, 3 \mathrm{H}) .{ }^{13} \mathrm{C} \mathrm{NMR}\left(\mathrm{CDCl}_{3}\right): 167.1,151.4,151.1,150.7(247,13 \mathrm{~Hz}), 149.4$ (248, $12 \mathrm{~Hz}), 141.7,139.0,136.4,133.5,132.6,129.0,128.8,128.7,127.1(13 \mathrm{~Hz}), 126.5(3 \mathrm{~Hz}), 126.2,123.5(7$, $5 \mathrm{~Hz}), 115.5(17 \mathrm{~Hz}), 110.5,109.0,106.9,32.0(2 \mathrm{~Hz}), 27.2,20.1$. HRMS $(m / z):[\mathrm{M}+\mathrm{H}]^{+}$calcd for $\mathrm{C}_{26} \mathrm{H}_{20} \mathrm{~F}_{2} \mathrm{~N}_{3} \mathrm{O}_{3}$ : 460.1473; found, 460.1473.

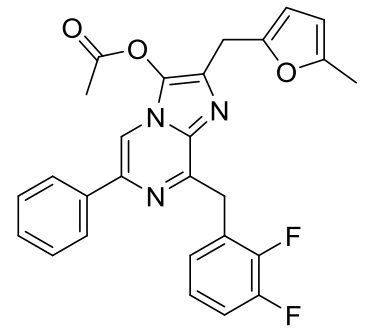

8-(2,3-Difluorobenzyl)-2-((5-methylfuran-2-yl)methyl)-6-phenylimidazo[1,2-a]pyrazin-3-yl acetate $25\{1,24,42\}$ (EC32712-043-1): Obtained as a solid $(0.22 \mathrm{~g}, 73 \%)$ after a recrystallization from $n$-heptane. ${ }^{1} \mathrm{H}$ NMR $\left(\mathrm{CDCl}_{3}\right)$ : $7.88(\mathrm{~s}, 1 \mathrm{H}), 7.85(\mathrm{~m}, 2 \mathrm{H}), 7.43(\mathrm{~m}, 2 \mathrm{H}), 7.38(\mathrm{~m}, 1 \mathrm{H}), 7.23(\mathrm{tt}, 1 \mathrm{H}, J=6.1,1.7 \mathrm{~Hz}), 7.05(\mathrm{~m}, 2 \mathrm{H}), 6.04(\mathrm{~d}, 1 \mathrm{H}, J=$ $3.0 \mathrm{~Hz}), 5.92(\mathrm{~m}, 1 \mathrm{H}), 4.72(\mathrm{~s}, 2 \mathrm{H}), 4.19(\mathrm{~s}, 2 \mathrm{H}), 2.38(\mathrm{~s}, 3 \mathrm{H}), 2.28(\mathrm{~s}, 3 \mathrm{H}) .{ }^{13} \mathrm{C} \mathrm{NMR}\left(\mathrm{CDCl}_{3}\right): 167.0,151.2,151.1$ $(247,13 \mathrm{~Hz}), 151.0,149.4(248,13 \mathrm{~Hz}), 149.4,138.9,136.5,133.4,133.0,129.0,128.8,128.6,127.1(13 \mathrm{~Hz})$, $126.5(3 \mathrm{~Hz}), 126.2,123.5(7,5 \mathrm{~Hz}), 115.5(17 \mathrm{~Hz}), 109.0,107.5,106.2,32.0(2 \mathrm{~Hz}), 27.3$, 20.1, 13.5. HRMS $(\mathrm{m} / \mathrm{z}):[\mathrm{M}+\mathrm{H}]^{+}$calcd for $\mathrm{C}_{27} \mathrm{H}_{22} \mathrm{~F}_{2} \mathrm{~N}_{3} \mathrm{O}_{3}$ : 474.1629; found, 474.1629.

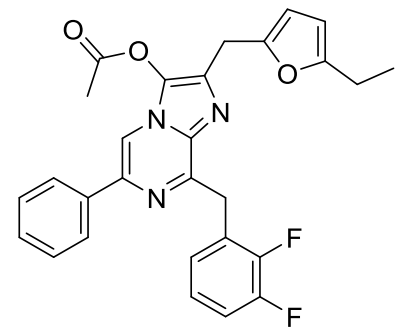

8-(2,3-Difluorobenzyl)-2-((5-ethylfuran-2-yl)methyl)-6-phenylimidazo[1,2-a]pyrazin-3-yl acetate $25\{1,24,44\}$ (EC32712-121-2): Obtained as a solid (0.32 g, 75\%) after a recrystallization from $n$-heptane. ${ }^{1} \mathrm{H} \mathrm{NMR}\left(\mathrm{CDCl}_{3}\right)$ : $7.88(\mathrm{~s}, 1 \mathrm{H}), 7.85(\mathrm{~m}, 2 \mathrm{H}), 7.44(\mathrm{~m}, 2 \mathrm{H}), 7.39(\mathrm{~m}, 1 \mathrm{H}), 7.23(\mathrm{~m}, 1 \mathrm{H}), 7.05(\mathrm{~m}, 2 \mathrm{H}), 6.05(\mathrm{~d}, 1 \mathrm{H}, J=3.0 \mathrm{~Hz}), 5.93$ $(\mathrm{d}, 1 \mathrm{H}, J=3.0 \mathrm{~Hz}), 4.72(\mathrm{~s}, 2 \mathrm{H}), 4.20(\mathrm{~s}, 2 \mathrm{H}), 2.63(\mathrm{q}, 2 \mathrm{H}, J=7.5 \mathrm{~Hz}), 2.37(\mathrm{~s}, 3 \mathrm{H}), 1.24(\mathrm{t}, 3 \mathrm{H}, J=7.6 \mathrm{~Hz}) .{ }^{13} \mathrm{C}$ NMR $\left(\mathrm{CDCl}_{3}\right): 167.0,157.1,151.0,150.7(247,13 \mathrm{~Hz}), 149.4(248,13 \mathrm{~Hz}), 149.2,138.9,136.5,133.4,133.0$, 129.0, 128.8, 128.6, 127.1 (13 Hz), 126.5 (3 Hz), 126.2, 123.5 (7, 5 Hz), 115.5 (17 Hz), 109.0, 107.3, 104.6, 32.0 (m), 27.4, 21.4, 20.1, 12.2. HRMS (m/z): $[\mathrm{M}+\mathrm{H}]^{+}$calcd for $\mathrm{C}_{28} \mathrm{H}_{24} \mathrm{~F}_{2} \mathrm{~N}_{3} \mathrm{O}_{3}$ : 488.1786; found, 488.1783. 


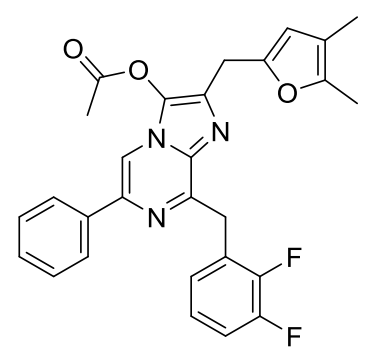

8-(2,3-Difluorobenzyl)-2-((4,5-dimethylfuran-2-yl)methyl)-6-phenylimidazo[1,2-a]pyrazin-3-yl

acetate 25 $\{1,24,48\}$ (EC32712-099-2): Obtained as a solid $(0.14 \mathrm{~g}, 74 \%)$ after a chromatography over silica gel (cyclohexane - ethyl acetate 5/1). ${ }^{1} \mathrm{H}$ NMR $\left(\mathrm{CDCl}_{3}\right)$ : $7.88(\mathrm{~s}, 1 \mathrm{H}), 7.85(\mathrm{~m}, 2 \mathrm{H}), 7.41(\mathrm{~m}, 3 \mathrm{H}), 7.22(\mathrm{~m}, 1 \mathrm{H}), 7.05$ $(\mathrm{m}, 2 \mathrm{H}), 5.94(\mathrm{~s}, 1 \mathrm{H}), 4.72(\mathrm{~s}, 2 \mathrm{H}), 4.15(\mathrm{~s}, 2 \mathrm{H}), 2.39(\mathrm{~s}, 3 \mathrm{H}), 2.19(\mathrm{~s}, 3 \mathrm{H}), 1.93(\mathrm{~s}, 3 \mathrm{H}) .{ }^{13} \mathrm{C} \mathrm{NMR}\left(\mathrm{CDCl}_{3}\right): 167.0$, 150.9, $150.7(247,13 \mathrm{~Hz}), 149.4(248,13 \mathrm{~Hz}), 148.0,146.3,139.0,136.4,133.3,133.0,128.9,128.8,128.7,127.1$ $(13 \mathrm{~Hz}), 126.5(3 \mathrm{~Hz}), 126.2,123.5(7,5 \mathrm{~Hz}), 115.5(17 \mathrm{~Hz}), 114.6,110.1,109.0,32.1(2 \mathrm{~Hz}), 27.1,20.0,11.2,9.8$. HRMS $(\mathrm{m} / z)$ : $[\mathrm{M}+\mathrm{H}]^{+}$calcd for $\mathrm{C}_{28} \mathrm{H}_{24} \mathrm{~F}_{2} \mathrm{~N}_{3} \mathrm{O}_{3}$ : 488.1786; found, 488.1784 .

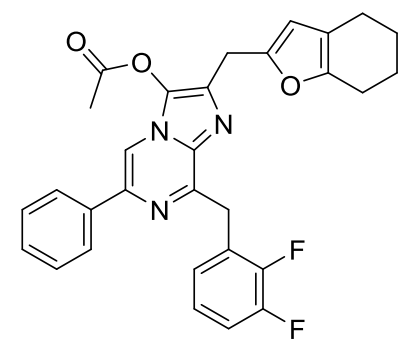

8-(2,3-difluorobenzyl)-6-phenyl-2-((4,5,6,7-tetrahydrobenzofuran-2-yl)methyl)imidazo[1,2-a]pyrazin-3-yl acetate 25 $\{1,24,49\}$ (YJ 33068-085-1): Obtained as a solid $(0.04 \mathrm{~g}, 12 \%)$ after a chromatography over silica gel (cyclohexane - ethyl acetate 6/1) and a recrystallization in $n$-heptane. ${ }^{1} \mathrm{H} \mathrm{NMR}\left(\mathrm{CDCl}_{3}\right): 7.88(\mathrm{~s}, 1 \mathrm{H}), 7.84(\mathrm{~m}$, $2 \mathrm{H}), 7.46-7.35(\mathrm{~m}, 3 \mathrm{H}), 7.21(\mathrm{~m}, 1 \mathrm{H}), 7.11-6.99(\mathrm{~m}, 2 \mathrm{H}), 5.97(\mathrm{~s}, 1 \mathrm{H}), 4.72(\mathrm{~s}, 2 \mathrm{H}), 4.18(\mathrm{~s}, 2 \mathrm{H}), 2.56(\mathrm{~m}, 2 \mathrm{H})$, $2.40(\mathrm{~m}, 2 \mathrm{H}), 2.39(\mathrm{~s}, 3 \mathrm{H}), 1.83(\mathrm{~m}, 2 \mathrm{H}), 1.72(\mathrm{~m}, 2 \mathrm{H}) .{ }^{13} \mathrm{C} \mathrm{NMR}\left(\mathrm{CDCl}_{3}\right): 167.1,150.9,150.7(13$ and $248 \mathrm{~Hz})$, 149.8, 149.5 (13 and $248 \mathrm{~Hz}), 148.6,139.1,136.4,133.2,132.9,129.9,128.8,128.7,127.0(13 \mathrm{~Hz}), 126.5(4 \mathrm{~Hz})$, 126.2, 123.5 (4 and $7 \mathrm{~Hz}), 117.6,115.5(17 \mathrm{~Hz}), 109.0,107.8$, . 32.1, 27.2, 23.2, 23.1 (two signals), 22.1, 22.1, 20.1. HRMS $(\mathrm{m} / \mathrm{z}):[\mathrm{M}+\mathrm{H}]^{+}$calcd for $\mathrm{C}_{30} \mathrm{H}_{26} \mathrm{~F}_{2} \mathrm{~N}_{3} \mathrm{O}_{3}: 514.1942$; found, 514.1949 .

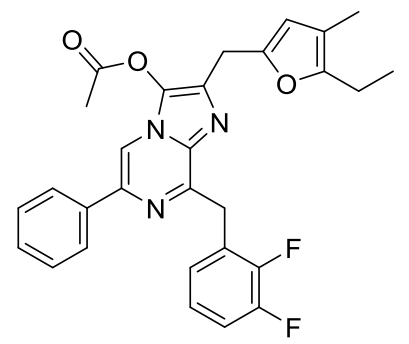

8-(2,3-Difluorobenzyl)-2-((5-ethyl-4-methylfuran-2-yl)methyl)-6-phenylimidazo[1,2-a]pyrazin-3-yl acetate 25 $\{1,24,50\}$ (YJ 33069-013-1): Obtained as a solid (0.28 g, 47\%) after a recrystallization from cyclohexane. ${ }^{1} \mathrm{H}$ NMR $\left(\mathrm{CDCl}_{3}\right): 7.88(\mathrm{~s}, 1 \mathrm{H}), 7.84(\mathrm{~m}, 2 \mathrm{H}), 7.43(\mathrm{~m}, 2 \mathrm{H}), 7.38(\mathrm{~m}, 1 \mathrm{H}), 7.22(\mathrm{~m}, 1 \mathrm{H}), 7.11-7.01(\mathrm{~m}, 2 \mathrm{H}), 5.93(\mathrm{~s}$, $1 \mathrm{H}), 4.73(\mathrm{~s}, 2 \mathrm{H}), 4.16(\mathrm{~s}, 2 \mathrm{H}), 2.57(\mathrm{q}, 2 \mathrm{H}, J=8.0 \mathrm{~Hz}), 2.37(\mathrm{~s}, 3 \mathrm{H}), 1.94(\mathrm{~s}, 3 \mathrm{H}), 1.19(\mathrm{t}, 3 \mathrm{H}, J=8.0 \mathrm{~Hz}){ }^{13} \mathrm{C}$ NMR $\left(\mathrm{CDCl}_{3}\right): 167.0,151.7,150.9,150.7(13$ and $248 \mathrm{~Hz}), 149.5(13$ and $248 \mathrm{~Hz}), 147.9,139.1,136.4,133.2$, 132.9, 128.9, 128.8, 128.7, $127.0(13 \mathrm{~Hz}), 126.5(4 \mathrm{~Hz}), 126.2,123.5(4$ and $7 \mathrm{~Hz}), 115.5(17 \mathrm{~Hz}), 113.7,110.2$, 109.0, 32.1, 27.1, 20.0, 19.3, 13.1, 9.7. HRMS (m/z): $[\mathrm{M}+\mathrm{H}]^{+}$calcd for $\mathrm{C}_{29} \mathrm{H}_{26} \mathrm{~F}_{2} \mathrm{~N}_{3} \mathrm{O}_{3}$ : 502.1942; found, 502.1943 . 


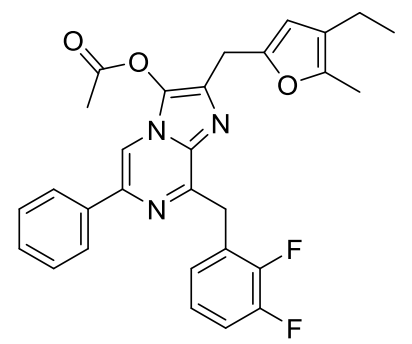

8-(2,3-Difluorobenzyl)-2-((4-ethyl-5-methylfuran-2-yl)methyl)-6-phenylimidazo[1,2-a]pyrazin-3-yl

acetate 25 $\{1,24,51\}$ (YJ33068-089-1): Obtained as a solid $(0.54 \mathrm{~g}, 62 \%)$ after a chromatography over silica gel (cyclohexane - ethyl acetate 6/1) and a recrystallization in $n$-heptane. ${ }^{1} \mathrm{H}$ NMR $\left(\mathrm{CDCl}_{3}\right): 7.88(\mathrm{~s}, 1 \mathrm{H}), 7.83(\mathrm{~m}$, $2 \mathrm{H}), 7.46-7.35(\mathrm{~m}, 3 \mathrm{H}), 7.21(\mathrm{~m}, 1 \mathrm{H}), 7.11-6.99(\mathrm{~m}, 2 \mathrm{H}), 5.99(\mathrm{~s}, 1 \mathrm{H}), 4.72(\mathrm{~s}, 2 \mathrm{H}), 4.16(\mathrm{~s}, 2 \mathrm{H}), 2.38(\mathrm{~s}, 3 \mathrm{H})$, $2.33(\mathrm{q}, 2 \mathrm{H}, J=7.4 \mathrm{~Hz}), 2.20(\mathrm{~s}, 3 \mathrm{H}), 1.13(\mathrm{t}, 3 \mathrm{H}, J=7.4 \mathrm{~Hz}) \cdot{ }^{13} \mathrm{C}$ NMR $\left(\mathrm{CDCl}_{3}\right): 167.0,150.9,150.7(13$ and 248 $\mathrm{Hz}), 148.2,149.5(13$ and $248 \mathrm{~Hz}), 145.6,139.0,136.4,133.3,133.0,129.0,128.8,128.6,127.0(13 \mathrm{~Hz}), 126.5(4$ $\mathrm{Hz}), 126.2,123.5(4$ and $7 \mathrm{~Hz}), 121.3,115.5(17 \mathrm{~Hz}), 109.0,108.4,32.0,27.2,20.1,18.1,14.9,11.3$. HRMS $(\mathrm{m} / z)$ : $[\mathrm{M}+\mathrm{H}]^{+}$calcd for $\mathrm{C}_{29} \mathrm{H}_{26} \mathrm{~F}_{2} \mathrm{~N}_{3} \mathrm{O}_{3}: 502.1942$; found, 502.1951.

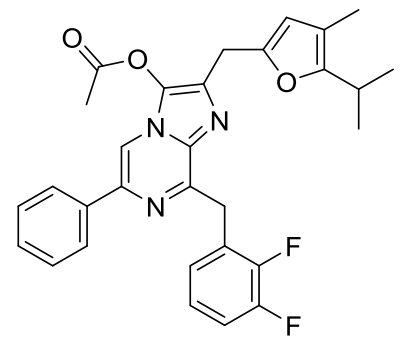

8-(2,3-Difluorobenzyl)-2-((5-isopropyl-4-methylfuran-2-yl)methyl)-6-phenylimidazo[1,2-a]pyrazin-3-yl acetate 25 $\{1,24,52\}$ (YJ 33069-063-1): Obtained as a solid (0.14 g, 26\%) after a (slow) recrystallization from $n$-heptane. ${ }^{1} \mathrm{H}$ NMR $\left(\mathrm{CDCl}_{3}\right): 7.88(\mathrm{~s}, 1 \mathrm{H}), 7.85(\mathrm{~m}, 2 \mathrm{H}), 7.43(\mathrm{~m}, 2 \mathrm{H}), 7.38(\mathrm{~m}, 1 \mathrm{H}), 7.22(\mathrm{~m}, 1 \mathrm{H}), 7.11-7.01(\mathrm{~m}, 2 \mathrm{H}), 5.90$ (s, 1H), $4.73(\mathrm{~s}, 2 \mathrm{H}), 4.16(\mathrm{~s}, 2 \mathrm{H}), 2.99(\mathrm{sept}, 1 \mathrm{H}, J=6.7 \mathrm{~Hz}), 2.36(\mathrm{~s}, 3 \mathrm{H}), 1.95(\mathrm{~s}, 3 \mathrm{H}), 1.23(\mathrm{~d}, 6 \mathrm{H}, J=6.7 \mathrm{~Hz})$. ${ }^{13} \mathrm{C}$ NMR $\left(\mathrm{CDCl}_{3}\right)$ : 167.0, 154.7, 150.9, $150.7(13$ and $248 \mathrm{~Hz}), 149.5(13$ and $248 \mathrm{~Hz}), 147.5,139.1,136.4,133.2$, 132.9, 128.9, 128.8, 128.7, $127.0(13 \mathrm{~Hz}), 126.5(4 \mathrm{~Hz}), 126.3,123.5(4$ and $7 \mathrm{~Hz}), 115.5(17 \mathrm{~Hz}), 112.6,110.3$, 108.9, 32.1, 27.1, 26.2, 21.4, 20.1, 9.7. HRMS $(\mathrm{m} / \mathrm{z})$ : $[\mathrm{M}+\mathrm{H}]^{+}$calcd for $\mathrm{C}_{30} \mathrm{H}_{28} \mathrm{~F}_{2} \mathrm{~N}_{3} \mathrm{O}_{3}$ : 516.2099; found, 516.2102.

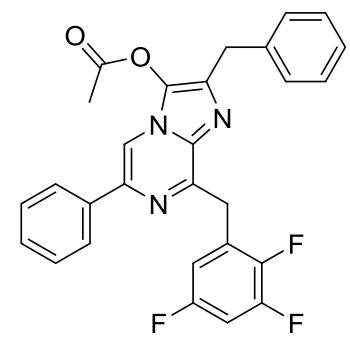

2-Benzyl-6-phenyl-8-(2,3,5-trifluorobenzyl)imidazo[1,2-a]pyrazin-3-yl acetate 25\{1,26,1\} (YJ 33067-135-1): ${ }^{1} \mathrm{H}$ NMR $\left(\mathrm{CDCl}_{3}\right)$ : Obtained as a solid $(0.04 \mathrm{~g}, 28 \%)$ after a recrystallization from $n$-heptane. $7.85(\mathrm{~s}, 1 \mathrm{H}), 7.84(\mathrm{~m}$, $2 \mathrm{H}), 7.43-7.26(\mathrm{~m}, 9 \mathrm{H}), 7.02(\mathrm{~m}, 1 \mathrm{H}), 6.84(\mathrm{~m}, 1 \mathrm{H}), 4.70(\mathrm{~s}, 2 \mathrm{H}), 4.21(\mathrm{~s}, 2 \mathrm{H}), 2.22(\mathrm{~s}, 3 \mathrm{H}) .{ }^{13} \mathrm{C} \mathrm{NMR}\left(\mathrm{CDCl}_{3}\right)$ : 167.0, $157.1(242,12$ and $3 \mathrm{~Hz}), 150.3(247,13$ and $15 \mathrm{~Hz}), 150.1,146.1(242,12$ and $4 \mathrm{~Hz}), 139.1,137.7,136.3$, 135.3, 133.2, 129.1, 129.0, 128.8, 128.7, 127.8 (9 and $14 \mathrm{~Hz}), 126.6,126.3,112.9$ (3 and $24 \mathrm{~Hz}), 109.2,103.8$ (21 and $28 \mathrm{~Hz}$ ), 34.1, 32.2, 19.8 (one signal missing). $\mathrm{HRMS}\left(\mathrm{m} / \mathrm{z}\right.$ ): $[\mathrm{M}+\mathrm{H}]^{+}$calcd for $\mathrm{C}_{28} \mathrm{H}_{21} \mathrm{~F}_{3} \mathrm{~N}_{3} \mathrm{O}_{2}: 488.1586$; found, 488.1583 . 


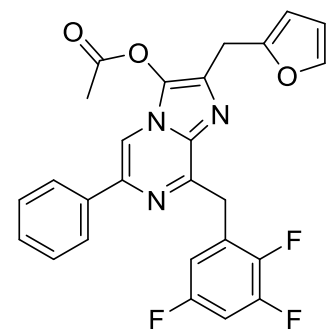

2-(Furan-2-ylmethyl)-6-phenyl-8-(2,3,5-trifluorobenzyl)imidazo[1,2-a]pyrazin-3-yl acetate $\mathbf{2 5}\{1,26,37\}$ (YJ 33067-137-1): Obtained as a solid $(0.41 \mathrm{~g}, 75 \%)$ after a recrystallization from a large amount of cyclohexane. ${ }^{1} \mathrm{H}$ NMR $\left(\mathrm{CDCl}_{3}\right): 7.89(\mathrm{~s}, 1 \mathrm{H}), 7.85(\mathrm{~m}, 2 \mathrm{H}), 7.47-7.36(\mathrm{~m}, 4 \mathrm{H}), 7.02(\mathrm{~m}, 1 \mathrm{H}), 6.85(\mathrm{~m}, 1 \mathrm{H}), 4.69(\mathrm{~s}, 2 \mathrm{H}), 4.23(\mathrm{~s}$, 2H), $2.38(\mathrm{~s}, 3 \mathrm{H}) .{ }^{13} \mathrm{C} \mathrm{NMR}\left(\mathrm{CDCl}_{3}\right): 167.0,157.1(242,12$ and $3 \mathrm{~Hz}), 151.2,150.3(247,13$ and $15 \mathrm{~Hz}), 150.2$, $146.1(242,12$ and $4 \mathrm{~Hz}), 141.7,139.1,136.3,133.3,132.8,129.0,128.8,128.7,127.8$ (9 and $14 \mathrm{~Hz}), 126.2,112.9$ (3 and $24 \mathrm{~Hz}), 110.5,109.2,106.9,103.8(21$ and $28 \mathrm{~Hz}), 32.1,27.1,20.1$. HRMS $(\mathrm{m} / \mathrm{z})$ : $[\mathrm{M}+\mathrm{H}]^{+}$calcd for $\mathrm{C}_{26} \mathrm{H}_{19} \mathrm{~F}_{3} \mathrm{~N}_{3} \mathrm{O}_{3}$ : 478.1378; found, 478.1374 .

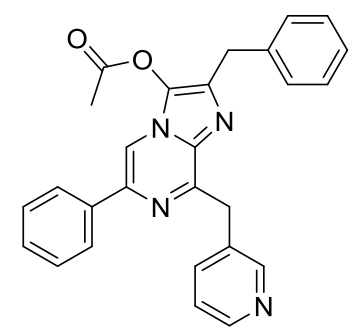

2-Benzyl-6-phenyl-8-(pyridin-3-ylmethyl)imidazo[1,2-a]pyrazin-3-yl acetate $\quad \mathbf{2 5}\{1,35,1\} \quad$ (YJ30367-045-2): Obtained as a white solid $(0.1 \mathrm{~g}, 37 \%)$ after a recrystallization from $n$-heptane. ${ }^{1} \mathrm{H}$ NMR $\left(\mathrm{CDCl}_{3}\right): 8.87(\mathrm{~d}, 1 \mathrm{H}, J=$ $2.0 \mathrm{~Hz}), 8.50(\mathrm{dd}, 1 \mathrm{H}, J=1.7,5.0 \mathrm{~Hz}), 7.92(\mathrm{~m}, 1 \mathrm{H}), 7.87(\mathrm{~m}, 2 \mathrm{H}), 7.82(\mathrm{~s}, 1 \mathrm{H}), 7.48-7.22(\mathrm{~m}, 9 \mathrm{H}), 4.63(\mathrm{~s}, 2 \mathrm{H})$, $4.21(\mathrm{~s}, 2 \mathrm{H}), 2.19(\mathrm{~s}, 3 \mathrm{H}) .{ }^{13} \mathrm{C} \mathrm{NMR}\left(\mathrm{CDCl}_{3}\right): 167.1,151.7,150.9,147.9,139.1,137.9,137.2,136.6,135.5,133.5$, 133.3, 129.1, 129.0, 128.8, 128.7, 128.5, 126.3, 123.2, 109.1, 35.5, 34.2, 19.9. HRMS $(\mathrm{m} / \mathrm{z}):[\mathrm{M}+\mathrm{H}]^{+}$calcd for $\mathrm{C}_{27} \mathrm{H}_{23} \mathrm{~N}_{4} \mathrm{O}_{2}$ : 435.1821; found, 435.1805 .

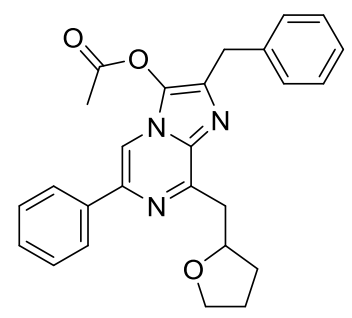

2-Benzyl-6-phenyl-8-((tetrahydrofuran-2-yl)methyl)imidazo[1,2-a]pyrazin-3-yl acetate $\mathbf{2 5}\{1,38,1\}$ (EC31093-0231): Obtained as a solid $(0.03 \mathrm{~g}, 30 \%)$ after a recrystallization from $n$-heptane and a small amount of toluene. ${ }^{1} \mathrm{H}$ NMR $\left(\mathrm{CDCl}_{3}\right): 7.90$ (, $\left.2 \mathrm{H} \mathrm{dt}, J=3.0,1.8 \mathrm{~Hz}\right), 7.78(\mathrm{~s}, 1 \mathrm{H}), 7.48-7.41(\mathrm{~m}, 2 \mathrm{H}), 7.40-7.35(\mathrm{~m}, 1 \mathrm{H}), 7.34-7.20$ $(\mathrm{m}, 5 \mathrm{H}), 4.85-4.75(\mathrm{~m}, 1 \mathrm{H}), 4.18(\mathrm{~s}, 2 \mathrm{H}), 3.98(\mathrm{td}, 1 \mathrm{H}, J=7.7,6.2 \mathrm{~Hz}), 3.78(\mathrm{td}, 1 \mathrm{H}, J=7.8,6.1 \mathrm{~Hz}), 3.64(\mathrm{dd}$, $1 \mathrm{H}, J=14.3,7.4 \mathrm{~Hz}), 3.38(\mathrm{dd}, 1 \mathrm{H}, J=14.3,6.1 \mathrm{~Hz}), 2.14(\mathrm{~s}, 3 \mathrm{H}), 2.21-1.74(\mathrm{~m}, 5 \mathrm{H}) .{ }^{13} \mathrm{C} \mathrm{NMR}\left(\mathrm{CDCl}_{3}\right): 167.2$, 152.2, 139.2, 138.1, 137.1, 135.2, 134.2, 129.2, 128.9, 128.7, 128.6, 126.7, 109.0, 77.6, 68.0, 39.3, 34.5, 31.7, 25.7, 20.0. HRMS $(m / z):[\mathrm{M}+\mathrm{H}]^{+}$calcd for $\mathrm{C}_{26} \mathrm{H}_{26} \mathrm{~N}_{3} \mathrm{O}_{3}, 428.1974$; found, 428.1936 .

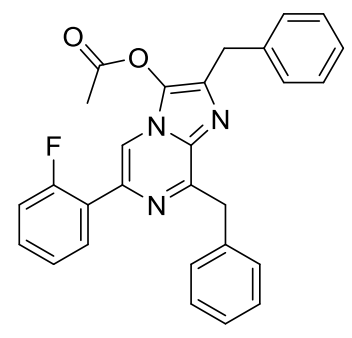


2,8-Dibenzyl-6-(2-fluorophenyl)imidazo[1,2-a]pyrazin-3-yl acetate 25\{2,1,1\} (YJ31134-091-1): Obtained as a solid $(0.21 \mathrm{~g}, 45 \%)$ after a recrystallization from $n$-heptane. ${ }^{1} \mathrm{H}$ NMR $\left(\mathrm{CDCl}_{3}\right): 8.20(\mathrm{~m}, 1 \mathrm{H}), 8.05(\mathrm{~s}, 1 \mathrm{H}), 7.62(\mathrm{~m}$, 2H), $7.32(\mathrm{~m}, 10 \mathrm{H}), 7.15(\mathrm{~m}, 1 \mathrm{H}), 4.63(\mathrm{~s}, 2 \mathrm{H}), 4.22(\mathrm{~s}, 2 \mathrm{H}), 2.18(\mathrm{~s}, 3 \mathrm{H}) .{ }^{13} \mathrm{C}$ NMR $\left(\mathrm{CDCl}_{3}\right): 167.0,160.0(\mathrm{~d}, J=$ $248 \mathrm{~Hz}), 152.9,138.0,137.6,135.4,133.5,133.3(\mathrm{~d}, J=2 \mathrm{~Hz}), 130.8$ (d, $J=2 \mathrm{~Hz}), 129.8,129.7,129.1,128.9$, 128.5, 128.3, 126.5, $124.6(\mathrm{~d}, J=3 \mathrm{~Hz}), 124.3(\mathrm{~d}, J=11 \mathrm{~Hz}), 115.9(\mathrm{~d}, J=23 \mathrm{~Hz}), 112.9(\mathrm{~d}, J=16 \mathrm{~Hz}), 39.4$, 34.2, 19.8 (one signal missing). HRMS $(m / z)$ : $[\mathrm{M}+\mathrm{H}]^{+}$calcd for $\mathrm{C}_{28} \mathrm{H}_{23} \mathrm{FN}_{3} \mathrm{O}_{2}, 452.1774$; found, 452.1838 .

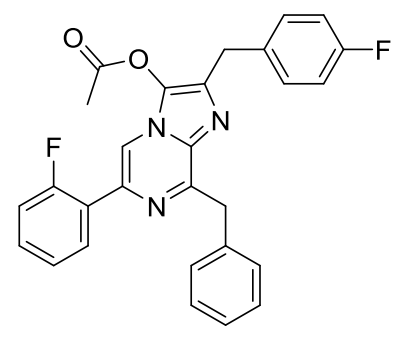

8-Benzyl-2-(4-fluorobenzyl)-6-(2-fluorophenyl)imidazo[1,2-a]pyrazin-3-yl acetate 25\{2,1,4\} (YJ30367-121-1): Obtained as a solid $(0.1 \mathrm{~g}, 63 \%)$ after a recrystallization from $n$-heptane. ${ }^{1} \mathrm{H} \mathrm{NMR}\left(\mathrm{CDCl}_{3}\right): 8.20(\mathrm{dt}, 1 \mathrm{H}, J=2.0$, $7.9 \mathrm{~Hz}), 8.06(\mathrm{~s}, 1 \mathrm{H}), 7.60(\mathrm{~m}, 2 \mathrm{H}), 7.38-7.21(\mathrm{~m}, 7 \mathrm{H}), 7.15(\mathrm{~m}, 1 \mathrm{H}), 7.01(\mathrm{~m}, 2 \mathrm{H}), 4.62(\mathrm{~s}, 2 \mathrm{H}), 4.16(\mathrm{~s}, 2 \mathrm{H}), 2.26$ (s, 3H). ${ }^{13} \mathrm{C} \mathrm{NMR}\left(\mathrm{CDCl}_{3}\right): 167.0,161.0(243 \mathrm{~Hz}), 160.0(248 \mathrm{~Hz}), 153.0,137.7,135.1,133.7(3 \mathrm{~Hz}), 133.5,133.4$ (2 Hz), $130.8(2 \mathrm{~Hz}), 130.4(8 \mathrm{~Hz}), 129.8$ (9 Hz), 129.7, 128.8, 128.3, 126.5, $124.6(3 \mathrm{~Hz}), 124.2(11 \mathrm{~Hz}), 115.9$ $(23 \mathrm{~Hz}), 115.1(22 \mathrm{~Hz}), 112.9(16 \mathrm{~Hz}), 39.4,33.2,19.9$. HRMS $(m / z):[\mathrm{M}+\mathrm{H}]^{+}$calcd for $\mathrm{C}_{28} \mathrm{H}_{22} \mathrm{~F}_{2} \mathrm{~N}_{3} \mathrm{O}_{2}, 470.1680$; found, 470.1670 .

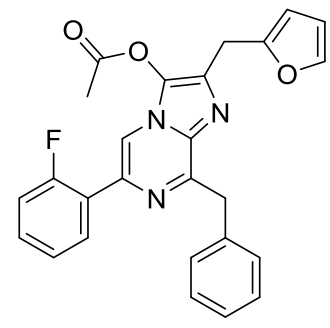

8-benzyl-6-(2-fluorophenyl)-2-(furan-2-ylmethyl)imidazo[1,2-a]pyrazin-3-yl acetate 25 2,1,37\} (YJ31134-093-2): Obtained as a solid $(0.15 \mathrm{~g}, 36 \%)$ after a recrystallization from $n$-heptane. ${ }^{1} \mathrm{H} \mathrm{NMR}\left(\mathrm{CDCl}_{3}\right): 8.20(\mathrm{~m}, 1 \mathrm{H}), 8.10(\mathrm{~s}$, $1 \mathrm{H}), 7.61(\mathrm{~m}, 2 \mathrm{H}), 7.31(\mathrm{~m}, 6 \mathrm{H}), 7.14(\mathrm{~m}, 1 \mathrm{H}), 6.35(\mathrm{~m}, 1 \mathrm{H}), 6.16(\mathrm{~m}, 1 \mathrm{H}), 4.63(\mathrm{~s}, 2 \mathrm{H}), 4.24(\mathrm{~s}, 2 \mathrm{H}), 2.35(\mathrm{~s}, 3 \mathrm{H})$. ${ }^{13} \mathrm{C} \mathrm{NMR}\left(\mathrm{CDCl}_{3}\right): 167.0,160.1(\mathrm{~d}, J=248 \mathrm{~Hz}), 153.1,151.5,141.6,137.7,133.5,133.4(\mathrm{~d}, J=2 \mathrm{~Hz}), 132.7$, $130.8(\mathrm{~d}, J=2 \mathrm{~Hz}), 129.8(\mathrm{~d}, J=9 \mathrm{~Hz}), 129.7,128.9,128.3,126.5,124.6(\mathrm{~d}, J=3 \mathrm{~Hz}), 124.2(\mathrm{~d}, J=11 \mathrm{~Hz}), 115.9$ $(\mathrm{d}, J=23 \mathrm{~Hz}), 112.9(\mathrm{~d}, J=16 \mathrm{~Hz}), 110.5,106.2,39.3,27.2,20.0$. HRMS $(m / z):[\mathrm{M}+\mathrm{H}]^{+}$calcd for $\mathrm{C}_{26} \mathrm{H}_{21} \mathrm{FN}_{3} \mathrm{O}_{3}$, 442.1567; found, 442.1631.

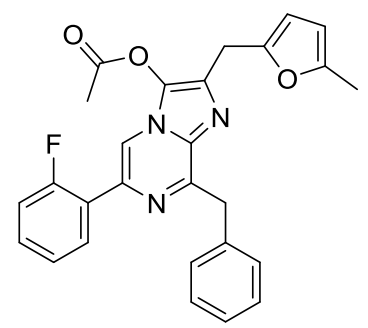

8-Benzyl-6-(2-fluorophenyl)-2-((5-methylfuran-2-yl)methyl)imidazo[1,2-a]pyrazin-3-yl acetate $\quad \mathbf{2 5}\{2,1,42\}$ (YJ30531-169-1): Obtained as a glass (0.08 g, 72\%). ${ }^{1} \mathrm{H}$ NMR $\left(\mathrm{CDCl}_{3}\right): 8.19(\mathrm{dt}, 1 \mathrm{H}, J=1.8,7.8 \mathrm{~Hz}), 8.09(\mathrm{~s}, 1 \mathrm{H})$, $7.61(\mathrm{~m}, 2 \mathrm{H}), 7.37-7.21(\mathrm{~m}, 5 \mathrm{H}), 7.15(\mathrm{ddd}, 1 \mathrm{H}, J=1.2,8.1,12.0 \mathrm{~Hz}), 6.02(\mathrm{~d}, 1 \mathrm{H}, J=3.0 \mathrm{~Hz}), 5.90(\mathrm{~m}, 1 \mathrm{H}), 4.63$ (s, 2H), $4.18(\mathrm{~s}, 2 \mathrm{H}), 2.35$ (s, 3H), $2.27(\mathrm{~s}, 3 \mathrm{H}) .{ }^{13} \mathrm{C} \mathrm{NMR}\left(\mathrm{CDCl}_{3}\right): 167.0,160.2(248 \mathrm{~Hz}), 153.0,151.2,149.5$, 137.7, 133.4 (2 Hz), 133.3, 133.0, $130.8(2 \mathrm{~Hz}), 129.8(9 \mathrm{~Hz}), 129.7,128.9,128.3,126.5,124.6$ (3 Hz), $124.2(11$ $\mathrm{Hz}), 115.9(23 \mathrm{~Hz}), 112.9(16 \mathrm{~Hz}), 107.5,106.2,39.3,27.2,20.0,13.5$. HRMS $(\mathrm{m} / z):[\mathrm{M}+\mathrm{H}]^{+}$calcd for $\mathrm{C}_{27} \mathrm{H}_{23} \mathrm{FN}_{3} \mathrm{O}_{3}, 456.1723$; found, 456.1734 . 


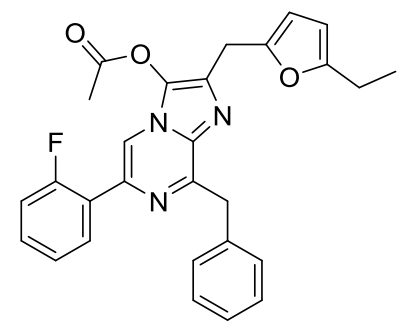

8-Benzyl-2-((5-ethylfuran-2-yl)methyl)-6-(2-fluorophenyl)imidazo[1,2-a]pyrazin-3-yl acetate $\quad \mathbf{2 5}\{2,1,44\}$ (YJ30367-111-1): Obtained as a wax (0.18 g, 95\%). ${ }^{1} \mathrm{H}$ NMR $\left(\mathrm{CDCl}_{3}\right): 8.19(\mathrm{dt}, 1 \mathrm{H}, J=1.8,7.8 \mathrm{~Hz}), 8.10$ (s, $\left.1 \mathrm{H}\right)$, $7.62(\mathrm{~m}, 2 \mathrm{H}), 7.39-7.22(\mathrm{~m}, 5 \mathrm{H}), 7.15(\mathrm{ddd}, 1 \mathrm{H}, J=1.2,8.1,12.0 \mathrm{~Hz}), 6.04(\mathrm{~d}, 1 \mathrm{H}, J=3.0 \mathrm{~Hz}), 5.93(\mathrm{~m}, 1 \mathrm{H}), 4.64$ $(\mathrm{s}, 2 \mathrm{H}), 4.21(\mathrm{~s}, 2 \mathrm{H}), 2.65(\mathrm{q}, 2 \mathrm{H}, J=7.6 \mathrm{~Hz}), 2.34(\mathrm{~s}, 3 \mathrm{H}), 1.24(\mathrm{t}, 3 \mathrm{H}, J=7.6 \mathrm{~Hz}) .{ }^{13} \mathrm{C} \mathrm{NMR}\left(\mathrm{CDCl}_{3}\right): 167.0$, $160.1(248 \mathrm{~Hz}), 157.0,153.0,149.3,137.7,133.4(2 \mathrm{~Hz}), 133.3,133.0,130.8(2 \mathrm{~Hz}), 129.8(9 \mathrm{~Hz}), 129.7,129.0$, 128.3, 126.5, $124.6(3 \mathrm{~Hz}), 124.2(11 \mathrm{~Hz}), 115.9(23 \mathrm{~Hz}), 112.9(16 \mathrm{~Hz}), 107.3,104.6,39.3,27.3,21.3$, 20.0, 12.2. HRMS $(m / z):[\mathrm{M}+\mathrm{H}]^{+}$calcd for $\mathrm{C}_{28} \mathrm{H}_{25} \mathrm{FN}_{3} \mathrm{O}_{3}, 470.1880$; found, 470.1884 .

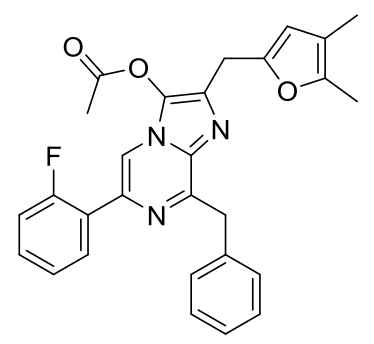

8-Benzyl-2-((4,5-dimethylfuran-2-yl)methyl)-6-(2-fluorophenyl)imidazo[1,2-a]pyrazin-3-yl acetate $\mathbf{2 5}\{2,1,48\}$ (YJ30367-177-1): Obtained as a wax (0.44 g, 95\%). ${ }^{1} \mathrm{H}$ NMR $\left(\mathrm{CDCl}_{3}\right): 8.20(\mathrm{dt}, 1 \mathrm{H}, J=1.8,8.0 \mathrm{~Hz}), 8.09$ (s, 1H), $7.61(\mathrm{~m}, 2 \mathrm{H}), 7.37-7.24(\mathrm{~m}, 5 \mathrm{H}), 7.15(\mathrm{ddd}, 1 \mathrm{H}, J=1.8,8.1,9.4 \mathrm{~Hz}), 5.91(\mathrm{~s}, 1 \mathrm{H}), 4.63(\mathrm{~s}, 2 \mathrm{H}), 4.41(\mathrm{~s}, 2 \mathrm{H}), 2.35$ (s, 3H), 2.19 (s, 3H), $1.92(\mathrm{~s}, 3 \mathrm{H}) .{ }^{13} \mathrm{C}$ NMR $\left(\mathrm{CDCl}_{3}\right): 167.0,160.0(248 \mathrm{~Hz}), 153.0,148.2,146.3,137.8,133.4(3$ $\mathrm{Hz}), 133.3,133.2,130.8$ (3 Hz), 129.8, 129.7 (three signals), 128.9, 128.3, $124.6(3 \mathrm{~Hz}), 124.3(11 \mathrm{~Hz}), 115.9(22$ $\mathrm{Hz}), 114.5,112.9(15 \mathrm{~Hz}), 110.1,39.3,27.3,20.0,11.2,9.8$. HRMS $(\mathrm{m} / \mathrm{z}):[\mathrm{M}+\mathrm{H}]^{+}$calcd for $\mathrm{C}_{28} \mathrm{H}_{25} \mathrm{FN}_{3} \mathrm{O}_{3}$, 470.1880; found, 470.1890 .

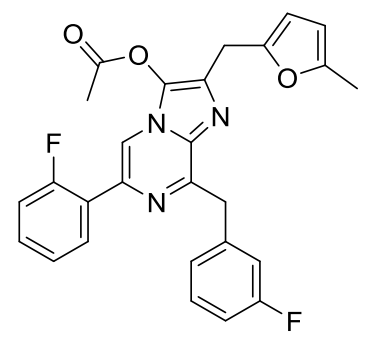

8-(3-fluorobenzyl)-6-(2-fluorophenyl)-2-((5-methylfuran-2-yl)methyl)imidazo[1,2-a]pyrazin-3-yl acetate 25 22,3,42\} (EC31095-115-2): Obtained as a solid (0.35 g, 70\%) after a chromatography over silica gel (cyclohexane - ethyl acetate 97/3). ${ }^{1} \mathrm{H}$ NMR $\left(\mathrm{CDCl}_{3}\right): 8.17(\mathrm{~m}, 2 \mathrm{H}), 7.32(\mathrm{~m}, 5 \mathrm{H}), 7.16$ (ddd, $1 \mathrm{H}, J=11.9,8.1,1.2$ $\mathrm{Hz}), 6.94(\mathrm{~m}, 1 \mathrm{H}), 6.03(\mathrm{~d}, 1 \mathrm{H}, J=2.8 \mathrm{~Hz}), 5.92(\mathrm{~m}, 1 \mathrm{H}), 4.62(\mathrm{~s}, 2 \mathrm{H}), 4.19(\mathrm{~s}, 2 \mathrm{H}), 2.36(\mathrm{~s}, 3 \mathrm{H}), 2.28(\mathrm{~s}, 3 \mathrm{H}) .{ }^{13} \mathrm{C}$ NMR $\left(\mathrm{CDCl}_{3}\right): 167.0,162.8(245 \mathrm{~Hz}), 160.1(248 \mathrm{~Hz}), 152.3,151.2,149.4,140.1(8 \mathrm{~Hz}), 133.4(3 \mathrm{~Hz}), 133.3(3$ $\mathrm{Hz}), 130.7(3 \mathrm{~Hz}), 129.9(9 \mathrm{~Hz}), 129.6(8 \mathrm{~Hz}), 129.0,125.4(3 \mathrm{~Hz}), 124.7(3 \mathrm{~Hz}), 124.2(11 \mathrm{~Hz}), 116.6(22 \mathrm{~Hz})$, $116.0(23 \mathrm{~Hz}), 113.4(21 \mathrm{~Hz}), 113.2,113.0,107.5,106.2,38.9(2 \mathrm{~Hz}), 27.3,20.0,13.5$. HRMS $(\mathrm{m} / \mathrm{z}):[\mathrm{M}+\mathrm{H}]^{+}$calcd for $\mathrm{C}_{27} \mathrm{H}_{22} \mathrm{~F}_{2} \mathrm{~N}_{3} \mathrm{O}_{3}$ : 474.1629; found, 474.1620. 


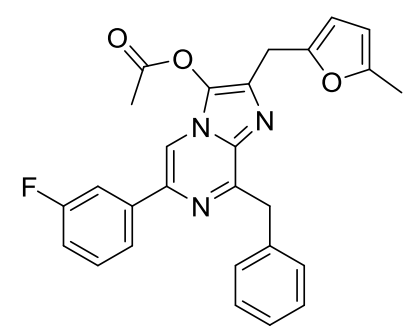

8-Benzyl-6-(3-fluorophenyl)-2-((5-methylfuran-2-yl)methyl)imidazo[1,2-a]pyrazin-3-yl $\quad$ acetate $\quad \mathbf{2 5}\{3,1,42\}$ (YJ30531-141-1): Obtained as white powder after a recrystallization in n-heptane $(0.018 \mathrm{~g}, 14 \%) .{ }^{1} \mathrm{H}$ NMR $\left(\mathrm{CDCl}_{3}\right): 7.85(\mathrm{~s}, 1 \mathrm{H}), 7.68(\mathrm{~m}, 2 \mathrm{H}), 7.60(\mathrm{~m}, 2 \mathrm{H}), 7.46-7.37(\mathrm{~m}, 2 \mathrm{H}), 7.34-7.21(\mathrm{~m}, 3 \mathrm{H}), 6.02(\mathrm{~d}, 1 \mathrm{H}, J=3.1 \mathrm{~Hz})$, $5.90(\mathrm{~m}, 1 \mathrm{H}), 4.62(\mathrm{~s}, 2 \mathrm{H}), 4.18(\mathrm{~s}, 2 \mathrm{H}), 2.37(\mathrm{~s}, 3 \mathrm{H}), 2.26(\mathrm{~s}, 3 \mathrm{H})$. HRMS $(\mathrm{m} / \mathrm{z}):[\mathrm{M}+\mathrm{H}]^{+}$calcd for $\mathrm{C}_{27} \mathrm{H}_{23} \mathrm{FN}_{3} \mathrm{O}_{3}$, 456.1723; found, 456.1732 .

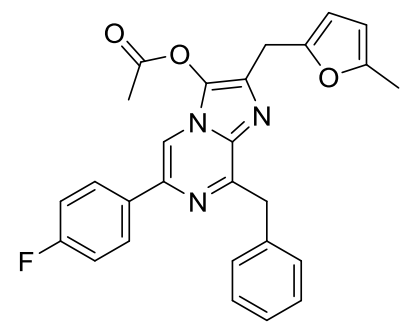

8-Benzyl-6-(4-fluorophenyl)-2-((5-methylfuran-2-yl)methyl)imidazo[1,2-a]pyrazin-3-yl acetate $\quad \mathbf{2 5}\{4,1,42\}$ (YJ30531-171-1): Obtained as a glass $(0.1 \mathrm{~g}, 83 \%) .{ }^{1} \mathrm{H}$ NMR $\left(\mathrm{CDCl}_{3}\right): 7.88(\mathrm{~m}, 2 \mathrm{H}), 7.78(\mathrm{~s}, 1 \mathrm{H}), 7.59(\mathrm{~m}, 2 \mathrm{H})$, 7.34-7.12 (m, 6H), $6.02(\mathrm{~d}, 1 \mathrm{H}, J=3.0 \mathrm{~Hz}), 5.90(\mathrm{~m}, 1 \mathrm{H}), 4.61(\mathrm{~s}, 2 \mathrm{H}), 4.18(\mathrm{~s}, 2 \mathrm{H}), 2.36(\mathrm{~s}, 3 \mathrm{H}), 2.27(\mathrm{~s}, 3 \mathrm{H}) .{ }^{13} \mathrm{C}$ NMR $\left(\mathrm{CDCl}_{3}\right)$ : $167.0,163.2(248 \mathrm{~Hz}), 153.1,151.2,149.4,138.3,137.7,133.4,133.0,132.9(3 \mathrm{~Hz}), 129.7,128.9$, $128.2(8 \mathrm{~Hz}), 128.1,126.5115 .6(21 \mathrm{~Hz}), 108.6,107.5,106.2,39.3,27.3,20.1,13.5$. HRMS $(\mathrm{m} / \mathrm{z}):[\mathrm{M}+\mathrm{H}]^{+}$calcd for $\mathrm{C}_{27} \mathrm{H}_{23} \mathrm{FN}_{3} \mathrm{O}_{3}, 456.1723$; found, 456.1721 .

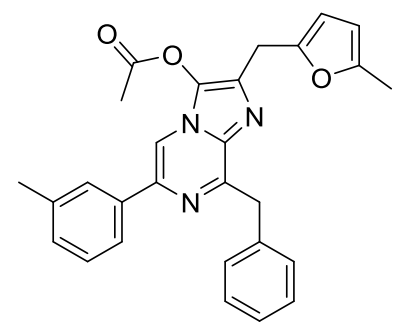

8-benzyl-2-((5-methylfuran-2-yl)methyl)-6-(m-tolyl)imidazo[1,2-a]pyrazin-3-yl acetate 25 \{5,1,42\} (YJ30367-751) Obtained as a white solid $(0.1 \mathrm{~g}, 53 \%)$ after a (slow) recrystallization from n-heptane. ${ }^{1} \mathrm{H}$ NMR $\left(\mathrm{CDCl}_{3}\right): 7.82(\mathrm{~s}$, $1 \mathrm{H}), 7.73(\mathrm{~m}, 1 \mathrm{H}), 7.79(\mathrm{~m}, 1 \mathrm{H}), 7.37-7.20(\mathrm{~m}, 5 \mathrm{H}), 6.01(\mathrm{~d}, 1 \mathrm{H}, J=3 \mathrm{~Hz}), 5.91(\mathrm{~m}, 1 \mathrm{H}), 4.62(\mathrm{~s}, 2 \mathrm{H}), 4.18(\mathrm{~s}$, $2 \mathrm{H}), 2.45(\mathrm{~s}, 3 \mathrm{H}), 2.36(\mathrm{~s}, 3 \mathrm{H}), 2.28(\mathrm{~s}, 2 \mathrm{H}) .{ }^{13} \mathrm{C} \mathrm{NMR}\left(\mathrm{CDCl}_{3}\right)$ : 167.0, 152.9, 151.2, 149.5, 139.3, 138.4, 137.9, 136.8, 133.5, 132.8, 129.7, 129.3, 128.9, 128.7, 128.2, 127.1, 126.4, 123.5, 108.8, 107.4, 106.2, 39.4, 27.4, 21.5, 20.1, 13.5. HRMS $(\mathrm{m} / \mathrm{z})$ : $[\mathrm{M}+\mathrm{H}]^{+}$calcd for $\mathrm{C}_{28} \mathrm{H}_{26} \mathrm{~N}_{3} \mathrm{O}_{3}, 452.1974$; found, 452.1981 .

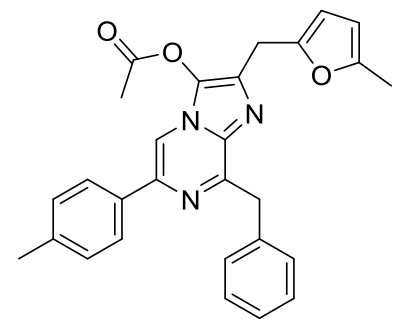

8-benzyl-2-((5-methylfuran-2-yl)methyl)-6-(p-tolyl)imidazo[1,2-a]pyrazin-3-yl acetate 25\{6,1,42\} (YJ30367-0471): Obtained as a white solid $(0.15 \mathrm{~g}, 70 \%)$ after a recrystallization from $\mathrm{n}$-heptane. ${ }^{1} \mathrm{H}$ NMR $\left(\mathrm{CDCl}_{3}\right): 7.82-7.79$ $(\mathrm{m}, 3 \mathrm{H}), 7.62(\mathrm{~m}, 2 \mathrm{H}), 7.33-7.20(\mathrm{~m}, 5 \mathrm{H}), 6.01(\mathrm{~d}, 1 \mathrm{H}, J=1 \mathrm{~Hz}), 5.90(\mathrm{~m}, 1 \mathrm{H}), 4.62(\mathrm{~s}, 2 \mathrm{H}), 4.17(\mathrm{~s}, 2 \mathrm{H}), 2.41(\mathrm{~s}$, $3 \mathrm{H}), 2.35$ (s, 3H), $2.28(\mathrm{~s}, 2 \mathrm{H}) .{ }^{13} \mathrm{C}$ NMR $\left(\mathrm{CDCl}_{3}\right): 167.0,152.9,151.1,149.6,139.2,138.5,137.9,134.0,133.5$, 
132.8, 129.7, 129.5, 128.8, 128.2, 126.4, 126.3, 108.4, 107.4, 106.2, 39.3, 27.3, 21.2, 20.1, 13.5. HRMS (m/z): $[\mathrm{M}+\mathrm{H}]^{+}$calcd for $\mathrm{C}_{28} \mathrm{H}_{26} \mathrm{~N}_{3} \mathrm{O}_{3}, 452.1974$; found, 452.1960.

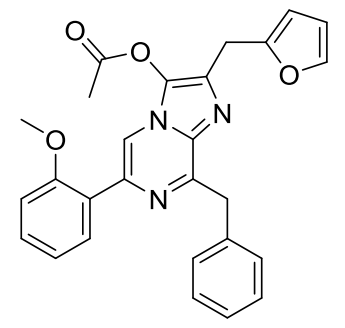

8-Benzyl-2-(furan-2-ylmethyl)-6-(2-methoxyphenyl)imidazo[1,2-a]pyrazin-3-yl acetate $\mathbf{2 5}\{7,1,37\}$ (EC31092057-3): Obtained as a beige solid $(0.27 \mathrm{~g}, 68 \%)$ after a recrystallization from $n$-heptane. ${ }^{1} \mathrm{H} \mathrm{NMR}\left(\mathrm{CDCl}_{3}\right): 8.21$ (s, $1 \mathrm{H}), 8.05(\mathrm{dd}, 1 \mathrm{H}, J=7.7,1.8 \mathrm{~Hz}), 7.59(\mathrm{dd}, 2 \mathrm{H}, J=7.9,0.9 \mathrm{~Hz}), 7.39-7.27(\mathrm{~m}, 4 \mathrm{H}), 7.23-7.18(\mathrm{~m}, 1 \mathrm{H}), 7.09$ (td, $1 \mathrm{H}, J=7.6,1.1 \mathrm{~Hz}), 6.99(\mathrm{dd}, 1 \mathrm{H}, J=8.3,0.9 \mathrm{~Hz}), 6.33(\mathrm{dd}, 1 \mathrm{H}, J=3.2,1.9 \mathrm{~Hz}), 6.16-6.10(\mathrm{~m}, 1 \mathrm{H}), 4.60(\mathrm{~s}$, 2H), 4.22 (s, $2 \mathrm{H}), 3.87$ (s, 3H), 2.32 (s, 3H). ${ }^{13} \mathrm{C}$ NMR $\left(\mathrm{CDCl}_{3}\right)$ : 167.2, 156.9, 152.5, 151.9, 141.7, 138.1, 135.6, 133.5, 132.4, 131.1, 129.9, 129.7, 128.8, 128.4, 126.5, 125.7, 121.4, 113.5, 111.7, 110.6, 106.9, 55.9, 39.5, 27.4, 20.1. HRMS $(m / z):[\mathrm{M}+\mathrm{H}]^{+}$calcd for $\mathrm{C}_{27} \mathrm{H}_{24} \mathrm{~N}_{3} \mathrm{O}_{4}, 454.1767$; found, 454.1785.

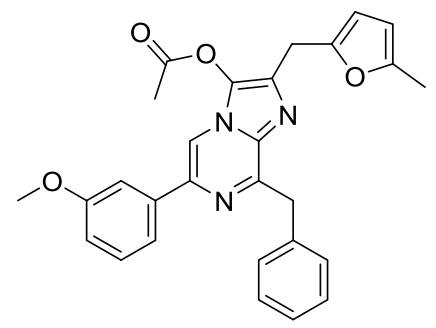

8-Benzyl-6-(3-methoxyphenyl)-2-((5-methylfuran-2-yl)methyl)imidazo[1,2-a]pyrazin-3-yl acetate $\mathbf{2 5}\{8,1,42\}$ (YJ30367-113-1): Obtained as a wax (0.08 g, 95\%). ${ }^{1} \mathrm{H}$ NMR $\left(\mathrm{CDCl}_{3}\right)$ : $7.84(\mathrm{~s}, 1 \mathrm{H}), 7.61(\mathrm{~m}, 2 \mathrm{H}), 7.52(\mathrm{~m}, 1 \mathrm{H})$, $7.45(\mathrm{~m}, 1 \mathrm{H}), 7.38-7.29(\mathrm{~m}, 3 \mathrm{H}), 7.23(\mathrm{~m}, 1 \mathrm{H}), 6.93(\mathrm{~m}, 1 \mathrm{H}), 6.02(\mathrm{~d}, 1 \mathrm{H}, J=3 \mathrm{~Hz}), 5.91(\mathrm{~m}, 1 \mathrm{H}), 4.63(\mathrm{~s}, 2 \mathrm{H})$, $4.18(\mathrm{~s}, 2 \mathrm{H}), 3.88$ (s, 3H), 2.35 (s, 3H), 2.28 (s, 3H). ${ }^{13} \mathrm{C} \mathrm{NMR}\left(\mathrm{CDCl}_{3}\right): 167.0,160.1,152.9,151.2,149.5,138.8$, 138.2, 137.8, 133.5, 132.9, 129.8, 129.7, 128.9, 128.2, 126.4, 118.5, 114.7, 112.3, 109.0, 107.5, 106.2, 55.3, 39.2, 27.3, 20.1, 13.5. HRMS (m/z): $[\mathrm{M}+\mathrm{H}]^{+}$calcd for $\mathrm{C}_{29} \mathrm{H}_{26} \mathrm{~N}_{3} \mathrm{O}_{4}, 468.1923$; found, 468.1928.

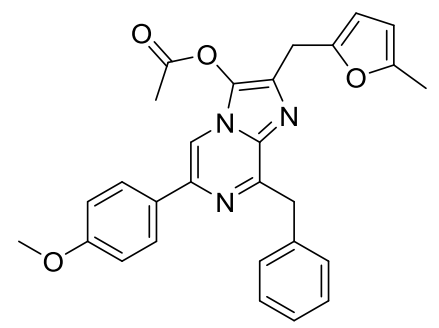

8-Benzyl-6-(4-methoxyphenyl)-2-((5-methylfuran-2-yl)methyl)imidazo[1,2-a]pyrazin-3-yl acetate $\mathbf{2 5}\{9,1,42\}$ (YJ30531-183-2): Obtained as a white solid after a recrystallization in $\mathrm{n}$-heptane $(0.11 \mathrm{~g}, 69 \%) .{ }^{1} \mathrm{H} \mathrm{NMR}\left(\mathrm{CDCl}_{3}\right)$ : $7.84(\mathrm{~m}, 2 \mathrm{H}), 7.75(\mathrm{~s}, 1 \mathrm{H}), 7.61(\mathrm{~m}, 2 \mathrm{H}), 7.32(\mathrm{~m}, 2 \mathrm{H}), 7.24(\mathrm{~m}, 1 \mathrm{H}), 7.0(\mathrm{~m}, 2 \mathrm{H}), 6.02(\mathrm{~m}, 1 \mathrm{H}), 5.91(\mathrm{~m}, 1 \mathrm{H}), 4.62$ (s, 2H), 4.17 (s, 2H), 3.87 (s, 3H), 2.35 (s, 3H), $2.28(\mathrm{~s}, 3 \mathrm{H}) .{ }^{13} \mathrm{C}$ NMR $\left(\mathrm{CDCl}_{3}\right): 167.0,160.1,152.8,151.1,149.6$, 139.0, 137.9, 133.4, 132.7, 129.7, 129.4, 128.8, 128.2, 127.6, 126.4, 114.2, 107.8, 107.4, 106.2, 55.4, 39.3, 27.3, 20.1, 13.5. HRMS $(\mathrm{m} / \mathrm{z})$ : $[\mathrm{M}+\mathrm{H}]^{+}$calcd for $\mathrm{C}_{29} \mathrm{H}_{26} \mathrm{~N}_{3} \mathrm{O}_{4}, 468.1923$; found, 468.1919 . 


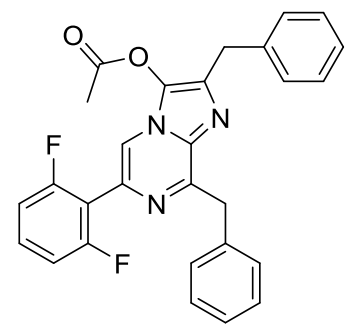

2,8-Dibenzyl-6-(2,6-difluorophenyl)imidazo[1,2-a]pyrazin-3-yl acetate 25\{10,1,1\} (MM34284-035-1): Obtained as a solid $(0.16 \mathrm{~g}, 61 \%)$ after a recrystallization from $n$-heptane. ${ }^{1} \mathrm{H} \mathrm{NMR}\left(\mathrm{CDCl}_{3}\right): 7.66(\mathrm{~s}, 1 \mathrm{H}), 7.63(\mathrm{~m}, 2 \mathrm{H}), 7.31$ $(\mathrm{m}, 9 \mathrm{H}), 7.02(\mathrm{~m}, 2 \mathrm{H}), 4.62(\mathrm{~s}, 2 \mathrm{H}), 4.21(\mathrm{~s}, 2 \mathrm{H}), 2.13(\mathrm{~s}, 3 \mathrm{H}) .{ }^{13} \mathrm{C} \mathrm{NMR}\left(\mathrm{CDCl}_{3}\right): 166.9,160.7(6,250 \mathrm{~Hz}), 153.3$, $137.9,137.6,135.4,133.3,130.2(10 \mathrm{~Hz}), 129.6,129.5,129.1,128.7,128.5,128.2,126.5,126.4,115.2(18 \mathrm{~Hz})$, 114.3, $111.8(6,19 \mathrm{~Hz}), 39.5,34.2,19.8$. HRMS $(\mathrm{m} / \mathrm{z})$ : $[\mathrm{M}+\mathrm{H}]^{+}$calcd for $\mathrm{C}_{28} \mathrm{H}_{21} \mathrm{~F}_{2} \mathrm{~N}_{3} \mathrm{O}_{2}, 470.1680$; found, 470.1630 .

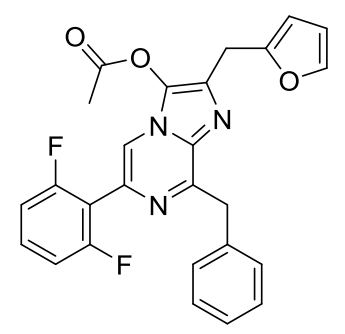

8-Benzyl-6-(2,6-difluorophenyl)-2-(furan-2-ylmethyl)imidazo[1,2-a]pyrazin-3-yl acetate 25 10,1,37\} (MM34284038-1): Obtained as a wax $(0.19 \mathrm{~g}, 95 \%) .{ }^{1} \mathrm{H}$ NMR $\left(\mathrm{CDCl}_{3}\right)$ : $7.70(\mathrm{~s}, 1 \mathrm{H}), 7.60(\mathrm{~m}, 2 \mathrm{H}), 7.37-7.19(\mathrm{~m}, 6 \mathrm{H}), 7.02$ $(\mathrm{m}, 2 \mathrm{H}), 6.35(\mathrm{~m}, 1 \mathrm{H}), 6.15(\mathrm{~m}, 1 \mathrm{H}), 4.62(\mathrm{~s}, 2 \mathrm{H}), 4.24(\mathrm{~s}, 2 \mathrm{H}), 2.31(\mathrm{~s}, 3 \mathrm{H}) .{ }^{13} \mathrm{C} \mathrm{NMR}\left(\mathrm{CDCl}_{3}\right): 166.9,160.7(6$, $250 \mathrm{~Hz}), 153.4,151.4,141.6,137.5,133.2,132.8,130.2(10 \mathrm{~Hz}), 129.7,129.6,128.7,128.2,126.5,115.2(18 \mathrm{~Hz})$, 114.3, $111.8(6,19 \mathrm{~Hz}), 110.4,106.8,39.4,27.2,20.0$. HRMS $(\mathrm{m} / \mathrm{z}):[\mathrm{M}+\mathrm{H}]^{+}$calcd for $\mathrm{C}_{26} \mathrm{H}_{19} \mathrm{~F}_{2} \mathrm{~N}_{3} \mathrm{O}_{3}, 460.1473$; found, 460.1430 .

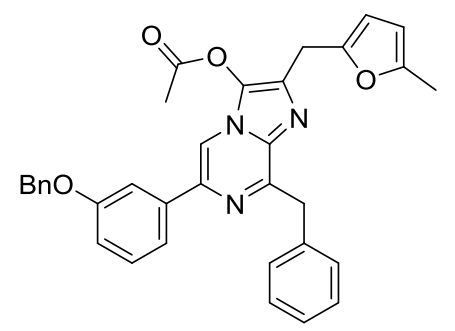

8-Benzyl-6-(3-(benzyloxy)phenyl)-2-((5-methylfuran-2-yl)methyl)imidazo[1,2-a]pyrazin-3-yl acetate 25\{11,1,42\} (RB32489-019-1): Obtained as an oil (0.46 g, still containing some $\mathrm{AcOH}, \mathrm{Ac}_{2} \mathrm{O}$ and $\left.\mathrm{EtOAc}\right)$ which was used directly in the next step. ${ }^{1} \mathrm{H}\left(\mathrm{CDCl}_{3}\right): 7.83(\mathrm{~s}, 1 \mathrm{H}), 7.61(\mathrm{~m}, 3 \mathrm{H}), 7.48(\mathrm{~m}, 3 \mathrm{H}), 7.44-7.30(\mathrm{~m}, 6 \mathrm{H}), 7.23(\mathrm{~m}, 1 \mathrm{H})$, $7.02(\mathrm{~m}, 1 \mathrm{H}), 6.03(\mathrm{~d}, 1 \mathrm{H}, J=3.0 \mathrm{~Hz}), 5.91(\mathrm{~m}, 1 \mathrm{H}), 5.14(\mathrm{~s}, 2 \mathrm{H}), 4.64(\mathrm{~s}, 2 \mathrm{H}), 4.20(\mathrm{~s}, 2 \mathrm{H}), 2.36(\mathrm{~s}, 3 \mathrm{H}), 2.28(\mathrm{~s}$, $3 \mathrm{H}) .{ }^{13} \mathrm{C}\left(\mathrm{CDCl}_{3}\right) 176.3,167.0,159.3,152.8,151.2,149.4,138.8,138.2,137.7,136.9,133.4,132.8,129.8,128.9$, 128.6, 128.3, 128.0, 127.6, 126.5, 118.8, 115.0, 113.3, 109.0, 107.5, 106.2, 70.2, 39.3, 27.2, 20.1, 13.5. HRMS $(\mathrm{m} / \mathrm{z})$ : $[\mathrm{M}+\mathrm{H}]^{+}$calcd for $\mathrm{C}_{34} \mathrm{H}_{30} \mathrm{~N}_{3} \mathrm{O}_{4}, 544.2236$; found, 544.2231.

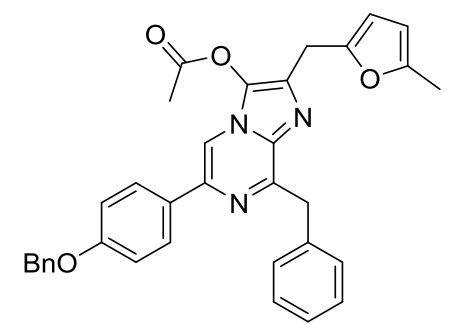

8-Benzyl-6-(4-(benzyloxy)phenyl)-2-((5-methylfuran-2-yl)methyl)imidazo[1,2-a]pyrazin-3-yl acetate $\mathbf{2 5}\{12,1,42\}$ (YJ 33069-171-1): Obtained as a white solid $(0.41 \mathrm{~g}, 91 \%)$ after diluting the THF solution in water, stirring for 15 
minutes, a filtration and drying under vacuum at $40{ }^{\circ} \mathrm{C} .{ }^{1} \mathrm{H}$ NMR $\left(\mathrm{CDCl}_{3}\right): 7.85(\mathrm{~m}, 2 \mathrm{H}), 7.76(\mathrm{~s}, 1 \mathrm{H}), 7.62(\mathrm{~m}$, 2H), $7.47(\mathrm{~m}, 2 \mathrm{H}), 7.42(\mathrm{~m}, 2 \mathrm{H}), 7.33(\mathrm{~m}, 3 \mathrm{H}), 7.22(\mathrm{~m}, 1 \mathrm{H}), 7.06(\mathrm{~m}, 2 \mathrm{H}), 6.03(\mathrm{~d}(\mathrm{br}), 1 \mathrm{H}, J=3.1 \mathrm{~Hz}), 5.91(\mathrm{~m}$, 1H), $5.15(\mathrm{~s}, 2 \mathrm{H}), 4.64(\mathrm{~s}, 2 \mathrm{H}), 4.20(\mathrm{~s}, 2 \mathrm{H}), 2.36(\mathrm{~s}, 3 \mathrm{H}), 2.28(\mathrm{~s}, 3 \mathrm{H}) .{ }^{13} \mathrm{C} \mathrm{NMR}\left(\mathrm{CDCl}_{3}\right):$. 167.0, 159.4, 152.7, $151.2,149.3,139.1,137.7,136.8,133.1,132.6,129.7,129.5,128.8,128.6,128.3,128.0,127.7,127.4,126.5$, $115.2,107.9,107.5,106.2,70.1,39.3,27.2,20.1,13.5$. HRMS $(m / z):[\mathrm{M}+\mathrm{H}]^{+}$calcd for $\mathrm{C}_{34} \mathrm{H}_{30} \mathrm{~N}_{3} \mathrm{O}_{4}, 544.2236$; found, 544.2232.

General procedure for the hydrolysis of esters 23 to prepare acids 24 via step xi. In a round bottomed flask, the considered ester $23(1.0 \mathrm{mmol})$ and powdered sodium hydroxide $(0.16 \mathrm{~g}, 4.0 \mathrm{~mol})$ were mixed. The atmosphere was replaced with argon and dry tetrahydrofuran $(5 \mathrm{~mL})$ was added. This was stirred overnight under an inert atmosphere, neutralized with a solution of ammonium chloride (1.1 equivalent). The aqueous phase was saturated with sodium chloride, extracted with ethyl acetate, the organic layer was washed with water, brine, dried over magnesium sulfate and concentrated to dryness to yield the corresponding acids $\mathbf{2 4}$ as described below which were used directly in the next step.

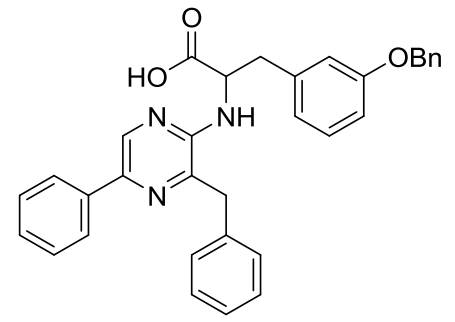

2-((3-Benzyl-5-phenylpyrazin-2-yl)amino)-3-(3-(benzyloxy)phenyl)propanoic acid 24\{1,1,18\} (YJ 31777-035-1): ${ }^{1} \mathrm{H}$ NMR (DMSO- $d_{6}$, very badly resolved, containing AcOEt): 8.32 (s (br), 1H), 7.79 (m, 2H), 7.43 - 6.93 (m, $14 \mathrm{H}), 6.84(\mathrm{~s}(\mathrm{br}), 1 \mathrm{H}), 6.72(\mathrm{~m}, 2 \mathrm{H}), 6.56(\mathrm{~s}(\mathrm{br}), 1 \mathrm{H}), 4.89(\mathrm{~s}, 2 \mathrm{H}), 4.64(\mathrm{~s}(\mathrm{br}), 1 \mathrm{H}), 4.15(\mathrm{~s}(\mathrm{br}), 1 \mathrm{H}), 3.90(\mathrm{~m}, 1 \mathrm{H})$, $3.30(\mathrm{~m}, 1 \mathrm{H}), 3.17(\mathrm{~m}, 1 \mathrm{H}) .{ }^{13} \mathrm{C}$ NMR (DMSO-d $)$ : 158.5, 151.7, 141.7, 141.5, 138.1, 137.7, 137.6, 136.7, 129.4, $129.1,129.0,128.7,128.6,128.1,128.0,127.6,126.5,125.1,122.4,116.4,112.2,69.5,57.4,39.2$, 37.5 (three signals missing). HRMS $(\mathrm{m} / z):[\mathrm{M}+\mathrm{H}]^{+}$calcd for $\mathrm{C}_{33} \mathrm{H}_{30} \mathrm{~N}_{3} \mathrm{O}_{3}: 516.2287$; found, 516.2283.

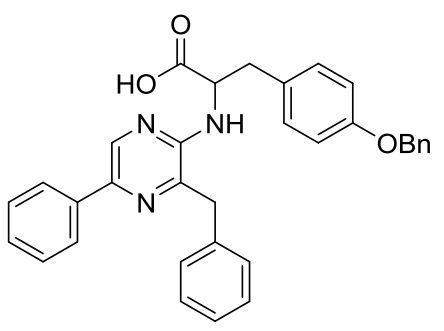

2-((3-Benzyl-5-phenylpyrazin-2-yl)amino)-3-(4-(benzyloxy)phenyl)propanoic acid 24\{1,1,19\} (YJ 31777-037-1): ${ }^{1} \mathrm{H}$ NMR (DMSO- $d_{6}$, very badly resolved, containing AcOEt): 8.34 (s (br), 1H), 7.81 (m, 2H), 7.41 - 7.10 (m, $13 \mathrm{H}), 6.99(\mathrm{~m}, 2 \mathrm{H}), 6.69(\mathrm{~m}, 2 \mathrm{H}), 6.47(\mathrm{~s}(\mathrm{br}), 1 \mathrm{H}), 4.91(\mathrm{~s}, 2 \mathrm{H}), 4.60(\mathrm{~s}(\mathrm{br}), 1 \mathrm{H}), 4.13(\mathrm{~s}(\mathrm{br}), 1 \mathrm{H}), 3.92(\mathrm{~m}, 1 \mathrm{H})$, $3.23(\mathrm{~m}, 1 \mathrm{H}), 3.10(\mathrm{~m}, 1 \mathrm{H}) .{ }^{13} \mathrm{C}$ NMR (DMSO- $\left.d_{6}\right): 157.0,151.6,141.6,138.1,137.7,137.6,136.8,131.8,130.8$, 129.3 , 129.0, 128.8, 128.7, 128.1, 128.0, 127.6, 126.6, 125.1, 114.5, 69.5, 57.4, 39.3, 36.5 (two signals missing). $\operatorname{HRMS}(\mathrm{m} / \mathrm{z}):[\mathrm{M}+\mathrm{H}]^{+}$calcd for $\mathrm{C}_{33} \mathrm{H}_{30} \mathrm{~N}_{3} \mathrm{O}_{3}: 516.2287$; found, 516.2286.

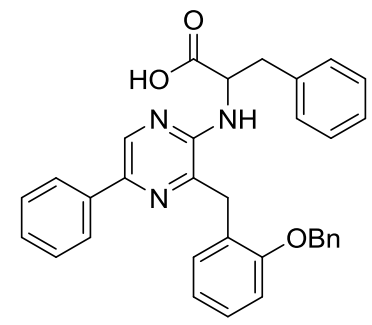

(3-(2-(Benzyloxy)benzyl)-5-phenylpyrazin-2-yl)phenylalanine 24\{1,17,1\} (YJ31776-155-1): ${ }^{1} \mathrm{H}$ NMR (DMSO-d6, very badly resolved, containing AcOEt): 8.38 (s (br), 1H), $7.86(\mathrm{~m}, 2 \mathrm{H}), 7.41-7.25$ (m, 8H), 7.12 - 6.97 (m, 6H), 
$6.93(\mathrm{~s}(\mathrm{br}), 1 \mathrm{H}), 6.82(\mathrm{~m}, 2 \mathrm{H}), 6.48(\mathrm{~s}(\mathrm{br}), 1 \mathrm{H}), 5.07(\mathrm{~m}, 2 \mathrm{H}), 4.62(\mathrm{~s}(\mathrm{br}), 1 \mathrm{H}), 4.02(\mathrm{~s}(\mathrm{br}), 2 \mathrm{H}), 3.29(\mathrm{~m}, 1 \mathrm{H}), 3.10$ (m, 1H). ${ }^{13} \mathrm{C}$ NMR (DMSO- $d_{6}$ ): 158.8, 151.6, 141.3, 139.7, 139.5, 138.1, 137.7, 137.6, 136.9, 129.9, 129.7, 129.4, 129.1, 128.8, 128.7, 128.2, 128.1, 128.0, 127.7, 126.0, 125.2, 115.9, 112.9, 69.6, 57.4, 39.3, 37.4. HRMS (m/z): $[\mathrm{M}+\mathrm{H}]^{+}$calcd for $\mathrm{C}_{33} \mathrm{H}_{30} \mathrm{~N}_{3} \mathrm{O}_{3}: 516.2287$; found, 516.2280.

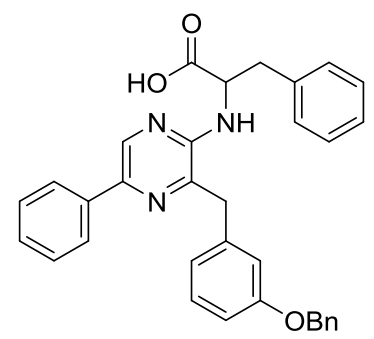

(3-(3-(Benzyloxy)benzyl)-5-phenylpyrazin-2-yl)phenylalanine 24 $\{1,18,1\}$ (YJ31776-153-1) ${ }^{1} \mathrm{H}$ NMR (DMSO- $d_{6}$, very badly resolved, containing AcOEt): $8.40(\mathrm{~s}(\mathrm{br}), 1 \mathrm{H}), 7.79(\mathrm{~m}, 2 \mathrm{H}), 7.37-7.14(\mathrm{~m}, 9 \mathrm{H}), 7.02(\mathrm{~m}, 7 \mathrm{H}), 6.81$ $(\mathrm{m}, 1 \mathrm{H}), 6.29(\mathrm{~s}(\mathrm{br}), 1 \mathrm{H}), 5.00(\mathrm{~s}, 2 \mathrm{H}), 4.52(\mathrm{~s}(\mathrm{br}), 1 \mathrm{H}), 4.12(\mathrm{~s}(\mathrm{br}), 1 \mathrm{H}), 3.94(\mathrm{~m}, 1 \mathrm{H}), 3.28(\mathrm{~m}, 1 \mathrm{H}), 3.13(\mathrm{~m}, 1 \mathrm{H})$. ${ }^{13}$ C NMR (DMSO- $\left.d_{6}\right)$ : $156.4,151.5,141.1,139.5,138.0,137.8,137.7,136.5,130.5,130.0,129.0,128.7,128.1$, 128.0, 127.9, 127.6, 127.5, 126.2, 126.0, 121.0, 112.6, 69.6, 56.9, 37.4, 33.7 (two signals missing). HRMS $(\mathrm{m} / \mathrm{z}$ ): $[\mathrm{M}+\mathrm{H}]^{+}$calcd for $\mathrm{C}_{33} \mathrm{H}_{30} \mathrm{~N}_{3} \mathrm{O}_{3}: 516.2287$; found, 516.2297.

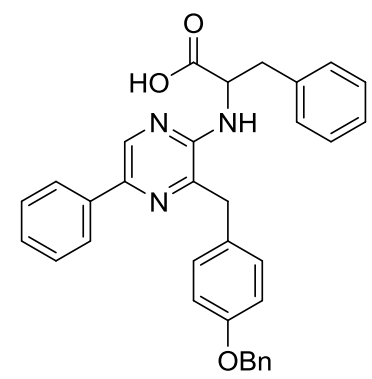

(3-(4-(Benzyloxy)benzyl)-5-phenylpyrazin-2-yl)phenylalanine 24\{1,19,1\} (YJ31776-151-1) ${ }^{1} \mathrm{H}$ NMR (DMSO- $d_{6}$, very badly resolved, containing AcOEt): $8.36(\mathrm{~s}(\mathrm{br}), 1 \mathrm{H}), 7.86(\mathrm{~m}, 2 \mathrm{H}), 7.44-7.27(\mathrm{~m}, 9 \mathrm{H}), 7.12(\mathrm{~m}, 2 \mathrm{H}), 7.05$ $(\mathrm{m}, 5 \mathrm{H}), 6.83(\mathrm{~m}, 2 \mathrm{H}), 6.43(\mathrm{~m}, 1 \mathrm{H}), 5.02(\mathrm{~s}, 2 \mathrm{H}), 4.54(\mathrm{~s}(\mathrm{br}), 1 \mathrm{H}), 4.07(\mathrm{~s}(\mathrm{br}), 1 \mathrm{H}), 3.90(\mathrm{~m}, 1 \mathrm{H}), 3.28(\mathrm{~m}, 1 \mathrm{H})$, $3.12(\mathrm{~m}, 1 \mathrm{H}) .{ }^{13} \mathrm{C}$ NMR (DMSO- $\left.d_{6}\right): 157.3,151.5,141.9,139.7,138.1,137.7,136.7,130.2,130.1,129.8,129.1$, 128.9, 128.2, 128.1, 128.0, 127.6, 126.0, 125.2, 115.2, 115.1, 69.6, 57.3, 38.5, 37.3 (one signal missing). HRMS $(\mathrm{m} / \mathrm{z}):[\mathrm{M}+\mathrm{H}]^{+}$calcd for $\mathrm{C}_{33} \mathrm{H}_{30} \mathrm{~N}_{3} \mathrm{O}_{3}: 516.2287$; found, 516.2288.

General procedure for the debenzylation of O-benzyl-bearing acids 24, preparation of the phenol-bearing acids 24 via step xiii. The considered O-benzyl derivative $24(1.0 \mathrm{mmol})$ and ammonium formate $(1.9 \mathrm{~g}, 30.0$ $\mathrm{mmol})$ were dissolved in ethanol $(30 \mathrm{~mL})$. To this was added $10 \%$ palladium over charcoal $(0.10 \mathrm{~g}, 0.1 \mathrm{mmol})$ and the suspension was heated to reflux for 90 minutes. This was cooled, filtered washed with a small amount of water and ethanol and the filtrate was concentrated to dryness under high vacuum to remove any unreacted ammonium formate as much as possible. This gave the corresponding crude deprotected acids $\mathbf{2 4}$ as described below which were used without further purifications in the next step.

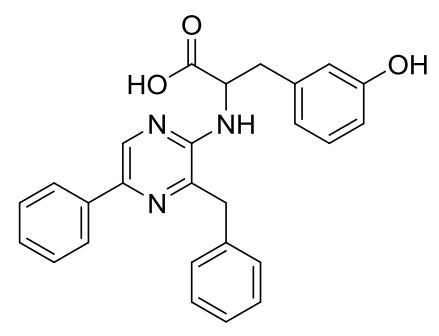

2-((3-Benzyl-5-phenylpyrazin-2-yl)amino)-3-(3-hydroxyphenyl)propanoic acid $\mathbf{2 4}\{1,1,62\}$ (YJ31777-045-1): ${ }^{1} \mathrm{H}$ NMR (DMSO-d $d_{6}$, containing EtOH): $8.45(\mathrm{~s}, 1 \mathrm{H}), 8.40(\mathrm{~s}, 1 \mathrm{H}), 7.89(\mathrm{~m}, 2 \mathrm{H}), 7.40(\mathrm{~m}, 2 \mathrm{H}), 7.30-7.14(\mathrm{~m}, 6 \mathrm{H})$, $6.91(\mathrm{~m}, 1 \mathrm{H}), 6.67(\mathrm{~m}, 1 \mathrm{H}), 6.52(\mathrm{~m}, 3 \mathrm{H}), 4.46(\mathrm{~s}(\mathrm{br}), 1 \mathrm{H}), 4.12(\mathrm{~d}, 1 \mathrm{H}, J=14.6 \mathrm{~Hz}), 3.94(\mathrm{~d}, 1 \mathrm{H}, J=14.6 \mathrm{~Hz})$, 
$3.19(\mathrm{dd}, 1 \mathrm{H}, J=4.8,13.9 \mathrm{~Hz}), 3.06(\mathrm{dd}, 1 \mathrm{H}, J=6.9,13.9 \mathrm{~Hz}) .{ }^{13} \mathrm{C}$ NMR (DMSO- $\left.d_{6}\right): 157.5,151.5,141.6,140.8$, 138.2, 138.1, 137.7, 136.9, 129.4, 129.1, 128.9, 128.7, 127.7, 126.7, 125.2, 120.5, 116.9, 113.3, 57.0, 39.2, 37.2 (one signals missing). HRMS (m/z): $[\mathrm{M}+\mathrm{H}]^{+}$calcd for $\mathrm{C}_{26} \mathrm{H}_{24} \mathrm{~N}_{3} \mathrm{O}_{3}$ : 426.1818; found, 426.1820 .

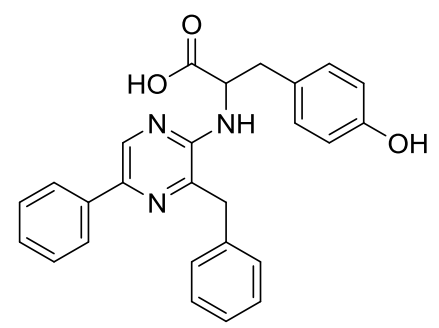

(3-Benzyl-5-phenylpyrazin-2-yl)tyrosine 24\{1,1,63\} (YJ31777-047-1): ${ }^{1} \mathrm{H}$ NMR (DMSO- $d_{6}$, containing EtOH): $8.41(\mathrm{~s}, 1 \mathrm{H}), 8.37(\mathrm{~s}, 1 \mathrm{H}), 7.88(\mathrm{~m}, 2 \mathrm{H}), 7.44-7.14(\mathrm{~m}, 9 \mathrm{H}), 6.91(\mathrm{~m}, 2 \mathrm{H}), 6.54(\mathrm{~m}, 3 \mathrm{H}), 4.48(\mathrm{~s}(\mathrm{br}), 1 \mathrm{H}), 4.17(\mathrm{~d}$, $1 \mathrm{H}, J=14.3 \mathrm{~Hz}), 3.95(\mathrm{~d}, 1 \mathrm{H}, J=14.3 \mathrm{~Hz}), 3.15(\mathrm{~m}, 1 \mathrm{H}), 3.03(\mathrm{~m}, 1 \mathrm{H}) .{ }^{13} \mathrm{C}$ NMR (DMSO-d $): 156.0,151.6$, 141.6, 138.3, 138.1, 137.7, 136.8, 130.6, 129.4, 129.3, 129.1, 128.7, 127.6, 126.6, 125.2, 115.1, 57.3, $39.2,36.4$ (one signals missing). HRMS $(m / z)$ : $[\mathrm{M}+\mathrm{H}]^{+}$calcd for $\mathrm{C}_{26} \mathrm{H}_{24} \mathrm{~N}_{3} \mathrm{O}_{3}$ : 426.1818; found, 426.1816.<smiles>O=C(O)C(Cc1ccccc1)Nc1ncc(-c2ccccc2)nc1Cc1ccccc1O</smiles>

(3-(2-Hydroxybenzyl)-5-phenylpyrazin-2-yl)phenylalanine 24\{1,61,1\} (YJ31776-157-1): ${ }^{1} \mathrm{H}$ NMR (DMSO-d6, very badly resolved, containing EtOH): 8.43 (s (br), 1H), $7.89(\mathrm{~m}, 2 \mathrm{H}), 7.43(\mathrm{~m}, 2 \mathrm{H}), 7.29(\mathrm{~m}, 2 \mathrm{H}), 7.12$ - $6.98(\mathrm{~m}$, $6 \mathrm{H}), 6.70(\mathrm{~m}, 2 \mathrm{H}), 6.60(\mathrm{~m}, 1 \mathrm{H}), 6.40(\mathrm{~m}, 1 \mathrm{H}), 4.53(\mathrm{~s}(\mathrm{br}), 1 \mathrm{H}), 4.07(\mathrm{~m}, 1 \mathrm{H}), 3.82(\mathrm{~m}, 1 \mathrm{H}), 3.25(\mathrm{~m}, 1 \mathrm{H}), 3.09(\mathrm{~m}$, 1H). ${ }^{13} \mathrm{C}$ NMR (DMSO- $\left.d_{6}\right): 157.9,151.4,141.5,139.4,139.2,138.3,137.7,136.8,129.9,129.7,129.1,128.1$, $127.7,126.1,125.2,119.9,116.1,113.9,57.1,39.2,37.3$ (one signal missing). HRMS $(\mathrm{m} / \mathrm{z}):[\mathrm{M}+\mathrm{H}]^{+}$calcd for $\mathrm{C}_{26} \mathrm{H}_{24} \mathrm{~N}_{3} \mathrm{O}_{3}$ : 426.1818; found, 426.1821.

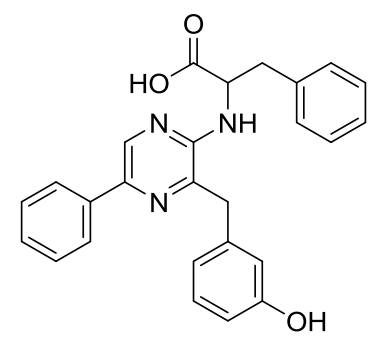

(3-(3-Hydroxybenzyl)-5-phenylpyrazin-2-yl)phenylalanine 24\{1,62,1\} (YJ31776-159-1): ${ }^{1} \mathrm{H}$ NMR (DMSO- $d_{6}$, very badly resolved, containing EtOH): 8.39 (s (br), 1H), $7.89(\mathrm{~m}, 2 \mathrm{H}), 7.39(\mathrm{~m}, 3 \mathrm{H}), 7.28(\mathrm{~m}, 2 \mathrm{H}), 7.18$ - $7.01(\mathrm{~m}$, $6 \mathrm{H}), 6.92(\mathrm{~m}, 1 \mathrm{H}), 6.69(\mathrm{~m}, 2 \mathrm{H}), 4.63(\mathrm{~s}(\mathrm{br}), 1 \mathrm{H}), 4.03(\mathrm{~m}, 2 \mathrm{H}), 3.28(\mathrm{~m}, 1 \mathrm{H}), 3.04(\mathrm{~m}, 1 \mathrm{H}) .{ }^{13} \mathrm{C}$ NMR (DMSO-d6): $155.3,151.5,141.8,140.8,139.3,138.3,137.6,136.5,130.5,129.8,129.1,128.2,127.7,126.2,125.2,123.9$, 119.5, 115.9, 57.1, 37.9, 33.4 (one signal missing). HRMS $(m / z)$ : $[\mathrm{M}+\mathrm{H}]^{+}$calcd for $\mathrm{C}_{26} \mathrm{H}_{24} \mathrm{~N}_{3} \mathrm{O}_{3}$ : 426.1818; found, 426.1821 . 


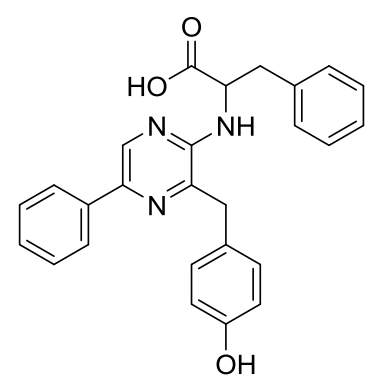

(3-(4-Hydroxybenzyl)-5-phenylpyrazin-2-yl)phenylalanine 24\{1,63,1\} (YJ31776-161-1): ${ }^{1} \mathrm{H}$ NMR (DMSO-d6, very badly resolved, containing EtOH): 8.40 (s (br), 1H), $7.89(\mathrm{~m}, 2 \mathrm{H}), 7.39(\mathrm{~m}, 2 \mathrm{H}), 7.29(\mathrm{~m}, 2 \mathrm{H}), 7.14$ - 7.01 (m, $7 \mathrm{H}), 6.64(\mathrm{~m}, 2 \mathrm{H}), 4.63(\mathrm{~s}(\mathrm{br}), 1 \mathrm{H}), 4.01(\mathrm{~m}, 2 \mathrm{H}), 3.84(\mathrm{~m}, 2 \mathrm{H}), 3.24(\mathrm{~m}, 1 \mathrm{H}), 3.12(\mathrm{~m}, 1 \mathrm{H}) .{ }^{13} \mathrm{C}$ NMR (DMSO-d $)$ : 156.3, 151.4, 142.2, 139.5, 138.2, 137.7, 136.6, 130.1, 129.9, 129.1, 128.1, 127.9, 127.7, 126.1, 125.2, 115.6, 57.1, 38.6, 37.3 (one signal missing). $\mathrm{HRMS}(\mathrm{m} / \mathrm{z})$ : $[\mathrm{M}+\mathrm{H}]^{+}$calcd for $\mathrm{C}_{26} \mathrm{H}_{24} \mathrm{~N}_{3} \mathrm{O}_{3}$ : 426.1818; found, 426.1822 .

General protocol for the cyclization and mono-acetylation of $\mathrm{OH}$-bearing acids 24 , preparation of phenolbearing $\boldsymbol{O}$-acetylated luciferins 25 via step xiv. The considered deprotected acid $(0.4 \mathrm{mmol})$ was dispersed in ethyl acetate $(10 \mathrm{~mL})$. To this was added acetic anhydride $(0.095 \mathrm{~mL}, 0.8 \mathrm{mmol})$ and the mixture heated to reflux for 30 minutes. The resulting solution was adsorbed over a small amount of silica under vacuum, without any heating and purified by a chromatography over silica gel without delay as described below.

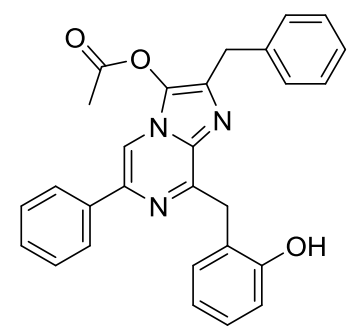

2-Benzyl-8-(2-hydroxybenzyl)-6-phenylimidazo[1,2-a]pyrazin-3-yl acetate 25 1,61,1\} (YJ31777-013-2): Obtained as a yellow powder $(0.06 \mathrm{~g}, 13 \%$ from $\mathbf{2 3}\{1,17,1\})$ after a chromatography over silica gel (cyclohexane - ethyl acetate 3/1). ${ }^{1} \mathrm{H}$ NMR (DMSO- $\left.d_{6}\right): 9.30(\mathrm{~s}, 1 \mathrm{H}), 8.73(\mathrm{~s}, 1 \mathrm{H}), 8.08(\mathrm{~m}, 2 \mathrm{H}), 7.51(\mathrm{~s}, 2 \mathrm{H}), 7.40(\mathrm{~m}, 1 \mathrm{H}), 7.30(\mathrm{~m}$, $4 \mathrm{H}), 7.22(\mathrm{~m}, 1 \mathrm{H}), 7.08(\mathrm{~m}, 1 \mathrm{H}), 6.91(\mathrm{~m}, 2 \mathrm{H}), 6.62(\mathrm{~m}, 1 \mathrm{H}), 4.40(\mathrm{~s}, 2 \mathrm{H}), 4.11(\mathrm{~s}, 2 \mathrm{H}), 2.25(\mathrm{~s}, 3 \mathrm{H}) .{ }^{13} \mathrm{C} \mathrm{NMR}$ (DMSO-d6): 168.5, 157.7, 152.2, 139.6, 138.9, 137.8, 136.7, 135.5, 133.5, 129.7, 129.3, 129.1, 128.9, 128.8, 126.7, 126.6, 120.4, 116.5, 113.9, 111.1, 39.2, 33.1, 20.7 (one signal missing). HRMS $(\mathrm{m} / z)$ : $[\mathrm{M}+\mathrm{H}]^{+}$calcd for $\mathrm{C}_{28} \mathrm{H}_{24} \mathrm{~N}_{3} \mathrm{O}_{3}$ : 450.1818; found, 450.1812 .

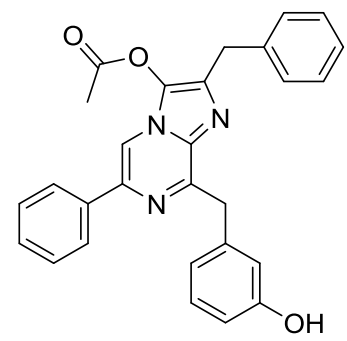

2-Benzyl-8-(3-hydroxybenzyl)-6-phenylimidazo[1,2-a]pyrazin-3-yl acetate 25\{1,62,1\} (YJ31777-015-1): Obtained as a pale pink powder $(0.12 \mathrm{~g}, 25 \%$ from $\mathbf{2 3}\{1,18,1\})$ after a chromatography over silica gel (cyclohexane - ethyl acetate 5/1) and a recrystallization in cyclohexane. ${ }^{1} \mathrm{H}$ NMR (DMSO- $\left.d_{6}\right): 9.66(\mathrm{~s}, 1 \mathrm{H}), 8.74(\mathrm{~s}, 1 \mathrm{H}), 8.00(\mathrm{~m}, 2 \mathrm{H})$, $7.46(\mathrm{~s}, 2 \mathrm{H}), 7.40(\mathrm{~m}, 1 \mathrm{H}), 7.30(\mathrm{~m}, 4 \mathrm{H}), 7.22(\mathrm{~m}, 2 \mathrm{H}), 7.08(\mathrm{~m}, 1 \mathrm{H}), 6.84(\mathrm{~m}, 1 \mathrm{H}), 6.74(\mathrm{~m}, 1 \mathrm{H}), 4.45(\mathrm{~s}, 2 \mathrm{H})$, 4.09 (s, 2H), 2.39 (s, 3H). ${ }^{13} \mathrm{C}$ NMR (DMSO-d $)$ : 168.6, 157.7, 152.4, 138.8, 137.8, 136.6, 135.2, 133.6, 131.1, 129.3, 129.2, 129.1, 128.9, 128.8, 128.2, 126.7, 126.5, 124.8, 119.5, 116.2, 111.1, 33.5, 33.0, 20.8. HRMS (m/z): $[\mathrm{M}+\mathrm{H}]^{+}$calcd for $\mathrm{C}_{28} \mathrm{H}_{24} \mathrm{~N}_{3} \mathrm{O}_{3}: 450.1818$; found, 450.1825 . 


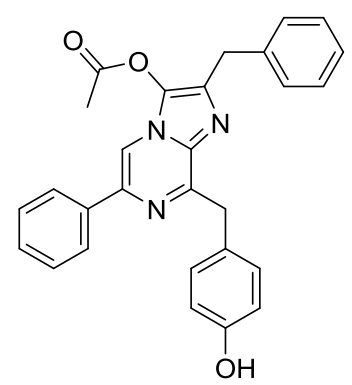

2-Benzyl-8-(4-hydroxybenzyl)-6-phenylimidazo[1,2-a]pyrazin-3-yl acetate 25 1,63,1\} (YJ31777-017-1): Obtained as a yellow powder $(0.18 \mathrm{~g}, 38 \%$ from $23\{1,19,1\})$ after a chromatography over silica gel (cyclohexane - ethyl acetate 3/1) and a dispersion in boiling cyclohexane. ${ }^{1} \mathrm{H}$ NMR (DMSO-d $): 9.20(\mathrm{~s}, 1 \mathrm{H}), 8.70(\mathrm{~s}, 1 \mathrm{H}), 8.07(\mathrm{~m}$, 2H), $7.49(\mathrm{~s}, 2 \mathrm{H}), 7.40(\mathrm{~m}, 1 \mathrm{H}), 7.31-7.19(\mathrm{~m}, 7 \mathrm{H}), 6.67(\mathrm{~m}, 2 \mathrm{H}), 4.36(\mathrm{~s}, 2 \mathrm{H}), 4.09(\mathrm{~s}, 2 \mathrm{H}), 2.38(\mathrm{~s}, 3 \mathrm{H}) .{ }^{13} \mathrm{C} \mathrm{NMR}$ (DMSO- $d_{6}$ ): $168.5,156.4,152.8,139.0,137.8,136.7,135.4,133.4,130.6,129.3,129.2,129.1,128.9,128.8,128.4$, 126.7, 126.5, 115.5, 111.0, 38.4, 33.0, 20.7. HRMS $(\mathrm{m} / \mathrm{z}):[\mathrm{M}+\mathrm{H}]^{+}$calcd for $\mathrm{C}_{28} \mathrm{H}_{24} \mathrm{~N}_{3} \mathrm{O}_{3}$ : 450.1818; found, 450.1807 .

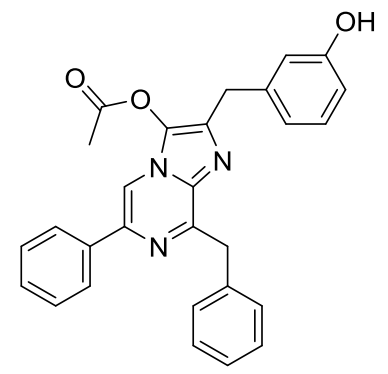

8-Benzyl-2-(3-hydroxybenzyl)-6-phenylimidazo[1,2-a]pyrazin-3-yl acetate 25\{1,1,62\} (YJ31777-051-1): Obtained as a yellow powder $(0.21 \mathrm{~g}, 43 \%$ from $\mathbf{2 3}\{1,1,18\})$ after a chromatography over silica gel (cyclohexane - ethyl acetate $3 / 1)$ and a dispersion in boiling cyclohexane. ${ }^{1} \mathrm{H}$ NMR $\left(\mathrm{CDCl}_{3}\right): 7.88(\mathrm{~m}, 2 \mathrm{H}), 7.78(\mathrm{~s}, 1 \mathrm{H}), 7.59(\mathrm{~m}, 2 \mathrm{H})$, $7.43(\mathrm{~m}, 3 \mathrm{H}), 7.26(\mathrm{~m}, 2 \mathrm{H}), 7.18(\mathrm{~m}, 1 \mathrm{H}), 7.13(\mathrm{~m}, 1 \mathrm{H}), 4.60(\mathrm{~s}, 2 \mathrm{H}), 4.10(\mathrm{~s}, 2 \mathrm{H}), 2.22(\mathrm{~s}, 3 \mathrm{H}) .{ }^{13} \mathrm{C} \mathrm{NMR}\left(\mathrm{CDCl}_{3}\right)$ : $167.0,156.8,152.7,139.5,139.0,137.5,136.5,134.8,133.2,129.7,129.6,129.0,128.8,128.7,128.3,126.5$, 126.4, 120.7, 116.1, 114.2, 108.7, 39.2, 33.5, 19.9. HRMS $(\mathrm{m} / z):[\mathrm{M}+\mathrm{H}]^{+}$calcd for $\mathrm{C}_{28} \mathrm{H}_{24} \mathrm{~N}_{3} \mathrm{O}_{3}$ : 450.1818; found, 450.1814 .

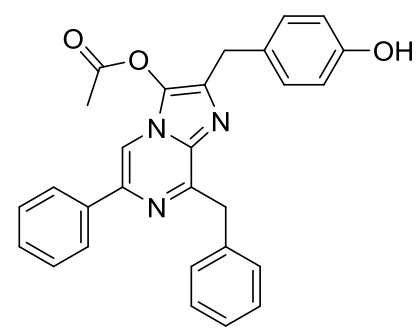

8-Benzyl-2-(4-hydroxybenzyl)-6-phenylimidazo[1,2-a]pyrazin-3-yl acetate 25 1,1,63\} (YJ31777-049-1): Obtained as a yellow powder $(0.14 \mathrm{~g}, 24 \%$ from $23\{1,1,19\})$ after a chromatography over silica gel (cyclohexane - ethyl acetate 3/1) and a dispersion in boiling cyclohexane. ${ }^{1} \mathrm{H}$ NMR (DMSO-d $): 9.20(\mathrm{~s}, 1 \mathrm{H}), 8.71(\mathrm{~s}, 1 \mathrm{H}), 8.06(\mathrm{~m}$, 2H), $7.48(\mathrm{~s}, 2 \mathrm{H}), 7.39(\mathrm{~m}, 1 \mathrm{H}), 7.28(\mathrm{~m}, 2 \mathrm{H}), 7.20(\mathrm{~m}, 1 \mathrm{H}), 7.10(\mathrm{~m}, 2 \mathrm{H}), 6.69(\mathrm{~m}, 2 \mathrm{H}), 4.49(\mathrm{~s}, 2 \mathrm{H}), 3.98(\mathrm{~s}, 2 \mathrm{H})$, 2.38 (s, 3H). ${ }^{13} \mathrm{C}$ NMR (DMSO-d $)$ : 168.5, 156.2, 152.1, 138.3, 137.7, 136.7, 136.2, 133.4, 130.2, 129.7, 129.2, $129.1,129.0,128.9,128.7,126.9,126.5,115.5,111.1,39.2,32.3,20.8$. HRMS $(\mathrm{m} / z):[\mathrm{M}+\mathrm{H}]^{+}$calcd for $\mathrm{C}_{28} \mathrm{H}_{24} \mathrm{~N}_{3} \mathrm{O}_{3}$ : 450.1818; found, 450.1814.

General procedure for the debenzylation of O-benzyl-bearing compound 25, preparation of the phenolbearing $\mathbf{O}$-acetylated luciferins 25 via step xvi. The considered $\mathrm{O}$-acetylated luciferin $\mathbf{2 5}$ ( $1 \mathrm{mmol}$ ) was dissolved in a mixture of ethyl acetate $(10 \mathrm{~mL})$, ethanol $(10 \mathrm{~mL})$ and acetic acid $(4 \mathrm{~mL})$. To this was added $10 \%$ palladium over charcoal $(0.1 \mathrm{~g}, 0.1 \mathrm{mmol})$, the atmosphere was replaced with hydrogen and the reaction stirred for 13 hours. This was filtered, concentrated to dryness and the residue purified by a chromatography over silica gel as described 
below (note 1). Note 1: the trace amount of palladium still present in the crude mixture, along with air, will over few hours, decompose the target luciferin. Similarly, adsorption of the residue over a small amount of silica should be done without any heating and the ensuing purification by chromatography undertaken without delay.<smiles>CC(=O)Oc1c(Cc2ccc(C)o2)nc2c(Cc3ccccc3)nc(-c3ccc(O)cc3)cn12</smiles>

8-benzyl-6-(4-hydroxyphenyl)-2-((5-methylfuran-2-yl)methyl)imidazo[1,2-a]pyrazin-3-yl acetate 25 $\{14,1,42\}$ (YJ 31776-083-2): This compound was obtained as a beige powder $(0.09 \mathrm{~g}, 27 \%)$ after a chromatography over silica gel (cyclohexane - ethyl acetate 4/1). ${ }^{1} \mathrm{H}$ NMR (DMSO-d $)$ : $9.66(\mathrm{~s}, 1 \mathrm{H}), 8.55(\mathrm{~s}, 1 \mathrm{H}), 7.90(\mathrm{~m}, 2 \mathrm{H}), 7.47(\mathrm{~m}, 2 \mathrm{H})$, $7.47(\mathrm{~m}, 2 \mathrm{H}), 7.30(\mathrm{~m}, 2 \mathrm{H}), 7.20(\mathrm{~m}, 1 \mathrm{H}), 6.87(\mathrm{~m}, 2 \mathrm{H}), 6.04(\mathrm{~d}(\mathrm{br}), 1 \mathrm{H}, J=3.0 \mathrm{~Hz}), 5.97(\mathrm{~m}, 1 \mathrm{H}), 4.47(\mathrm{~s}, 2 \mathrm{H})$, 4.07 (s, 2H), 2.40 (s, 3H), $2.20(\mathrm{~s}, 3 \mathrm{H}) .{ }^{13} \mathrm{C} \mathrm{NMR}$ (DMSO-d $): 168.3,158.5,152.0,150.8,150.0,138.4,138.3$, $133.1,132.8,129.6,129.2,128.8,127.9,127.5,126.8,115.9,109.3,107.8,106.8,39.1,26.6,20.7$, 13.7. HRMS $(\mathrm{m} / z):[\mathrm{M}+\mathrm{H}]^{+}$calcd for $\mathrm{C}_{27} \mathrm{H}_{54} \mathrm{~N}_{3} \mathrm{O}_{4}$ : 454.1767; found, 454.1766.<smiles>CC(=O)Oc1c(Cc2ccc(C)o2)nc2c(Cc3ccccc3)nc(-c3cccc(O)c3)cn12</smiles>

8-benzyl-6-(3-hydroxyphenyl)-2-((5-methylfuran-2-yl)methyl)imidazo[1,2-a]pyrazin-3-yl acetate $\mathbf{2 5}\{13,1,42\}$ (RB32489-025-2): This compound was obtained as a red powder $(0.15 \mathrm{~g}, 41 \%)$ after a chromatography over silica gel (cyclohexane - ethyl acetate 4/1). ${ }^{1} \mathrm{H}\left(\mathrm{CDCl}_{3}\right)$ : $7.81(\mathrm{~s}, 1 \mathrm{H}), 7.57(\mathrm{~m}, 2 \mathrm{H}), 7.41(\mathrm{~m}, 2 \mathrm{H}), 729-7.24(\mathrm{~m}, 3 \mathrm{H}), 7.18$ $(\mathrm{m}, 1 \mathrm{H}), 6.85(\mathrm{~m}, 1 \mathrm{H}), 6.01(\mathrm{~d}, 1 \mathrm{H}, J=2.5 \mathrm{~Hz}), 5.89(\mathrm{~m}, 1 \mathrm{H}), 4.61(\mathrm{~s}, 2 \mathrm{H}), 4.19(\mathrm{~s}, 2 \mathrm{H}), 2.34(\mathrm{~s}, 3 \mathrm{H}), 2.25$ (s (br), $3 \mathrm{H}) .{ }^{13} \mathrm{C}\left(\mathrm{CDCl}_{3}\right)$ 167.0, 156.6, 152.8, 151.2, 149.3, 139.0, 138.1, 137.6, 133.4, 132.9, 129.9, 129.6, 129.0, 128.3, 126.5, 118.3, 115.9, 113.8, 109.1, 107.6, 106.2, 39.2, 27.2, 20.0, 13.5. HRMS (m/z): $[\mathrm{M}+\mathrm{H}]^{+}$calcd for $\mathrm{C}_{27} \mathrm{H}_{54} \mathrm{~N}_{3} \mathrm{O}_{4}$ : 454.1767; found, 454.1760 .

\section{Synthetic scheme for the preparation of hydroxypyrazines $19\{1,60\}, 19\{1,38\}$ and $19\{1,35\}$}

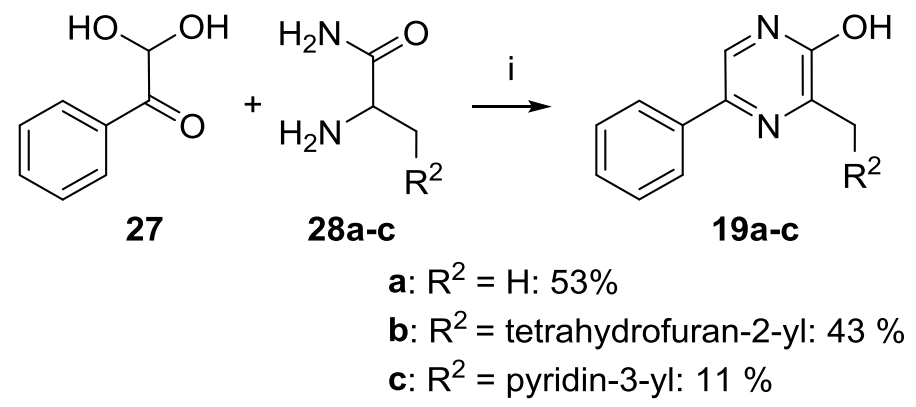

Scheme 3. i: a) $\mathrm{NaOH}, \mathrm{MeOH},-78^{\circ} \mathrm{C}$, b) $\mathrm{H}_{3} \mathrm{O}^{+} \mathrm{Cl}^{-}$

Preparation of aminoamides 28 from $\square$-amino esters. In a steel reactor, the considered $\square$-amino ester (0.065 mol, free base, note 1) was dispersed in $7 \mathrm{~N}$ methanolic ammonia $(50 \mathrm{~mL})$. This was properly stirred and heated at $70{ }^{\circ} \mathrm{C}$ for 20 hours, concentrated to dryness as described below and used directly in the next step. Note 1: the hydrochloride salts were also used in other instances. 


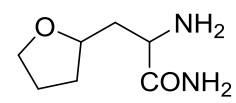

2-Amino-3-(tetrahydrofuran-2-yl)propanamide 28\{38\} (YJ31134-019-1): Obtained as an oil which solidified (1.7 g, 93\%). ${ }^{1} \mathrm{H} \mathrm{NMR}\left(\mathrm{CDCl}_{3}\right)$ : mixture of isomer: $7.23(\mathrm{~s}, 1 \mathrm{H}), 5.63(\mathrm{~s}, 1 \mathrm{H}), 4.02(\mathrm{~m}, 2 \mathrm{H}), 3.88(\mathrm{~m}, 1 \mathrm{H}), 3.74(\mathrm{~m}$, $1 \mathrm{H}), 3.56(\mathrm{~m}, 1 \mathrm{H}), 2.07(\mathrm{~m}, 1 \mathrm{H}), 1.89(\mathrm{~m}, 5 \mathrm{H}), 1.54(\mathrm{~m}, 1 \mathrm{H}) .{ }^{13} \mathrm{C} \mathrm{NMR}\left(\mathrm{CDCl}_{3}\right):$ 178.3, 79.3, 68.0, 55.6, 40.3, 32.3, 25.2. HRMS $(m / z):[\mathrm{M}+\mathrm{H}]^{+}$calcd for $\mathrm{C}_{7} \mathrm{H}_{15} \mathrm{~N}_{2} \mathrm{O}: 159.1134$; found, 159.1122 .

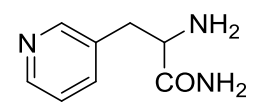

2-Amino-3-(pyridin-3-yl)propanamide 28\{35\} (YJ30367-005-2): Obtained as an orange solid (3.46 g, 99\%). ${ }^{1} \mathrm{H}$ NMR (DMSO- $\left.d_{6}\right): 8.42(\mathrm{~m}, 1 \mathrm{H}), 8.40(\mathrm{dd}, 1 \mathrm{H}, J=1.9,4.6 \mathrm{~Hz}), 7.62(\mathrm{~m}, 1 \mathrm{H}), 7.26(\mathrm{~m}, 2 \mathrm{H}), 6.95(\mathrm{~s}(\mathrm{br}), 1 \mathrm{H}), 4.02$ $(\mathrm{m}, 2 \mathrm{H}), 3.35(\mathrm{dd}, 1 \mathrm{H}, J=5.3,8.0 \mathrm{~Hz}), 2.90(\mathrm{dd}, 1 \mathrm{H}, J=5.2,13.6 \mathrm{~Hz}), 2.64(\mathrm{dd}, 1 \mathrm{H}, J=8.0,13.6 \mathrm{~Hz}), 1.75(\mathrm{~m}$, $2 \mathrm{H}) .{ }^{13} \mathrm{C}$ NMR (DMSO- $\left.d_{6}\right): 176.9,150.9,147.7,137.2,134.9,123.6,56.3,38.6 . \mathrm{HRMS}(\mathrm{m} / \mathrm{z}):[\mathrm{M}+\mathrm{H}]^{+}$calcd for $\mathrm{C}_{8} \mathrm{H}_{12} \mathrm{~N}_{3} \mathrm{O}$ : 166.0980; found, 166.0984 .

General procedure for the condensation between phenyl glyoxal 27 and amino amides $\mathbf{2 8}$. These compounds were prepared by following the protocols previously described. ${ }^{[5]}$ Phenyl glyoxal hydrate 27 (1.33 g, $\left.8.74 \mathrm{mmol}\right)$ and the corresponding amino amide $28(8.74 \mathrm{mmol})$ were dispersed in methanol $(50 \mathrm{~mL})$. This was cooled to -78 ${ }^{\circ} \mathrm{C}$ with a dry ice bath and sodium hydroxide $(0.45 \mathrm{~g}, 11.36 \mathrm{mmol}$, note 1$)$ dissolved in water (3 ml) was added drop-wise. The solution was stirred at $-78{ }^{\circ} \mathrm{C}$ for 15 minutes allowed to warm back to $0{ }^{\circ} \mathrm{C}$ while stirring for 90 minutes and then further stirred at $20^{\circ} \mathrm{C}$ for 30 minutes. This was made acid with $37 \%$ hydrochloric acid, diluted in water brought back to neutrality with solid sodium hydrogenocarbonate and filtered. When a precipitation occurred (note 2), the collected solid was washed with water and dry under vacuum ate $60{ }^{\circ} \mathrm{C}$ to yield the hydroxypyrazines 19 as described below. Note 1: if a hydrochloric salt of the aminoamide is used and additional equivalent of sodium hydroxide must be used. Note 2: see below for the isolation procedure of 5-phenyl-3(pyridin-3-ylmethyl)pyrazin-2-ol (19 $\{1,35\})$.

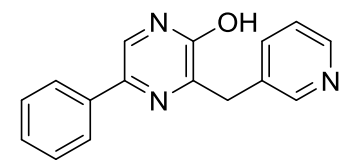

5-Phenyl-3-(pyridin-3-ylmethyl)pyrazin-2-ol 19\{1,35\} (YJ30367-008-5): Obtained as a white solid (0.57 g, 11\%) although an extraction of the reaction medium with ethyl acetate had to be made. The resulting residue was dispersed in dichloromethane and the insoluble material further purified by a chromatography over silica gel (dichloromethane - ethanol 95/5 to 9/1). ${ }^{1} \mathrm{H}$ NMR (DMSO- $\left.d_{6}\right): 12.47$ (s(br), $\left.1 \mathrm{H}\right), 8.57$ (d, $\left.1 \mathrm{H}, J=1.7 \mathrm{~Hz}\right), 8.43$ $(\mathrm{dd}, 1 \mathrm{H}, J=1.7,4.8 \mathrm{~Hz}), 7.89(\mathrm{~s}, 1 \mathrm{H}), 7.92(\mathrm{~m}, 2 \mathrm{H}), 7.74(\mathrm{~m}, 1 \mathrm{H}), 7.40-7.25(\mathrm{~m}, 4 \mathrm{H}), 4.10(\mathrm{~s}, 2 \mathrm{H}) .{ }^{13} \mathrm{C} \mathrm{NMR}$

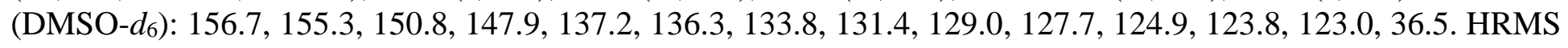
$(\mathrm{m} / z):[\mathrm{M}+\mathrm{H}]^{+}$calcd for $\mathrm{C}_{16} \mathrm{H}_{14} \mathrm{~N}_{3} \mathrm{O}, 264.1137$; found, 264.1122 .

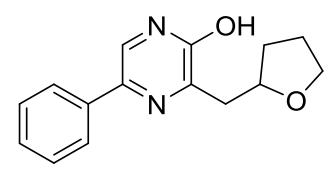

5-Phenyl-3-((tetrahydrofuran-2-yl)methyl)pyrazin-2-ol 19\{1,38\} (YJ31134-061-1): Obtained as a pink solid (1.02 $\mathrm{g}, 45 \%)$ which was used without any further purification in the step viii described above. ${ }^{1} \mathrm{H}$ NMR $\left(\mathrm{CDCl}_{3}\right): 13.22$ $(\mathrm{s}(\mathrm{br}), 1 \mathrm{H}), 7.81(\mathrm{~m}, 2 \mathrm{H}), 7.63(\mathrm{~s}(\mathrm{br}), 1 \mathrm{H}), 7.44(\mathrm{~m}, 2 \mathrm{H}), 7.35(\mathrm{~m}, 1 \mathrm{H}), 4.60(\mathrm{p}, 1 \mathrm{H}, J=6.6 \mathrm{~Hz}), 3.99(\mathrm{~m}, 1 \mathrm{H})$, $3.80(\mathrm{~m}, 1 \mathrm{H}), 3.30(\mathrm{dd}, 1 \mathrm{H}, J=7.0,14.8 \mathrm{~Hz}), 3.05(\mathrm{dd}, 1 \mathrm{H}, J=6.1,14.8 \mathrm{~Hz}), 2.17(\mathrm{~m}, 1 \mathrm{H}), 1.98(\mathrm{~m}, 2 \mathrm{H}), 1.76(\mathrm{~m}$, 1H). ${ }^{13} \mathrm{C} \mathrm{NMR}\left(\mathrm{CDCl}_{3}\right): 157.2,156.8,135.9,134.4,128.8,128.0,125.1,120.3,76.9,67.8,39.0,31.4,25.6$. HRMS $(\mathrm{m} / \mathrm{z}):[\mathrm{M}+\mathrm{H}]^{+}$calcd for $\mathrm{C}_{15} \mathrm{H}_{17} \mathrm{~N}_{2} \mathrm{O}_{2}, 257.1290$; found, 257.1281 . 
POH

3-Methyl-5-phenylpyrazin-2-ol 19\{1,60\} (VHE30612-133-2): This compound was obtained using alanine amide hydrochloride ${ }^{[6]}$ as a light orange solid $(4.08 \mathrm{~g}, 53 \%)$ which was used without any further purification in the step vii described above. ${ }^{1} \mathrm{H}$ NMR (DMSO- $\left.d_{6}\right) 12.31(\mathrm{~s}, 1 \mathrm{H}), 7.83(\mathrm{~m}, 3 \mathrm{H}), 7.39(\mathrm{~m}, 2 \mathrm{H}), 7.28(\mathrm{~m}, 1 \mathrm{H}), 2.36(\mathrm{~s}, 3 \mathrm{H})$. ${ }^{13} \mathrm{C}$ NMR (DMSO- $\left.d_{6}\right): 156.3,155.8,136.5,131.2,129.0,127.6,124.9,122.3,20.9$. HRMS $(\mathrm{m} / z):[\mathrm{M}+\mathrm{H}]^{+}$calcd for $\mathrm{C}_{11} \mathrm{H}_{11} \mathrm{~N}_{2} \mathrm{O}$ : 187.0871 ; found, 187.0808 .

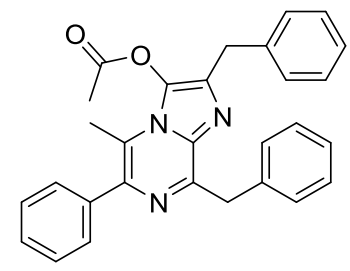

Preparation of 2,8-dibenzyl-5-methyl-6-phenylimidazo[1,2-a]pyrazin-3-yl acetate (30), the following synthetic pathway was used:

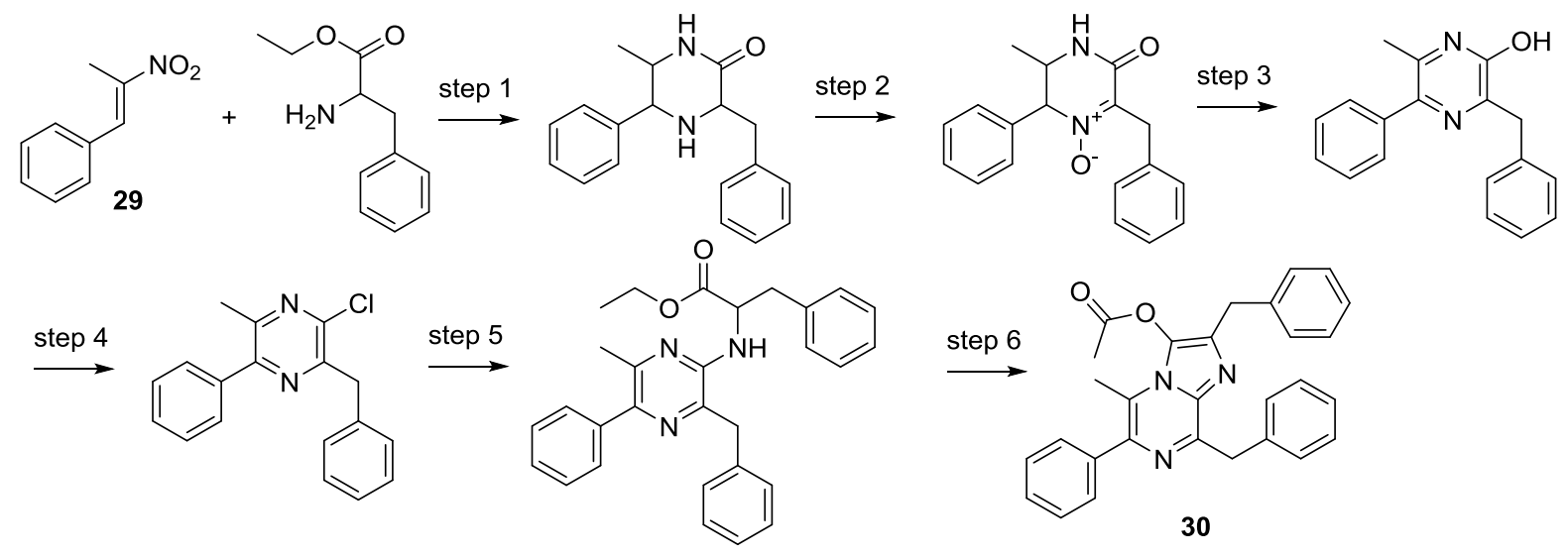

First step, preparation of 3-benzyl-6-methyl-5-phenylpiperazin-2-one: By using the general protocol described above (steps i, ii and iii) for the synthesis compounds 17, using compound 29 (5.94 g, 0.036 mol) and phenylalanine ethyl ester $\mathbf{1 4}\{1,1\}$ a crude mixture of the four possible diastereoisomers was obtained as an oil and used directly in the N-oxidation step. Note: in another trial, removal of unreacted phenylalanine ethyl ester was achieved under high vacuum at $180{ }^{\circ} \mathrm{C}$ and a chromatography over silica gel (dichloromethane - methanol 98/2 to 95/5) led to fractions containing unseparated mixture of these diastereoisomers. One of these fractions could be recrystallized in cyclohexane to give a single diastereoisomer $(0.8 \mathrm{~g}, 7 \%)$ with an undetermined configuration. (GG 30532-125-1) ${ }^{1} \mathrm{H}$ NMR $\left(\mathrm{CDCl}_{3}\right): 7.38-7.28(\mathrm{~m}, 7 \mathrm{H}), 7.24-7.20(\mathrm{~m}, 3 \mathrm{H}), 6.62(\mathrm{~s}, 1 \mathrm{H}), 3.90-3.87(\mathrm{dd}, 1 \mathrm{H}, J=10.2$, $3.8 \mathrm{~Hz}), 3.70-3.63(\mathrm{~m}, 2 \mathrm{H}), 3.30-3.16(\mathrm{~m}, 2 \mathrm{H}), 1.73(\mathrm{~s}, 1 \mathrm{H}), 1.03-0.98(\mathrm{~m}, 3 \mathrm{H}) .{ }^{13} \mathrm{C} \mathrm{NMR}\left(\mathrm{CDCl}_{3}\right): 172.0,139.5$, $138.0,129.3,128.9,128.7,128.4,127.9,126.8,59.6,59.5,54.8,37.7,18.9$. HRMS $(\mathrm{m} / z)$ : $[\mathrm{M}+\mathrm{H}]^{+}$calcd for $\mathrm{C}_{18} \mathrm{H}_{21} \mathrm{~N}_{2} \mathrm{O}: 281.1654$, found: 281.1659 .

Second step, preparation of 6-benzyl-3-methyl-5-oxo-2-phenyl-2,3,4,5-tetrahydropyrazine 1-oxide. By using the general protocol described above (steps v) for the synthesis compounds 18, using the crude mixture of the four possible diastereoisomers described above $(10.11 \mathrm{~g})$, a $1 / 7$ mixture of the two possible diastereoisomers $(2.63 \mathrm{~g}$, $24 \%$ from 29) was obtained after after a chromatography over silica gel (cyclohexane - ethyl acetate 3/2 to 1/2). (YJ 31777-011-3) ${ }^{1} \mathrm{H}$ NMR ( $\mathrm{CDCl}_{3}$, minor isomer): 10.18 (s, 1H), 7.36-7.18 (m, 10H), 5.27 (d (br), 1H, J = 2.2 $\mathrm{Hz}), 4.08(\mathrm{~m}, 1 \mathrm{H}), 4.01(\mathrm{~m}, 2 \mathrm{H}), 1.35(\mathrm{~d}, 3 \mathrm{H}, J=6.4 \mathrm{~Hz}) .{ }^{13} \mathrm{C} \mathrm{NMR}\left(\mathrm{CDCl}_{3}\right): 157.3,140.2,136.9,136.2,129.1$, 129.0, 128.8, 127.0, 126.8, 77.8, 59.5, 30.5, 17.0 (one signal missing). ${ }^{1} \mathrm{H}$ NMR ( $\mathrm{CDCl}_{3}$, major isomer): 8.47 (d (br), $1 \mathrm{H}, J=3.9 \mathrm{~Hz}), 7.36-7.18(\mathrm{~m}, 10 \mathrm{H}), 5.09(\mathrm{~d}(\mathrm{br}), 1 \mathrm{H}, J=4.1 \mathrm{~Hz}), 3.98(\mathrm{~s}, 2 \mathrm{H}), 3.84(\mathrm{~m}, 1 \mathrm{H}), 1.21(\mathrm{~d}, 3 \mathrm{H}, J=$ $6.6 \mathrm{~Hz}) .{ }^{13} \mathrm{C} \mathrm{NMR}\left(\mathrm{CDCl}_{3}\right)$ : 159.9, 140.4, 137.2, 136.1, 129.3, 128.9, 128.8, 1287, 127.3, 126.8, 77.0, 49.4, 30.1, 20.7. HRMS (m/z): $[\mathrm{M}+\mathrm{H}]^{+}$calcd for $\mathrm{C}_{18} \mathrm{H}_{19} \mathrm{~N}_{2} \mathrm{O}_{2}, 317.1266$; found, 317.1262 . 
Third step, preparation of 3-benzyl-6-methyl-5-phenylpyrazin-2-ol: By using the general protocol described above (steps vi) for the synthesis compounds 19, this compound was obtained as a pale-yellow solid $(1.70 \mathrm{~g}, 70 \%)$ after a chromatography over silica gel (dichloromethane - ethanol 98/2). (YJ 31777-023-4) ${ }^{1} \mathrm{H}$ NMR (DMSO- $d_{6}$ ): 12.29 (s, 1H), $7.48(\mathrm{~m}, 2 \mathrm{H}), 7.41(\mathrm{~m}, 2 \mathrm{H}), 7.34-7.25(\mathrm{~m}, 5 \mathrm{H}), 7.17(\mathrm{~m}, 1 \mathrm{H}), 4.01(\mathrm{~s}, 2 \mathrm{H}), 2.25(\mathrm{~s}, 3 \mathrm{H}) .{ }^{13} \mathrm{C}$ NMR (DMSO$\left.d_{6}\right): 155.9,154.3,138.8,138.1,133.9,131.0,129.4,129.3,128.7,128.5,127.5,126.6,38.8,17.4$. HRMS $(\mathrm{m} / \mathrm{z})$ : $[\mathrm{M}+\mathrm{H}]^{+}$calcd for $\mathrm{C}_{18} \mathrm{H}_{17} \mathrm{~N}_{2} \mathrm{O}: 277.1341$; found, 277.1344.

Fourth step, preparation of 2-benzyl-3-chloro-5-methyl-6-phenylpyrazine: By using the general protocol described above (steps vii) for the synthesis compounds $\mathbf{2 0}$, and heating for 12 hours, this compound was obtained as an oil $(0.83 \mathrm{~g}, 77 \%)$ after a chromatography over silica gel (cyclohexane - ethyl acetate 96/4). (YJ 31777-039-2) ${ }^{1} \mathrm{H}$ NMR $\left(\mathrm{CDCl}_{3}\right)$ : $7.61(\mathrm{~m}, 2 \mathrm{H}), 7.50(\mathrm{~m}, 3 \mathrm{H}), 7.39(\mathrm{~m}, 2 \mathrm{H}), 7.32(\mathrm{~m}, 2 \mathrm{H}), 7.25(\mathrm{~m}, 1 \mathrm{H}), 4.36(\mathrm{~s}, 2 \mathrm{H}), 2.6(\mathrm{~s}, 3 \mathrm{H})$. ${ }^{13} \mathrm{C}$ NMR $\left(\mathrm{CDCl}_{3}\right): 151.5,150.7,149.3,145.6,137.6,137.5,129.1,129.0,128.9,128.5,128.4,126.6,43.7,22.4$. HRMS $(\mathrm{m} / \mathrm{z}):[\mathrm{M}+\mathrm{H}]^{+}$calcd for $\mathrm{C}_{18} \mathrm{H}_{16} \mathrm{ClN}_{2}, 295.1002$; found, 295.1003.

Fifth step, preparation of ethyl (3-benzyl-6-methyl-5-phenylpyrazin-2-yl)phenylalaninate (GG30532-130-3): Obtained as an oil $(0.4 \mathrm{~g}, 84 \%)$ using the $\mathrm{N}$-arylation protocol (step $\mathrm{x}$ ) described above for the preparation of compounds 23, after a chromatography over silica gel (cyclohexane - ethyl acetate 94/6). ${ }^{1} \mathrm{H} \mathrm{NMR}\left(\mathrm{CDCl}_{3}\right)$ : 7.60$7.57(\mathrm{~m}, 2 \mathrm{H}), 7.48-7.44(\mathrm{~m}, 2 \mathrm{H}), 7.39-7.35(\mathrm{~m}, 1 \mathrm{H}), 7.31-7.20(\mathrm{~m}, 8 \mathrm{H}), 7.03-7.00(\mathrm{~m}, 2 \mathrm{H}), 5.00-4.95(\mathrm{q}, 1 \mathrm{H}, J=$ 7.0 Hz), 4.80-4.78 (m, 1H), 4.18-4.09 (m, 4H), 3.17-3.12 (m, 1H), 3.08-3.03 (m, 1H), $2.46(\mathrm{~s}, 3 \mathrm{H}), 1.22-1.18(\mathrm{t}$, $3 \mathrm{H}, J=7.9 \mathrm{~Hz}) .{ }^{13} \mathrm{C} \mathrm{NMR}\left(\mathrm{CDCl}_{3}\right): 172.7,149.5,146.3,141.4,139.7,138.3,137.0,136.5,129.3,129.1,128.8$, 128.5, 128.4, 128.2, 127.3, 126.8 (two signals?), 61.0, 54.9, 40.4, 38.0, 22.4, 14.1. HRMS $(\mathrm{m} / \mathrm{z}):[\mathrm{M}+\mathrm{H}]^{+}$calcd for $\mathrm{C}_{29} \mathrm{H}_{30} \mathrm{~N}_{3} \mathrm{O}_{2}, 452.2338$; found, 452.2347 .

Sixth step, preparation of 2,8-dibenzyl-5-methyl-6-phenylimidazo[1,2-a]pyrazin-3-yl acetate (30) (GG30532-1371): This compound was obtained as a red oil $(0.30 \mathrm{~g}, 76 \%)$ after a using the general protocol described for the synthesis of compound 25 and a concentration to dryness. ${ }^{1} \mathrm{H}$ NMR $\left(\mathrm{CDCl}_{3}\right): 7.62(\mathrm{~m}, 2 \mathrm{H}), 7.51-7.39(\mathrm{~m}, 5 \mathrm{H}), 7.30$ $(\mathrm{m}, 8 \mathrm{H}), 4.58(\mathrm{~s}, 2 \mathrm{H}), 4.16(\mathrm{~s}, 2 \mathrm{H}), 2.15(\mathrm{~s}, 3 \mathrm{H}) .{ }^{13} \mathrm{C} \mathrm{NMR}\left(\mathrm{CDCl}_{3}\right): 168.4,150.8,139.1,138.4,138.3,138.1$, 135.9, 134.6, 130.0, 129.9, 129.6, 129.1, 128.4, 128.3, 128.2, 127.9, 126.4, 126.3, 122.5, 34.1, 20.1, 14.8 (one signal missing). $\mathrm{HRMS}(\mathrm{m} / \mathrm{z})$ : $[\mathrm{M}+\mathrm{H}]^{+}$calcd for $\mathrm{C}_{29} \mathrm{H}_{26} \mathrm{~N}_{3} \mathrm{O}_{2}, 448.2025$; found, 448.2020.

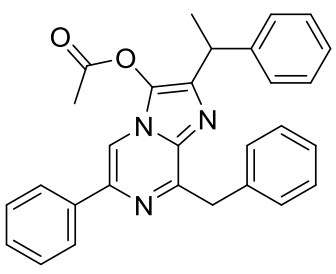

Synthesis of 8-benzyl-6-phenyl-2-(1-phenylethyl)imidazo[1,2-a]pyrazin-3-yl acetate (32), the following synthetic pathway was used:

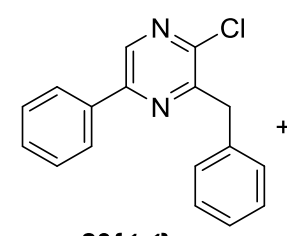

$20\{1,1\}$

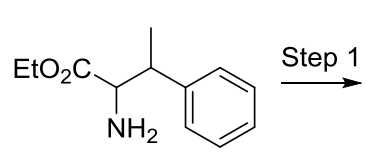

31
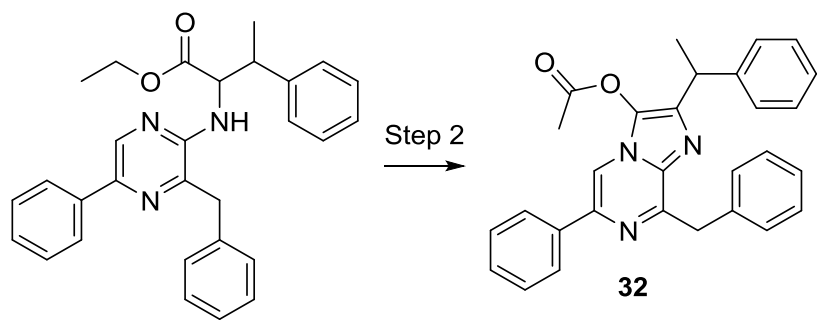

First step: preparation of ethyl 2-((3-benzyl-5-phenylpyrazin-2-yl)amino)-3-phenylbutanoate (YJ31067-019-2): This compound was obtained using the $\mathrm{N}$-arylation protocol (step $\mathrm{x})$ described above as an oil $(0.3 \mathrm{~g}, 67 \%)$ after a chromatography over silica gel (cyclohexane - ethyl acetate 96/4). ${ }^{1} \mathrm{H}$ NMR (diastereoisomeric mixture, 0.6 / 0.4 ratio) $\left(\mathrm{CDCl}_{3}\right): 8.40(\mathrm{~s}, 0.6 \mathrm{H}), 8.34(\mathrm{~s}, 0.3 \mathrm{H}), 7.94(\mathrm{~m}, 2 \mathrm{H}), 7.46(\mathrm{~m}, 2 \mathrm{H}), 7.39-7.19(\mathrm{~m}, 7 \mathrm{H}), 7.21(\mathrm{~m}, 1 \mathrm{H}), 7.16$ $(\mathrm{m}, 1 \mathrm{H}), 7.05(\mathrm{~m}, 2 \mathrm{H}), 4.95(\mathrm{~m}, 1 \mathrm{H}), 4.89(\mathrm{~d}, 0.4 \mathrm{H}, J=7.7 \mathrm{~Hz}), 4.69(\mathrm{~d}, 0.6 \mathrm{H}, J=8.3 \mathrm{~Hz}), 4.20(\mathrm{~m}, 0.8 \mathrm{H}), 4.08$ $(\mathrm{m}, 0.8 \mathrm{H}), 4.08(\mathrm{~m}, 2.6 \mathrm{H}), 3.37(\mathrm{~m}, 0.6 \mathrm{H}), 3.0(\mathrm{~m}, 0.4 \mathrm{H}), 1.28(\mathrm{~d}, 1.8 \mathrm{H}, J=7.1 \mathrm{~Hz}), 1.24(\mathrm{~d}, 0.8 \mathrm{H}, J=7.3 \mathrm{~Hz})$, $1.16(\mathrm{t}, 1.8 \mathrm{H}, J=7.5 \mathrm{~Hz}), 1.08(\mathrm{t}, 0.8 \mathrm{H}, J=7.3 \mathrm{~Hz}) \cdot{ }^{13} \mathrm{C} \mathrm{NMR}\left(\mathrm{CDCl}_{3}\right)$ : too many signal overlaps. HRMS $(\mathrm{m} / z)$ : $[\mathrm{M}+\mathrm{H}]^{+}$calcd for $\mathrm{C}_{29} \mathrm{H}_{30} \mathrm{~N}_{3} \mathrm{O}_{2}, 452.2338$; found, 452.2378 . 
Second step: preparation of 8-Benzyl-6-phenyl-2-(1-phenylethyl)imidazo[1,2-a]pyrazin-3-yl acetate (32) (YJ31067-23-1): This compound was obtained using the cyclization/O-acetylation protocol described above (step xii) as a white powder after a recrystallization from $\mathrm{n}$-heptane $(0.12 \mathrm{~g}, 44 \%) .{ }^{1} \mathrm{H}$ NMR $\left(\mathrm{CDCl}_{3}\right): 7.90(\mathrm{~m}, 2 \mathrm{H}), 7.74$ (s, 1H), $7.68(\mathrm{~m}, 2 \mathrm{H}), 7.46(\mathrm{~m}, 2 \mathrm{H}), 7.40(\mathrm{~m}, 1 \mathrm{H}), 7.33(\mathrm{~m}, 5 \mathrm{H}), 7.25(\mathrm{~m}, 2 \mathrm{H}), 4.68(\mathrm{~d}, 1 \mathrm{H}, J=13.8 \mathrm{~Hz}), 4.63(\mathrm{~d}$, $1 \mathrm{H}, J=13.8 \mathrm{~Hz}), 4.34(\mathrm{q}, 1 \mathrm{H}, J=6.7 \mathrm{~Hz}), 2.14(\mathrm{~s}, 3 \mathrm{H}), 1.83(\mathrm{~d}, 3 \mathrm{H}, J=6.7 \mathrm{~Hz}) .{ }^{13} \mathrm{C} \mathrm{NMR}\left(\mathrm{CDCl}_{3}\right): 167.1,153.0$, 143.9, 139.5, 139.0, 138.0, 137.0, 133.4, 129.8, 128.7, 128.5, 128.4, 128.2, 127.8, 126.4 (three signals), 108.9, 39.6, 38.5, 20.7, 19.8. HRMS (m/z): $[\mathrm{M}+\mathrm{H}]^{+}$calcd for $\mathrm{C}_{29} \mathrm{H}_{26} \mathrm{~N}_{3} \mathrm{O}_{2}, 448.2025$; found, 448.2031.

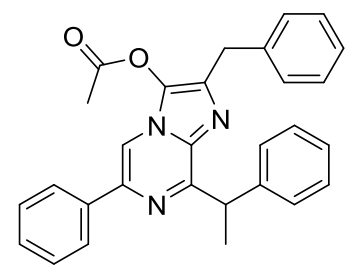

Preparation of 2-benzyl-6-phenyl-8-(1-phenylethyl)imidazo[1,2-a]pyrazin-3-yl acetate (34), the following synthetic pathway was used:

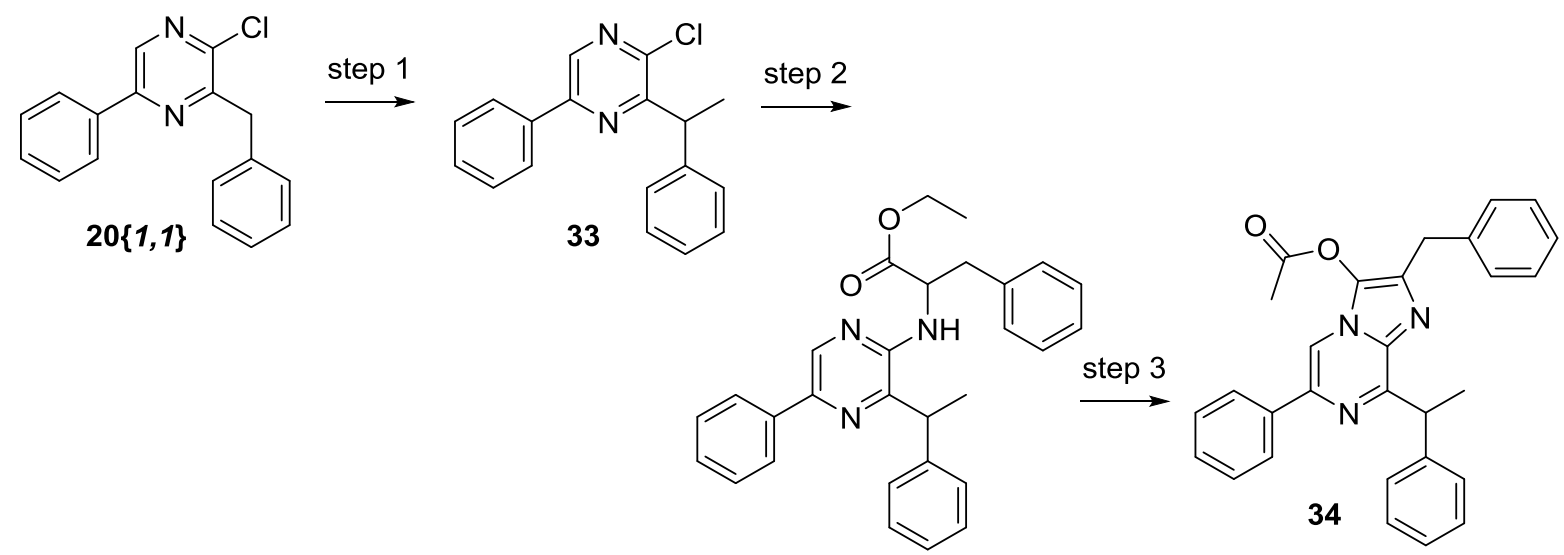

Step 1, preparation of 2-chloro-5-phenyl-3-(1-phenylethyl)pyrazine (33) (YJ 31777-025-2): Under an argonprotected atmosphere, compound $20\{1,1\}(0.94 \mathrm{~g}, 3.34 \mathrm{mmol})$ was dissolved in dry tetrahydrofuran $(15 \mathrm{~mL})$. This was cooled to $-78{ }^{\circ} \mathrm{C}$, and a $2 \mathrm{M}$ solution of lithium diisopropylamide $(2 \mathrm{~mL}, 4.02 \mathrm{mmol}$, in THF, $n$-heptane and ethylbenzene) was injected. This was stirred at $-78{ }^{\circ} \mathrm{C}$ for 10 minutes (immediate blackening of the solution) and methyl iodide $(0.27 \mathrm{~mL}, 4.35 \mathrm{mmol})$ was then injected. This was stirred for 10 minutes at $-78{ }^{\circ} \mathrm{C}$ and allowed to warm back to room temperature for 20 minutes. The resulting solution was diluted in water and ethyl acetate, the organic layer was washed with water, brine, dried over magnesium sulfate and concentrated to dryness. The residue was purified by a chromatography over silica gel (cyclohexane - dichloromethane 3/1) to yield compound 33 as a solid $(0.73 \mathrm{~g}, 73 \%)$. ${ }^{1} \mathrm{H}$ NMR $\left(\mathrm{CDCl}_{3}\right): 8.67(\mathrm{~s}, 1 \mathrm{H}), 8.11(\mathrm{~m}, 2 \mathrm{H}), 7.54(\mathrm{~m}, 3 \mathrm{H}), 7.42(\mathrm{~m}, 2 \mathrm{H}), 7.33(\mathrm{~m}$, $2 \mathrm{H}), 7.22(\mathrm{~m}, 1 \mathrm{H}), 4.76(\mathrm{q}, 1 \mathrm{H}, J=7.0 \mathrm{~Hz}), 1.81(\mathrm{~d}, 3 \mathrm{H}, J=7.0 \mathrm{~Hz}) .{ }^{13} \mathrm{C}$ NMR $\left(\mathrm{CDCl}_{3}\right): 156.5,150.0,146.7$, $143.0,138.1,135.6,130.0,129.1,128.5,128.0,126.8,126.7,43.0,20.9$. HRMS $(m / z):[\mathrm{M}+\mathrm{H}]^{+}$calcd for $\mathrm{C}_{18} \mathrm{H}_{16} \mathrm{ClN}_{2}$, 295.1002; found, 295.1006.

Step 2, preparation of ethyl (5-phenyl-3-(1-phenylethyl)pyrazin-2-yl)phenylalaninate (YJ 31777-041-1): By using the general $\mathrm{N}$-arylation protocol described above for the preparation of compounds $\mathbf{2 3}$ (step $\mathrm{x}$ ), this compound was obtained as an oily $1 / 1$ mixture of two diastereoisomers $(0.33 \mathrm{~g}, 52 \%)$ using toluene as a solvent and a chromatography over silica gel (cyclohexane - ethyl acetate 97/3). ${ }^{1} \mathrm{H}$ NMR $\left(\mathrm{CDCl}_{3}\right)$ : 8.44 (s, 0.5H), 8.42 (s, $0.5 \mathrm{H}), 8.06(\mathrm{~m}, 2 \mathrm{H}), 7.49(\mathrm{~m}, 2 \mathrm{H}), 7.38(\mathrm{~m}, 1 \mathrm{H}), 7.40-7.11(\mathrm{~m}, 9 \mathrm{H}), 6.81(\mathrm{~m}, 1 \mathrm{H}), 4.95(\mathrm{~m}, 3 \mathrm{H}), 4.81(\mathrm{~d}(\mathrm{br}), 1 \mathrm{H}, J$ $=8.0 \mathrm{~Hz}), 4.20-3.97(\mathrm{~m}, 3 \mathrm{H}), 3.19(\mathrm{dd}, 0.5 \mathrm{H}, J=5.9,13.6 \mathrm{~Hz}), 3.09(\mathrm{~m}, 1 \mathrm{H}), 2.99(\mathrm{dd}, 0.5 \mathrm{H}, J=5.5,13.6 \mathrm{~Hz})$, $1.77(\mathrm{~m}, 3 \mathrm{H}), 1.25(\mathrm{t}, 1.5 \mathrm{H}, J=7.1 \mathrm{~Hz}), 1.09(\mathrm{t}, 1.5 \mathrm{H}, J=7.1 \mathrm{~Hz}) .{ }^{13} \mathrm{C} \mathrm{NMR}\left(\mathrm{CDCl}_{3}\right): 172.5,172.3,149.8,149.6$, $144.1,143.7,143.1,142.9,140.4,140.3,137.7,136.5,135.8,135.7,129.4,129.1,129.0,128.9,128.7,128.5$, 
$128.5,128.4,127.9,127.8,127.7,127.6,127.5,126.9,126.7,125.5,61.2,61.0,55.0,54.8,42.9,42.8,37.9,21.0$, 20.8, 14.1, 14.0. HRMS (m/z): $[\mathrm{M}+\mathrm{H}]^{+}$calcd for $\mathrm{C}_{29} \mathrm{H}_{30} \mathrm{~N}_{3} \mathrm{O}_{2}, 452.2338$; found, 452.2332 .

Step 3, preparation of 2-benzyl-6-phenyl-8-(1-phenylethyl)imidazo[1,2-a]pyrazin-3-yl acetate (34) (YJ 31777-0531): By using the general protocol described above for the preparation of compounds $\mathbf{2 5}$, this $O$-acetylated luciferin was obtained as a white cotton $(0.10 \mathrm{~g}, 32 \%)$ after a recrystallization in $n$-heptane. ${ }^{1} \mathrm{H}$ NMR $\left(\mathrm{CDCl}_{3}\right): 7.99(\mathrm{~m}$, 2H), $7.79(\mathrm{~s}, 1 \mathrm{H}), 7.71(\mathrm{~m}, 2 \mathrm{H}), 7.50(\mathrm{~m}, 2 \mathrm{H}), 7.42(\mathrm{~m}, 1 \mathrm{H}), 7.35-7.28(\mathrm{~m}, 6 \mathrm{H}), 7.26-7.19(\mathrm{~m}, 2 \mathrm{H}), 5.30(\mathrm{q}, 1 \mathrm{H}, J=$ $7.1 \mathrm{~Hz}), 4.22(\mathrm{~s}, 2 \mathrm{H}), 2.16(\mathrm{~s}, 3 \mathrm{H}), 1.91(\mathrm{~d}, 3 \mathrm{H}, J=7.1 \mathrm{~Hz}) .{ }^{13} \mathrm{C} \mathrm{NMR}\left(\mathrm{CDCl}_{3}\right): 167.1,156.2,143.5,139.0,137.9$, 136.9, 134.6, 133.0, 129.1, 128.8, 128.7, 128.6, 128.5, 128.4, 128.2, 126.5, 126.3, 108.4, 41.2, 34.1, 20.2, 19.8 (one signal missing). HRMS $\left(\mathrm{m} / \mathrm{z}\right.$ ): $[\mathrm{M}+\mathrm{H}]^{+}$calcd for $\mathrm{C}_{29} \mathrm{H}_{26} \mathrm{~N}_{3} \mathrm{O}_{2}, 448.2025$; found, 448.2038. 
General hydrolysis protocol of $\mathrm{O}$-acetylated luciferins 25, to generate solutions of the luciferins 26 (or 35-37) via step xv. In a closed plastic vial (even closed $50 \mathrm{~mL}$ falcon tubes were fine on large scale), the considered Oacetylated luciferin $(1 \mathrm{mg})$ was dissolved in DMSO $(0.2 \mathrm{~mL})$ and then diluted by adding a solution of acidic ethanol $(0.3 \mathrm{ml})$ made from the addition of $37 \%$ hydrochloric acid $(100 \mu \mathrm{l})$ on $100 \%$ ethanol $(12 \mathrm{~mL})$. This $0.5 \mathrm{~mL}$ solution was incubated at $50{ }^{\circ} \mathrm{C}$ for 2 hours to give a stock solution which was then stored at $-20^{\circ} \mathrm{C}$. As depicted in the figure below, the LC/MS monitoring of the hydrolysis of the $O$-acetylated luciferin $\mathbf{2 5}\{1,1,44\}$ into the corresponding luciferin $\mathbf{2 6}\{1,1,44\}$ under these conditions was complete and fairly clean in less than two hours.

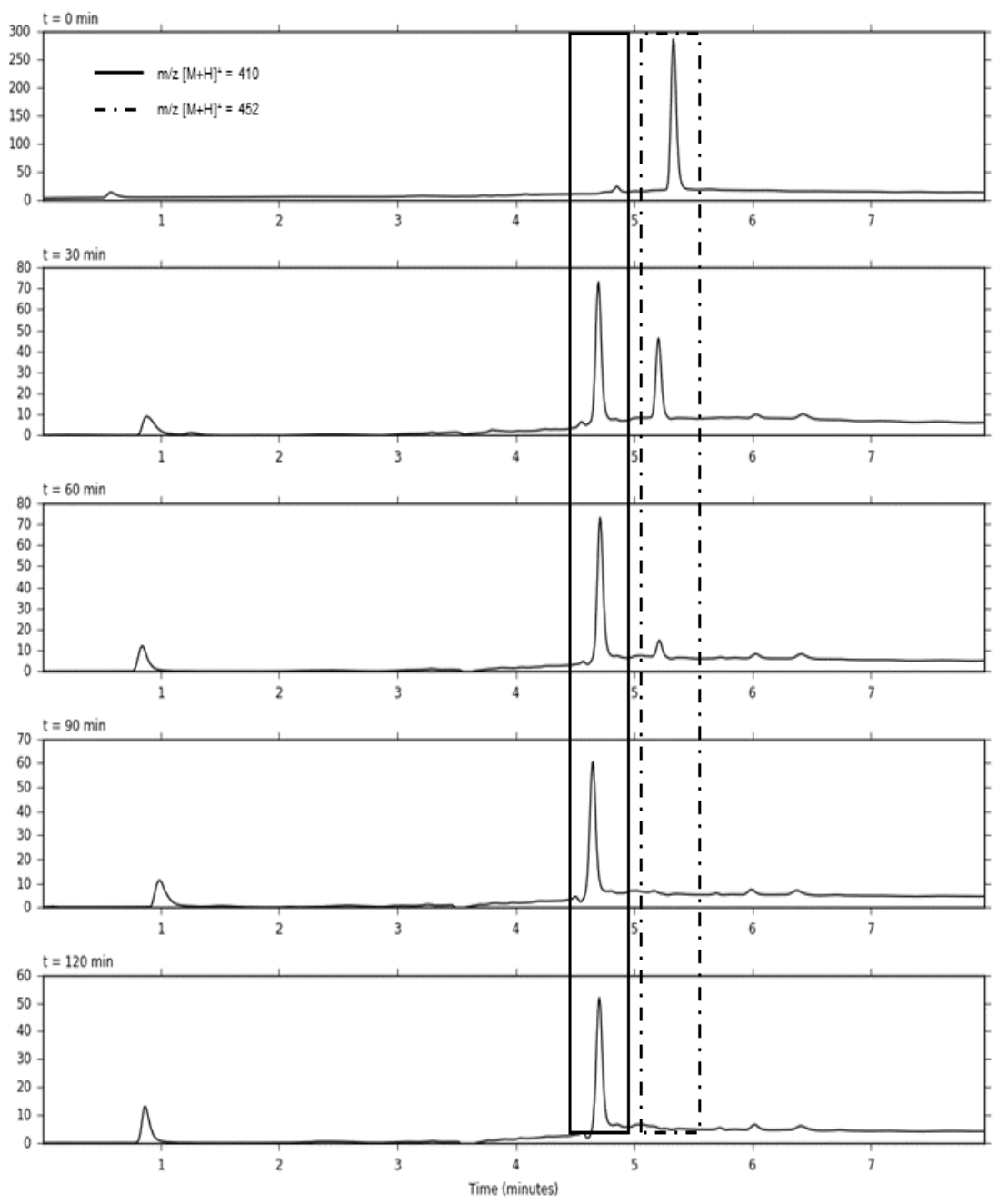

HPLC profiles over time for the acetyl hydrolysis of proluciferin $\mathbf{2 5}\{1,1,44\}$ into the corresponding luciferin $\mathbf{2 6}\{1,1,44\}$. Obtained on an Agilent apparatus, with a $3.5 \mu \mathrm{m}$ XDB-C18 column and a water / methanol gradient (containing $5 \mathrm{mM}$ ammonium formate, from $95-5$ to 5-95 in $3.5 \mathrm{~min}$ and then $3.5 \mathrm{~min}$ back at 5-95) UV monitoring set at $254 \mathrm{nM}$.

Note: in general, the resulting acid solution of these luciferins can be used within few hours or should be stored at $20^{\circ} \mathrm{C}$ to avoid their relatively slow decay (see below for more extensive LC/MS monitoring). On the other hand, upon dilution in buffers, the resulting solutions are decaying much faster and should be used within a short time frame.

\section{HPLC/MS monitoring of the hydrolysis products of the 0 -acetylated luciferins 25 described in table 8 .}

The hydrolysis of each of the proluciferins (hikarazines) 25 listed in table 8 and leading to $5 \mathrm{mM}$ solution of the corresponding luciferins $\mathbf{2 6}$ was undertaken following the protocols described above for. 
An initial LC/MS analysis was run on diluted (1/10 in DMSO) samples of the solutions right after the hydrolysis step, a second one was done16 hours after and a third one 14 days after all this while keeping the stock solutions at $18^{\circ} \mathrm{C}$.

As depicted below, we provide in each cases the reaction involved along with, when relevant, hypothetical structures corresponding to some additional mass peak detected. This is followed by the HPLC profiles, as detected by UV at $254 \mathrm{~nm}$, and the TIC profile (mass between 180 and 800) along with the corresponding spectra obtained from the main HPLC peak as well as the lesser one. The first set corresponds to the analysis right after the hydrolysis, the second corresponds to the same analysis but after leaving the mother solution standing at $18^{\circ} \mathrm{C}$ for 16 hours.

The HPLC profile were obtained on an Agilent 1200 series LC/MSD system using an Agilent Jet-Stream atmospheric electrospray ionization system, with a $3.5 \mu \mathrm{m}$ XDB-C18 column and a water / acetonitrile gradient (containing $5 \mathrm{mM}$ ammonium formate, from $95-5$ to 5-95 in $3.5 \mathrm{~min}$ and then $3.5 \mathrm{~min}$ back at 5-95) UV monitoring set at $254 \mathrm{nM}$. 
Hydrolysis products of hikarazine-1 $(25\{1,1,37\})$
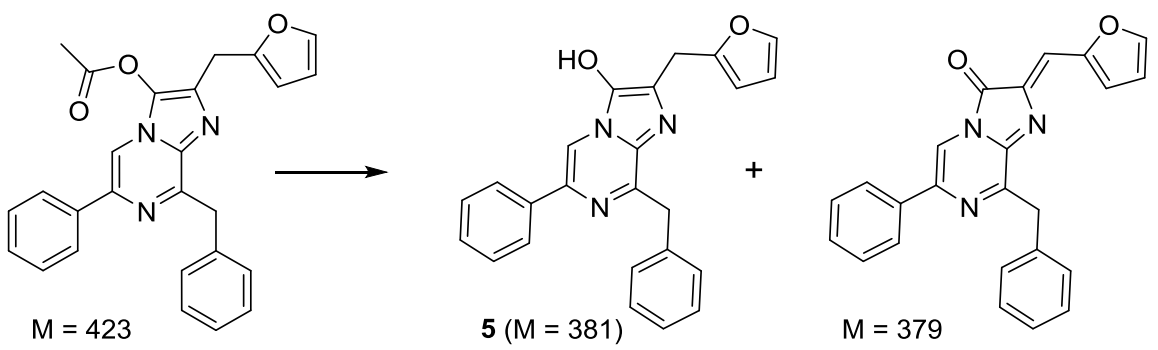

$\mathrm{t}=0$
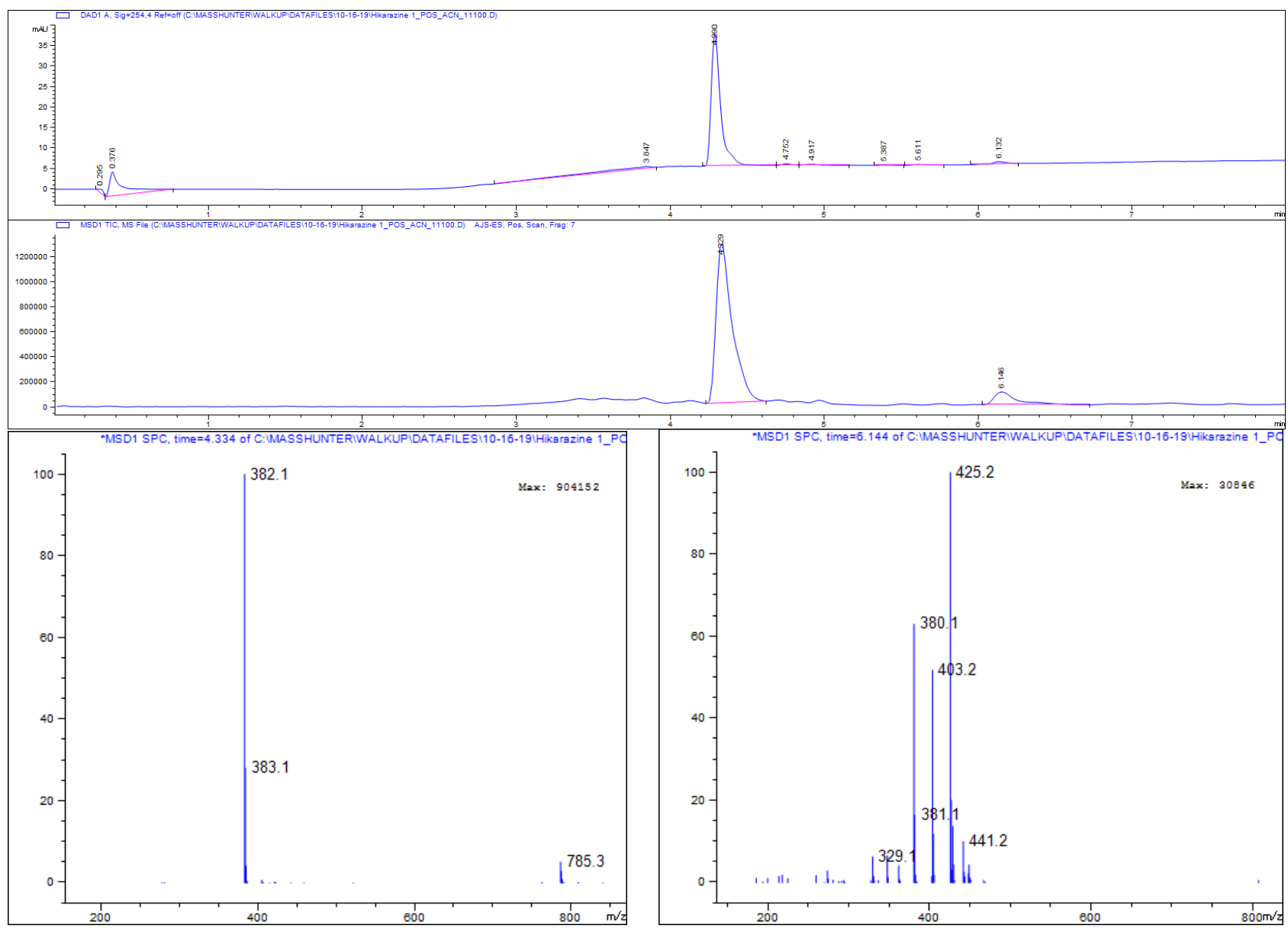
$t=16 h$
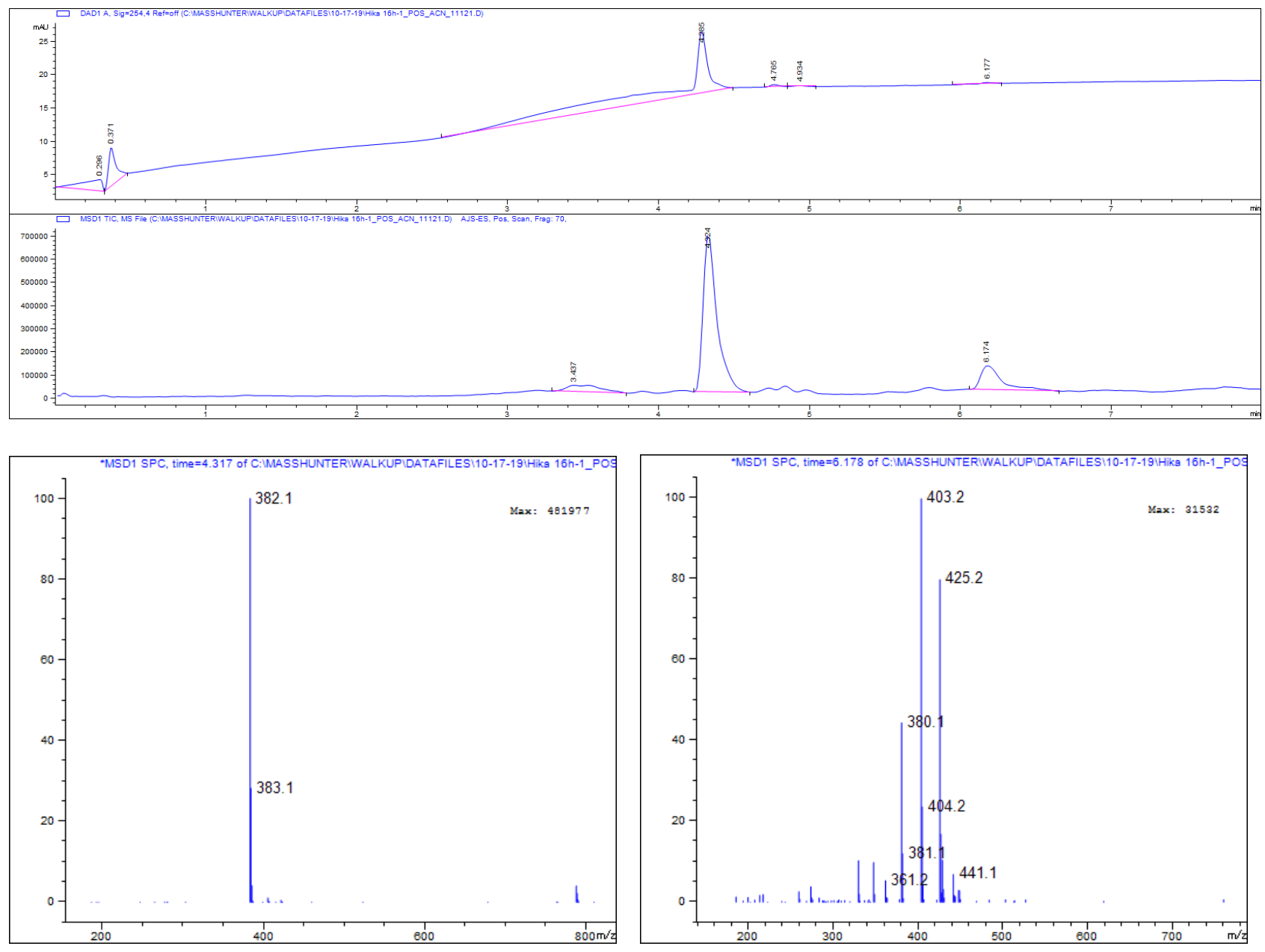


\section{$\mathrm{t}=14$ days}
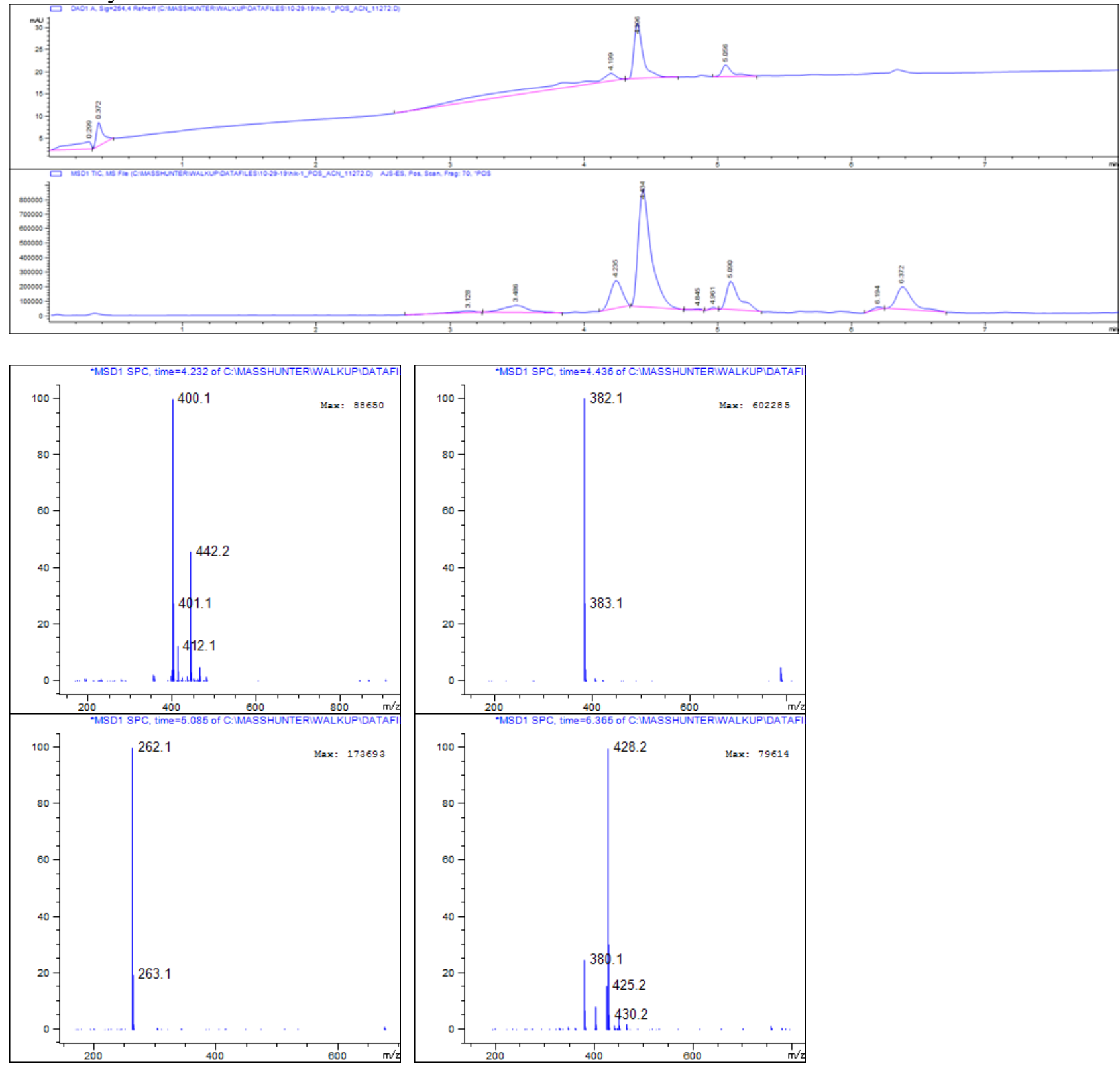
Hydrolysis products of hikarazine-2 $(\mathbf{2 5}\{\mathbf{1}, \mathbf{1}, \mathbf{1}\})$
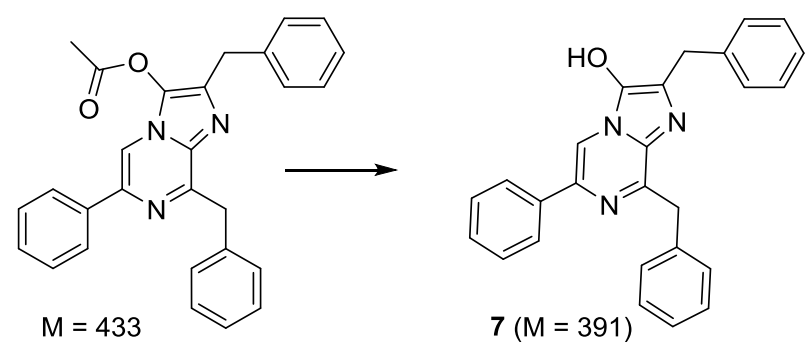

$\mathrm{t}=0$
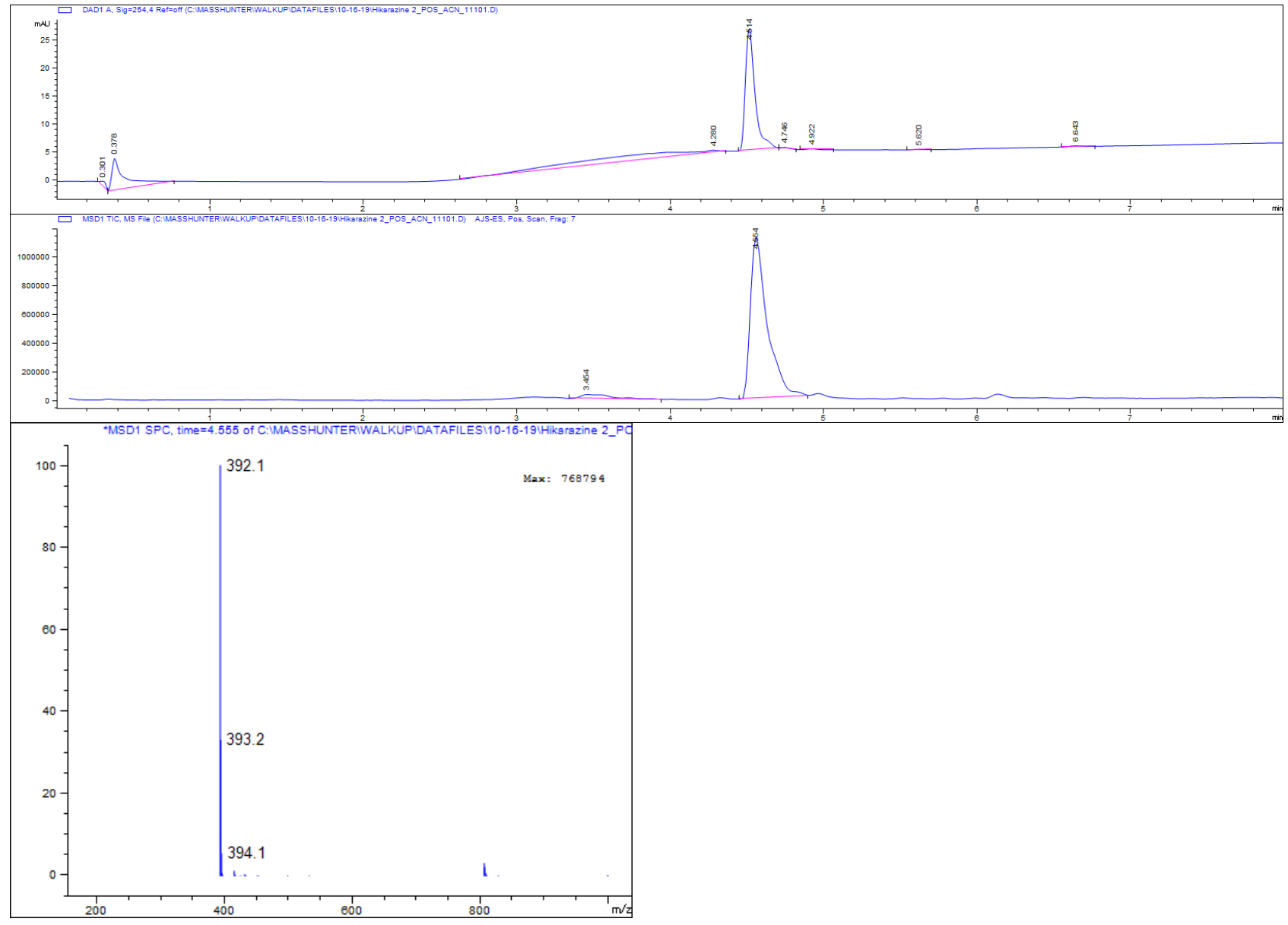
$t=16 h$
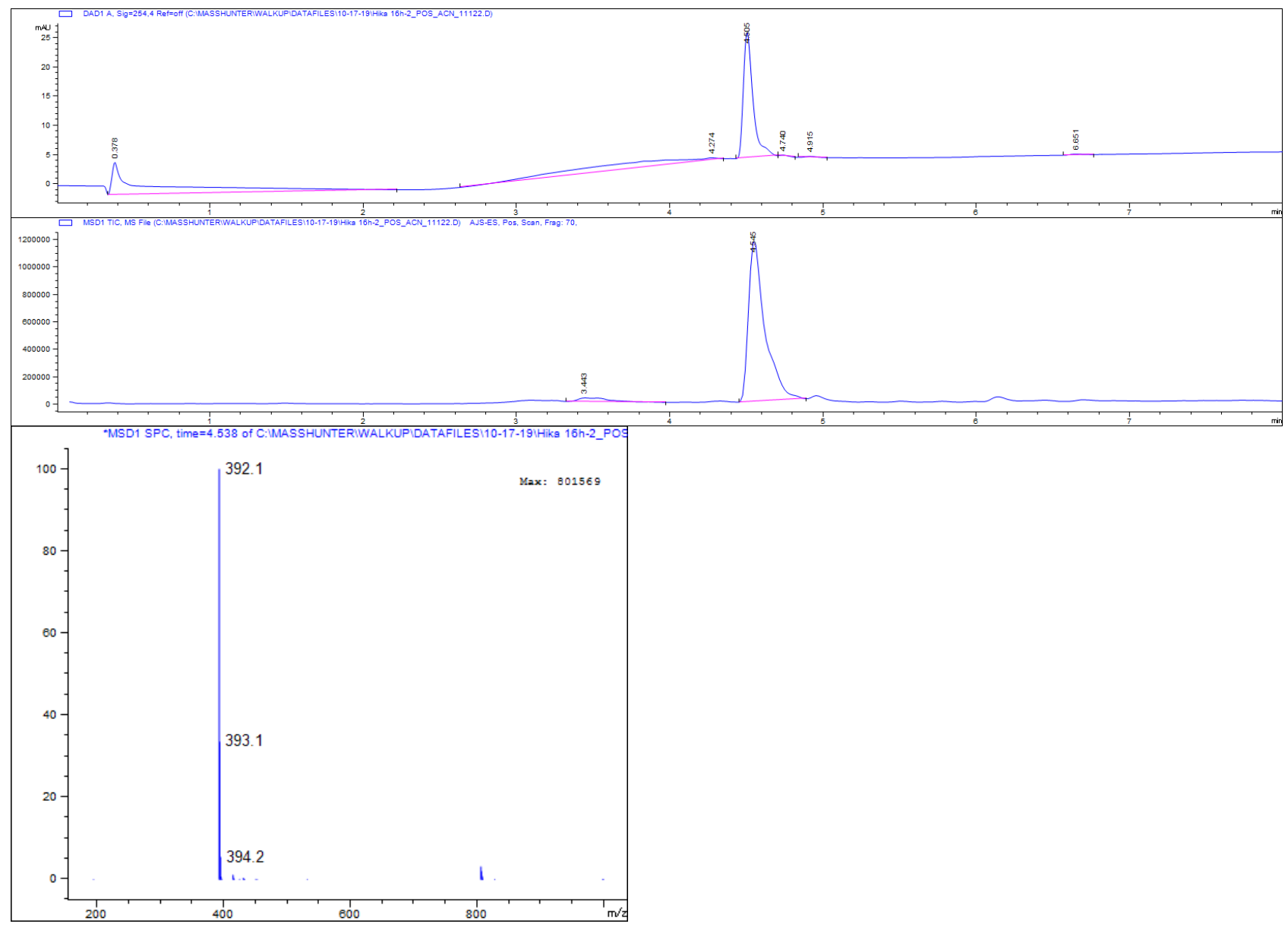


\section{$\mathrm{t}=14$ days}
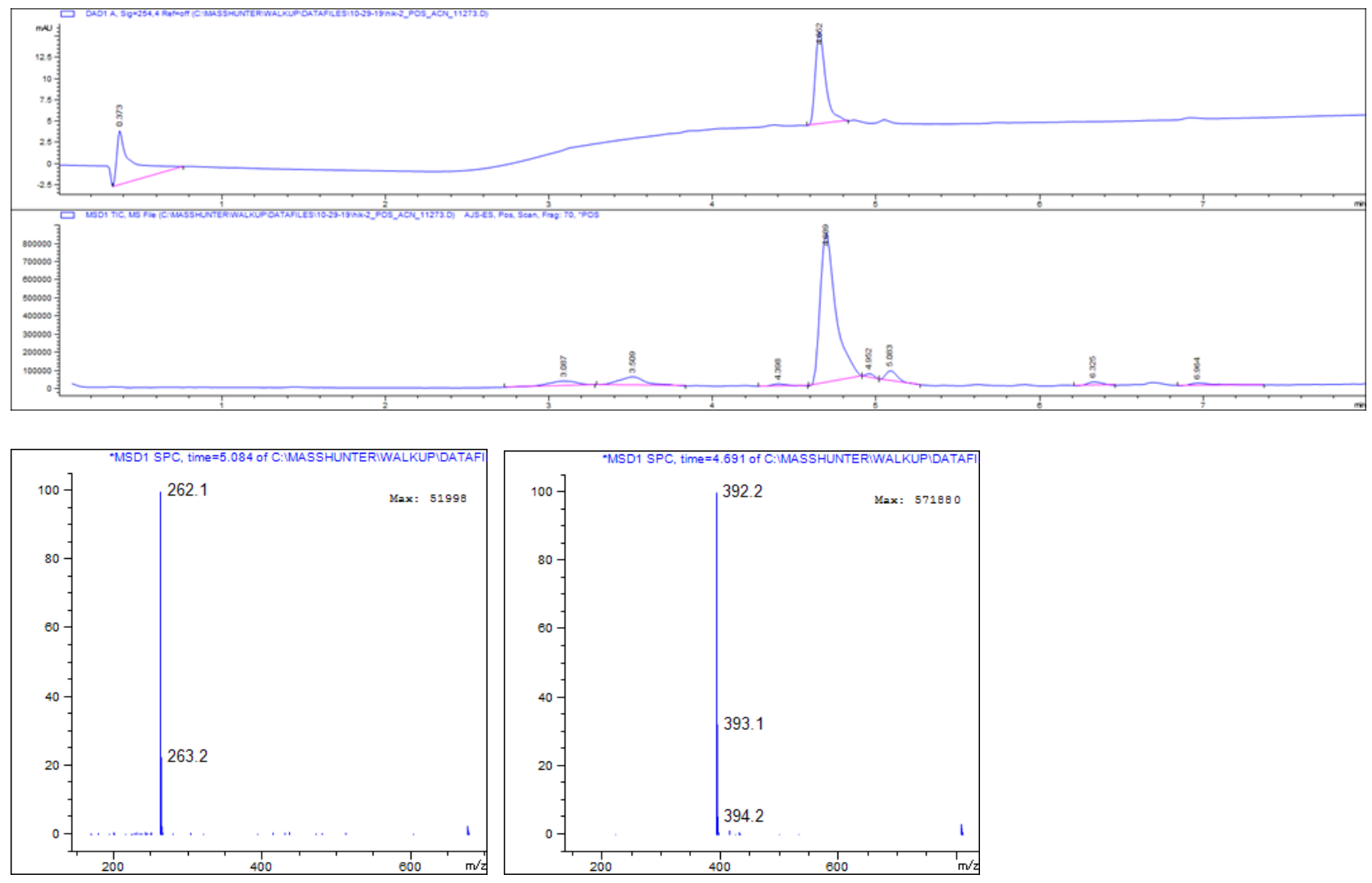
Hydrolysis products of hikarazine-3 $(25\{1,1,42\})$<smiles>CC(=O)Oc1c(Cc2ccc(C)o2)nc2c(Cc3ccccc3)nc(-c3ccccc3)cn12</smiles>
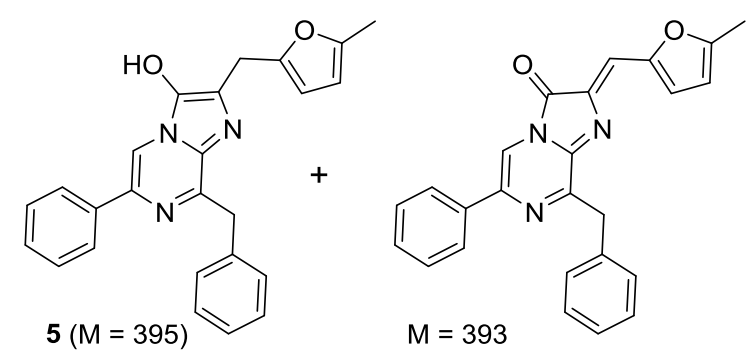

$$
\mathrm{t}=0
$$
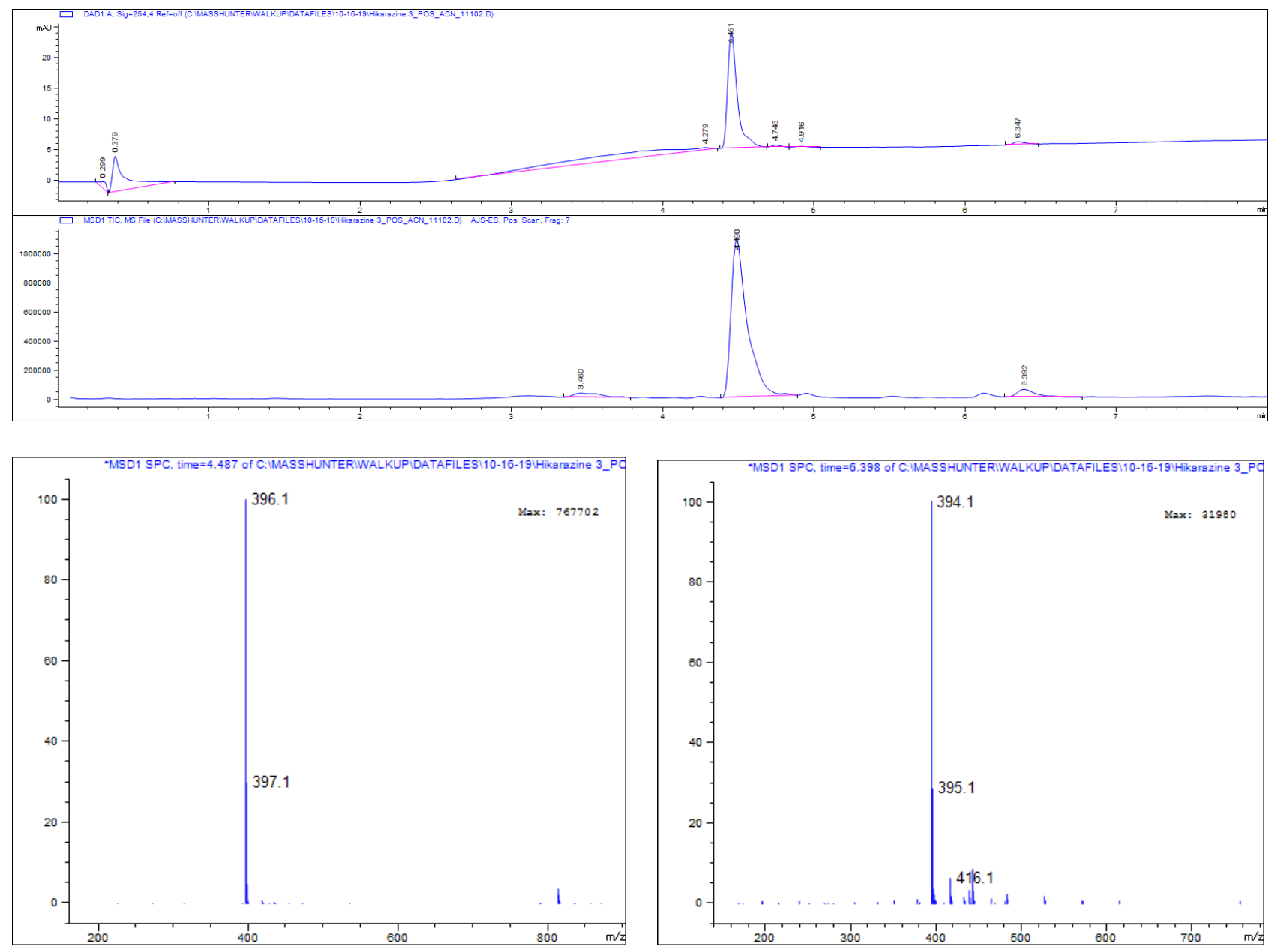

154 
$t=16 h$
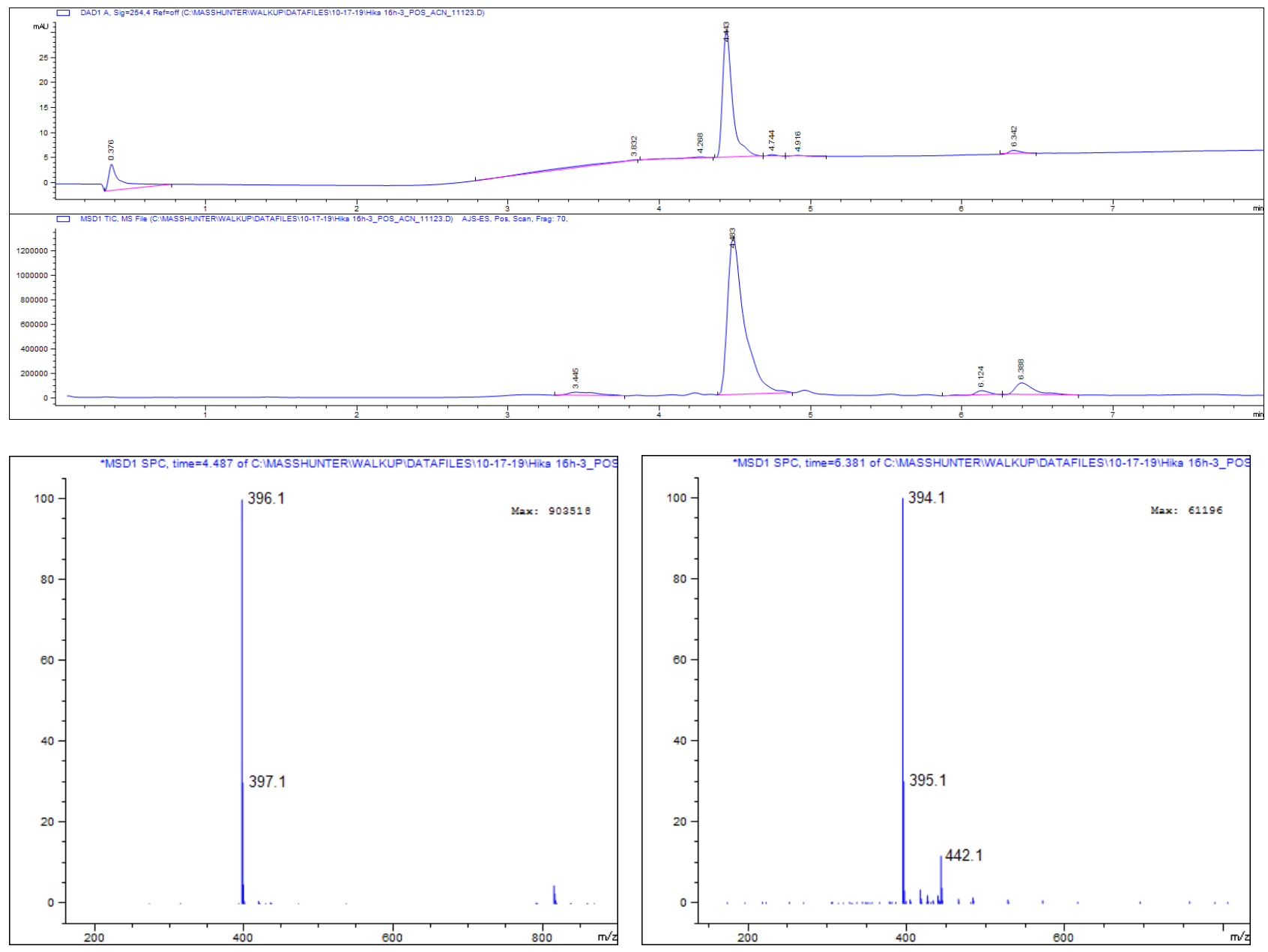


\section{$\mathrm{t}=14$ days}
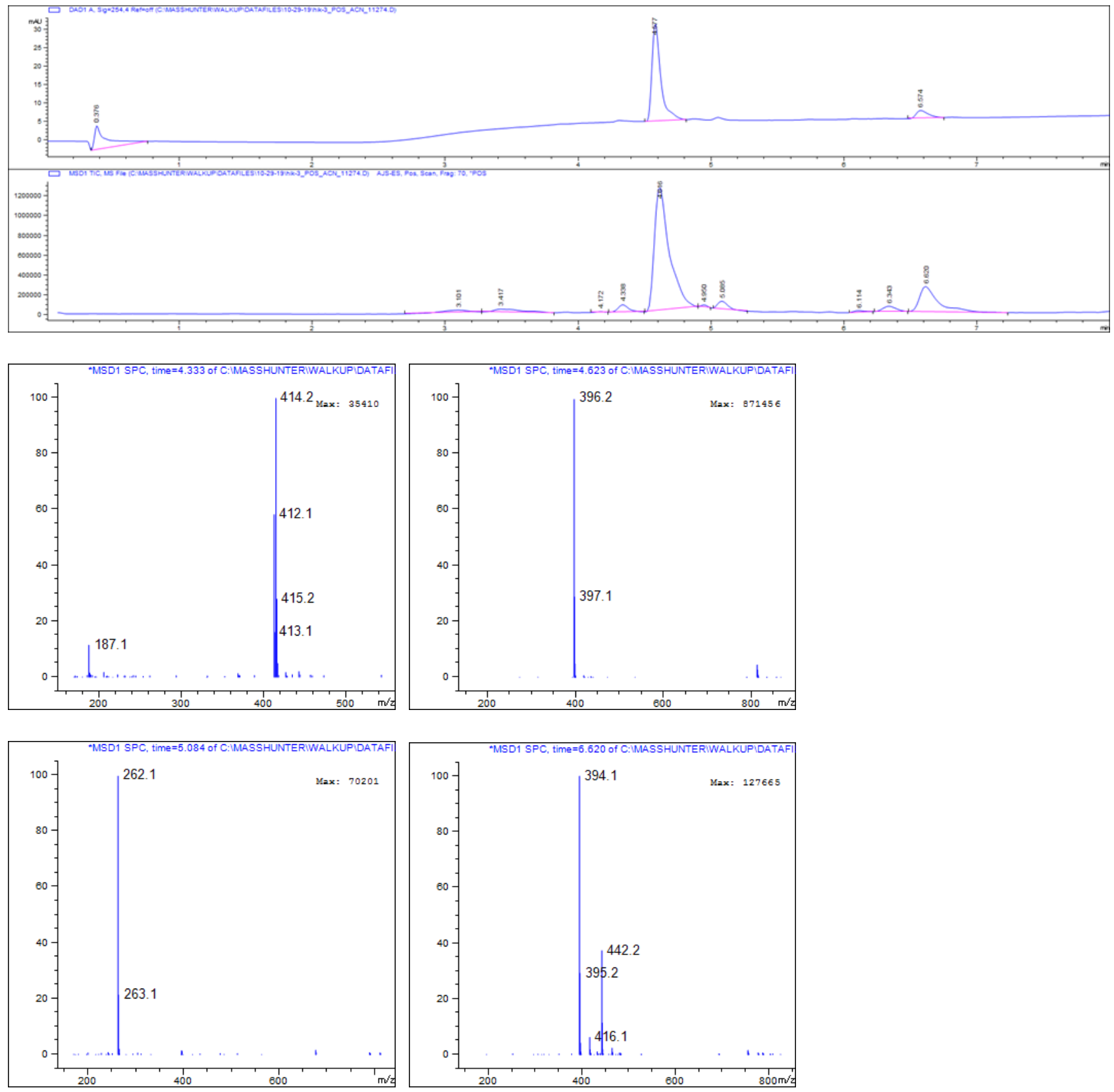
Hydrolysis products of hikarazine-14 $(\mathbf{2 5}\{\mathbf{1}, \mathbf{1}, \mathbf{1 2}\})$
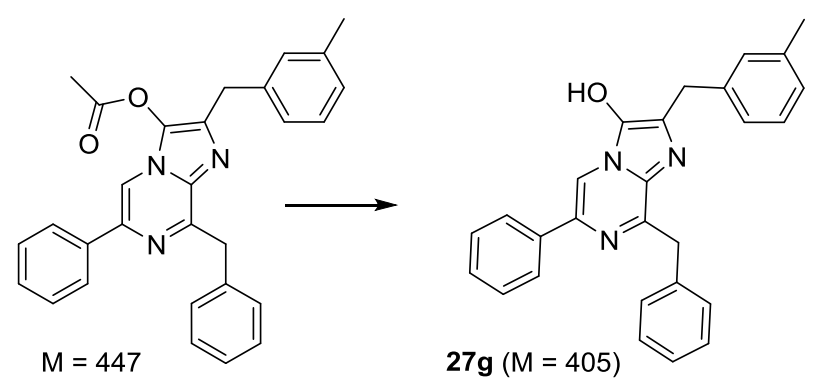

$t=0$
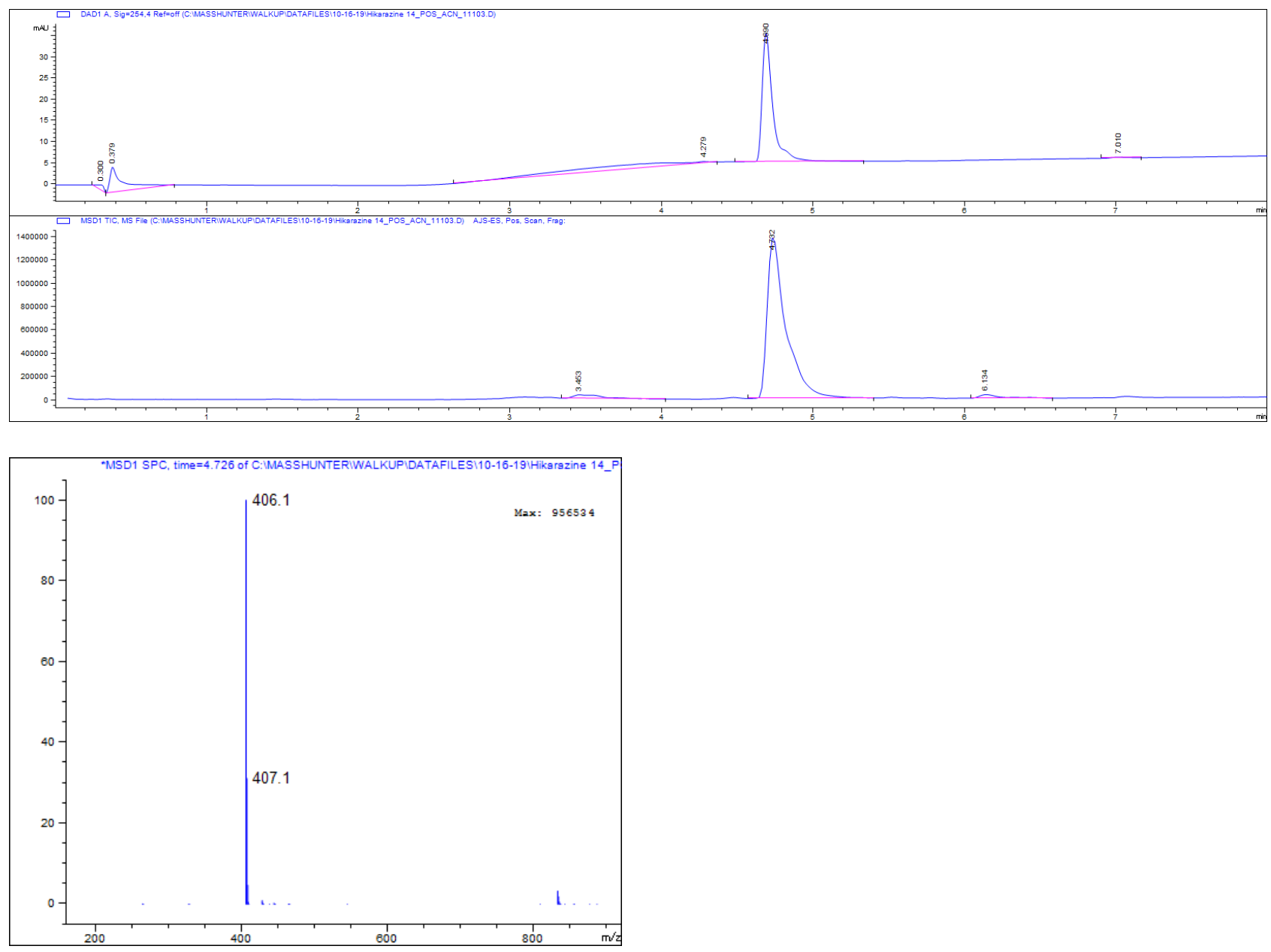
$t=16 h$
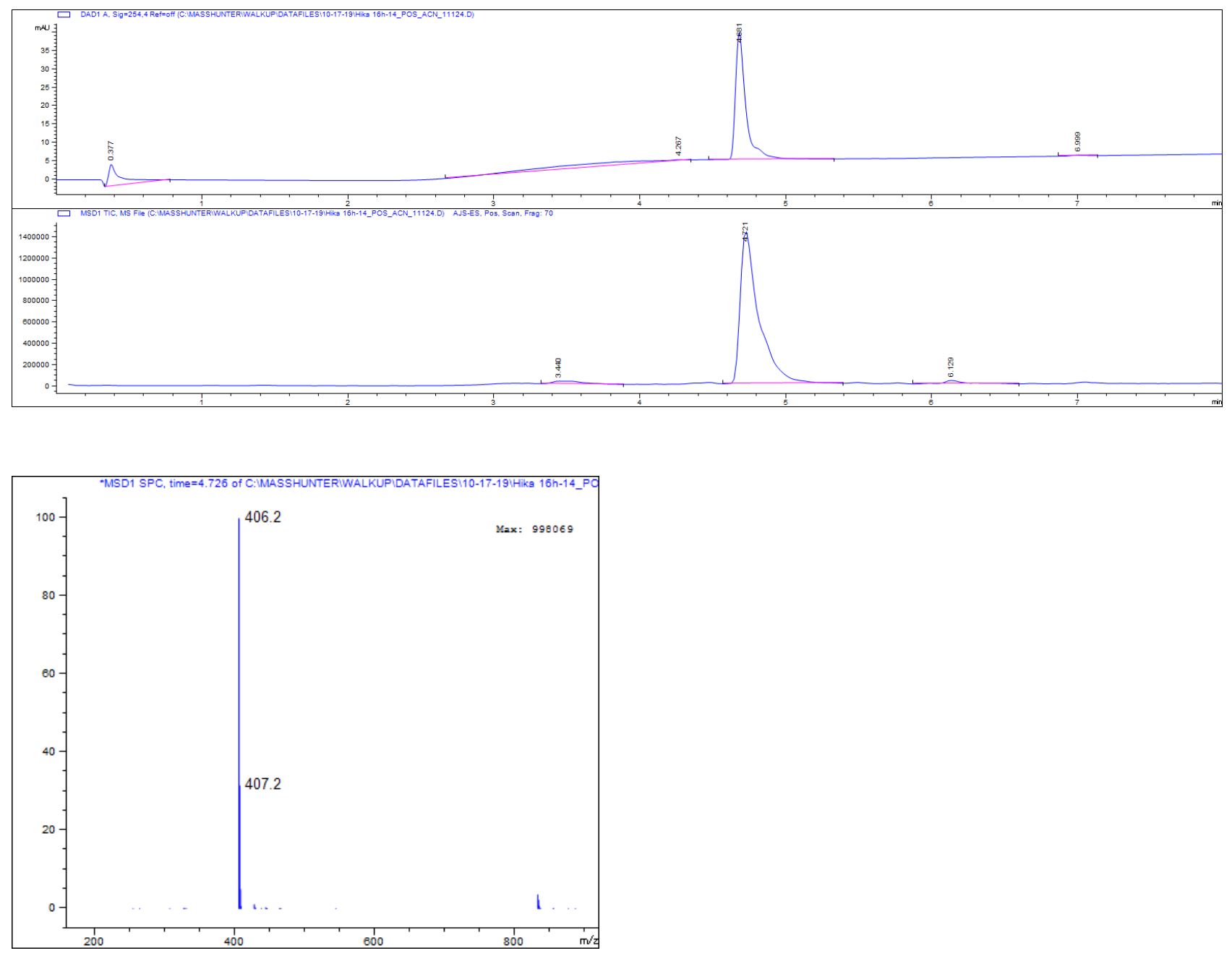

158 


\section{$\mathrm{t}=14$ days}
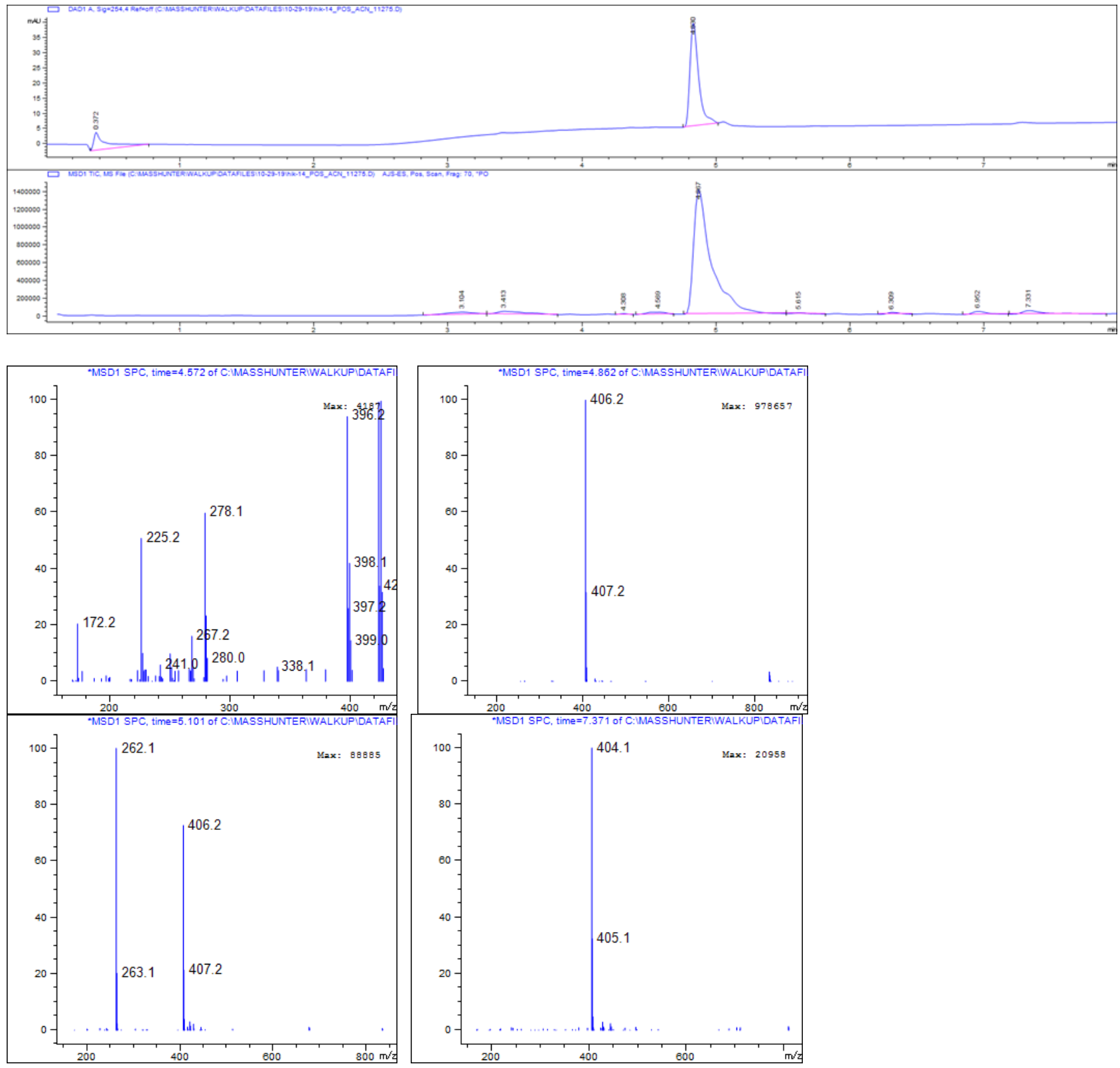
Hydrolysis products of hikarazine-75 $(\mathbf{2 5}\{\mathbf{1 4}, \mathbf{1}, \mathbf{1}\})$

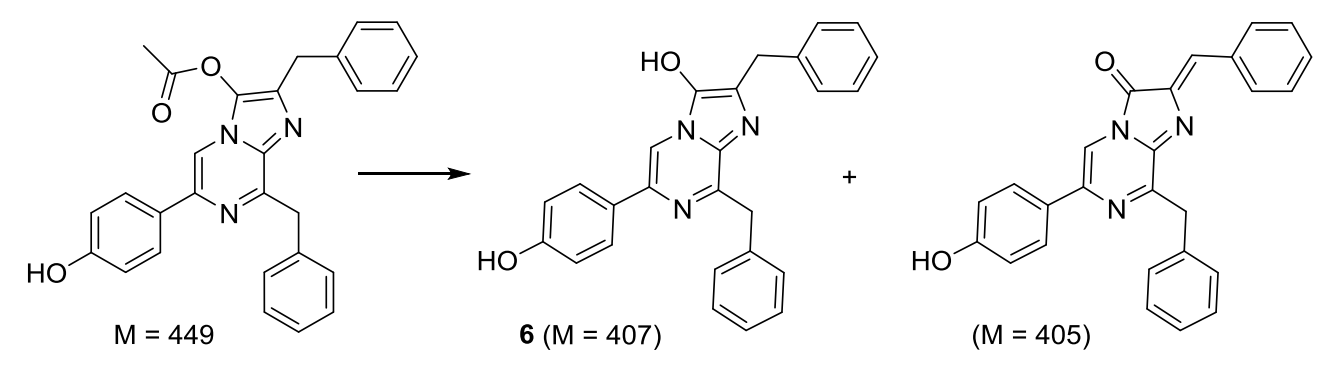

$\mathrm{t}=0$
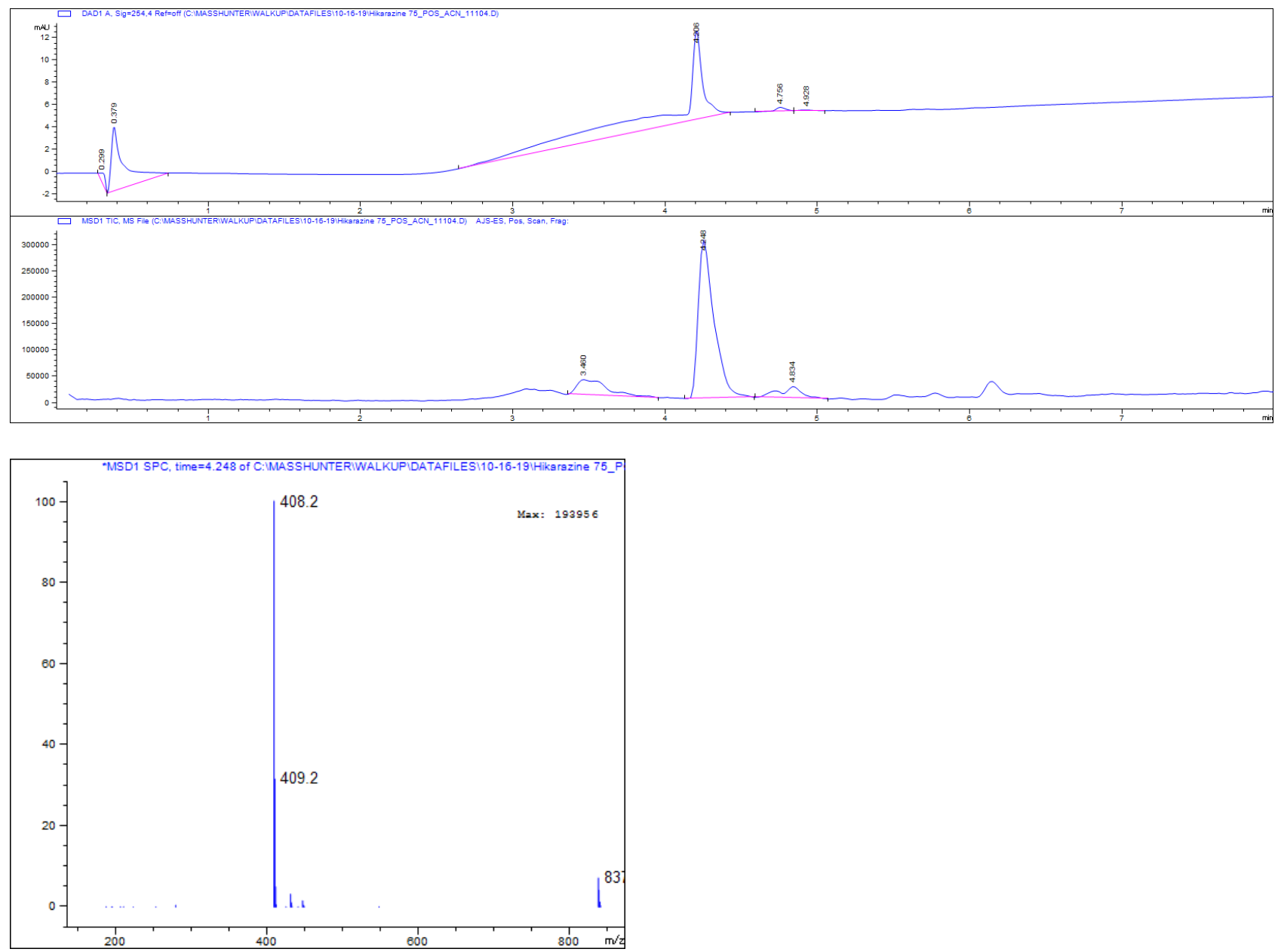
$t=16 h$
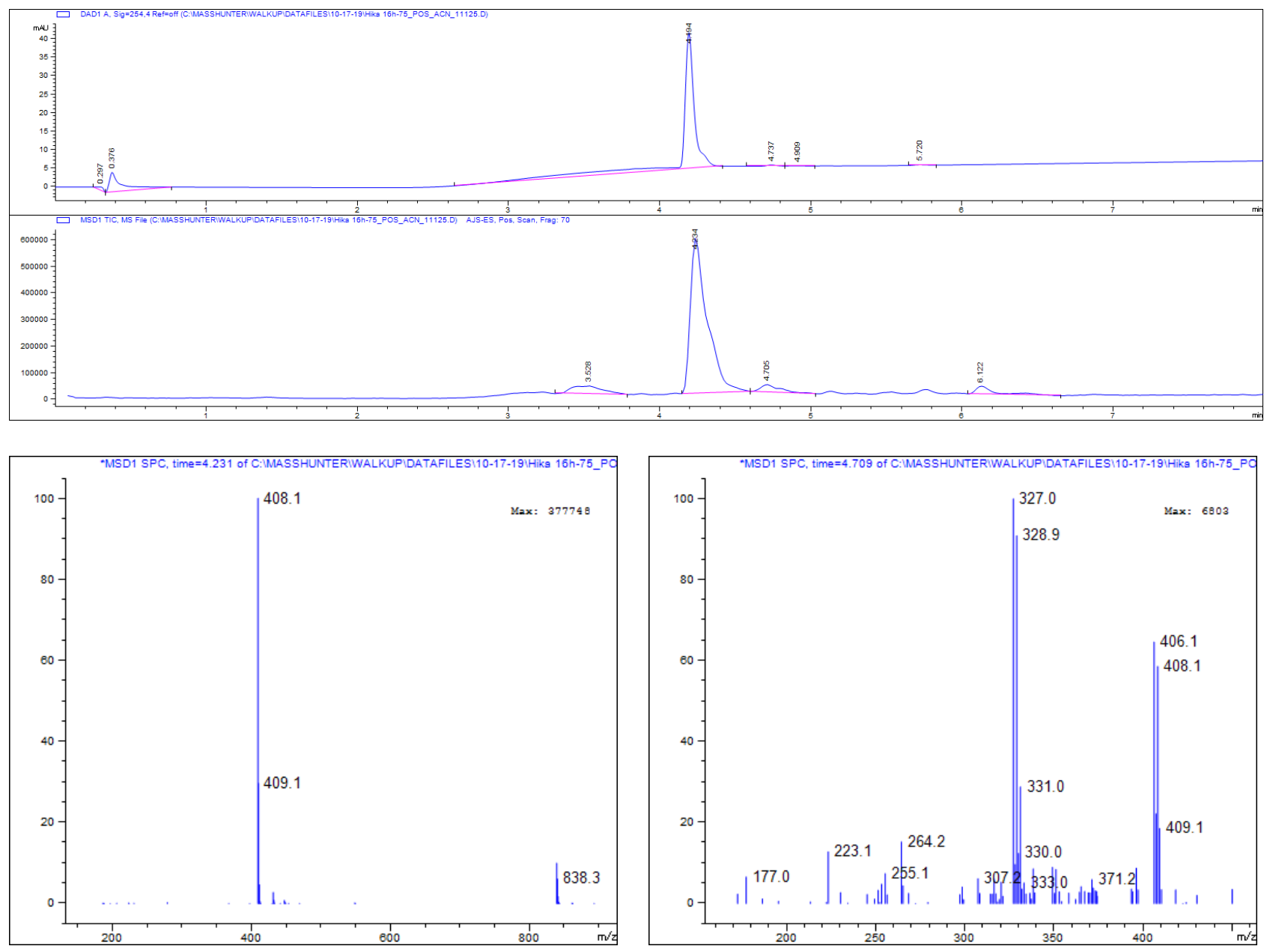


\section{$\mathrm{t}=14$ days}
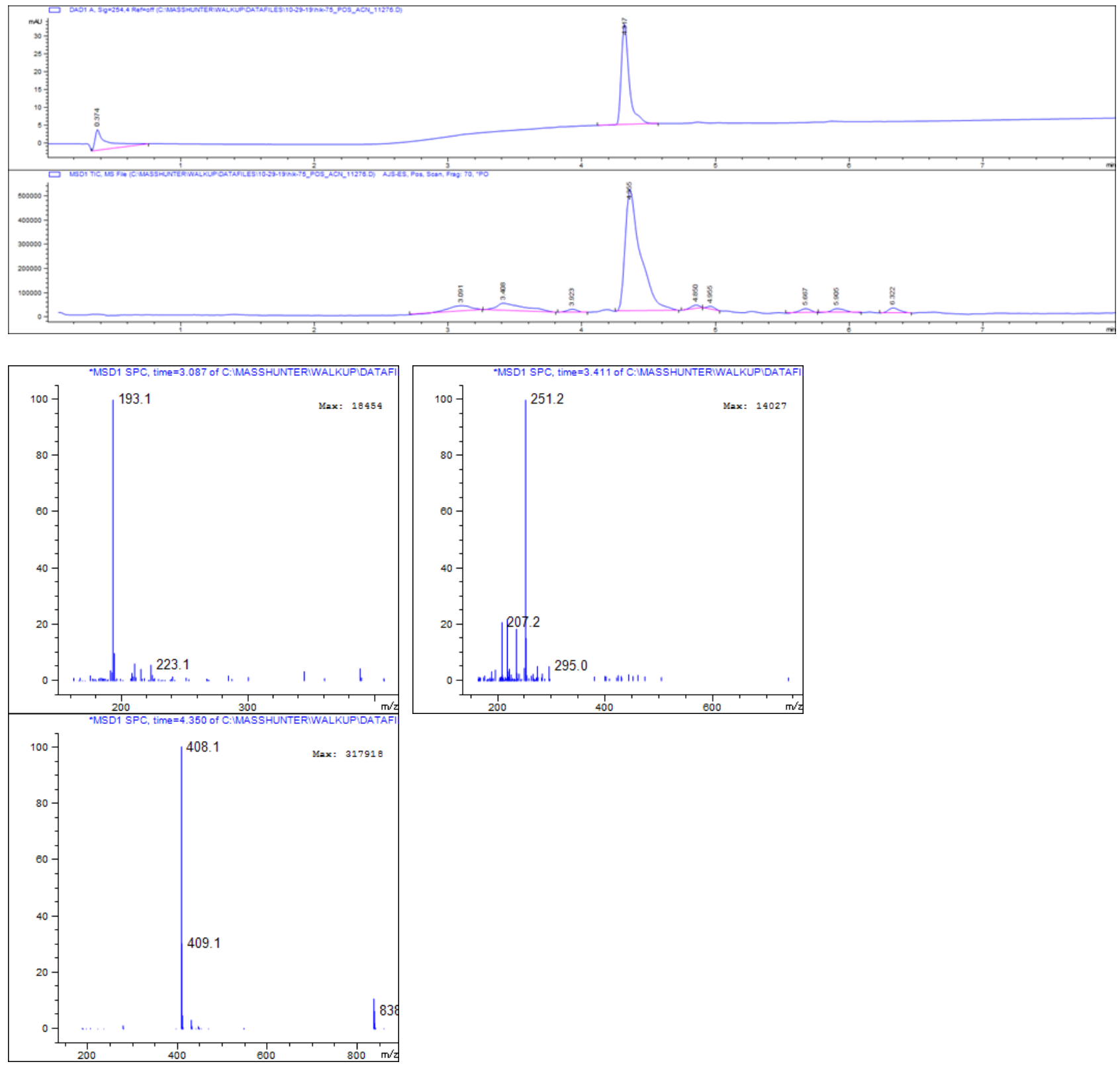
Hydrolysis products of hikarazine-80 $(\mathbf{2 5}\{\mathbf{1 4}, \mathbf{1}, \mathbf{3 7}\})$<smiles>CC(=O)Oc1c(Cc2ccco2)nc2c(Cc3ccccc3)nc(-c3ccc(O)cc3)cn12</smiles>

$t=0$
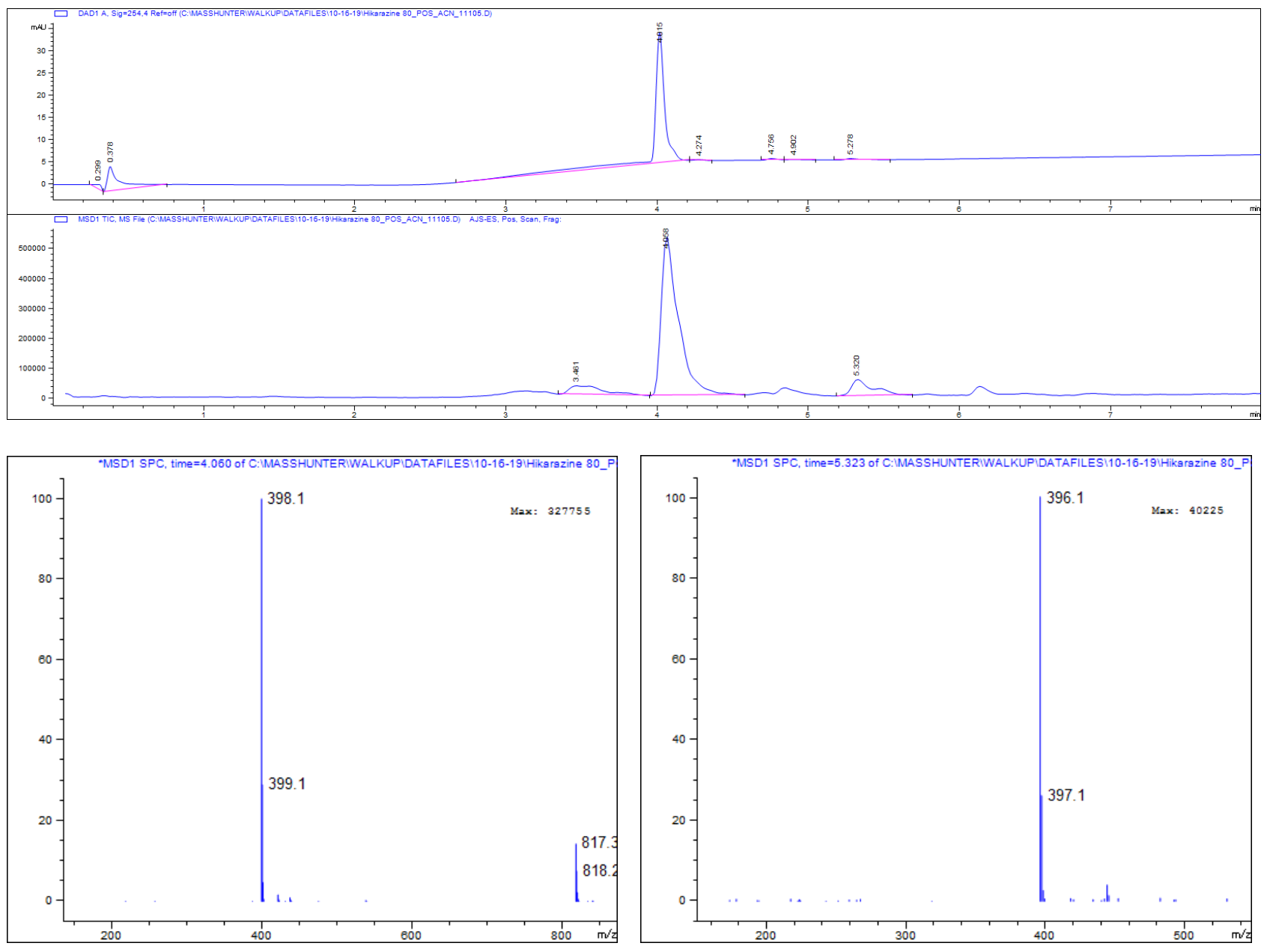
$t=16 h$
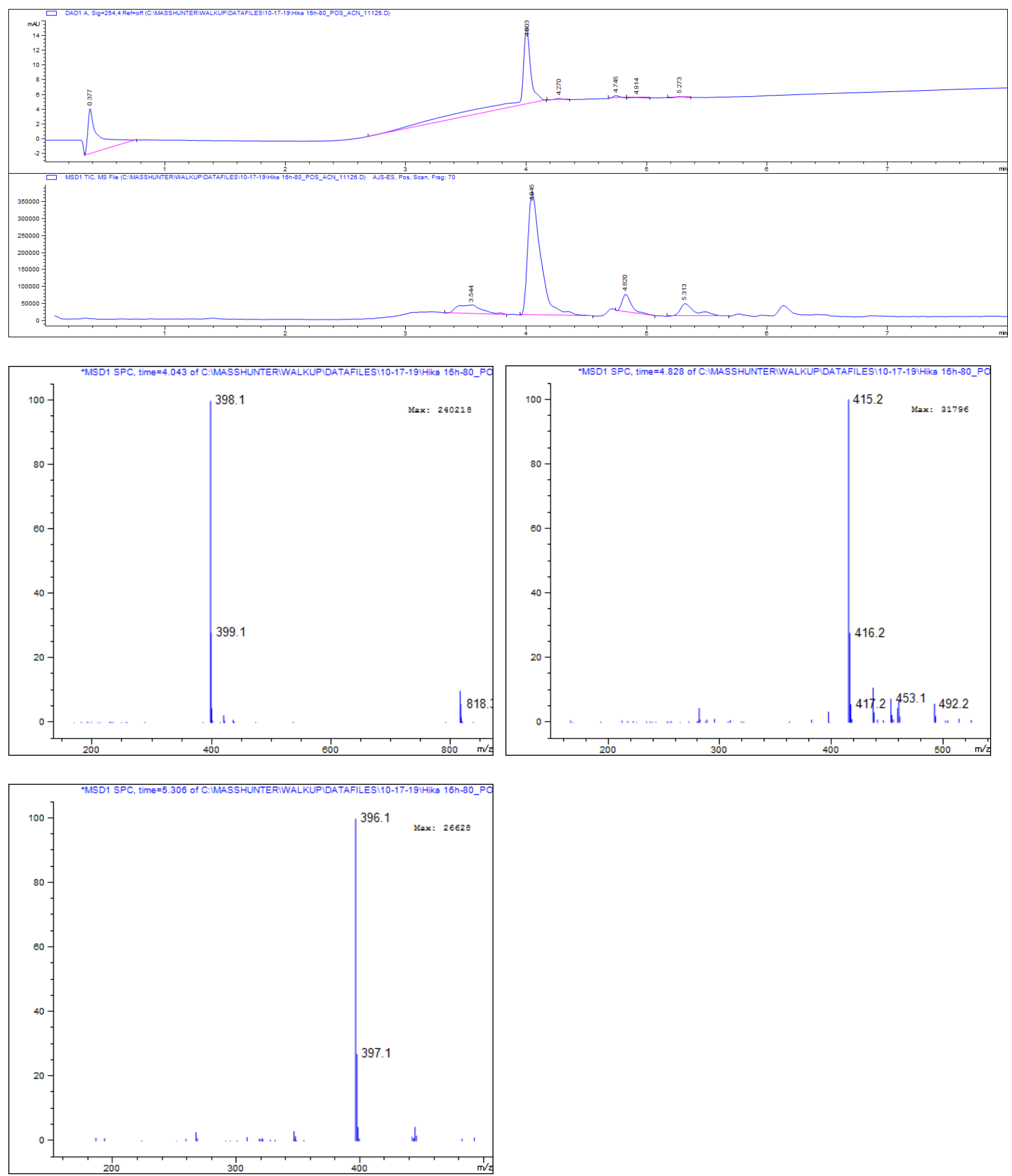


\section{$\mathrm{t}=14$ days}
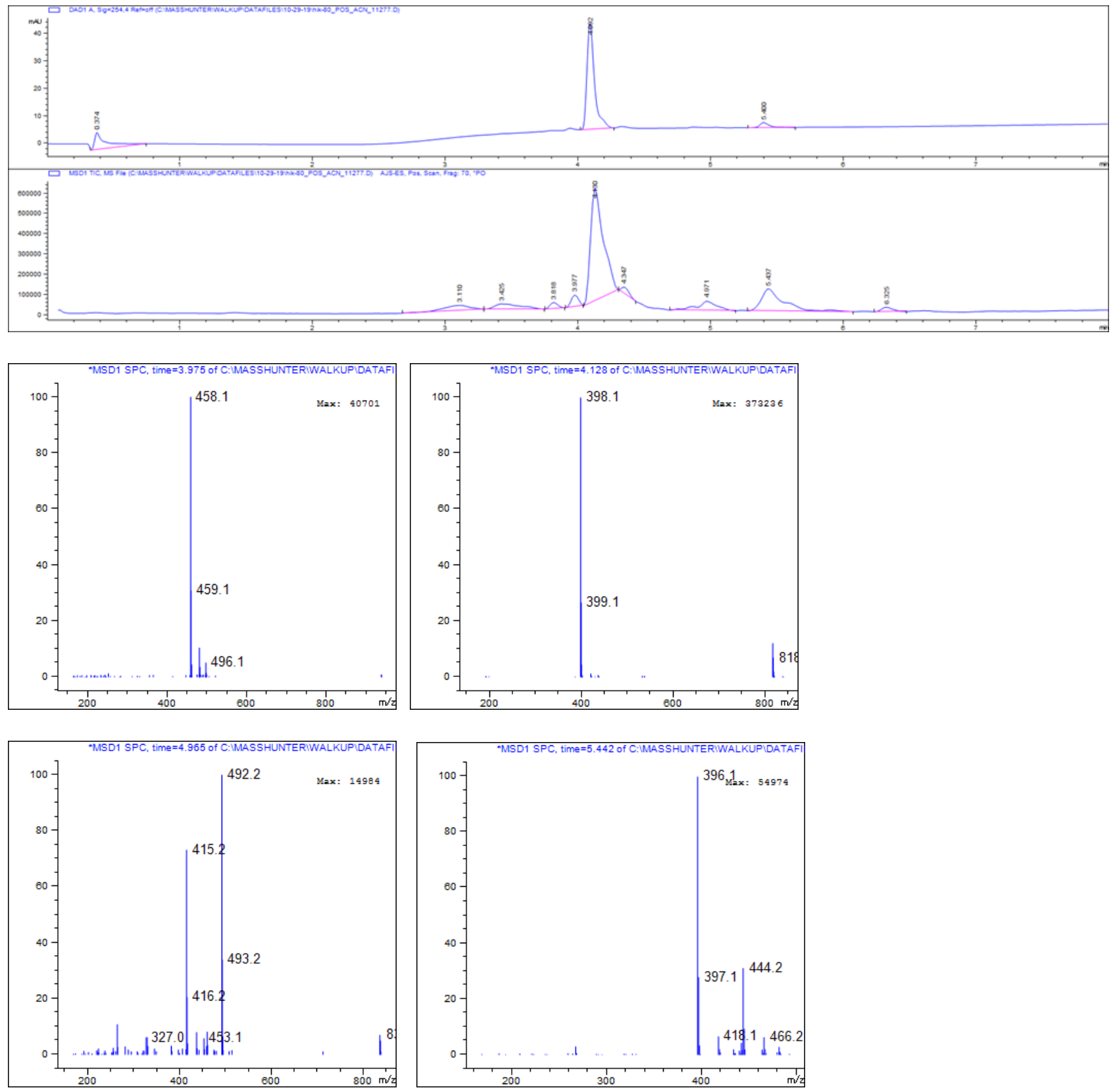
Hydrolysis products of hikarazine-85 $(\mathbf{2 5}\{1,2,42\})$
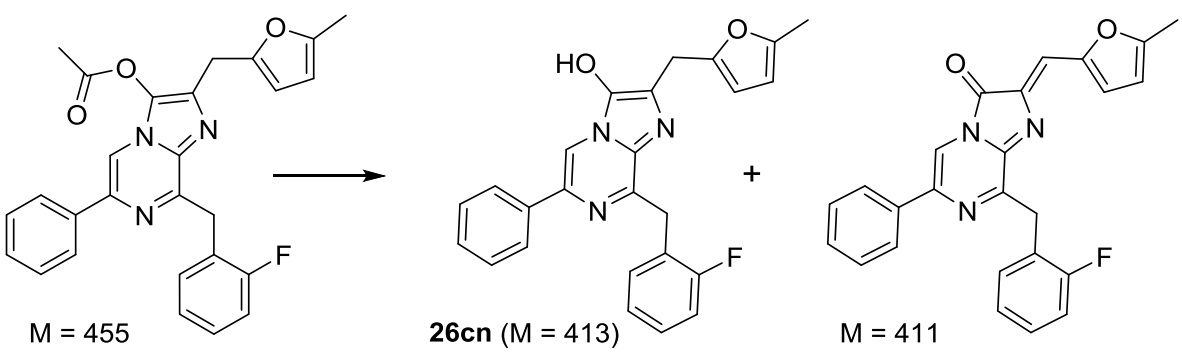

$t=0$
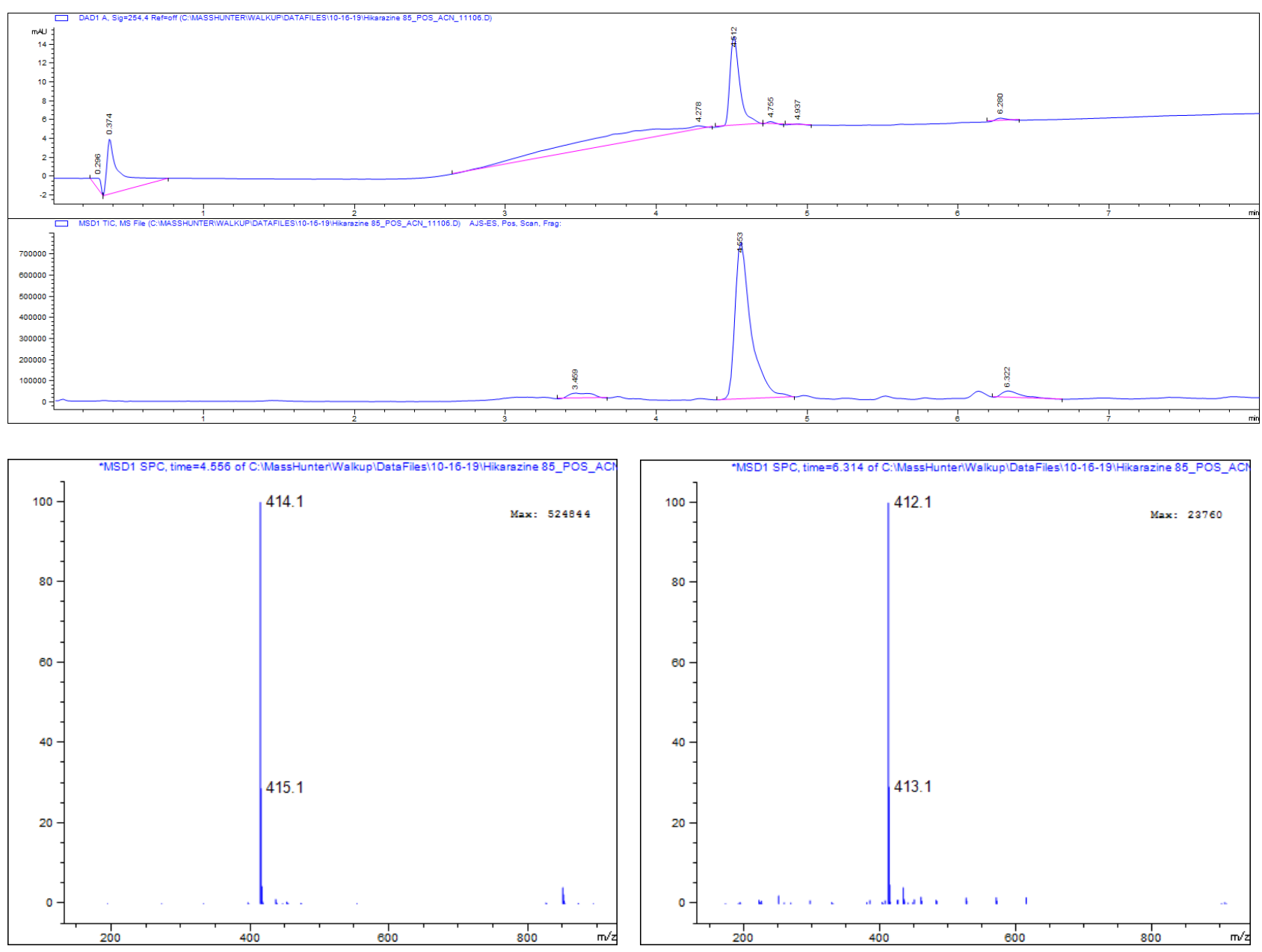
$t=16 h$

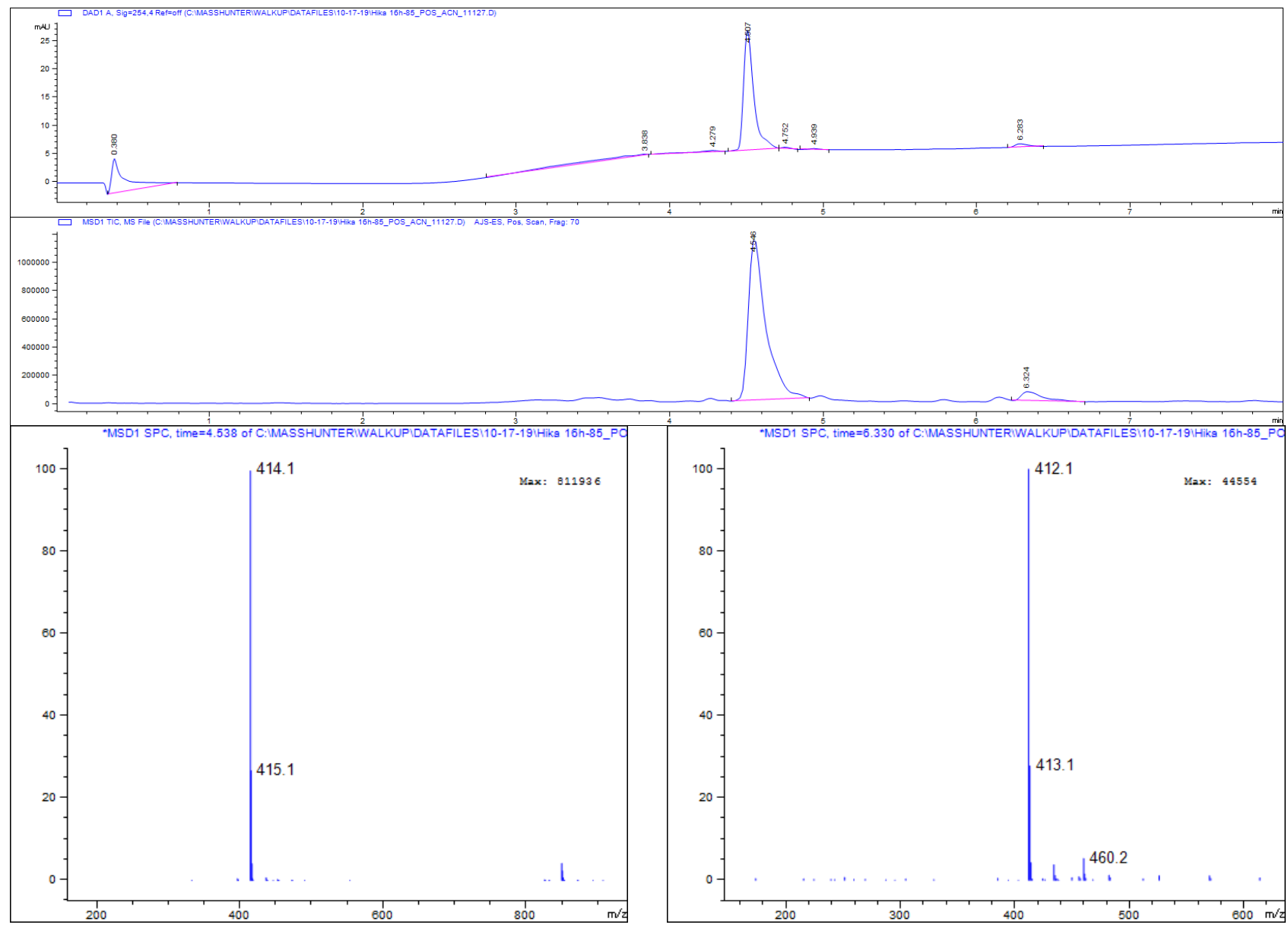




\section{$\mathrm{t}=14$ days}
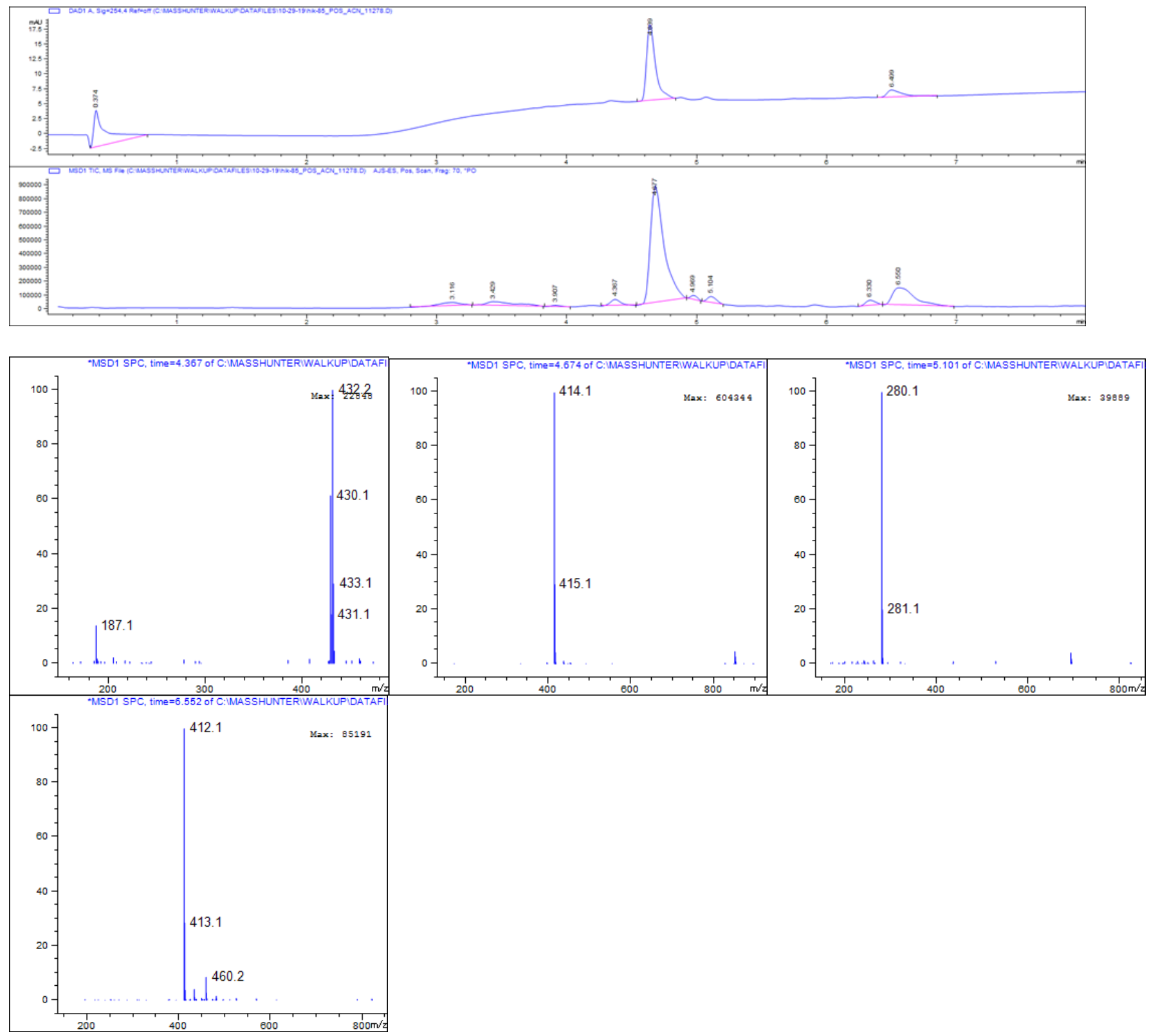
Hydrolysis products of hikarazine-86 $(\mathbf{2 5}\{14,1,63\})$

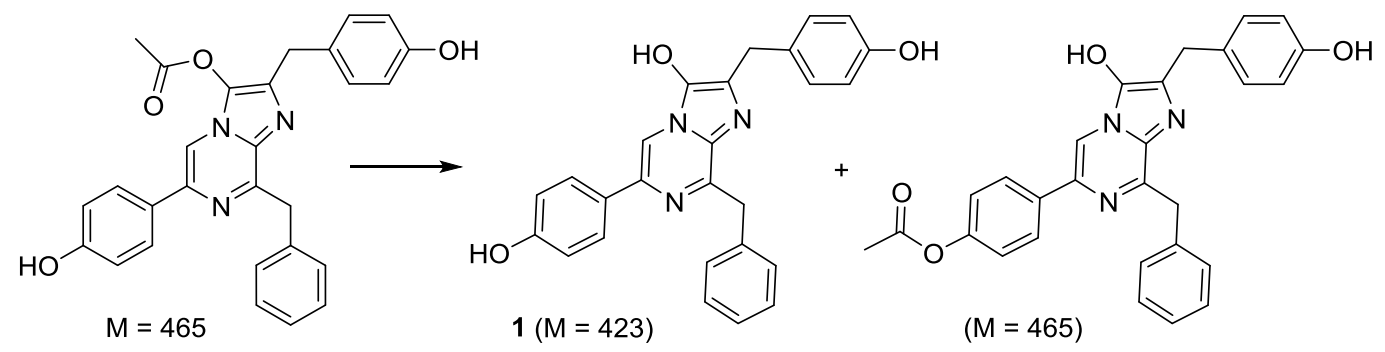

$t=0$
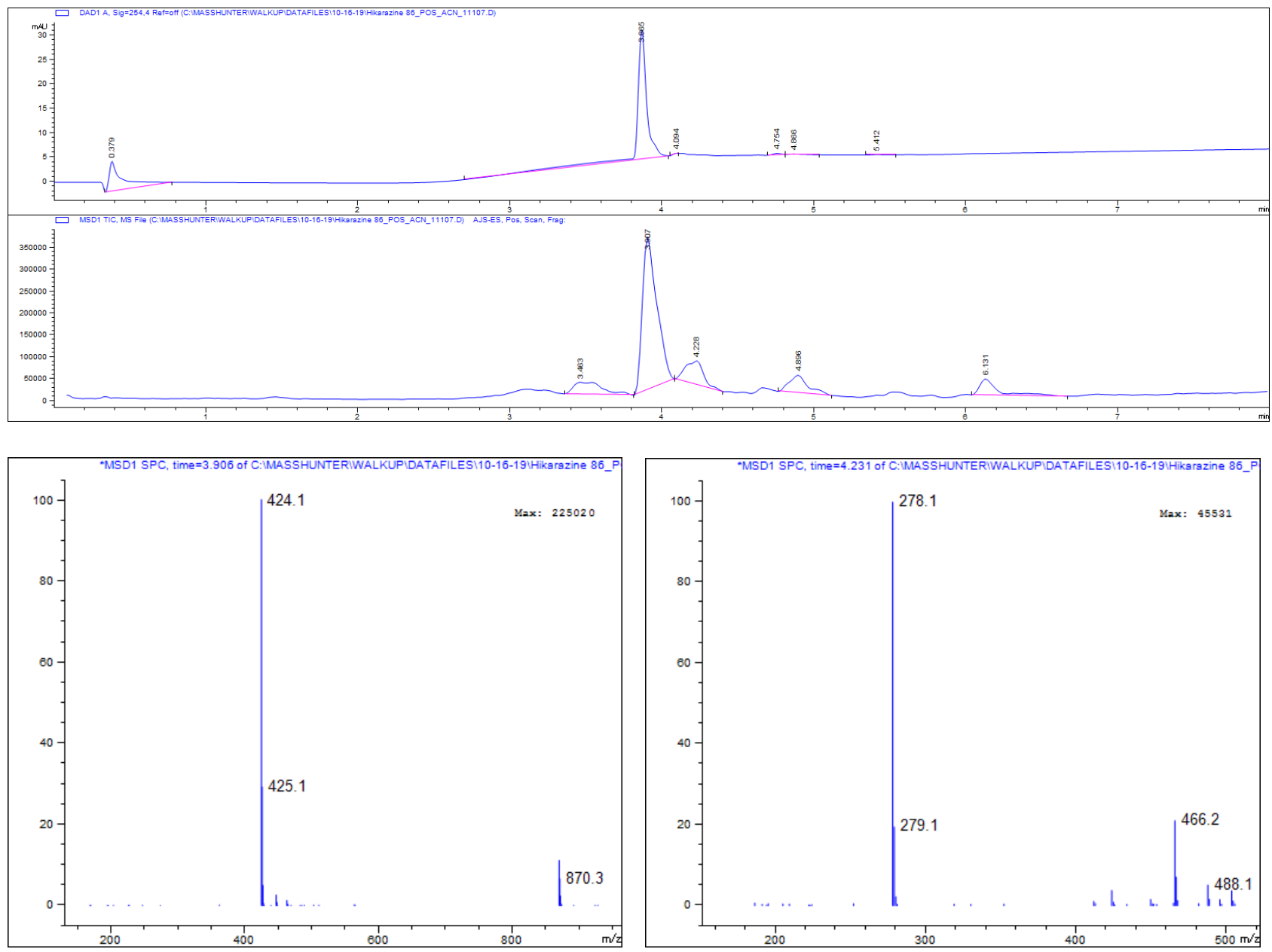
$t=16 h$
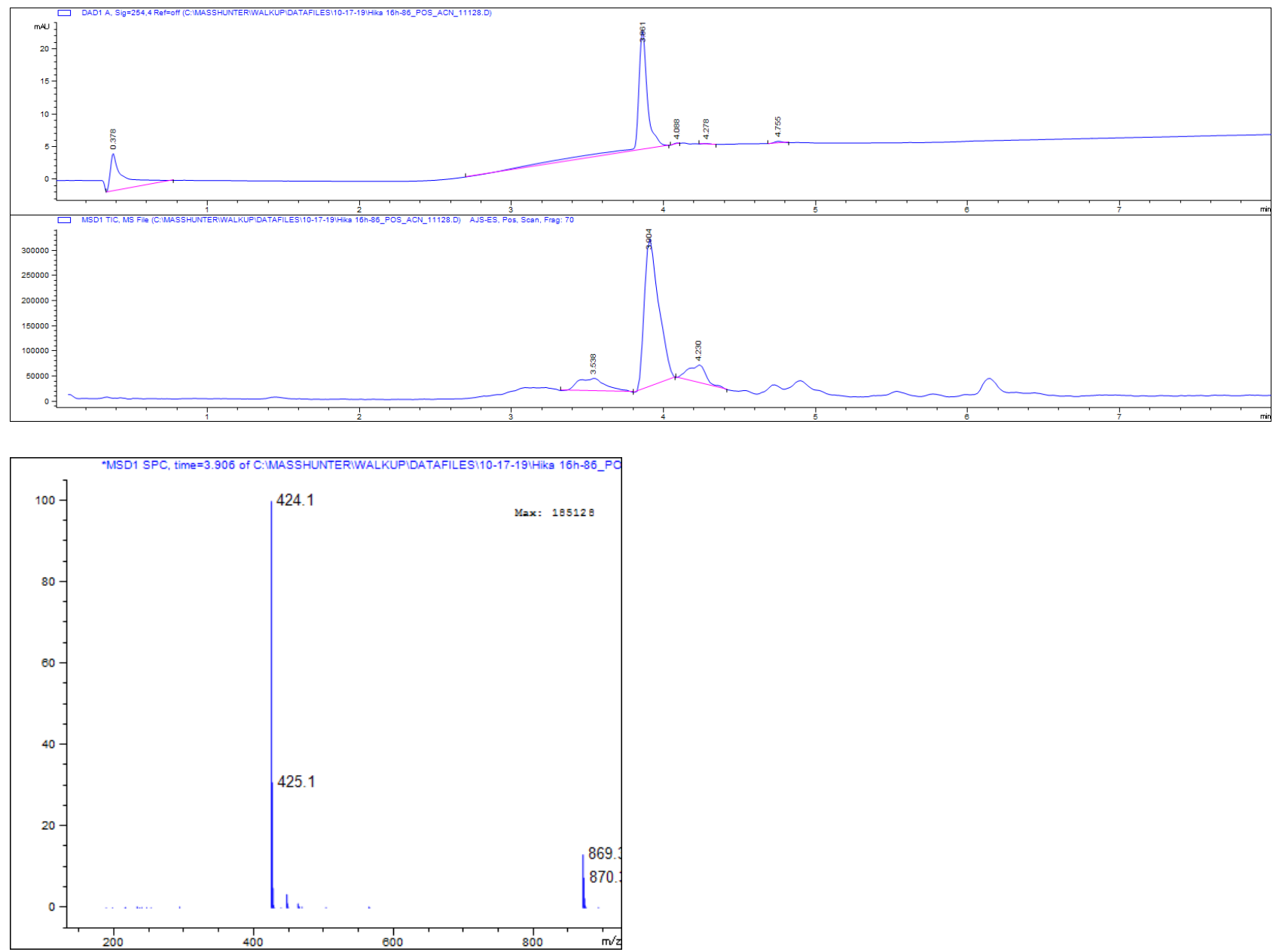


\section{$\mathrm{t}=14$ days}
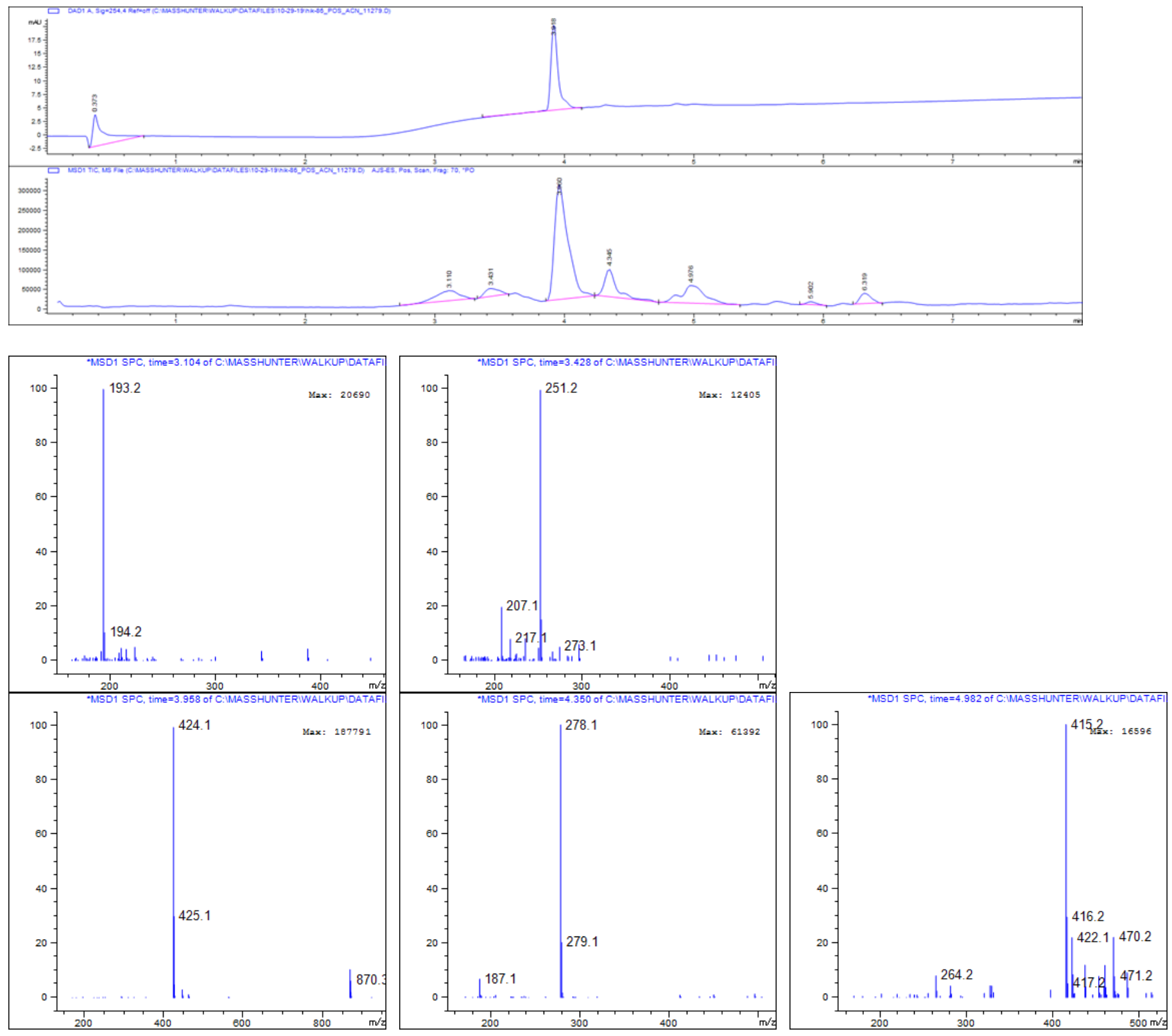
Hydrolysis products of hikarazine-96 $(\mathbf{2 5}\{1,2,44\})$
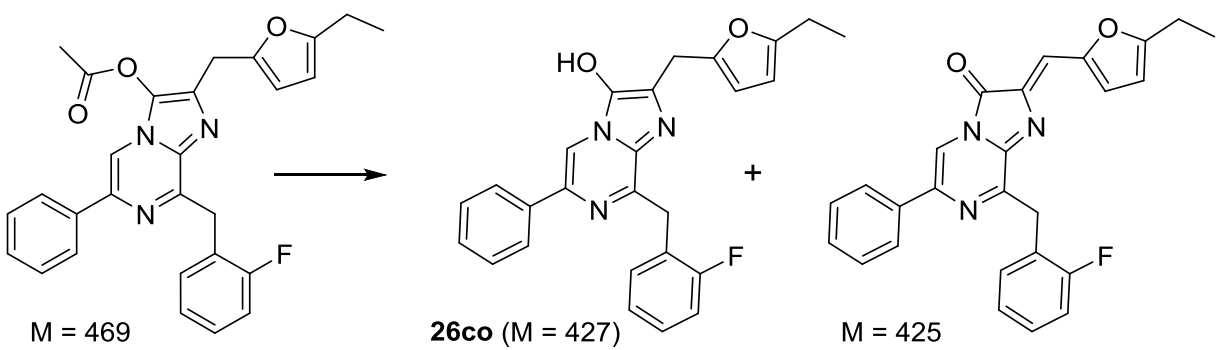

$\mathrm{t}=0$
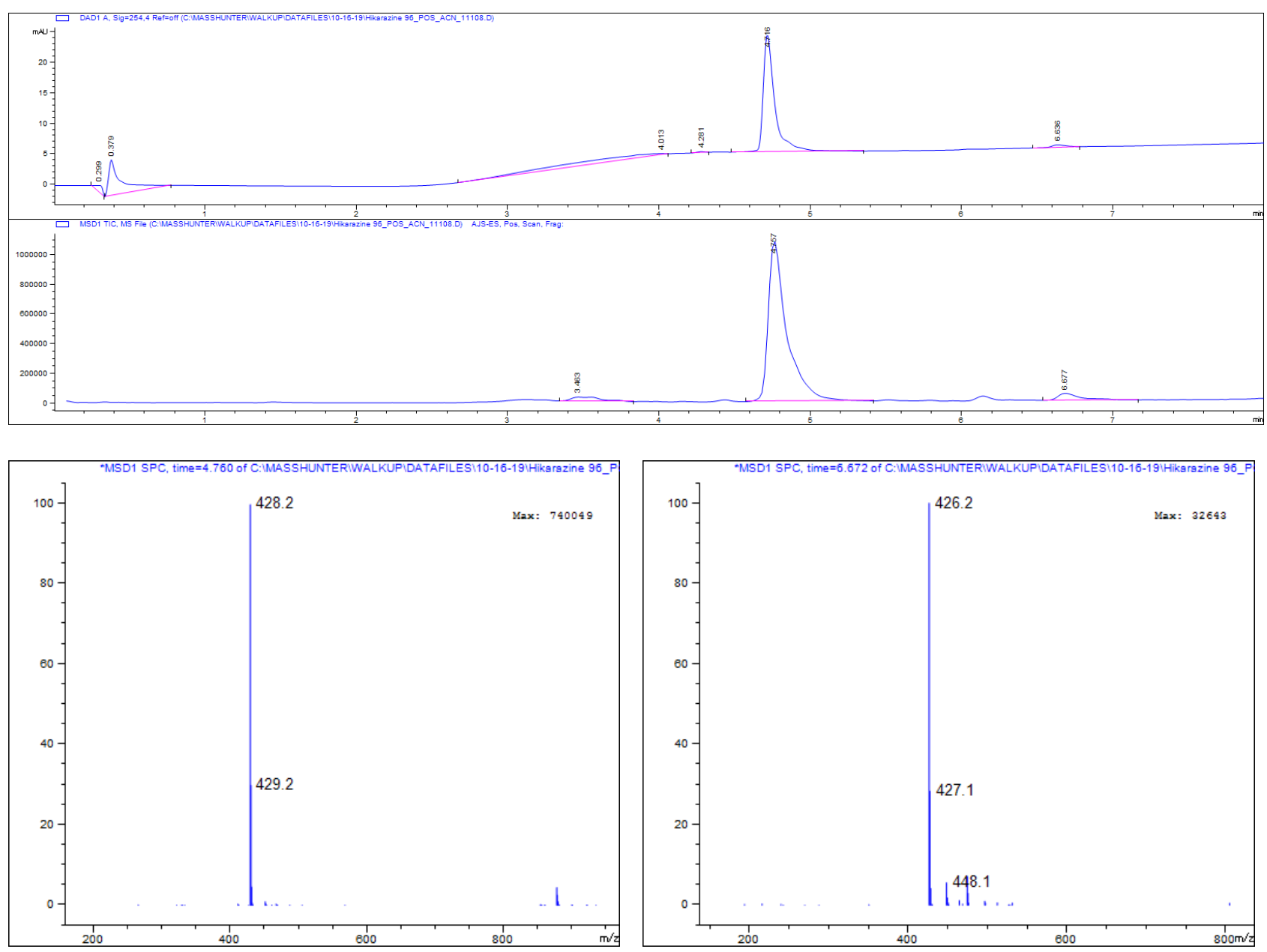
$t=16 h$
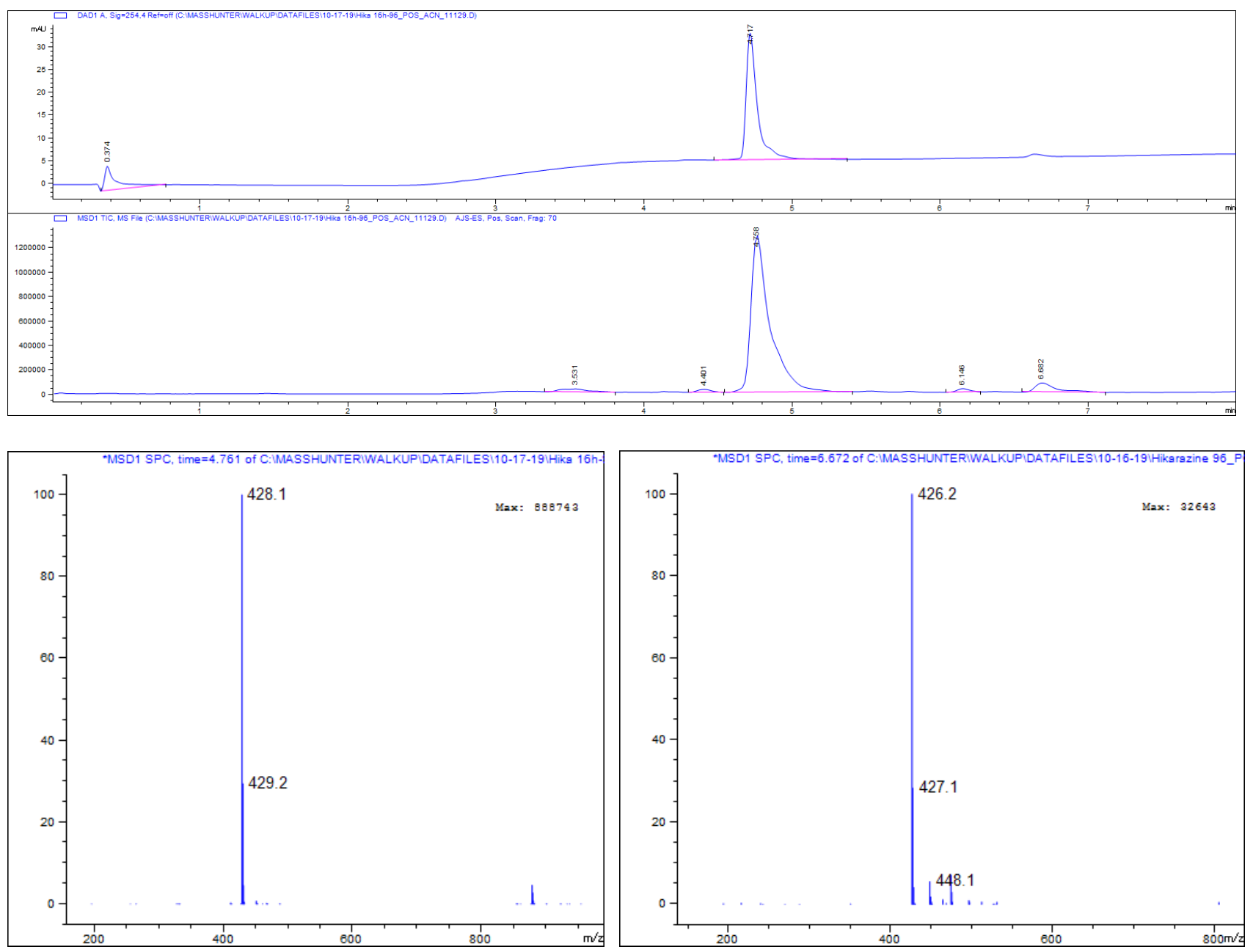


\section{$\mathrm{t}=14$ days}
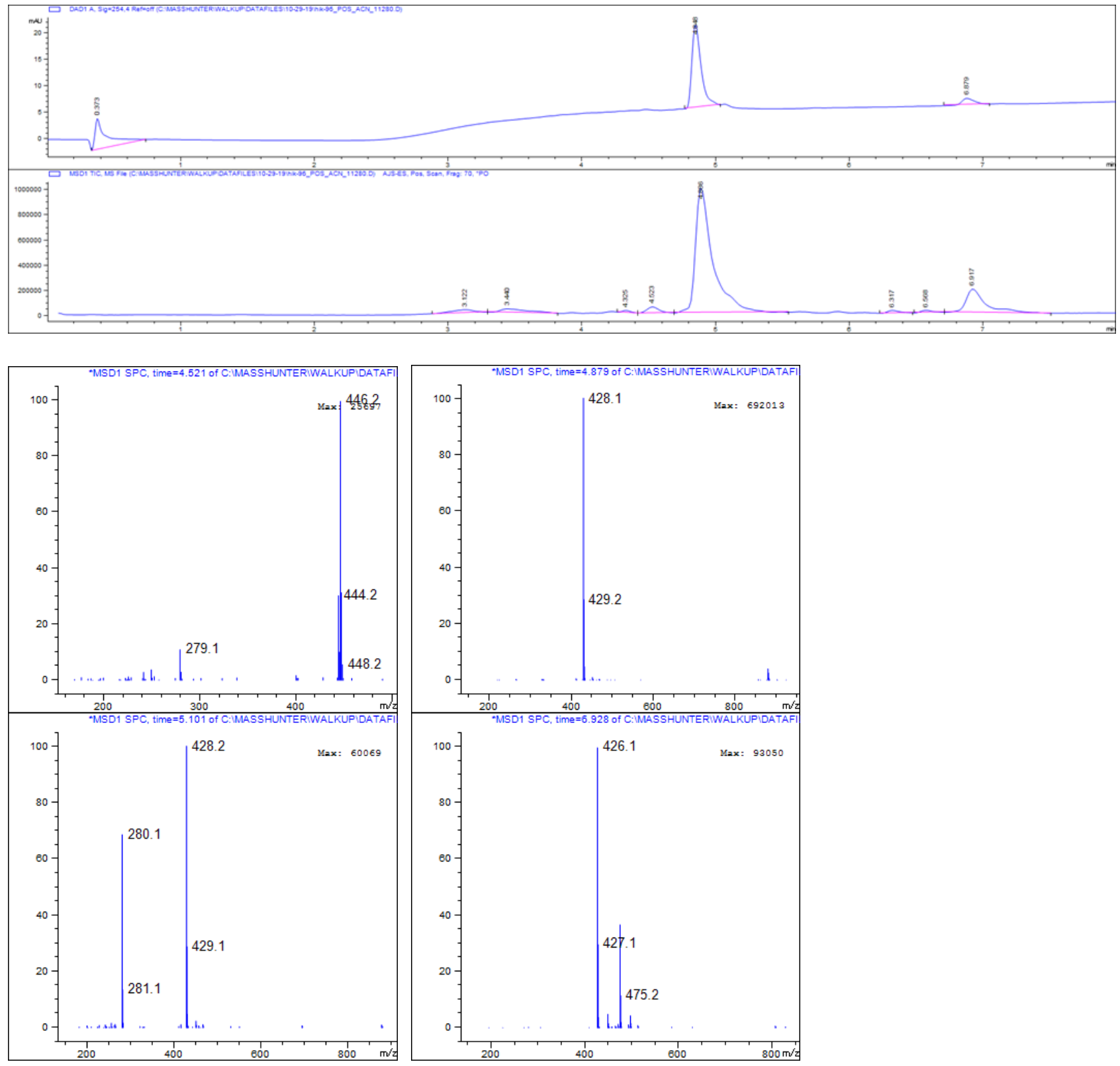
Hydrolysis products of hikarazine-97 $(\mathbf{2 5}\{\mathbf{1 , 3}, \mathbf{4 2}\})$
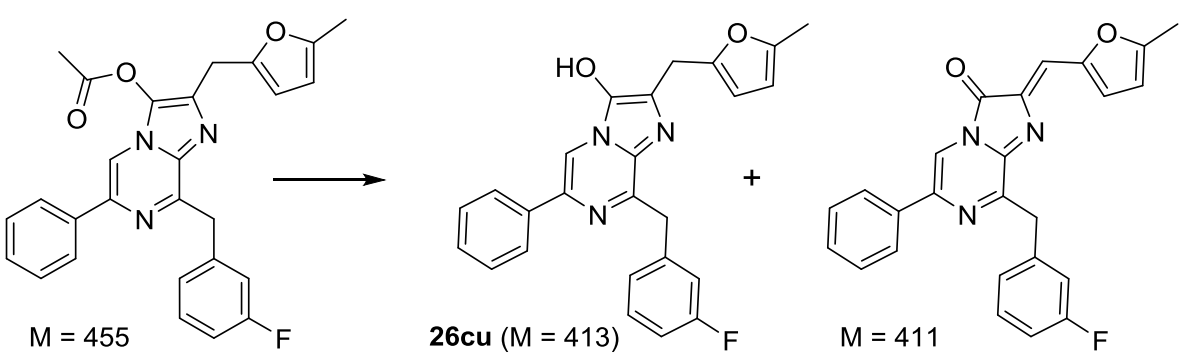

$\mathrm{t}=0$
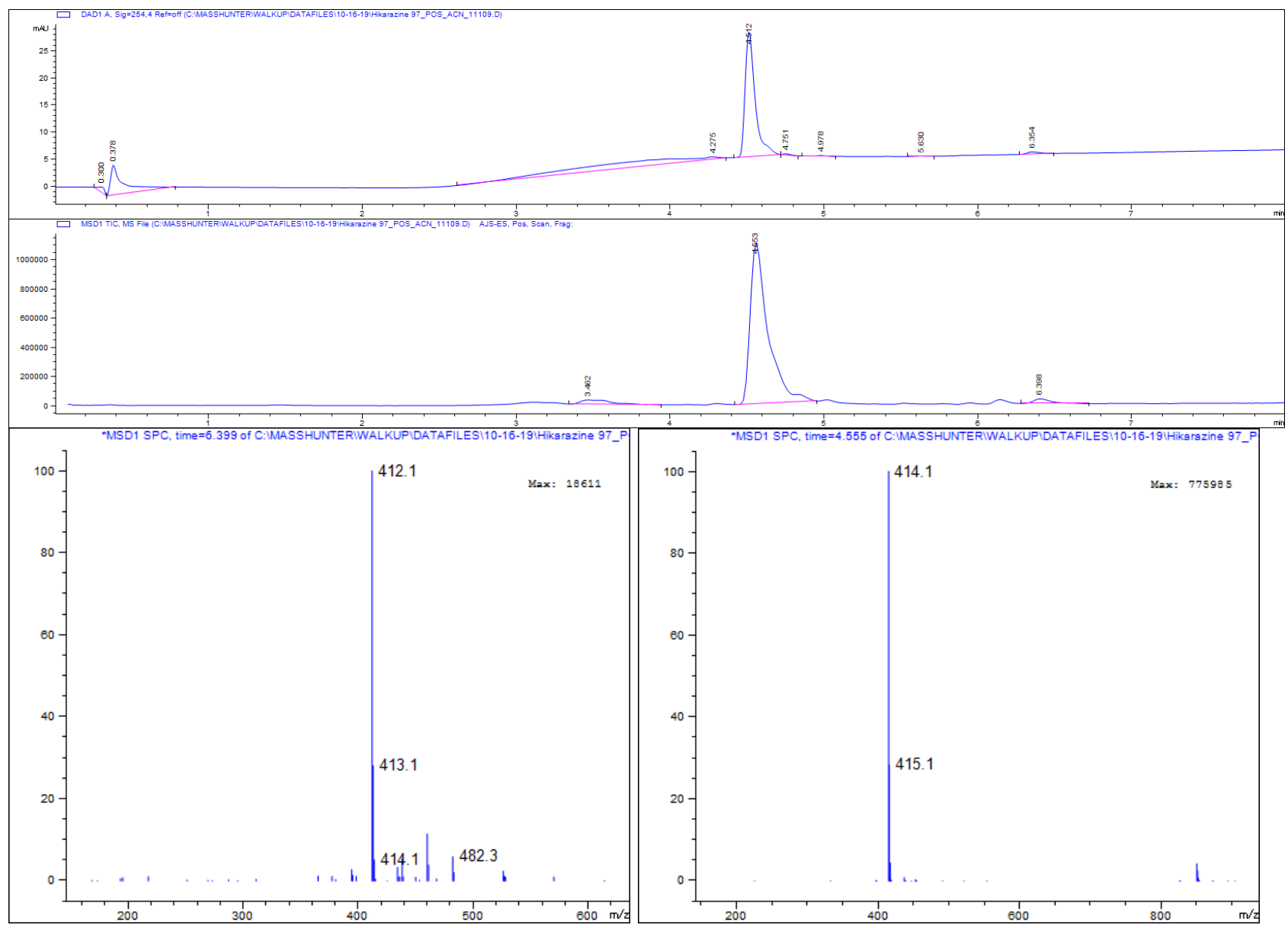
$t=16 h$
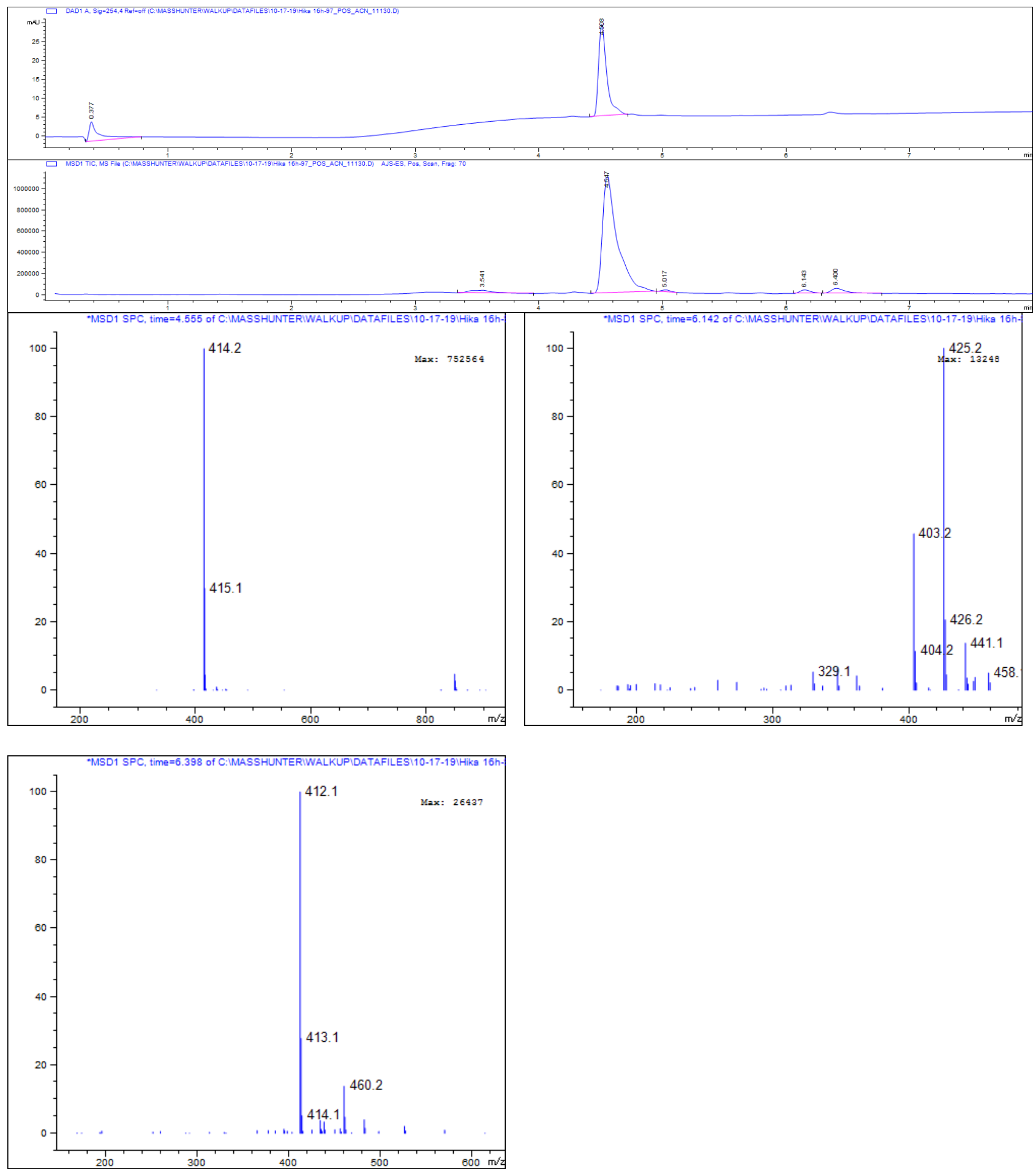


\section{$\mathrm{t}=14$ days}
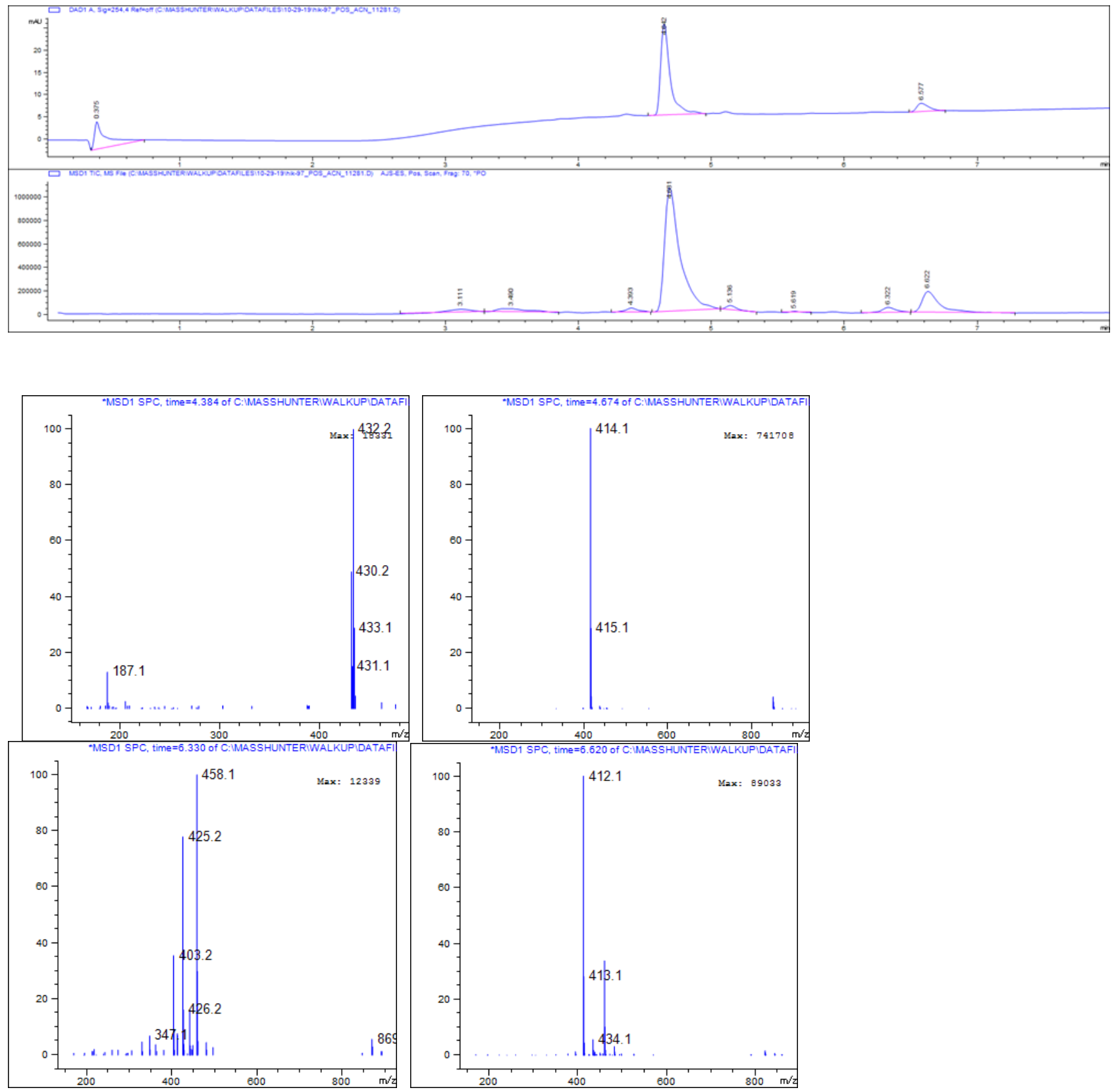
Hydrolysis products of hikarazine-99 $(\mathbf{2 5}\{1,3,44\})$
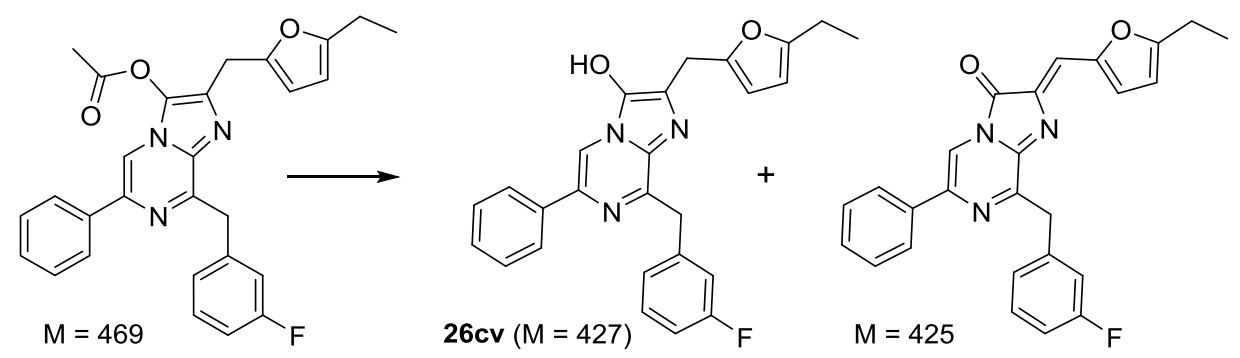

$\mathrm{t}=0$
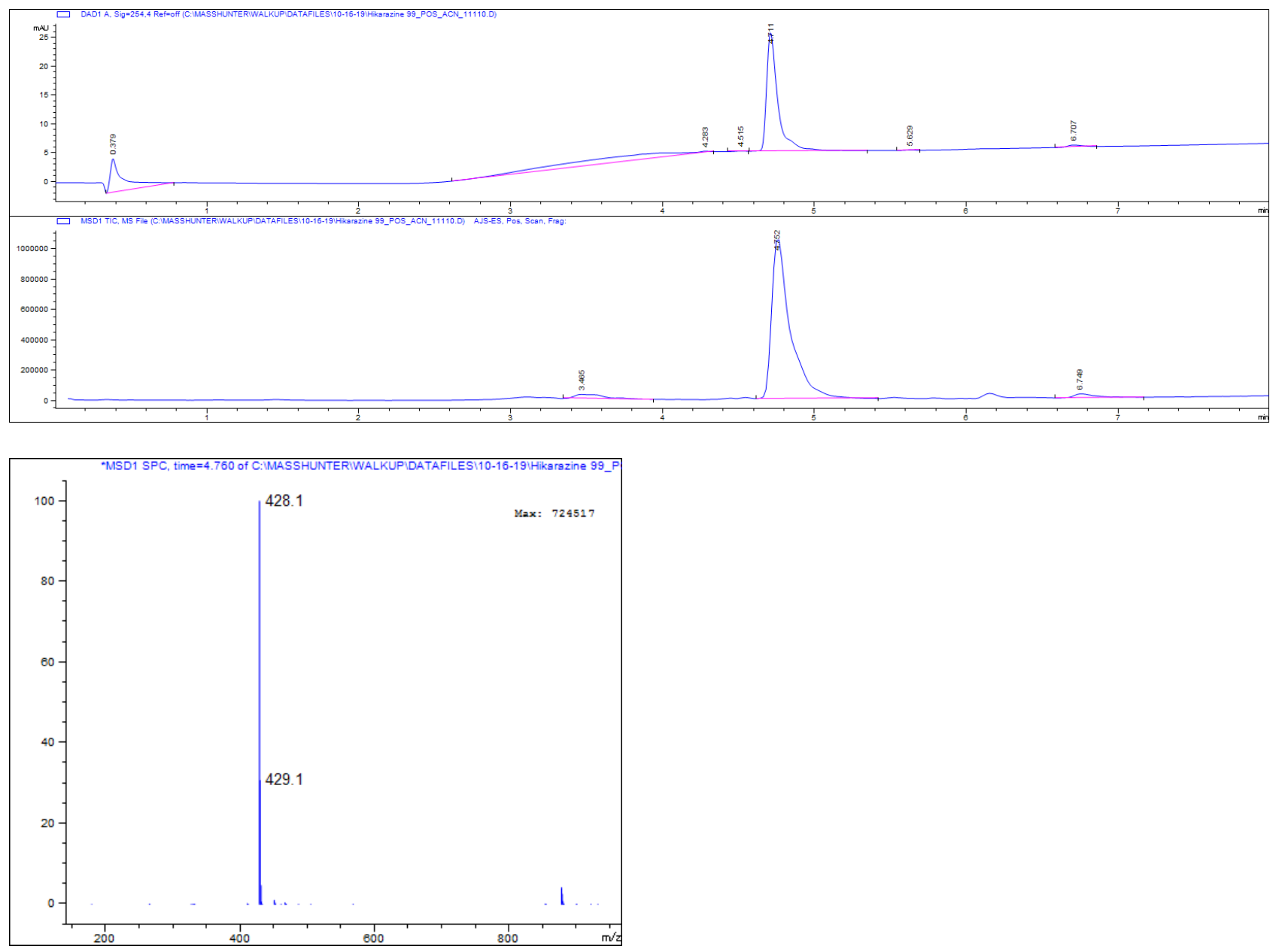

178 
$t=16 h$
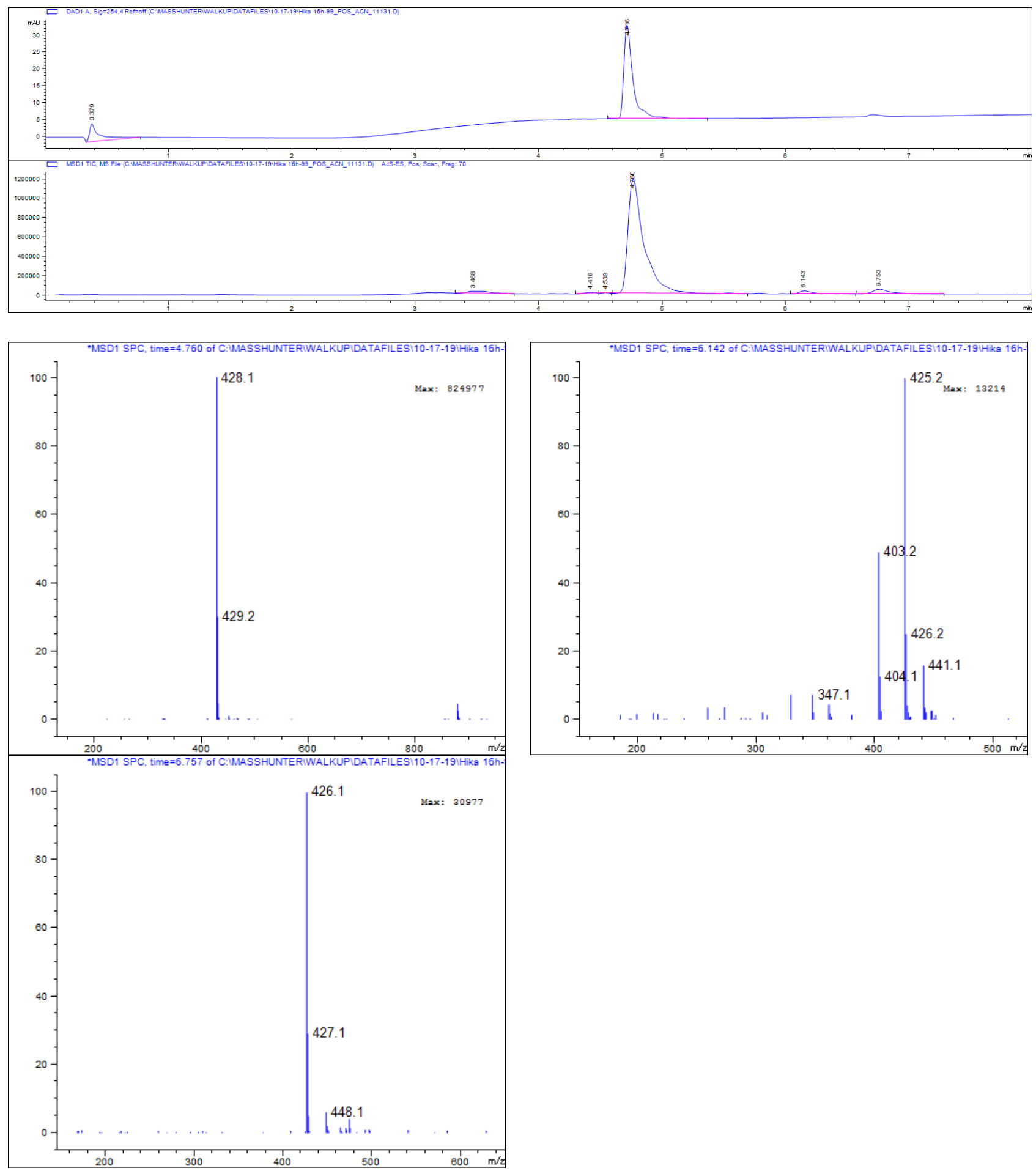


\section{$\mathrm{t}=14$ days}
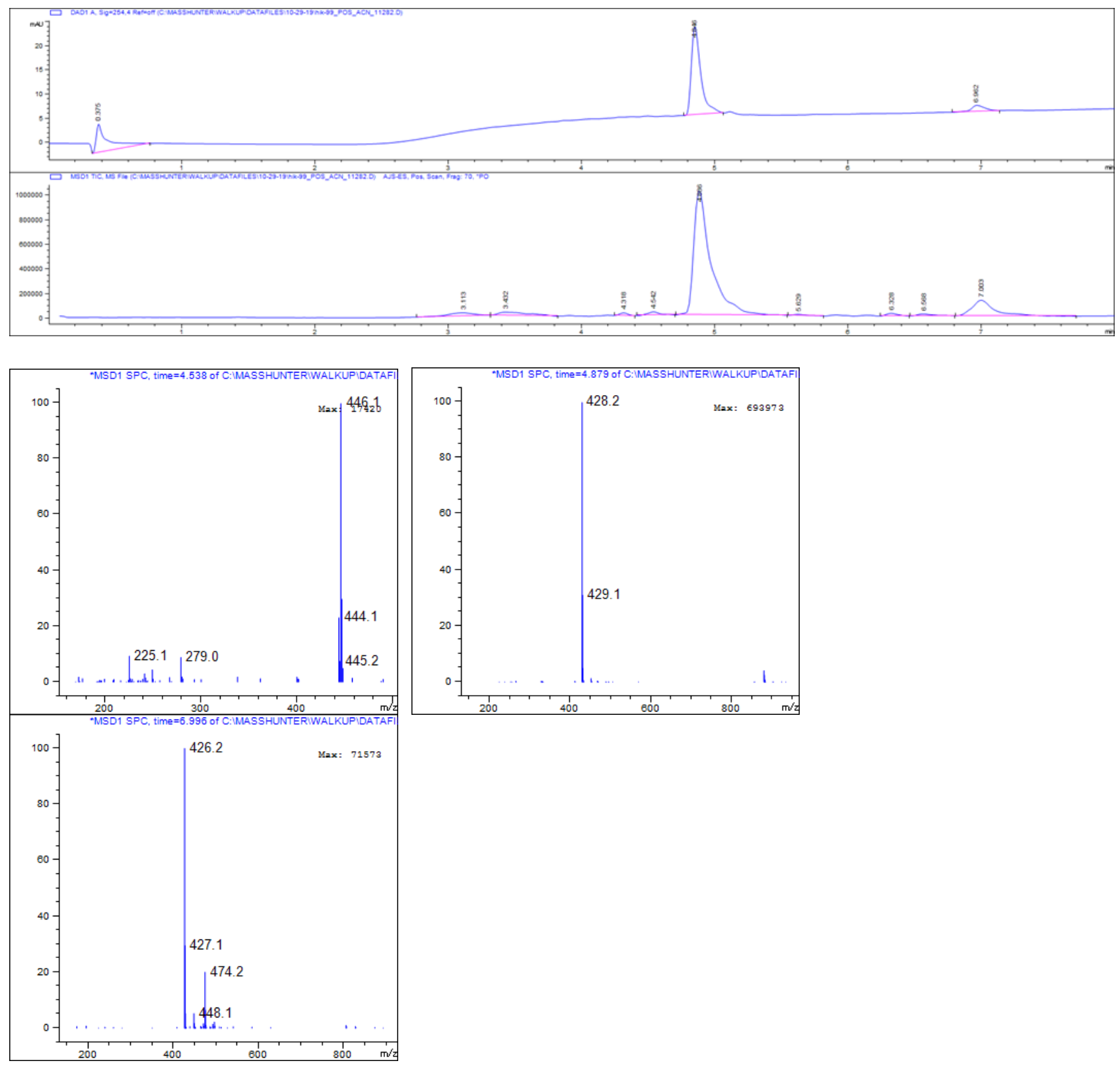
Hydrolysis products of hikarazine-103 (26db $\{1,22,37\})$<smiles>CC(=O)Oc1c(Cc2ccco2)nc2c(Cc3cc(F)cc(F)c3)nc(-c3ccccc3)cn12</smiles>

$$
M=459
$$

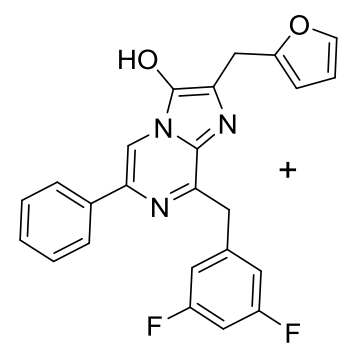

$26 d b(M=417)$

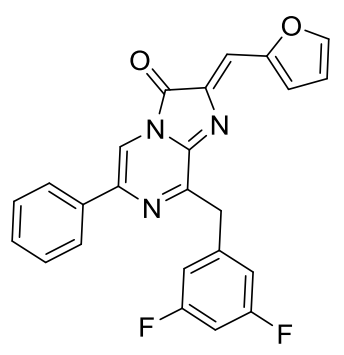

$M=415$

$\mathrm{t}=0$
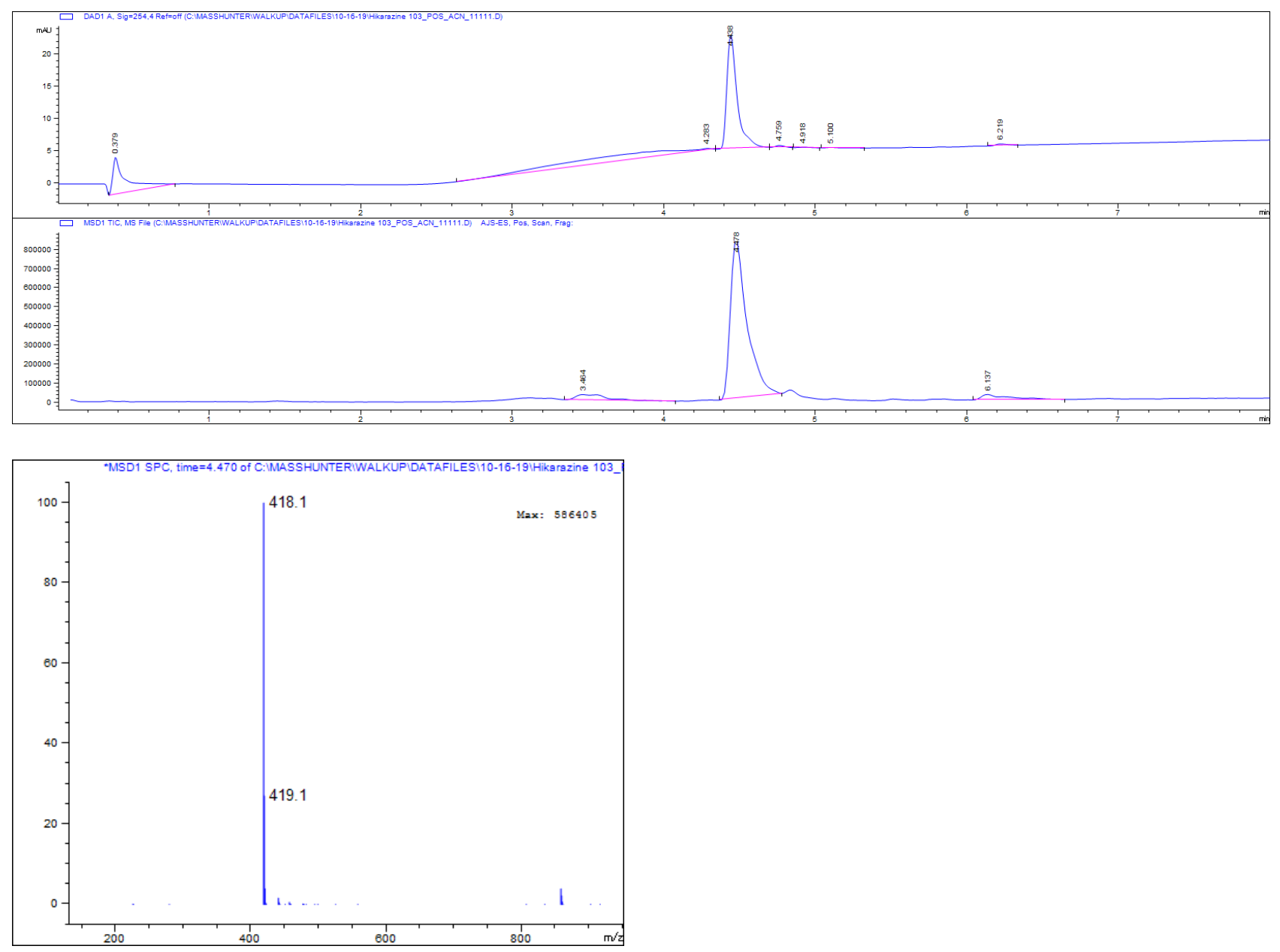

181 
$t=16 h$
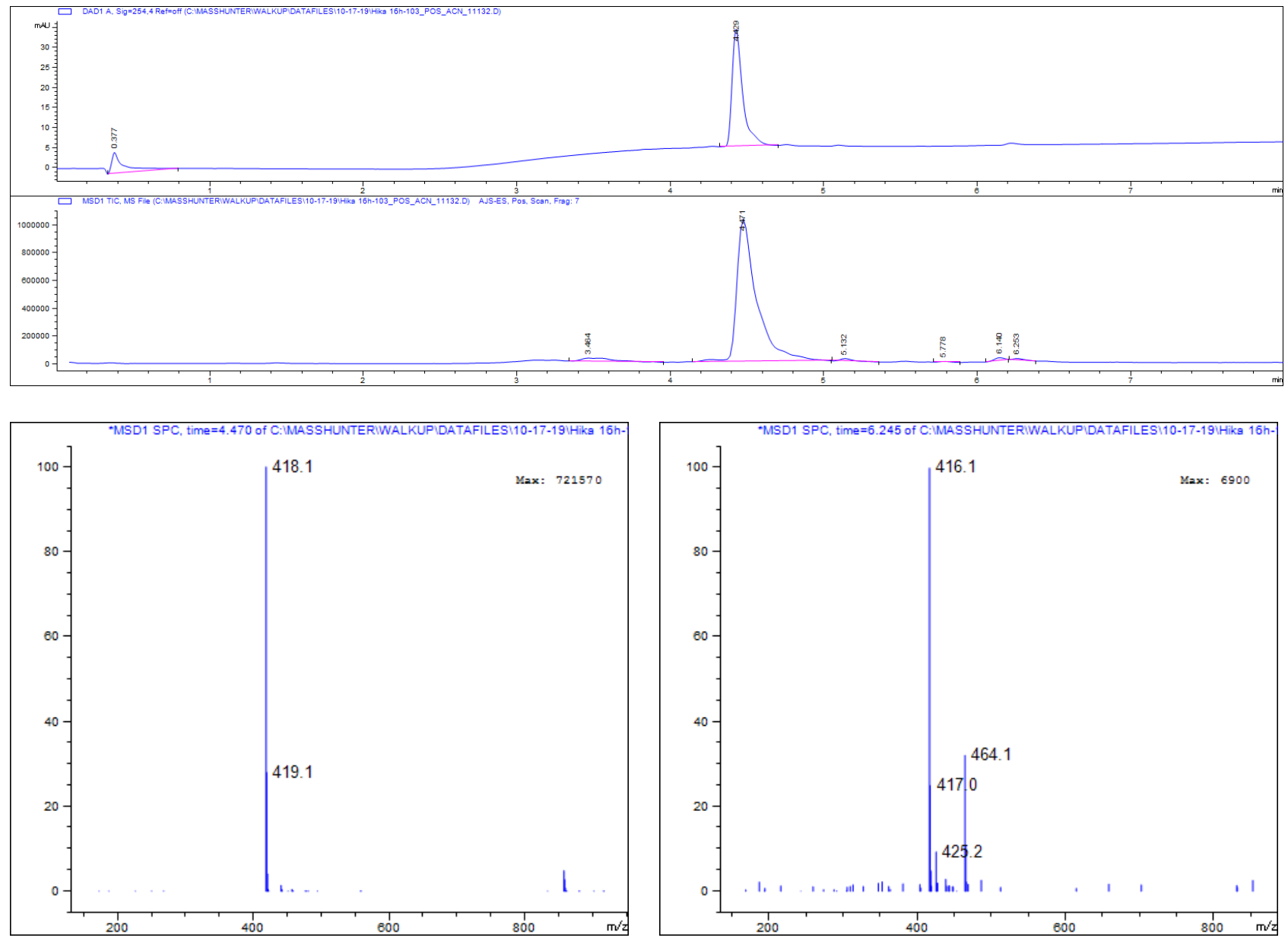


\section{$\mathrm{t}=14$ days}
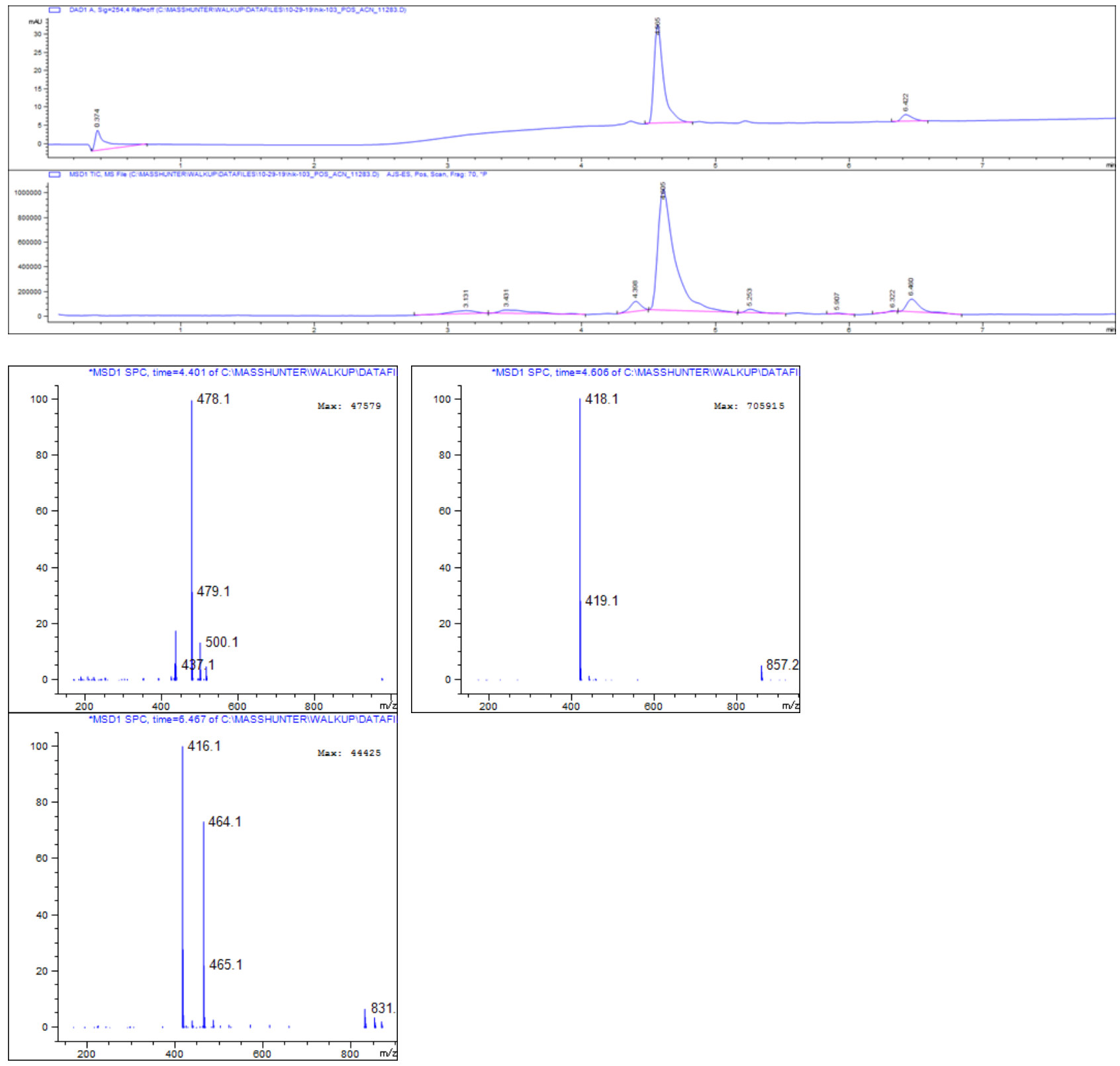
Hydrolysis products of hikarazine-105 (26dw $\{2,3,42\})$

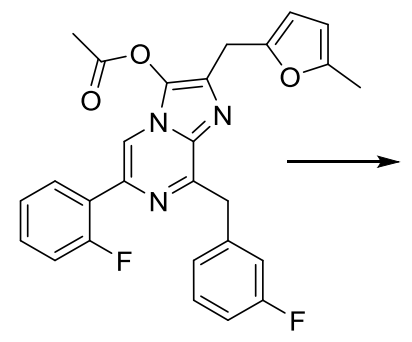

$M=473$

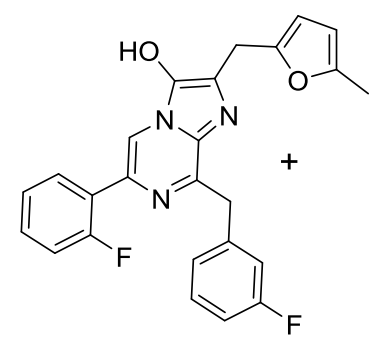

26dw $(M=431)$

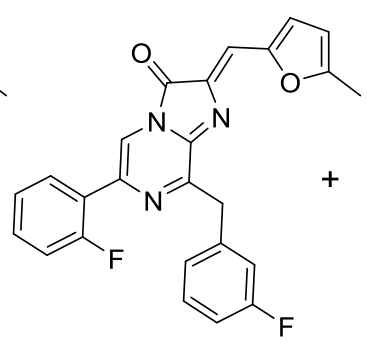

$M=429$

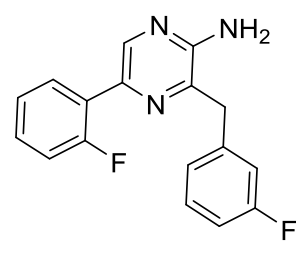

$M=297$

$\mathrm{t}=0$
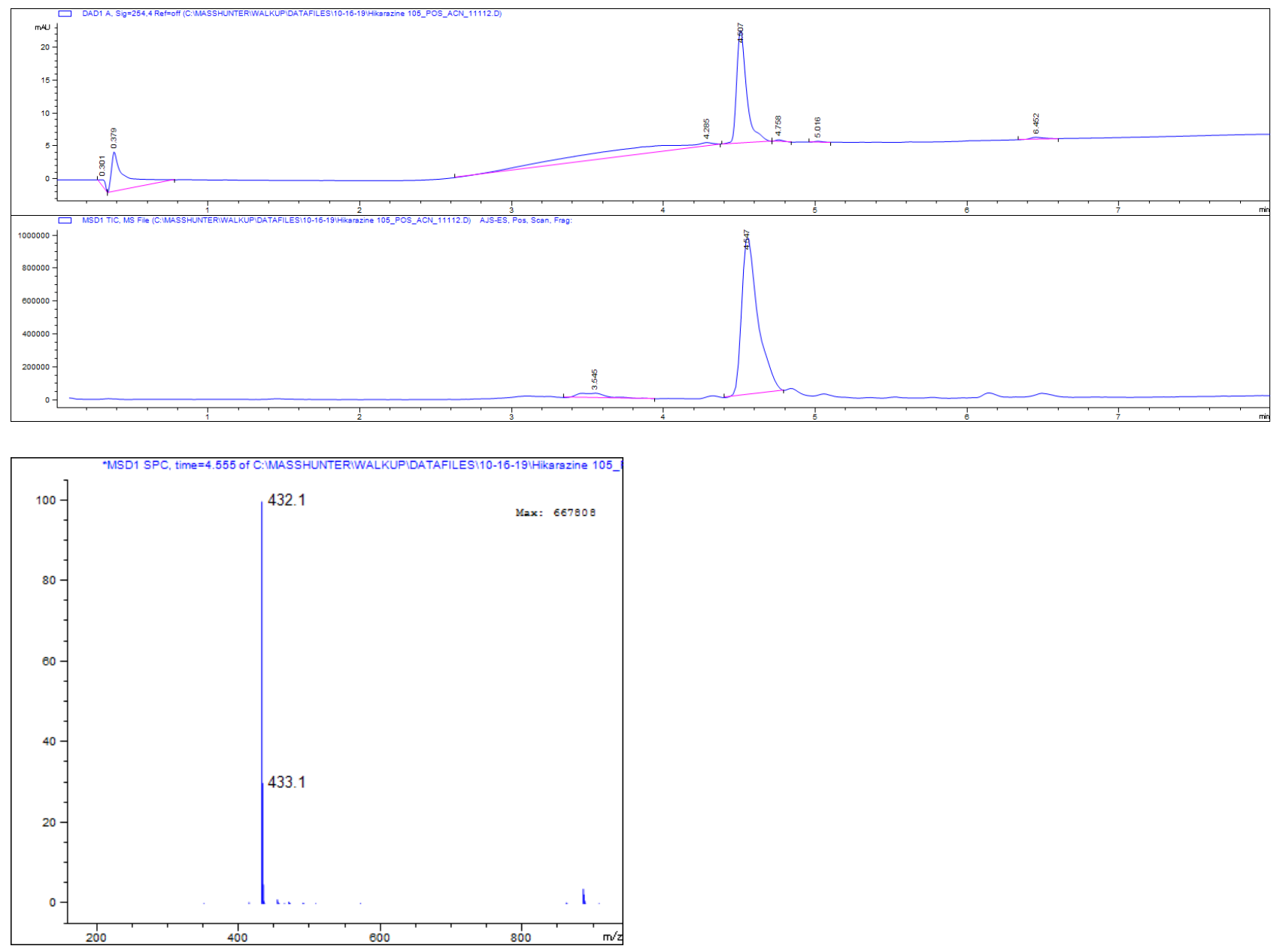

184 
$t=16 h$
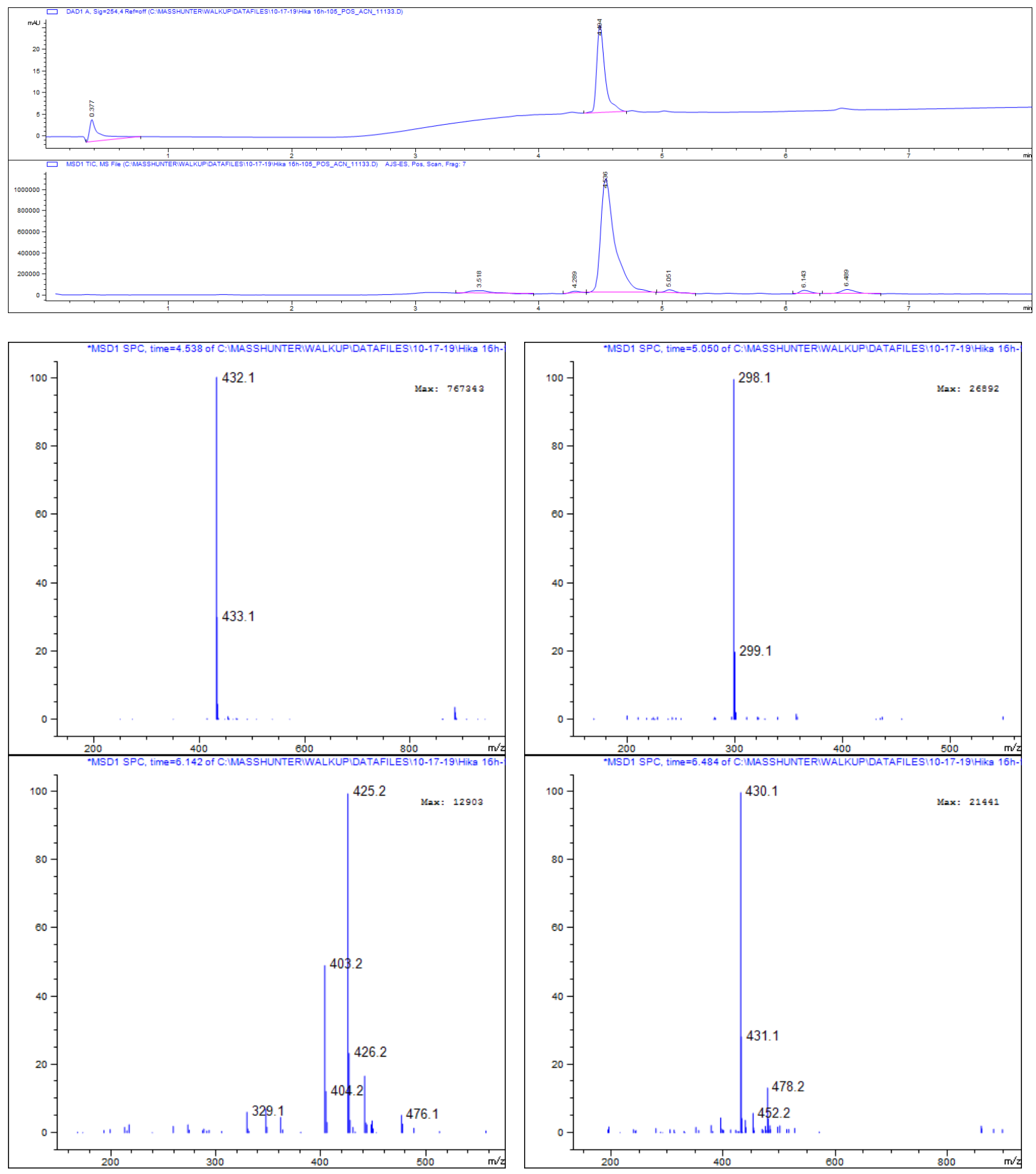


\section{$\mathrm{t}=14$ days}
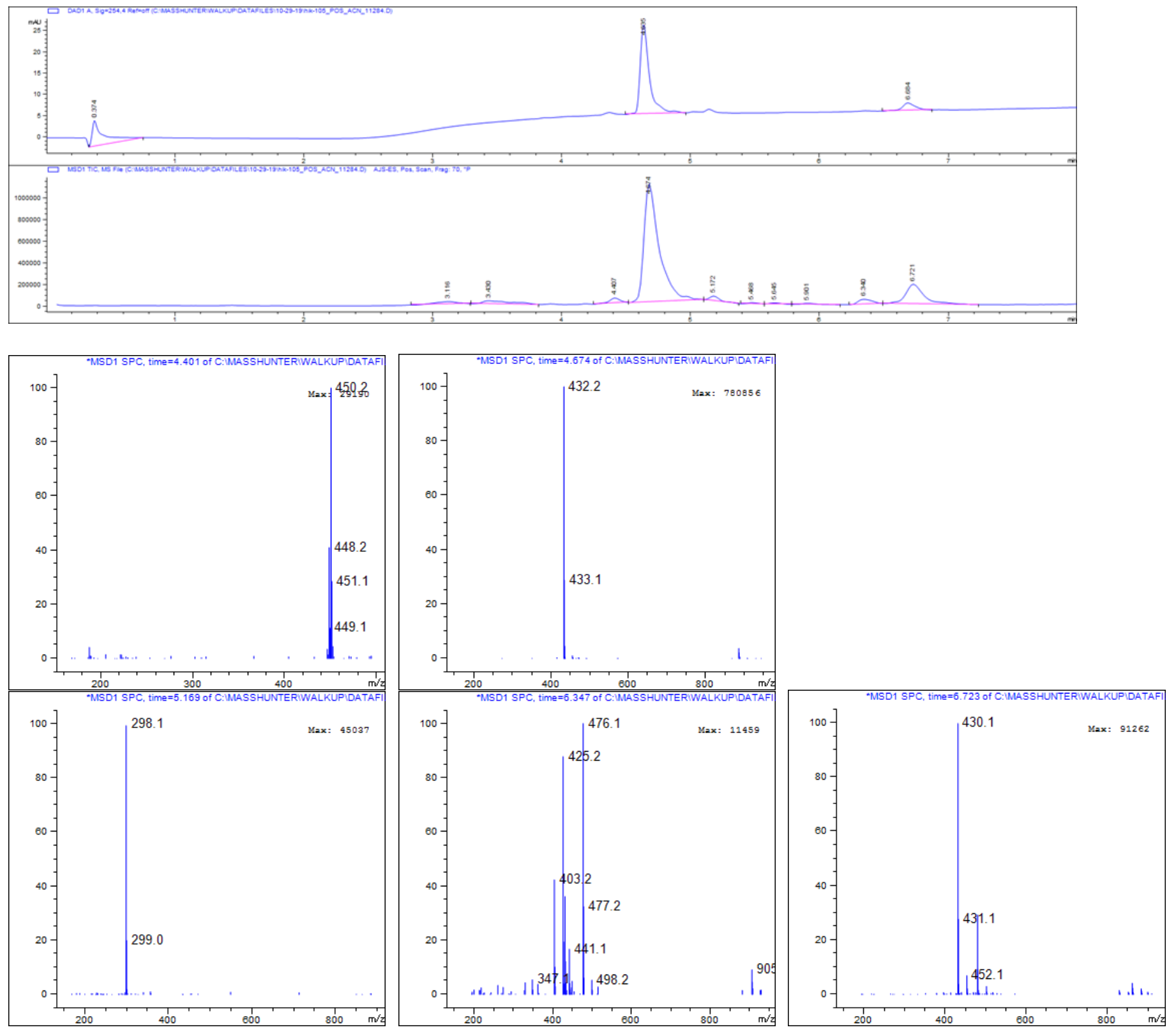
Hydrolysis products of hikarazine-108 $(\mathbf{2 5}\{1,24,42\})$

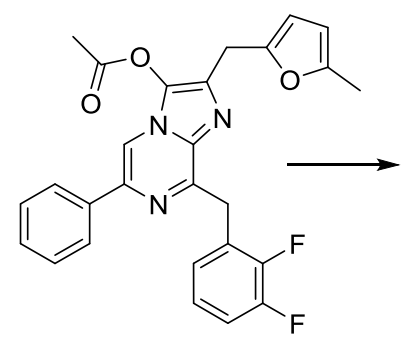

$M=473$

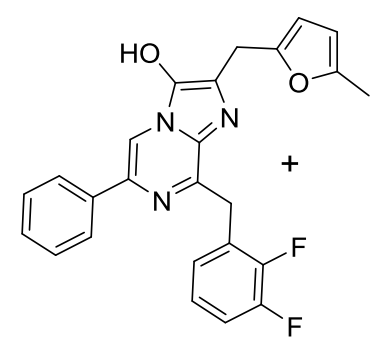

26dl ( $M=431)$

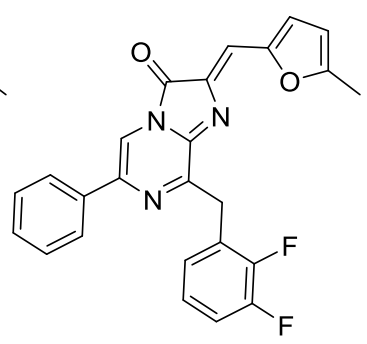

$M=429$

$t=0$
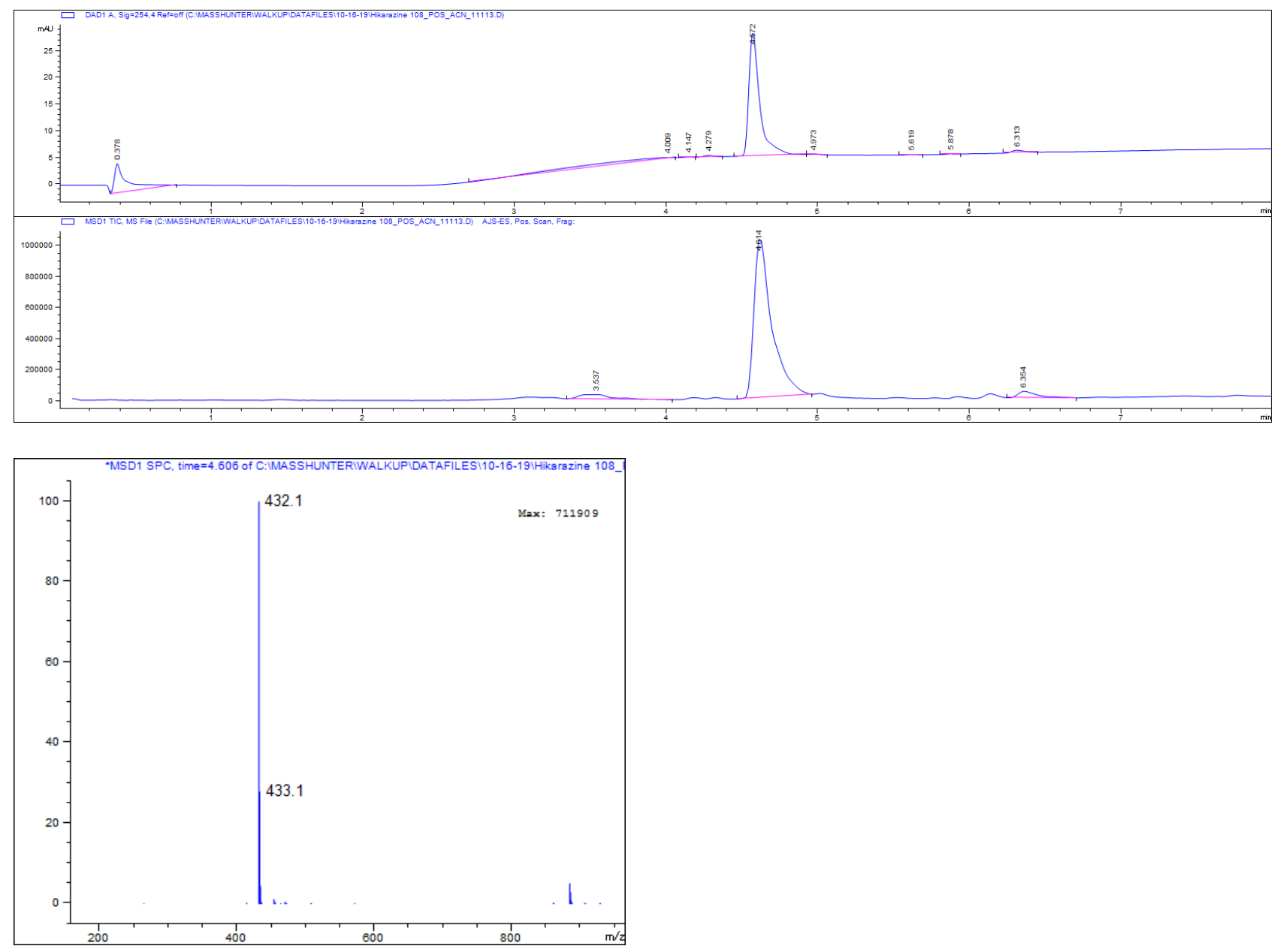

187 
$t=16 h$
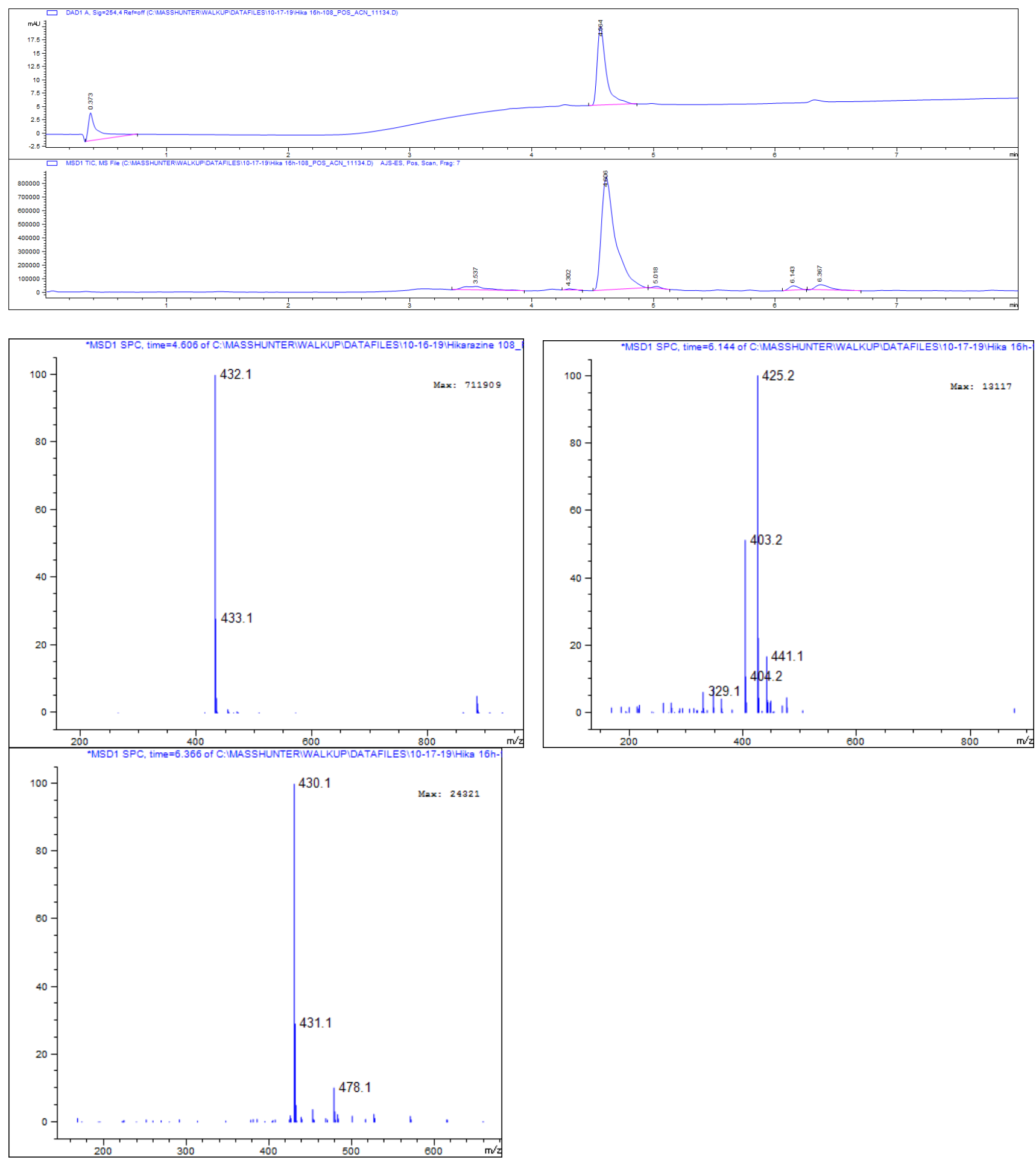


\section{$\mathrm{t}=14$ days}
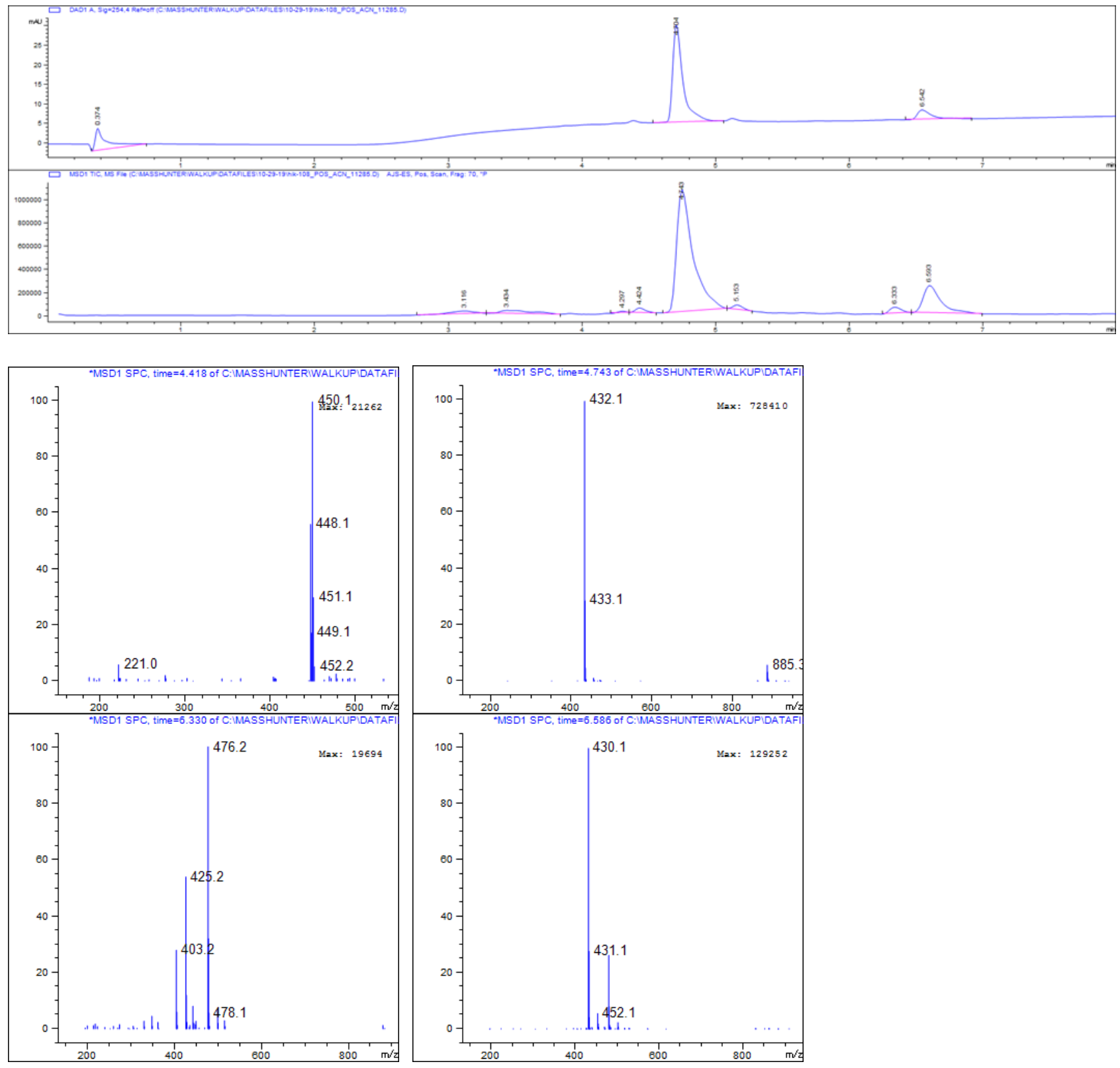
Hydrolysis products of hikarazine-113 $(\mathbf{2 5}\{1,22,42\})$

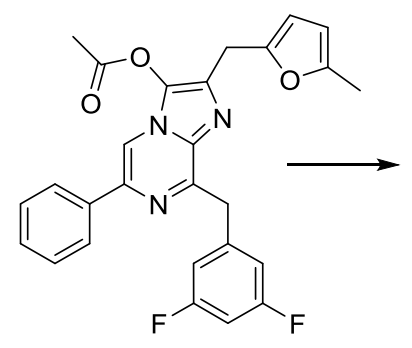

$M=473$

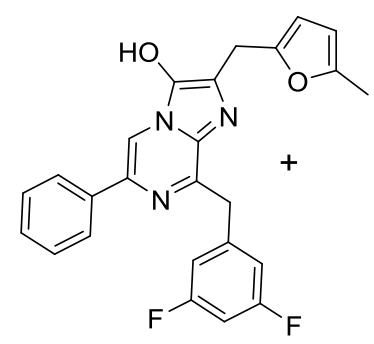

26dc $(M=431)$

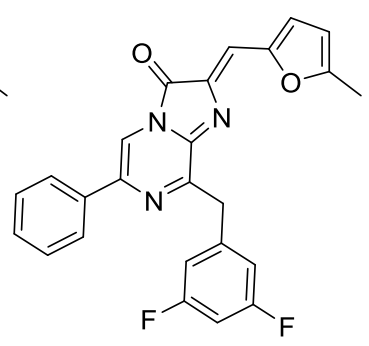

$M=429$

$t=0$
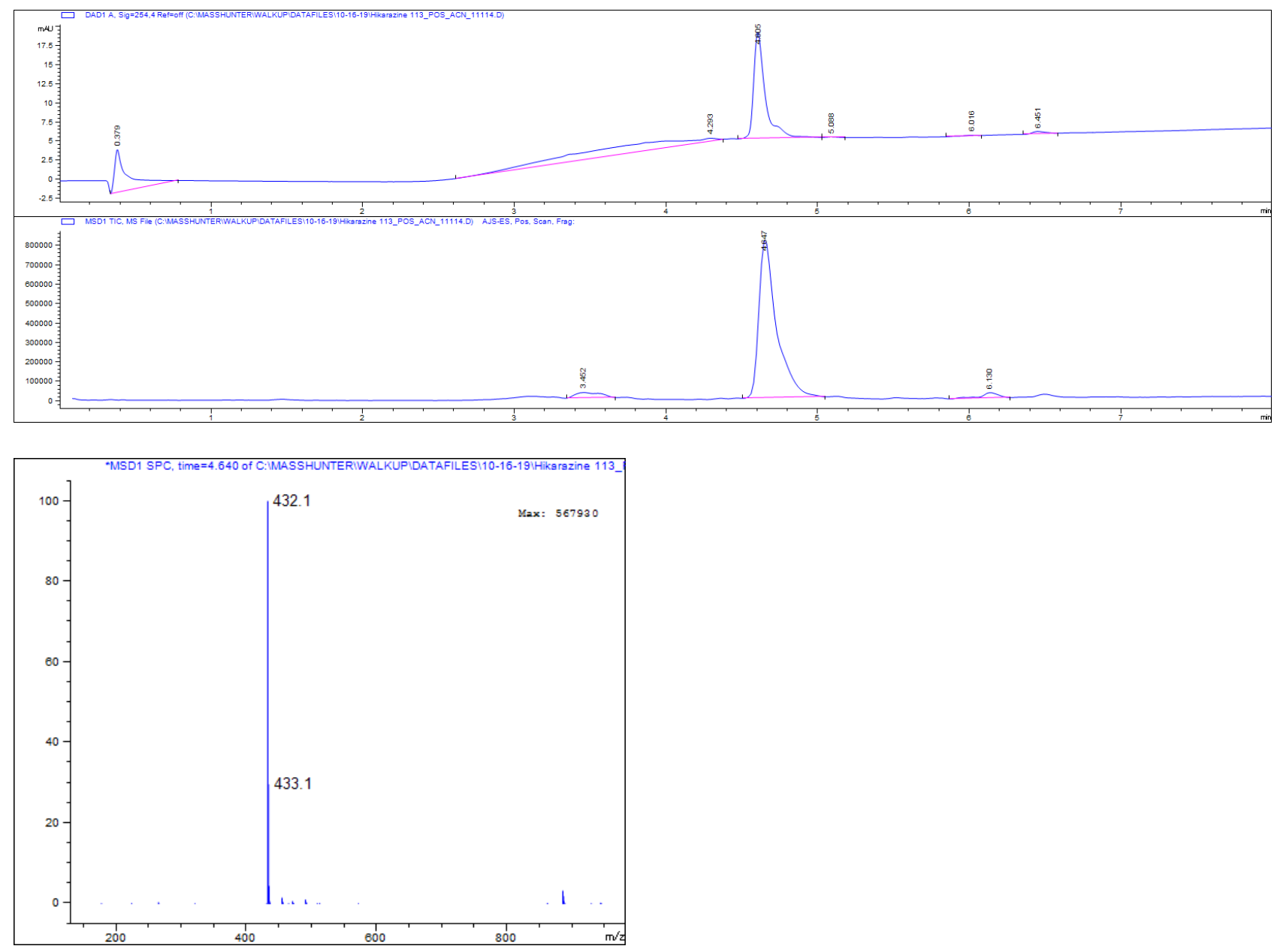

190 
$t=16 h$
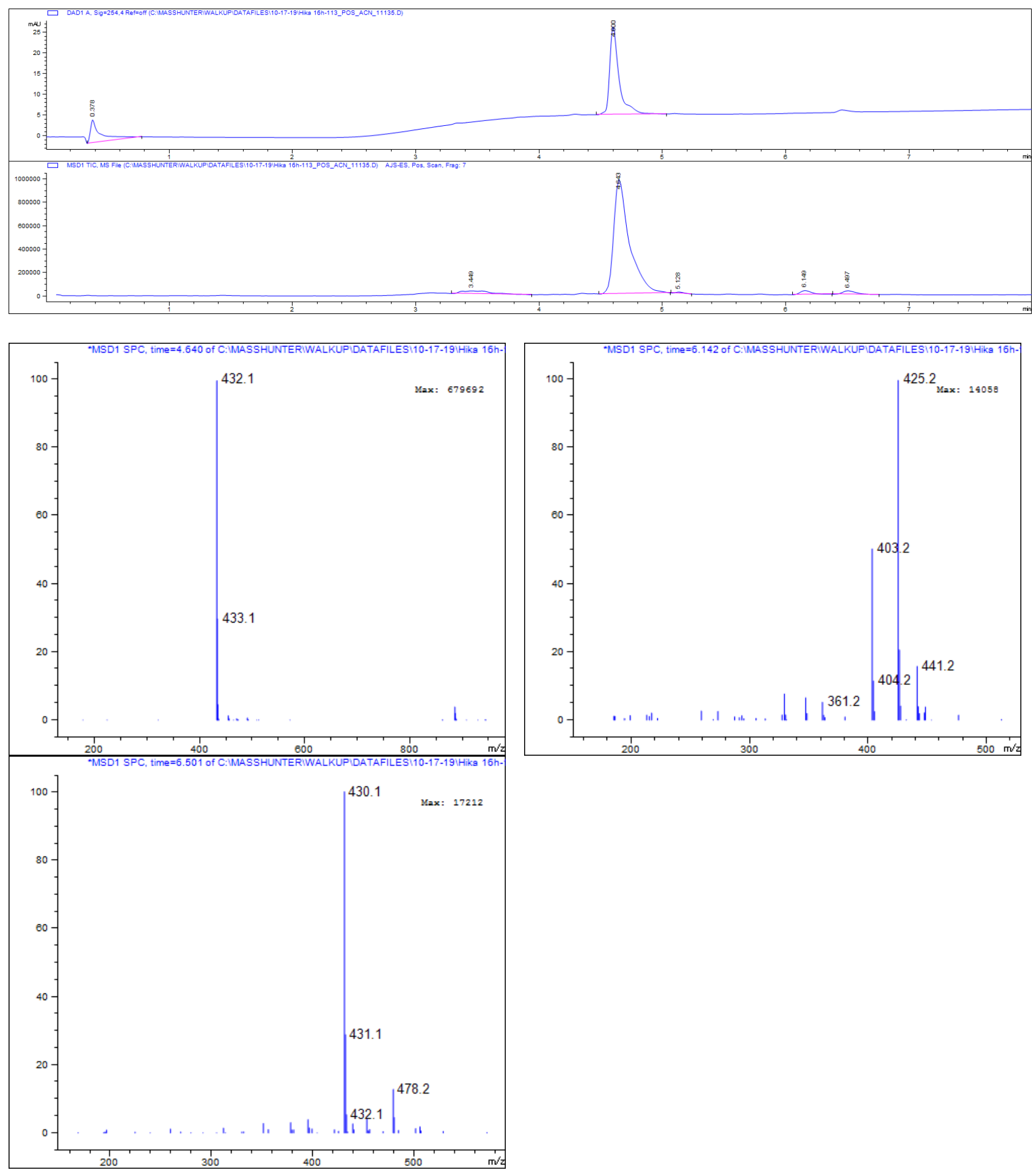


\section{$\mathrm{t}=14$ days}
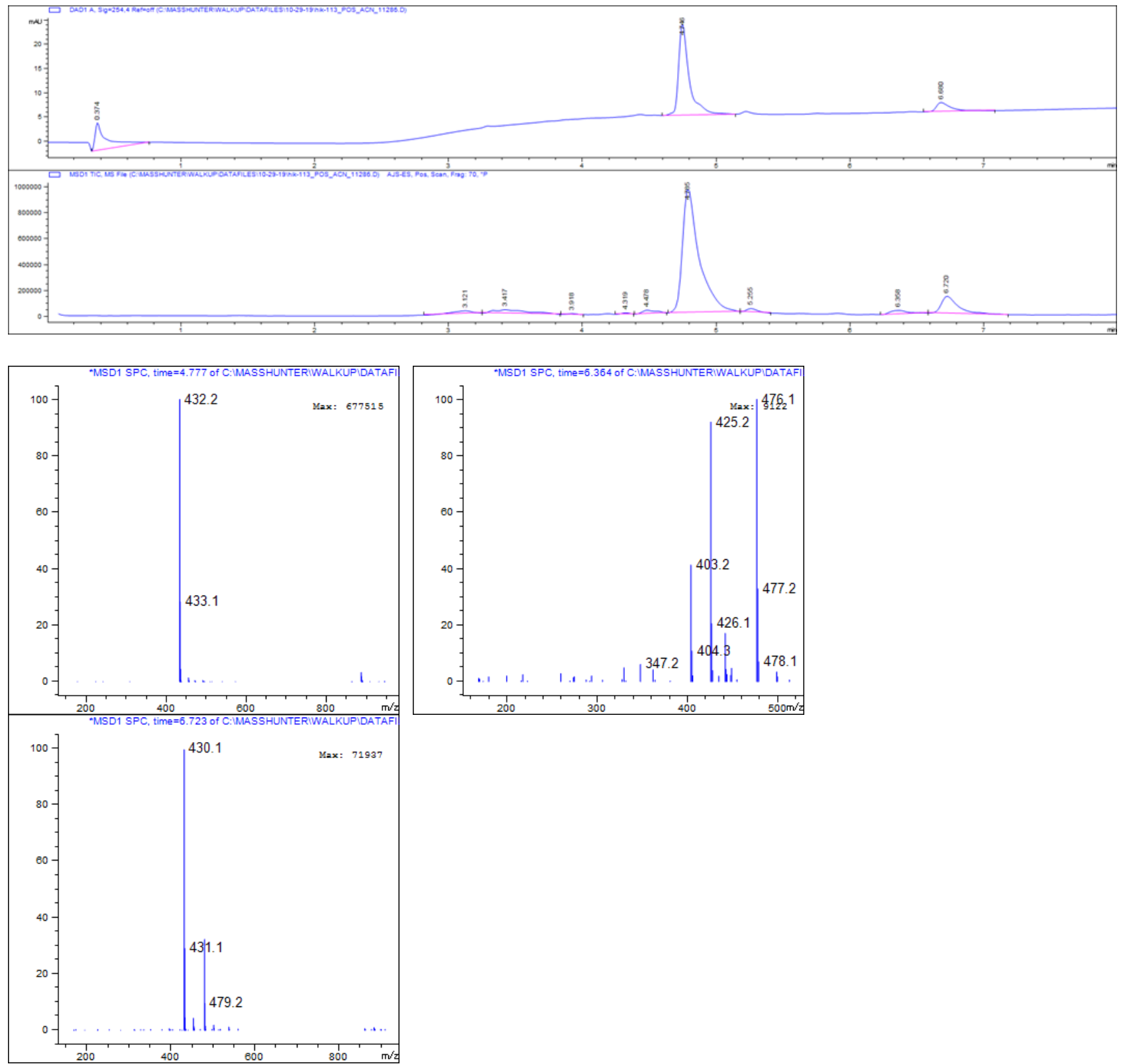
Hydrolysis products of hikarazine-149 (25 $\{13,1,37\})$<smiles>CC(=O)Oc1c(Cc2ccco2)nc2c(Cc3ccccc3)nc(-c3cccc(O)c3)cn12</smiles>

$M=439$
$\mathrm{HO}$<smiles>CC(O)=C(C)Cc1ccco1</smiles><smiles>C/C=C\Cc1nc2c(Cc3ccccc3)nc(-c3cccc(OC(C)=O)c3)cn2c1O</smiles>

$M=439$

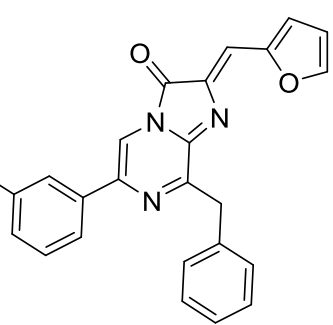

$M=395$

$\mathrm{t}=0$

26bh ( $M=397)$
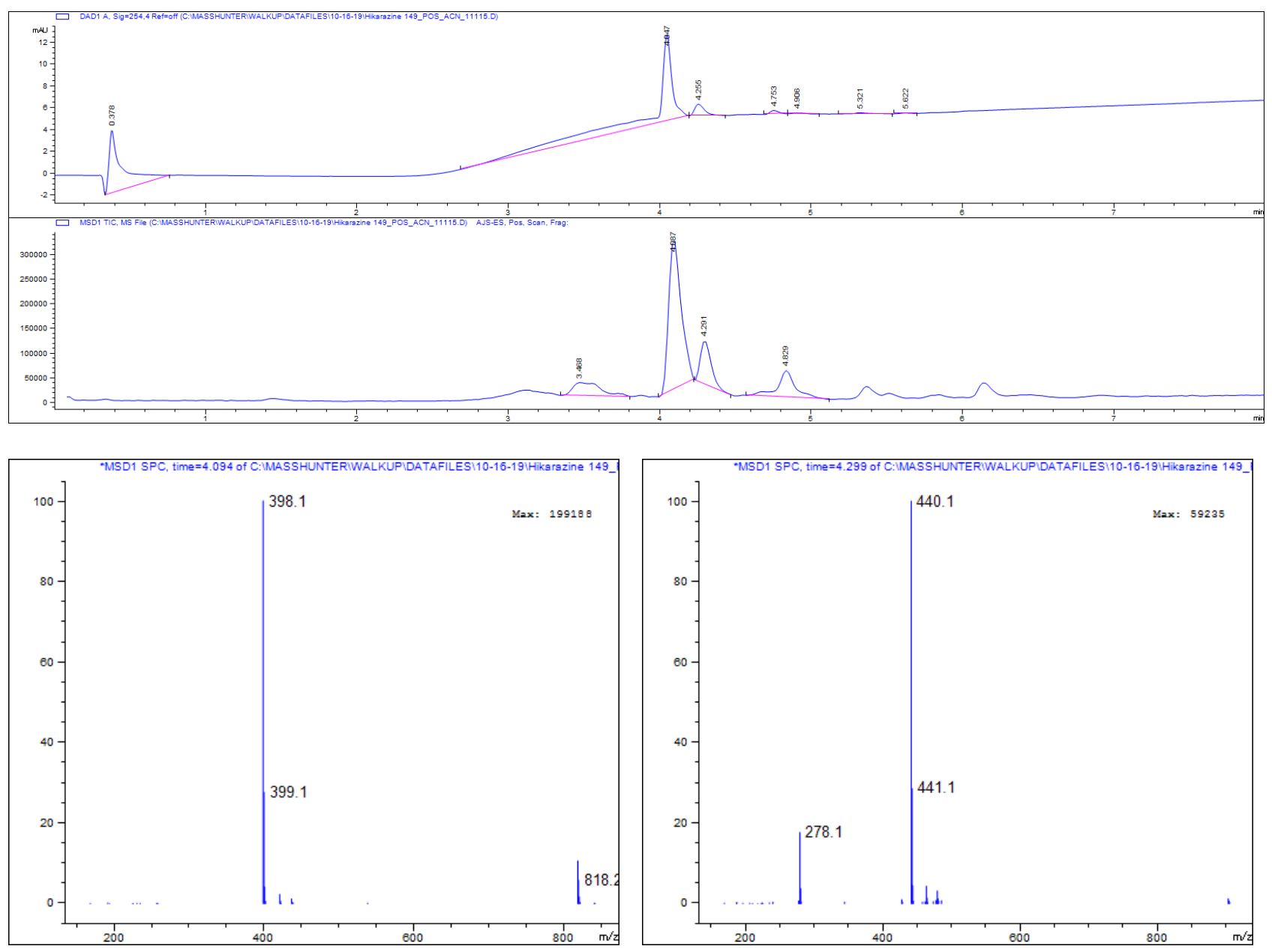
$t=16 h$
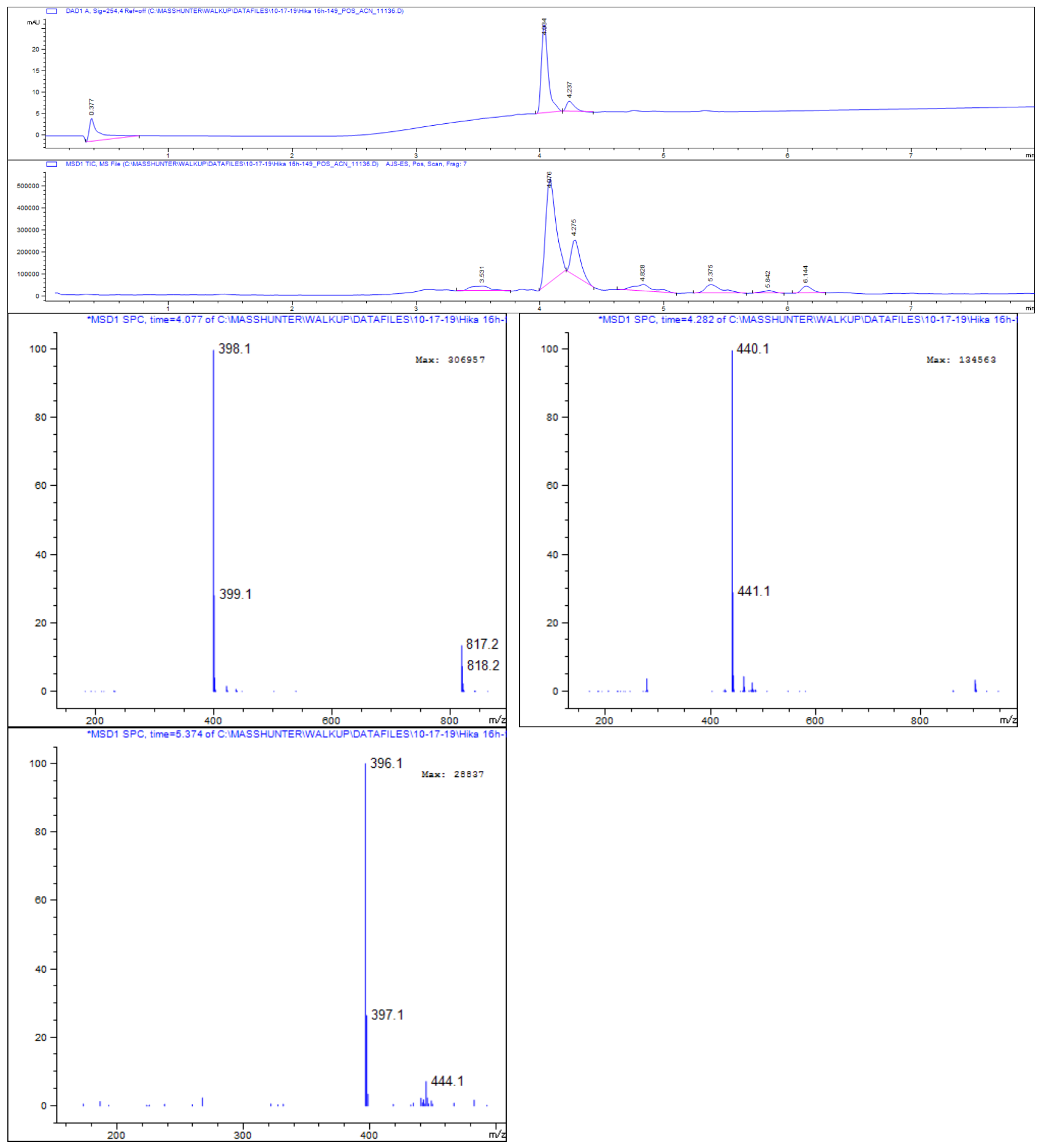


\section{$\mathrm{t}=14$ days}
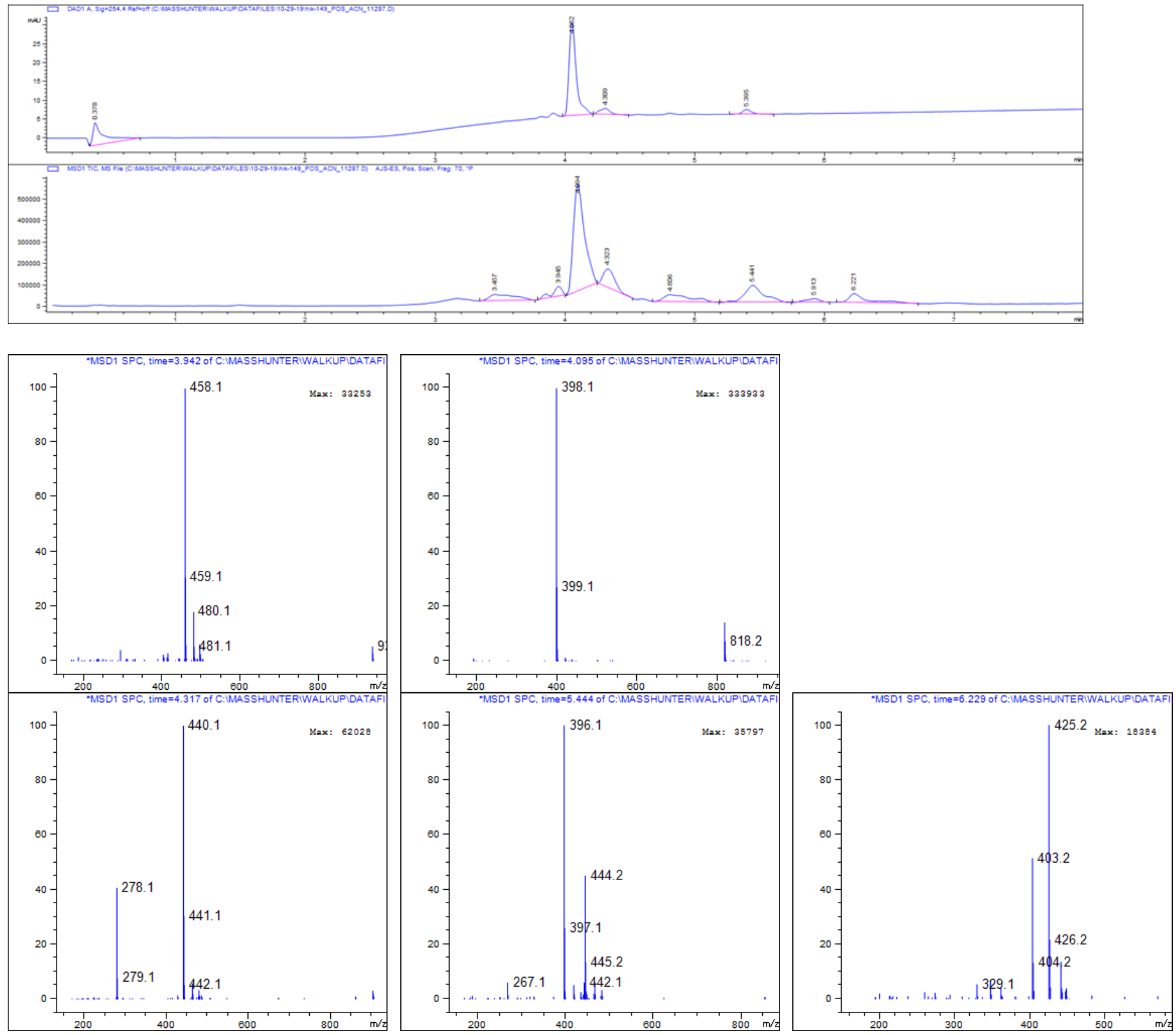
Hydrolysis products of hikarazine-150 (25 $\{13,1,63\})$

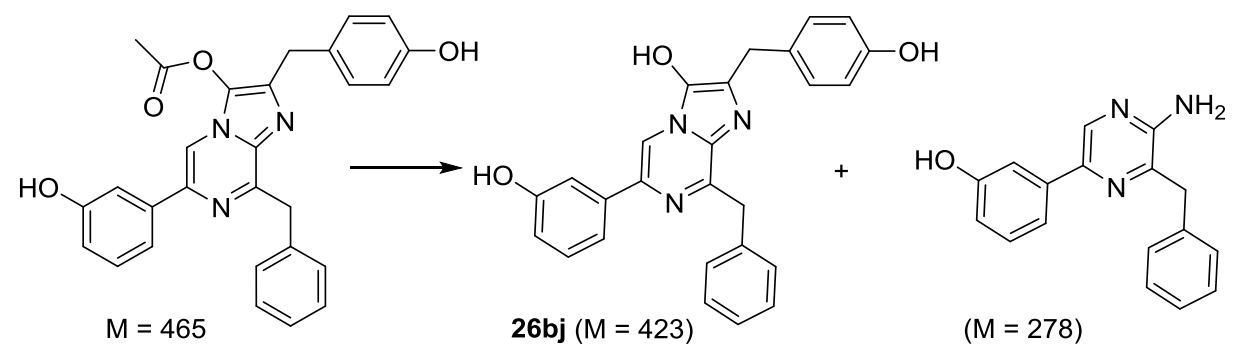

$\mathrm{t}=0$
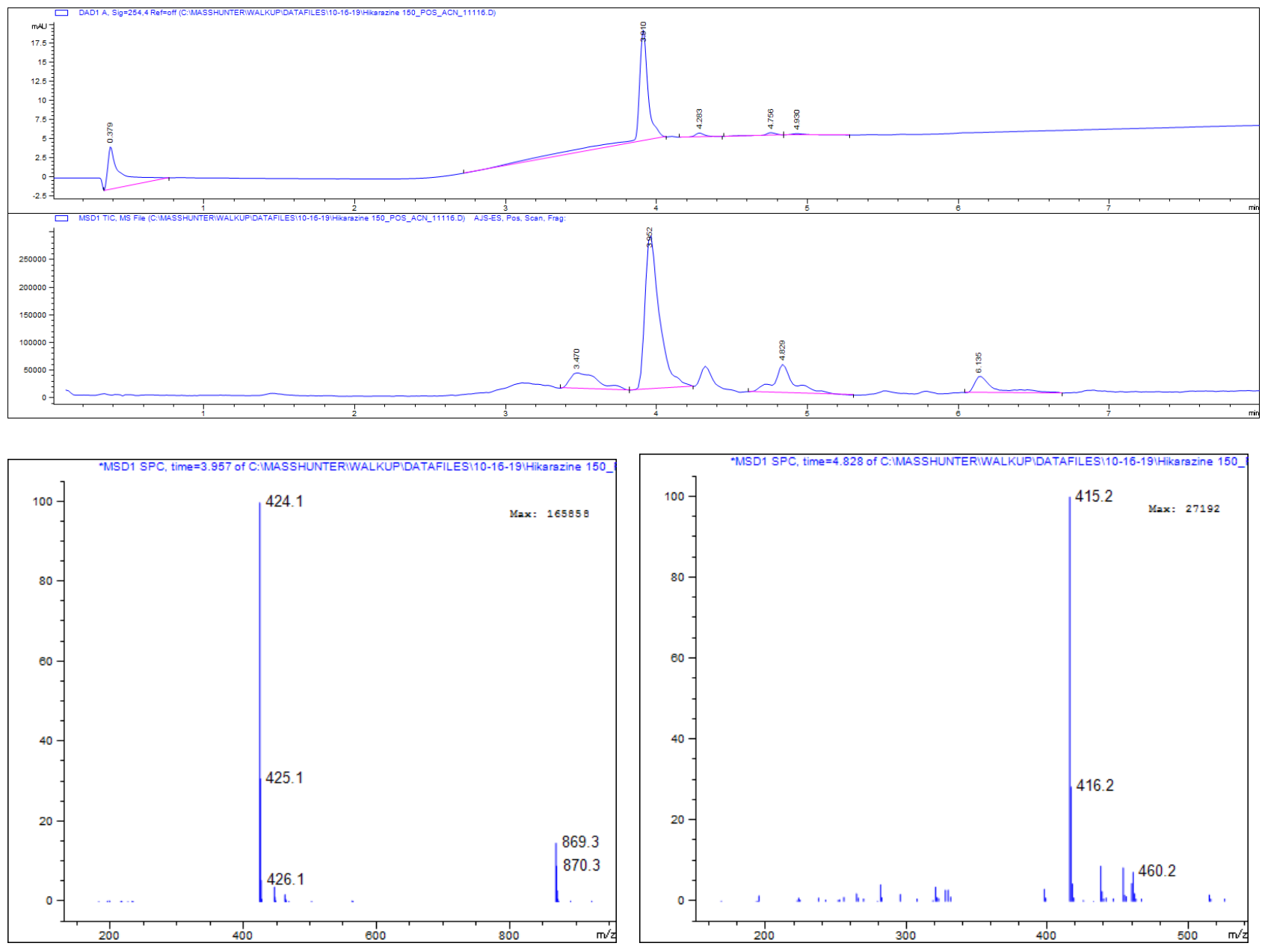
$t=16 h$
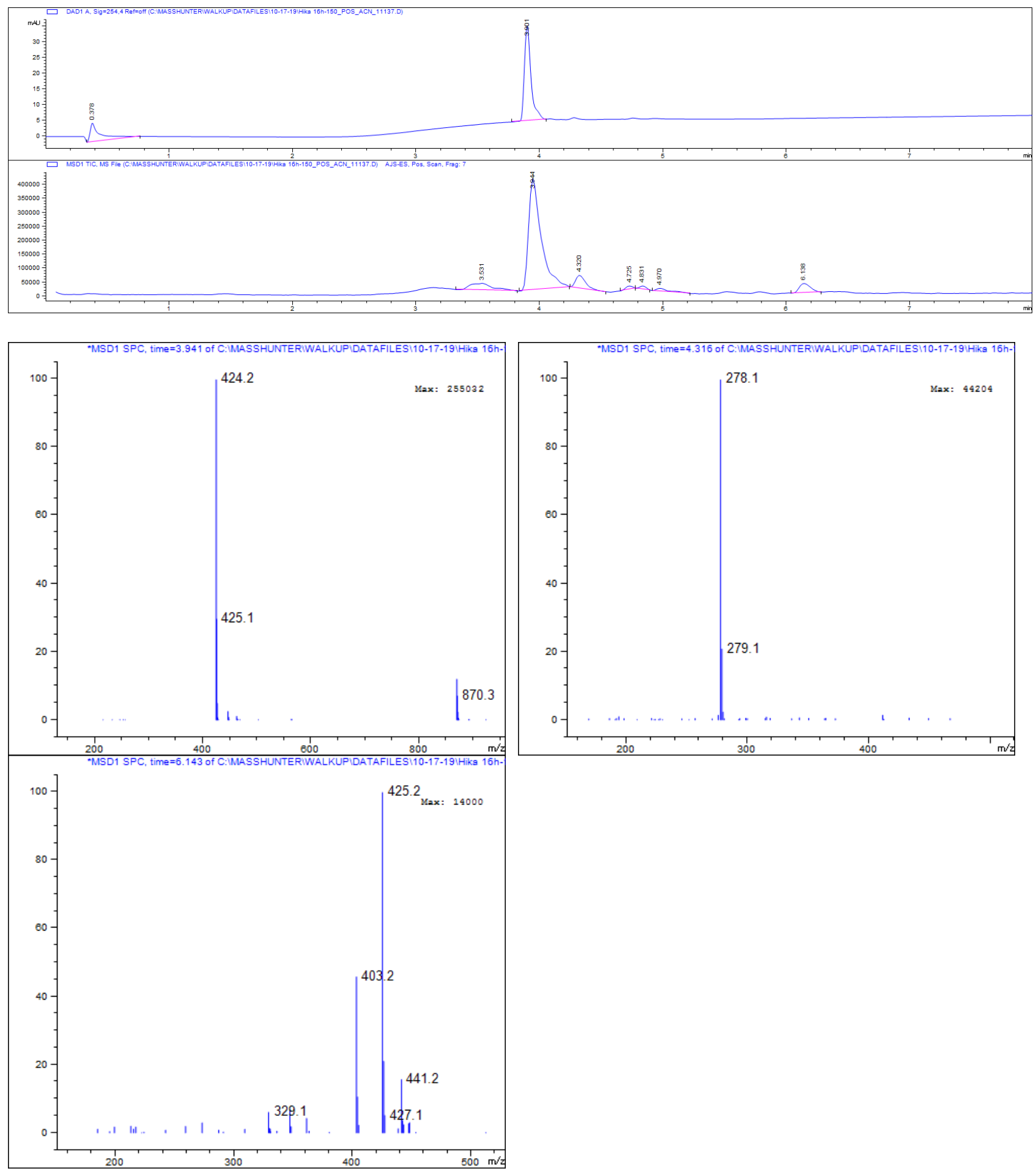


\section{$\mathrm{t}=14$ days}
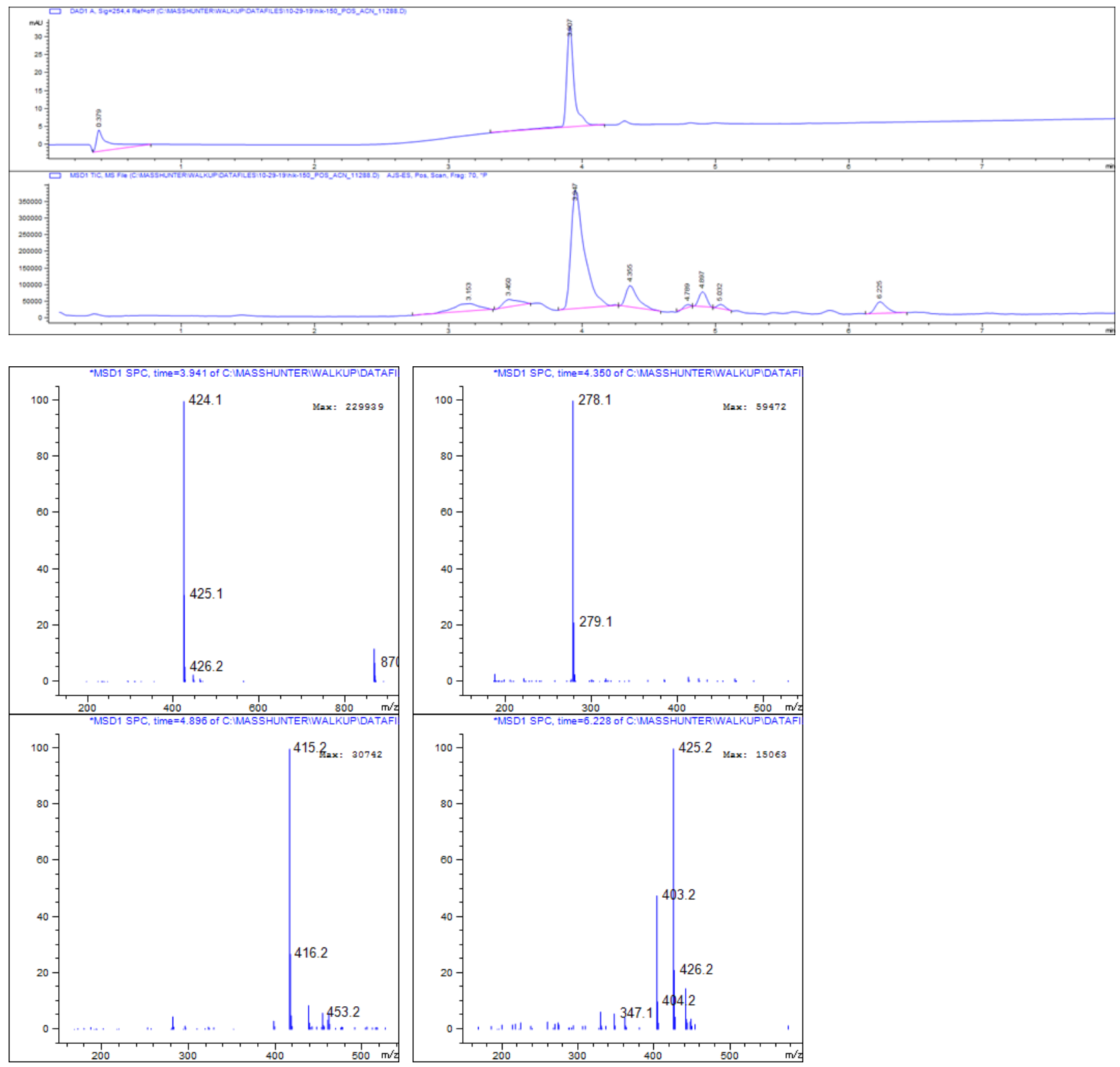
Hydrolysis products of hikarazine-162 (25 $\{14,1,42\})$<smiles>[Y16]C=Cc1nc(-c2ccc(O)cc2)cn2c(OC(C)=O)c(Cc3ccccc3)nc12</smiles><smiles>C=C(O)Cc1nc2c(Cc3ccccc3)nc(-c3ccc(O)cc3)cn2c1O</smiles><smiles>C/C=C(/O)c1ccc(O)cc1</smiles><smiles>CC(C)=CC1=NC(C(=N)Cc2ccccc2)NC1=O</smiles><smiles>CC(=O)Oc1ccc(C2=CN3C(=O)C(=Cc4ccc(C)o4)N=C3C(Cc3ccccc3)=N2)cc1</smiles>

$t=0$
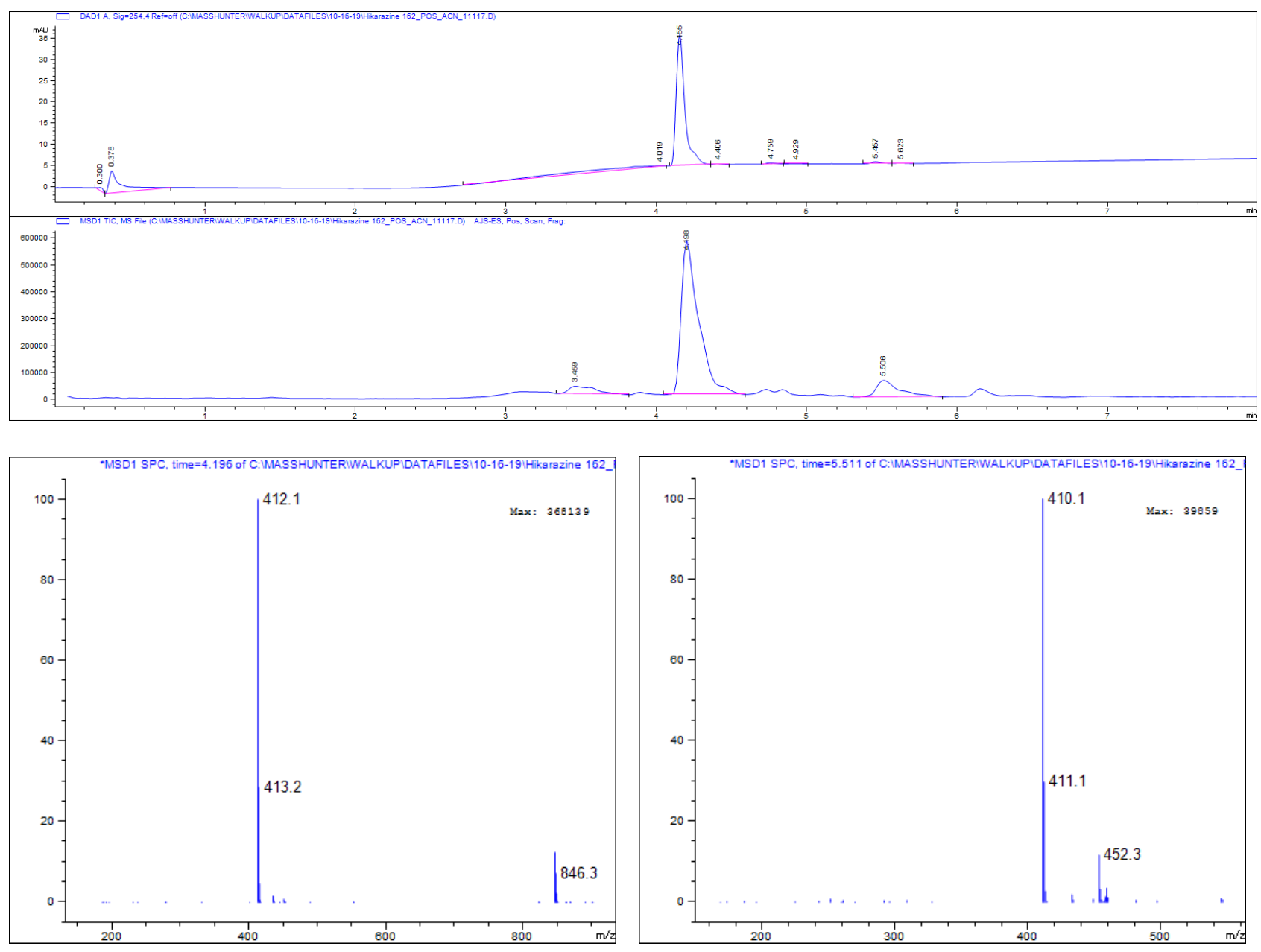
$t=16 h$
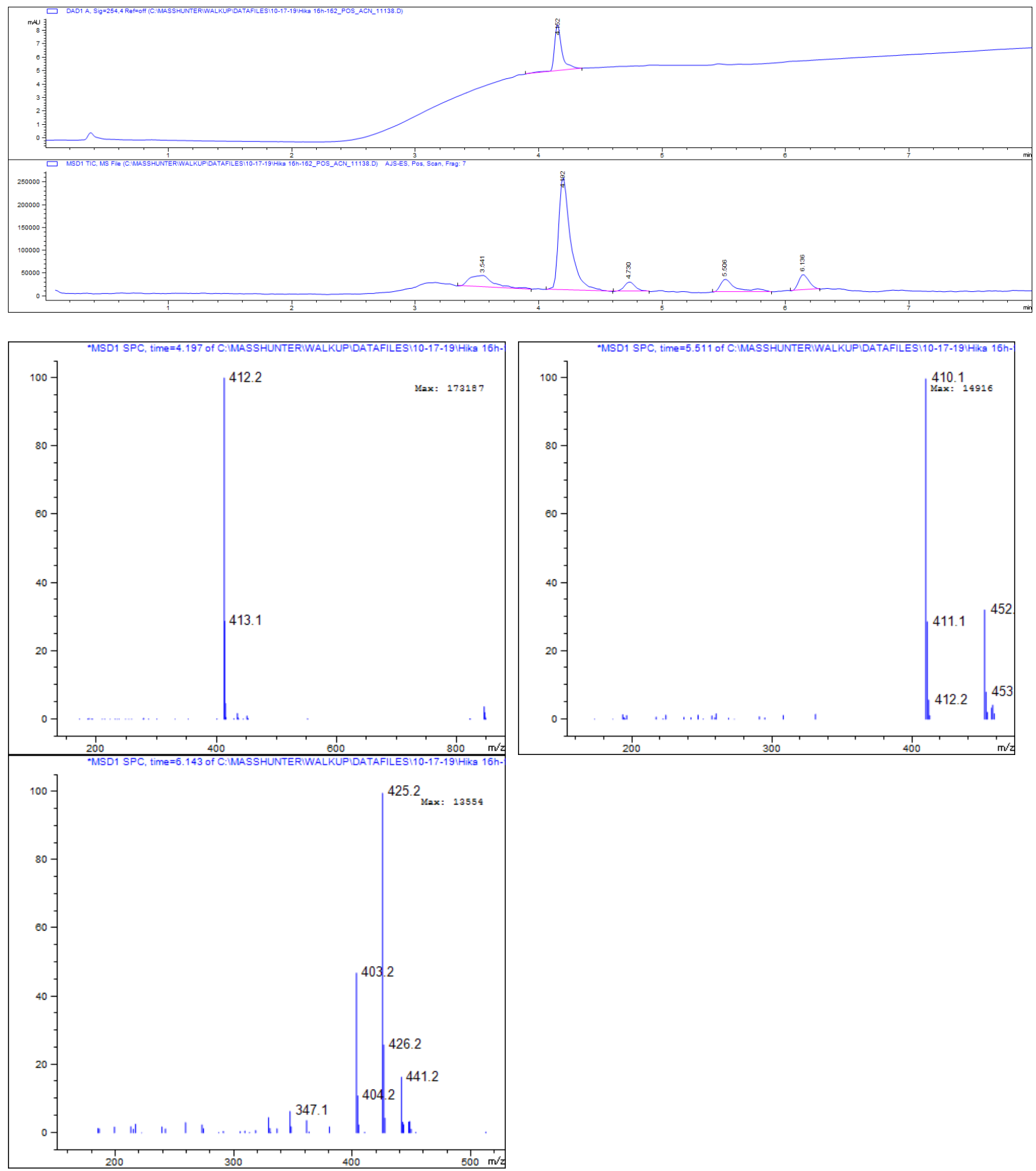


\section{$\mathrm{t}=14$ days}
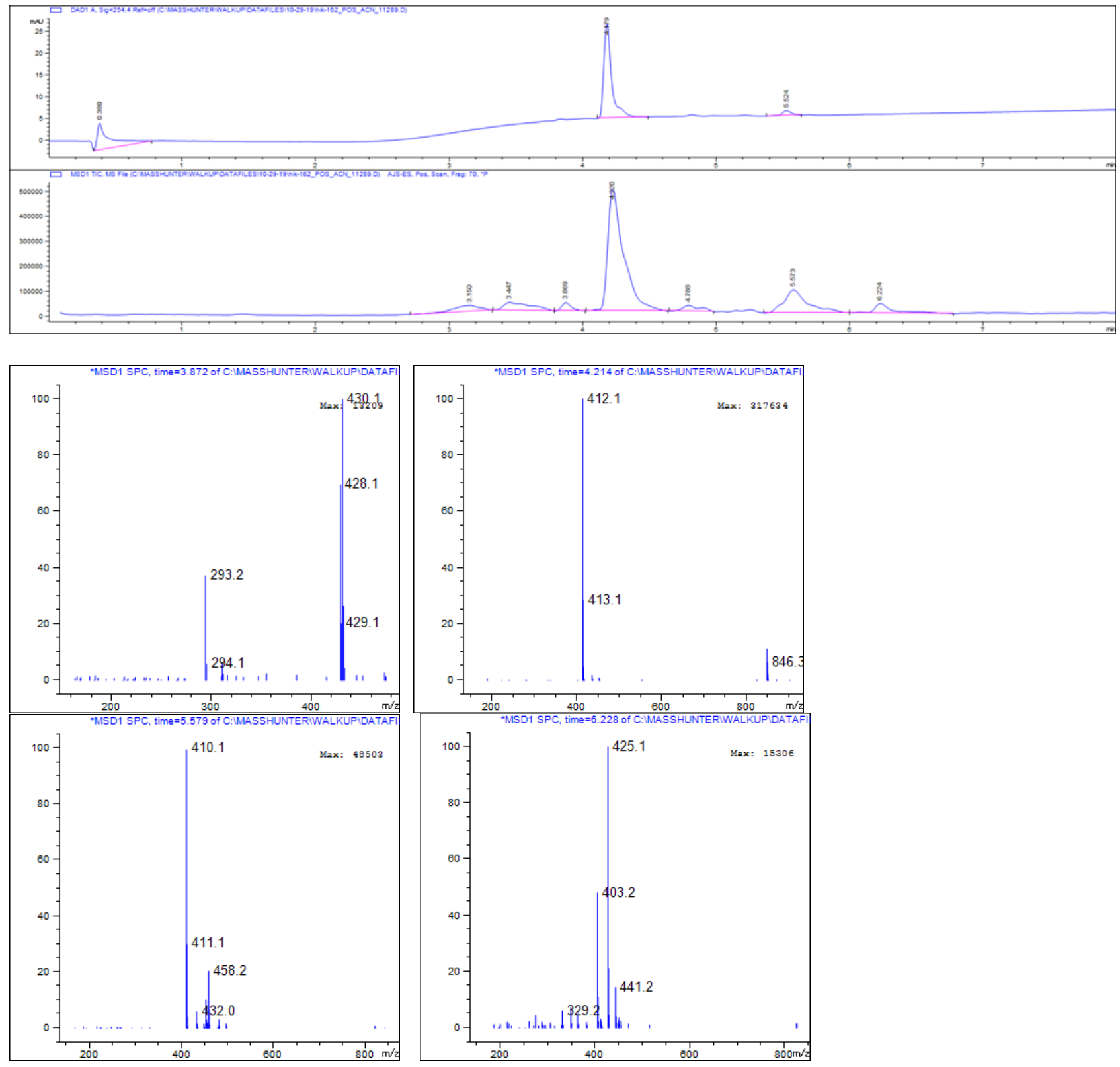


\section{Biology}

Recombinant nanoKAZ/NanoLuc was produced as described before. ${ }^{[4]}$

\section{Bioluminescence assays}

\section{- Protocol for the results described in tables 1-7 (a Berthold CentroXS3 luminometer was used for these experiments).}

Buffer A: Dulbecco's Phosphate-Buffer Saline (DPBS) 1X, no calcium, no magnesium + 0.1\%v/v Tween® 20 .

1. Preparation of 96-well daughter plates and deprotection of $\mathrm{O}$-acetylated luciferins $\mathbf{3 5}$

- Move 96-well mother plates containing $10 \mathrm{mM}$ stock solutions of preluciferins in DMSO from $20{ }^{\circ} \mathrm{C}$ to room temperature.

- Pipette $5 \mu \mathrm{L}$ from mother plates into wells of polypropylene $\mathrm{V}$-shaped 96-well daughter plates.

- Pipette $5 \mu \mathrm{L}$ from a stock $0.84 \% \mathrm{v} / \mathrm{v}$ ethanolic solution of $37 \%$ hydrochloric acid (leading to a $0.1 \mathrm{M} \mathrm{HCl}$ concentration) into wells of daughter plates.

- Thermo-seal daughter plates, centrifuge 60 seconds at $2000 \mathrm{rpm}$, and store overnight at room temperature to allow deprotection of preluciferins.

- Store daughter plates at $-20{ }^{\circ} \mathrm{C}$ until use. Note: the luciferins being intrinsically unstable, it is advised to immediately use daughter plates, or to minimize as much as possible the storage duration.

2. Dilutions for bioluminescence assays

- Move prepared 96-well daughter plates containing $5 \mathrm{mM}$ solutions of luciferins in DMSO/ethanol/HCl from -20 ${ }^{\circ} \mathrm{C}$ to room temperature.

- Dilute luciferins 1:20 by adding $190 \mu \mathrm{L}$ of Buffer A into wells and mixing, leading to $250 \mu \mathrm{M}$ solutions of luciferins.

- Pipette $10 \mu \mathrm{L}$ of the $250 \mu \mathrm{M}$ solutions of luciferins into white polystyrene flat-bottomed 96-well plates (e.g. Greiner Lumitrac ${ }^{\circledR} 200$ ). This plate P1 will be used for determination of the autoluminescence of luciferins in Buffer A.

- Pipette $10 \mu \mathrm{L}$ of the $250 \mu \mathrm{M}$ solutions of luciferins into white polystyrene flat-bottomed 96-well plates (e.g. Greiner Lumitrac ${ }^{\circledR} 200$ ). This plate P2 will be used for determination of the luminescence of luciferins with the desired luciferase.

3. Bioluminescence Assays

- For autoluminescence measurements, dilute luciferins 1:10 adding $90 \mu \mathrm{L}$ of Buffer A into wells of P1 and mixing.

- Measure autoluminescence signal over 5 minutes, integrating $1 \mathrm{~s} /$ well.

- For luminescence measurements, dilute luciferins 1:10 adding $90 \mu \mathrm{L}$ of enzyme solution into wells of P2.

Note: in our case, the nanoKAZ solution (at $50 \mathrm{ng} / \mathrm{L}$ ) was prepared diluting 1:2.000.000 adding $2 \mu \mathrm{L} \mathrm{of} \mathrm{a} 0.1 \mathrm{~g} / \mathrm{L}$ solution in $198 \mu \mathrm{L}$ Buffer A, mixing, then adding $2 \mu \mathrm{L}$ of this $1 \mathrm{mg} / \mathrm{L}$ solution in $198 \mu \mathrm{L}$ Buffer $\mathrm{A}$, mixing, then adding $100 \mu \mathrm{L}$ of this $10 \mu \mathrm{g} / \mathrm{L}$ solution in $19.9 \mathrm{~mL}$ Buffer A.

- Shake 10 seconds, then measure luminescence signal over 2 hours, integrating $0.5 \mathrm{~s} /$ well.

The bioluminescence assays were carried out two times, starting from the same mother plate. Data were normalized to multiple wells containing furimazine, then averaged over the two runs. The final concentrations were $25 \mu \mathrm{M}$ for each substrate and $45 \mathrm{ng} / \mathrm{L}$ for the nanoKAZ luciferase.

Given the design of the experiment, the first measurement of luminescence occurred between 30 seconds and 1 minute after addition of the luciferase; maximum intensities can thus be under-estimated for extremely fastdecaying substrates. For most of the substrates, however, the maximum of intensity was reached after 2 to 4 minutes. 


\section{Kinetics analysis described in table 8.}

In these experiments and as described before, ${ }^{5}$ a Berthold Centro LB 960 luminometer was used. In order to get the maximum intensities for extremely fast-decaying substrates, the kinetics were acquired individually for each of substrates for 4 minutes, starting 1 second after the direct injection of the enzyme in the well of the plate already inside the plate reader and a one second period of shaking.

The apparent reaction rate ( $\left.\mathrm{v}^{\prime}\right)$ is given by the measured light intensity (RLU/s) reflecting counted photons/s, depending on the real catalytic rate $\left(k_{c a t}\right)$ and the yield of the measurement $(\rho$, the number of molecules catalyzed per collected RLU). The kinetics have been fitted with Michaelis-Menten model drawn below considering 1) the luciferins ( $\mathrm{S})$ as the limiting substrates and $\mathrm{O}_{2}$ as saturating substrate in the experimental assay conditions $(100 \mu \mathrm{L})$ in a $6 \mathrm{~mm}$-diameter well of multi-well plates, 2) the inhibition of the enzyme E by excess of substrate through the binding of a second substrate (ESS) on the Michaelis' complex (ES) with the dissociation constant $K_{\mathrm{I}}$ and the on $\left(k_{S S, o n}\right)$ and off $\left(k_{S S, o f f}\right)$ binding constants, and 3) the stochastic inactivation of the enzyme (E*) along the reaction turn over decreasing exponentially the active enzyme population with the kinetic constant $k_{\text {inact }} . K^{\prime}{ }_{M}$ is the apparent constant of Michaelis and $\mathrm{V}^{\prime}{ }_{\text {max }}$ the apparent maximal reaction velocity.

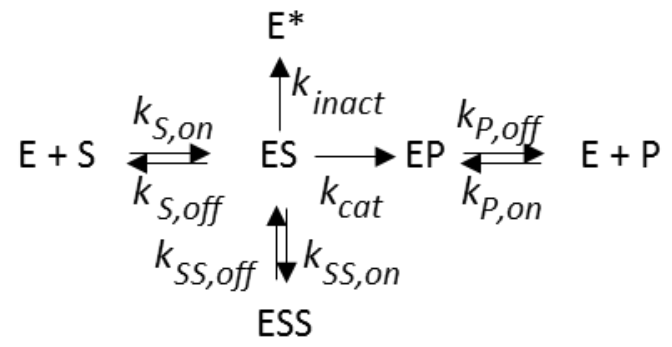

with

$\mathrm{d}[\mathrm{ES}] / \mathrm{dt}=[\mathrm{E}][\mathrm{S}] k_{S, \text { on }}-[\mathrm{ES}] k_{S, \text { off }}-[\mathrm{ES}] k_{\text {cat }}-[\mathrm{ES}][\mathrm{S}] k_{S S, \text { on }}+[\mathrm{ESS}] k_{S S, \text { off }}-[\mathrm{ES}] k_{\text {inact }}$

$K_{\mathrm{I}}=k_{S S, \text { off }} / k_{S S, \text { on }}$ in $\mathrm{M}$

$K_{\mathrm{M}}=\left(k_{S, \text { off }}+k_{\text {cat }}\right) / k_{S, \text { on }}=K_{\mathrm{M}}{ }^{\prime} /\left(1+[\mathrm{S}]^{2} / K_{\mathrm{I}}\right)$ expressed in $\mathrm{M}$

$\mathrm{V}_{\max }=\left[\mathrm{E}_{0}\right][\mathrm{S}] k_{\text {cat }}=\mathrm{V}^{\prime}{ }_{\text {max }}\left([\mathrm{S}]+K_{\mathrm{M}}+\left([\mathrm{S}]^{2} / K_{\mathrm{I}}\right)\right) /[\mathrm{S}]$ expressed in $\mathrm{mol} / \mathrm{s}$

$\mathrm{v}^{\prime}=[\mathrm{E}][\mathrm{S}] k_{\text {cat }} /\left([\mathrm{S}]+K_{\mathrm{M}}+\left([\mathrm{S}]^{2} / K_{\mathrm{I}}\right)\right)$ expressed in RLU/s

$[\mathrm{E}]=\left[\mathrm{E}_{0}\right] \mathrm{e}^{-\mathrm{t} \cdot \text { kinact }}$ with $k_{\text {inact }}$ expressed in $\mathrm{s}^{-1}$ and the time $t$ in $\mathrm{s}$.

$\mathrm{v}=\mathrm{v}, \rho$ in $\mathrm{mol} / \mathrm{s}$

$\rho=[\mathrm{S}] N_{a} \mathrm{vol} / \Sigma$ I in molecules/RLU with $N_{a}=6.02 \cdot 10^{23}$ and the sample volume vol in L 
As an illustration of the inhibition of reaction by an excesses of substrates, the two figures below are providing the curves obtained (in plain) for compounds $\mathbf{5}$ and $\mathbf{2 6} \mathbf{d l}$ as well as the fitting with calculated curves taking in account (or not) this inhibition. Time frame of these experiences was of four minutes.

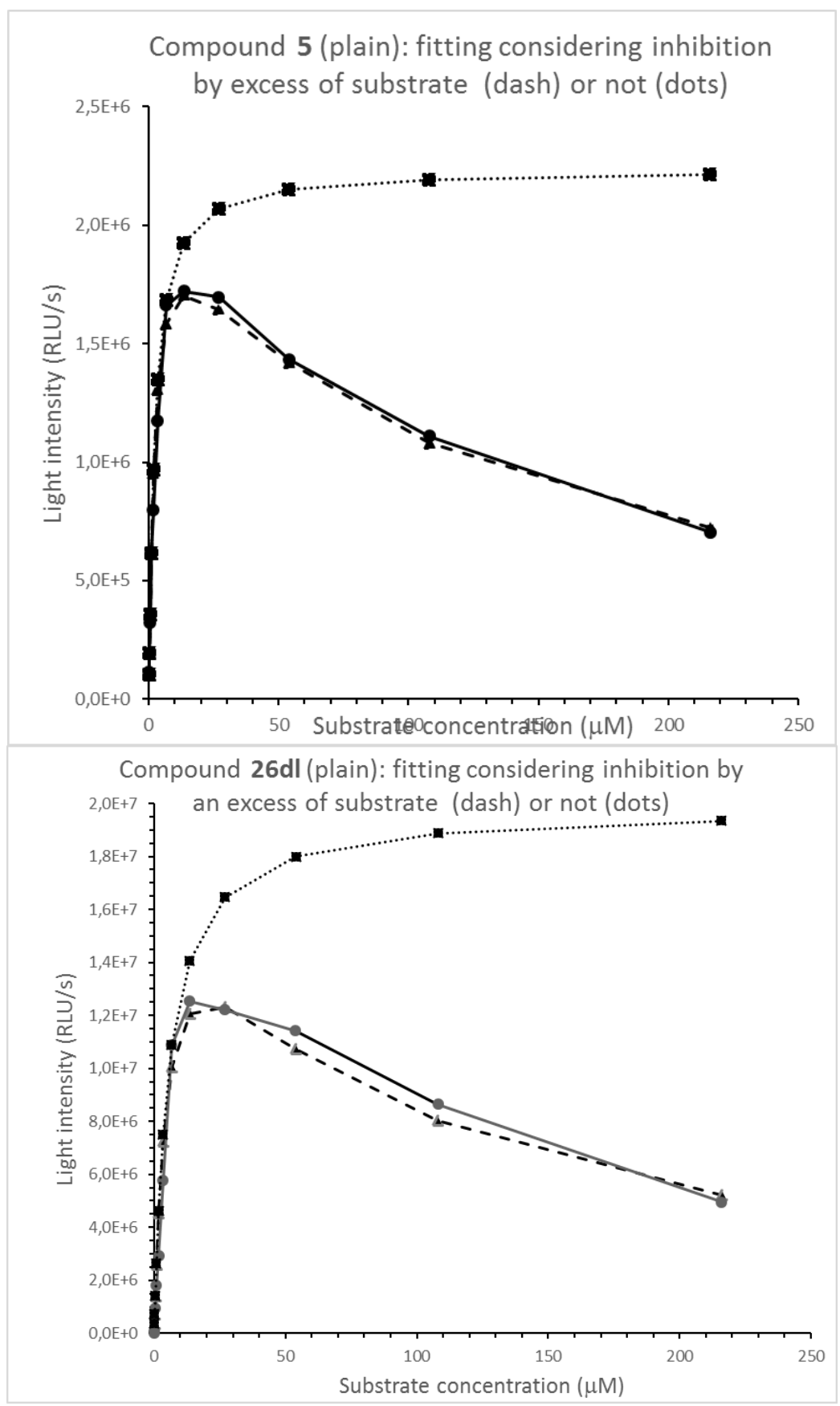


As an illustration of the irreversible inhibition over time, the two following figure are depicting the kinetics (plain) and their fit (dash), again for compound $\mathbf{5}$ and 26dl, when taking account an enzyme inactivation. The first point was measured after one second.
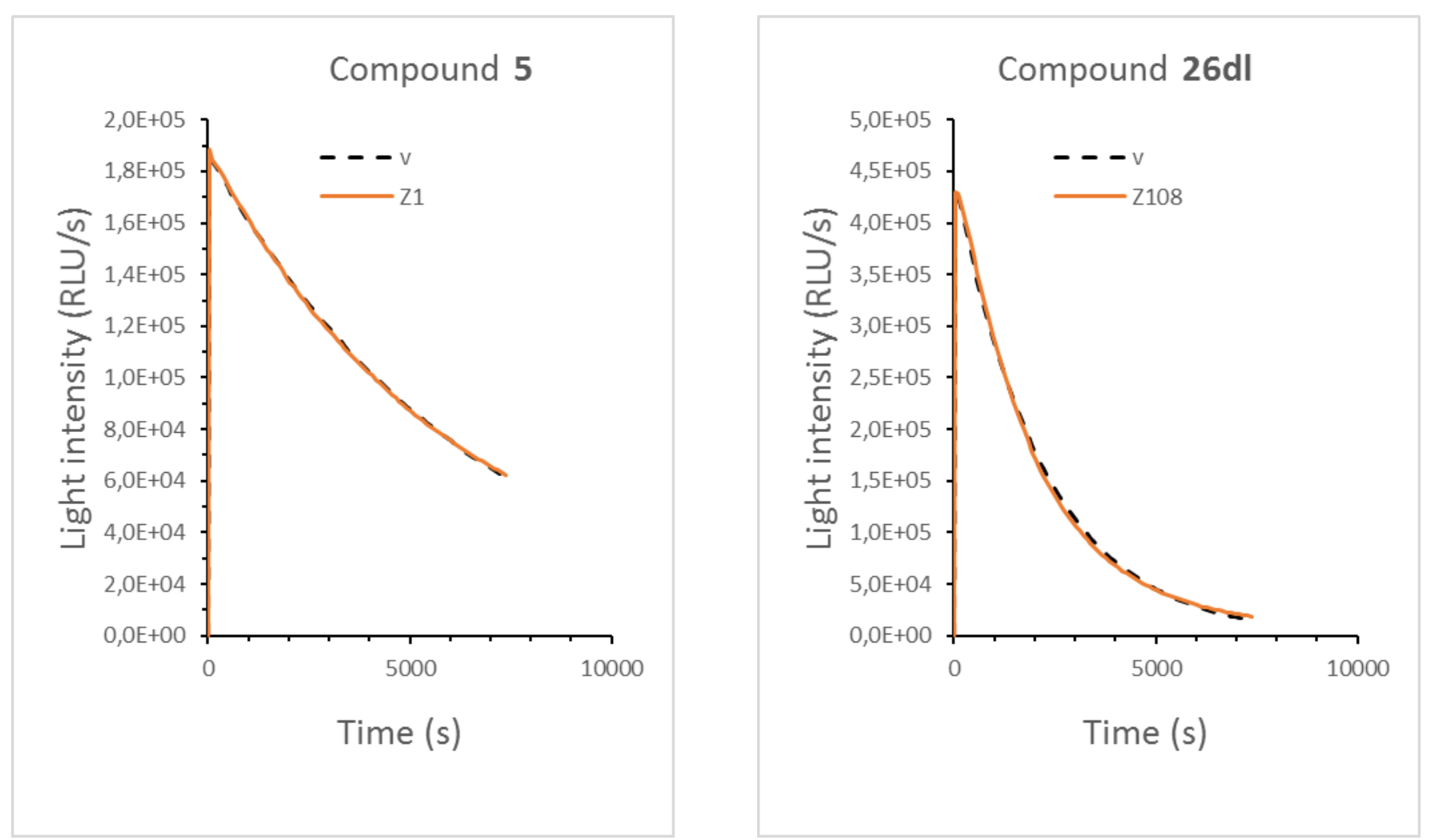

Enzyme inactivation kinetics. The kinetics are plotted versus time with the same enzyme concentration and the same substrate concentration. These plots compare the fitting of the experimental data (red plain line) with the theoretical reaction velocity computed according to the described Michaelis-Menten model taking into consideration the irreversible inactivation of the enzyme (dashed black lines). 
Finally, as another illustration of this enzyme death over time, we provide in the following the signal profile over time for compound $\mathbf{5}$ and for the flashy compound $\mathbf{2 6 b f}$ (which we have already published ${ }^{[4]}$ ). Each of the intensity increases marks the addition of fresh enzyme in the reaction media.
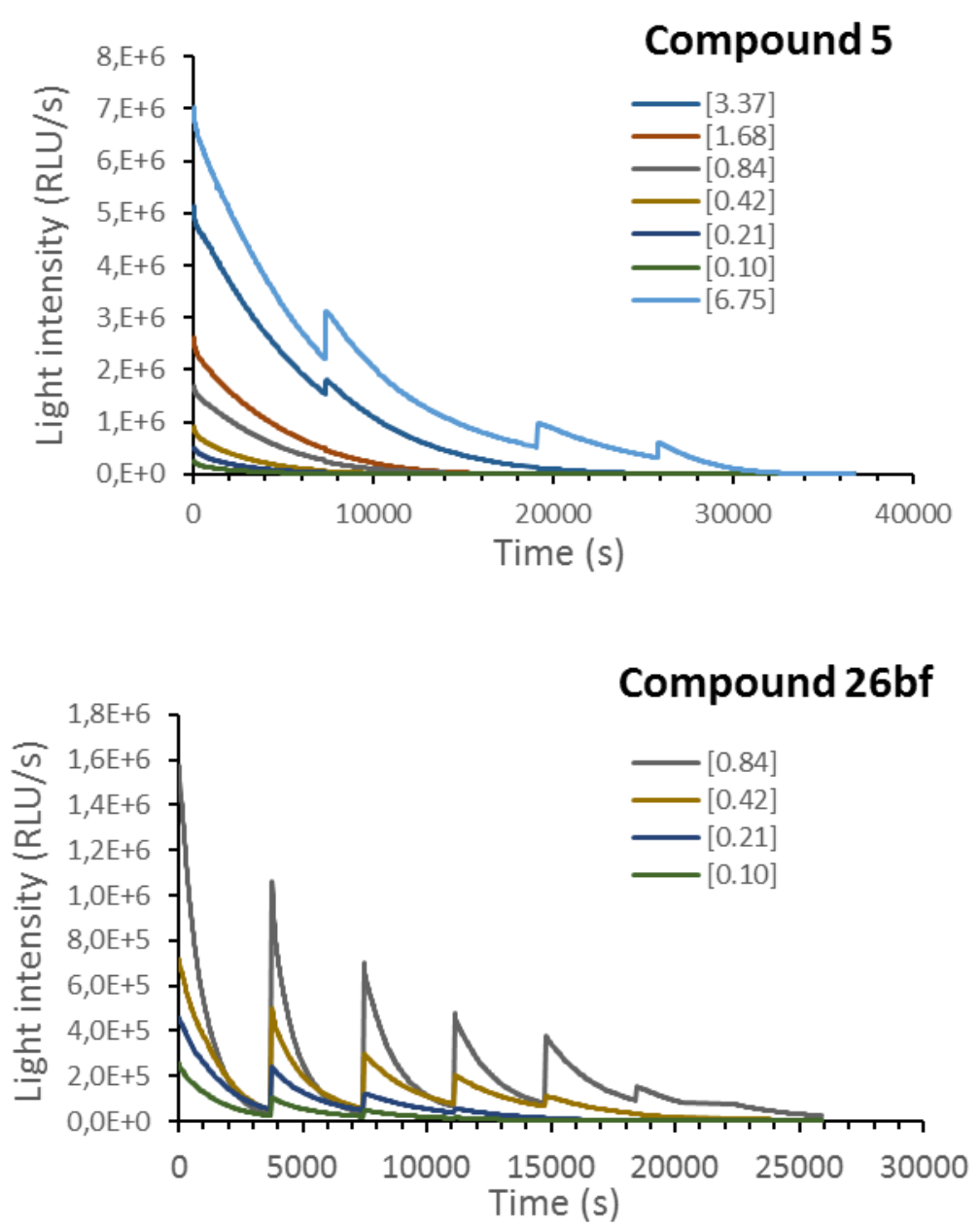

Light emission intensity decreases with time: steeply for the "flashy" compound 26bf and much more slowly for compound 5 a "glow" substrates. To check if the reaction rate indicated by the photon emission (RLU/s) decreases with time, the same enzyme amount $\left(62 \cdot 10^{-18} \mathrm{~mol}\right)$ was added as seen by the intensity increases for different concentrations of substrates (in $\mu \mathrm{M}$ ) as indicated in the legend between brackets. 
The wavelengths at maximum emission $\left(\lambda_{\max }\right)$ were determined using a JASCO FP-6300 spectrofluorometer, the following pictures is depicting the superposition of the 18 compounds listed in table 8, their intensities were normalized to compare the wavelength max as well as the spread.

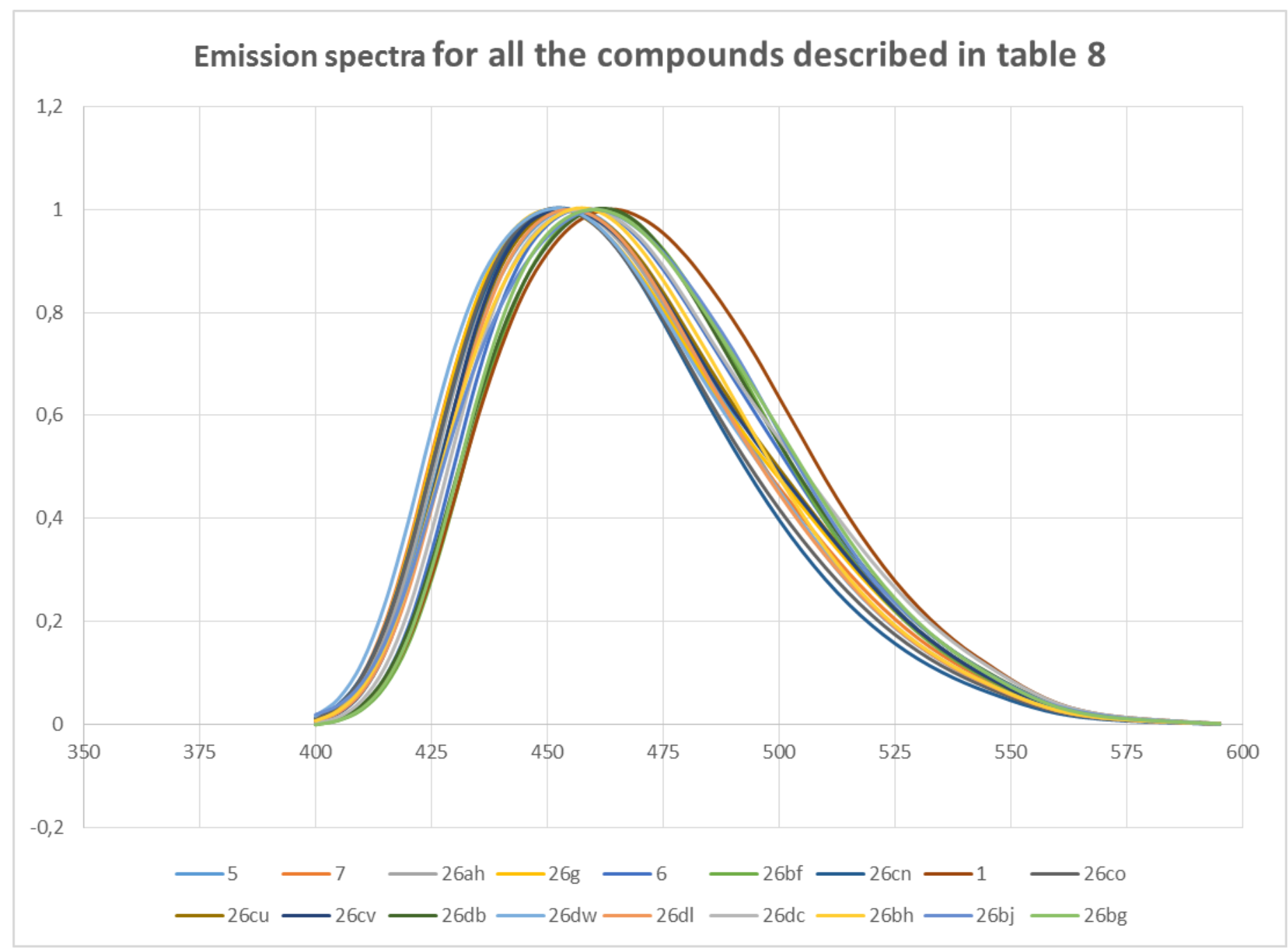




\section{References}

[1] H. Chen, X. Han, N. Quin, L. Wei, Y. Yang, L. Rao, B. Chi, L. Feng, Y. Ren and J. Wan, Bioorg. Med. Chem. 2016, 24, 1225-1230.

[2] R. Jacobo, A. Cota, E. Rogel, J. D. Garcia, I. A. Rivero, L. H. Hellberg and R. Somanathan, J. Fluorine Chem. 1994, 67, 253-255.

[3] a) G. Gagnot, V. Hervin, E. P. Coutant, S. Desmons, R. Baatallah, V. Monnot and Y. L. Janin, Beilstein J. Org. Chem. 2018, 14, 2846-2852; b) E. P. Coutant, V. Hervin, G. Gagnot, C. Ford, R. Baatallah and Y. L. Janin, Beilstein J. Org. Chem. 2018, 2853-2859.

[4] E. P. Coutant, S. Goyard, V. Hervin, G. Gagnot, R. Baatallah, T. Rose, Y. Jacob and Y. L. Janin, Org. Biomol. Chem. 2019, 17, 3709-3713.

[5] a) R. G. Jones, J. Am. Chem. Soc. 1949, 71, 78-81; b) N. Schwartz and R. J. Mohrbacher, (McNeil Laboratories Inc.), US 4046763, 1977

[6] G. Karmas and P. E. Spoerri, J. Am. Chem. Soc. 1952, 74, 1580-1584. 